\title{
Intracellular Delivery by Membrane Disruption: Mechanisms, Strategies, and Concepts
}

\author{
Martin P. Stewart*1,2, Robert Langer*1,2, Klavs F. Jensen*1 \\ *martstew@mit.edu \\ *rlanger@mit.edu \\ *kfjensen@mit.edu \\ ${ }^{1}$ Department of Chemical Engineering, Massachusetts Institute of Technology, Cambridge, Massachusetts 02139, USA. \\ ${ }^{2}$ The Koch Institute for Integrative Cancer Research, Massachusetts Institute of Technology, Cambridge, Massachusetts \\ 02139, USA.
}

\begin{abstract}
:
Intracellular delivery is a key step in biological research and has enabled decades of biomedical discoveries. It is also becoming increasingly important in industrial and medical applications ranging from biomanufacture to cell-based therapies. Here, we review techniques for membrane disruption-based intracellular delivery from 1911 until the present. These methods achieve rapid, direct, and universal delivery of almost any cargo molecule or material that can be dispersed in solution. We start by covering the motivations for intracellular delivery and the challenges associated with the different cargo types - nucleic acids, proteins/peptides, small molecules, synthetic nanomaterials, and large cargo. The review then presents a broad comparison of delivery strategies followed by an analysis of membrane disruption mechanisms and the biology of the cell response. We cover mechanical, electrical, thermal, optical, and chemical strategies of membrane disruption with a particular emphasis on their applications and challenges to implementation. Throughout, we highlight specific mechanisms of membrane disruption and suggest areas in need of further experimentation. We hope the concepts discussed in our review inspire scientists and engineers with further ideas to improve intracellular delivery.
\end{abstract}




\section{Table of Contents}

1. Introduction

2. Intracellular Delivery Cargo \& Applications

2.1. Overview of Key Applications

2.1.1 Intracellular Delivery is Moving Beyond Traditional Transfection

2.1.2 Intracellular Delivery for Cell-Based Therapies

2.1.3 Intracellular Delivery in Stem Cell Reprogramming

2.2. Cargo Categories for Intracellular Delivery

2.2.1 Small Molecules

2.2.2 Proteins \& Peptides

2.2.3 Nucleic Acid Transfection

2.2.4 Synthetic Nanomaterials \& Devices

2.2.5 Large Cargo

3. Approaches for Intracellular Delivery

\subsection{Carrier-Mediated}

3.2 Membrane Disruption-Mediated

3.2.1 Direct Penetration

3.2.2 Permeabilization

4. Membrane Disruption-Mediated Delivery: Background Concepts

4.1 Cell Structure \& Properties

4.2 Defect Formation in Lipid Membranes

4.3 Cell Response to Membrane Disruption

5. Intracellular Delivery by Direct Penetration

5.1 Microinjection

5.2 Penetrating Projectiles (Biolistics)

5.3 Nanowires \& Nanostraws

6. Intracellular Delivery by Permeabilization

6.1. Mechanical Membrane Disruption

6.1.1 Mechanical: Solid Contact

6.1.2 Mechanical: Fluid Shear

6.1.3 Mechanical: Pressure Changes

6.2. Electrical Membrane Disruption (Electroporation)

6.2.1 Mechanisms of Membrane Disruption \& Cargo Entry

6.2.2 Electroporation Challenges \& Technical Advancements

6.2.3 In Vitro \& Ex Vivo Applications of Electroporation

6.3. Thermal Membrane Disruption

6.4. Optical Membrane Disruption (Optoporation)

6.5. Biochemical Membrane Disruption

6.5.1 Organic Solvents \& Penetration Enhancers

6.5.2 Detergents

6.5.3 Membrane-Active Peptides

6.5.4 Pore-Forming Proteins \& Toxins

6.5.5 Chemical Destabilization

7. Gated Channels \& Valves

8. Summary \& Outlook 


\section{Introduction}

Cells transmit information through molecules. Just as computer chips process information using electronic signals, the currency of information exchange in cells is molecules. DNA encodes RNA and proteins. Proteins perform work, transmit signals, and act as building blocks of cellular structure. Lipids form membranes and store energy. The cell is infinitely more complex than an electronic device - we are still learning how it works. In addition to the natural molecules that comprise cells, new technologies are enabling synthetic materials to be deployed within cells. Introducing molecules and materials into cells is an important step in decoding cell function, guiding cell fate, and reprogramming cell behaviour. Thus, intracellular delivery is central to our ability to understand biology and treat disease.

This review is intended for anyone interested in intracellular delivery: the biologist looking for the most appropriate method for their project, the chemist investigating a novel molecule that requires verification in live cells, the engineer searching to develop innovative new intracellular delivery technology, the cell physiologist seeking a deeper understanding of the mechanisms underlying membrane disruption-based deliver; or the biomanufacturing expert examining ways to improve production efficiency. This review seeks to deconstruct the literature into a clear and understandable framework. More than 1500 papers are referenced but we've examined almost 4000 in the process of compiling this paper.

The scope of this review is focused on membrane disruption-based intracellular delivery, as opposed to carrier-mediated methods. There are many more reviews on carriers (also known as vectors, vehicles, nanocarriers, and delivery nanoparticles), particularly for nucleic acid delivery ${ }^{1-9}$, including in this journal ${ }^{10-15}$. Comparatively fewer reviews exist on membrane disruption-based delivery, possibly due to the diverse array of approaches for creating holes in membranes. Part of the scope of the review also covers the different cargo types that researchers seek to deliver to the intracellular space. This analysis helps to frame why and how such diverse methods of intracellular delivery have come about, and the reasons behind membrane disruption-based delivery becoming a key approach.

In this review we cover literature from 1911 to the present. However, the field of membrane disruption-mediated intracellular delivery was small until the mid 1980's, which coincided with the rise of electroporation along with other 
means of cell permeabilization. We have narrowed the discussion of membrane disruption-mediated delivery primarily to cells in vitro, as opposed to in vivo scenarios. The review will focus mostly on cells of animal and human origin, although we will sometimes venture beyond this scope to highlight particular examples in bacteria, microorganisms, and plants.

To begin the review, we will first cover the types of cargo that researchers seek to deliver and their applications. This includes an overview of cargo chemical and dimensional properties, as these characteristics are inextricably linked to the challenges involved in their delivery. We then survey all methods of cargo delivery, defining what is membrane disruption-mediated and what is not. Specifically, we break this into two areas: 1) carrier-mediated delivery, which comprises endocytic and fusion entry pathways, and 2) membrane disruption-based intracellular delivery, which includes direct penetration and plasma membrane permeabilization mechanisms. Next, we provide relevant background on cell membranes, their function, and mechanisms of disruption and cell recovery. We then explore each membrane disruption technique in depth, highlighting its history, the mechanisms by which it operates, pros and cons, and where appropriate, a perspective on opportunities and potential feasibility.

\section{Intracellular Delivery Cargo \& Applications}

\subsection{Overview of Key Applications}

For decades researchers have been developing, synthesizing, and adapting molecules and materials for deployment to the intracellular environment. Most of these "cargo" are membrane impermeable and thus require intracellular delivery. In this section, we provide an overview of the key applications of intracellular delivery and the categories of cargo that researchers seek to deliver along with related challenges.

\subsubsection{Intracellular Delivery is Moving Beyond Traditional Transfection}

Transfection refers to the intracellular delivery of nucleic acids: DNA and RNA. Historically, nucleic acids have been the most popular cargo for intracellular delivery experiments performed at a population scale. Genetic modulation with DNA or RNA is viewed as a robust route for controlling cell function. Increasingly, however, researchers are discovering new ways to manipulate cells with other forms of cargo, for example, genome-editing nucleases ${ }^{15-18}$, synthetic intracellular 
probes $^{19,20}$, and combinations of proteins and/or inhibitors that guide cell fate ${ }^{21,22}$. This reflects a transition from the narrowly focused delivery of nucleic acids to a wider concept of "intracellular delivery". To illustrate this, figure 1 depicts the diversity of cargo that can be delivered into cells and the potential outcomes. The schematic highlights the progression from input cargo to cellular output states and end-point applications. In all these cases, the prime challenge is that impermeable cargo must be introduced to the cell interior without untoward damage or perturbation to the cell. The five horizontal tiers in figure 1 are not mutually exclusive, having significant overlap between inputs and output. This "menu" of options reflects the combinatorial potential of intracellular delivery to analyze cell behaviour and engineer cell function. 

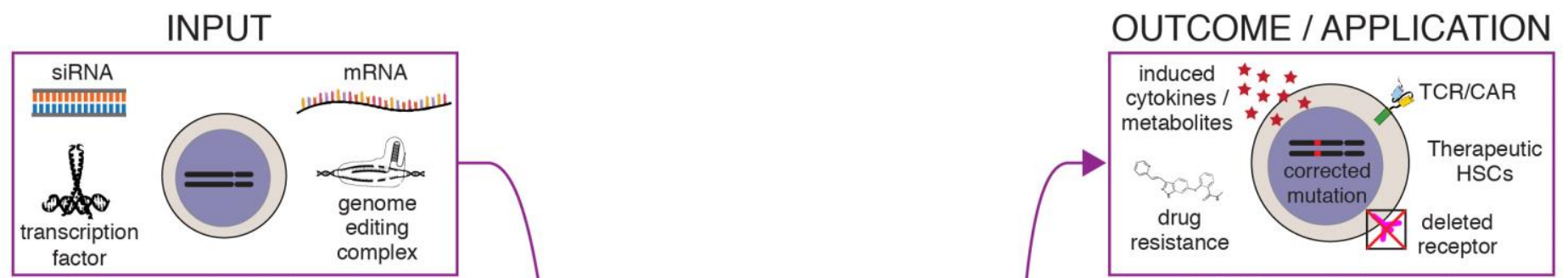

Therapeutic Molecules

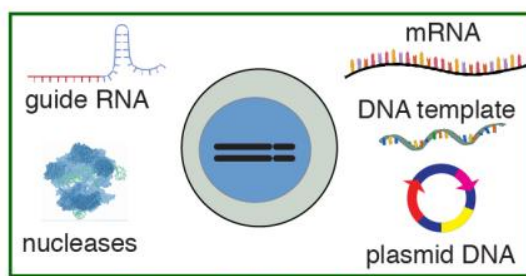

Gene Editing Tools

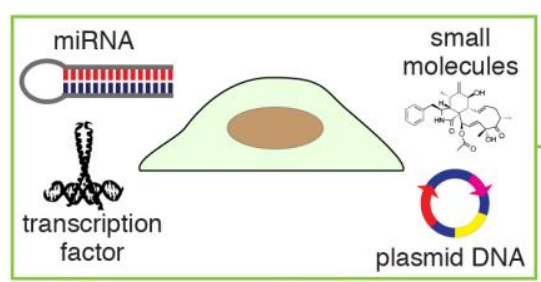

Reprogramming Factors

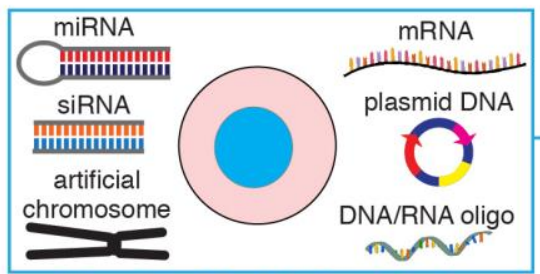

Genetic Modulation Tools

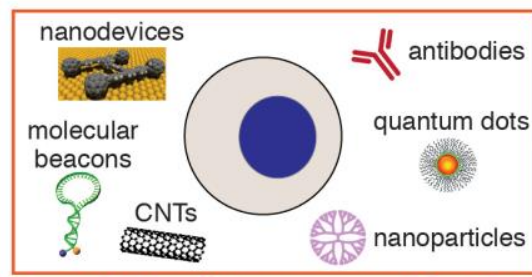

Intracellular Sensors and Devices

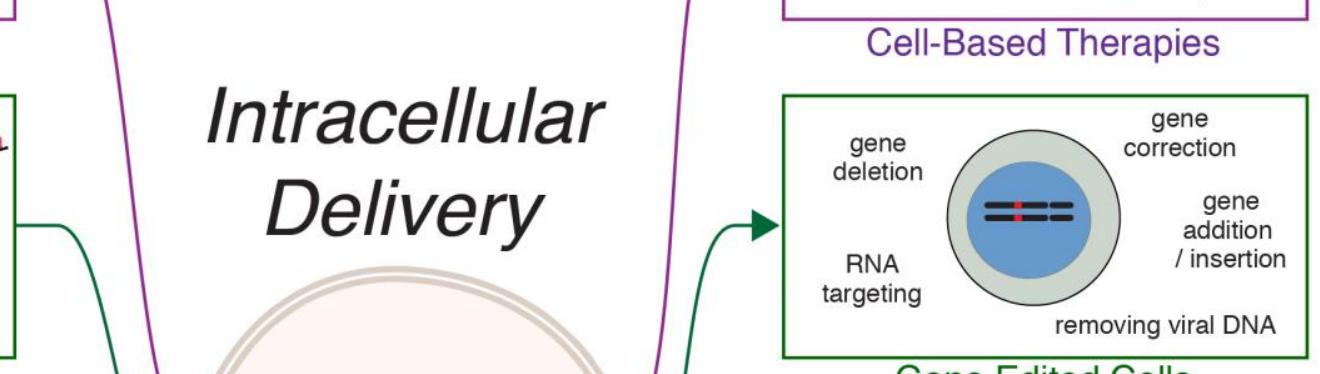

Gene Edited Cells

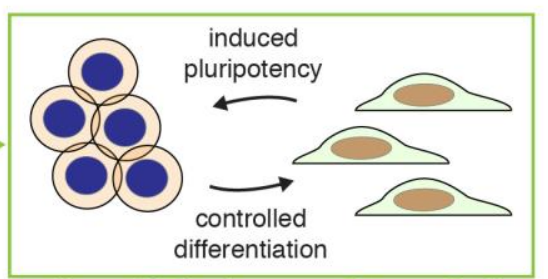

Induced Pluripotent Stem Cells
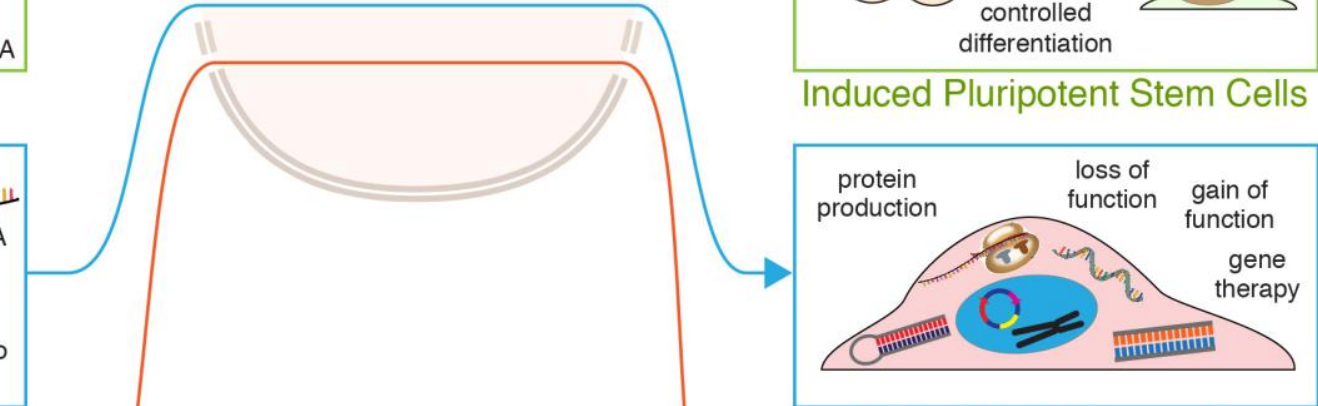

Control Gene Expression

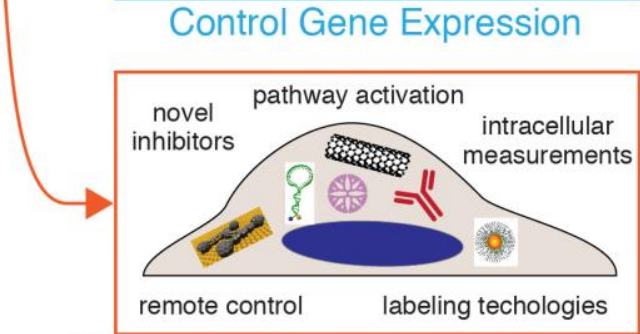

Probing the Intracellular Environment

Figure 1. Example motivations for intracellular delivery. Combinations of cells and cargo molecules / materials are shown on the left. Through intracellular delivery these cargoes are able to confer the outcome or application depicted on the right. The horizontal tiers are not mutually exclusive and substantial overlap exists between them. Abbreviations: $T C R=T$ cell receptor. $C A R=$ chimeric antigen receptor. CNT = carbon nanotube. $\mathrm{HSCs}=$ hematopoietic stem cells.

\subsubsection{Intracellular Delivery for Cell-Based Therapies}

In cell-based therapies, cells can be viewed as a living drug to be administered to the patient. Cells that have been modified, repaired or reprogrammed are introduced into a patient to confer a therapeutic effect or restore lost function. For example, when endogenous immune cells lose their ability to eliminate cancer cells, modified $\mathrm{T}$ cells can be introduced to 
compensate $^{23}$. In the case of CAR-T cells, novel function is conferred through induced expression of specific $\mathrm{T}$ cell receptors (TCRs) or chimeric antigen receptors (CARs) that guide the $\mathrm{T}$ cells to bind to, and attack, specific cancer cells $^{24,25}$. Recent clinical trials against B cell malignancies validate the power of this approach ${ }^{26}$, which was approved in 2017 by the United States Food and Drug Administration (FDA) ${ }^{27}$.

Currently, most cell-based therapies are carried out through ex vivo manipulation, where cells are extracted from the patient are manipulated in vitro, and then reintroduced to the body to produce a therapeutic effect ${ }^{28}$. Intracellular delivery critical to the in vitro manipulation step. Ex vivo cell-based therapies have demonstrated efficacy in treating several human diseases in clinical trials ${ }^{28,29}$. Examples include hematopoietic stem cell (HSC) transplantation ${ }^{30}$ and engineering of immune cells for cancer immunotherapy ${ }^{23,25,31,32}$, as mentioned above. Disease-causing mutant HSCs can be genetically corrected with ex vivo gene therapy, whereby stable genomic modifications are used to confer a durable therapeutic effect $^{28}$. Recent successes include viral vector-mediated gene therapy for correction of monogenic diseases such as severe combined immunodeficiency (SCID-X1), Wiskott-Aldrich syndrome (WAS), and $\beta$-thalassemia ${ }^{29}$. The future delivery of genome editing components for precise gene correction is anticipated to improve the safety and efficiency of HSC gene therapy above what is currently attained with viral vectors ${ }^{16,33,34}$.

\subsubsection{Intracellular Delivery in Stem Cell Reprogramming}

In 2006 it was shown that expressing a combination of transcription factors can induce a state of pluripotency in somatic cells, now known as induced pluripotent stem cells or iPSCs ${ }^{35}$. Early results were achieved with expression from potentially mutagenic viral vectors, an approach that is considered problematic for medical applications. To address this concern, iPSCs have since been produced via direct intracellular delivery of proteins ${ }^{36}, \mathrm{mRNA}^{37,38}$, and microRNA ${ }^{39}$ together with small molecules ${ }^{40}$. Medical applications of iPSCs include in vitro expansion for drug screening of patient

cells and gene therapy before re-implantation ${ }^{41}$. Reprogrammed iPSCs also offer potential for cell-based regenerative medicine $^{42}$, for example to generate immune-compatible organs for patient transplants ${ }^{43}$, off-the-shelf $\mathrm{T}$ cells for cancer immunotherapy ${ }^{44}$, or gene-edited endothelial cells to correct hemophilia ${ }^{45}$.

\subsection{Cargo Categories for Intracellular Delivery}


Cargoes of interest for intracellular delivery are highly variable in size, shape, architecture and chemical properties (Figure 2). They range from small hydrophilic molecules around $1 \mathrm{~nm}$, such as the cryoprotectant trehalose, to large micron-sized organelles and microorganisms approaching the size of the cell itself. This scale represents more than 3 orders of magnitude. It also encompasses a diversity of origins, from typical biomolecules like proteins, DNA, and RNA, to synthetic materials such as carbon nanotubes (CNTs), quantum dots, nanoparticles, and microdevices. In the following, we categorize these cargoes for discussion of their properties, delivery challenges, and intracellular applications. This analysis sheds light on how such diverse methods of intracellular delivery came about, and the factors underpinning the emergence of membrane disruption-based delivery as a key approach. 

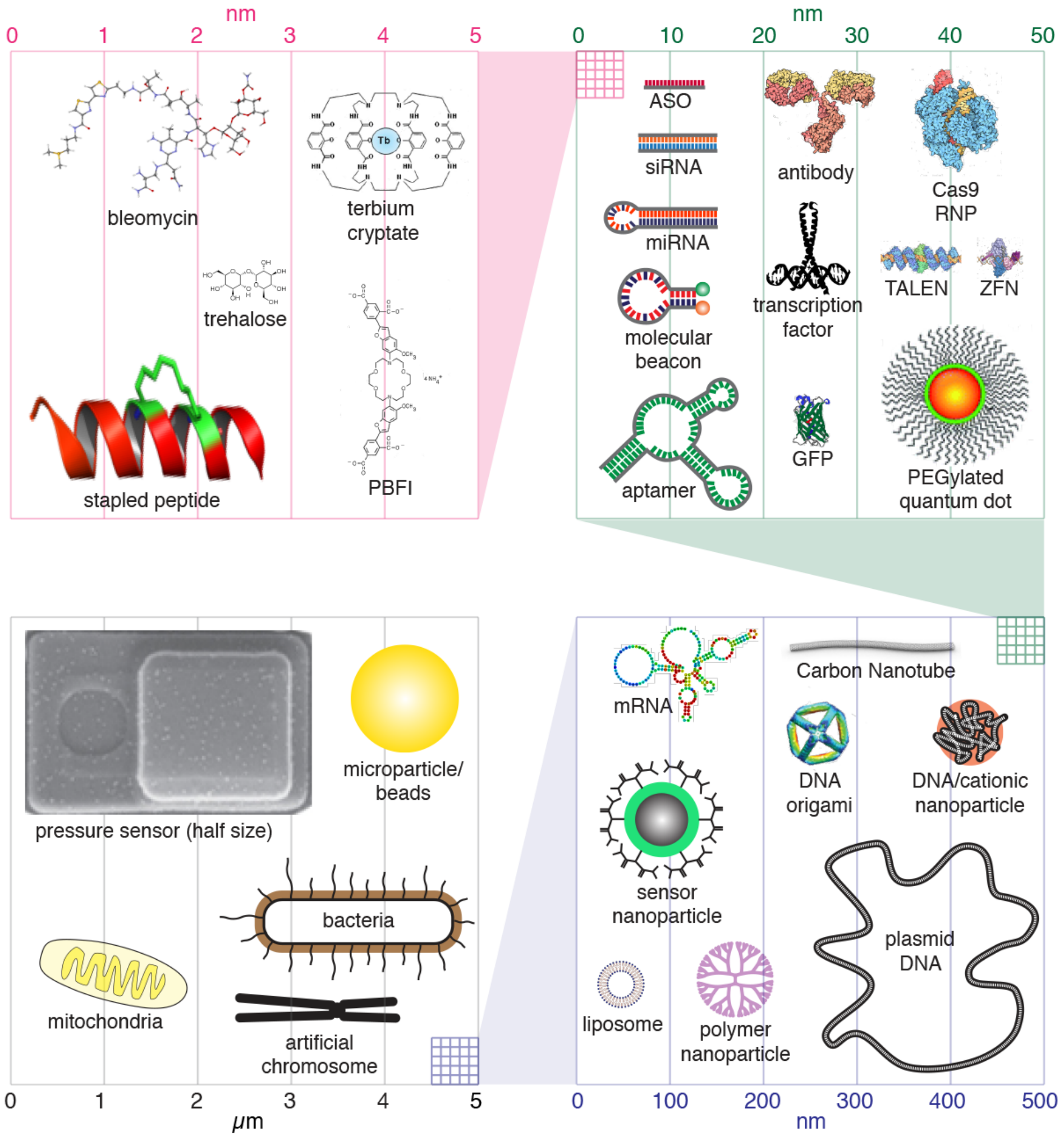

Figure 2. Size scale of cargoes of interest for intracellular delivery. The top left quadrant represents $5 \mathrm{~nm}$ in scale. The top right quadrant represents $50 \mathrm{~nm}$ in scale, including a pink box showing the size of the $5 \mathrm{~nm}$ quadrant. The bottom right quadrant represents $500 \mathrm{~nm}$ in scale, including a green box showing the size of the $50 \mathrm{~nm}$ quadrant. The bottom left quadrant represents 5 $\mu \mathrm{m}$ in scale, including a blue box showing the size of the $500 \mathrm{~nm}$ quadrant. The properties of each of the cargoes and their applications are discussed throughout section 2. PBFI is a potassium indicating dye. ASO means antisense oligonucleotide. siRNA is small interfering RNA. miRNA is micro RNA. GFP stands for green fluorescent protein. RNP stands for ribonucleoprotein. TALEN means Transcription activator-like effector nuclease. ZFN means zinc finger nuclease. The pressure sensor is actually $6 \mu \mathrm{m}$ long but is 
scaled to half size for presentation purposes. Several images are reprinted with permission from Ref ${ }^{46}$, Copyright 2012 Frontiers; Ref

47, Copyright 2012 RSC, Ref ${ }^{48}$, Copyright 2015 Springer Nature, Ref ${ }^{49}$, Copyright 2013 Springer Nature.

\subsubsection{Small Molecules}

\subsubsection{Small Molecule Drugs}

Small molecule drugs are organic compounds of 900 Da or less, a molecular weight which corresponds to a physical size of 1 nanometer or less (Table 1). The first small molecule drugs were natural products isolated from plants, microbes, marine invertebrates, or other lifeforms. An early example is morphine, a metabolite purified from opium extract in 1815 and dispensed by Merck as pain relieving medicine from $1827^{50}$. Today thousands of small molecule drugs are used as medicines. Advances in chemistry have enabled the purification of countless natural products, production of derivatives and mimics of them, or production of completely synthetic compounds ${ }^{50}$.

If a drug target is intracellular, one of three scenarios makes it feasible 1) passive diffusion across the membrane, 2) active transport via membrane proteins, or 3) intracellular delivery. Small molecules that exhibit passive membrane permeability usually align with Lipinski's classic "rule of 5 "51. Such molecules should ideally be less than 500 Da, of intermediate lipophilicity, of limited hydrogen bonding capacity, and uncharged. These requirements have been used to narrow drug discovery efforts to candidates that are likely to be bioavailable. This is especially important for synthetic molecules, which lack endogenous transport processes. On the other hand, a number of natural products undergo active transport, and in these cases do not need to be permeable or obey Lipinski's rule of $5^{52}$. Oxidized ascorbate, for example, is membrane impermeable due to its hydrophilic nature but readily undergoes transport into cells through GLUT1, a glucose transporter that is overexpressed in many cancer cells ${ }^{53}$.

In instances where small molecules are neither permeable nor actively transported, intracellular delivery is required. One of the simplest strategies is to administer the molecule alongside a solvent such as ethanol or DMSO. Not only do these solvents improve the solubility of the small molecule, but they also increase the incidence of nanoscale membrane defects that assist the passage of small molecules across membranes ${ }^{54}$. Alternatively, several small molecule anti-cancer drugs have been encapsulated in nanocarriers such as liposomes to improve their intracellular delivery ${ }^{1}$. Intracellular delivery enables the deployment of drugs that are larger than $500 \mathrm{Da}$. An example is bleomycin $\left(\mathrm{M}_{\mathrm{w}} 1.4 \mathrm{kDa}, \sim 2 \mathrm{~nm}\right.$ diameter), an 
anti-cancer drug with poor permeability due to its positive charge and hydrophilicity. By using the intracellular delivery with electroporation, bleomycin potency can be increased more than a hundred fold ${ }^{55,56}$. This strategy has been demonstrated both in vitro and in vivo ${ }^{56}$.

\subsubsection{Small Molecule Probes}

In addition to drugs, another category where small molecules are useful is as intracellular probes ${ }^{57}$. Probe molecules are capable of optically reporting membrane potential ${ }^{58,59}, \mathrm{pH}^{60,61}$, and intracellular concentrations of $\mathrm{K}^{+62-65}, \mathrm{Na}^{+64}$, or $^{\mathrm{Ca}^{2+66,67}}$ by changing their fluorescent properties according to concentration or other stimuli. Most of these probes require intracellular delivery. One example is PBFI $(\sim 1 \mathrm{kDa})$, a fluorescent dye that can be employed for the measurement of intracellular potassium concentration, however, it is naturally cell impermeable ${ }^{63,64}$. The native form of PBFI can be loaded into cells via intracellular delivery methods such as osmotic lysis of pinosomes, microinjection, or electroporation. Alternatively, it can be acetoxymethyl-esterified (AM-esterified) to neutralize the carboxyl groups, as described by Roger Tsien in the early $1980 \mathrm{~s}^{68}$. This process shields the charge of the dye molecule, making it cell-permeable. Once the molecules are inside cells, the acetoxymethyl ester is hydrolyzed by esterases (intracellular enzymes) and the dye molecule returns to the natural, impermeable state ${ }^{63}$. This approach has become a standard practice for loading cells with PBFI to monitor intracellular potassium concentrations. Other probe molecules, such as the calcium-sensitive dyes fura-2, fluo-4 and indo-1, can also be acetoxymethyl-esterified for intracellular delivery and accumulation. Recently, the strategy of acetoxymethyl-esterification has also been used for modification and delivery of small signaling molecules, such as inositol trisphosphate ${ }^{69}$. Another example of small molecule probes requiring intracellular delivery are terbium cryptates $(\sim 1 \mathrm{~nm})^{70}$. Researchers have delivered these to the cytosol by osmotic lysis of pinosomes or transient permeabilization with pore-forming toxins ${ }^{71,72}$. Upon loading, the terbium-based probe TMP-Lumi4 enables luminescence resonance energy transfer (LRET) for imaging of specific protein-protein interactions in live cells ${ }^{72}$.

\section{Cryoprotectants}

Cryoprotectants are chemicals used to protect biological cells and tissues from freezing damage incurred by ice crystal formation. Membrane permeable cryoprotective agents include DMSO, glycerol, and ethylene glycol, and are typically non-toxic, low molecular weight molecules that can penetrate the cell membrane. Unfortunately, these agents are limited 
in their cryoprotective capability. Impermeable sugars may be better cytoprotectants but are highly hydrophilic and do not readily diffuse across cell membranes. For example, trehalose $\left(\mathrm{M}_{\mathrm{w}} 342 \mathrm{Da}\right)$ is a natural disaccharide synthesized by a range of organisms to withstand desiccation or freezing. Studies have shown that intracellular loading of trehalose into animal cells at concentrations up to $0.2 \mathrm{M}$ can provide superior cryoprotection to animal cells when compared to alternative methods ${ }^{73,74}$. Techniques for intracellular delivery of trehalose include influx during thermal shock ${ }^{75}$, stimuliresponsive nanocarriers ${ }^{76}$, engineered pores $^{77}$, and electroporation ${ }^{78,79}$.

Table 1. Characteristics of common cargo molecules of interest for intracellular delivery. The cargoes are ordered down the table in approximate size order. RNP = ribonucleoprotein. $\mathrm{ASO}$ = antisense oligonucleotide.

\begin{tabular}{|l|l|l|l|l|}
\hline Cargo & Typical size (units) & Approx. Mass (Da) & $\begin{array}{l}\text { Dimensions in solution } \\
(\mathbf{n m})\end{array}$ & Charge at neutral pH \\
\hline Small molecules & N/A & $<900 \mathrm{Da}$ & $<1 \mathrm{~nm}$ & $\begin{array}{l}\text { Variable. Often neutral } \\
\text { to promote permeability }\end{array}$ \\
\hline Peptides & $<40$ amino acids & $\sim 110$ Da per amino acid & $\sim 0.2-3 \mathrm{~nm}$ & $\begin{array}{l}\text { Varies according to } \\
\text { amino acid composition }\end{array}$ \\
\hline Proteins & $\begin{array}{l}20 \text { to } 1000 \text { 's of amino } \\
\text { acids }\end{array}$ & $\sim 110$ Da per amino acid & $\sim 2-25 \mathrm{~nm}$ & $\begin{array}{l}\text { Varies according to } \\
\text { amino acid composition }\end{array}$ \\
\hline Cas9 RNP & $\begin{array}{l}\sim 1400 \text { amino acids, } \sim 100 \\
\text { base RNA }\end{array}$ & $\begin{array}{l}\sim 188 \mathrm{kDa}(\sim 158 \mathrm{kDa} \\
\text { protein, } \sim 30 \mathrm{kDa} \text { RNA) }\end{array}$ & $\sim 12-15 \mathrm{~nm}$ & $\begin{array}{l}\sim-80(+22 \text { protein, }-100 \\
\text { RNA) }\end{array}$ \\
\hline Nucleic acids & & & $\begin{array}{l}\text { Length of } 4-8 \mathrm{~nm} \text { if } \\
\text { linear }\end{array}$ & -1 per base \\
\hline ASO & $\begin{array}{l}13-25 \text { bases (single } \\
\text { stranded) }\end{array}$ & $4-8 \mathrm{kDa}$ & 2 wide $x 7.5 \mathrm{~nm}$ long & -1 per base \\
\hline siRNA / miRNA & $21-23$ basepair duplex & $13-15 \mathrm{kDa}$ & Tens to hundreds of $\mathrm{nm}$ & -1 per base \\
\hline mRNA & $\begin{array}{l}0.5-10 \text { kilo-bases RNA } \\
\text { (single stranded) }\end{array}$ & $\sim 320$ Da per base & $\begin{array}{l}\text { Hundreds of } \mathrm{nm}- \\
\text { depends on supercoiling }\end{array}$ & -1 per base \\
\hline plasmid DNA & $\begin{array}{l}2-10 \text { kilo-basepairs } \\
\text { DNA (double stranded) }\end{array}$ & $\sim 650$ Da per base pair &
\end{tabular}

\subsubsection{Proteins \& Peptides}

Proteins are polymers of amino acids that self-organize into three-dimensional, tertiary, structures with specific biological functions. Proteins catalyze biochemical reactions, transmit signals, form receptors and transporters in membranes, and provide intracellular and extracellular structural support. Peptides are smaller than proteins, with generally less than 40 amino acids. Depending on the peptide, they may or may not form defined three-dimensional structures.

\subsubsection{Brief History of Intracellular Delivery of Proteins}

Intracellular delivery of purified proteins began in the 1960s, even before the advent of nucleic acid transfection. In proofof-concept demonstrations, amoebae were microinjected with ferritin $(450 \mathrm{kDa})^{80}$ and mouse eggs with bovine albumin 
$(67 \mathrm{kDa})^{81}$. In the $1970 \mathrm{~s}$, more advanced studies used intracellular delivery of proteins conjugated with fluorescent dyes to investigate intracellular processes and structures ${ }^{82-86}$. During this time, further innovation for the intracellular delivery of proteins was also reported using methods that encapsulate proteins within red cell ghosts ${ }^{87-89}$ and liposomes ${ }^{90,91}$. This was followed by methods that induce transient permeabilization of cell membranes including hypotonic shock ${ }^{92,93}$, osmotic lysis of pinosomes ${ }^{94,95}$, Paul McNeil's scrape ${ }^{96}$, bead ${ }^{97}$ and syringe ${ }^{98}$ loading methods, detergent exposure ${ }^{99}$, electroporation ${ }^{100,101}$, and treatment with the pore-forming toxin Streptolysin O (SLO) ${ }^{102,103}$. Since 2000, a new generation of membrane disruption delivery techniques has been developed using microfluidics and nanotechnology ${ }^{19,104-107}$, such as cell squeezing ${ }^{108}$ and nanowires ${ }^{109,110}$.

Reagents for the intracellular delivery of proteins were adapted from reagents that were initially used for the delivery of nucleic acids, including lipid and polymer compounds first used for delivery of nucleic acids in the 1990s (see review ${ }^{111}$ ). Protein delivery mediated by chemical carriers is also referred to as protein transduction, or less often by the misnomers protein transfection or profection ${ }^{111}$. The following categories have been reported: 1) Lipid and polymer compounds analogous to transfection reagents ${ }^{112-114}, 2$ ) Cell penetrating peptides (CPPs), also known as protein transduction domains (PTDs) $)^{115,116}$, 3) Bacterial toxins and viral components ${ }^{117-121}$, and 4) Engineered nanocarriers ${ }^{122-124}$. Lipid and polymer reagents, while successful for some proteins, are not appropriate for all situations. Unlike DNA and RNA, proteins are vastly different in size, charge, and structure. Thus, lipid and polymeric reagents for intracellular delivery have a limited efficacy for use across a range of different proteins ${ }^{111}$. On the other hand, PTDs and CPPs, can be attached to most proteins but they are prone to endocytic entrapment, cell toxicity, and poor efficiency of cytosolic delivery ${ }^{125}$. Despite promise, the history of PTD and CPP research is troubled by disagreement regarding delivery mechanisms ${ }^{116,126,127}$. Intracellular delivery of proteins using bacterial toxins and viral components is similar in many ways to PTDs and CPPs, but with more precise, well-defined mechanisms ${ }^{118,121}$. Use of bacterial toxins and viral components aims to the mimic pathogenic entry processes by targeting a protein of interest to a particular endocytic pathway, and then triggering natural mechanisms of endosome escape. Unfortunately, this strategy has to be tailored to particular cell types, however, and can be excessively labor-intensive or inaccessible for most researchers seeking to perform protein delivery. The final category of engineered nanocarriers has seen a huge rise in interest over the last 15 years. They can be designed as higher ordered structures with multifunctional and stimuli-responsive properties. Such nanocarriers are constructed from, and functionalized with, combinations of biomolecules, lipids, polymers, and inorganic materials. They have yet to be 
translated into commercial products. The intracellular delivery of proteins lacks straightforward universal techniques, and typically requires significant amounts of work for robust effects to be observed. The limited success of the abovementioned approaches to intracellular delivery of proteins is reflected by the fact that a comparative study of available techniques for antibody delivery indicated that electroporation is the leading option ${ }^{125}$.

\subsubsection{Motivations for Intracellular Delivery of Proteins}

Straightforward intracellular delivery of proteins and peptides holds significant, yet currently unrealized, potential for many areas of science and medicine ${ }^{21,22}$. Delivery of proteins into living cells, such as genome-editing nucleases ${ }^{128}$, active inhibitory antibodies ${ }^{125}$, or stimulatory transcription factors ${ }^{36}$, represents a powerful toolset for manipulating and analyzing cell function ${ }^{21,22}$. For example, the localization and visualization of engineered antibodies within living cells and perturbation of their associated cellular processes may allow a more direct study and functional analysis at a level not possible with genetic methods ${ }^{125}$. As well as classical antibodies $(\sim 150 \mathrm{kDa})$, a number of recombinant small antibodybased molecules such as immunoglobulin (Ig) derived Fab $(\sim 50 \mathrm{kD})$ and $\mathrm{scFv}(\sim 25 \mathrm{kD})$, non-Ig derived monobodies $(\sim 10 \mathrm{kD})$, nanobodies $(\sim 14 \mathrm{kDa})$, and affibodies $(\sim 6.5 \mathrm{kD})$ have been developed ${ }^{129}$. When combined with fluorescent labels these antibodies are able to serve as precise functional probes for intracellular imaging applications ${ }^{130}$. Further, there are applications where direct protein delivery is favorable over indirect expression from nucleic acids, for example to avoid the risk of insertional mutagenesis associated with DNA transfection. However, one significant challenge is that the amount of protein delivered has to be sufficient to generate the desired effect, whereas plasmid DNA can be amplified by replication. Unlike nucleic acids, with their uniform properties, one-size-fits-all protein delivery has been elusive due to the inherent variance in size, structure and charge amongst proteins ${ }^{21,22,131}$.

\subsubsection{Expanding Protein Therapeutics Through Intracellular Delivery}

Since the advent of human recombinant insulin in 1982, the number of protein therapeutics has been growing rapidly ${ }^{132}$. There are now more than 200 FDA-approved protein therapeutics, of which around half are monoclonal antibodies. According to market reports, annual worldwide revenue from protein therapeutics is anticipated to reach USD 200 billion by 2020. Protein therapeutics can be grouped into molecular types that include antibody-based drugs, anticoagulants, blood factors, bone morphogenetic proteins, engineered protein scaffolds, enzymes, Fc fusion proteins, growth factors, 
hormones, interferons, interleukins, and thrombolytics ${ }^{132,133}$. Rather than an intracellular site, these therapeutics exert their action outside the cell, by modulating molecular interactions in the blood, interstitial fluids, or at the cell membrane. Part of the success of protein therapeutics is due to their precision as inhibitors or binding partners. In particular, proteins and peptides can generate surfaces capable of recognizing targets that their small molecule counterparts fail to ${ }^{21}$.

Around two thirds of the human proteome lies inside the cell, inaccessible to binding by impermeable molecules ${ }^{134}$. Because of this, intracellular proteins have a limited potential for therapeutic modulation. While an extensive discussion of intracellular protein delivery in vivo is beyond the scope of this review, protein delivery has been critical to medical developments and scientific understanding when used in ex vivo cell-based therapies. One example is the preparation of anti-tumor vaccines for cancer immunotherapy. Loading mutant tumor proteins into dendritic cells can program an immune response that primes cytotoxic $\mathrm{T}$ cells to attack and kill tumor cells that exhibit those same mutant proteins. This strategy has been verified in animal models ${ }^{135,136}$ and is beginning to be tested for safety and feasibility in clinical trials $^{137,138}$.

\subsubsection{Gene Editing Through Intracellular Delivery of Nucleases \& RNPs}

Gene editing allows precise, targeted changes in the genomic DNA of a cell ${ }^{17}$. Recent advances rely on enzymes known as nucleases, protein machinery that can cut or alter DNA. Key examples include zinc fingers (ZFNs), transcription activator-like effector nucleases (TALENs), meganucleases, and the clustered regularly interspaced short palindromic repeats (CRISPR)/Cas system of RNA-guided nucleases. CRISPR-based gene editing is usually performed with the bacterial nuclease Cas9, which forms a complex, or ribonucleoprotein (RNP), with a single guide RNA (sgRNA) to become targetable and active ${ }^{139}$. Genome editing requires that nucleases enter the nucleus to exert their action on genomic DNA $^{17,140}$. In the case of CRISPR, initial studies in live cells introduced Cas9 indirectly via expression from plasmids or mRNA $^{140,141}$. Subsequent experimentation with delivery of the pre-formed Cas9 RNP indicates this to be a more efficient and straightforward approach ${ }^{142,143}$, particularly when used with therapeutically relevant cells types, such as iPSCs, primary T cells and HSCs ${ }^{144-146}$. 
Since the initial reports in 2014, Cas9 RNPs have been delivered by methods as diverse as electroporation ${ }^{143,144,146,147}$, microinjection $^{148,149}$, lipid nanoparticle formulations ${ }^{150}$, osmotically-induced endocytosis followed by endosome disruption $^{151}$, microfluidic deformation ${ }^{152}$ and CPPs $^{153}$. Typically, sgRNA is about 100 base pairs of single-stranded RNA ( $\sim 30 \mathrm{kDa},-100$ charges) while native Cas9 is $\sim 158 \mathrm{kDa}(\sim 10 \mathrm{~nm}$ diameter $)$ with theoretical net charges of $+22^{150,154,155}$. Thus, the resultant RNP complex has about -80 negative charges, $\sim 188 \mathrm{kDa}$ mass, and is up to $15 \mathrm{~nm}$ in size (Table 1). These properties make electroporation methods particularly successful for RNP delivery, as the negative charge facilitates electrophoretic delivery ${ }^{128}$. Furthermore, the negative charge on Cas9 RNPs makes them electrostatically amenable to complexation with cationic lipid and polymer reagents for carrier-mediated delivery ${ }^{15,150,156}$. Indeed, other types of RNPs have previously been delivered with cationic polymer reagents ${ }^{157}$. RNP delivery strategies are currently a topic of intense research for the purpose of therapeutic genome editing, especially for ex vivo cell-based therapies ${ }^{15,16,18,34}$. Recently, CRISPR-based gene therapy for correction of disease-causing genes was achieved in human embryos ${ }^{158}$. The correction of a common 4 base pair deletion in the MYBPC3 gene known to cause hypertophic cardiomyopathy, was achieved through microinjection of Cas9-sgRNA RNPs and a 200-mer ssODN correction template into zygotes ${ }^{158}$.

\subsubsection{Delivery-Relevant Properties of Proteins \& Peptides}

The molecular weight of most proteins is in the range of $5 \mathrm{kDa}$ up to several hundred $\mathrm{kDa}$. This corresponds to physical dimensions of 2-20 nm, $\sim 10 \mathrm{x}$ smaller than the encoding mRNA. Peptides are smaller than proteins with a typical molecular weight below $5 \mathrm{kDa}$ and physical dimensions less than $3 \mathrm{~nm}$ in size. The molecular weights and dimensions of some common proteins include green fluorescent protein (GFP, $28 \mathrm{kDa}$, a $2 \times 4 \mathrm{~nm}$ barrel), bovine serum albumin (BSA, $67 \mathrm{kDa}$, a 12x4x4nm rod), Cas9 (158 kDa, a globular endonuclease of $>10 \mathrm{~nm}$ diameter), and immunoglobulin antibody $(\sim 150 \mathrm{kDa}, 14 \times 8 \times 4 \mathrm{~nm})^{159}$. Because the structure of proteins is critical to function, any modification for intracellular delivery including chemical modifications or packaging in carrier particles should not compromise protein structure and function. Accordingly, the varied charge, dimensional and structural properties of each protein may be considered as unique and requiring a custom solution for efficacious intracellular delivery ${ }^{160}$. This is particularly important when comparing proteins to nucleic acid, as proteins denature much more easily than nucleic acids (e.g. due to heat, salt concentrations or $\mathrm{pH}$ changes) restricting the treatments that can be used in their formulations. 


\subsubsection{Effect of Charge on Intracellular Delivery of Proteins}

The overall charge of proteins and peptides is dependent on their amino acid composition. Generally, in a solution of neutral $\mathrm{pH}$ arginine and lysine will infer a positive charge, whereas glutamate and aspartate will infer a negative charge. The majority of proteins, such as antibodies, carry a mild negative charge in physiological solutions. However, the charge of peptides can be highly variable. Molecular charge is an important consideration as molecules with a positive charge tend to be more efficient at penetrating negatively charged cell membranes to gain entry to cells. Examples include socalled supercharged proteins ${ }^{161}$, cationic cell-penetrating peptides (CPPs) such as the arginine-rich TAT peptide from $\mathrm{HIV}^{162}$, and cationic lipids and polymers commonly used as transfection agents ${ }^{10}$. Cationic molecules are thought to associate robustly to the cell surface (for example via attachment to anionic proteoglycans) where they induce endocytosis, and/or generate membrane defects ${ }^{116}$. However, this mechanism of cell entry is problematic as strongly charged molecules may have to overcome unfavourable energetic barriers to diffuse through holes in the plasma membrane unless there is an electrophoretic driving force, such as voltage pulses supplied during electroporation ${ }^{163}$.

\subsubsection{Permeability of Peptides}

Unlike nucleic acids and proteins, some peptides possess an intrinsic ability to permeate through cell membranes and into the cell. However, such membrane permeable peptides typically have permeability coefficients substantially below typical small molecule drugs. One example is the 11 amino acid cyclic peptide cyclosporin $\mathrm{A}\left(\mathrm{M}_{\mathrm{w}} \sim 1.2 \mathrm{kDa}\right)$, which is a useful inhibitor of cyclophilin in T cells. Cyclosporin A is a feasible drug for oral delivery due to its relatively high permeability coefficient that is similar to that of small molecules $\left(2.5 \times 10^{-7} \mathrm{~cm} \mathrm{~s}^{-1}\right)$, its low concentration required for intracellular activity $(7-10 \mathrm{nM})^{164}$, and relative chemical stability conferred by its cyclic conformation. Despite the success of cyclosporine A, most inhibitory peptides are limited in their usefulness due to inconsistent or low cell permeability, or sensitivity to degradation by proteases. To this end, researchers in the field have sought to understand the rules governing peptide permeability in the hope of applying this knowledge to design better peptides for intracellular delivery ${ }^{116,165-167}$. Understanding peptide permeability is complicated by observations that suggest many different entry mechanisms are possible. The simplest mechanism is passive diffusion as a result of the foreign molecule partitioning the hydrophobic cores of membranes, such as is believed to be the case for cyclosporin $\mathrm{A}^{168}$. Alternatively, transmembrane transporters have been proposed to shuttle short peptides across the membrane ${ }^{168}$. Other peptides are believed to induce endocytosis 
and subsequent endosomal escape. Most cell-penetrating peptides (CPPs) are thought to enter cells via endocytosis ${ }^{116}$, although other routes such as direct translocation across the membrane, inverted micelle formation, transient pore formation, adaptive translocation, and local electroporation-like effects have been suggested ${ }^{127}$.

Some general characteristics have been found to promote peptide permeability. For example, most CPPs are between 8 and 20 amino acids long and possess somewhere between 5 and 8 positively charged residues (usually arginines) in various configurations that confer a favourable charge for membrane interactions and cell entry ${ }^{116}$. Other strategies involve the use of 'stapled peptides', where a synthetic brace (typically a covalent crosslink between two residues) is added to lock small peptides into an active conformation (most often an alpha-helix) ${ }^{169,170}$. Using stapled peptides, Verdine and colleagues produced a synthetic, cell-permeable, stabilized alpha-helical peptide of 16 amino acids that targets a critical protein-protein interface in the difficult-to-drug NOTCH transactivation complex ${ }^{171}$. Ongoing research efforts are expected to decode the size, conformation, charge, polarity and amphiphilicity that optimize the intracellular delivery of peptides and their cargo.

\subsubsection{Nucleic Acid Transfection}

The word transfection is derived from the terms transformation and infection. It has paradoxically come to refer to nonviral (i.e. non-infectious) methods of nucleic acid delivery. Transfection has mainly been performed with plasmid DNA, mRNA, and oligonucleotides, with more recent use of nucleic acid-based constructs/devices. The analogous term transduction refers to the introduction of nucleic acids to the intracellular space by viruses or viral vectors. Viral mediated delivery of nucleic acids is the gold standard for their intracellular delivery. The use of viruses for transduction leverages the naturally occurring mechanisms that viruses use to enter cells.

\subsubsection{Brief History \& Motivations}

Starting from the 1960s, researchers observed that mixing nucleic acids, which are negatively charged, with cationic molecules leads to the formation of macromolecular complexes that can enter cells and degrade, thereby releasing nucleic acids inside of a cell. Two early examples of transfection complexes are the polymer diethylaminoethyl-dextran/nucleic acid combination (1968) ${ }^{172-174}$ and the insoluble ionic salt calcium phosphate/nuclei acid precipitant $(1973)^{175}$. Today, the 
most widespread regents used for transfection use a complex formed between lipids and nucleic acids. The first reports of lipid-based transfections were as early as the 1980s, first with liposomes (1980) ${ }^{176,177}$ and then via 'lipofection' with cationic lipids(1987) ${ }^{178}$. The most effective methods were commercialized, with the launch of the cationic lipid-based product lipofectamine in 1993. This was shortly followed with dendrimers like PAMAM"180 from 1993 ("superfect" reagent launched in late 1990s) and cationic polymers such as PEI in $1995^{179}$ (marketed as "polyjet" soon after). Cationic polymers such as polybrene ${ }^{181}$ and poly-L-lysine ${ }^{182,183}$ also formed the basis of several transfection technologies. The technique of electroporation was first used for DNA transfection in the early $1980 \mathrm{~s}^{184}$. This technique remains particularly useful for hard-to-transfect cell types and was commercialized from the mid-1980s by Biorad and others. Today, most transfection is performed with lipid reagents, while polymer reagents and electroporation are the next most popular options. The popularity of these techniques over more efficacious virally mediated transduction methods is due to the relative simplicity of transfection procedures, lower cost, and smaller time investment.

By 2020 the transfection market is predicted to be worth one billion USD, with applications in three areas: 1) basic research, 2) biomanufacture, and 3) cell-based therapies (Figure 3). Because genetic material underlies almost all biological function, transfection is central to biological research, in both academic and industrial settings. Transfection impacts fields from cell biology and genetics to immunology and drug discovery. In the context of biomanufacture, transfection is used for bio-production of proteins, antibodies, viral vectors, and virus-like particles for vaccines. In cellbased therapies, transfection has been critical to ex vivo gene therapy (correcting aberrant genes) ${ }^{29}$, hematopoietic stem cell engineering ${ }^{30,185}$, production of induced pluripotent stem cells ${ }^{37}$, and preparation of cells for immunotherapy ${ }^{186-188}$. As shown in figure 3, nucleic acid transfection is currently the dominant category of intracellular delivery. In future, however, demand for delivery of non-nucleic acid materials (for example, antibodies, genome editing nucleases, and synthetic materials) is expected to compete with transfection in several applications ${ }^{15,18,19,21,22,107}$. 


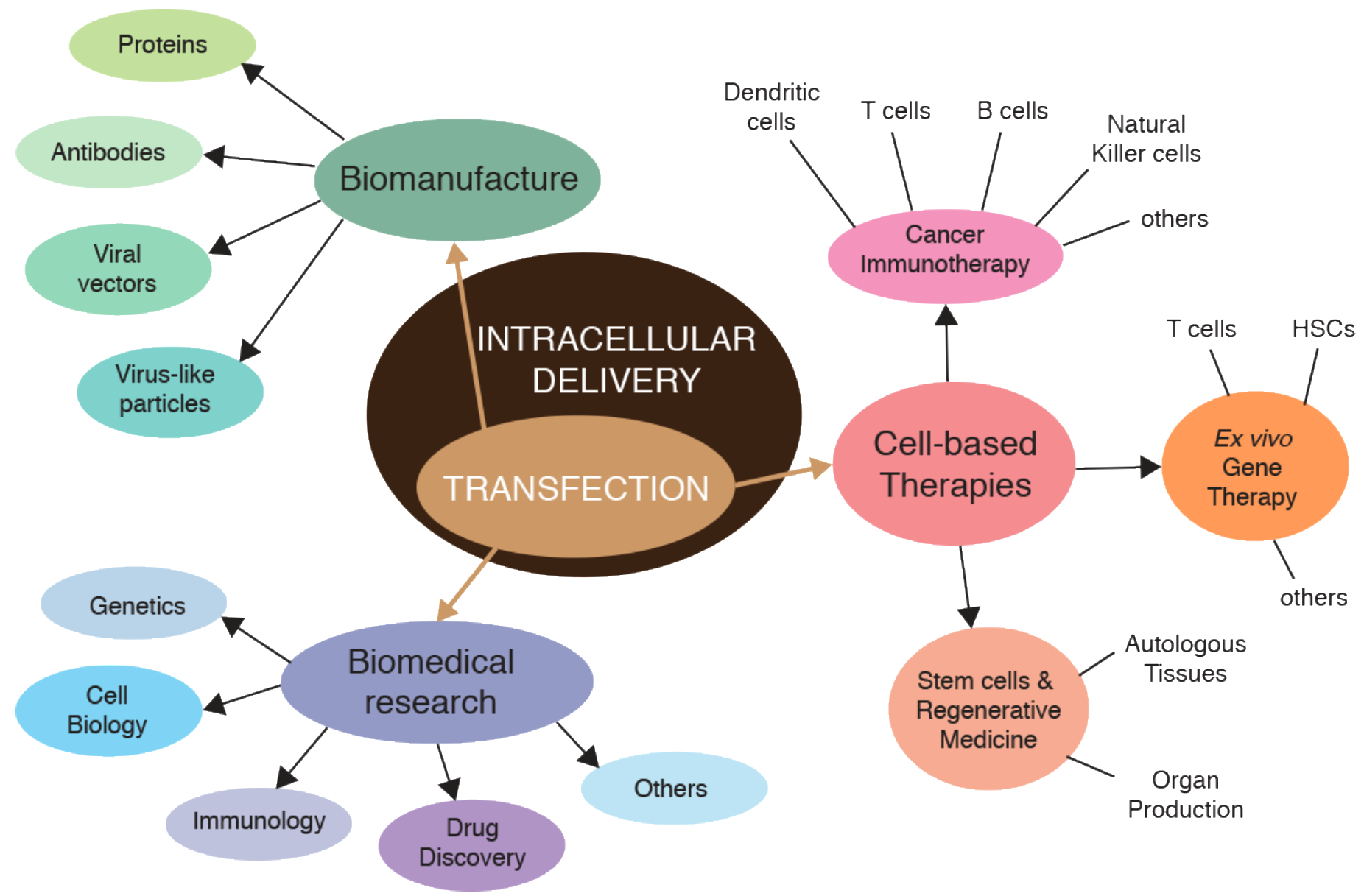

Figure 3. Concept map displaying the main application areas of transfection. In terms of market share and research, medical, or industrial activity, transfection is the largest sub-component of intracellular delivery. HSCs = hematopoietic stem cell.

\subsubsection{DNA Vectors}

A vector is a DNA molecule that acts as a vehicle for the expression or replication of DNA. Some examples of different types of vectors include plasmids, cosmids, viral vectors, and artificial chromosomes. Plasmids are circular doublestranded DNA molecules that were originally discovered in bacteria ${ }^{189}$. Cosmids are similar to plasmids but exhibit the ability to be packaged into a phage capsids ${ }^{190}$. Viral vectors pack a limited amount of DNA within a viral envelope - an efficient configuration that confers self-delivery through viral-mediated cell entry ${ }^{191}$. Artificial chromosomes have a larger DNA capacity than other vectors, containing up to a million base pairs with dimensions in the micron size range. Artificial chromosomes are used in specialized situations where their larger capacity and natural chromosome-like behaviour are advantageous $^{192}$. 
The most commonly used vectors are plasmids, which are usually around 5-10 kilo-base pairs. DNA engineering techniques enable the manipulation of all vectors through recombination, thus allowing specific sequences to be cut and paste into them. Pioneering studies in the 1970s inserted foreign DNA into viral vectors ${ }^{193}$ and plasmids ${ }^{194}$ for subsequent intracellular delivery and gene expression. By decoding the genetic elements of vectors, such as expression promoters and origins of replication, it became possible to introduce and express genes from one organism into another and vice versa ${ }^{195}$. For example, plasmids were exploited to first express eukaryotic genes in bacteria ${ }^{196,197}$, then foreign genes in animal cells, via calcium phosphate transfection ${ }^{198,199}$ or microinjection ${ }^{200-202}$. That plasmids must enter the nucleus to undergo expression was established by microinjection experiments that compared cytoplasmic with nuclear injection ${ }^{202}$.

A 5-10 kilo-basepair plasmid is >100 nanometers in diameter when uncondensed ${ }^{203,204}$ (Table 1). Each nucleotide carries a single negative charge due to repeating phosphate groups along the DNA polymer backbone. Cationic compounds, such as lipids and polymer reagents, condense plasmids into solid nanoparticles with dimensions of tens of nanometers ${ }^{10,205,206}$. Condensation of DNA promotes cellular uptake by reducing the plasmid size and balancing its negative charge. The level of supercoiling also influences the durability and compaction. In general plasmids bearing a smaller footprint are capable of better transfection and expression ${ }^{207,208}$.

\subsubsection{Oligonucleotides}

Oligonucleotides are single- or double-stranded sequences of DNA or RNA, generally less than 30 nucleotides in length. Antisense oligonucleotides (ASOs) were first discovered in 1978, when it was shown that a single-stranded 13-mer of DNA hybridized with complementary mRNA to inhibit its translation ${ }^{209}$. Antisense inhibition occurs because mRNA is either sterically blocked and thereby unavailable for translation or designated for enzymatic degradation. In the 1980s ASOs were established as tools for performing genetic loss of function studies ${ }^{210-212}$. In these cases, ASOs were either expressed from plasmids or microinjected after in vitro transcription. Thereafter, several companies began developing antisense therapeutics, with the first approved medication in 1998 being fomivirsen, a 21 -mer oligonucleotide that blocks the translation of cytomegalovirus mRNA ${ }^{213,214}$. 
The discovery of RNA interference (RNAi) by Fire and Mello in $1998^{215}$ led to the revelation of double-stranded RNA for silencing gene expression. Subsequently, it was shown that RNAi in mammalian cells could be mediated by intracellular delivery of short 21-22 base pair duplexes, termed small interfering RNAs (siRNAs) ${ }^{216}$. Once in the cytoplasm, siRNAs bind to protein machinery known as the RNAi-induced silencing complex (RISC), which binds with matching RNA to enzymatically degrade it. Micro RNAs (miRNAs), discovered in $1993^{217}$, represent the endogenous mechanism of gene silencing. Small 'hairpin miRNA', named as such due to their shape, are processed by enzymes within the cell into smaller pieces similar to siRNAs, which then silence genes through antisense or RNAi mechanisms.

Overall, oligonucleotides may modify cell behaviour through a number of mechanisms ${ }^{218}$. These include: (1) activating toll-like receptors in the endosome (non-specific), (2) siRNAs, (3) miRNA mimics, (4) antagomirs, sterically blocking endogenous miRNA, (5) ASOs such as gapmers, inducing RNase H degradation or sterically blocking RNA, (6) oligonucleotides directed against nuclear regulatory RNA species such as long noncoding RNAs (lncRNAs), (7) splice switching oligonucleotides that perturb mRNA maturation, (8) anti-gene oligonucleotides that bind to genomic DNA, perturbing transcription or binding of other proteins, and (9) aptamers, which to bind, and alter the function of, proteins ${ }^{218}$. Aptamers are distinct from the rest of these oligonucleotides in that they form higher order structures with conformations exhibiting affinities to specific target molecules. With the exception of the first mechanism (activation in endosomes), oligonucleotides must enter the cytoplasm or nucleus to exert their effects.

The chemical and dimensional properties of oligonucleotides affect their function and use for intracellular delivery. Because oligonucleotides are negatively charged polar molecules in the size range of small proteins (Figure 2), their propensity for interaction with negatively charged cell membranes and consequential cellular permeability is poor. siRNA duplexes have approximate dimensions of $7.5 \mathrm{~nm}$ length by $2 \mathrm{~nm}$ diameter $^{219}$ (Table 1). miRNA is only slightly larger than siRNA because it is a single stranded hairpin shape with an extraneous loop. An ASO of 16 bases is about $5 \mathrm{~nm}$ long by $1 \mathrm{~nm}$ wide. In addition to their large size and relative negative charge, challenges associated with oligonucleotide delivery include susceptibility to enzymatic degradation and binding to undesirable targets ${ }^{220}$. One approach to improve unwanted oligonucleotide degradation and chemical cleavage is chemical modification ${ }^{9,221}$. Other methods to improve intracellular delivery of oligonucleotides are to neutralize the negatively charged portions of the polymer backbone conferring more favourable interactions between the oligonucleotides and cell membranes. Oligonucleotides can be 
neutralized by replacement of natural bases with morpholinos ${ }^{222}$, peptide nucleic acids (PNAs) $)^{223}$, or by the addition of specific functional groups ${ }^{224}$.

Of the different approaches for intracellular delivery of oligonucleotides, lipid reagents have been the most prevalent ${ }^{229-}$

${ }^{231}$. However, delivery strategies can also include combinations of those the approaches listed above, including chemical modification of the oligonucleotide itself, use of lipid or polymeric nanocarriers, and linking oligonucleotides to cell targeting agents such as carbohydrates, peptides or aptamers 220,225 . In these approaches, it is thought that the biological effects of oligonucleotides are due to the small amount of oligonucleotides that escape from endosomes and reach key cytosolic or nuclear compartments ${ }^{226-228}$. In cells that are recalcitrant to such reagents, success has been obtained with electroporation ${ }^{232-237}$ and pore-forming agents ${ }^{238,239}$.

\subsubsection{Intracellular Delivery of Messenger RNA}

One alternative to the delivery of DNA vectors is the use of messenger RNA or mRNA. This alternative is particularly attractive for therapeutic applications (discussed below). Pioneering studies for the intracellular delivery of mRNA expression were conducted in the 1970 s via microinjection methods ${ }^{240-242}$. Following that, mRNA was transfected into mammalian cells using the cationic polymer DEAE-dextran ${ }^{243,244}$ and with cationic lipid complexes ${ }^{245,246}$, the latter of which became the standard approach ${ }^{247}$. Transfection of mRNA via electroporation has also been demonstrated as an effective option in a number of cell types ${ }^{248,249}$.

Protein expression from the intracellular delivery of mRNA has a number of advantages when compared to that of DNA vectors $^{250,251}$. First, there is no risk of adverse genomic integration that can occur after the intracellular delivery of DNA. Second, mRNA expression is based upon interaction with ribosomes located in the cytoplasm, and thus does not require delivery across the nuclear envelope as DNA does. Third, protein expression resulting from mRNA delivery is dosedependent and rapid, commencing within minutes. Fourth, additional control over the location of protein expression can be conferred by using mRNA, as its specific subcellular delivery can localise protein expression ${ }^{252}$. Fifth, mRNA can be less toxic and less immunogenic than DNA vectors in sensitive cells, making it a preferred option for sensitive cells or applications that require cell high viability. These beneficial aspects of mRNA delivery make it attractive for therapeutic 
applications $^{253-255}$. One such therapeutic application has used intracellular delivery of mRNA for expression of antigens in dendritic cells and T cells ex vivo; a promising immunotherapy for treatment of cancer ${ }^{188,256-259}$. Electroporation of mRNA has become a preferred option for delivery of mRNA in therapeutic cell types that are difficult to transfect with cationic lipids, such as dendritic cells ${ }^{260-262}$.

Similar to DNA, mRNA is a large negatively charged polymer that can be condensed into cationic nanoparticles to promote uptake ${ }^{247,255}$. Messenger RNA is a single stranded nucleic acid that usually forms secondary structures featuring various loops and hairpins (Figure 2). The dimensions of mRNA are normally $\sim 10$ times larger than the protein it encodes, putting it in the range of $20-200 \mathrm{~nm}^{263}$. The disadvantages of using mRNA are that it may invoke an immune response or be unstable, however, these disadvantages can be circumvented with appropriate chemical modifications ${ }^{9,264}$.

\subsubsection{Nucleic Acid-Based Constructs \& Devices}

One emerging area of nucleic acid research involves DNA constructs and devices engineered form higher-order two- or three-dimensional shapes. These precision DNA nanostructures have become known as DNA origami, a concept that rose to prominence in $2006^{265}$. Using DNA origami, precise nanostructures of calculable size and shape can be assembled into template structures via specific folding interactions. Tian et al. recently developed DNA octahedrons of $\sim 60 \mathrm{~nm}$ with encoded sites for molecular positioning, allowing multiple nanoparticles with different functions to be integrated into a single structure ${ }^{48}$. In another example, DNA icosahedra found use as vehicles the delivery of quantum dots ${ }^{266}$. DNA origami, with a defined number of binding sites, has recently been used to calibrate fluorescence for determination of protein copy number inside cells ${ }^{267}$. Oligonucleotides may also be deployed inside cells as probes. For example, oligonucleotide-based molecular beacons are short ( 25 base) hairpins featuring internally quenched fluorophores that alter their fluorescence upon hybridizing with a target sequence ${ }^{268,269}$. Aptamers, described above as inhibitors, can also be used as conjugates, receptor-targeting moieties, intracellular biosensors, and imaging probes ${ }^{270-273}$.

\subsubsection{Hard-to-Transfect Cells}

Effective transfection remains a significant hurdle for many primary cells. Moreover, even when high transfection efficiencies are achieved, toxic and off-target effects may confound results. This is a well-known barrier to the study of 
immune cells, where cells types such as T cells, B cells, natural killer cells, dendritic cells, and macrophages are known to be difficult to transfect ${ }^{234,274-286}$. Primary stem cells, cells of the hematopoietic lineage, and neurons are other cell types that have historically proven difficult to transfect ${ }^{287-290}$. The ability to conduct biological studies in these important cell types is often restricted by limitations on transfection efficiency and tolerance to treatment. Thus, while there has been a huge amount of work on refining transfection approaches over the last decades, there is still significant room for improvement.

\subsubsection{Synthetic Nanomaterials \& Devices}

Synthetic nanomaterials and devices represent another frontier where demand for suitable intracellular delivery solutions exceeds supply ${ }^{19,20}$. Probes engineered from functional nanomaterials, including carbon nanotubes $(\mathrm{CNTs})^{291-293}$, quantum $\operatorname{dots}^{294,295}$, magnetic nanoparticles, and various fluorescent reporter systems ${ }^{19,296-299}$ have potential as sensors for intracellular processes. Yet, limitations to their successful intracellular delivery, a poor understanding of their interaction with biological environments, and the toxicity issues of these novel materials have retarded their deployment inside the cell. Many of these materials and devices still await systematic intracellular testing due to ineffective delivery, and as a consequence, their true effectiveness remains unknown ${ }^{19,20,300}$. The delivery challenges of these molecules and unconventional materials must first be addressed before their potential in research or therapeutic and diagnostic applications can be fully realized. Below we highlight several examples of progress in the field.

CNTs have been proposed as sensors, labels, electrical field enhancers, and next-generation devices in biological applications $^{292,301}$. The smallest single-walled configurations exhibit diameters from $1.2 \mathrm{~nm}$ and lengths spanning from tens of nanometers up to microns ${ }^{302}$. Chemical functionalization can be employed to increase the solubility and biocompatibility of $\mathrm{CNTs}^{302}$, however their toxicity profiles and suitability for intracellular applications are still a matter of controversy ${ }^{303}$. One example where they have been useful in probing the intracellular environment was published by Fakhri et al. in which functionalized CNTs were loaded into cells by electroporation ${ }^{293}$. By tracking the near-infrared luminescence of kinesin-targeted single-walled CNTs, they observed a regime of non-equilibrium stirring dynamics driven by active cellular motors ${ }^{293}$. Another recent study used microinjection to load high concentrations of single-walled CNTs of length $\sim 150 \mathrm{~nm}$ into frog embryos ${ }^{304}$. The localization of CNTs and potential toxicity were tracked throughout the growth of the animal. They found CNTs tended to localize to the perinuclear region within most cells, however, there 
were no obvious structural defects, developmental abnormalities or toxicity to report ${ }^{304}$. These studies suggest CNTs might be safe for intracellular applications.

Quantum dots are semiconductor crystal configurations in the size range $<10 \mathrm{~nm}$. Due to their advantageous optical properties, intracellular labeling and analysis applications have been proposed ${ }^{295,305}$. Quantum dots are usually negatively charged and surface passivation with a poly-ethylene glycol (PEG) shell is a standard strategy to increase the biocompatibility of the structure, with a final diameter of $20 \mathrm{~nm}$ being typical for this configuration ${ }^{300}$. An early study compared microinjection, electroporation, and lipid transfection reagents for quantum dot delivery into cultured cells ${ }^{294}$. The investigators found that lipid reagents and electroporation failed to disperse the dots homogenously into cells, instead leading to aggregation or endosomal entrapment. On the other hand, low-throughput microinjection was able to deliver quantum dots homogeneously to the cytoplasm. Since then a number of approaches have been tested for quantum dots delivery. They include osmotic loading of pinosomes ${ }^{306}, \mathrm{CPPs}^{307}$, microfluidic cell squeezing ${ }^{308}$, controlled laser-induced cavitation $^{309,310}$, detergent permeabilization ${ }^{311}$, and successful examples of electroporation ${ }^{312,313}$. We point the reader to dedicated reviews on intracellular delivery of quantum dots for further information ${ }^{300,314,315}$.

Magnetic nanoparticles in biomedical science are useful for magnetic resonance imaging (MRI), generating local heat effects, and providing a handle for attractive forces ${ }^{316}$. In some of these applications it is necessary for magnetic nanoparticles to be delivered to the cell interior. One group developed a cell labeling approach using short cell penetrating peptides (HIV-Tat) to derivatize $\sim 45 \mathrm{~nm}$ superparamagnetic nanoparticles ${ }^{317}$. The particles were internalized into hematopoietic stem cells before being reintroduced for in vivo homing to the bone marrow where they were subsequently detected and retrieved ${ }^{317}$. In another example, Nitin et al. developed $\sim 10 \mathrm{~nm}$ superparamagnetic iron oxide nanoparticles with a PEG-modified, phospholipid micelle coating, functionalized with cell-penetrating peptides ${ }^{318}$. The particles were demonstrated to enter cells and act as MRI contrast agents for intracellular molecular imaging in deep tissue ${ }^{318}$. Furthermore, polymer nanoparticles with iron oxide cores have been deployed for DNA transfection purposes ${ }^{319}$. The magnetic core enables their uptake and localization to be tracked by $\mathrm{MRI}^{319}$. In another case, the mechanical and physiological properties of fibroblasts were measured as a function of intracellular uptake of biocompatible magnetic nanoparticles and the applied magnetic field ${ }^{320}$. 
Various nanoparticle systems have also been deployed as intracellular temperature probes ${ }^{321}$. In one report, temperatureresponsive nanodiamonds of approximately 100 nanometer were introduced into cells via nanowires ${ }^{322}$. The nanodiamonds were then used as local temperature gauges to perform nanometer-scale thermometry in living cells at microkelvin resolution ${ }^{322}$. Another study used smaller, but less accurate, particles for intracellular temperature measurements ${ }^{323}$. Okabe et al. prepared a fluorescent polymeric thermometer of $\sim 9 \mathrm{~nm}$ diameter, functionalized it with hydrophilic residues, and microinjected it into the cytoplasm of living cells. With a temperature measurement resolution of 0.18-0.5 K, they claimed to measure temperature differences between various organelles ${ }^{323}$.

\subsubsection{Large Cargo}

Relative to most cells, large cargo is anything from hundreds of nanometers up the range of the cell itself (usually tens of microns). Examples of large cargo that have been delivered into cells are shown in the bottom left quadrant of Figure 3, and include bacteria, mitochondria, whole chromosomes, microbeads, sperm, nuclei, and micro-electro-mechanical systems (MEMS) devices. The first demonstration of large cargo delivery occurred alongside the invention of microinjection itself in $1911^{324}$. Marshall Barber demonstrated that a single bacterium, once inside the cytoplasm of a plant cell, was sufficient to kill it ${ }^{324,325}$.

For a century microinjection has been the dominant method for introducing large cargo into cells. Microinjection was used for the first nuclear transplant experiments that surgically dissected the nucleus from blastula cells and inserted them into living frog eggs ${ }^{326}$. To the amazement of the researchers, these eggs then had the potential to grow and produce a new animal. Building on this breakthrough, John Gurdon and colleagues showed that nuclei transplanted from fully differentiated somatic cells were capable of generating a new animal ${ }^{327,328}$. Based on this work. Gurdon later shared the Nobel prize for "the discovery that mature cells can be reprogrammed to become pluripotent". Microinjection was also required for the nuclear transplant that led to the first mammalian cloning, as exemplified by the birth of Dolly the sheep in $1997^{329}$. Furthermore, in an unconventional form of gene therapy, transplant of pronuclei from human eggs with pathological mitochondria to donor eggs with functional mitochondria has been shown to correct diseases of mitochondrial inheritance ${ }^{330}$. 
Other examples emphasizing the importance of microinjection in biotechnology include in vitro fertilization (IVF) and chromosome or mitochondrial transplantation. IVF occurs through the artificial delivery of sperm into eggs cells. The IVF concept was first demonstrated through microinjection of sperm into sea urchin eggs ${ }^{331}$. Subsequently, IVF generated the first human pregnancies in the early $1990 \mathrm{~s}^{332}$. Chromosome transplantation techniques have also been described with microinjection apparatus ${ }^{333}$. Indeed, artificial chromosomes have been engineered and transferred into cells by microinjection for transgenic studies or proof-of-concept gene therapy ${ }^{334,335}$. In another example of large cargo delivery, transplant of mitochondria $(\sim 1-2 \mu \mathrm{m})$ via microinjection has been demonstrated in several different cell types and model systems $^{336-338}$.

While microinjection has traditionally dominated large cargo delivery, it is not the only option. Indeed, several rival methods have arisen mainly out of the need for greater throughput. For example, Chiou and colleagues pioneered an approach using laser-triggered cavitation bubbles to deliver $\sim 2 \mu \mathrm{m}$ bacteria into cultured cells at both single cell ${ }^{339}$ and high throughput scales ${ }^{340}$. The same approach was extended to delivery of functional mitochondria for studies of mitochondrial dysfunction in metabolic diseases ${ }^{341}$. Another method of mitochondrial transfer is cell fusion, where the mitochondria are supplied from donor cells $\mathrm{s}^{342,343}$. In studies involving gene therapy with human artificial chromosome they can also be transferred by cell fusion, in a process termed microcell-mediated chromosome transfer $\left(\right.$ MMCT) ${ }^{192,344-}$ 347. Engineered CHO donor cells carry the human chromosome and are triggered to fuse with the acceptor cell, thus transferring the genetic material ${ }^{344}$.

Apart from delivery of organelles and subcellular components, insertion of large synthetic materials and devices is another area of interest. As a case in point, micron-scale particles, spheres, and beads have been loaded into cells for intracellular microrheology studies that analyze the internal mechanics and dynamics of cells. So far they have been delivered by microinjection $^{348,349}$ or ballistic propulsion ${ }^{350-353}$. A recent study microinjected PEGylated tracer beads of up to $0.5 \mu \mathrm{m}$ into cells to show that motor-driven cytoplasmic mixing substantially enhances intracellular movement of both small and large components $^{354}$. In another study of cell mechanics, $\sim 1 \mu \mathrm{m}$ melamine particles coated with PEG were fired into HeLa cells by a gene gun to study glassy dynamics in the cytosol ${ }^{355}$. Magnetic cargoes have also been used to probe the intracellular environment. Moch et al. loaded $2.8 \mu \mathrm{m}$ magnetic beads into the cytoplasm of mammalian cells for probing cell mechanical properties by magnetic tweezers ${ }^{356}$. Garzon-Coral et al. measured mitotic spindle forces one- and two-cell 
Caenorhabditis elegans embryos in vivo by injecting $1.0 \mu \mathrm{m}$ diameter superparamagnetic beads and using magnetic tweezers to exert calibrated forces of up to $200 \mathrm{pN}$ on mitotic spindles ${ }^{357}$. In other instances, MEMS can measure intracellular properties, such as cytoplasmic pressure ${ }^{49}$. One group deployed a MEMS-based intracellular hydrostatic pressure sensor, about $6 \mu \mathrm{m}$ in size, that was claimed to be delivered into HeLa cells via lipofection ${ }^{49}$. The same researchers also microinjected silicon MEMS barcodes up to $10 \mu \mathrm{m}$ in length into mouse embryos for tracking and labeling purposes ${ }^{358}$.

\section{Approaches for Intracellular Delivery}

As outlined in the previous section, a wide range of cargos has been introduced to the intracellular space through a diverse range of delivery approaches. Here, we categorize these approaches according to the mechanism at the plasma membrane

(Figure 4), rather than traditional classifications of biological, physical, and chemical techniques ${ }^{105,359-362}$. As the cell is agnostic to our distinction between scientific disciplines, we believe this categorization is more mechanistically relevant ${ }^{107}$. Broadly, methods may involve either 1) disruption of the cell membrane to facilitate entry of cargo, or 2) packaging with carriers, which then undergo uptake into endosomal trafficking routes or fuse with the target cell membrane. Although chemical or structural modifications can be used to increase the passive permeability of some small molecules or short peptides, most cargo molecules and materials require an active intracellular delivery method.

\subsection{Carrier-Mediated}

Most of the early developments in carrier-mediated delivery were directed towards nucleic acid transfection, particularly for DNA plasmids. As mentioned in the transfection section (see section 2.2.3), cationic lipids and polymers can condense plasmids and other nucleic acids into compact nanoparticles with dimensions down to tens of nanometers ${ }^{10,205,206}$. This makes the task of delivering these molecules significantly more manageable. Moreover, the positive charge of these particles facilitates their interactions with the cell surface, which is negatively charged due to the typical -35 to $-80 \mathrm{mV}$ membrane potential of cells. The positive charge may also promote binding to certain receptors ${ }^{10}$. Upon binding, subsequent internalization via endocytosis is thought to be most efficient for particles in the size range below $100 \mathrm{~nm}^{363}$. Complexation into nanoparticles also confers protection for nucleic acids against degradation in the cytoplasm ${ }^{364}$. Potential disadvantages of complexation, however, may include delayed unpacking (making it inaccessible for gene 
expression $^{365}$ ) or excessive toxicity ${ }^{366}$. In the last two decades researchers have expanded the scope of transfection strategies to include carriers designed from lipids, liposomes, polymers, inorganic nanomaterials, carbon nanotubes, protein-based nano-assemblies and functionalization with various peptides, ligands, and chemical modifications $6,7,9,10,364$.

\section{PHYSICAL}

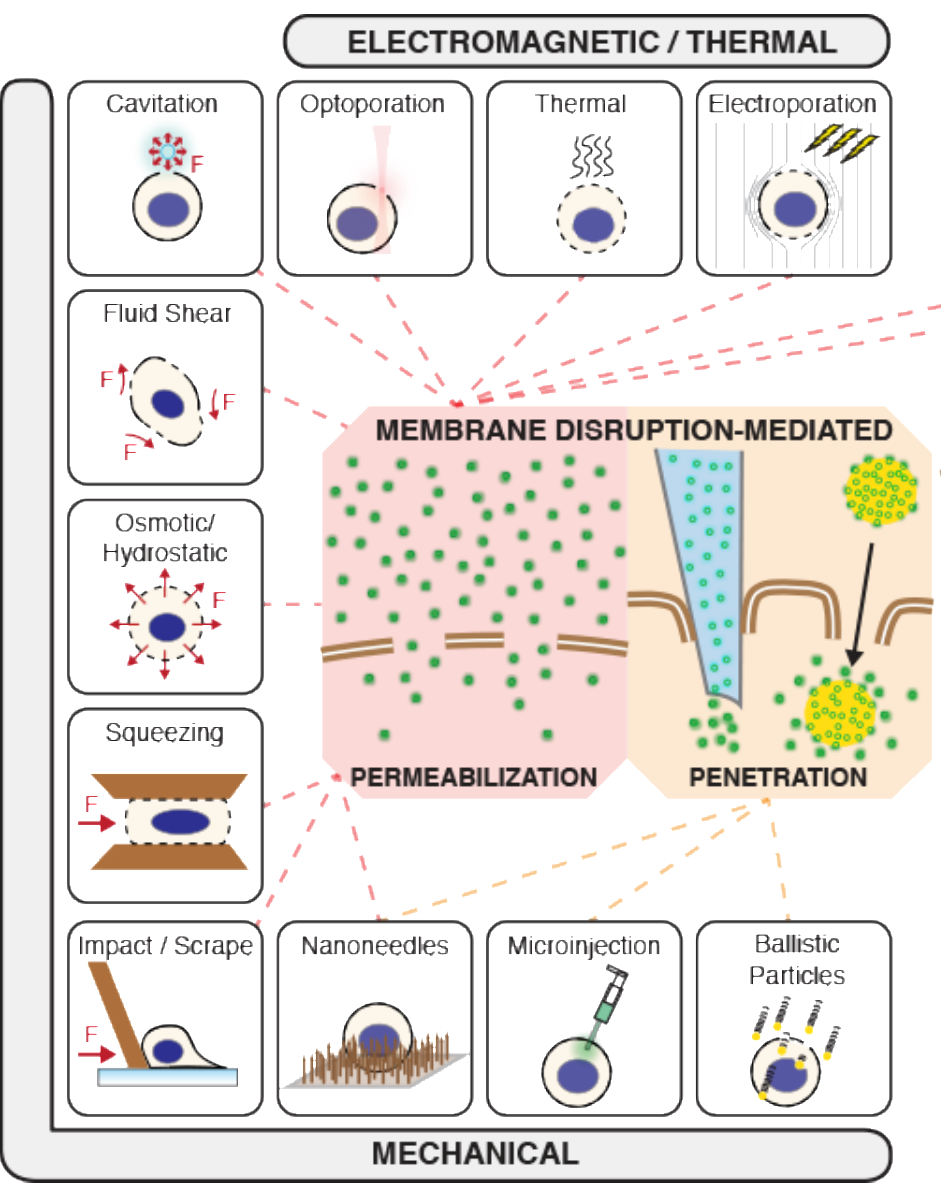

\section{BIOCHEMICAL}

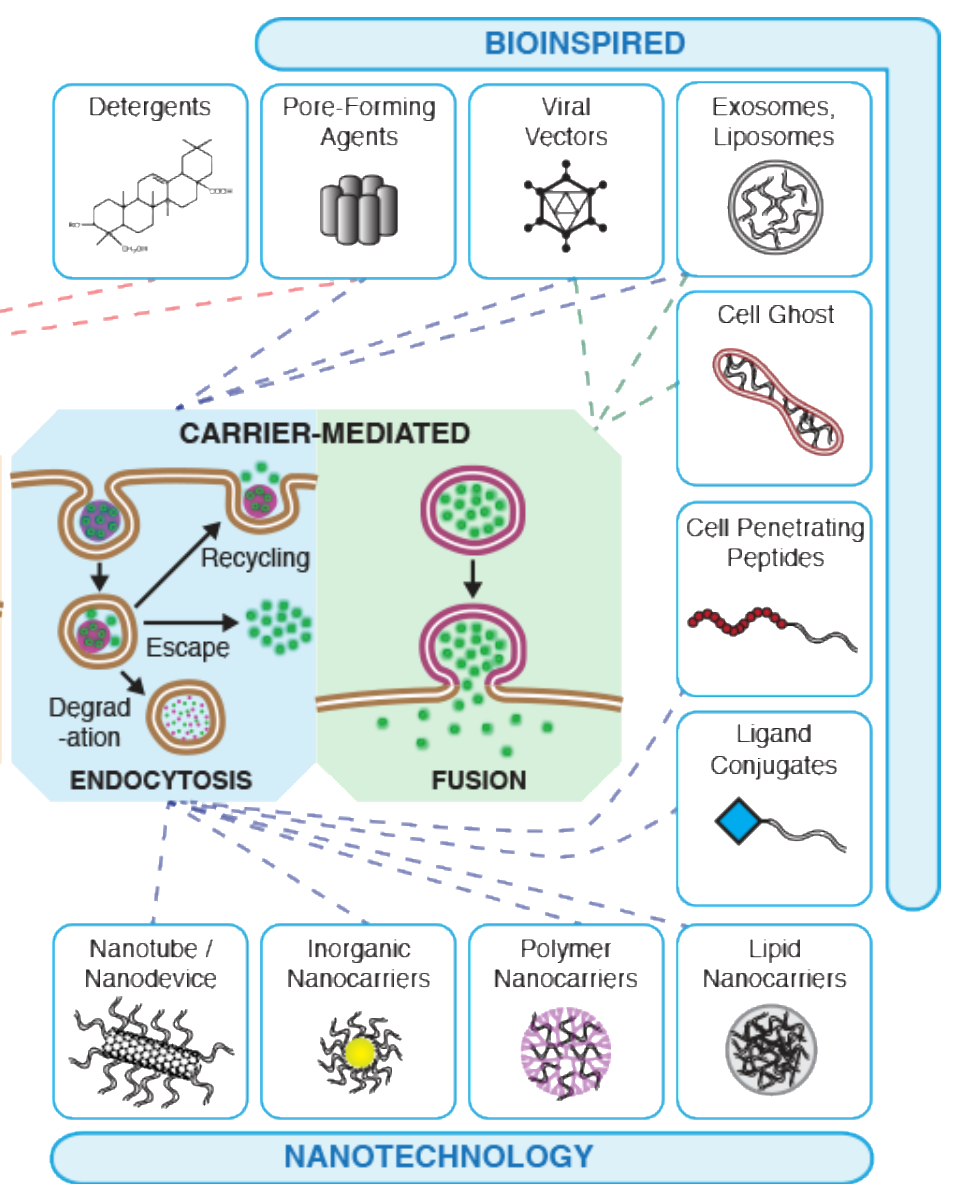

Figure 4. A map of intracellular delivery methods and their mechanisms. Current intracellular delivery methods are shown sorted within the four indicated mechanisms: 1) permeabilization, 2) penetration, 3) endocytosis, and 4) fusion. Methods that overlap on more than one mechanism may promote intracellular delivery via multiple pathways depending on the context. For example, most viral vectors are believed to go through endocytosis but a limited number fuse directly with the plasma membrane.

The other major type of carriers for nucleic acid delivery are viral vectors, which exploit the viral infection pathway to enter cells but avoid the subsequent expression of viral genes that leads to replication and pathogenicity ${ }^{191}$. This is done by deleting coding regions of the viral genome and replacing them with the DNA to be delivered, which either integrates into host chromosomal DNA or exists as an episomal vector. At present, viral vectors are the most clinically advanced nucleic acid delivery agents owing to their high efficiency and specificity. They were first employed from the 1970 s - constructed from SV40 ${ }^{367}$ or retroviruses ${ }^{368,369}$. Newer generations of viral vector platforms have been produced based on components 
from lentivirus, retrovirus, adenovirus or adeno-associated virus, and other viruses ${ }^{370-372}$. While highly efficient for DNA delivery, key weaknesses of viral vectors are: 1) labor-intensive and expensive protocols; 2) safety issues; 3) risk of causing immune/inflammatory responses; 4) integration into the genome with recombinant vectors; 5) risk of insertional genotoxicity; and 6) limited packaging capacity (Adeno and AAV typically restricted to carry 5 to $7.5 \mathrm{~kb})^{290,373}$. The problems with viral vectors continue to motivate the development of non-viral carriers ${ }^{9,10,374}$.

Beyond nucleic acid transfection, researchers initially explored protein delivery through the use of red cell ghosts ${ }^{87-89}$ and liposomes $^{90,91}$. Newer generations of nanocarriers are now being designed to address intracellular delivery of proteins on a broader scale ${ }^{6,124,131,375}$, although these developments are more at a nascent stage. Intracellular delivery of genome editing complexes is a particular application that is driving the evolution of next-generation nanocarriers ${ }^{15,18,156}$.

Mechanistic investigations indicate that most carriers enter cells via endocytosis before escaping into the

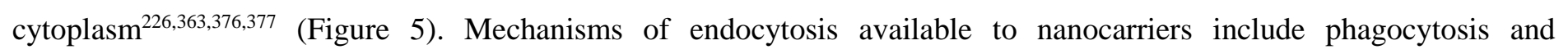
pinocytosis through clathrin-dependent and clathrin-independent pathways ${ }^{363,377}$. The pathways employed by target cells are dependent upon the particle size, shape, material composition, surface chemistry and/or charge en, $263,376-378^{\text {. Cargo not }}$ able to escape endosomes are trafficked through lysosomes for degradation or recycled back out to the cell surface ${ }^{379-381}$. Maximal efficiencies of around $1 \%$ endosomal escape have been reported for the most advanced non-viral carrier strategies, including lipid nanoparticles ${ }^{380,382}$ and cell-penetrating peptides ${ }^{116}$. Moreover, the exact mechanisms of endosome escape remain unclear and are a matter of ongoing research $379-381,383$.

Alternatively, some carriers are able to fuse directly with the plasma membrane. These systems were first inspired by viruses that deploy specialized surface proteins to induce fusion with target membranes ${ }^{89,384}$. Fusogenic carriers are bound by a phospholipid bilayer that hosts the fusion machinery or confers fusion intrinsic properties. Examples include 1) cell ghosts, dead cells that have had their cytoplasm replaced with cargo ${ }^{89,384}, 2$ ) virosomes, cargo-loaded vesicles reconstituted to display functional viral proteins ${ }^{385}$, and 3) fusogenic liposomes ${ }^{386,387}$. In the latter case, a new generation of liposomes have been reported to become fusogenic by modulating the lipid composition without any need for fusogenic protein or peptide conjugation 387,388 . 
Recently, a subset of natural cell-derived vesicles known as exosomes have been discovered to fuse with target cell membranes for the exchange of RNA and proteins between immune cells ${ }^{389}$. Although the exact fusion mechanisms are yet to be described, it is anticipated that exosome-inspired systems may represent a new generation of vehicles for efficient and biocompatible intracellular delivery ${ }^{390,391}$.

A

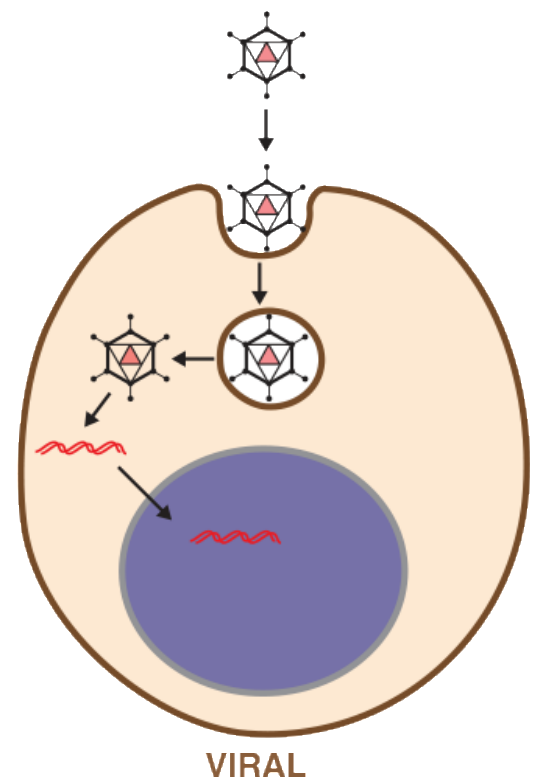

B

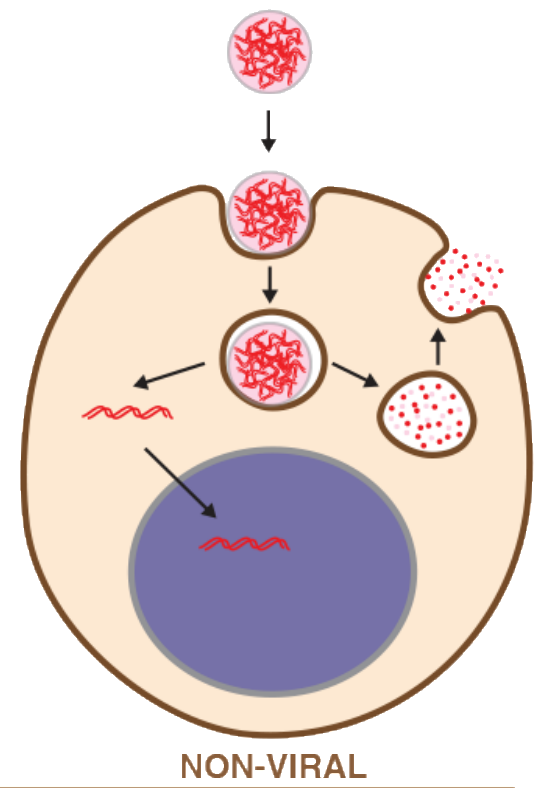

CARRIER-MEDIATED
C Nucleic Protein Nano- Small acid Protein particle molecule
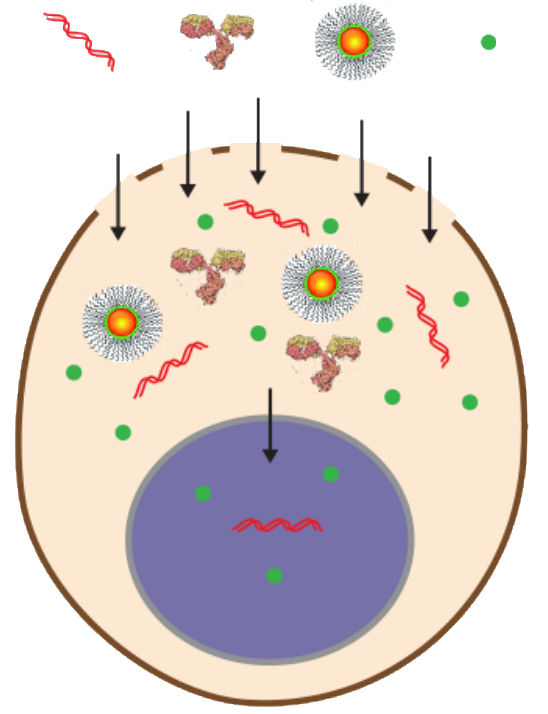

MEMBRANE DISRUPTION-MEDIATED

Figure 5. Cargo delivery trajectories for the main intracellular delivery categories. (A) Viral vectors only deliver nucleic acids but do so very efficiently (endocytosis example). (B) Most non-viral carriers are optimized for nucleic acid delivery although some adaptations can carry other materials. Non-viral carriers are endocytosed into the cell with small amounts of nucleic acid breaking out into the cytoplasm while the majority are degraded in lysosomes or recycled back out to the extracellular space. (C) Membrane disruption is able to deliver almost any cargo that can be dispersed in solution provided it is small enough to fit through transient openings in the plasma membrane. Nucleus is depicted in purple.

\subsection{Membrane Disruption-Mediated}

Unlike carriers that may be restricted in the feasibility of cargo-carrier combinations, membrane disruption-mediated strategies are near-universal, being able to rapidly deliver almost any cargo that can be dispersed in solution (Figures 4 \& 5). In this review the term "membrane disruption" refers to the generation of any hole that would increase the permeability of the plasma membrane to cargo. This includes pores, defects, inhomogeneities, tears, lesions, holes, and perforations of all sizes and shapes. 
The challenge for membrane disruption-mediated approaches is 1) to open up the right kind of holes in the plasma membrane to achieve substantial delivery of the cargo, and 2) to avoid undesirable cell perturbation or death associated with membrane damage. The main two ways membrane disruption is accomplished are through direction penetration or permeabilization.

\subsubsection{Direct Penetration}

Strategies involving direct penetration use a conduit or vehicle to break through the membrane, thereby creating a passage for the cargo. Prevalent examples are microinjection, ballistic particles, and nanoneedles, as shown in Figure 4. Microinjection was the first intracellular delivery method to be invented and represents a classic case of a direct penetration strategy 325,392 . The cell membrane is disrupted with a miniaturized pipette-like element, which is then used to pump fluid containing the molecule of interest inside the cell. Nanoneedles operate on a similar principle except that they are scalable in parallelized arrays and typically consist of finer, more intricately fabricated structures ${ }^{109,110,393}$. Ballistic particles are coated with the material to be delivered and fired at high velocity into the cell ${ }^{394}$. They are categorized as membrane disruption in this review (rather than carriers) due to the critical role of active force in puncturing the cell membrane to achieve access. In all direct penetration strategies, the target cell must respond to repair damage sustained to the plasma membrane or other cellular structures.

\subsubsection{Permeabilization}

In contrast to direct penetration, permeabilization strategies make the cell transiently permeable to cargo present in the extracellular solution. The plasma membrane is considered permeable when membrane disruptions are of sufficient size and lifetime to permit passage of the cargo molecules or materials. Thus, the threshold level of permeabilization needed depends on the properties of the cargo.

As seen in Figure 4, many different permeabilization strategies have been attempted. They range from mechanical and laser-based to electrical and chemical ${ }^{105,395-397}$. The key events associated with permeabilization-based intracellular delivery are shown in Figure 6. First, the cargo of interest is dispersed into solution at a concentration conducive to influx. Second, the cells are exposed to the membrane disruption event. Physical methods of permeabilization generally have 
better control of the intensity, duration, and placement of the membrane disruption effect ${ }^{105,396}$. Biochemical methods, such as exposure to pore-forming toxins or detergents, are more scalable but can be harder to control since they are not associated with a discrete event ${ }^{395}$. Upon membrane disruption, cargo begins to diffuse into the cell according to its concentration gradient while some cytoplasmic contents are lost. In certain cases, additional effects, such as electrophoretic force, can also be harnessed to augment influx of cargo. Third, it is important to note that the imposed membrane disruptions are not stable, and thus only allow passage of cargo for a limited time. Upon membrane insult, the target cell responds with active membrane repair processes. Healing of the plasma membrane can take from a few seconds up to several minutes to complete. Once membrane integrity is restored, the cell may engage metabolic and transport processes to restore cytoplasmic composition and bring itself back to full health ${ }^{398,399}$. Most permeabilization strategies apply specific conditions, such as temperature and buffer composition, to first promote permeabilization and delivery, and subsequently facilitate cell recovery. The membrane disruption effect must not be too severe or prolonged, otherwise the cells will be unable to repair and recover. Effective permeabilization strategies must therefore find a balance, optimizing both the membrane damage and cell treatment conditions.

The remainder of this review will focus on membrane disruption-based delivery. This exploration will mostly be centered around animal and mammalian cells in vitro and ex vivo. In the next section we will discuss background concepts helpful in understanding how and why membrane disruption can be a successful approach. Following that, we will offer a detailed appraisal of the various delivery methods. Each section will cover content areas that include history, mechanisms, feasibility, performance, toxicity, applications, technical advances, and envisaged future opportunities. 


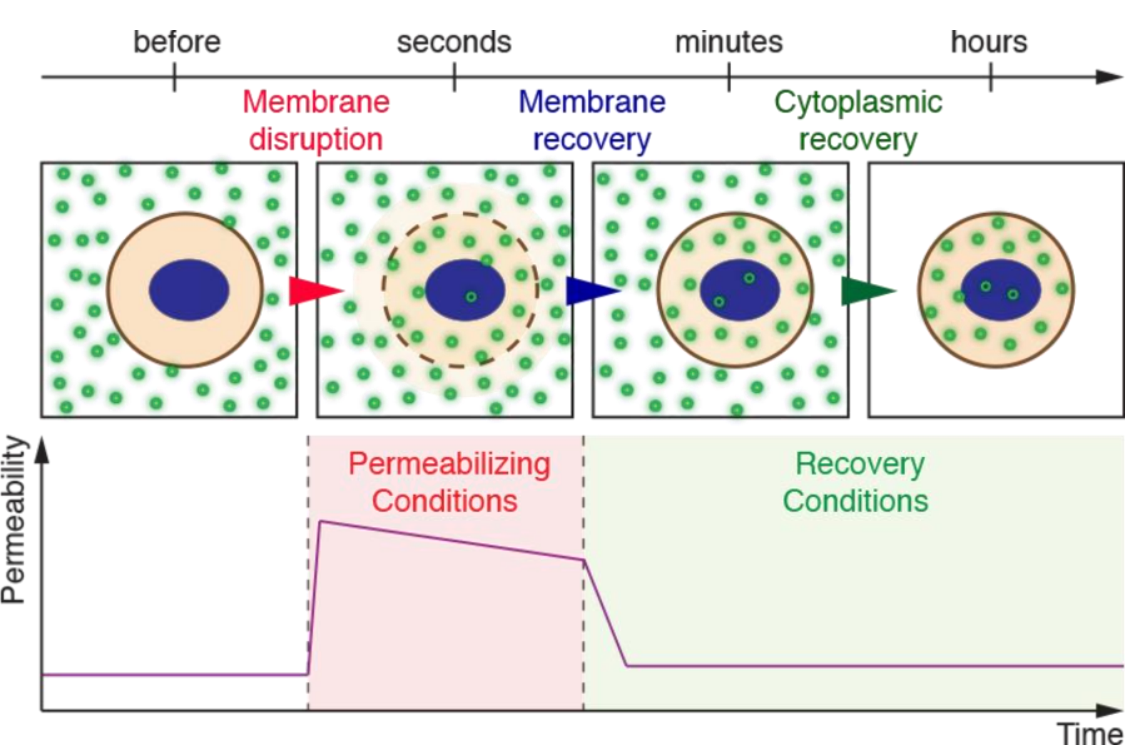

Figure 6. Key events associated with permeabilization-based intracellular delivery. Acute membrane disruption triggers an increase in permeability to the cargo of interest (green). For a limited window of time (seconds), cargo diffuses into the cell according to its concentration gradient while some cytoplasmic materials may be lost (orange). Upon membrane disruption the cell responds with membrane active repair processes that can take from a few seconds up to several minutes to complete. Once membrane integrity is restored, the cell engages metabolic and transport processes to restore membrane and cytoplasmic homeostasis. It may take hours for the cell to return to the pre-perturbation state.

\section{Membrane Disruption-Mediated Delivery: Background Concepts}

In this section we will cover the basics of: 1) cell and membrane properties, 2) mechanisms of membrane disruption, and 3) cell response to membrane disruption. These background concepts lay a foundation to explore the common issues that arise in membrane disruption-based intracellular delivery. The following sections then examine direct penetration (section 5) and permeabilization (section 6) methods.

\subsection{Cell Structure \& Properties}

\subsubsection{Plasma Membrane Function}

The primary barrier to intracellular delivery is the plasma membrane, which defines the essential boundary between the inside and outside of a cell. The plasma membrane enables cells to control their composition and properties. Its central component is a $\sim 5 \mathrm{~nm}$ thick phospholipid bilayer with polar heads facing the aqueous environment and fatty acyl chains pointing inward to form a hydrophobic core. This hydrophobic core is the main limiting barrier to the passage of macromolecules and polar molecules. The permeability of a given molecule or cargo material across the plasma membrane depends on the properties of the membrane (e.g. composition, heterogeneity, thickness), active cellular 
regulation of the plasma membrane (e.g. protein activity, cytoskeletal interaction), the properties of the molecule itself (e.g. charge, size, polarity), and environmental factors (e.g. temperature) $)^{168,400}$.

The plasma membrane allows compartmentalization of electrolyte concentrations between the cell interior and external solutions (Figure 7A). For example, relatively high intracellular potassium (140 mM) and low sodium $(5-15 \mathrm{mM})$ are generated by the action of the $\mathrm{Na}^{+} / \mathrm{K}^{+}$ATPase, a plasma membrane-embedded transport protein ${ }^{401}$. Intracellular chloride, calcium, and magnesium are all lower than their corresponding extracellular concentrations. The maintenance of these electrolyte gradients is key for the typical negative membrane potential ( -35 to $-80 \mathrm{mV}$ ) of most animal cells and a host of other essential functions. The cell also has elevated intracellular concentrations of metabolites such as ATP (typically 2-5 $\mathrm{mM}$ ), amino acids and other biomolecules. The difference between intracellular and extracellular composition is an important consideration in membrane disruption-based intracellular delivery, as strategies that take this into account can lead to more efficient treatments and better cell health. Minimizing the depletion of intracellular contents, for example, can improve cell treatment outcomes (see section 4.3). 
A
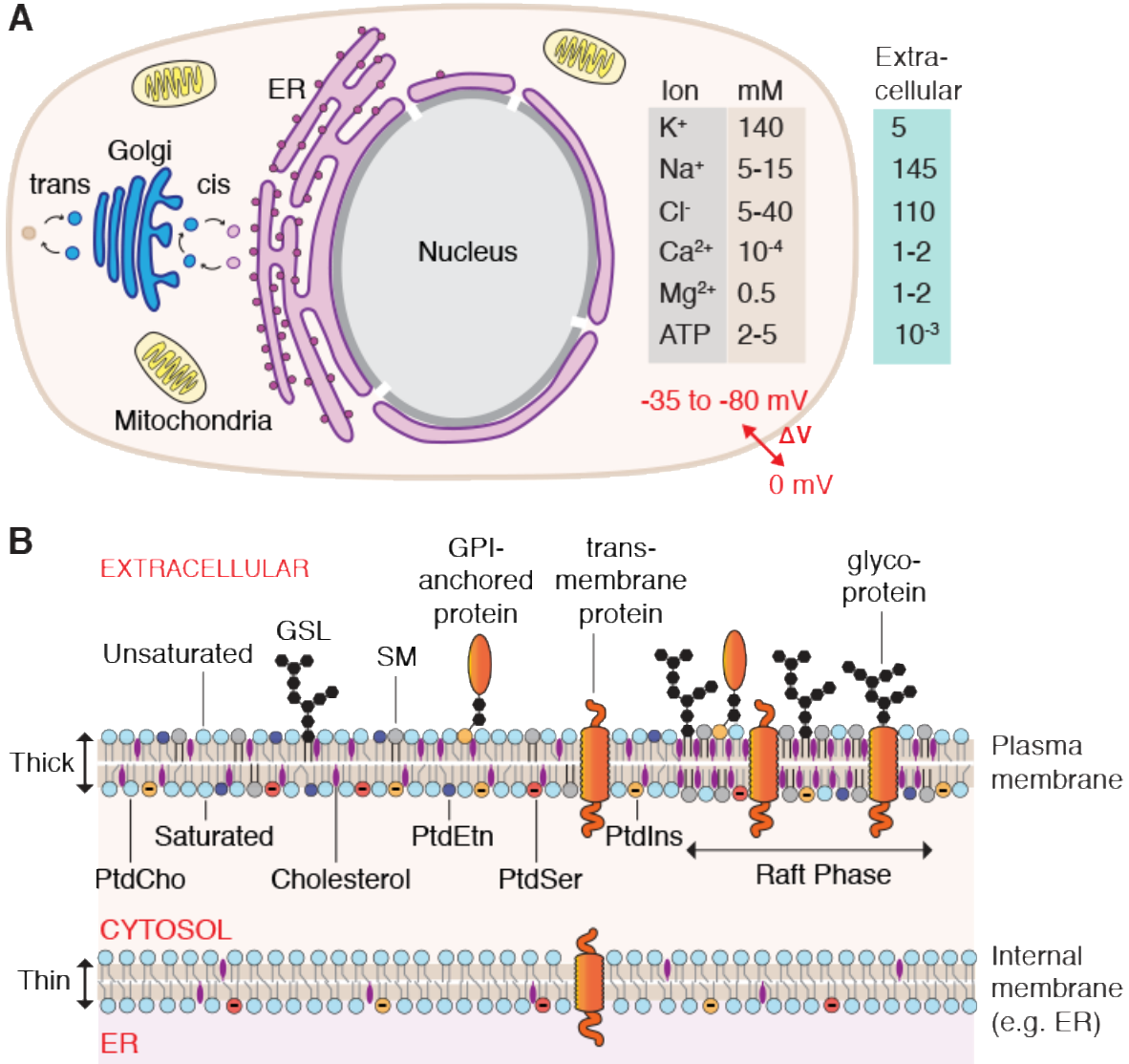

C

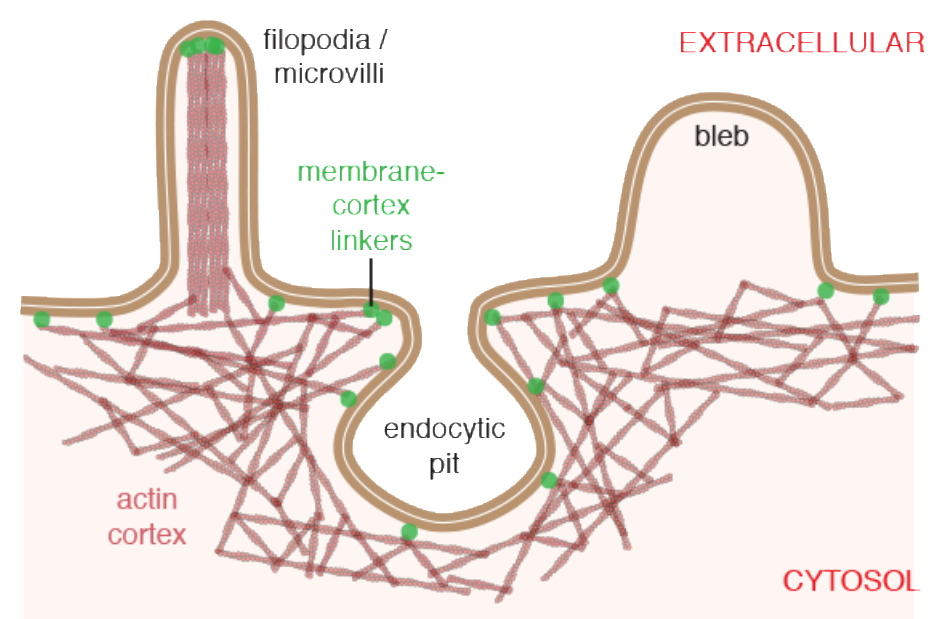

Figure 7. Relevant structure and properties of the cell interior and surface. (A) Overview of typical animal cell structure with basic organelles, intra- and extracellular ion concentrations, and negative membrane potential $(\Delta V)$. ER: endoplasmic reticulum. (B) Features of the plasma membrane including lipid asymmetry across bilayer leaflets and lateral segregation into domains, such as raft phases. Abbreviations are: phosphatidylcholine (PtdCho), phosphatvidylethanolamine (PtdEtn), phosphatidylserine (PtdSer), and phosphatidylinositol (Ptdlns), sphingomyelin (SM), glycosphingolipids (GSL). Carbohydrate residues are depicted in black, cholesterol in purple. Note the highly regulated heterogeneous distribution of molecules between different types of membranes, lateral domains and leaflets. As a result, the ER membrane is thinner and sparser than the plasma membrane, with more unsaturated lipid tails. (C) Plasma membrane reservoirs and their relationship with the underlying actin cortex, which is connected by membranecortex linker proteins (green). Actin rods support filopodia and microvilli. Blebs are typically devoid of actin until they are pulled back in. Remodeling of the actin cytoskeleton facilitates formation and stabilization of endocytic pits. 


\subsubsection{Plasma Membrane Composition \& Properties}

The plasma membrane has characteristic properties distinct from other types of lipid membranes (Figure 7B). It is much more complex and dynamic than pure lipid bilayers, containing hundreds of different lipid species and up to $50 \%$ membrane proteins by weight. Proteins associated with the plasma membrane include various transporters, receptors, and enzymes. They may span the membrane via transmembrane domains or be anchored to one side via lipophilic appendages. The spatial organization of plasma membranes feature both lateral heterogeneity (lipid domains) and uneven distribution between inner and outer leaflets (lipid asymmetry $)^{402}$. Cells use up to $5 \%$ of their genes for synthesis of a diverse array of lipids, reflecting the importance of the functions arising from this diversity ${ }^{403}$.

The different types of lipids are distributed in a highly regulated and distinct manner across the various membranes of the cell ${ }^{402}$. This gives the different membranes unique properties (Figure 7B). In eukaryotes there are three main categories of membrane lipids: glycerophospholipids, sphingolipids, and sterols. Glycerophospholipids are the major structural lipids of membranes, of which common species are phosphatidylcholine (PtdCho), phosphatidylethanolamine (PtdEtn), phosphatidylserine (PtdSer), and phosphatidylinositol (PtdIns). Their hydrophobic tail is a diacylglycerol (DAG), which contains saturated or cis-unsaturated fatty acyl chains of varying lengths. Unsaturated tails don't pack as tightly, increasing the lateral space between lipids and promoting lateral fluidity in the membrane. PtdCho is the most common lipid, accounting for $>50 \%$ of the phospholipids in most eukaryotic membranes ${ }^{402}$. PtdSer and PtdIns exhibit negatively charged head groups and localize to the inner (cytoplasmic) leaflet. The major sphingolipids in mammalian cells are sphingomyelin (SM) and sugar-decorated glycosphingolipids (GSLs). The sphingolipids feature a ceramide as their hydrophobic backbone, having saturated (or trans-unsaturated) tails so they tend to form a taller, narrower cylinder shape than their glycerophospholipid counterparts.

Sterols are highly abundant in the plasma membrane, contributing greatly to barrier function and lateral organization ${ }^{404,405}$. In mammals, the predominant species of sterol is cholesterol, which represents up to $40 \%$ of the lipid molecules in the plasma membrane ${ }^{405}$. This is in contrast to other internal membranes, such as the endoplasmic reticulum (ER), where the corresponding number is only $\sim 5 \%$. Cholesterol tends to straighten out hydrophobic chains and fill in structural defects in membranes. Thus, it serves to stiffen and thicken the plasma membrane, improving its durability. Cholesterol is also 
essential to the formation of lipid rafts, which are characterized by the assemblage potential of sterol-sphingolipid interactions and particular proteins that have affinity for the raft phase (i.e. raft proteins $)^{405}$. These lateral raft domains are thought to serve as platforms for key structural, signaling and membrane trafficking phenomena, such as the nucleation of caveolae pits in the plasma membrane ${ }^{406}$. In contrast to the plasma membrane, internal membranes, such as the ER, feature less cholesterol, more unsaturated lipids, and less diversity of lipid species ${ }^{402}$. Internal membranes are thinner, sparser, and less durable, being more adapted for biogenesis rather than the comparatively robust and stable barrier function of the plasma membrane $e^{402}$.

The unique characteristics of the plasma membrane are a key factor in certain membrane disruption strategies. For example, specific pore-forming toxins, such as cholesterol-dependent cytolysins $(\mathrm{CDCs})^{407}$, and detergents, such as saponins ${ }^{408}$, are specific for high cholesterol-containing membranes. This makes it possible to disrupt plasma membranes in a relatively specific manner without damaging internal membranes ${ }^{397}$.

\subsubsection{Intrinsic Membrane Permeability}

Although the plasma membrane comprises a highly regulated barrier to control the intracellular composition, it is naturally permeable to certain substances. Phospholipid bilayers are permeable to gas molecules such as $\mathrm{O}_{2}, \mathrm{CO}_{2}, \mathrm{~N}_{2}$ (permeability coefficients $10^{1}-10^{-2} \mathrm{~cm} \mathrm{~s}^{-1}$ ), solvents such as $\mathrm{H}_{2} \mathrm{O}$, ethanol, and dimethylsulfoxide (DMSO) (permeability coefficients $10^{-3}-10^{-4} \mathrm{~cm} \mathrm{~s}^{-1}$ ), and to some extent other small uncharged polar molecules like urea and glycerol (permeability coefficients $\left.10^{-6}-10^{-7} \mathrm{~cm} \mathrm{~s}^{-1}\right)^{168,409}$. Most cell-penetrant small molecule drugs and peptides have permeability coefficients approaching a maximum of about $10^{-6} \mathrm{~cm} \mathrm{~s}^{-1} .{ }^{168}$ Despite their small size, the cations $\mathrm{Na}^{+}$and $\mathrm{K}^{+}$ are relatively impermeable with coefficients of $10^{-14}-10^{-15} \mathrm{~cm} \mathrm{~s}^{-1}$.

In live cells it is often a challenge to decipher whether permeability arises due to the passive properties of the plasma membrane, the presence of membrane transporters and solute carriers, or fluctuations in transient bilayer defects (such as can be promoted by ethanol or DMSO $)^{168,410}$. In many instances the apparent permeability of a molecule is actually regulated by the cell. For example, membrane proteins called aquaporins increase the transmembrane flux of water and glycerol $^{411}$, the expression of which can vary significantly across a cell population or between cell types. The cell actively 
opens and closes sodium channels to dynamically alter the $\mathrm{Na}^{+}$permeability during action potentials. Furthermore, many small molecule drugs have been postulated to enter cells via metabolite transporters whose structures they often mimic ${ }^{412}$. In other cases, peptide transporters, such as PepT1 and OATP, have been reported to pump small peptides and peptidebased drugs into or out of cells ${ }^{168}$. Regardless of the mechanisms, few candidate drug molecules exhibit passive permeability or are amenable to active uptake by the cell. Chemical modifications or conjugations can be conferred to increase the permeability in some cases, but this is not feasible for most macromolecular cargo, particularly for those larger than one nanometer in size.

\subsubsection{Structure \& Properties of the Cell Surface}

The durability of the plasma membrane may be reinforced by intra- or extra-cellular scaffolds. Some lipids (e.g. glycosphingolipids) and proteins (glycoproteins) have extracellular carbohydrate domains. When sufficiently dense, these carbohydrate moieties can form a thick outward coating known as the glycocalyx, which is prominent in animal epithelial/endothelial cells and some types of bacteria ${ }^{413}$. On the interior side, the plasma membrane may be reinforced by the underlying actin cytoskeleton, and the proteins that link it to the membrane, to form a cortical structure hundreds of nanometers thick ${ }^{414}$ (Figure 7C). Other cytoskeletal elements such as microtubules, intermediate filaments, septins, spectrins and clusters of cell adhesion molecules (e.g. integrins) can also assemble into supporting structures that affect membrane properties. Because the actin cortex is often more mechanically robust than the plasma membrane, in many cases it is thought to control cell shape and apparent surface area ${ }^{414}$. Indeed, the plasma membrane features a plethora of small folds, wrinkles, and reservoirs in the form of outward-protruding actin-filled filopodia/microvilli and actin-void blebs or inward-bending endocytic pits, such as caveolae. The excess of plasma membrane surface area is thought to be in the range 2-10 fold the apparent cell surface area ${ }^{414}$. These excess reservoirs allow the cell to accommodate rapid shape and volume changes without tearing the membrane ${ }^{415,416}$, a key property to ensure durability of the cell in mechanically challenging environments.

In cases where the plasma membrane is significantly reinforced by other components, it may become more difficult to mechanically disrupt. This is an important factor to consider particularly for mechanical membrane disruption techniques. 
For example, the cell surface has been reported to exhibit an impressive ability to conform to nanoneedles and other penetrating objects, making intracellular delivery less efficient than anticipated ${ }^{417,418}$.

As living cell membranes are much more complicated, dynamic, and heterogeneous than artificial lipid bilayers, insights from simplified model systems and simulations must be interpreted with caution ${ }^{410}$. The full complexity of the properties and behaviour of the cell surface must be accounted for when thinking about intracellular delivery approaches and the cell response. Furthermore, plasma membrane variability across cell types is a frontier that must be addressed in order to better understand how to target specific cell types.

\subsection{Defect Formation in Lipid Membranes}

Membrane disruption-based delivery approaches rely on various methods to nucleate and expand defects in the plasma membrane. Mechanistically, the most well-studied examples are electroporation and mechanical tension, partly due to their relative simplicity and ease of modeling and simulating. There are also a host of molecules that can bind to and disrupt membranes by chemical means. Here we provide a theoretical overview of the various mechanisms underlying membrane disruption. Further details on the individual disruption methods are discussed later in their respective sections.

\subsubsection{Mechanical \& Electrical}

Theories seeking to explain the energetics and formation of membrane disruptions by mechanical tension and electrical potential have arrived at similar models $\mathrm{s}^{419-421}$. At near-physiological temperatures, there is a finite probability of thermally-driven defect formation in lipid membranes. This defect formation is associated with random lateral diffusion of lipids, which is itself made possible by fluctuations in the void volume between lipid molecules. Thermally-driven defects take the form of a so-called hydrophobic pore, where a small gap opens up between hydrophobic tails (Figure 8A). Hydrophobic pores are thought to be at a local free energy maximum when the radius is around $0.5 \mathrm{~nm}$, which is slightly larger than the width of one lipid headgroup. From there, further lateral growth permits the rearrangement of hydrophobic tails into a hemispherical conformation at the edge of the pore. Once polar head groups face the aqueous solution, the pore becomes hydrophilic, thereby permitting the passage of water and becoming conductive to electrical charge. Hydrophilic 
pores are thought to occupy a local energy minimum and thus exhibit notable stability at a minimum radius of around 0.8 $\mathrm{nm}$, which is the width of around 2 to 2.5 lipid head groups ${ }^{422}$.

Over time the most likely scenario is that thermal fluctuations lead to closure of a hydrophilic pore. This happens through a reversal over the energy barrier represented by the hydrophobic pore, thus returning the membrane to a defect-free state. Conversely, there is the low probably of crossing the much larger energy barrier towards destruction of the whole membrane bilayer via infinite expansion of the pore. Increased input of mechanical tension or electrical potential into the system tilts the energy landscape towards the possibility of total destruction. Opposing pore expansion is line tension, an inward-directed force produced around the rim of a hydrophilic pore (Figure 8B, C). Under certain conditions, line tension has been observed to drive closure of micron-scale holes in giant vesicles and is directly related to the composition of the membrane, being boosted by the incorporation of cholesterol, for example ${ }^{423}$. The line tension may also be influenced by supporting structures, such as the actin cortex, which can be regulated by the cell to modulate membrane resealing ${ }^{424}$. 
A

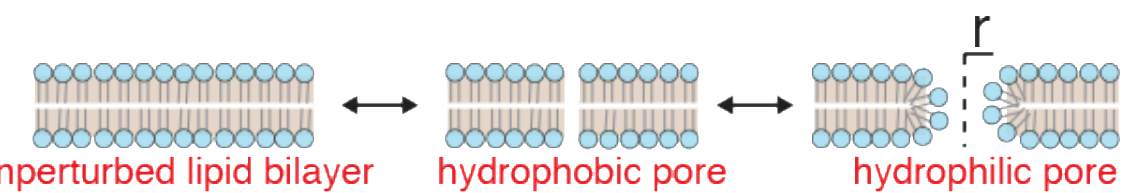
unperturbed lipid bilayer hydrophobic pore hydrophilic pore

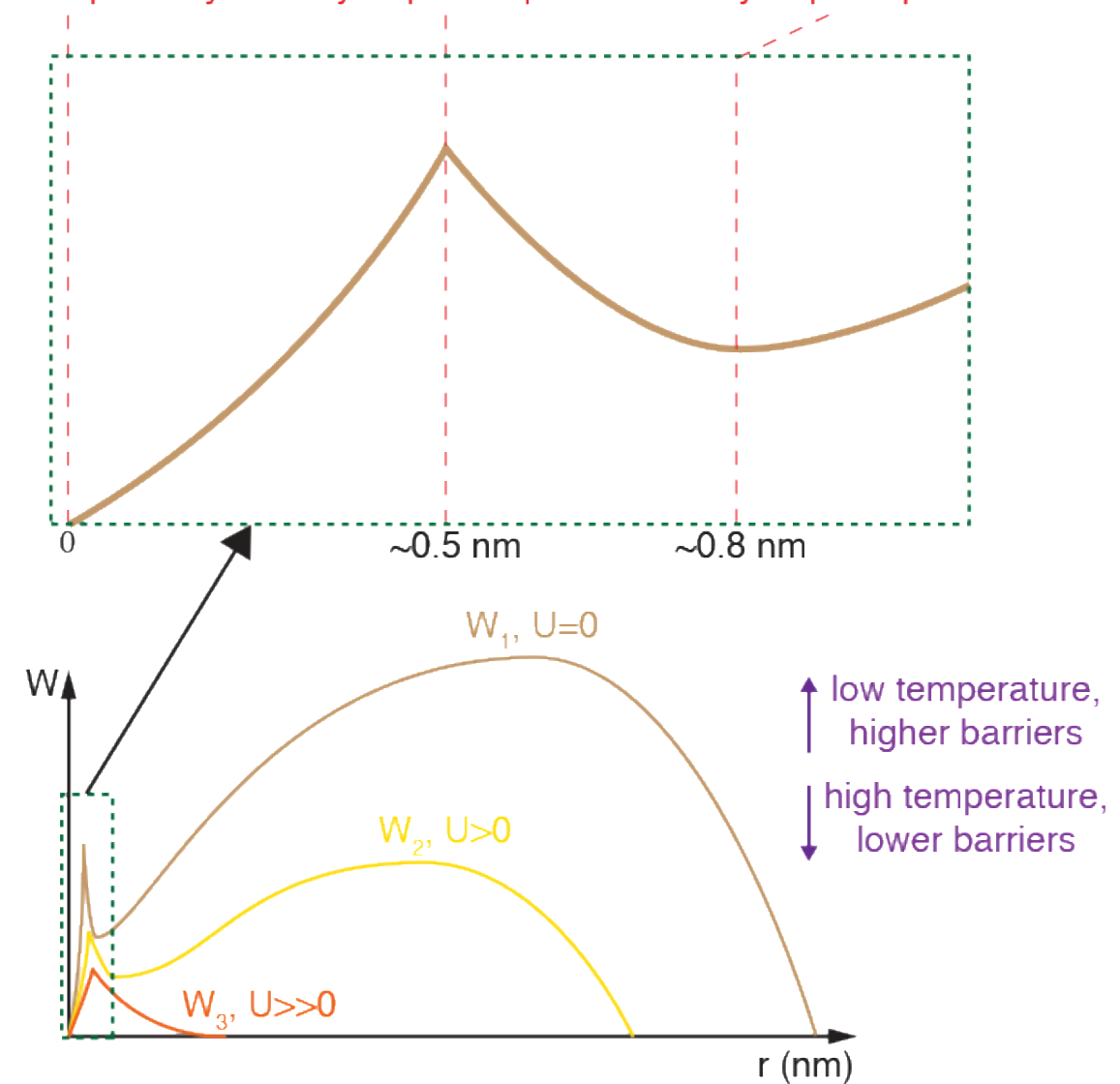

B Mechanical stress
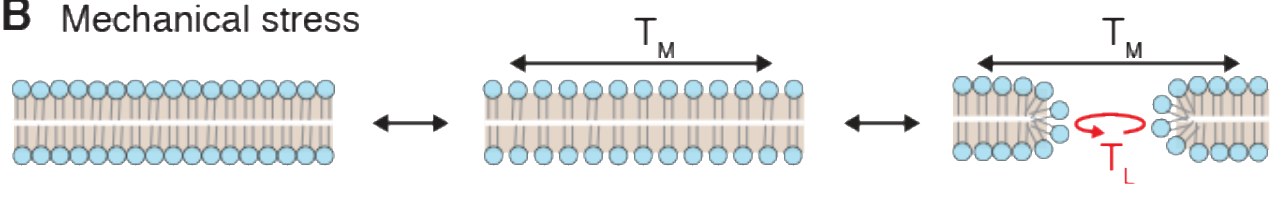

\section{Electroporation}
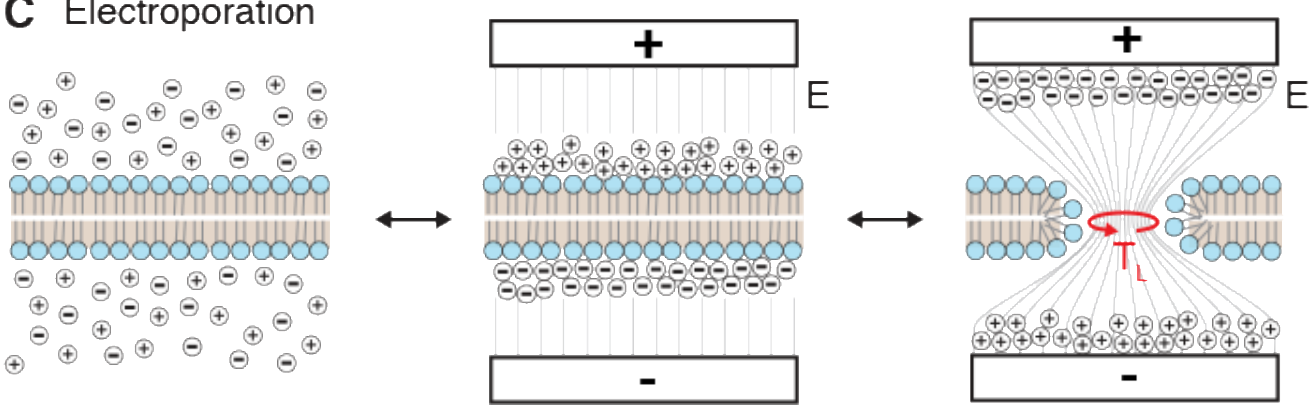

Figure 8. Theory of mechanical and electrical disruption of lipid bilayers according to energy landscape of defect formation. (A) Energy landscape according to hydrophilic pore theory. Energy is required to open up hydrophobic defects with radius $\sim 0.5$ nm. Further growth to a hydrophilic, toroidal pore with lipid head groups facing inward is associated with a local energy minimum at pore radius $\sim 0.8 \mathrm{~nm} . W_{1}$ represents the energy landscape at rest with no external mechanical or electrical input, $W_{2}$ (yellow) represents an intermediate mechanical of electrical stress, while $W_{3}$ (orange) indicates the effect of a large mechanical or electrical potential. Low temperature is synonymous with increased barrier heights while high temperature favors membrane destabilization. (B) Illustration of pore formation due to mechanical stress where the membrane is first stretched before pore formation. The applied in plane tension ( $\left.T_{M}\right)$ and the line tension $\left(T_{L}\right)$ within a lipid pore are diametrically opposed. (C) Illustration of pore formation due to application of electrical potential normal to membrane where $E$ is the electric field strength and $T_{L}=$ line tension within a hydrophilic pore. Hydrophilic pores are conducting, thus leading to relaxation of charge buildup and a reduction of entropy in the system. 
Thus, electroporation and mechanical disruption can be described according to the following. For a given cell, the combined effects of temperature, expansive electrical or mechanical forces, and line tension within the pores conspire to yield a population of hydrophilic defects of various sizes that can be modeled by a probability density function ${ }^{420}$. In real world numbers, biomembranes can generally withstand up to $3 \%$ mechanical area strain ${ }^{425}$ or $200 \mathrm{mV}$ electrical potential ${ }^{419}$ before persistent loss of membrane integrity occurs.

For mechanical membrane disruption in live cells that possess membrane reservoirs, further considerations are the deformation force and velocity. In the case of a nanowire impaling a small local area (Figure 9), it is evident that the deformation velocity, rather than the final deformation magnitude, determines whether a membrane disruption event occurs. Slow impalement of a nanowire allows sufficient time for lipids to flow in from the surrounding reservoirs (such as blebs and folds) to compensate for the local increase in membrane tension. On the other hand, rapid impalement does not give the system sufficient time to pull lipids from adjacent areas. Instead, the local membrane tension ramps up to a point where formation of a hole/pore becomes energetically favorable ${ }^{426}$. This helps to explain why larger contact forces are known to increase the probability of membrane penetration when deforming membranes with sharp AFM tips or nanowires ${ }^{427},{ }^{428},{ }^{429},{ }^{430,431},{ }^{432}$ - because the size of the impaling object and the force upon contact determine the local deformation rate, which is in turn related to the probability of membrane disruption. Further mechanistic studies are required to quantify these parameters in different types of cells. 


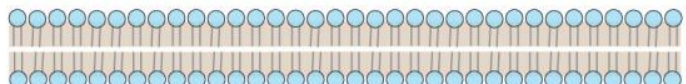

Slow

Impalement

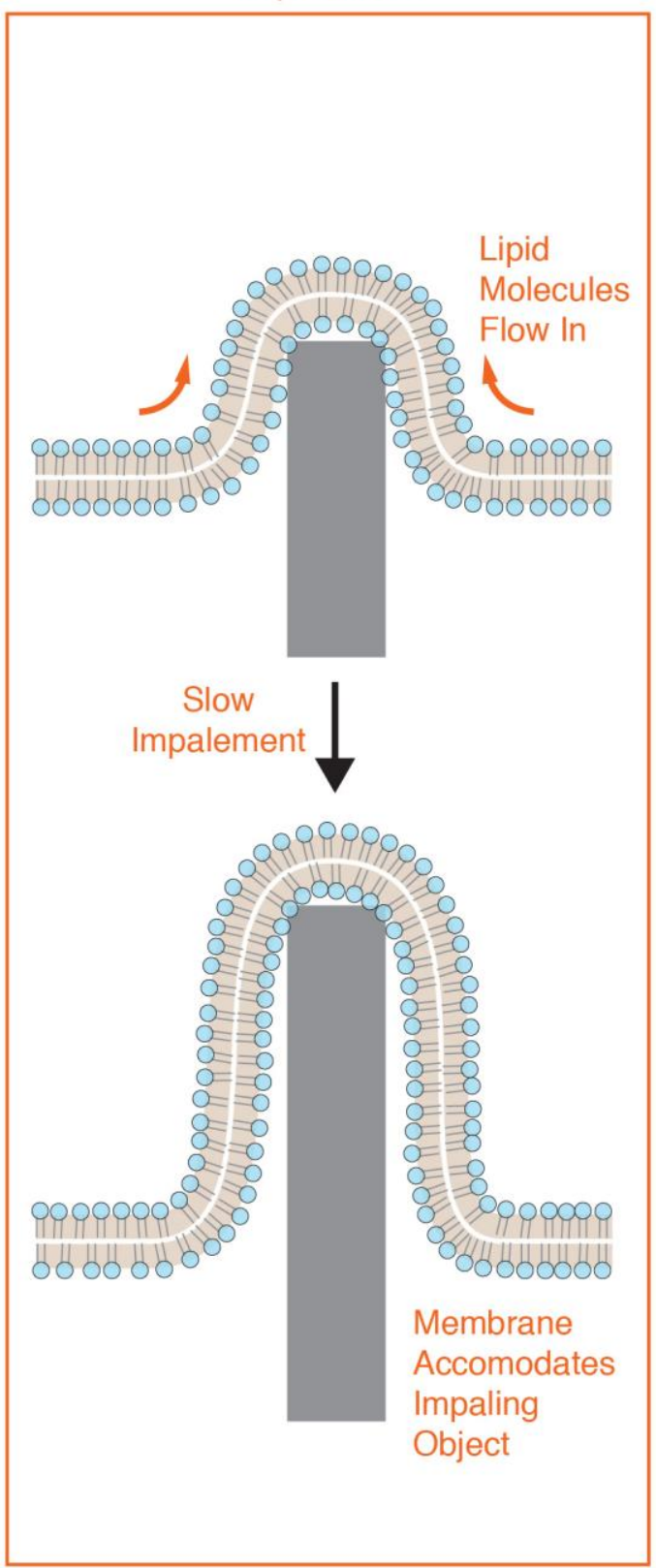

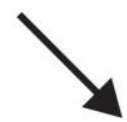

Rapid Impalement

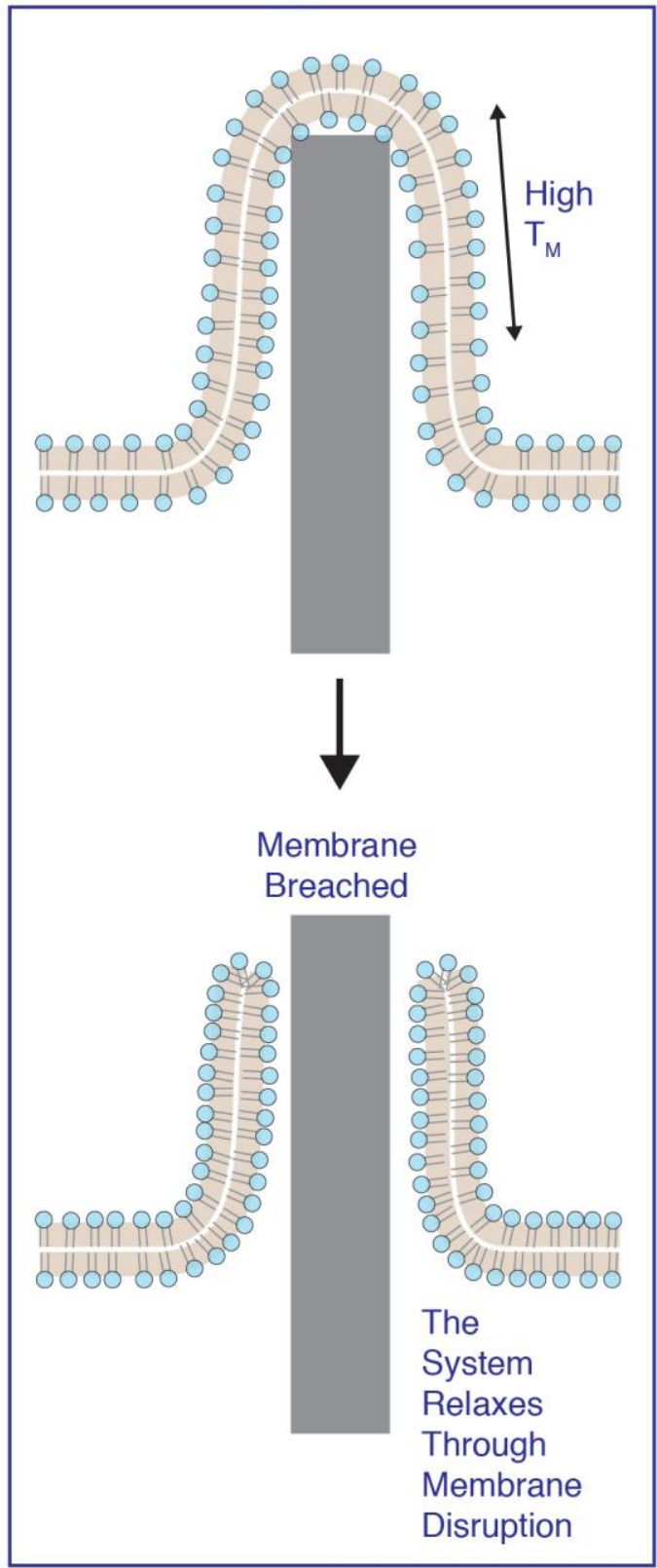

Figure 9. The dependence of deformation rate on mechanical membrane disruption. In the schematic, impalement with a solid nanowire only leads to membrane disruption in the case of rapid impalement. Slow impalement allows the system sufficient time for lipids to flow in from adjacent reservoirs. Rapid deformation ramps up the local membrane tension $\left(T_{M}\right)$ to a point where membrane disruption (hole formation) becomes energetically favorable. 


\subsubsection{Chemical}

Apart from physical insults, a host of chemical agents and effects can lead to membrane perforation (Figure 10). Chemical disruption of lipid barriers can occur through modification of constituent lipids, for example by oxidation, insertion of pore-forming proteins and peptides, and exposure to agents acting as detergents and surfactants. Because the modeling of these phenomena is more complicated, energy landscapes have not been described for most of these scenarios ${ }^{433}$. Instead, simulations are increasingly being exploited to capture, model, and visualize critical molecular events ${ }^{410,434}$.

Membrane disruption can proceed via localized chemical reactions, especially oxidation/peroxidation ${ }^{435}$ (Figure 10A). Simulations and experiments suggest that oxidized lipids exhibit distorted hydrophobic tails that decrease the lateral ordering of lipids and cause an increased area per lipid head. This, in turn, triggers bilayer thinning and variations in the lateral diffusion coefficients, which is associated with a decline in bending rigidity and increase in membrane deformation and permeability ${ }^{436-438}$. If these effects are sufficiently localized, it can lead to formation of membrane pores, as suggested by simulations ${ }^{410,438}$.

Another biochemical trigger for membrane disruption involves the exposure of bilayers to pore-forming agents, predominantly in the form of amphiphilic peptides or proteins (Figure 10B). Subunits associate with the membrane before assembling into a pore complex with variable size ranges, some being as large as several tens of nanometers ${ }^{439,440}$. Membrane disruption can also occur via detergents or surfactants (Figure 10C). These amphiphilic molecules integrate into the membrane and distort or buckle the bilayer, inducing conformational stresses that relax via pore formation and loss of bilayer integrity ${ }^{441,442}$. Detergents and surfactants thereby solubilize membranes in a concentration-dependent manner ${ }^{441,442}$. 

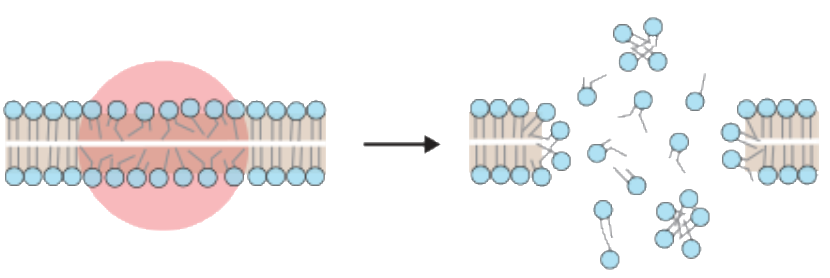

\section{B Pore-forming agents}

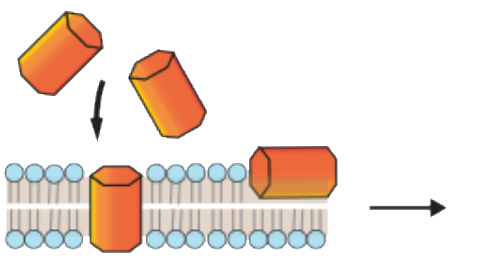

\section{Surfactants / Detergents}
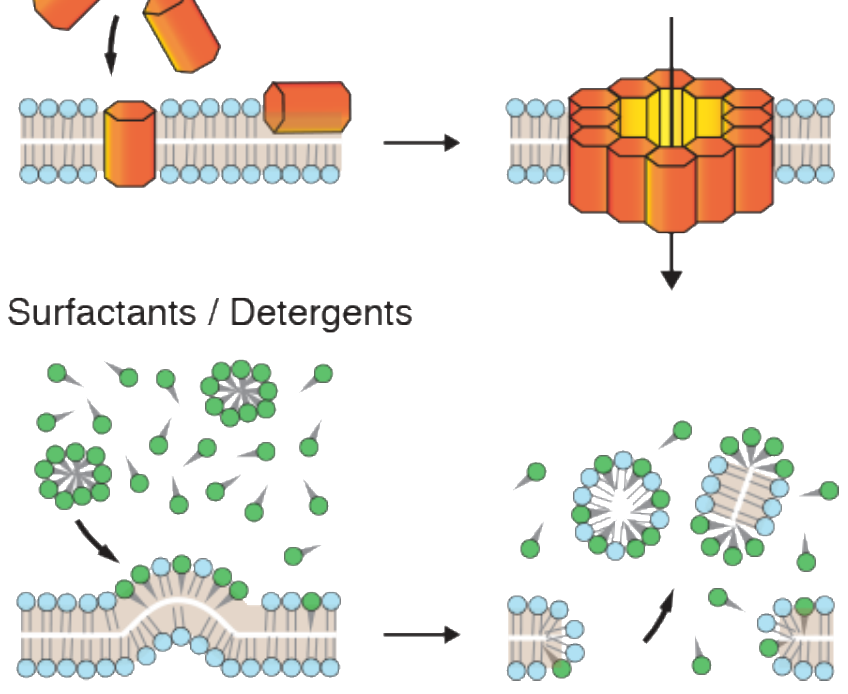

Figure 10. Chemical mechanisms for generating disruptions in lipid bilayers. (A) Localized chemical effects within a specific region (red circle) can lead to a change in the structure of lipid molecules, triggering their dissociation from the bilayer as free molecules or micelle-like formations. The dissociated molecules then leave behind a hole in the membrane. As an example, an intense laser pulse can break bonds within lipid tails or cause them to become distorted through unsaturation. (B) Pore-forming agents can interact with a membrane to assemble an oligomeric pore that allows the passage of cargo molecules and materials. (C) Surfactants and detergents can embed into the bilayer and induce curvatures that distort the membrane, leading to pore formation and loss of bilayer integrity.

\subsection{Cell Response to Membrane Disruption}

The previous sub-sections (4.1 and 4.2) covered the basic properties of cells and their membranes as well as mechanisms of how membranes can be disrupted. Here, we will examine how cells respond to membrane disruption (summarized in Figure 11). The first response is an urgent call to action to repair the breached membrane. If this is not accomplished rapidly, the cell will die. The second major response from the cell is after membrane repair, where it seeks to rebalance the homeostasis of its membrane composition and intracellular contents. This response takes place over minutes to hours and will determine whether the cell returns to its previous state, lives with permanent alterations, or dies through a form of programmed cell death. This section provides an overview of these events and the strategies and concepts associated with their manipulation in order to optimize membrane disruption-based intracellular delivery. 


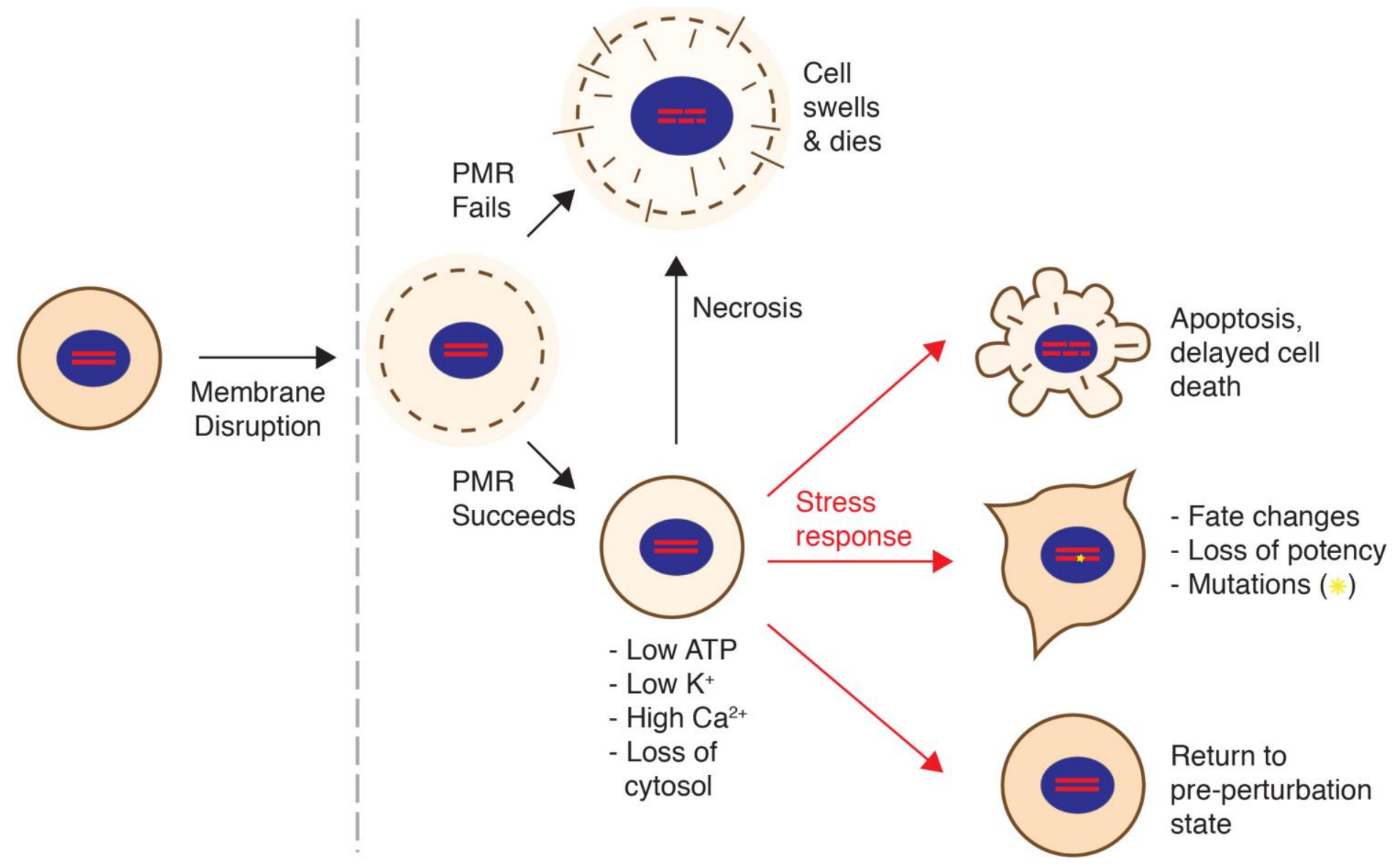

Figure 11. Cell response to membrane disruption. First, plasma membrane repair (PMR) engages within seconds to rescue the cell. If PMR fails the cell depolarizes, swells, and dies. Shown are the altered cytoplasmic contents that eventuate if membrane disruption is conducted in a physiological buffer. If PMR is successful, the cell is left in a perturbed state with loss of cytosol. Stress response guides the cell to return to the pre-perturbation homeostatic state or into apoptosis. In some cases, trauma or off-target damage involved with disruption-recovery cycle may cause mutations, fate changes, or loss of cell potency.

\subsubsection{Plasma Membrane Repair}

Plasma membrane resealing was thought to be a passive process until the mid 1990s when Steinhardt and colleagues discovered that rapid exocytosis drives plasma membrane repair ${ }^{443}$. In a mechanism analogous to neurotransmitter release, exocytosis was found to be triggered by calcium influx ${ }^{443}$. The calcium concentration difference between inside $(\sim 100$ $\mathrm{nM})$ and outside $(\sim 1 \mathrm{mM})$ is $\sim 4$ orders of magnitude, and serves as an acute alarm signal to detect and repair plasma membrane breaches ${ }^{444}$.

Since Steinhardt's discovery, a number of different mechanisms and pathways have been implicated in membrane repair.

The topic has been discussed in detail in recent reviews ${ }^{398,445-453}$. Overall, up to six repair variations have been proposed ${ }^{447}$. As illustrated in figure 12, the mechanisms include contraction, patching, plugging, exocytosis, internalization, and 
externalization $^{447}$. Multiple membrane repair processes may cooperate together to achieve resealing at timescales of anywhere from a few seconds to several minutes ${ }^{447}$. The type of membrane repair is thought to depend on factors such as environmental conditions (e.g. temperature, extracellular ions), size of the hole, and cell type.

Contraction

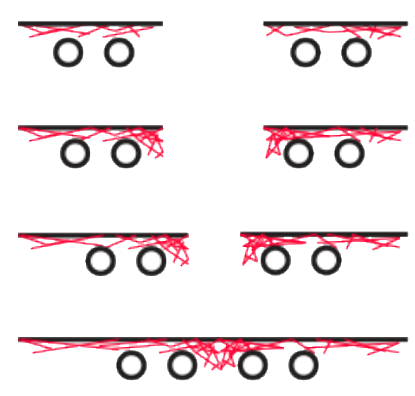

\section{Exocytosis}

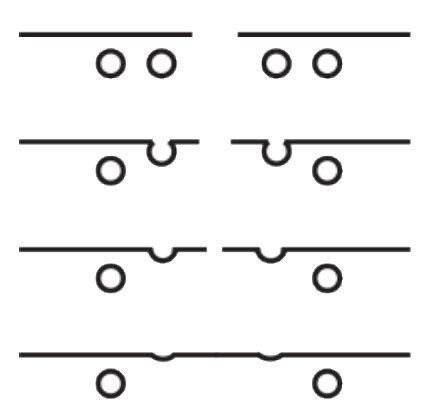

Patching

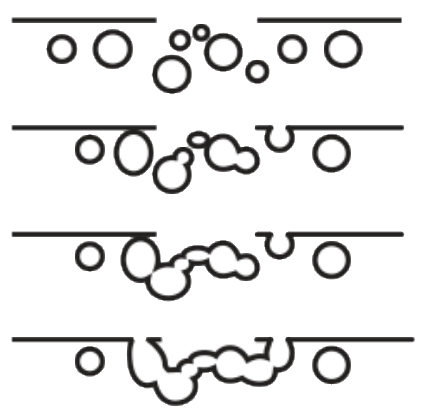

Internalization

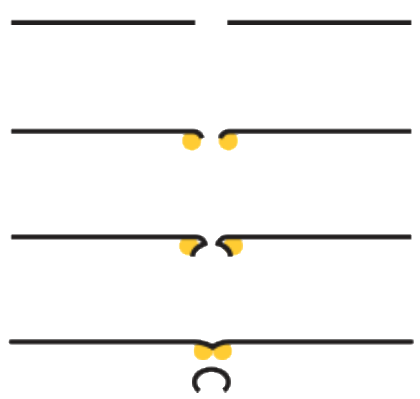

Plugging

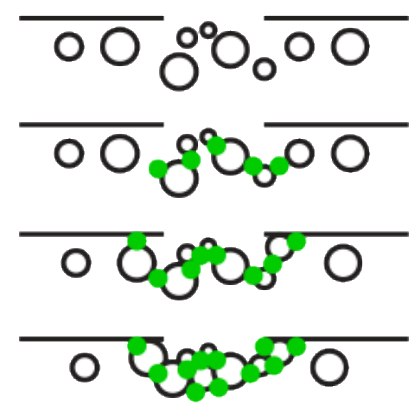

Externalization

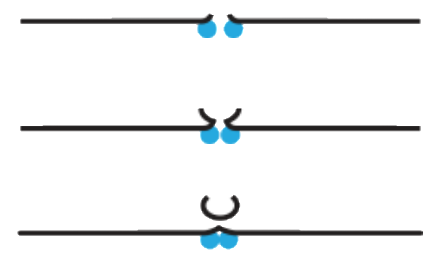

Figure 12. Proposed mechanisms of membrane resealing. In each case, the black line with gap represents the plasma membrane with a wound-induced hole and healing progresses from top to bottom. Black circles represent vesicles in the cell. Red lines in "Contraction" represent cortical cytoskeleton; orange dots in "Internalization" represent machinery powering endocytic invagination and pinching; blue dots in "Externalization" represent ESCRT machinery powering scission; green dots in "Plugging" represent proteins crosslinking membranous compartments. Adapted from ref ${ }^{447}$, Copyright 2015, with permission from Elsevier.

Studies have shown that, while large holes $(>0.2 \mu \mathrm{m})$ cause more immediate trauma in cells, they tend to be detected and repaired more quickly ${ }^{399,444,454}$. Rapid exocytosis, plugging, and patching are typical mechanisms that cells deploy to repair large holes ${ }^{444}$. For smaller disruptions, internalization through endocytosis or externalization through shedding serves to extract lesions into a disposable vesicle ${ }^{454-456}$. Very small holes, particularly from electroporation or lingering pore-forming toxins, can persist for longer durations and drain the cell of resources ${ }^{399,454,457,458}$. Thus, strategies to reseal small disruptions post-treatment should be of benefit to membrane permeabilization-based methods. In this regard, additives such as PEG, poloxamer 188 and other poloxamers (which may also exhibit antioxidant activity ${ }^{459}$ ) have shown potential as cell recovery agents ${ }^{98,460-465}$. Vitamin C, E and lipid antioxidants represent further options for promoting restoration of membrane integrity after delivery ${ }^{4661,467,468}$. 


\subsubsection{Cell Swelling}

Although rarely mentioned in the membrane disruption literature, cells tend to swell when their membranes are disrupted in physiological buffers. From Figure 7A one can see that $\mathrm{Na}^{+}$and $\mathrm{Cl}^{-}$will flow into a compromised cell while only $\mathrm{K}^{+}$ ions will exit. The net influx of osmolytes and osmotically obliged water causes cell swelling through a colloid osmotic effect, a process that goes hand-in-hand with depolarization of the cell membrane potential. Cell swelling has been observed with electroporation ${ }^{469-478}$, microinjection ${ }^{479}$, laser optoporation ${ }^{480-486}$, and exposure to cavitation ${ }^{487}$ or fluid shear $^{488}$. In these reports swelling usually reaches a maximum within 1-2 minutes of membrane disruption before plasma membrane repair and regulatory volume mechanisms synergize to bring cells back to normal volume.

Interestingly, cells can survive up to $50 \%$ volume increase and still recover ${ }^{469,470,475,477,485,489}$. Above that, the risk of instant death from bursting becomes imminent ${ }^{490}$. It is known that swelling activates specific stress signaling events ${ }^{491}$ and is a classic hallmark associated with necrotic cell death ${ }^{492,493}$. Inhibition of cell swelling has been explored as a strategy to improve cell function during and after membrane disruption-based intracellular delivery ${ }^{490}$. Related to this notion, cell shrinkage has been observed in electroporation conditions where the induced membrane disruptions are small and the buffer is composed of osmolytes that are too big to flow into the cells (for example, an isotonic large molecular weight PEG buffer ${ }^{475,494}$. Unlike physiological media, such a buffer is devoid of electrolytes that can flow into the cell, thus $\mathrm{K}^{+}$ and $\mathrm{Cl}^{-}$ions exit the cytoplasm along with water ${ }^{475}$. Such results demonstrate how buffer composition can affect the volume response of disrupted cells. Despite this, exactly how volume changes influence delivery performance and cell survival is yet to be extensively explored.

\subsubsection{The State of the Resealed Cell}

When the plasma membrane is compromised to allow cargo influx, there is uncontrolled exchange of molecules between the inside and outside of the cell. In standard physiological buffer (see Figure 7A), disrupted cells will sustain elevated $\mathrm{Na}^{+}, \mathrm{Cl}^{-}$, and $\mathrm{Ca}^{2+}$, and reduced levels of $\mathrm{K}^{+}$, ATP, metabolites, amino acids, proteins, and other intracellular contents (Figure 11). Even after plasma membrane integrity is fully restored, cells may still undergo necrosis, a type of cell death caused by irreversible disturbance of cellular homeostatic mechanisms ${ }^{399}$. In particular, dramatically reduced levels of 
ATP and potassium can trigger necrotic cell death due to deregulation of mitochondrial activity ${ }^{399}$. Necrotic cell death is almost indistinguishable from an initial failure to reseal, also being characterized by swelling and loss of membrane integrity $^{492}$.

Once the cell reseals its plasma membrane, homeostatic processes will kick in to restore intracellular contents. In this regard, the most critical molecules are thought to be ATP, potassium, and calcium ${ }^{399}$. ATP is a particularly crucial metabolite as it is the primary energy source for the cell. Studies have shown it can take from two ${ }^{495,496}$ to five ${ }^{497}$ hours to recuperate ATP levels after electroporation ${ }^{496}$ or treatment with pore-forming toxins ${ }^{495,497}$. Potassium has been observed to drop from $\sim 140 \mathrm{mM}$ to $\sim 20 \mathrm{mM}$ when cells are exposed to transient membrane damage ${ }^{498}$ and recovery can take from minutes to hours ${ }^{399}$. Influx of calcium can be viewed as a double-edged sword, although it assists the cell in detecting and repairing damage, excessive amounts can be toxic and lead to cell death ${ }^{456,499-501}$. High intracellular calcium serves as an activator of certain proteases, such as calpains, enzymes that promote apoptosis and degradation of cytoplasmic components $^{399}$.

Membrane disruption and recovery is often paralleled by cytoskeletal disruption and recovery. In particular, microtubule depolymerization has been observed upon electroporation ${ }^{502-505}$, mechanical wounding ${ }^{506,507}$, and exposure to pore-forming toxins $^{508}$. Microtubule depolymerization manifests locally around the wound sites due to calcium influx ${ }^{506,507}$. This is in congruence with the observation that electroporation does not alter microtubule structure in media devoid of calcium ${ }^{503}$. In standard calcium conditions recovery of microtubule integrity has been reported to take from minutes up to an hour ${ }^{503,504,507}$. In some cases, membrane disruption also appears to cause depolymerization of F-actin and intermediate filaments ${ }^{504,509}$.

\subsubsection{Stress Response After Membrane Disruption}

A number of secondary consequences occur as a result of the perturbations associated with membrane disruption ${ }^{399,495}$. For example, a decrease in cytosolic potassium can lead cells into a quiescent state characterized by autophagy (recycling of cellular building blocks), formation of lipid droplets to conserve energy, and arrest in global translation ${ }^{495}$. Time taken to restore intracellular potassium homeostasis correlates with the duration of these effects ${ }^{495}$. Furthermore, a drop in 
potassium is thought to be responsible for activation of MAP kinase stress response and proteolytic signaling cascades including the inflammasome, which in turn trigger downstream effectors including caspase proteins and the unfolded protein response ${ }^{399,510-513}$. In all systems tested so far, pore-forming toxins activate the three main MAP kinase stress response pathways: p38, JNK, and ERK ${ }^{399,514-517}$. Cell permeabilization in media containing high potassium prevents MAP kinase activation, indicating that potassium depletion is the key trigger ${ }^{518,519}$. MAP kinase and its downstream effectors promote cell survival and their inhibition appears to worsen cell death after membrane disruption ${ }^{518,519}$. Whether or not pre-activation of MAP kinase pathways can improve survival upon membrane disruption-mediated delivery remains unexplored.

Many of the characteristic responses elicited from pore forming toxins are also shared with electroporation and mechanical wounding, further reinforcing that membrane disruption is the key event ${ }^{399}$. In the early days of the field, McNeil and colleagues witnessed that expression of c-fos and NF- $\mathrm{BB}$, two transcriptional activators, are strongly and selectively increased in cells that suffered and resealed a mechanically-induced membrane disruption ${ }^{520}$. Detectable NF$\mathrm{\kappa B}$ and innate inflammatory responses were also measured in endothelial cells subject to membrane attacks with poreforming toxins ${ }^{521}$. Furthermore, mechanical micropuncture was found to activate MAP kinases, CREB1, and protein kinase C (PKC) to promote cell survival ${ }^{522-524}$. Interestingly, engagement of PKC is thought to prime cells to cope with future membrane wounding events ${ }^{522}$, and has similarly been observed upon SLO exposure ${ }^{512}$ and electroporation ${ }^{525}$. Recently, electroporation was also demonstrated to activate MAP kinase pathways ${ }^{526}$ and trigger transcriptional changes to support MAP kinase activity, membrane repair, and recovery from oxidative stress ${ }^{527}$. Complementing this picture, reports have emerged that electroporation triggers autophagy in response to nanosecond pulsed electric fields ${ }^{528}$.

A key implication in all of these findings is that activation of stress response pathways prioritizes cell survival and threat surveillance at the expense of proliferation and anabolism. If stress levels reach a critical threshold, cells trigger a shutdown response via apoptosis or other forms of regulated cell death ${ }^{492}$. In certain cell types delayed cell death has been a significant problem after electroporation, even when the initial membrane repair is successful ${ }^{490,529}$. In other cases, cell outcomes may be improved by adding inhibitors of apoptosis ${ }^{530}$. As more inhibitors of specific cell death processes become available, they may find use in such situations. 


\subsubsection{Manipulating Cell Response to Optimize Outcomes}

The concept of optimizing intracellular delivery by manipulating cell response has received sporadic attention over the past decades. As mentioned above, some positive results have been reported from supplementation with membrane healing polymers ${ }^{98,460-465}$ and/or antioxidants ${ }^{461,4661,467,468}$. Most of the work to date, however, has focused on engineering the permeabilization buffer. The electroporation literature, in particular, has extensively explored this aspect in an effort to optimize cargo delivery and cell health outcomes.

An analysis of 300 membrane disruption-based delivery papers analyzed in the process of compiling this review reveals four main types of buffers: 1) Na-rich "physiological" buffers such as PBS; 2) Cell media, which is essentially physiological buffer plus nutrients; 3) K-rich "intracellular" buffers; and 4) buffered sugar solutions. In our analysis, cell media (37\%) and Na-rich buffers (34\%) are the most popular, ahead of buffered sugar solutions (17\%) and K-rich buffers (9\%) (Table 2). Deconstructing these trends by modes of membrane disruption reveals further insights. For example, buffered sugar solutions have historically been used by the electroporation community to avoid electrolytic effects associated with higher conductivity salt-based buffers ${ }^{531,532}$. Their origins can be traced back to the beginnings of the field in the late 1970s and onwards ${ }^{502,531,533-538}$. In contrast, physical non-electroporation-based methods, such as mechanical wounding and optoporation, have mostly opted for cell media (58\%) or Na-rich buffers $(32 \%)$. Biochemical methods, of which detergents and pore-forming toxins are the main options, have been the most likely to experiment with K-rich “intracellular" buffers $(22 \%)$ but most often used their Na-rich counterparts (43\%). Biochemical permeabilization methods, which have less control over the timing of membrane disruption, seem more concerned with maintaining intracellular homeostasis through implementation of K-rich buffers ${ }^{539,540}$.

Table 2. Disruption buffers from papers analyzed in the process of compiling this review. Note that some papers use multiple buffers so percentages may not add to $100 \%$. Not specified is likely to be cell media or Na-rich buffer by default.

\begin{tabular}{|l|l|l|l|l|l|}
\hline $\begin{array}{l}\text { MODE OF MEMBRANE } \\
\text { DISRUPTION: }\end{array}$ & Cell media & $\begin{array}{l}\text { Na-rich } \\
\text { “physiological” } \\
\text { buffer }\end{array}$ & $\begin{array}{l}\text { K-rich } \\
\text { “intracellular” } \\
\text { buffer }\end{array}$ & $\begin{array}{l}\text { Buffered sugar } \\
\text { solution }\end{array}$ & $\begin{array}{l}\text { Not } \\
\text { specified / } \\
\text { other }\end{array}$ \\
\hline ALL & $37 \%(n=110)$ & $34 \%(n=101)$ & $9 \%(n=27)$ & $17 \%(n=52)$ & $12 \%(n=35)$ \\
\hline Electroporation & $28 \%(n=47)$ & $31 \%(n=52)$ & $7 \%(n=12)$ & $25 \%(n=42)$ & $17 \%(n=28)$ \\
\hline $\begin{array}{l}\text { Physical (non- } \\
\text { electroporation) }\end{array}$ & $58 \%(n=49)$ & $32 \%(n=27)$ & $7 \%(n=6)$ & $5 \%(n=4)$ & $7 \%(n=6)$ \\
\hline Biochemical & $28 \%(n=13)$ & $43 \%(n=20)$ & $22 \%(n=10)$ & $15 \%(n=7)$ & $0 \%(n=0)$ \\
\hline
\end{tabular}


K-rich buffers have been in use since the pioneering days of membrane permeabilization, with detergents ${ }^{541}$, electroporation ${ }^{542}$, and mechanical scraping ${ }^{543}$ being early examples. The argument in favor of these buffers is simple - by mimicking the intracellular composition as closely as possible, homeostasis and cell health should theoretically be maintained ${ }^{397,542,544}$. One study compared K-rich buffers to Na-rich ones, concluding that K-rich are superior for gene expression and cell recovery after delivery by mechanical membrane disruption ${ }^{545}$. A different investigation found that electroporation in buffers designed to match intracellular contents (with appropriate levels of ATP, GTP, amino acids, $\mathrm{K}^{+}$, $\mathrm{Mg}^{2+}$, and $\left.\mathrm{Ca}^{2+}\right)$ accelerated recovery of protein synthesis to within 5 minutes compared to from $>1$ hour for standard $\mathrm{PBS}^{546}$. Another group observed electroporation in intracellular mimicking buffer featuring high $\mathrm{K}^{+}, \mathrm{Mg}^{2+}, \mathrm{ATP}$ and glutathione promoted cell survival compared to cell media or PBS ${ }^{547,548}$. Furthermore, a cold-storage solution for organ transplants, containing high $\mathrm{K}^{+}, \mathrm{Mg}^{2+}$, and antioxidants, was reported to markedly improve survival of electroporated cells $^{549}$. Although most of the commercial electroporation buffers today are based on high sodium ${ }^{550}$, nucleofection offers a K-rich variant with high magnesium, ATP and glucose, which appears to be useful in treating primary human cells ${ }^{146}$. Whether K-rich intracellular mimicking buffers are under-utilized in membrane disruption-mediated delivery remains to be established.

Commercial electroporation systems such as nucleofection appear to have placed significant effort into optimizing proprietary buffers, mostly arriving at formulations featuring high $\mathrm{Na}^{+}, 10-20 \mathrm{mM} \mathrm{Mg}^{2+}$, strong pH buffering, and extra organic osmolytes ${ }^{550}$. Several academic groups have lifted the lid on these formulations and screened their effectiveness in an attempt to lower costs ${ }^{551-553}$. Indeed, several studies testing nucleofection buffers found only marginal benefits over $\mathrm{PBS}^{554}$ or cell media ${ }^{555}$, suggesting that the high cost of these proprietary buffers may not be justified. On the other hand, Biorad electroporation guides recommend more basic options such as cell media, strongly buffered Na-rich saline, or buffered sugar solutions ${ }^{556,557}$. Neon electroporation buffers seem to be based on PBS bolstered by extra pH buffering, sugar, and magnesium ${ }^{558,559}$. Interestingly, many of the electroporation-based pre-clinical or clinical studies simply use OPTIMEM (a popular cell media) in place of commercial electroporation buffers ${ }^{187,560}$.

Taken together, consistent benefits seem to be obtained by supplementing buffers with $\mathrm{Mg}^{2+}$ ATP, glucose, antioxidants, and by lowering or avoiding $\mathrm{Ca}^{2+}$. Additionally, strong $\mathrm{pH}$ buffering probably helps to negate potential detrimental effects of electrolytic reactions in the case of electroporation. Magnesium is slightly antagonistic to calcium, possibly helping to 
blunt some of the damaging aspects of calcium influx ${ }^{443}$. It is also a co-factor to hundreds of intracellular enzymes, including those involved in energy metabolism and stabilization of mitochondrial membranes ${ }^{561,562}$. ATP supplementation might be beneficial not only in preventing its loss from the cytoplasm ${ }^{397}$, but also in engaging extracellular receptors to activate 'purinergic' signaling, which is thought to prime cells against the danger of membrane disruption ${ }^{456,563}$. As an example of its potential benefits, electroporation buffers supplemented with ATP help to achieve faster gene expression after plasmid delivery ${ }^{564}$. Glucose is added to some buffer formulations ${ }^{146,551}$ and would tend to prevent cell energy depletion due to cytoplasmic leakage. Anti-oxidants have been reported to promote membrane repair and overall cell health by neutralizing reactive oxygen species (ROS) ${ }^{466-468}$. ROS may damage proteins, lipids, and nucleic acids, the latter of which can lead to mutations in DNA. Most of the optimized buffers also tend to contain little or no $\mathrm{Ca}^{2+}$. Although it is the prime trigger for membrane repair, precise studies have shown that only $\sim 5-20 \mu \mathrm{M}$ is required ${ }^{565-567}$. Normal extracellular $\mathrm{Ca}^{2+}$ levels $(\sim 1 \mathrm{mM})$ are probably only helpful when cells are returned back to standard media for final recovery.

Other potential supplements for augmenting cell health could be addition of Zinc ${ }^{568}$ and recombinant proteins that participate in membrane repair - such as MG53 $3^{568-571}$, annexins ${ }^{572}$, and ASMase ${ }^{573}$. Conducting cell membrane disruption and/or recovery in the presence of certain inhibitors may also be beneficial in guiding cell fate, however, has received little attention to date. Recombinant proteins and inhibitors might be worth using in clinical scenarios, such as a critical $e x$ vivo cell-based therapy.

Temperature is a core consideration for any in vitro cell treatment procedure, and deliberate membrane disruption is no exception. Despite this, there is no consensus in the literature on which temperatures are best for membrane disruptionbased intracellular delivery. An analysis of more than 300 membrane disruption-based delivery papers analyzed in compiling this review reveals three categories of temperature that have been used: 1 ) $\leq 4{ }^{\circ} \mathrm{C} ; 2$ ) room temperature (usually in the range $18-25{ }^{\circ} \mathrm{C}$ ); and 3) $\sim 37{ }^{\circ} \mathrm{C}$ (Table 3). The rationale for treating cells at $\leq 4{ }^{\circ} \mathrm{C}$ is that it can facilitate a preservative effect. Most stress responses and programmed cell death pathways are inhibited at $4{ }^{\circ} \mathrm{C}$, so unless the cell is killed by the treatment itself, the long-term cell survival may be improved. One detergent-based protocol credited low temperature and intracellular buffer as the two main factors increasing cell survival ${ }^{539}$. Biochemical protocols employed $\leq 4{ }^{\circ} \mathrm{C} 38 \%$ of the time compared with $11 \%$ for electroporation and $12 \%$ for physical non-electroporation. Low 
temperatures probably slow down membrane repair, but it also makes cells more resistant to disruption, particularly electroporation ${ }^{574,575}$. Furthermore, many pore-forming toxins do not assemble at $4{ }^{\circ} \mathrm{C}$, so a switch to warmer conditions can be used as a trigger to control the timing of permeabilization ${ }^{576}$.

Table 3. Disruption temperatures used in papers analyzed in the process of compiling this review. Note that some papers use multiple temperatures so percentages may not add to $100 \%$. RT denotes room temperature and varies considerably between publications from 18 to $25^{\circ} \mathrm{C}$. Not specified is most likely to be room temperature by default.

\begin{tabular}{|l|l|l|l|l|}
\hline MODE OF MEMBRANE DISRUPTION: & $\mathbf{3 7}{ }^{\circ} \mathbf{C}$ & RT $\left(\sim 18-25^{\circ} \mathbf{C}\right)$ & $<\mathbf{4}^{\circ} \mathbf{C}$ & Not Specified \\
\hline ALL & $22 \%(n=65)$ & $46 \%(n=139)$ & $16 \%(n=47)$ & $16 \%(n=49)$ \\
\hline Electroporation & $9 \%(n=15)$ & $67 \%(n=112)$ & $11 \%(n=19)$ & $13 \%(n=22)$ \\
\hline Physical (non-electroporation) & $34 \%(n=30)$ & $25 \%(n=22)$ & $12 \%(n=11)$ & $27 \%(n=24)$ \\
\hline Biochemical & $43 \%(n=20)$ & $13 \%(n=6)$ & $38 \%(n=18)$ & $6 \%(n=3)$ \\
\hline
\end{tabular}

The rationale for treating cells at room temperature is simply convenience, as it does not require any additional temperature control equipment. Membrane repair in mammalian cells seem to proceed quite normally at $25{ }^{\circ} \mathrm{C}$, as evidenced by studies of annexin-mediated resealing $500,566,577,578$. Electroporation protocols, in particular, favor room temperature (67\% of papers analyzed). Because Joule heating associated with electroporation can spike the temperature of a solution by up to $20{ }^{\circ} \mathrm{C}^{579}$, using a baseline of $37^{\circ} \mathrm{C}$ may be harmful to cells undergoing electroporation. On the other hand, the rationale for treating cells at $37^{\circ} \mathrm{C}$ is maintenance of physiological function. Most non-electroporation protocols choose to employ such physiological conditions, with biochemical procedures using $37{ }^{\circ} \mathrm{C} 43 \%$ of the time and physical non-electroporation 34\% (Table 3). Membrane repair and stress response are expected to be at their most efficient at 37 ${ }^{\circ} \mathrm{C}$.

\subsubsection{Semi-Intact Cells}

Although most applications of intracellular delivery by membrane permeabilization aim for a transient permeabilization from which the cell recovers, there are situations where a persistent ongoing permeabilization is opted for. Such systems have been referred as semi-intact cells ${ }^{580}$, semipermeable cells ${ }^{581}$ or perforated cells ${ }^{582}$. They involve irreparable disruption of cell membranes by mechanical ${ }^{580-582}$ or biochemical means ${ }^{583-590}$. Strategies such as low temperature and low calcium concentrations may be employed to deliberately prevent membrane resealing ${ }^{580}$. Efflux of cytoplasmic constituents follows, but the extracellular media is manipulated to "reconstitute" the cytoplasmic composition replete with desired inhibitors, activators, antibodies, metabolites, ATP-regenerating systems, and other macromolecules of interest ${ }^{586,587,591}$. 
Semi-intact systems have therefore been useful for functionally reconstituting intracellular processes while being able to manipulate the buffer. Apart from high potassium, such buffers usually contain high magnesium, low calcium, ATP at $\mathrm{mM}$ concentrations, strong buffering, and reducing agents or anti-oxidants. The major concern in using these methods is that it has been difficult to assess to what extent the semi-intact cells are a valid model for intact cells ${ }^{395}$. The concept of semi-intact cells illustrates the lengths biologists have pursued to address intracellular delivery and manipulation challenges. Despite their limitations, these reconstituted systems have been key in discovering fundamental mechanisms of secretory pathways and principles underlying trafficking of proteins, lipids, and nucleic acids between intracellular organelles, for example to decode the rules that govern nuclear import ${ }^{592,593}$. Semi-intact cells remain popular for certain types of studies, such as probing mitochondrial function in muscle cells $\mathrm{s}^{594}$.

\section{Intracellular Delivery by Direct Penetration}

Direct penetration mechanisms are utilized in the techniques of microinjection, particle bombardment, and nanowires / nanostraws. In each of these cases, penetrating elements provide direct access to the intracellular space. Microinjection is the classic embodiment of the direct penetration mechanism and was the first intracellular delivery technique to be introduced in the early 1900s. Particle bombardment and nanowires / nanostraws were invented in the late 1980s and early 2000s respectively. In this section we discuss the key details of each of these methods.

\subsection{Microinjection}

In 1911 Marshall Barber reported the invention of microinjection ${ }^{324}$. By pulling glass capillaries over a flame, Barber generated pipettes with sharp micron-sized ends suitable for injection into living cells. Combined with micromanipulators and pressure control systems, dual pipettes were demonstrated with holding, dissecting, extraction, and injection capabilities. The apparatus was used to extract nuclei from living amoebae, inject various fluids into cells, and deliver single bacteria into plant cells ${ }^{325}$. Barber rightly predicted that "The introduction of foods, poisons, stains, and fixatives is made possible and cells may be probed or dissected under high powers, methods which may be of use in the study of the structure, chemistry, and physiology of cells. Finally, materials may be withdrawn from one cell and injected into another, and it is possible that investigations on fertilization and heredity may be extended by this technic". After inventing microinjection, Barber trained others in its use before leaving the field ${ }^{325}$. In 1915, Kite used it to inject dyes 
into the cytoplasm of living animal and plant cells to investigate their permeability ${ }^{595}$. Chambers then introduced an improved version of the instrument in the early 1920s, featuring more precise micromanipulators and pressure control ${ }^{596}$.

As microinjection spread to other researchers, it was initially adopted by plant, developmental, and micro-biologists, for example to determine cytoplasmic $\mathrm{pH}$, introduce viruses into cells, or perform nuclear transplants ${ }^{597-600}$. Moreover, it became the basis for patch clamp and a host of similar pipette-based cell manipulation and analysis techniques ${ }^{325,601}$. As covered in section 2.2.5, microinjection has long been the dominant method for intracellular delivery of large cargo. It was used for the first nuclear transplants in $1952^{326}$, cloning frogs in $1958^{327}$, cloning mammals in $1997^{329}$, mitochondrial transplants in $1974^{336}$, chromosome transplant protocols in $1973^{333}$, intracellular delivery of sperm into egg cells in $1962^{331}$, and the first human pregnancies achieved by IVF in $1992^{332}$. More recent examples of large cargo delivery include micron-sized beads for intracellular microrheology analysis ${ }^{348,349,354}$, magnetic beads for application of forces $^{356,357}$, and silicon MEMS barcodes up to $10 \mu \mathrm{m}$ in $\operatorname{size}^{358}$.

Although microinjection was employed for large cargo delivery from the beginning, it took more than half a century for it find routine use for intracellular delivery of proteins, DNA, and other such biomolecules in animal cells. Purified proteins began to be injected into animal cells in the 1960s. The protein ferritin was introduced into amoebae to monitor its intracellular distribution ${ }^{80}$. Then mouse oocytes injected with bovine gamma globulin were shown as capable of developing into defect-free animals ${ }^{81}$. In 1972, the calcium sensitive protein aequorin was injected into the squid giant synapse to determine intracellular calcium ${ }^{602}$. Other studies in the 1970 s used fluorescently labeled proteins and dextrans to study nuclear permeability ${ }^{82,83}$ and autophagy ${ }^{84}$. Microinjection of peptides also emerged around that time ${ }^{603}$. Fluorescently labeled $\operatorname{actin}^{85}$ and alpha-actinin ${ }^{86}$ were injected into cells to visualize and elucidate their role in the cytoskeleton. A classic example where intracellular delivery of a protein led to discovery of its function was the case of vinculin $^{604}$. Microinjection of the uncharacterized protein labeled with fluorescent dyes was used to identify its role as a mediator of cytoskeletal adhesion assemblies by observing localization dynamics in living fibroblasts ${ }^{604}$.

Along with protein delivery, researchers began experimenting with microinjection of DNA and RNA. The first mRNA expression studies were carried out by microinjection from 1973 onward $240-242,605$. Viral DNA was injected into cells to investigate its ability to transform cells ${ }^{606}$. Recombinantly engineered plasmids were expressed in cells post-injection in 
$1977^{200}$. Several years later, Capecchi demonstrated that nuclear injection of plasmid DNA encoding thymidine kinase was successfully expressed in $50-100 \%$ of cells. Yet the same plasmid injected into the cytoplasm led to $0 \%$ expression in hundreds of cases ${ }^{202}$. Thus, microinjection studies were used to prove that plasmids must be delivered to the nucleus to undergo expression. In 1980, transgenic mice were successfully produced by microinjection of recombinant plasmid DNA into the nucleus of fertilized ooctyes ${ }^{607}$. Following the elucidation of antisense oligonucleotides in the 1980s, antisense RNA was injected into cells to inhibit protein expression in studies of developmental biology ${ }^{211,608}$. The Nobel Prize winning experiments that elucidated RNAi were performed by microinjection of double stranded RNA into C. Elegans cells in $1998^{215}$.

As illustrated in the above examples, microinjection is a versatile delivery platform, being able to deliver almost any cargo to most cell types. In its current form, microinjection is commonly performed with commercial systems fitted with glass micropipettes of diameter 0.3 to $1.0 \mu \mathrm{m}$ (Figure 13A). It is important to note that microinjection does suffer some degree of cell type-dependence. Small cells, such as blood cells with diameters less then $10 \mu \mathrm{m}$, can be challenging to microinject due to their small volume and poor tolerance for needle penetration ${ }^{609}$. For non-adherent or suspension cells an additional holding pipette is used to keep cells in place (Figure 13B), but this adds to the complexity and timeconsuming nature of the procedure. Researchers and clinicians most often use microinjection for experiments or procedures involving single cells or small batches of cells where high fidelity of intracellular delivery is ensured. For example, due to its accuracy and control, microinjection has been a routine technique to achieve human pregnancies by in vitro fertilization.

A

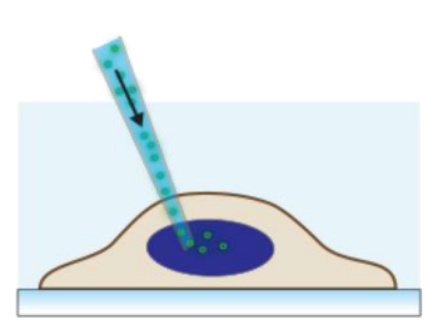

microinjection

(adherent)
B

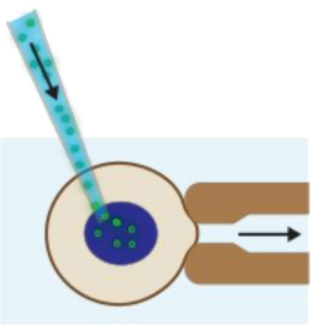

microinjection

(dual pipette)
C

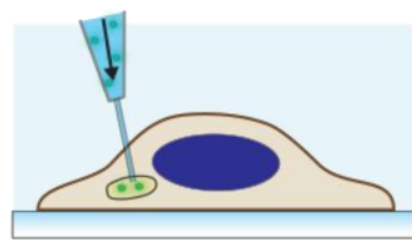

nanopipette injection (nanoinjection)

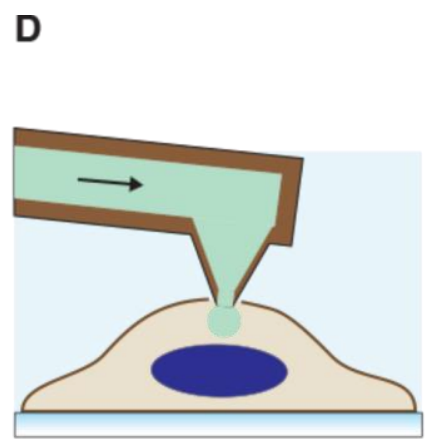

FluidFM

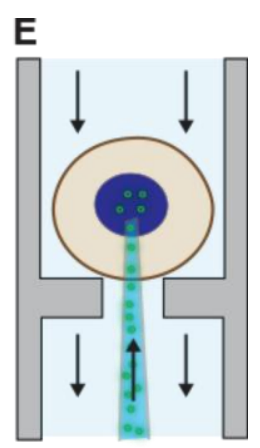

microfluidic microinjection

Figure 13. Intracellular delivery via microinjection. (A) Depiction of an adherent cell being microinjected with a glass micropipette. (B) Microinjection of a suspended cell that is held in place by a secondary holding pipette. (C) Nanopipette injection (nanoinjection) 
where the penetrating aperture consists of a nanotube. In this illustration an intracellular organelle is being injected. (D) Use of a hollow AFM cantilever to inject cells (FluidFM). (E) Microfluidic microinjection where a cell is pushed onto a sharp micropipette via flow. Pressure is then generated in the micropipette to deliver fluid into the cell. Reversing the flow of the main microfluidic channel can be used to eject the cell.

\subsubsection{Advances in Technical Precision of Microinjection}

Significant advantages of microinjection include precise control of dosage volume and injection location. In one innovation, organelle targeting was demonstrated with an ultra-fine tip and femtoliter to attoliter volume control provided by a galinstan expansion syringe ${ }^{610}$. Using a tip diameter of $\sim 100 \mathrm{~nm}$, researchers were able to inject single chloroplasts in plant cells without dissipation of intracellular turgor pressure or untoward impact on other cellular structures (Figure 13C). Exploiting a different mechanism of volume control, an electrochemical attosyringe with tip diameter of $100-400$ $\mathrm{nm}$ achieved picoliter to attoliter volume control ${ }^{611}$. Such fine electrochemical control of fluid motion allowed the accurate dispensation of precise volumes from the fabricated 'nanopipette' ${ }^{611}$. Another group employed carbon nanotubes as the pipette. The device, termed a nanotube endoscope, demonstrated delivery of fluorescent molecules to subcellular localizations at a resolution down to $100 \mathrm{~nm}^{612}$. Recently a system that takes advantage of electrophoretic cargo propulsion was claimed to provide higher cell viability post-injection ${ }^{613}$. This was based on $100 \mathrm{~nm}$ diameter nanoinjector that drives materials into cells via electrophoretic force rather than bulk pumping of fluid ${ }^{613}$.

Some interesting adaptations to the microinjection concept have been produced by modifying atomic force microscope (AFM) systems to allow injection or extraction ${ }^{614}$. One technology, called FluidFM, was first demonstrated by the use of hollow cantilevers with fluid control capabilities for force-controlled injection of soluble materials into cells (Figure 13D) ${ }^{615}$. AFM force feedback was reported to enable unprecedented control of contact force thereby facilitating the determination of required penetration forces ${ }^{614}$. Recently, the FluidFM system has been used for non-destructive sampling from cells for time-resolved analysis of molecular composition ${ }^{616}$ and metabolite profiles ${ }^{617}$. It also features the precision

to deliver or extract from the nucleus ${ }^{616,618}$. In a similar approach to FluidFM, another group used a scanning probe system to detect cell surfaces and provide voltage pulses to deliver fluorescent dyes into individual cells ${ }^{619}$. 


\subsubsection{Attempts Toward Higher Throughput Microinjection}

The primary limitation of standard microinjection is the serial, low-throughput, and tedious nature of the process. Even an experienced operator is limited to approximately one successful injection per minute. An early attempt at automated microinjection was published in 1988, with a reported throughput of 1500 cells per hour when performed on adherent cells $^{620,621}$. For unknown reasons, this innovation was not widely adopted. Other attempts at high-throughput microinjection include a vacuum-enabled embryo holding array, which allows injections based on robotic motion control and image recognition by computer vision processing ${ }^{622}$. The reported throughput of 15 cells per minute was demonstrated to yield a high survival rate (98\%) for large non-adherent cells such as embryos and oocytes. In a semi-automatic approach, a micro-robotic system achieved up to 25 injections per minute on adherent endothelial cells ${ }^{623}$. The human operator selects injection destinations through mouse clicking on a computer screen and the system executes with a purported survival rate of $>95 \%$ and a success rate of $>80 \%{ }^{623}$.

Apart from automation, microfluidic systems have been explored to address microinjection throughput challenges. Adamo and colleagues reported a microfluidic version of microinjection that works by suction of cells onto a $0.5 \mu \mathrm{m}$ diameter hollow-tip glass needle embedded in a PDMS device (Figure 13E ${ }^{624}$. Several picoliters of liquid could be injected into the

cell in $\sim 0.5$ seconds followed by flow reversal of the main channel to dislodge the cell ${ }^{624}$. The cell could be then routed through an exit channel for collection after delivery ${ }^{624}$. Problems with cell clogging and fouling from biological debris, however, prevented the device from achieving consistent operation. A follow-up concept sought to address this problem with high-pressure fluid jet injection, but synchronization of jet firing with cell passage at the injection nozzle presented a significant unsolved challenge ${ }^{625}$.

\subsubsection{Microinjection Summary}

Microinjection was the first intracellular delivery method to be invented and has been in use for over a hundred years. It is a method of choice to deliver almost any cargo, whether large or small, to single cells or small groups of cells $(<100)$. Despite technical advances, however, the intrinsic low-throughput of microinjection remains a serious limitation for the 
great majority of applications. An effective platform for high-throughput microinjection would be ground-breaking but remains elusive.

\subsection{Penetrating Projectiles (Biolistics)}

Biolistic intracellular delivery employs high-velocity microprojectiles to deliver nucleic acids and other substances into intact cells and tissues. The microprojectile particles are accelerated to sufficient velocity by use of a gas shock wave, which can be generated by various means. For example, it may be derived by chemical explosion (gunpowder), highvoltage electronic discharge, release of pressurized inert gas, or helium shock generated via a rupture-membrane mechanism ${ }^{626-628}$. The gas shock wave is used to either 1) accelerate a macroprojectile into a stopping plate to dislodge adhered microprojectiles, or 2) blast the microprojectiles off the surface of screen or the inside of a barrel ${ }^{626-628}$. Particles then collide with target cells, busting through the plasma membrane and releasing cargo molecules from their surface into the cytosol (Figure 14A). Biolistic intracellular delivery has been referred to as the biolistic process, ballistic particle delivery, microprojectile bombardment, and, in certain embodiments, the 'gene gun'.

Biolistic delivery came onto the scene in 1987, where it was first invented for the purpose of DNA transfection in plants $^{394}$. In the late 1980s and early 1990s it was adapted for transfection of diverse microorganisms (yeast, fungi, algae, bacteria), many of which are difficult to transfect with other methods ${ }^{626,627}$. It was also attempted for transfection of an assortment of animal cells and tissues. Given the limited penetration distance of particle bombardment into tissue, it was initially tested with cell cultures in vitro and skin or exposed tissue sections in vivo ${ }^{627,629-631}$. For cell cultures in vitro, particles are sprayed down on a monolayer of adherent cells or a thin dispersion of suspension cells. As a rule of thumb, particle sizes should be no larger than one tenth the size of the target cells ${ }^{626}$. Heavy metal particles are durable, dense, and do an excellent job of maintaining the momentum needed for breaching the plasma membrane ${ }^{626}$. Particles used in biolistic systems tend to be tungsten (occasionally toxic), gold or silver (less toxic) and in the size range 0.5 to $2 \mu \mathrm{m}^{632}$. 


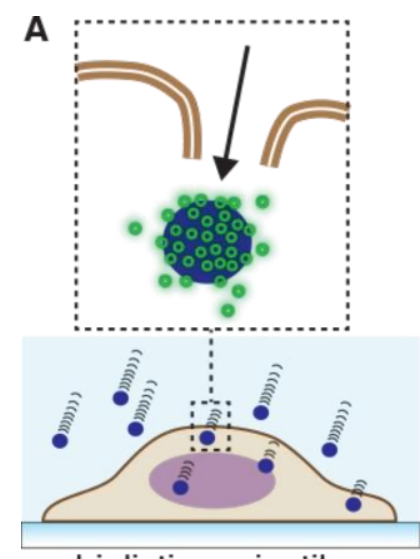

biolistic projectiles

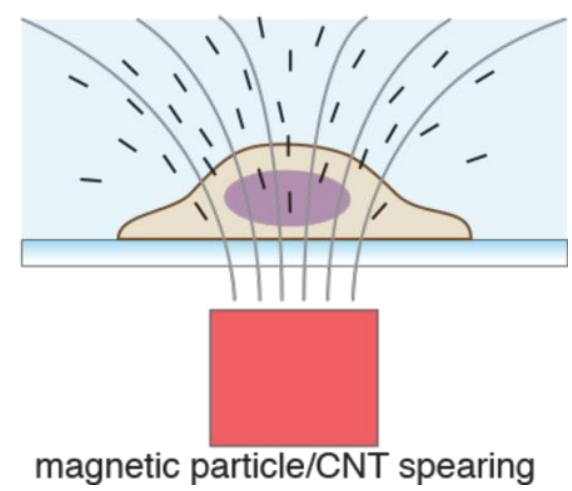

Figure 14. Intracellular delivery via penetrating projectiles. (A) Biolistic projectiles consisting of metal beads are propelled towards a cell with enough force to burst through the plasma membrane. The metal beads are coated with cargo, which then releases inside the cell. Inset shows an example of a single cargo-covered bead disrupting the plasma membrane. (B) A magnetic field is used to attract magnetically functionalized particles (such as modified CNTs) through the plasma membrane into the target cell for delivery of attached cargo.

\subsubsection{Cell Type Applicability}

Several early studies on biolistic intracellular delivery sought to test applicability to hard-to-transfect mammalian cells, particularly immune cells, blood cells, and neurons. It was shown that both adherent and suspension cell cultures can be transfected with plasmid-coated metal particles. Transfection efficiencies in $\mathrm{T}$ cells were reported to be a maximum of $2 \%{ }^{633}, 6 \%{ }^{634}$ and $3 \%{ }^{635}$ respectively. In ex vivo HSCs, efficiencies were either not directly reported ${ }^{636}$ or achieved a maximum of $6 \%$ alongside $75 \%$ viability ${ }^{637}$.

Both adherent and suspension tumor cells could be transfected with the plasmid-coated $\sim 1-2 \mu \mathrm{m}$ gold particles shot from a helium driven gene gun ${ }^{638}$. But this study reported only the yield of expressed protein and not percentage cells transfected $^{638}$. A comparison across many cell types observed from $2 \%$ to $40 \%$ transfection efficiency depending on cell line $^{639}$. Upper limits of 30-40\% were obtained for common adherent cell lines such as prostate cancer cell lines ${ }^{640}$ or HEK $^{-}$ cells $^{628}$. Due to the random spray of particles over a cell sample, it is unlikely that particles will penetrate the nucleus of every cell to deliver DNA cargo for subsequent expression. For large cells that 'catch' many particles, such as myotubes, $20-70 \%$ transfection can be obtained ${ }^{641}$.

Some reports claim biolistic delivery is a highly efficient DNA transfection method in mammalian cells ${ }^{642}$. However, it is only efficient in its use of DNA, not necessarily in the percentage of cells treated. It has been estimated that about 200 
plasmids are delivered per gold particle ${ }^{643}$. Hence, the amount of DNA required to produce a given yield of protein can be very efficient ${ }^{644}$. In comparison, electroporation and lipid reagents are highly wasteful of DNA (most is lost in solution) but produce a large proportion of cells that are successfully transfected. Empirical optimizations aimed at improving the performance of biolistic delivery in animal cells identified parameters such as size of the particles, the target distance, extent of vacuum, and the size of the cell culture plate ${ }^{626,642}$. Tuning of such parameters, however, has yielded limited success. Thus, after an initial period of excitement surrounding biolistic transfection, electroporation and viral vectors have risen to prominence as the preferred methods for hard-to-transfect cells such as HSCs and immune cells.

One area where the biolistic process gained notable traction is delivery to neurons and organotypic brain slices ${ }^{628,645-650}$. Neurons are regarded as very difficult to transfect with conventional methods. Early studies of plasmid delivery into neural cell cultures have achieved transfection efficiencies of $<2 \%{ }^{651}, 2-8 \%$, depending on the type of neurons ${ }^{648}$, and up to $10 \%{ }^{647}$. Although most of the protocols hover below $10 \%^{647-651}$, maximums of $20-30 \%$ were reported with a highly optimized protocol ${ }^{628}$. As the alternatives are generally poor, such performance has proven sufficient to carry out several interesting studies in neuronal cultures ${ }^{628}$. Particle bombardment has been particularly useful in organotypic brain slices, where alternative methods such as electroporation lack access to cells ${ }^{650}$.

After three decades of experimentation, the main cells and tissues that have proven amenable to biolistic delivery are: 1) plants, especially for generating transgenic $\operatorname{crops}^{394}, 2$ ) neurons and organotypic brain slices ${ }^{628,645-650}, 3$ ) microorganisms that are difficult to transfect with other methods ${ }^{626}$, 4) inoculation of skin or muscle for applications such as vaccination $^{629,652-654}$. Efficient DNA immunizations against influenza have been achieved by using a gene gun to deliver DNA-coated gold beads to the epidermis in mice and chicken ${ }^{655}$. Projectile bombardment is suitable for these applications because the immunization is thought to be effective even when only a small fraction of cells are transfected. For intracellular delivery to skin cells, there is a notable trade-off between power, size and number of bombarding particles, and cell viability ${ }^{656}$. 


\subsubsection{Cargo Applicability}

The biolistic process has been used mainly for plasmid transfection. Additionally, it has proven particularly advantageous for delivery of larger DNA vectors such as cosmids and artificial chromosomes ${ }^{643,651,657}$. In the early 2000 s researchers successfully experimented with attaching dyes and indicators to the projectiles ${ }^{658-661}$, mostly for delivery to neural cell types and brain slices. Following that, mRNA and siRNA were shown to be feasible for transfection into a variety of cells and organisms ${ }^{662-666}$. Biolistic methods have also been deployed for delivery of large beads to the cytoplasm for analysis of intracellular mechanical properties. In these cases cytoplasmic microrheology was assessed by monitoring fluctuations in polymer beads within the cytoplasm ${ }^{350-353}$. In a one example, $\sim 1 \mu \mathrm{m}$ melamine particles coated with PEG were shot into HeLa cells to study glassy dynamics in the $\operatorname{cytosol}^{355}$. More recently, protein delivery has been demonstrated with particle bombardment, first in plants ${ }^{667,668}$, then in mammalian scenarios ${ }^{669}$. Protein delivery protocols have been further adapted for biolistic Cas9 RNP delivery ${ }^{670}$. RNPs were dried onto gold particles and fired into immature wheat embryos to produce gene-edited crops $^{670}$.

\subsubsection{Biolistic Systems \& Variations}

Biorad is the main supplier of commercial biolistic delivery platforms. The gene gun is a hand-held device with a 'point and fire' mode of operation. The more advanced biolistic systems employ a vacuum chamber for higher momentum and evenness of microparticle dispersion. The vacuum systems are typically used for in vitro applications where the sample is more amenable to manipulation. A major weakness of biolistic delivery is the damage that high velocity particles can cause to cells. This is one of the reasons why it is popular for plants, which have stiff cell walls that can tolerate harsh mechanical impacts ${ }^{626}$. Damage from gene guns has been identified as a key limiting factor in treatment of cell cultures in vitro, as well as skin and muscle tissues ${ }^{671}$. In general, damage is intensified as the projectile diameter increases relative to the cell size. Nanoparticles of $\sim 40 \mathrm{~nm}$ have been tested with the biolistic method and found to provide better cell survival, especially with small cells ${ }^{672} . \sim 80 \mathrm{~nm}$ silver nanoparticles were also evaluated and found to transmit less damage to cells $^{673}$. In both cases delivery efficiency of cargo was not reported to be adversely compromised by using nanoparticles instead of micro-sized beads, and the higher surface area to volume ratio of nanoparticles could be a potential advantage. Overall, implementation of biolostic particle bombardment approaches to animal cells requires a number of empirically 
determined parameters to be optimized. These include size of particles, distribution, density, impact speed and loading technique ${ }^{396}$.

In a nano-inspired adaptation of the projectile delivery approach, Cai et al. used DNA-carrying nickel-embedded nanotubes propelled by magnetic fields to "spear" cells ${ }^{674}$. Nanotubes in solution were attracted to a magnet placed underneath the substrate, thus creating the driving force for penetration of cells placed on the substrate (Figure 14B). With this method they demonstrated efficient GFP expression in primary mouse B cells and neurons with minimal cell death ${ }^{674}$. More recently, magnetic nanospears composed of $\mathrm{Au} / \mathrm{Ni} / \mathrm{Si}$ with dimensions of $\sim 5 \mu \mathrm{m}$ long and $<50 \mathrm{~nm}$ diameter have been used to transfect adherent cell lines with absorbed plasmids at $>80 \%$ efficiency ${ }^{675}$. Thus, for in vitro and ex vivo applications, smaller projectiles that minimize damage to cellular structures may present an opportunity for projectilemediated intracellular delivery.

\subsubsection{Penetrating Projectiles (Biolistics) Summary}

Since its introduction in 1987, a range of different types of cargo-laden projectiles have been fired into cells for the purpose of transfection and intracellular delivery. The field is witnessing a trend towards smaller, less damaging projectiles and attempts to improve the consistency of cell treatment. If limitations around 1) cargo and cell type applicability, and 2) consistency of cell treatment can be overcome, biolistic intracellular delivery has the potential to break new ground.

\subsection{Nanowires \& Nanostraws}

Nanowires, also referred to as nanoneedles, nanosyringes, nanofibers and high aspect ratio nanostructures, are thin elongated structures typically with diameters of hundreds of nanometers or less and lengths on the micrometer scale. For intracellular delivery at high-throughput, nanowires are fabricated into vertically aligned arrays that can interfaced with thousands of cells. Nanostraws are hollow versions of nanowires, which can deliver fluid from an external reservoir directly to the intracellular space. 
Intracellular delivery by penetrating nanowires was first demonstrated by McKnight and colleagues in the early $2000 \mathrm{~s}^{109,676}$. They produced conical spikes of $6-10 \mu \mathrm{m}$ in length, tip diameters of $20-50 \mathrm{~nm}$ and base diameters of $\sim 1 \mu \mathrm{m}$.

These carbon/nitrogen-based structures were grown via plasma-enhanced chemical vapor deposition off nickel-spotted silicon wafers ${ }^{109}$. The first cargo to be delivered with them was DNA plasmids, which were physically absorbed or covalently tethered to the tips of the conical nanowires. $\mathrm{CHO}$ cells were then forced against the array by centrifugation at $600 \mathrm{~g}$ followed by sandwiching against an opposing substrate. This provided an active force for penetration, which proved to be necessary for efficient transfection in this system (Figure 15A). The nanowires were able to achieve nuclear penetration as evidenced by rapid GFP expression. Interestingly, GFP plasmids that were physically absorbed to the nanowires were passed on to cell progeny while covalently tethered plasmids were not, suggesting that the former dissociate in the cell interior while the latter are able to mediate gene expression even though they remained attached to the nanostructures. In follow-up studies the same researchers extended the application of nanowires to include spatially indexed substrates for long-term cell tracking ${ }^{676}$ and simultaneous delivery of multiple different plasmids ${ }^{677}$.

A

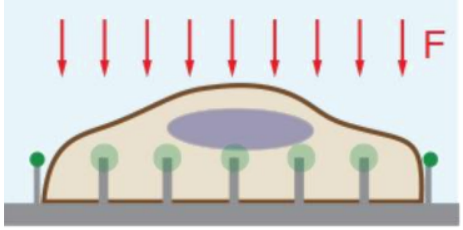

nanowires

+ active force
B

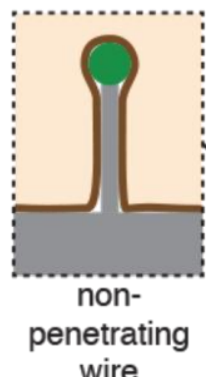

wire

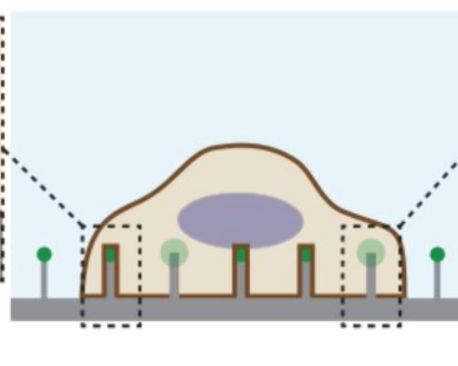

nanowires

(passive)

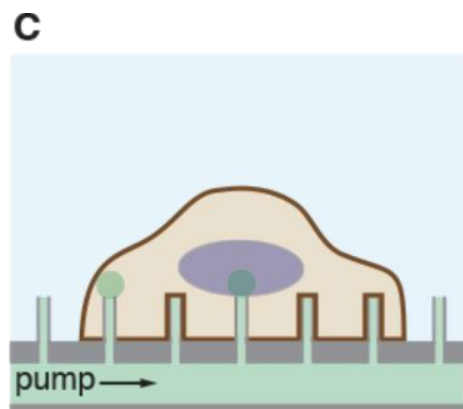

nanostraws

(passive)

Figure 15. Intracellular delivery via penetrating nanowires/nanoneedles and nanostraws. (A) Cell pushed onto an array of nanowires with active force (F), such as centrifugation. The number of penetrating nanowires increases given the same needles as in $B$. (B) Passive settling and adhesion of a cell onto an array of nanowires coated with cargo molecules at the tip (green). In this case some nanowires may penetrate through the plasma membrane into the cytosol to release their contents inside the cell (green cloud). (C) Hollow nanowires (nanostraws) used for intracellular delivery by pumping cargo from a fluid reservoir connected to the nanostraws.

\subsubsection{Expanding the Repertoire of Deliverable Cargo}

As mentioned above, nanowire arrays were first used for DNA transfection ${ }^{109,676-678}$. Since then delivery of siRNA ${ }^{110,679-}$ ${ }^{681}$, proteins ${ }^{110,427,680,682}$, molecular beacons ${ }^{683}$, quantum dots ${ }^{684}$, DNA nanocages ${ }^{685}$, and impermeable drugs ${ }^{110}$ have been further demonstrated. One of the first such examples was achieved by Park et al., who produced nanosyringes of $50 \mathrm{~nm}$ outer diameter and $120 \mathrm{~nm}$ height ${ }^{684}$. The cup-like hollow nanostructures were pre-filled with DNA or $\sim 3 \mathrm{~nm}$ quantum 
dots, which were then released into cells upon penetration ${ }^{684}$. This was one of the first reports where passive settling of cells onto penetrating nanostructures appeared sufficient for efficient delivery (Figure 15B).

In 2010 Shalek et al. showcased the multifaceted potential of nanowires by demonstrating successful intracellular delivery of a wide range of materials to various cell types. Functional siRNA, plasmid DNA, peptides, proteins, and membrane impermeable drugs were non-covalently and non-specifically bound to the surface of silicon nanowire arrays and cells were allowed to settle on top, thus taking advantage of passive penetration. These materials were successfully introduced into a range of immortalized cell lines and primary cell types, including hard-to-transfect mammalian neurons ${ }^{110}$. Patterning of target molecules on the nanostructure arrays is a further advantage of this approach, as it can enable spatially encoded delivery of cargo materials ${ }^{110}$. Shalek et al.'s nanowire platform was then adapted for hard-to-transfect primary immune cells ${ }^{679,686}$. By screening nanowire density and height against different cell types and sizes, optimal parameters were supposedly established for each cell type. Efficient delivery of molecules to primary B cells, dendritic cells, macrophages, natural killer cells, and T cells was reported without the adverse immune responses that confound common transfection reagents ${ }^{679}$.

Kim et al. also used a nanowire strategy to deliver molecular beacons for the quantitative detection of mRNA ${ }^{683}$. In their strategy, $\mathrm{ZnO}$ nanowires were incorporated into a PDMS device whereby pneumatic pumping provided the force to push cells down onto nanowires. Another group reported the delivery of peptide-functionalized DNA nanocages by passive incubation of cells on $1 \mu \mathrm{m}$ long $150 \mathrm{~nm}$ diameter cargo-coated nanowire arrays ${ }^{685}$. Other modes of nanowire delivery have been shown to be capable of intracellular loading of proteins such as Cre recombinase ${ }^{682}$ and antibodies against cytoskeletal proteins ${ }^{427}$. Apart from large cargo, nanowire arrays have been proven to deliver most categories of macromolecules into the cytosol of various cell types.

\subsubsection{Nanowire Penetration Mechanisms}

Despite the reports of successful delivery of multiple cargo types, it is not fully understood how nanowires breach the plasma membrane. Indeed, the mechanisms and efficiency of nanowire penetration have been a matter of debate for almost a decade. For example, several groups claim that active force is not required if the density, length, and diameter of 
nanowire arrays is optimized for a particular cell type ${ }^{110,678,679,687}$. On the other hand, conflicting reports indicate that a majority of nanowires fail to penetrate cells that passively settle on top ${ }^{688-690}$. For example, nanowires ranging from 2 to 11 $\mu \mathrm{m}$ in length and $100 \mathrm{~nm}$ diameter were found to be excluded from the cytoplasm as observed by confocal imaging ${ }^{690}$. TEM images also revealed that both the plasma membrane and nuclear envelope resist nanowire penetration, and overall DNA transfection efficiency was low in the absence of active forces ${ }^{688}$. Using $\sim 100 \mathrm{~nm}$ diameter hollow nanostraws to conduct a time-resolved GFP quenching assay, researchers from the Melosh lab determined that only $7 \pm 3 \%$ of features were penetrant, even in adherent cells ${ }^{418}$. Studies of the mechanism suggest that puncture does not occur upon initial cell contact, but requires active cell spreading and coincident accumulation of traction forces from focal adhesions ${ }^{418,678,691}$. Once penetrant, however, a given nanowire continues to provide sustained intracellular access as long as the cell remains adherent $^{418}$.

The majority of the literature indicates that provision of active forces is necessary or at least helpful for penetration and subsequent cargo delivery. In several studies with hard-to-transfect immune cells, it was found that intracellular delivery of plasmid DNA, siRNA, and proteins was only possible with the addition of g-forces to push cells against vertically aligned nanowires ${ }^{680,684}$. This was the case even when the same nanowire architecture was previously successful with common cell lines ${ }^{680,684}$. This raises the possibility that some cell types, particularly those that naturally exist in a nonadherent state, may require active forces to achieve nanowire-mediated intracellular delivery. Larger penetration forces would also increase the velocity of impalement upon contact with the cell membrane, thereby elevating the chance of membrane disruption events (Figure 9).

Several strategies have been used to provide active forces for nanowire penetration. As mentioned above, one technique is to generate g-forces from centrifuging cells onto nanowire arrays ${ }^{109,676,680}$. Another method is to sandwich the cells between nanowires and an opposing surface. For example, DNA delivery in hard-to-transfect algae was augmented by using an engineered PDMS microvalve to press cells against an array of $\mathrm{ZnO}$ nanowires ${ }^{692}$. Other strategies have been inspired by cell printing, whereby jetting velocity upon ejection from the printing nozzle is directly proportional to penetration force and can be tuned to balance efficiency of cell impalement versus cell bursting ${ }^{693}$. Movement of nanoneedles by a piezoelectrically actuated stage has also been tested ${ }^{682}$. In this case an inverted array of nanoneedles was oscillated with an amplitude of $10 \mu \mathrm{m}$ against an immobile monolayer of cells to improve plasmid transfection ${ }^{682}$. 
How large are the forces required for nanowire penetration? Researchers have attempted to address this question with a number of different methods and calculations. Using a model that estimates traction forces associated with long term cell adhesion, calculations of 1.5 to $6 \mathrm{nN}$ were obtained for cells cultured on $\sim 100 \mathrm{~nm}$ diameter nanowires ${ }^{428}$. In another case, active centrifugation of a grid of diamond nanowires was used to poke holes in cells for diffusive delivery of cargo from the extracellular solution ${ }^{694}$. They estimated a force of $\sim 2 \mathrm{nN}$ was needed to breach the membrane with $\sim 400$ nm diameter nanowires. Other groups have used AFM to directly quantify the forces of penetration for different diameter objects. For example, it was observed that 30-40 nm wide multi-walled CNTs had a penetration force of 100-200 $\mathrm{pN}$ and required an indentation depth of only $100-200 \mathrm{~nm}^{429}$. Obataya et al. found that silicon AFM tips sculpted into thin nanowires of 200$800 \mathrm{~nm}$ diameter exhibited penetration forces in the range of 0.65 to $1.9 \mathrm{nN}$ when tested on cultured human epidermal cells $^{430,431}$. Nanowires of $200 \mathrm{~nm}$ were found to breach the plasma membrane after $\sim 1-2 \mu \mathrm{m}$ indentation, and be much more efficient at both plasma membrane and nuclear envelope penetration compared to pyramidal tips ${ }^{430,431}$. As evidence of penetration, a $200 \mathrm{~nm}$ diameter nanowire inserted into the nuclei of HEK cells successfully induced expression of attached plasmid DNA ${ }^{695}$. Another study with larger AFM probe tips estimated that the forces required to penetrate supported lipid membranes range from $5 \mathrm{nN}$ for a sharp $(<300 \mathrm{~nm}$ diameter) nanoneedle probe to $20 \mathrm{nN}$ for a standard pyramidal tip ${ }^{432}$. However, the supported lipid membranes may be more difficult to break through than a cell plasma membrane, depending on approach speed, temperature, and other factors. One group used antibodies attached to nanowires to detect membrane penetration and found that lowering temperature to $4{ }^{\circ} \mathrm{C}$ appeared to improve nanowire penetration by reducing membrane adaptability ${ }^{427}$. Together, mechanistic studies indicate that biological membranes under physiological conditions are able to passively adjust to nanowire conformations, and therefore factors such as small tip area, low temperature, high forces and/or approach velocities may facilitate effective penetration of the plasma membrane.

\subsubsection{Nanowire Effects on Cells}

Long-term culture of cells on nanowires is not thought to be damaging, however, there are concerns over unexpected changes in the behaviour of cells cultured on nanowires ${ }^{696}$. Early studies indicated that nanowires altered the growth rate and cell cycle progression of cells ${ }^{676}$. Nanowire arrays have also been reported to interfere with cell division in fibroblasts 
and lead to a higher frequency of multinuclear cells, an effect that was more pronounced with longer nanowires ${ }^{697}$. Moreover, when nanowire density increases, it may inhibit stable cell adhesion and trigger cells into a more motile and less proliferative state ${ }^{698}$. On the other hand, Bonde et al. obtained results suggesting that the growth rate of HEK cells may be stimulated by arrays of nanoneedles ${ }^{699}$. Although nanowire induced-perturbations appear minor in most reports, details of their effects on cell physiology should remain open for further investigation.

\subsubsection{Nanostraw Arrays for Injection \& Extraction}

Nanostraws, which are essentially hollow nanowires, can be used for injection of cargo-laden fluid from an external reservoir (Figure 15C). In one of the first examples of nanostraw delivery, researchers from the Melosh lab fabricated beds of aluminium nanostraws on polycarbonate track-etched substrates followed by seeding of HeLa cells and CHO cells. By controlling the composition and pressure of the fluidic reservoir underneath the nanostraws, temporal control over delivery of dyes and quenching agents was achieved, thus providing direct fluidic access to the cell interior ${ }^{700}$. In a different study, hollow nanostraws were fabricated from silicon oxide. Only nanostraws that pumped a mixture of membrane-perturbing saponin and cargo were able to introduce fluorescently labeled dextran, indicating that nanostraws acted to localize the membrane permeabilizing effects of saponin and to function as conduits for delivery into cells ${ }^{701}$. In an analogous fashion, nanostraws have been reported to localize the membrane-perturbing effects of electric fields ${ }^{702}$. Low voltage pulses acted as a gating mechanism to enable access to the cytosol for delivery of membrane impermeable dyes and plasmid $\mathrm{DNA}^{702}$. A key benefit of hollow nanostraws, as opposed to solid nanowires, is the temporal control over delivery, volume, and dosage concentration.

In further studies of nanostraw technology, intracellular administration of calcium with complex signal patterns, such as oscillations over time ${ }^{703}$ and delivery of cell impermeable small molecule probes ${ }^{704}$ has been achieved. Nanostraws were also adapted for cytoplasmic extraction, being capable of continuous time-resolved sampling from the intracellular space for up to five days ${ }^{705}$. In another example, $\sim 6 \mu \mathrm{m}$ long conical nanostraws were employed for delivery of $\sim 10 \mathrm{~nm}$ quantum dot to microalgal organisms ${ }^{706}$. Moreover, Golshadi et al. showed that an array of short, dense, nanotubes of $200 \mathrm{~nm}$ outer diameter, $140 \mathrm{~nm}$ inner diameter and $180 \mathrm{~nm}$ protrusion height were capable of intracellular dye delivery and efficient 
plasmid transfection in HEK cells ${ }^{707}$. Because of the dense clustering of these structures, fully adherent cells could cover almost 1000 nanotubes ${ }^{707}$.

\subsubsection{Mechanisms of Cargo Delivery by Penetrating Elements}

The mechanisms by which nano- and micro-scale penetrating elements deliver molecules into cells are threefold: injection, dissociation, and permeabilization (Table 4). Microinjection, mostly featuring tip diameters of $\sim 0.3-1 \mu \mathrm{m}$, is the classic example of delivery by injection (see section 5.1). Advanced versions of microinjection have also been introduced with $100 \mathrm{~nm}$ diameter tips (nanoinjection ${ }^{610-613}$ ) and AFM control (FluidFM ${ }^{615}$ ). Nanostraws can be considered a highly parallelized adaptation of the microinjection mechanism with capability for much higher throughput ${ }^{418,700-702}$. However, some degree of control over the penetration and injection process is sacrificed.

Table 4. Cargo delivery mechanism versus scale of throughput for nano- and micro-mechanical membrane disruption techniques. For injection mechanisms, the nano- or micro-mechanical element is hollow, thus allowing injection of cargo. Dissociation-based delivery works by enabling cargo to detach from the penetrating element once inside the cell. For permeabilization, the cargo is in the extracellular solution and flows into the cell by diffusion upon withdrawal of the penetrating element. References for each example are included.

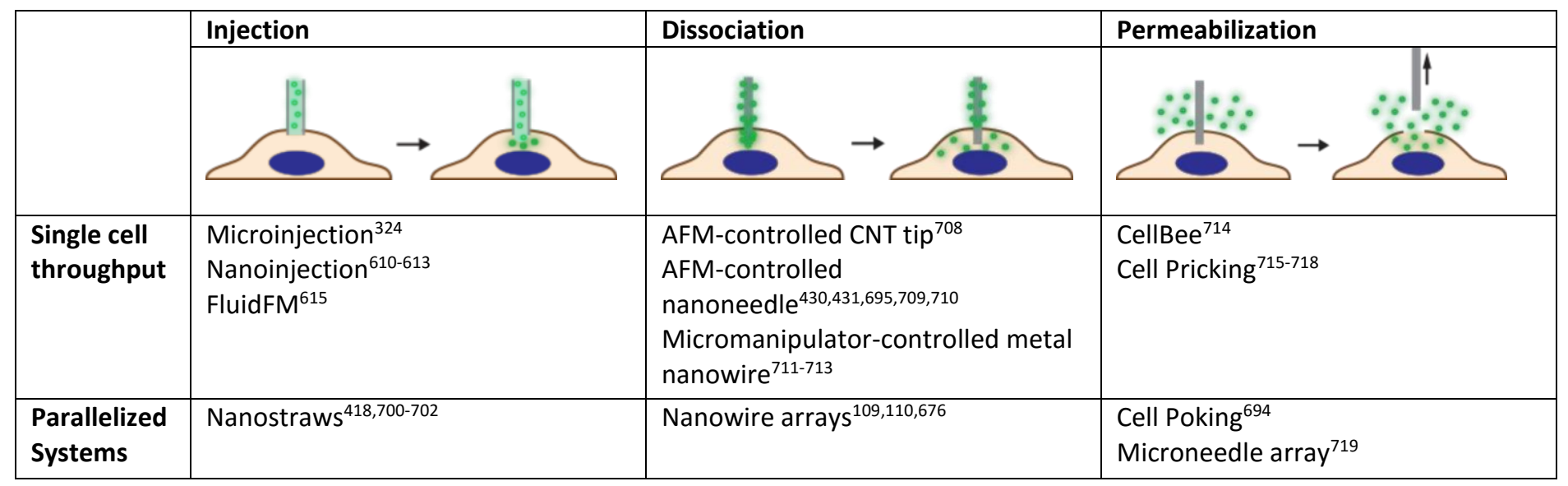

To date, most of the nanowire systems deliver cargo by dissociation. These include the original nanowire arrays introduced by McKnight et al. ${ }^{109,676}$ and Shalek et al. ${ }^{110,679}$ for simultaneous treatment of thousands of cells as discussed above. Single cell versions of nanowire delivery have also been explored. One system attached multi-walled CNTs of 10$20 \mathrm{~nm}$ diameter and up to $1.5 \mu \mathrm{m}$ length to AFM tips to deliver quantum dots to selected single cells ${ }^{708}$. Dissociation was achieved by the action of intracellular enzymes that cleave the linker holding the cargo to the penetrating $\mathrm{CNT}^{708}$. AFMcontrolled nanoneedles sculpted by focused ion beams have been shown to provide nuclear penetration and mediate gene 
expression $^{430,431,695,709}$. Another method used a $~ 500 \mathrm{~nm}$ diameter gold nanowire to penetrate mouse embryos and release plasmids inside. The plasmids are released through dissociation triggered by an electric pulse. Because the technique is thought to be less violent, embryo survival was reported to be significantly higher than traditional microinjection ${ }^{713}$.

Finally, nanowire delivery can also be mediated by permeabilization whereby the mechanism involves diffusive influx of cargo from the extracellular solution. In this case the penetrating element is withdrawn from the cell and the influx occurs before completion of plasma membrane repair (see Figure 6). Both single cell ${ }^{714}$ and parallelized ${ }^{694}$ versions of this approach have been published. They will be further discussed in section 6.1 .1 below, which deals with delivery by permeabilization.

\subsubsection{Nanowire \& Nanostraw Summary}

In the reported nanowire and nanostraw delivery modalities demonstrated thus far, the cargo material is delivered by (1) dissociation from the penetrating structure upon cytosolic entry, (2) direct injection through hollow nanostraws, or (3) permeabilization of the plasma membrane (Table 4). In most cases active forces and/or rapid approach velocities improve penetration and resultant delivery efficiency. So far, high aspect ratio nanowires for intracellular delivery have been successfully fabricated out of carbon, diamond, silicon, silicon oxide, zinc oxide, gold, and various other inorganic semiconductors, metals, and metal oxides ${ }^{696,720-722}$. Polymer coatings have been suggested to improve delivery performance and cell health, for example, in the case of siRNA delivery ${ }^{681}$ and DNA transfection ${ }^{723}$. The physiological effect of exposing nanowire materials to the intracellular space will be essential knowledge if nanowires are to proceed toward biomedical applications. Furthermore, open questions remain regarding the membrane conformation adopted around nanowires and the subsequent degree of penetration. Understanding the effect of nanowire dimensions and density, the requirement of active forces, surface functionalization and chemistry, as well as the influence of culture conditions, cell properties, and cell type will be key information for the future implementation of nanowires and nanostraws.

\section{Intracellular Delivery by Permeabilization}

As specified in section 3, permeabilization methods work by transiently permeabilizing the cell to cargo in the extracellular solution. Here we will discuss methods for intracellular delivery that rely on mechanical, electrical, optical, 
thermal, and chemical means of permeabilizing the plasma membrane. A major advantage of permeabilization-based delivery is that it is near-universal, being able to deliver almost any material that can be dispersed in solution. Because most cells can recover from micron-sized membrane disruptions ${ }^{444}$, delivery of large cargo is also feasible.

\subsection{Mechanical Membrane Disruption}

Mechanical methods of membrane permeabilization have been performed by (1) solid contact of foreign objects with cells, such as is the case for direct penetration mediated delivery discussed in the previous section. Membranes have also been permeabilized without solid contact, such as with (2) fluid shear forces and (3) hydrostatic or osmotic pressure changes. These three mechanisms of membrane permeabilization are categorized and discussed separately below.

\subsubsection{Mechanical: Solid Contact}

\subsubsection{Scrape \& Bead Loading}

Among the earliest reported mechanical cell permeabilization methods were those published by Paul McNeil and colleagues in the 1980s, which include scrape loading ${ }^{96}$ and glass bead loading ${ }^{97}$. In scrape loading, a rubber spatula is passed over a cell-laden substrate to dislodge adherent cells and bring them into solution, hence the technique is only applicable to adherent cells (Figure 16A). Moreover, the amount of damage to each cell is stochastic, with some cells being instantly killed while others remain almost unaffected. In cells that receive optimal amounts of membrane damage, cargo molecules dispersed in solution diffuse through transient membrane disruptions to achieve delivery. Glass bead loading involves shaking the adherent cells with medium containing glass beads and the cargo to be delivered (Figure 16B). The impact of collisions between beads and cells imparts sufficient strain to generate disruptions in the plasma membrane. Again, the magnitude of plasma membrane damage that each cell sustains is highly variable and may lead to inconsistent delivery and cell survival. The generation of cellular and biological debris may be another problematic aspect of cell scraping and bead loading. Moreover, delivery of expensive reagents that need to be concentrated into small volumes can be difficult to achieve with these protocols. On the other hand, potential benefits include the low-cost and accessible nature, as these methods can be performed with common lab equipment. In applications where high cell viability is not a priority, scraping and bead loading may represent convenient options. A later adaption of bead loading 
termed 'immunoporation' used beads functionalized with antibodies to bind to cells and permeabilize them by ripping off bits of their membranes ${ }^{724-730}$.

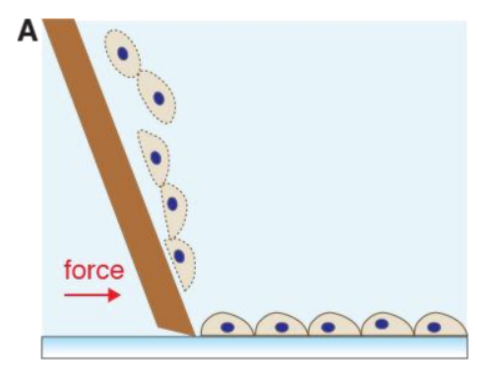

scrape loading

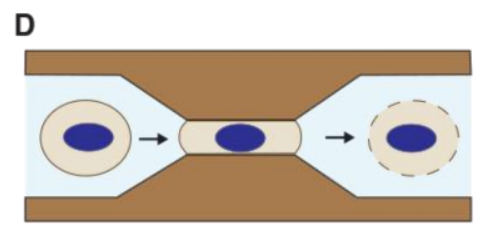

microfluidic squeezing

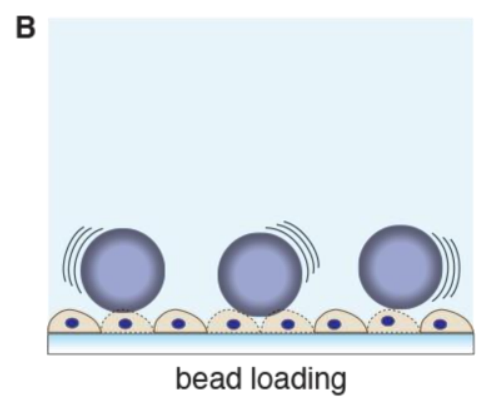

$E(i)$

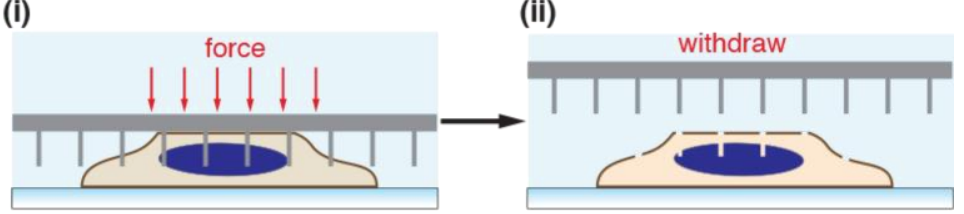

nanoneedle penetrate-withdraw

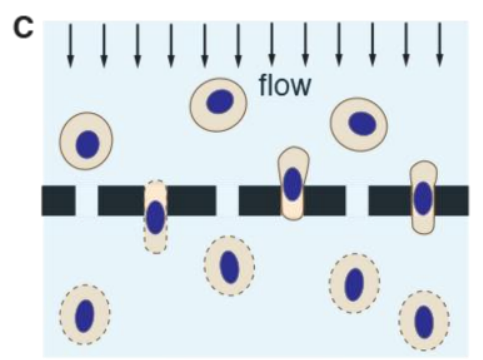

filtroporation

(ii)

Figure 16. Mechanical membrane permeabilization by direct contact. (A) Scrape loading, where a rubber spatula or similar scraping object can be used to simultaneously dislodge cells and permeabilize them. (B) Bead loading, wherein micron-scale beads can be rolled across a cell monolayer for controlled cell injury via collisions. (C) Filtroporation, where a solution of cells is passed through holes in filter membranes, such as a track-etched polycarbonate filter. (D) Microfluidic cell squeezing, where cells membranes are disrupted by rapid deformations in cell shape that occur with passage through microfabricated constrictions. (E) Permeabilization with nanoneedle/nanowire arrays. (i) The array is first centrifuged or otherwise pressed against cells adhered to a rigid substrate. (ii) The array is then removed to enable cargo influx through membrane disruptions in the target cells.

Bead and scrape loading techniques have been used to deliver a variety of cargoes into cells. Bead loading has been used to deliver dye-conjugated dUTP for fluorescent visualization of chromosome formation ${ }^{731}$, antibody loading into macrophages $^{732,733}$ and fibroblasts ${ }^{734}$, intracellular delivery of proteins ${ }^{735-737}$, peptides ${ }^{738}$, fab fragments ${ }^{739,740}$, peptide nucleic acid probes ${ }^{741}$, SNAP-reactive dyes ${ }^{742}, \mathrm{CNTs}^{743}$, and quantum dots up to $15 \mathrm{~nm}$ in several cell lines ${ }^{744}$. Scrape loading has achieved intracellular delivery of proteins ${ }^{96,543,745-751}$, antibodies ${ }^{752-754}$, peptides ${ }^{755,756}$, morpholinos ${ }^{757}$, high molecular weight dextrans ${ }^{96,758}$, lipopolysaccharides ${ }^{759}$, dyes ${ }^{760,761}, \mathrm{pH}$-sensitive probes $^{762}$, and transfection of plasmids ${ }^{545}$.

A variant of the scrape loading technique is scratch loading ${ }^{763}$. Also introduced by Paul McNeil, it involves dragging a needle or other kind of sharp object across a layer of adherent cultured cells. The cells that brush the edge of the needle undergo membrane damage but remain adherent to the substrate. Intracellular delivery of dextrans ${ }^{763}$, dyes ${ }^{764}$, fluorescently-labeled nucleotides ${ }^{765}$, and quantum dots ${ }^{744}$ has been achieved in cells adjacent to the scratch zone. Although the method is lower throughput than scrape loading, one advantage of scratch loading is that cells remain adherent for immediate analysis. 


\subsubsection{Sudden Cell Shape Changes \& Protease Treatments}

Sudden contraction of cells from an adherent, elongated shape to a rounded shape has the potential to generate membrane disruptions. Grinnell and colleagues found that the sudden contraction involved in the fibroblast-driven collapse of collagen matrices is able to induce permeabilization and uptake of dextrans up to $150 \mathrm{kDa}$ in size $\mathrm{f}^{766,767}$. In this approach, fibroblast-colonized collagen matrices that are stabilized by substrate attachment are peeled away from their support. The isometric contractile forces collectively generated by the fibroblasts then trigger compaction of the collagen matrix into a dense body one tenth of its original size ${ }^{767}$. This process induces plasma membrane disruptions in the contracting fibroblasts. Membrane permeabilization is thought to be due to the tearing of focal adhesion sites associated with rapid cell shape change and compression of the collagen matrix ${ }^{576,766}$. The lesions are resealed in a $\mathrm{Ca}^{2+}$-dependent fashion, with the fibroblasts reported to be impermeable to uptake several seconds after return to standard physiological media ${ }^{576,766}$. Fibroblasts that detach from their substrates to round up in mitosis also exhibit permeability to dextrans up to $150 \mathrm{kDa}$, peptides, proteins, or oligonucleotides ${ }^{768}$. This observation is in congruence with other studies that have observed plasma membrane damage and dye uptake during mitotic cell rounding ${ }^{769,770}$.

In what could be a related phenomena, permeabilization has been observed when attached fibroblasts are treated with strong doses of the proteases trypsin, pronase, or collagenase ${ }^{771,772}$. Cytoplasmic delivery of the proteins insulin $(6 \mathrm{kDa})$, lysozyme $(14 \mathrm{kDa})$, BSA $(76 \mathrm{kDa})$, and thyroglobulin $(660 \mathrm{kDa})$ were achieved with this simple treatment. Although the mechanisms were not investigated, cells presumably become permeable as they detach from the substrate ${ }^{766}$. Indeed, membrane ripping has previously been observed when certain cell types move across or detach from surfaces ${ }^{766}$. However, intracellular delivery of proteins by protease permeabilization has been reported for both adherent ${ }^{773}$ and nonadherent cell types ${ }^{774}$. If protease-mediated permeabilization is not due to membrane ripping during detachment, it could be that cells are permeabilized cells through the action of the proteases themselves. Trypsin can trigger signaling events that culminate in vigorous contractile activity at the cell surface and loss of coherence between the cortex and plasma membrane ${ }^{775}$. Such events could potentially induce transient plasma membrane disruptions. Thus, further studies may be needed to identify the mechanisms of membrane disruption by rapid cell shape changes and the action of proteases, and whether these phenomena can be made more widely useful for intracellular delivery. 


\subsubsection{Projectile Permeabilization}

Sautter et al. pioneered a variation of the biolistic approach that retains free DNA in solution ${ }^{776}$. It is distinct from the projectile bombardment methods covered in section 5.2 in that the particles are used to permeabilize the cells rather than carry cargo. Projectiles are accelerated towards target cells in a Bernoulli air stream as a fine mist of droplets. The projectile particles create membrane disruptions that allow influx of plasmid DNA dispersed within the droplets. This stream of droplets can be targeted toward $150 \mu \mathrm{m}$ areas of cells or tissue for localized targeting with dynamic adjustment of particle density and velocity.

\subsubsection{Filtroporation}

In 1999 a constriction-based method for generating disruptions in the plasma membrane was reported ${ }^{777}$. The technique, termed "filtroporation", works by forcing cell suspensions through uniformly-sized micropores in commercially available track-etched polycarbonate filters (Figure 16C). In the reported study, a polycarbonate filter of approximately $12 \mu \mathrm{m}$ thick with pore sizes ranging from 5-18 $\mu \mathrm{m}$ was used. Plasmid DNA and dextran-conjugates up to $500 \mathrm{kDa}$ were successfully delivered into $\mathrm{CHO}$ cells of nominal diameter $\sim 13 \pm 2 \mu \mathrm{m}$. The cell suspensions were driven through the polycarbonate filter by a pressure regulator supplying constant pressures of 0 to $175 \mathrm{kPa}$. Delivery efficiency and cell damage were both increased as a function of driving pressure. Severity of the treatment also increased as the micropore diameter was decreased when all other parameters were held constant. By tuning parameters, optimal conditions of $8 \mu \mathrm{m}$ pore size and driving pressure of $35 \mathrm{kPa}$ were identified in $\sim 13 \mu \mathrm{m}$ CHO cells. Thus, the cells experienced $40 \%$ constriction of their diameter as they passed through the polycarbonate filter. These conditions permitted uptake of a luciferase reporter plasmid, which resulted in transfection of the cells with a reported transfection efficiency above $50 \%$ after 2 days in culture. Despite these results, further work on filtroporation is absent from the literature as the technique does not appear to have gained traction.

\subsubsection{Microfluidic Cell Squeezing}

Microfluidic and lab-on-chip methods of plasma membrane perturbation offer the opportunity for precise control of the membrane disruption process ${ }^{19,104,106,107}$. In 2013, Sharei and colleagues reported on the development of a microfluidic platform for intracellular delivery by rapid cell deformation (or squeezing) through channel constrictions (Figure 16D). 
This innovative method has demonstrated delivery of diverse macromolecular materials into a wide range of cell types $^{108,308,778}$. The delivery mechanism is via diffusion of macromolecular cargo through membrane disruptions generated by rapid deformations of cell shape (Figure 17A). The device is comprised of parallel constrictions generated by deep reactive ion etching in silicon wafers, followed by bonding to pyrex glass and drilling holes for inlet and outlets. Gas pressures of $10-100 \mathrm{kPa}$ are then used to drive cell suspensions through constrictions of 4 to $8 \mu \mathrm{m}$ width, 10 to $50 \mu \mathrm{m}$ length, and $20 \mu \mathrm{m}$ channel depth. The ability to engineer angle of entry and repeated constrictions is also possible. In the first published study, the bona fide cytoplasmic delivery of unaggregated quantum dots was demonstrated in HeLa cells ${ }^{308}$. Then a wider range of cell types was screened to showcase efficacy with primary blood derived immune cells (T cells, B cells, and macrophages), primary dendritic cells, embryonic stem cells, and primary fibroblasts, as well as a panel of immortalized cell lines ${ }^{108}$. Efficient cytosolic delivery of siRNA, carbon nanotubes, quantum dots, antibodies, transcription factors and dextran-conjugated dyes was observed in many of these cell types.

A major strength of cell squeezing is the simplicity of the approach - no moving parts or external power are required, simply a pressure source and controller to modulate flow rate. Weaknesses include cell type and size dependence for a particular device geometry, and the potentially narrow range of flow rates required to achieve optimal balance between delivery and viability. However, a variety of constriction geometries have been developed to address a broad range of cell types. Furthermore, experiments with buffer composition (e.g. $\mathrm{Ca}^{2+}$ concentration) indicate that it can successfully be tuned to optimize membrane recovery kinetics and cell survival ${ }^{779}$. In line with what is known from the cell biology of membrane repair (see section 4.3), it was observed that buffers with calcium promoted rapid ( 30 s) closure of membrane wounds while no calcium conditions allowed the membrane to remain open for several minutes ${ }^{779}$. By modulating treatment parameters as well as temperature, a further demonstration of immune cell engineering with siRNA, antibodies and proteins was shown in T cells, B cells, dendritic cells, and monocytes/macrophages at throughputs of millions of cells per second ${ }^{780,781}$. These results suggest cell squeezing might be a promising path towards engineering cell function for immune cell therapy at high-throughput.

The cell squeezing platform has been used for protein delivery to primary mammalian plasmacytoid dendritic cells with a device consisting of $10 \mu \mathrm{m}$ long and $4 \mu \mathrm{m}$ wide constrictions repeated 5 times in series ${ }^{782}$. Zoldan and colleagues employed microfluidic cell squeezing to perform high throughput delivery of fluorescently labeled tRNAs into multiple 
myeloma cells with a transfection efficiency of $\sim 45 \%{ }^{783}$. Delivery of fluorescently labeled tRNAs enabled monitoring protein synthesis inside the cells in real time ${ }^{783}$. Delivery of otherwise impermeable JAK inhibitors into human primary cells was achieved by squeezing cells through constrictions of $10 \mu \mathrm{m}$ long and $4 \mu \mathrm{m}$ wide ${ }^{784}$. Intracellular delivery of small fluorescent tags for protein labeling and subsequent live cell imaging has also been demonstrated by cell squeezing $^{785}$.

Because of the sensitivity of cells to constriction size, it was tested whether the squeeze platform could exploit size differences of cells to facilitate selective intracellular delivery ${ }^{786}$. As a proof of concept, Saung et al. showed that the system is able to selectively deliver molecules to pancreatic cancer cells within a heterogeneous mixture containing $\mathrm{T}$ cells $^{786}$. One future application of this concept would be the selective tagging of CTCs or other abnormal large cells in the blood $^{786}$. 
A

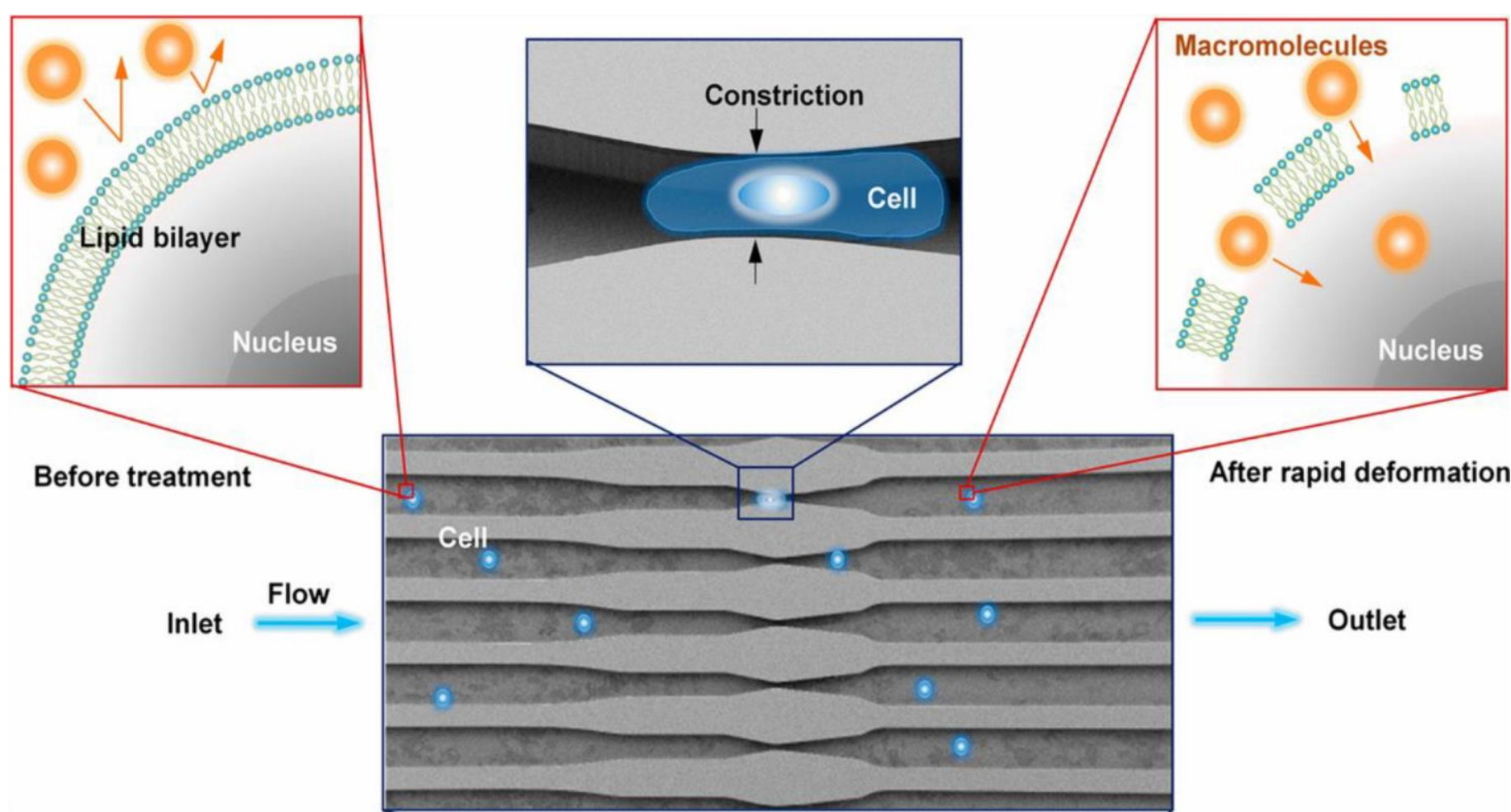

B

(i)

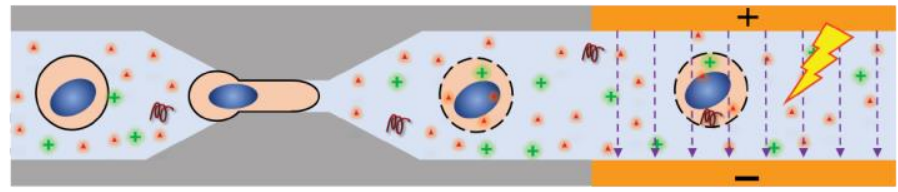

(ii)

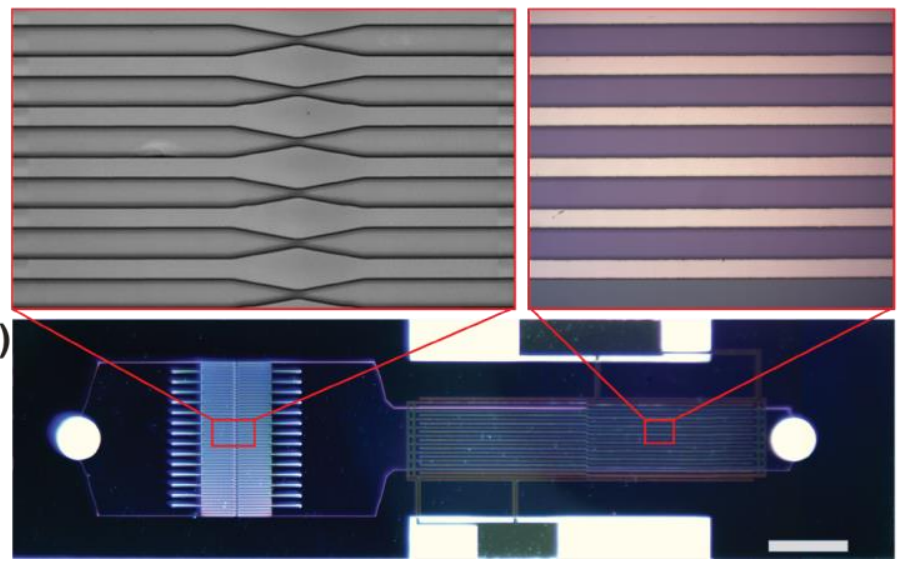

C(i)
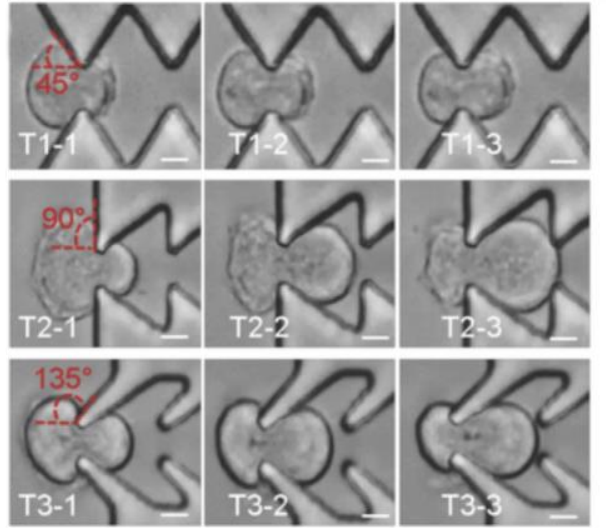

(ii)

Max: 2.79
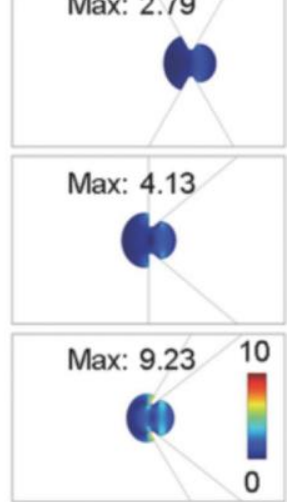

Figure 17. Different variations of cell squeezing for intracellular delivery by mechanical membrane permeabilization. (A) The original microfluidic platform for cell squeezing ${ }^{108}$. The rapid deformation a cell experiences upon passage through a constriction transiently permeabilizes the plasma membrane, allowing influx of macromolecules into the cytosol. Reprinted with permission from Reference 108. Copyright 2013 PNAS. (B) Similar to cell squeezing in panel A but with addition of a downstream electric field. The electric field enhances delivery of large nucleic acids, such as plasmid DNA, into the cell by electrophoretic forces. In this case the device was optimized for delivery of plasmids into the cell nucleus at high throughput ${ }^{787}$. Panel (i) shows the delivery concept. Panel (ii) shows the close-up architecture of the constriction and electrode zones on the chip. Panel (iii) shows a view of the whole chip including holes (white) for inlet (left) and outlet (right). Scale bar: $1 \mathrm{~mm}$. Reprinted with permission from Reference ${ }^{787}$. Copyright 2017 Springer Nature. (C) Cell squeezing with different constriction geometries in a PDMS device. (i) Comparison of $45^{\circ}$ pyramidal pattern, $90^{\circ}$ saw tooth pattern, and $135^{\circ}$ reverse wishbone pattern of repeated constrictions. (ii) COMSOL modeling indicates the stress ( $\mathrm{N}$ $\mathrm{m}^{-2}$ ) that the cell membrane would undergo upon passage through the different shapes of constrictions. Experiments and modeling show the reverse wishbone pattern as the most effective for localized membrane disruption in this device ${ }^{788}$. Figure reprinted from ref ${ }^{788}$, Copyright 2017, with permission from John Wiley and Sons. 


\subsubsection{Electric Field-Enhanced Microfluidic Cell Squeezing}

Like most other mechanical membrane disruption techniques, DNA transfection efficiencies upon cell squeezing are generally quite low in many cell types. Ding and co-workers explored the idea of adding a downstream electric field to investigate whether it could improve DNA transfection results (Figure 17B) ${ }^{787}$. The strategy, termed 'disruption and field enhancement' (DFE), was compared with standard cell squeezing, microfluidic flow-based electroporation, commercial electroporation (Neon - Thermo Fisher), microinjection directly to the nucleus, and lipofection ${ }^{787}$. In HeLa cells, DFE was able to achieve similar transfection efficiencies as lipofection and commercial electroporation. Surprisingly, plasmid expression approached its maximum within 1-2 hours of treatment, which was also the case with microinjection. This contrasts with the delayed onset on expression after lipofection and standard electroporation, which can take 24 hours or longer due to requirement of endocytosis and other intracellular trafficking processes to deliver DNA to the nucleus ${ }^{787}$. Fixation and imaging of cells directly after treatment indicated that DFE, like microinjection, could deliver plasmids directly into nucleus for immediate expression. To determine whether DFE was permeabilizing the nuclear envelope to permit DNA uptake, a HeLa cell line expressing the protein CHMP4B-GFP was imaged with confocal microscopy. CHMP4B is a component of the ESCRT-III complex, recently discovered to be involved in repair of both plasma membrane and nuclear envelope disruptions ${ }^{454,789-791}$. While squeezing and standard electroporation only permeabilized the plasma membrane, DFE was found to also generate disruptions in the nuclear envelope. After treatment, nuclear envelope repair appeared to be completed within $\sim 15$ minutes, in agreement with previous studies ${ }^{789,790}$. It was speculated that, by first disrupting the plasma membrane, subsequent exposure to the electric field was able to electroporate the nucleus. Indeed, specific types of electroporation have previously been found to selectively permeabilize intracellular compartments (reviewed $\mathrm{in}^{579}$ ). DFE thus represents a useful strategy for high-throughput nuclear delivery and rapid expression of $\mathrm{DNA}^{787}$. Further work should clarify the exact mechanisms of cargo influx upon two-step mechanical/electrical hybrid treatments such as DFE.

\subsubsection{Variations on Microfluidic Cell Squeezing and Cell Deformation Strategies}

In 2015 the Qin lab introduced microfluidic intracellular delivery devices featuring various types of PDMS-based microconstrictions ${ }^{792}$. Until this point, most results had been obtained in microfabricated silicon devices ${ }^{108}$. By using repeated arrays of constrictions fabricated from PDMS, Qin and co-workers reported delivery of single-stranded DNA, siRNA, and 
plasmids into HEK cells and several other cell lines ${ }^{792}$. Moreover, they demonstrated genome editing in MCF7 and HeLa cells via delivery of plasmids that express Cas9 and gRNA, although transfection efficiencies were not directly reported $^{792}$. In a subsequent study, the group modified their device architecture to perform siRNA delivery to cancer cells with a repeated pattern of $5 \mu \mathrm{m}$ constrictions in a reverse wishbone configuration ${ }^{788}$ (Figure 17C). Experiments and simulations both indicate that the sharper constrictions conferred by the reverse wishbone intensified the local stress on the plasma membrane to increase the magnitude of membrane disruption ${ }^{788}$. Another of this group's publications featured sharp star-shaped constrictions to facilitate delivery of dextrans, siRNA, and Cas9 RNPs to the intracellular space of hardto-transfect suspension cell lines and T cells ${ }^{152}$. By delivering RNPs targeting GFP, they were able to achieve CRISPRmediated GFP knockout in several standard cell lines. Also demonstrated was low-efficiency CRISPR-mediated knock-in editing of the PD-1 gene in primary T cells, an application that could be relevant for cell-based therapies ${ }^{152}$.

So far, the results on cell squeezing indicate that the rapid deformation of cells in suspension is able to create holes in the plasma membrane in a relatively well-controlled and reproducible manner. In an extension of this concept, it is possible to asymmetrically deform cells by flowing them past an abrasive object positioned on one side of a microfluidic channel. Such a strategy would presumably disrupt the plasma membrane in a more localized manner, preferentially permeabilizing on one side of the cell. To explore this idea, the Qin lab introduced a device with sharp silicon nanoblades protruding from one side of PDMS microfluidic channels ${ }^{793}$. The protruding edge of the silicon nanoblade essentially formed a spike of $\sim 200 \mathrm{~nm}$ radius, creating a gap of $\sim 2 \mu \mathrm{m}$ for cell passage. By optimizing the flow rate and number of nanoblade constrictions, they achieved $\sim 70 \%$ delivery efficiency of $70 \mathrm{kDa}$ dextan with $\sim 80 \%$ cell viability in hard-totransfect $\mathrm{HSCs}^{793}$. Compared to electroporation, the delivery efficiency was the same, however, survival and ability of HSCs to remain pluripotent were claimed to be superior with the nanoblade device. Cas9 RNPs were successfully delivered into HSCs, but the actual gene editing efficiencies as a percentage of total cells treated were not reported ${ }^{793}$.

Another method of microfluidically-controlled cell deformation is to flow cells through a T-junction and cause them to collide with the channel wall ${ }^{794}$. A recent report from Deng et al. shows that cells can be propelled into a spike-like structure protruding from the channel wall to permeabilized them at a throughput of a million cells per minute ${ }^{794}$. As with cell squeezing, the extent of permeabilization is proportional to the flow speed of the cells ${ }^{794}$. Using this strategy, 
intracellular delivery of dextrans, Cas9 RNPs, siRNA, plasmid DNA, and DNA nanostructures were demonstrated at efficiencies around $50 \%$ in several cells lines ${ }^{794}$.

\subsubsection{Potential Off-Target Effects of Cell Squeezing}

Cell squeezing strategies often rely on significant cell deformations - sometimes up to $70 \%$ of the cell diameter. An unresolved issue is whether off-target damage may be inflicted upon intracellular structures, such as the cytoskeleton, nucleus, and even genomic DNA. For example, it has been observed that cells migrating through tight constrictions undergo transient nuclear ruptures and DNA damage ${ }^{789}$. As the stiffest large object in the cell, the nucleus is widely regarded as the determining factor governing passage of cells through micron-sized constrictions ${ }^{795,796}$. It has also been observed that apoptotic and cell stress response can significantly impact cell survival after passage of cells through constrictions $^{797}$. Lamins, which mechanically reinforce the nuclear envelope, play a protective role in physically buffering the nucleus from mechanical stress and their depletion is known to make cells more vulnerable to death after passage through constrictions ${ }^{797}$. Moreover, DNA damage has previously been observed with imposed cyclic mechanical stresses in certain cell types ${ }^{798}$. Experiments from Ding and colleagues that visualized nuclear disruptions with CHMP4B-GFP indicated that squeezing HeLa cells (nuclear diameter $\sim 8-12 \mu \mathrm{m}$ ) through $7 \mu \mathrm{m}$ constrictions did not disrupt the nuclear envelope $^{787}$. Because disruption of the nuclear envelope can be associated with DNA damage, it indicates genomic DNA may be safe even when cells are squeezed by more than $50 \%$ of their initial diameter. Moreover, measurements of DNA damage with a high throughput COMET assay ${ }^{799}$ failed to indicate significant DNA damage in HeLa cells forced through $6 \mu \mathrm{m}$ constrictions (unpublished observations). However, further investigations with different types of cells (particularly those of clinical relevance) may be required to fully address the question of off-target DNA damage.

\subsubsection{Nanowires for Transient Permeabilization}

Arrays of sharp nanowires have been used to permeabilize cells by transiently piercing their plasma membranes. In these cases, nanowires are thrusted into the cells followed by withdrawal to promote diffusive influx from the surrounding media (Figure 16E). This mode of plasma membrane penetration is similar to the nanowires/nanostraws described in section 5.3, except that the delivery mechanism is via diffusion through a permeabilized plasma membrane rather than dissociation from the nanoneedles themselves. In one notable example, a grid of diamond nanowires was centrifuged onto 
cultured cells at controlled forces using standard lab centrifuges ${ }^{694}$. Thin diamond nanowires were fabricated by first depositing a nanodiamond film on silicon wafers followed by microwave plasma chemical vapor deposition to grow a uniform field of needles. In the versions used for experiments, dimensions were optimized to $\sim 300 \mathrm{~nm}$ diameter and $\sim 4.5$ $\mu \mathrm{m}$ height with straight sidewalls at a density of $\sim 6$ nanowires per $10 \times 10 \mu \mathrm{m}^{2}$. It was found that nanowires of diameter $>800 \mathrm{~nm}$ caused excessive damage to cells, but those $<400 \mathrm{~nm}$ produced a suitable balance between delivery efficiency and cell damage. For this geometry, it was calculated that centrifugation at 300 r.p.m. yields $\sim 2 \mathrm{nN}$ penetration force per nanowire, which was claimed to be an ideal penetration force for monolayers of cells grown in culture. Upon withdrawal of nanowires from cells, influx of IgG antibodies, $\sim 20 \mathrm{~nm}$ quantum dots, and $\sim 200 \mathrm{~nm}$ polystyrene nanoparticles into the cytoplasm of primary neurons was demonstrated. Furthermore, by packaging DNA with lipid-based lipofectamine complexes, plasmid transfection in neurons was boosted from around 1-5\% (lipofectamine alone) to almost 50\% with additional nanowire permeabilization. If nanowire permeabilization were used with naked DNA alone, transfection efficiency was $<1 \%$, suggesting that: 1) centrifuged nanowires did not consistently permeabilize the nucleus, and 2) that lipid complexes may facilitate nuclear targeting and protect the DNA from premature degradation. Thus, direct cytosolic delivery of DNA-lipid complexes may boost transfection efficiency in otherwise difficult-to-transfect cells such as neurons.

Several other groups have also used arrays of nanowires to permeabilize cells for intracellular delivery. In one case arrays of silicon lances were pressed against cell monolayers with a compliant suspension system in lieu of centrifugation ${ }^{719}$. The silicon lances were larger than typical nanowires, with lengths of $8 \mu \mathrm{m}$, diameters around 0.5 to $1.0 \mu \mathrm{m}$, and sharpened tips. Although this setup yielded diffusion-based intracellular delivery of propidium iodide, delivery of larger molecules of biological interest was not tested ${ }^{719}$. Matsumoto produced nanowire arrays of $25 \mu \mathrm{m}$ length and 200 nm diameter ${ }^{800}$. They were attached to a piezoelectric actuator stage and lowered onto cell monolayers before being vertically oscillated at a frequency of $5 \mathrm{kHz}$ and an amplitude of $\sim 0.5 \mu \mathrm{m}$ for up to 2 minutes ${ }^{800}$. Continuous delivery of molecules from solution appeared to be augmented by the agitation associated with nanowire oscillation. Up to $50 \%$ of cells retained detectable levels of $70 \mathrm{kDa}$ dextran after treatment. Efficiency of plasmid transfection, however, was only 7\%, which was less than the $18 \%$ achieved when plasmids are directly attached to nanowires ${ }^{682}$. Interestingly, the abovementioned examples of nanowire permeabilization are essentially scaled-up versions of single cell permeabilization previously performed with sharpened AFM tips. In 2006, a method introduced by Hara et al. demonstrated stab and withdraw permeabilization by 
using AFM tips that had been sharpened by focused ion beam technology ${ }^{714}$. Expression of plasmid DNA that flowed into cells from the culture media was achieved with serial penetrations of sharpened tips into HeLa cell nuclei using a computer controlled device called the "CellBee""

\subsubsection{Summary of Permeabilization by Mechanical: Solid Contact}

Classic methods of mechanical contact-mediated permeabilization such as scrape and bead loading provide low-cost, accessible and crude solutions for delivery of certain cargoes, especially proteins, small molecules, and oligonucleotides. However, delivery efficiency and cell survival may not be sufficient for certain applications, particularly in sensitive cell types. Recent progress in solid contacted-mediated mechanical membrane disruption takes advantage of the increased precision afforded by MEMS, microfluidics, lab-on-chip, and nanotechnology capabilities to finely control the level of cell injury ${ }^{19,104-107}$. Prominent examples include microfluidic constrictions for squeezing of cells in suspension ${ }^{108,787}$ and nanowires to transiently permeabilize adherent cell monolayers for high throughput intracellular delivery ${ }^{694}$.

\subsubsection{Mechanical: Fluid Shear}

Lipid bilayers can be disrupted by fluid shear forces in a number of ways. If water molecules flow parallel to a membrane surface at a sufficiently rapid velocity, it can tilt the lipid heads in the direction of the shear and lead to buckling instabilities that eventuate in bilayer rupture ${ }^{801}$. Alternatively, a jet of water molecules propelled perpendicularly into a membrane can pierce it in an analogous way to a mechanical object ${ }^{802}$. Unlike membrane disruption via solid contact (discussed above), fluid shear forces are less invasive. On the flipside, fluid shear forces in aqueous environments tend to be more difficult to control. In this section we discuss the strategies and methods that have been used to perform membrane disruption-based intracellular delivery by harnessing fluid shear forces. First, we will explore shear forces generated by flow of fluid past microscale channels and objects. Second, acoustic sonoporation, which is thought to depend mainly on the forces associated with cavitation bubbles, will be discussed. Third, we will cover laser-induced cavitation as a strategy for generating highly localized and intense zones of fluid shear. 


\subsubsection{Syringe Loading}

One of the simplest approaches for generating zones of high fluid shear force is to drive a liquid through tight constrictions. In 1992 Paul McNeil and colleagues introduced an intracellular delivery method called syringe loading, where cell suspensions mixed with high concentrations of a cargo to be loaded are repeatedly aspirated and expelled through fine-gauge syringe needles to transiently permeabilize cells (Figure 18A) ${ }^{98}$. The flow rate is a critical parameter as it determines the velocity of the cells traversing the constriction zone and thus the shear forces they experience. A typical syringe loading protocol consists of eight passes of cell suspension through a $1 \mathrm{ml}$ syringe affixed with a $30 \mathrm{G}$ needle, which has an inner diameter of $160 \mu \mathrm{m}^{98}$. In the initial publication, delivery of cargo sizes up to $150 \mathrm{kDa}$ were obtained in several mammalian cell lines ${ }^{98}$. Furthermore, the addition of pluronic F-68 (also known as poloxamer 188) was found to increase the tolerance of cells to membrane permeabilizing shear forces, thereby enabling the cells to undergo harsher treatments and improve cell survival. In the cell types tested, syringe loading in the presence of pluronic F-68 appeared more efficient than both bead and scrape loading ${ }^{98}$. Low-volume versions of the protocol were also developed, using a 25 $\mu 1$ Hamilton syringe with $25 \mathrm{G}$ fixed needle (inner diameter $260 \mu \mathrm{m}$ ) for 80 passes. A $5 \mu$ l version of the protocol was described with a $10 \mu 1$ micropipette tip (inner diameter not reported) involving 60 passes.

In subsequent reports, syringe loading has demonstrated utility in a variety of delivery applications, mostly to conduct studies in basic biology. In one example, it has been used to perform DNA transfection ${ }^{803}$. Using a selection strategy, stable integration of plasmid DNA into the genome of host $\mathrm{CHO}$ and mouse Ltk(-) cells was estimated in approximately one of every 50,000 cells treated, which was considered a success given the low cost of the technique ${ }^{803}$. Ghosh and colleagues found that syringe loading could deliver neutrally charged antisense phosphorodiamidate morpholinos into cells for the purpose of gene silencing ${ }^{804}$. Moreover, the same delivery strategy has been used for loading of small molecular weight nucleotides, GTP and GDP $(\sim 0.5 \mathrm{kDa})$, and their analogues to explore G-protein biology in immune

cells and endothelial cells ${ }^{805,806}$. In another application, fluorescent labeling of the neuronal cytosol was achieved when trypsinized ganglia were syringe-loaded with dye-conjugated $10 \mathrm{kDa}$ dextrans ${ }^{807}$. 
A

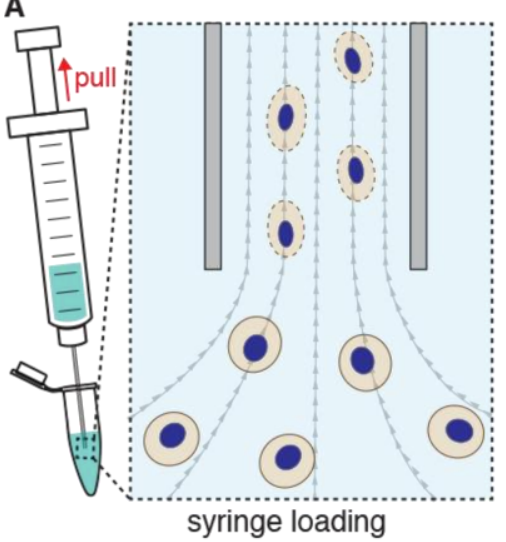

D

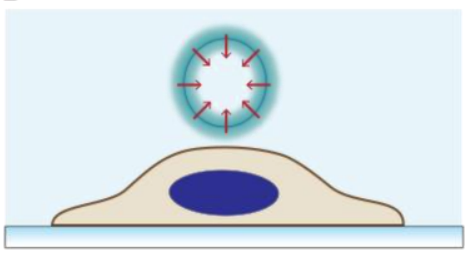

$\downarrow$

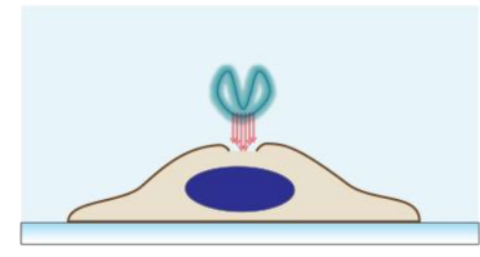

micro-jetting:

inertial cavitation

(collapse)
B

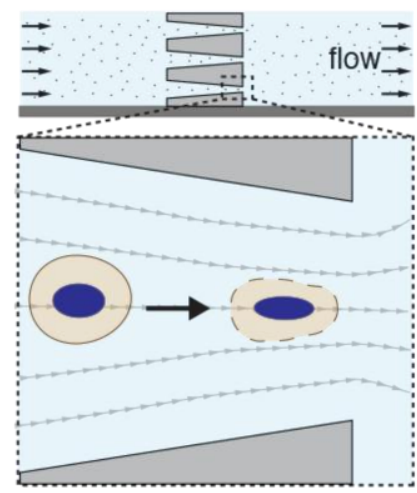

microfluidic shear force

E

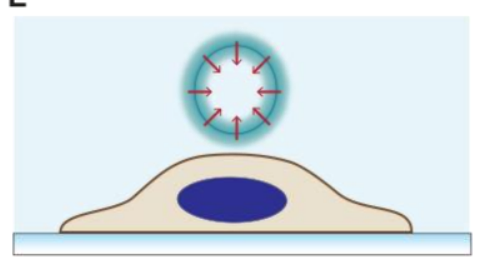

$\uparrow$

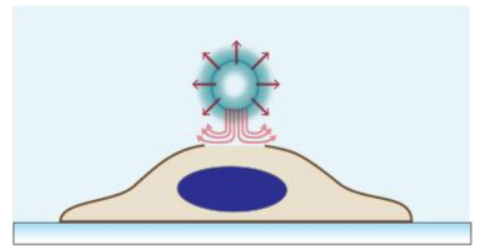

micro-streaming:

stable cavitation

(oscillation)
C

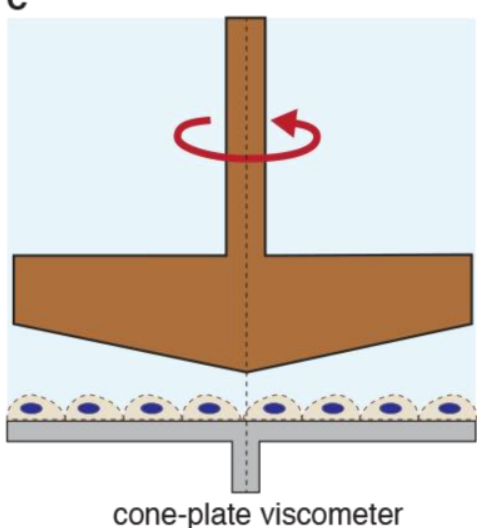

$\mathbf{F}$
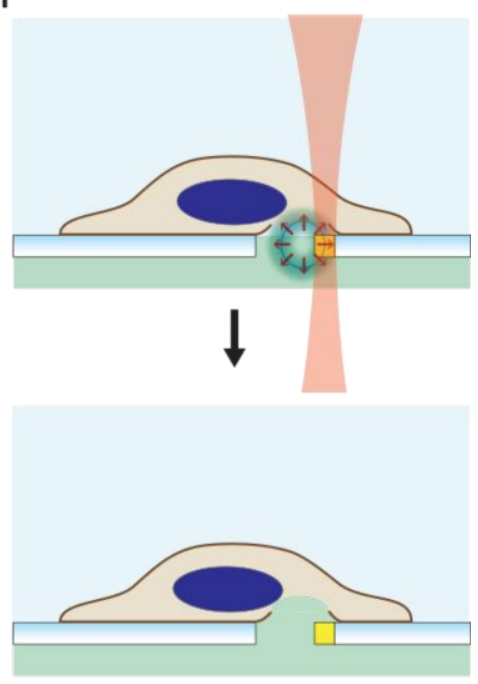

laser-induced cavitation from seed structure

Figure 18. Mechanical membrane permeabilization by fluid shear forces. (A) Syringe loading, where a cell solution is repeatedly aspirated and ejected through the terminal aperture of a syringe needle. Shear forces at the nozzle promote cell membrane disruption. The inset illustrates cell deformation associated with shear forces. (B) Microfluidic shear-based permeabilization. Similar to syringe loading but exploiting the increase of shear forces associated with flow through narrowing microfluidic channels. The inset illustrates cell deformation upon flow through a single constriction. (C) Cone-plate viscometer. Generation of permeabilizing shear forces via rotation of a viscometer plate above a monolayer of cells. (D) Generation of local shear forces via collapse of a cavitation bubble. (E) Generation of local shear forces via oscillation of a cavitation bubble. (F) Induction of cavitation bubbles on the basal side of a cell through a seed structure that absorbs laser energy. The cavitation bubble can produce a large hole in the plasma membrane that allows influx from a separate fluid reservoir underneath the cell.

The most common application of syringe loading has been delivery of proteins and antibodies to the intracellular space.

GST-FAK fusion proteins were loaded into fibroblasts by passing them through a 30 gauge syringe needle 30 times ${ }^{808}$.

HEp-2 cells were loaded with monoclonal antibodies by 20 cycles through a 27 gauge needle ${ }^{809}$. A modified version of the protocol was employed by Sydor et al. to deliver fluorescently-labeled antibodies into trypsinized neurons by using $\sim 100$ cycles of aspiration-expulsion though pipette tips ${ }^{810}$. For delivery of monoclonal antibodies to fibroblasts, a mixture of cells and antibodies was cycled 20 times through a 30 gauge needle ${ }^{811}$. Kasier et al. syringe loaded a fluorescently labeled version of the protein profilin into amoebas and human cells to study its binding to intracellular actin ${ }^{812}$. In other studies of 
the actin cytoskeleton, FITC-conjugated anti-fascin immunoglobulins were delivered into $29 \%$ of fibroblasts or myoblasts by 4 passages through a $1 \mathrm{ml}$ syringe fitted with a 25 gauge needle ${ }^{813}$. Researchers from the Schwartz lab loaded endothelial cells with alexa-labeled versions of the p21 binding domain of PAK1 to investigate mechanobiology of the Rac1 pathway ${ }^{814,815}$. Several studies have also employed syringe loading to study the effect of bacterial and viral proteins inside cells. For example, fibroblasts were syringe-loaded with HIV proteins to examine their impact on intracellular and nuclear architecture ${ }^{816}$. In another case, CHO cells were drawn up and expelled slowly $\left(\sim 0.2 \mathrm{ml} \mathrm{s}^{-1}\right)$ through a 30 gauge syringe needle 6 times for intracellular delivery of the bacterial toxin ExoU ${ }^{817}$. Moreover, $\mathrm{Xu}$ et al. delivered the Legionella pneumophila protein SidK into macrophages by 100 cycles of pipetting through a $200 \mu 1$ pipette tip $^{818}$. In studies of herpes simplex virus replication, herpes virus and nucleoporin antibodies were introduced into Vero cells by 50 passages through a 27 gauge needle ${ }^{819}$.

\subsubsection{Microfluidic Control of Shear Forces}

Syringe loading presumably works by creating regions of significant shear force around the entrance and exit of the syringe needle (Figure 18A). Because the fluid flow is controlled manually, however, it may require extensive empirical testing and skill to reproducibly obtain optimal cell treatments ${ }^{820}$. Improved precision and reproducibility could potentially be achieved by using microfluidic devices to generate consistent zones of fluid shear. Along these lines, Prausnitz and colleagues fabricated a simple flow-through microfluidic device with parallel constrictions ${ }^{821}$ (Figure 18B). Lasers were used to bore out 50 - $300 \mu \mathrm{m}$ conical microchannels from 100 to $250 \mu \mathrm{m}$ thick mylar sheets and syringe pumps were employed to flow cell suspensions through the channels at controlled flow rates, thereby subjecting cells to well-defined shear forces. The resultant loading of fluorescently labeled dextrans and proteins into DU145 prostate cancer cells, as well as the viability, however, turned out to be less favorable than syringe loading. Further attempts towards plasma membrane permeabilization through microfluidic control of shear forces have not been reported and therefore present an opportunity for future investigations.

\subsubsection{Other Examples of Cell Permeabilization Through Shear Forces}

Driving fluid through narrow constrictions is not the only way to generate fluid shear forces for cell permeabilization. Indeed, researchers have used cone-plate viscometers to produce hydrodynamic shear forces above cell monolayers, 


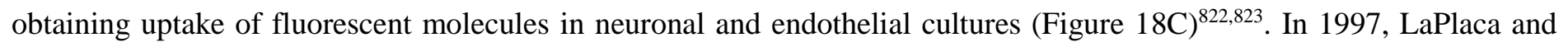
colleagues confirmed permeabilization of neurons by observing an increase in intracellular $\mathrm{Ca}^{2+}$, release of intracellular enzymes to the extracellular solution, and cell swelling ${ }^{822}$. Later, Blackman and colleagues used a modified cone-plate setup to expose endothelial cell monolayers to consistent fluid shear forces ${ }^{823}$. When forces were too high, cells peeled away from the substrate. After empirical optimization, however, conditions were identified where all cells remained attached to the substrate yet $16 \%$ of cells retained $4 \mathrm{kDa}^{823}$. The Blackman cone-plate viscometer was then used to permeabilize cultured neurons, investigate their physiological response, and test strategies to improve post-injury neuron survival $^{824}$. Relative permeabilization efficiency was analyzed by influx of small molecular weight fluorescent dyes ${ }^{824}$.

Intense pulses of fluid shear can be directed at cells by firing jets of pressurized inert gas toward them ${ }^{825,826}$. Similar to the case of cone-plate viscometers, it was found that excessive shear forces can rip cells away from the underlying substrate, but if modulated just below this range, were capable of permeabilizing cell membranes while leaving adherent cells in place. With the appropriate optimizations, intracellular delivery of dextrans, plasmids and other cargo has been demonstrated in common adherent cell lines permeabilized by inert gas jets ${ }^{825,826}$.

\subsubsection{Sonoporation}

Sonoporation is the disruption of cell membranes by acoustic pressure waves, mostly in the ultrasound frequency range $(20 \mathrm{kHz}$ to $\mathrm{GHz}$ ). Its deployment for intracellular delivery purposes first arose in the mid 1980s through the use of ultrasound to permeabilize cultured cells ${ }^{545,827-829}$. Permeabilization was achieved by placing cell suspensions in a plastic tube and applying 3 half-second pulses of the ultrasonic transducer directly to the tube. With this rudimentary approach,

Fechheimer et al. demonstrated intracellular loading of dextrans and proteins into Amoebae ${ }^{545,827,829}$. Ultrasound-mediated permeabilization was compared head-to-head with scrape loading ${ }^{545}$. However, scrape loading was found to yield superior delivery of dextran-conjugated dyes and DNA plasmids into HeLa cells, hepatic tissue cultures, and mammalian fibroblasts ${ }^{545}$.

About a decade later, sonoporation began to be taken seriously as a method for DNA transfection ${ }^{830-832}$. Several factors converged to motivate this trend ${ }^{833}$. First, high intensity focused ultrasound was gaining prominence as a non-invasive 
method for therapeutic treatment of targeted cells and tissues in vivo ${ }^{834,835}$. Examples include local tissue ablation, local drug delivery stimulated by ultrasound, and, gene therapy by targeted nucleic acid transfection ${ }^{836}$. Second, the mechanisms of ultrasonic effects were being increasingly clarified, with cavitation bubbles implicated as the prime instigators of membrane disruption ${ }^{837}$. These mechanistic insights enabled a more rational approach toward sonoporation that greatly boosted its efficiency. Particularly key was the deployment of gas body ultrasound contrast agents to act as cavitation nuclei. This modification was found to drastically improve transfection efficiency compared to ultrasound alone $^{831,838}$. For example, commercially available microbubbles were mixed with cultured immortalized human chondrocytes and exposed to $1.0 \mathrm{MHz}$ ultrasound transmitted through the bottom of a six well culture plate. The addition of microbubble cavitation nuclei, along with other empirical optimizations, enhanced DNA transfection nearly 20 -fold over previous reports and indicated that ultrasound could be a feasible DNA transfection technique ${ }^{838}$.

\subsubsection{Mechanisms of Sonoporation}

As the field currently stands, hundreds of studies have been published on the subject of understanding and improving sonoporation. Although non-invasive in vivo applications may be the final goal, many of these efforts have exploited in vitro experiments for in-depth mechanistic investigations and proof-of-principle studies. Recent reviews have covered the sonoporation field in detail ${ }^{839-844}$. The mechanisms underlying sonoporation are diverse and may involve: 1) microstreaming caused by stable cavitation, whereby a cavitation bubble oscillates in synchrony with the acoustic field (Figure 18E), 2) jetting forces from inertial cavitation, which is triggered by the collapse of a cavitation bubble (Figure 18D), 3) a shrinking cavitation bubble pulling against the plasma membrane ${ }^{845}$, 4) an expanding cavitation bubble pushing against the plasma membrane $\left.{ }^{845}, 5\right)$ bubble translation, whereby acoustic radiation forces push a bubble through the plasma membrane, 6) nucleation of a cavitation bubble between bilayer leaflets, rupturing the membrane upon expansion, and 7) non-bubble acoustic effects, such as acoustic streaming due to pressure differences of the acoustic field ${ }^{840,842,846}$. The literature consensus indicates that the first two mechanisms are the most prevalent. Below we discuss how these cavitation phenomena generate membrane disruptions.

Cavitation bubbles form and/or expand when the low-pressure part of the acoustic wave passes through a liquid medium.

Conversely, the high pressure peak of the wave corresponds with compression and/or implosion of cavitation 
bubbles $^{840,842,846,847}$. The bubbles may be created by the pressure waves themselves or provided by the supplementation of stabilized microbubbles in the form of commercially available contrast agents. A bubble that expands and contracts in synchrony with the acoustic field (stable cavitation) generates local oscillatory shear forces due to microstreaming ${ }^{848,849}$. The microstreaming forces are sufficiently potent to permeabilize nearby cells. On the other hand, a bubble that implodes (inertial cavitation) can trigger extreme phenomena including electromagnetic radiation (sonoluminescence), severe temperature spikes up to thousands of degrees, sonochemical reactions such as the production of free radicals, and intense microjetting. Although any of those phenomena can perturb lipid bilayers, the permeabilizing effects of bubble collapse have primarily been ascribed to the potent fluid shear forces generated by microjetting ${ }^{840,842,846}$. As a cavitation bubble implodes, surrounding water molecules rush in to fill the void. If there is a surface nearby (such as a lipid membrane) less water molecules are available to flow from that region. This biases the flow towards that surface and results in the microjet being oriented in that direction. Thus, imploding cavitation bubbles can result in the selective puncture of an adjacent cell (Figure 17D). High pressure ultrasound is more likely to trigger inertial cavitation while low pressure procedures bias the system toward stable cavitation ${ }^{842}$.

\subsubsection{Cargo Delivered by Sonoporation}

Because of the variation in magnitude and mode of fluid shear phenomena related to sonoporation, it is perhaps not surprising that the resultant holes have been reported to range from nanometers up to several micron ${ }^{840,842,846,850-852}$. Under conditions where large holes are generated, sonoporation can be expected to enable delivery of small and large cargos alike. Because of the motivation for gene therapy, significant efforts have gone into optimizing sonoporation for DNA transfection over the last two decades ${ }^{830,831,833,838,853-859}$. Transfection of other nucleic acids, such as antisense oligonucleotides ${ }^{860}$, siRNA ${ }^{861,862}$, and $\mathrm{mRNA}^{863}$ have received less attention, but have also been demonstrated. To study mechanisms, much work in the field has exploited delivery of fluorescently labeled dextrans of varying molecular weight ( 1-30 nm hydrodynamic radius $)^{545,827,828,849-851,864-872}$ and low molecular weight dyes $(<1 \mathrm{~nm})^{802,845,850,851,857,865,871,873-881}$. Also demonstrated has been intracellular delivery of small molecule drugs ${ }^{871,882-886}$, polymer nanoparticles of $25-75 \mathrm{~nm}^{867}$, viral particles ${ }^{887}$, proteins ${ }^{865}$, antibodies ${ }^{888}$, and peptides ${ }^{889}$. In some cases delivery has been ascribed to endocytosis and not influx after permeabilization ${ }^{868}$. This explanation could be applicable to larger cargo such as plasmid DNA, where delayed expression kinetics akin to electroporation have been observed ${ }^{846}$. As with other mechanical delivery methods ${ }^{787}$, 
the plasmid transfection efficiency of acoustic shear-based methods may be improved by addition of downstream electrophoretic forces to augment delivery of charged cargoes ${ }^{890}$.

The majority of reports on sonoporation-mediated delivery have focused on technology development and not its use to carry out basic research. In the early days of sonoporation in the late 1980s and early 1990s, however, there were several examples of biologists using it to carry out basic research $827,829,873,887,888$. Although at least one commercial sonoporation system has been available for more than a decade (Sonidel SP100), its use for intracellular delivery appears confined within the ultrasound community ${ }^{891,892}$. The most significant challenge for sonoporation in vitro remains the random and uncontrolled nature of cavitation events leading to excessive cell damage and death ${ }^{844}$. A 2012 review of 26 published studies conducted over more than a decade concluded that conventional in vitro sonoporation with nucleation agents almost never yielded above $50 \%$ for both delivery efficiency and cell viability ${ }^{844}$. Poor viabilities are perhaps due to cavitation-related side effects such as high local temperatures and generation of reactive oxygen species ${ }^{893}$. Thus, bulk sonoporation may be inherently limited as a delivery approach in vitro. In vivo applications have been more promising ${ }^{836}$, especially in skin where optimal parameters have been identified and barriers to delivery of therapeutic cargo are more on the tissue, rather than cellular, level ${ }^{839}$.

\subsubsection{Shock Wave-Mediated Permeabilization}

Shock waves differ from acoustical waves in that they are higher pressure and propagate at supersonic speed ${ }^{894}$. They are best known as the by-products of explosions. Various devices and strategies have been employed for producing shock waves to permeabilize cell membranes. They include shock wave lithotripters ${ }^{895-898}$, shock tubes ${ }^{899-901}$, underwater spark discharge $e^{902}$, and laser-induced shock waves ${ }^{900,901,903-909}$. These systems mostly administer pulses one at a time instead of the continuous waves characteristic of acoustic ultrasound. Lithotripters generate potent high pressure pulses that are used to break down tissue obstructions such as kidney stones (requiring up to 4000 individual pulses). In 1994, Gambihler and colleagues placed polypropylene vials containing a mixture of suspended mouse L1210 lymphocytic leukemia cells and fluorescent dextrans under the focal point of lithotripter shock waves ${ }^{897}$. After treatment, the uptake and retention of dextran molecules was detected by flow cytometry. Although the authors admitted electroporation was more consistent 
and efficient, lithotripter treatment showed a significant uptake of $2000 \mathrm{kDa}$ dextran $(\sim 50 \mathrm{~nm}$ diameter $)$ with reasonable cell survival.

Kodama et al. employed shock tubes to generate intense shock waves in cell suspensions and obtain intracellular delivery of labeled dextrans ${ }^{899-901}$. Shock tubes generate a mechanical pulse when a thin diaphragm between a high pressure and low pressure chamber ruptures. The pulse then propagates through a second diaphragm and is focused into the cell solution via a reduction nozzle, thereby achieving membrane permeabilization ${ }^{900}$.

A number of studies have employed laser-induced shock waves for cell membrane permeabilization ${ }^{900,905-909}$. Laserinduced stress waves can be generated by optical breakdown, ablation, or rapid heating of an absorbing medium ${ }^{907}$. In one configuration, laser irradiation of an absorbing polymer film produces shock waves that emanate into a solution containing cells and cargo ${ }^{906,908,909}$. Depending on experimental conditions, the mechanism of cell membrane disruption may or may not rely on cavitation. In one set of examples, the rise time of the stress wave and its duration was associated with membrane permeabilization, probably due to shear forces involved with the wavefront itself ${ }^{900,905-907,909}$. Conversely, in other studies cavitation was implicated as the critical determinant of shock wave-induced membrane damage ${ }^{895,903,910 .}$

\subsubsection{Laser-Induced Cavitation Bubbles}

So far, we have covered membrane disruption arising from acoustic pressure waves and shock waves, as well as cavitation phenomena triggered by these stimuli. Cavitation can also be triggered and/or controlled in a more direct manner by the action of lasers incident upon an absorbent agent in an aqueous environment ${ }^{911,912}$. The absorbent agent may be the membrane itself, a photoabsorbent molecule added to solution, a particle suspended in solution, or a material interfacing with the solution (Figure 19). When the plasma membrane absorbs laser energy and is disrupted (Figure 19A), this is known as optoporation and is covered in section 6.4. If the absorbing agent is in direct contact with the plasma membrane, the membrane will likely be perforated by a complex combination of secondary effects including extreme heat, near-field plasmas, and phenomena related to growth and collapse of cavitation bubbles (Figure 19B). If the absorbing agent is distant from the plasma membrane, membrane disruption is much simpler and cleaner: it most likely occurs by fluid shear (Figure 19C) as thermal effects and near-field plasmas do not propagate very far in an aqueous environment. In any of the 
above three scenarios (Figure 19A-C), the membrane may be disrupted by laser-induced cavitation. Upon absorption, the energy supplied by the laser is transduced into heat and/or chemical effects that lead to vaporization of surrounding liquid to create cavitation bubbles ${ }^{911,912}$. The bubbles disrupt cell membranes in the same way as sonoporation, either by microjetting after collapse (Figure 18D), through microstreaming from bubble oscillation (Figure 18E), or through secondary effects. Most reports of laser-induced cavitation suggest bubble collapse, but there are a few cases where laser pulsing regimes can be tuned to sustain bubble oscillations ${ }^{913}$.

A

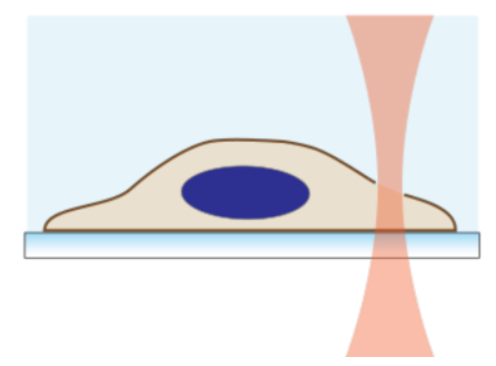

B

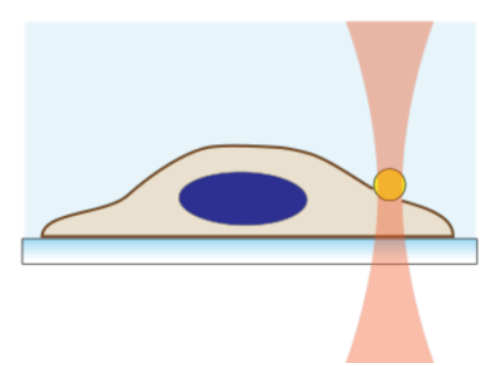

C

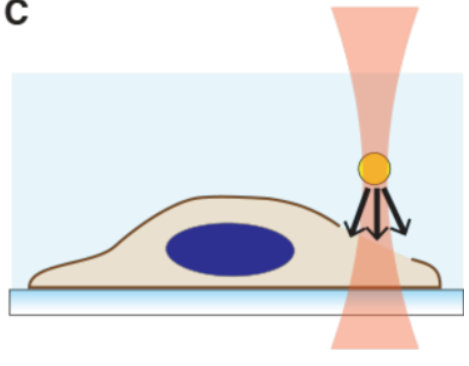

Figure 19. Mechanisms of laser-induced membrane disruption. (A) Laser optoporation occurs when incident energy is absorbed by the plasma membrane, directly disrupting it. This is known as "Optoporation" and is covered in section 6.4. (B) Membrane disruption eventuates as the result of laser absorption by an absorbing agent in contact with the cell (such as a particle or interface), which then generates secondary effects (heat, fluid shear, near-field plasma etc.) to disrupt the plasma membrane. (C) Membrane disruption eventuates through laser absorption by an absorbing agent distant from the plasma membrane. In these cases, fluid shear from cavitation and/or shock waves is the most likely cause of membrane disruption.

In a series of studies by $\mathrm{Ohl}$ and colleagues, microfluidic confinement was used to investigate the relationship between membrane damage and proximity to laser-induced cavitation bubbles ${ }^{914}$. The photo-absorbent molecule phenol red was added to solution to allow generation of cavitation bubbles from the laser focal region. Their results showed that the probability of cell permeabilization by cavitation bubble collapse could be modeled as a function of the distance of cells from the bubble and maximum cavitation bubble radius ${ }^{914}$. In a follow up study, they took advantage of arrayed microfluidic cell traps to immobilize myleoma cells and systematically analyze the conditions for controlled permeabilization at single cell level ${ }^{487}$. Again, phenol red was used as an absorbing agents to facilitate the production of laser-induced cavitation bubbles that expand to $\sim 100 \mu \mathrm{m}$ diameter and collapse within tens of microseconds ${ }^{487}$. High frame rate imaging clearly visualized the expansion and shrinkage of cavitation bubbles in a non-symmetric manner due to the presence of a nearby structure. During bubble collapse, a fast microjet was directed toward the cell to generate a single large pore with diameters ranging from 0.2 to several $\mu \mathrm{m}$. The diffusive uptake of trypan blue dye into the cell then took place over several seconds. If the standoff distance between cell and bubble were greater than $30 \mu \mathrm{m}$, no membrane disruption occurred. One concern highlight by the authors was whether cavitation bubbles perturb cells through 
temperature spikes. To address this issue, Ohl and colleagues performed another study with fluorescence-based thermometry to measure local temperature gradients around laser-induced bubbles ${ }^{915}$. Under similar conditions as their previous experiments, it was found that the temperature rises are moderate $\left(<12.8{ }^{\circ} \mathrm{C}\right)$, localized $(<15 \mu \mathrm{m})$ and short lived $(<1.3 \mathrm{~ms})$. Thus, by developing a cavitation regime that damages cell membranes purely through mechanical forces, laser-induced cavitation may be amenable to implementation on a wider scale. It was suggested that arraying cells in microfluidic traps would allow for potential scale-up with pre-determined laser protocols to control the size and position of membrane-permeabilizing cavitation bubbles.

\subsubsection{Laser-Induced Cavitation via Absorbent Particles}

To transduce laser energy into cavitation, some approaches employ a deliberate seed particle to absorb the laser energy. One of the first papers to do this was published by Pitsillides et $\mathrm{al}^{916}$. They labeled lymphocytes with antibodyfunctionalized metal microspheres and irradiated them with a $565 \mathrm{~nm}$ laser at a fluence of $0.35 \mathrm{~J} \mathrm{~cm}^{-2}$ and pulse duration of $20 \mathrm{~ns}^{916}$. Rapid eminence of microbubbles was observed around the seed particles and cell membranes were subsequently permeabilized. By adjusting particle numbers, size, and laser energy delivered to the metal microspheres it was possible to tune the treatment either toward killing cancer cells for potential therapeutic purposes or transiently increasing the permeability of the plasma membrane for intracellular delivery ${ }^{916}$. Another group used femtosecond laser irradiation of gold nanoparticles to produce plasmonic nanobubbles and permeabilize primary human cells for ex vivo intracellular delivery ${ }^{917,918}$. Selective delivery of plasmids and dextrans was demonstrated in primary human cancer cells, T cells, and hematopoietic stem cells with reportedly good cell viability ${ }^{917,918}$.

In 2010, Prausnitz and colleagues introduced an intracellular delivery strategy involving laser irradiation of dispersed carbon black nanoparticle ${ }^{919}$. Adherent cells were exposed to the cargo molecule to be delivered and sprinkled with $\sim 200$ $\mathrm{nm}$ aggregates of carbon black followed by irradiation with femtosecond lasers ${ }^{919}$. Rather than thermal effects, they proposed that the mechanism of membrane disruption was primarily due to a carbon-steam reaction at the particle surface, which subsequently propagates cavitation-related acoustic forces ${ }^{919,920}$. Delivery of dyes, proteins, siRNA and plasmid DNA were achieved with acceptable cell viabilities in several cancer cell lines ${ }^{919,921}$. Control experiments verified that neither the carbon black particles nor laser exposure alone were sufficient to enable molecular uptake ${ }^{919}$. This intracellular 
delivery concept was then extended beyond adherent cells to homogenous suspensions of carbon black nanoparticles and cells, which may be more amenable to treatment at higher throughputs ${ }^{922}$.

In a different strategy from Braeckmans and co-workers, gold nanoparticles were employed as absorbing agents and laser excitation parameters were screened to test for and manipulate the balance between pure heating and bubble nucleation ${ }^{923}$. By tuning the laser energy, they identified conditions where it was possible to produce vapor nanobubbles around $\sim 70 \mathrm{~nm}$ gold nanoparticles without transfer of heat to the surrounding environment. Comparing these two strategies revealed that vapor nanobubbles enabled superior delivery and siRNA transfection with less cytotoxicity ${ }^{923}$. Building on this approach, the same group delivered quantum dots into cells at high-throughput with efficiencies and viabilities above $80 \%{ }^{310}$. Furthermore, in primary human $\mathrm{T}$ cells the vapor nanobubble approach was reported to yield greater siRNA transfection efficiency and cell survival when compared with nucleofection ${ }^{924}$. In congruence with these results, other groups have presented experimental and theoretical work that demonstrates nanobubble formation from the generation of a nanoscale plasma around the particle due to the enhanced near-field rather than from the heating of the particle $\mathrm{e}^{925,926 .}$

\subsubsection{Laser-Induced Cavitation at an Interface}

Absorbing materials can be placed at a solid-liquid interface to convert laser energy into membrane-perturbing cavitation bubbles or shock waves. In recent studies, Ohta and colleagues fabricated a channel of defined height, with cells cultured on one side apposing an optically absorbing composite layer of $1 \mu \mathrm{m}$ amorphous silicon and $200 \mathrm{~nm}$ indium tin oxide ${ }^{927}$. Instead of generating an exploding bubble, they oscillated a bubble using a $980 \mathrm{~nm}$ laser with $90 \mu$ s pulses over a duration of 10-15 seconds. Up to 3 oscillations of 8-10 $\mu \mathrm{m}$ without collapse were able to induce microstreaming shear forces to trigger plasma membrane permeabilization in adjacent cells. In this configuration, the bubble had to be pressed tightly against the cell to induce membrane disruption. For $70 \mathrm{kDa}$ fluorescently labeled dextran, up to $80 \%$ delivery at $>95 \%$ viability was achieved. The pore-size was estimated to be about $30 \mathrm{~nm}$ based on exclusion of $500 \mathrm{kDa}$ dextran and the closure dynamics indicated plasma membrane healing within $~ 20$ seconds. In a follow-up study, the same authors lowered the channel height to $10 \mu \mathrm{m}$ and generated stronger shear forces over 0.4 seconds with $60-100 \mu$ s pulses applied at a frequency of $50 \mathrm{~Hz}^{913}$. By generating larger pores with a more powerful shear forces, delivery efficiency of $500 \mathrm{kDa}$ dextran improved to $70 \%$ and expression of $5.7 \mathrm{~kb}$ DNA plasmid was recorded at $86 \%$. 
Permeabilization of adherent cells can be achieved by culturing them on patterned thermoplasmonic substrates followed by laser irradiation ${ }^{509,928,929}$. In a strategy introduced by Mazur and colleagues, a thermoplasmonic substrate patterned with microscale gold-coated pyramids was fabricated by photolithography and template-stripping. A nanosecond pulsed laser was then scanned across the substrate to produce intense heating at the apex of each pyramid, thereby generating bubbles through plasmonic effects ${ }^{930}$. A large beam spot can be scanned across the substrate to permeabilize millions of cells over the course of minutes ${ }^{929}$. Growth and collapse of the bubbles presumably disrupts cell membranes by mechanical shear forces, although plasmonic chemical effects or heat cannot be ruled out. Delivery of dextrans up to $2000 \mathrm{kDa}$ have been obtained with high cell viabilities ${ }^{929}$ through holes estimated to be in the range of $20 \mathrm{~nm}^{509}$.

In a different approach, the Chiou lab developed a "photothermal nanoblade" capable of addressing single cells ${ }^{339}$. A metallic nanostructure was placed at the tip of a micropipette as a seed structure to harvest short laser pulse energy and convert it into highly localized explosive vapor bubbles. Upon placement of the device next to cells, laser irradiation triggered cavitation events that yielded controlled pore sizes of up to several microns on the apical surface of adherent cells. Delivery of large cargo such as $\sim 2 \mu \mathrm{m}$ bacteria, mRNA, plasmid DNA, polystyrene beads, and quantum dots was achieved $^{309,339}$. Furthermore, in an intriguing biological application, the photothermal nanoblade was used for mitochondrial transplants between cells ${ }^{341}$. By delivering functional mitochondria to cells with normally dysfunctional mitochondria, it was possible to identify mechanisms involved in restoration of metabolism ${ }^{341}$. Consistent with what is known about membrane repair in healthy cells, electrical impedance measurement showed that it takes 1-2 minutes to recovery membrane integrity after treatment with the photothermal nanoblade ${ }^{931}$.

A high throughput version of the photothermal nanoblade concept was unveiled in $2015^{340}$. Substrates arrayed with pores lined by metallic absorbers were irradiated to generate exploding cavitation bubbles underneath the basal side of adherent cells (Figure 18F). Membrane permeabilization was synchronized with active pumping of cargo through the pores to successfully introduce living bacteria into the cytoplasm of several cell types. Showcasing the potential of the approach, it was discovered that the $i g l C$ gene from the bacterial species $F$. novicida is required for intracellular proliferation after cytosolic delivery. Such a high-throughput strategy to deliver micron-sized cargo clearly has broad utility with adherent cells, showcasing the power of well-controlled fluid shear forces to permeabilize vast populations of cells. 


\subsubsection{Summary of Permeabilization by Mechanical: Fluid Shear}

Methods to exploit fluid shear forces to permeabilize cell membranes range from constriction-mediated fluid shear zones

in microfluidic devices and syringe nozzles to cone-plate viscometers and cavitation-related phenomena. The use of cavitation bubbles for plasma membrane disruption has received the most attention to date due to the ability to control them remotely via acoustic fields and lasers. Because of the inherently unstable and powerful nature of cavitation, challenges remain in how to harness them for reproducible and consistent cell treatments. Cavitation bubbles, however, have demonstrated the ability to delivery almost any cargo to a wide range of cell types. In future we anticipate intriguing and innovative devices able to deploy highly localized fluid shear forces for precise membrane disruption, perhaps through the use of lab-on-chip, microfluidic, and nanotechnological systems.

\subsubsection{Mechanical: Pressure Changes}

Osmotic and hydrostatic pressure gradients can be imposed across cell membranes leading to their rupture. The geometry of these gradients can vary, for example between a suspended cell and the extracellular solution, across a select part of the plasma membrane (such as the apical membranes of an adherent cell monolayer), or between an intracellular vesicle (e.g. endosome) and the surrounding cytosol. Although difficult to control in time and space, transient pressure gradients achieved by osmotic or hydrostatic means represent a low-cost and simple avenue for membrane disruption-mediated intracellular delivery. These methods have not been heavily pursued to date, however, perhaps due to a poor understanding of their effects and hesitance of researchers to excessively perturb cells ${ }^{932}$.

\subsubsection{Osmotic Shock and Plasma Membrane Disruption}

One of the simplest perturbations that a cell can experience is an osmotic shock, whereby a hydrostatic pressure is generated across the cell membrane due to differences in osmotic potential. Most mammalian cells normally exist in an aqueous environment of $\sim 300$ mOsm and significant deviations from this condition will induce the flow of water molecules into (hypotonic swelling) or out of (hypertonic shrinkage) the cell. When a cell is placed into a low osmolarity solution water rushes into the cell through the plasma membrane and aquaporin channels to solvate impermeable intracellular electrolytes and osmolytes. The subsequent swelling of cell volume leads to the unfolding of loose 
membrane, followed by well-described lipid bilayer rupture if area strain exceeds 2-3\% (Figure 20A). Cells have been reported to possess membrane reservoirs of 2-10x their apparent surface area depending on the cell type and state ${ }^{415}$. Caveolae, endocytic pits, membrane folds, filopodia and microvilli are all examples of membrane reservoirs that can unfold to buffer membrane strain and accommodate cell surface area increase ${ }^{414,416}$. It is thought that these reservoirs should be exhausted globally or locally before membrane stretch can result in rupture.

A
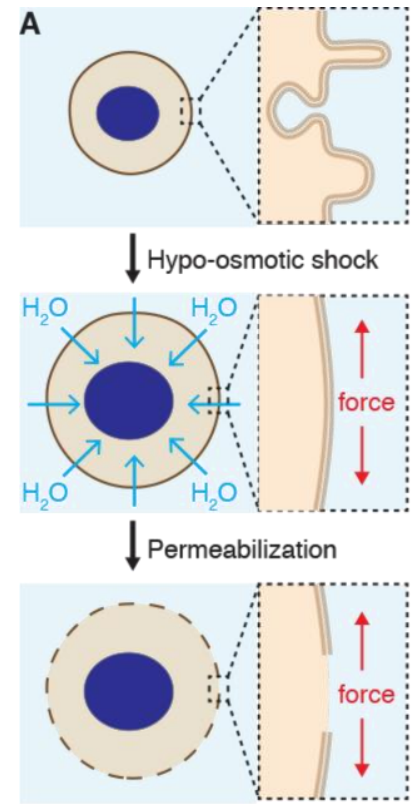

Hypo-osmotic shock (suspension)

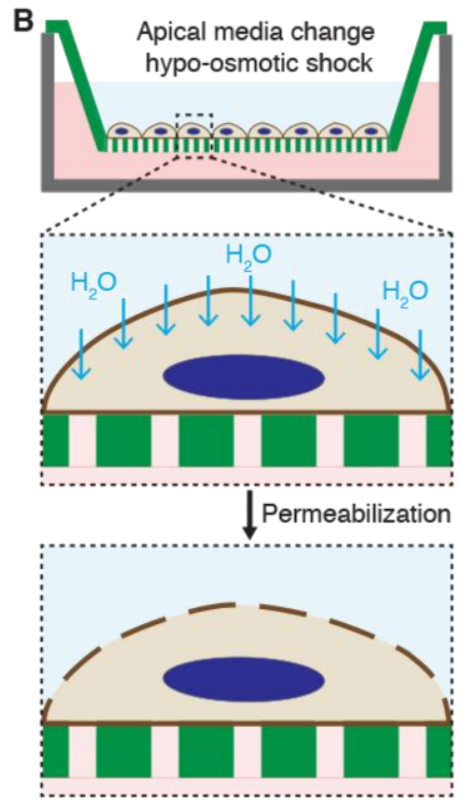

Hypo-osmotic shock (apical)

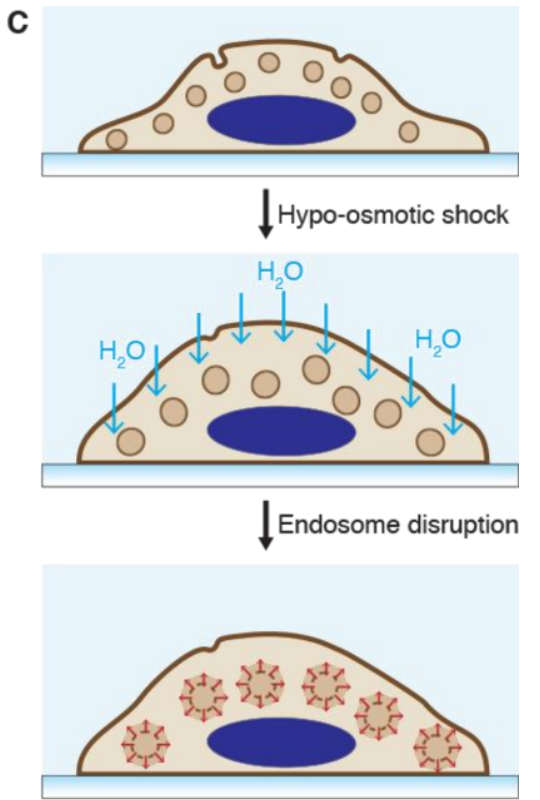

Osmotic lysis of endosomes

Figure 20. Mechanical membrane disruption via osmotic pressure changes. (A) Cells in suspension subject to hypotonic shock will first swell, which unravels membrane reservoirs. If the membrane strain is sufficient, permeabilization will occur. The inset shows microscale conformation of the plasma membrane. (B) Cells in an adherent monolayer cultured on a porous substrate can be subject to a perturbing osmotic gradient via hypotonic shock at their apical surface. Swelling and subsequent permeabilization occur similarly as in panel A but the permeabilization is localized to the apical side of the cell. (C) In a scenario where endosomes are preloaded with osmolytes and cargo to be delivered, a hypotonic shock can be used to cause lysis of endosomes and release of cargo to the cytoplasm.

\subsubsection{Hypotonic Loading of Red Blood Cell Ghosts}

If the magnitude and duration of osmotic shock is optimal, partially burst cells can recover membrane integrity in the form of hollowed out "ghosts". Although dead, ghosts can reseal and regain a limited set of functions. The concept was first established in red blood cells (RBCs) throughout the $1960 \mathrm{~s}^{933-935}$. Although RBCs possess little surface reservoirs compared to most nucleated cells, their capacity to reseal after a brief hypotonic shock is well-proven ${ }^{936,937}$. Indeed, RBC ghosts were able enclose molecular cargo and even retain some basic biological functions despite being hollowed out of cytoplasmic components ${ }^{937,938}$. In one early study, by adding ferritin at various times after the onset of hemolysis, it was 
determined that most cells were permeable for 15-25 seconds after hypotonic shock ${ }^{939}$. Furthermore, the size and shape of membrane disruptions, as seen in fixed cells by SEM imaging, resembled long, narrow tears up to $1 \mu \mathrm{m}$ long ${ }^{940}$. Later more accurate studies, however, indicated smaller holes around tens of nanometers or less ${ }^{941}$. Further adaption of the technique optimized the hypotonic lysis procedures to result in high efficiency loading of proteins and enzymes into RBC ghosts $^{938,942,943}$. Partly due to such ease of hypotonic loading and autologous biocompatibility, RBC ghosts have been proposed as drug carriers for decades ${ }^{944-947}$. Furthermore, fusion of loaded RBC ghosts into recipient cells was a popular method of intracellular delivery in the $1970 \mathrm{~s}$ and $1980 \mathrm{~s}^{87,938,948,949}$ before falling out of favor with the rise of electroporation and other alternatives ${ }^{544}$.

\subsubsection{Hypotonic Shock for Intracellular Delivery}

Unlike RBCs that can passively reseal, most cell types mobilize active repair processes to recover from membrane disruption ${ }^{424}$. It wasn't until the early 1980 s that osmotic delivery methods would be translated beyond RBCs into other cell types. In 1982, Borle and Snowdowne devised a simple procedure to deliver the calcium-sensitive protein aequorin $(21 \mathrm{kDa})$ into nucleated cells ${ }^{92,93}$. Washed pellets of monkey kidney cells were suspended and immersed in a $\sim 10 \mathrm{mOsm}$ hypotonic solution consisting of $3 \mathrm{mM}$ MgATP, $3 \mathrm{mM}$ HEPES buffer, and a given concentration of aequorin for 2 minutes at $4{ }^{\circ} \mathrm{C}$. This was followed by sufficient addition of buffered $\mathrm{KCl}$ to restore isotonicity. Cells were then incubated in standard cell media for 1 hour at $37{ }^{\circ} \mathrm{C}$ to promote restoration of homeostasis before experiments. Optical readouts of aequorin activity indicated that it had been loaded successfully into fully functional cells, and it was used to measure accurate intracellular calcium concentrations of $\sim 50 \mathrm{nM}$.

Citing Borle and Snowden's method as an inspiration, the hypo-osmotic approach for cytoplasmic delivery of aequorin was re-examined in greater detail by Klabusay et $\mathrm{al}^{950}$. They were motivated by the need to accurately measure intracellular calcium dynamics in follicular lymphoma B cells, an application where the aequorin protein offers higher signal-to-noise ratio, better dynamic range, and more reliable calcium readouts than commonly used small fura dyes. In their method, cell suspensions of $30 \mu \mathrm{l}$ were added to $200 \mu \mathrm{l}$ of $\mathrm{pH}$ buffered hypo-osmotic solution $(\sim 2 \mathrm{mOsm})$ and 0.1 $\mathrm{mg} \mathrm{mL}-1$ aequorin before gentle mixing. After a pre-determined duration of hypotonic exposure, addition of $230 \mu 1$ hyperosmotic solution was used to bring the suspension back to isotonic conditions and enable membrane recovery. To 
test the cell response to hypo-osmotic exposure, the time between addition of hypo-osmotic and return to isotonic conditions was varied from 10 seconds to 10 minutes. They found that treatment times of 10-30 seconds were ineffective in loading aequorin $(21 \mathrm{kDa})$ or GFP $(28 \mathrm{kDa})$. Upon two minutes exposure, long term cell viability up to 18 hours was more than $50 \%$ with sufficient delivery to determine intracellular calcium concentrations, which turned out to be $\sim 0.9 \mu \mathrm{M}$ in follicular lymphoma cells. 10 minutes exposure led to robust delivery but a gradual loss of viability in almost all cells after 10 hours, probably due to delayed cell death responses (see section 4.3). One major advantage of Klabusay's protocol is its applicability to treat difficult-to-transfect suspension cells and that it appears agnostic to cell size and type of cargo material to be delivered.

In 1999 Koberna and co-workers unveiled a method based on a 'hypotonic shift' to achieve intracellular delivery of modified nucleotides, nucleosides, dyes, and peptides into a wide range of cell types ${ }^{951}$. The hypotonic buffer consists of $10 \mathrm{mM}$ HEPES for $\mathrm{pH}$ buffering and $30 \mathrm{mM} \mathrm{KCl}(\sim 70 \mathrm{mOsm})$. Cells were exposed to the hypotonic buffer for 5 mins before a return to isotonic media for recovery. After treatment, metabolic production of DNA, RNA, and protein was inhibited and took $\sim 4$ hours to return to normal levels. No loss of viability or apoptosis was observed. The hypotonic shift method was reported to be highly effective for smaller molecules $\sim 1 \mathrm{kDa}$ but efficiency decreased for cargo of greater molecular weight. For example, the procedure was unable to deliver large proteins such as labeled antibodies. Koberna et al.'s hypotonic shift approach has been particularly popular for intracellular delivery of labeled nucleotides ${ }^{952-960}$. It has also been adapted for the successful loading of the peptide actinomycin $\mathrm{D}^{961}$, dye-conjugated dextrans ${ }^{962}$, and $5 \mathrm{~nm}$ gold particles ${ }^{963}$.

Apart from severe hypotonic shock, intracellular delivery has also been accomplished with milder hypotonic shocks in the range of $\sim 150$ mOsm. Mills et al. used hypotonic swelling for intracellular loading of antibodies into rat submandibular acini cells ${ }^{964}$. This application is notable in that cells are not individually isolated in suspension - acini are small clusters of cells organized in a quasi-circular arrangement to form a hollow duct in the center. In the procedure, acini were exposed to a mild hypotonic solution $(\sim 150 \mathrm{mOsm})$ containing $5 \mathrm{mM}$ ATP and the antibody of interest for 1 minute following a switch back to isotonic conditions. The loaded antibody was found capable of inhibiting its target CTFR protein in the cytoplasm, verifying that delivery had indeed occurred. The procedure has also been used to deliver the calcium chelator BAPTA $^{965}$ and enzymes ${ }^{966}$ into acini cells. 
In studies that require intracellular delivery of lanthanum-based contrast agents, milder hypotonic shocks ( 90-160 mOm) have been used to load normally impermeable tracers, such as the Gadolinium ion, into adherent or suspension cells ${ }^{967}$. In this case, a 30 minute $\sim 160$ mOsm hypotonic exposure at $37{ }^{\circ} \mathrm{C}$ was used for cytoplasmic delivery of lanthanide complexes and dyes in various macrophage and cancer cell lines ${ }^{967}$. A comparison with electroporation and osmotic lysis of pinosomes concluded that hypotonic shock was the most advantageous method for delivery of these small $(<1 \mathrm{~nm})$ molecules ${ }^{967}$. Other reports appear to verify this strategy, as Gadolinium complexes have been delivered into HeLa cells with the same protocol ${ }^{968}$. In further cases, a more severe shock of $\sim 90-110$ mOsm for 60 minutes at $37{ }^{\circ} \mathrm{C}$ produced loading of Lanthanide complexes into HeLa cells ${ }^{969,970}$. Interestingly, iron oxide nanoparticles of up to $60 \mathrm{~nm}$ were loaded into RBCs with hypotonic shocks of 90-110 $\mathrm{mOsm}^{969}$. Other reports in RBCs employed a 30 minute $160 \mathrm{mOsm}$ hypotonic shock at $4{ }^{\circ} \mathrm{C}$ to load gadolinium-based complexes into the cytoplasm without loss of cell functionality ${ }^{971-973}$.

In a strategy that synergizes hypotonic shock with the membrane perturbing effects of detergents, Medepalli and coworkers demonstrated quantum dot loading into adherent H9C2 cells by exposure to a mild hypotonic buffer (150-200 mOsm) combined with low concentrations of the detergent $\operatorname{saponin}^{311}$. Presumably saponin reduces the threshold for induction of plasma membrane defects under hypotonic stretch, thereby synergizing the permeabilization effects of both approaches. After delivery, quantum dots of hydrodynamic diameter 20-25 nm were observed to be evenly dispersed in the cytoplasm of treated cells.

\subsubsection{Osmotic Gradients Acting on Part of the Plasma Membrane}

When cells form a tight monolayer across a porous substrate, they form an impermeable barrier between two bodies of liquid media. An osmotic shock in one of those solutions creates an osmotic gradient across the cells. Taking advantage of this principle, Widdicombe et al. cultured epithelial or endothelial cells into confluent polarized monolayers on substrates with $0.45 \mu \mathrm{m}$ pore size ${ }^{974}$. The apical media was then exchanged with water containing macromolecules to be loaded while retaining the basal media as physiological saline (Figure 20B). This resulted in a $\sim 300$ mOsm osmotic gradient across the cell monolayer. Disruption of the apical cell membrane was evidenced by uptake of $67 \mathrm{kDa}$ fluorescent albumin and $2000 \mathrm{kDa}$ dextrans but was reversible within $\sim 5$ mins when apical water was replaced with normal cell culture media. 
By adding fluorescently labeled molecules at different times after hypotonic shock, it was found that the majority of uptake occurred within the first 4 minutes. This technique was reported to be temperature insensitive, working equally well at 4 or $37{ }^{\circ} \mathrm{C}$, thereby indicating that endocytic activity had a minimal role and suggesting plasma membrane disruption as the prime mechanism. After the procedure, cell layers were able to recover full trans-epithelial resistance within several hours.

In a complementary study by Widdicombe's co-workers, Tawa et al. demonstrated successful transfection of airway barrier cells in rat lungs by exposure to apical water containing $\mathrm{DNA}^{975}$. A follow-up report argued that the hypotonic transfection of DNA to airway barrier cells could be due to active uptake by membrane trafficking, which is known to stimulate exocytosis and endocytosis associated with regulatory volume mechanisms ${ }^{976}$. However, this model would not fit with the original observation of rapid delivery by Widdicombe et al. In an analogous situation, hypotonic aerosols have been observed to facilitate intracellular delivery of PEI-complexed DNA by a membrane permeabilization mechanism in mouse airway epithelium ${ }^{977}$. Thus, a hypo-osmotic delivery principle may prove feasible when applied to exposed cell monolayers in vivo, particularly in the lungs.

\subsubsection{Hydrostatic Pressure and Hydrodynamic Delivery}

Membrane disruption due to a sudden increase in hydrostatic pressure is believed to be the mechanism of so-called 'hydrodynamic delivery', where a rapid injection of fluid into the cardiovascular system causes transient disruption in the plasma membrane of cells in certain tissues. A prime example is tail vein injection, where robust transfection of hepatocytes and sometimes other cardiovascular tissues has been observed in rodents ${ }^{978,979}$. In a mouse model, transfection is achieved by fast injection ( $\sim 5$ seconds) of almost $2 \mathrm{~mL}$ of DNA-containing saline solution into a $20 \mathrm{~g}$ animal. The introduced solution is close to $10 \%$ of the body weight, thus representing a rapid expansion of blood volume that cannot be immediately pumped through the vena cava of the heart. This causes sudden distension and hydrostatic pressure buildup in the surrounding tissues. A weak point is typically retrograde flow into the liver, where it has been observed that fenestrations in hepatic tissue expand to generate disruptions in cell membranes, thereby allowing influx of cargo

molecules from the blood directly into the cytosol of hepatocytes ${ }^{980}$. High delivery efficiencies have been achieved using hydrodynamic tail vein injection, with up to $40 \%$ transfection of liver hepatocytes from a single injection ${ }^{979}$. Rapid 
intracellular delivery of other macromolecules such as labeled dyes, proteins, oligonucleotides, siRNA, bacterial artificial chromosomes, and linear or circular DNA fragments as large as $175 \mathrm{~kb}$ have also been delivered to rodent hepatocytes by this method, lending similar credence to a membrane permeabilization mechanism without reliance on endocytosis ${ }^{980-987}$. More recently, hydrodynamic tail vein injections have found use in CRISPR-based genome editing in mouse liver, albeit at lower efficiencies ${ }^{988-991}$. A major limitation of hydrodynamic injection is that it is only available in rodents.

Apart from injection into veins, intracellular delivery of nucleic acid cargo has been observed by direct injection of solutions into skeletal muscle, heart, thyroid, skin, and liver ${ }^{992}$. Mechanistic studies indicate that this also occurs by membrane permeabilization, but a role for endocytosis has not been completely ruled out ${ }^{992-995}$. The degree to which membrane permeabilization or active uptake processes underlie delivery is probably dependent upon the properties of the solution and manner in which the injection is performed ${ }^{992,994}$.

In 1999 Mann et al. introduced a method for hydrostatic pressure-mediated transfection in human vein segments and rat myocardium ex vivo ${ }^{996}$. 1-2 cm segments of veins were cannulated, encased in a plastic sleeve to prevent distension, and infused with pressurized solutions of up to $\sim 100 \mathrm{kPa}$ above baseline pressure ${ }^{996} .10$ minutes of this treatment was reported to yield intracellular delivery of fluorescently-labeled antisense oligonucleotides into $\sim 90 \%$ of endothelial cells lining the vein segment ${ }^{996}$. Moreover, ex vivo treatment of rat hearts pressurized inside and out at up to $\sim 200 \mathrm{kPa}$ showed $\sim 50 \%$ transfection in myocardial cells ${ }^{996}$. Although the exact delivery mechanisms were not revealed, imaging of cells after treatment suggested it was non-endocytic ${ }^{996}$. Variants of this technique have been used to achieve intracellular delivery of siRNA ${ }^{997}$, antisense oligonucleotides ${ }^{996,998-1002}$, plasmid DNA ${ }^{998,1003,1004}$ and $\sim 100$ nm polystyrene microspheres ${ }^{1005}$.

\subsubsection{Disruption of Endosomes by Osmotic Forces}

In 1982 Okada and Rechsteiner described an intracellular delivery technique, termed osmotic lysis of pinosomes. It works by harnessing osmotic forces to rupture endosomes pre-loaded with cargo of interest, thereby obtaining cytosolic delivery (Figure 20C) $)^{94}$. In the first step, endocytic uptake is promoted by a $\sim 10$ minute incubation of cells in a $\sim 800 \mathrm{mOsm}$ hypertonic buffer containing $0.5 \mathrm{M}$ sucrose, 10\% polyethylene glycol (PEG)-1000 and molecules to be delivered. Exchange to a hypotonic solution $(\sim 180 \mathrm{mOsm})$ consisting of diluted media for $\sim 2$ minutes then generates a rush of water 
into the cell. During this hypotonic shock phase endosomes laden with cargo and osmolytes expand and rupture, thus releasing their contents. The pendulum swing from hypertonic to hypotonic conditions may also disrupt the plasma membrane, however cells are generally able to release osmolytes to counteract swelling ${ }^{491}$. Endosomes, on the other hand, have no volume regulation and therefore swell uncontrollably until bursting ${ }^{1006,1007}$. This means the imposed hypotonic shock may impact endosomes significantly more than the plasma membrane. Okada and Rechsteiner reported that the osmotic lysis of pinosomes method was capable of introducing antibodies, various proteins, and $70 \mathrm{kDa}$ labeled dextrans into the cytosol of L292, 3T3 fibroblasts, and HeLa cells ${ }^{94}$.

Following in the example of the original paper, osmotic lysis of pinosomes has been particularly useful for intracellular delivery of proteins ${ }^{94,95,1007-1021}$, antibodies ${ }^{1013,1022-1026}$, dextrans ${ }^{94,1026-1028}$, and peptides ${ }^{1029-1031}$. In a landmark paper in 1988, osmotic lysis of pinosomes was used to prove that cytosolic loading of proteins could mediate their presentation as antigens through the major histone compatibility I pathway to invoke a specific immune response ${ }^{95}$. In other reports, osmotic lysis of pinosomes has found success in intracellular delivery of cell lysates ${ }^{1032}$, hyaluronan ${ }^{1033,1034}$, trehalose $^{1035}$, Lanthanide imaging probes ${ }^{71,72}$, various small molecule dyes ${ }^{1026,1036}$, uridine triphosphate-glucuronic acid ${ }^{1037}$, antisense oligonucleotides ${ }^{1038}$, antisense morpholinos ${ }^{804}$, virus particles ${ }^{1039}$, and nanomaterials such as quantum dot-labeled motor proteins for biophysical studies $306,1040,1041$.

With the advent of RNAi-mediated gene silencing in the early 2000s, researchers tested the ability to perform siRNA transfection via osmotic lysis of pinosomes. By using up to $1.6 \mu \mathrm{M}$ siRNA in solution, gene silencing of $>50 \%$ was reproducibly achieved in common cell lines such as $\mathrm{HEK}$ and $\mathrm{HeLa}^{1042}$. In a subsequent study by a different group, improved RNAi transfection was demonstrated in hard-to-transfect immune cell lines ${ }^{1043}$. Their modified procedure was more extreme, involving hypertonic sucrose solutions of up to $2 \mathrm{M}$ and siRNA concentrations of $10 \mu \mathrm{M}^{1043}$. Difficult-totransfect immune cell lines including mouse macrophage RAW264.7 and J774.1 as well as the T lymphocyte cell line DO11.10 were all shown to be transfectable with this approach. Other benefits were minimal cytotoxicity and immunomodulatory responses compared to the synthetic cationic lipid reagents lipofectamine and oligofectamine, or the polymer reagent jetPEI. In a microfluidic adaption of the approach, a device was deployed for rapidly cycling suspended cells through the various solutions to induce osmotic lysis of pinosomes, thus avoiding the need for centrifugation to 
exchange solutions ${ }^{1028}$. Results were reported to be superior to the conventional protocol for loading fluorescent dextrans into Jurkat cells ${ }^{1028}$.

It is important to note that the osmotic lysis of pinosomes method has several caveats: 1) cell stress, 2) delivery capacity is limited by extent of endocytosis, and 3) absence of reports on larger cargo such as plasmid DNA and mRNA. First, the hypertonic media imposes significant stress on cells and has been observed to actually inhibit endocytosis in some cell types ${ }^{1044}$. Second, the extent of endocytosis during the hypertonic exposure window is a limiting factor that affects the final concentration of cargo delivered ${ }^{1044}$. Multiple rounds of the procedure may be conducted to boost delivery efficiency but are time-consuming and must be balanced with considerations of cell stress ${ }^{1027}$. Several publications indicate that cell function and health may be compromised as a function of duration and intensity of the osmotic challenges ${ }^{94,932}$. The third consideration is that certain combinations of cell types and cargo molecules appear to be unfeasible with the procedure. This can be due to degradation of cargo in the acidic environment of endosomes or an unmet need for destabilizing agents to assist with endosome rupture, a role that PEG was later suggested to play ${ }^{1044}$.

Interestingly, an in vivo application of the osmotic lysis of pinosomes concept was accomplished in rat arteries. Without surgical removal, isolated, pressurized mesenteric arteries of the rat were cycled through hypertonic and hypotonic solutions. Endothelial cells were found to take up dyes, dextrans, peptides, and labeled antibodies into the cytoplasm without comprising the structure and function of the surrounding tissues ${ }^{1026}$. This strategy was used to identify a critical role for connexin 40 protein in EDHF-mediated dilation of endothelial cells in rat mesenteric arteries.

\subsubsection{Induced Transduction by Osmocytosis}

Motivated by limitations of the osmotic lysis of pinosomes method in primary cell types, D'Astolfo et al. introduced an adaptation, termed iTOP, which stands for induced transduction by osmocytosis and propanebetaine ${ }^{151}$. Instead of relying on hypotonic solutions for endosome disruption, propanebetaine appears sufficient to trigger cargo leakage specifically from macropinosomes. The method relies on NaCl-related hypertonicity of extracellular medium to induce macropinocytosis followed by spontaneous endosomal leakage. A high extracellular concentration of $\mathrm{Na}^{+}$ions was shown to be necessary to stimulate NHE1-mediated macropinocytosis. Unlike osmotic lysis of pinosomes, however, no discrete 
trigger was required for endosomal rupture. Instead, intracellular macropinosome leakage was a stochastic event promoted by the presence of propanebetaine or other compounds with similar physicochemical properties. The osmotic pressure created by hypertonic endosomes may also contribute to destabilize endosomes. Using iTOP, RNPs of Cas9-sgRNA were delivered into KBM7 cells and H1 human embryonic stem cells to produce CRISPR-mediated gene deletions. Various other proteins were also delivered, demonstrating efficient delivery of several cargo materials into a variety of primary cell types.

\subsubsection{Summary of Permeabilization by Mechanical: Pressure Changes}

Rapid changes in hydrostatic and osmotic pressure are an obvious, straightforward, and low-cost strategy for placing cell membranes under mechanical force. This insult has been harnessed against both the plasma membrane (external) and endosomal membrane (internal) systems for the purposes of intracellular delivery. Because current procedures rely on buffer changes, however, there is a significant stress placed on the entire cell. Concepts for better controlling the spatiotemporal application of mechanical pressure changes at the scale of the cell may provide more precise membrane permeabilization in future.

\subsection{Electrical Membrane Disruption (Electroporation)}

In the 1980s, electroporation, which involves the transient permeabilization of cell membranes with electric pulses, rose to prominence as a powerful approach for intracellular delivery, applicable to a wide range of cell types, from animal cells to plants and lower organisms. Prior to its introduction, the stage had been set by more than a decade of research exploring the effect of voltage pulses on artificial lipid bilayers, vesicles ${ }^{1045,1046}$ and red blood cells $\mathrm{s}^{533,534,1047}$. In nucleated mammalian cells, Eberhard Neumann and colleagues published a groundbreaking report in 1982 which demonstrated that electroporation led to the efficient transfection of plasmid DNA in mouse lyoma cells ${ }^{184}$. The study also stimulated electroporation theory by introducing a generalized van't Hoff relationship to model the extent of permeabilization in an electroporated cell, whereby poration phenomena is viewed as structural rearrangements of lipids and water ${ }^{184}$. Electroporation, while initially emphasized for DNA transfection, has subsequently shown utility for delivery of a huge variety of cargo: from small molecule drugs, dyes, and tracers, to larger proteins/antibodies and multiple forms of DNA 
and RNA ${ }^{163,1048-1050}$. In this section we first cover the mechanisms of electroporation before exploring the challenges, technical advances and applications.

\subsubsection{Mechanisms of Membrane Disruption \& Cargo Entry}

\subsubsection{Mechanisms of Membrane Pore Formation}

Mechanistically, electroporation is the formation of pores in a membrane by the application of a potential difference across that membrane. When the potential difference reaches a critical magnitude of voltage, the probability of electroporation taking place drastically increases. According to theory, the increase in electric field energy within the membrane and ever-present thermal fluctuations combine to create and expand a heterogeneous population of pores ${ }^{163,419,1051}$. Although there is no fixed voltage threshold that triggers electroporation, the critical parameter of electroporation is the trans-membrane potential. This is because the maintenance of a trans-membrane electrical potential incurs a probability of generating a membrane defect for a given field strength, time, and temperature. Membrane defects originate as so-called hydrophobic pores of radius $<0.5 \mathrm{~nm}$, which form due to random thermal fluctuations of the individual lipid molecules that make up the membrane (Figure 21A). Fueled by the external electrical energy provided, these defects may then traverse their energy landscape to become hydrophilic pores, which are typically lined by at least 8 - 10 phospholipid head groups and defined by their ability to permit free passage of water molecules (Figure 21B). Hydrophilic pores $(\mathrm{r}>0.5 \mathrm{~nm})$ can be stable because the energy barrier also exists in the reverse direction.
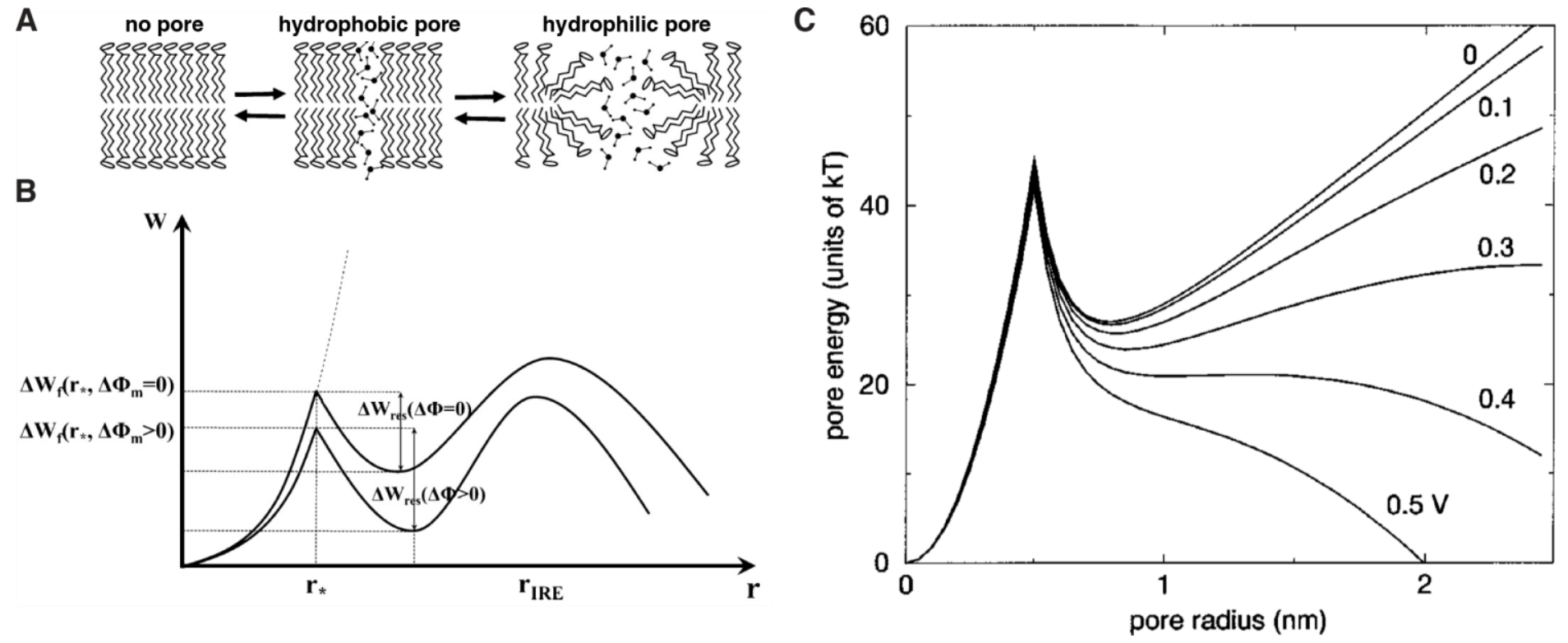
Figure 21. Energy landscapes and theory of pore formation in membranes by electric fields. (A) Schematic of pore formation showing the transition from a hydrophobic pore to a hydrophilic (conducting) pore. (B) Graphs of relationship between free energy of pores $\Delta W$ and pore radius $r$ for $\Delta \Phi_{m}=0$ (upper curve) and at $\Delta \Phi_{m}>0$ (lower curve). $r *$ is the critical radius corresponding to the transition from hydrophobic to hydrophilic pore. $\Delta W_{f}$ corresponds to the height of the energy barrier for pore formation while $\Delta W_{\text {res }}$ relates to the energy barrier height for pore resealing. $r_{\mathrm{IRE}}$ is the pore radius corresponding to state of irreversible electroporation. $\Delta \Phi_{m}$ is the electrical potential difference across the membrane. The higher the electrical potential, the more probability of pore formation. Figure reprinted by permission from Springer Nature from ref ${ }^{163}$, Copyright 2015. (C) Calculations of the effect of applied voltage on the energy landscape of pore formation with transmembrane potentials ranging from 0 to $0.5 \mathrm{~V}$. Reprinted figure with permission from ref ${ }^{420}$ and the authors, Copyright 1999 by the American Physical Society.

Current theory posits that small pores are not very good conductors; hence the continued application of an electric field is not only critical for their formation, but also their enlargement ${ }^{419,1051}$. Pore formation and expansion are energetically favorable because it relaxes the charge buildup that would otherwise become entropically unfavorable. As the pores become better conductors, however, the electrical expanding pressure decreases, resulting in a decay in the rate of their growth. This explains two phenomena characteristic of electroporation: 1) longer pulses (usually tens of ms) are required to grow larger pores, and 2) electroporation is not very good at producing large (e.g. $>50 \mathrm{~nm}$ ) pores ${ }^{579,1052}$.

Apart from the energy landscape of electroporation, theoretical models and simulations have been used to decipher the chemical thermodynamic and kinetic aspects of pore formation ${ }^{184,1053-1058}$. Upon application of an electric field across a lipid bilayer, an early event is tilting of the electrical dipoles associated with the lipid headgroups to align with the direction of the applied electric field ${ }^{184,1053,1058}$. This causes rotation of lipid molecules, thus thinning the bilayer, perturbing its organization, and facilitating the entry of water molecules into the hydrophobic core ${ }^{184,1053,1058}$. Pore formation follows, and the rate of transition between pore states is subject to a hysteresis where their opening (microseconds range) is thought to be much faster than the timescales of their closure (seconds to minutes) ${ }^{1057}$. Such models of electroporation have been developed to a degree where analytical expressions are available to optimize electroporation in several biotechnological and medical applications ${ }^{1054-1056}$. For further explanation of the formulas and analytical frameworks see the abovementioned publications ${ }^{184,1053-1058}$.

Electroporation phenomena are thought to be primarily related to changes in electrical conductance, but chemical, thermal, and electromechanical membrane deformation effects may also contribute ${ }^{458,1059}$. The application of mechanical tension has been observed to lower the electric voltage threshold required for membrane disruption ${ }^{1060,1061}$. This is because mechanical forces contribute to bias the energy landscape toward defect formation (see Figure 8). In keeping with this notion, lower temperatures have been observed to increase the field strength required for electroporation ${ }^{575,1062}$ and slow 
the kinetics of resealing ${ }^{1054,1055}$. Furthermore, mathematical descriptions and models have been developed to assess, for example, the effect of applied voltage on the distribution of pore radii ${ }^{420,1063}$ (Figure 21C). More recently, simulations have also assisted in illuminating the molecular events associated with electroporation, although (due to limitations in computational power) they currently only cover very short time scales on the order of nanoseconds or less ${ }^{1053,1064,1065}$. It is imperative to note, however, that many of the mathematical models and simulations are challenging to verify experimentally.

\subsubsection{Electroporation in Cell Suspensions}

In suspensions of isolated cells electroporation is observed with applied trans-membrane potentials in the range of 0.2 to 1.5 volts. Pulse times are typically on the order of microseconds to almost a second. The membrane charges like a capacitor with a characteristic charging time proportional to the surface area of the enclosed membranous body ${ }^{579}$. For conventional cuvette-style parallel plate setups, a cell suspension in conducting buffer is placed between two electrodes connected to a generator of high electric voltage (Figure 22). This type of setup produces a homogeneous electric field across the cell suspension. Upon application of voltage, the various regions of the plasma membrane take different times to reach their characteristic trans-membrane threshold potentials. This results in growth of a heterogeneous distribution of pores over the cell surface, both in terms of number and size. Moreover, because of the negative resting potential of cells (-35 to $80 \mathrm{mV}$ for most cell types - see Figure 7A), permeabilization occurs first at the hyperpolarized side of the cell facing the positive electrode ${ }^{1048}$. This creates an inherent anisotropy in the area and degree of permeabilization between the two poles ${ }^{1066}$. The hyperpolarized side of the cell is supposed to carry smaller but more numerous pores. The depolarized half, which faces the negative electrode, has fewer pores due to fewer nucleation events. The pores on the depolarized side may, however, be larger in diameter as the prolonged electrical field exposure is focused on expanding a less numerous population of defects ${ }^{1067}$. In general, it is thought that coverage area of permeabilization is controlled by pulse strength while the pore growth size is more strongly correlated with the pulse duration ${ }^{1048}$. Once pores are formed and begin conducting, the local electroporation effect diminishes somewhat as charge is free to flow through these defects. Therefore the amount of energy channeled into the growth of pores declines through the lifetime of a particular pulse $\mathrm{e}^{458}$. 


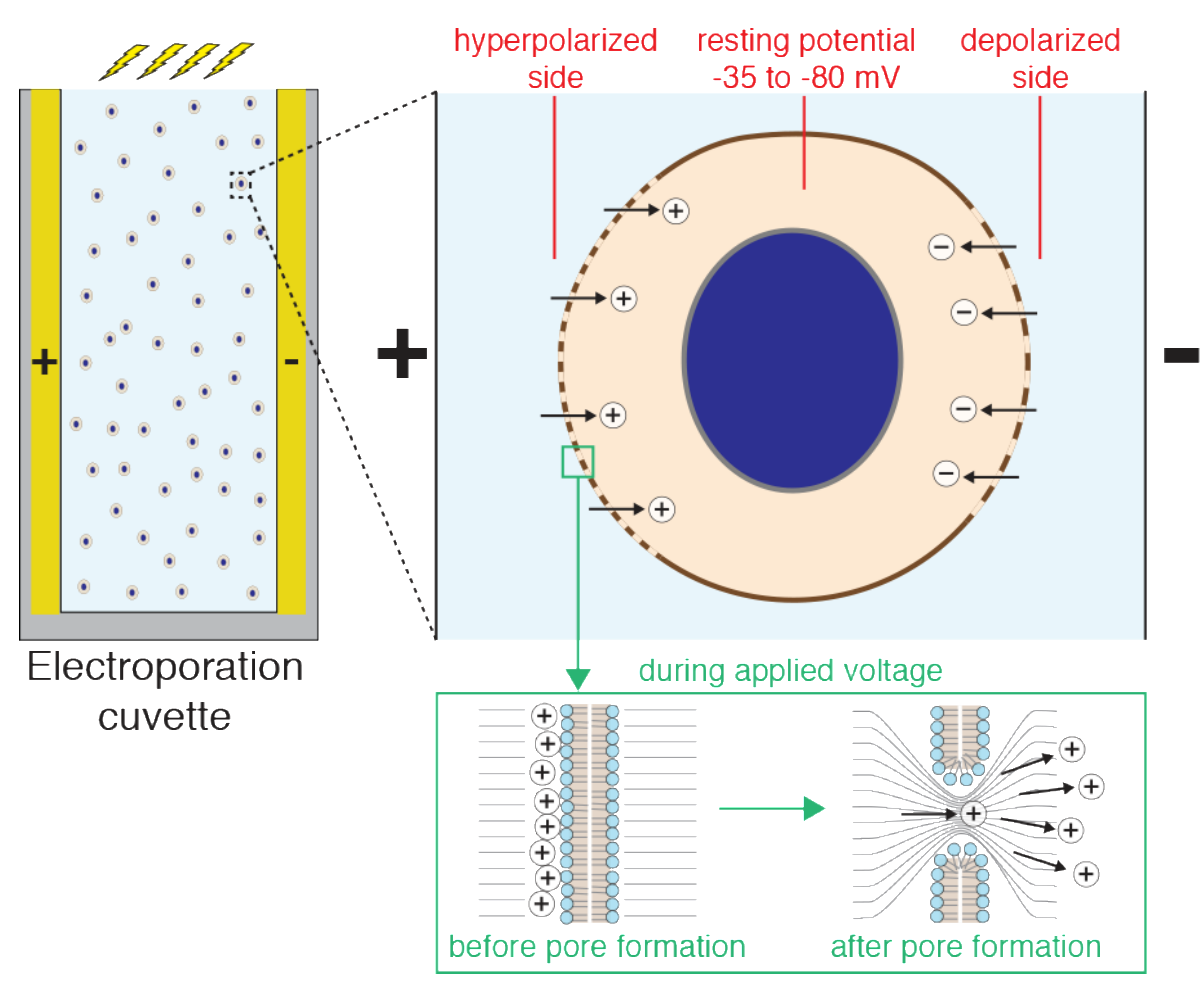

Figure 22. A conventional parallel plate cuvette configuration for electroporation of suspended cells (left). Zoom-in (right) shows the approximate distribution of pores over the cell surface as a function of orientation and polarization under applied electric field. The surface area of poration and number of pores is greater on the hyperpolarized side compared to the depolarized side. Further zoomin (bottom) illustrates the capacitor-like function of the lipid bilayer before poration and the flow of positive charges once a conducting pore is formed (opposite movement of negative charges not shown). Electric field lines are displayed in grey.

Upon electroporation, the response within cell populations and between cell types is somewhat heterogeneous, reflecting differences in cell size, orientation, surface area, and physiological state, as well as variances in membrane composition and the presence of local inhomogeneities in the electric field itself. The microenvironment of the cell surface is characterized by the distribution of nearby or adhered macromolecules, membrane proteins, lipid phases and lateral domains, extracellular protrusions, membrane reservoirs, and underlying cytoskeletal linkages (see Figure 7B, C). It is currently not well understood how these complexities influence the generation of defect nucleation and growth under an electric field ${ }^{1049}$. A recent study to visualize the behaviour of membrane defects in artificial planar bilayers found that electropores form preferentially in the liquid disordered phase ${ }^{1068}$. This preference is also likely to be true in live cells, but lack of experimental methods to measure such phenomena has made it challenging to validate ${ }^{1068}$. Another mystery is the lifetimes of electropores in live cells. Once hydrophilic pores of $>1 \mathrm{~nm}$ open up in the plasma membrane, they are thought to either spontaneously close or require active cellular processes for the bilayer to heal. For active repair processes, many researchers observe timescales of seconds to minutes ${ }^{1048,1069}$. The electroporation literature, however, suggests rapid shrinkage of pores after cessation of the electric field ${ }^{1070}$. A memory effect, where changes in the membrane porosity 
remain on a longer time scale of hours has also been suggested ${ }^{1069}$. For further reading on the theory and mechanisms of electroporation as pertaining to live cells, we recommend other more comprehensive reviews on the topic $^{163,419,544,579,1048,1049,1051,1054,1055,1069-1072}$.

\subsubsection{Targeting Cellular Structures Across the Pulse Strength-Duration Space}

The parameter space for electroporation is vast. As discussed, there is no fixed threshold electroporation voltage because formation of electropores depends on a combination of voltage strength, pulse duration, number of pulses, pulse waveform, temperature, buffer conductivity, and cell properties ${ }^{1048,1049}$. This large variable space presents a challenge in optimizing electroporation. All other variables being held constant, most approaches focus on tuning the "pulse strengthduration space" ${ }^{, 579}$. Manipulating this parameter space can exert a measure of spatiotemporal control over which cellular membranes are permeabilized (Figure 23). In general, high voltage ultrashort pulses have been purported to perturb internal and organelle membranes while longer and milder pulses emphasize permeabilization of the plasma membrane and bias the effect toward larger cell types ${ }^{579}$.

The charging time for the plasma membrane is on the order of $1 \mu \mathrm{s}$. It is even shorter in highly conductive buffers such as PBS. Pulses of duration less than the plasma membrane charging time are thought not to efficiently porate the plasma membrane of a cell ${ }^{419}$. For example, high voltage ultrashort pulses in the nanosecond range may rupture subcellular structures and organelles while leaving the plasma membrane essentially untouched ${ }^{458,579}$. A pioneering study by Schoenbach et al. in 2001 demonstrated short nanosecond pulses at $>10 \mathrm{kV} \mathrm{cm}^{-1}$ field strength selectively target intracellular organelles ${ }^{1073}$. Specifically, human eosinophils were exposed to a field strength of $53 \mathrm{kV} \mathrm{cm}^{-1}$ applied in a train of 5 pulses of 60 nanoseconds each. In response, the cells formed intracellular granules even without extensive plasma membrane permeabilization. Follow up studies by the same group indicated these nanosecond pulses induced apoptosis, as signified by exposure of annexin-V at the cell surface and the absence of ethidium homodimer fluorescence ${ }^{1074}$. Further hallmarks of apoptosis were observed with fluorescent probes that report on caspase activation and the release of mitochondria-associated protein cytochrome c into the cytoplasm. It is thought that apoptosis occurs due to a release of cytotoxic factors from permeabilized mitochondria and breakdown of intracellular calcium stores. It was therefore concluded that apoptosis triggered by nanosecond pulsed electroporation can occur in the absence of 
disruption to the plasma membrane. This is of widespread interest for two reasons: 1) the targeted induction of apoptosis by ultrashort electrical pulses could avoid the immune response associated with lysing or necrotic cells ${ }^{1075}$. 2) For intracellular delivery applications it is an effect that should be avoided to maintain cell survival. Unwanted disruption of intracellular organelles could explain observations of delayed cell death that sometimes occur after high field strength electroporation.

As nanosecond pulses increase in duration, the chance of permeabilizing the plasma membrane also increases ${ }^{1076}$. Pulses in the $1-10 \mathrm{~ns}$ range have less chance of permeabilizing the plasma membrane, while pulses in the $10-1000 \mathrm{~ns}$ range tend to generate very small pores $(\leq 1 \mathrm{~nm})^{477,1076,1077}$.

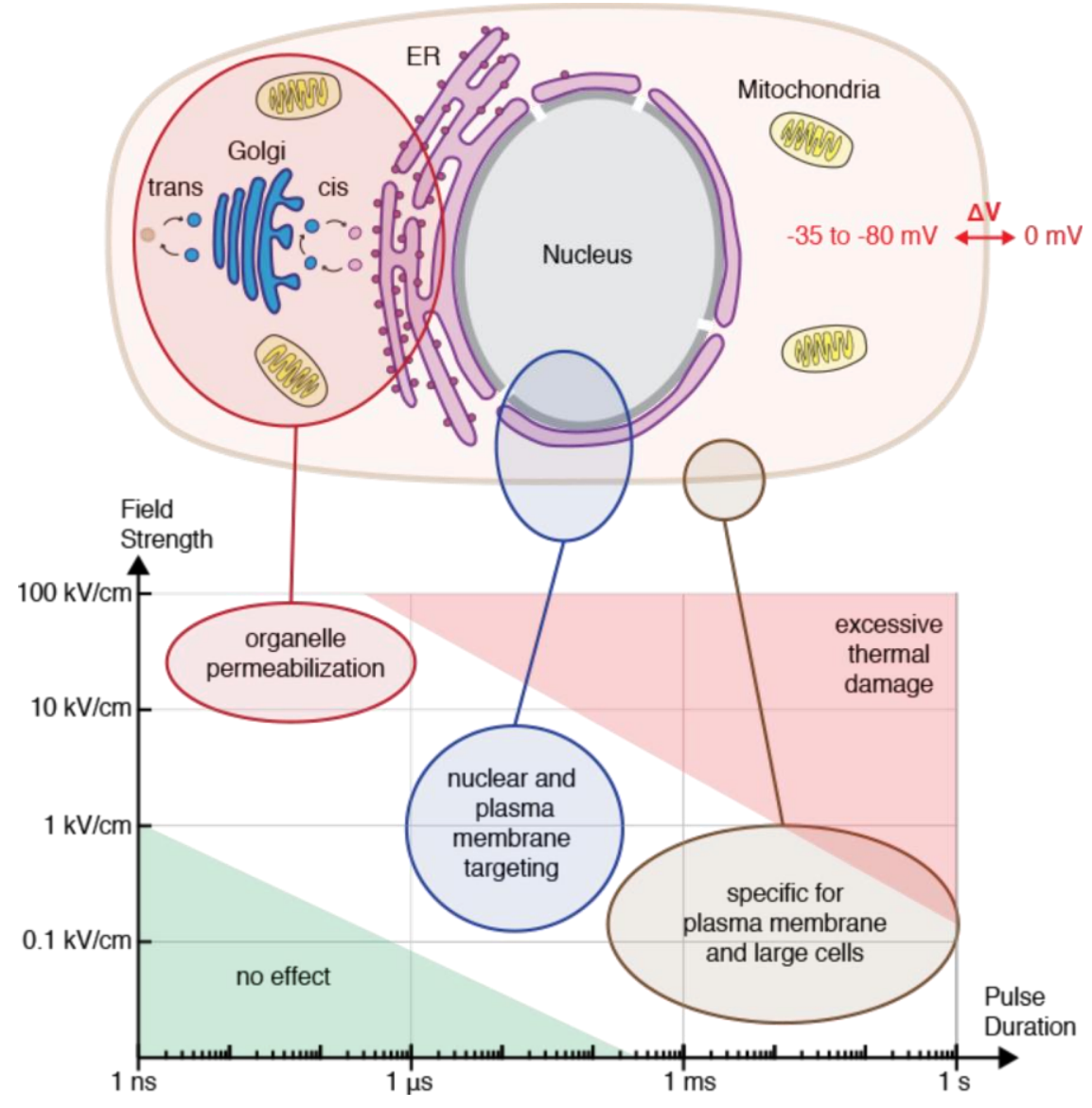

Figure 23. Relationship between the pulse strength-duration parameter space and subcellular targeting. High intensity short pulses are biased toward perturbing small membrane bound bodies like organelles while milder, longer pulses are more specific for the plasma membrane and larger cells. At large field strengths and longer durations thermal damage due to heating becomes an issue, being also dependent on buffer conductivity.

Conventional electroporation systems almost exclusively target the plasma membrane. Short pulses in the microsecond to 
millisecond range result in numerous, but smaller sized pores distributed evenly over the poles of the plasma membrane and sometimes nucleus ${ }^{579}$. The longer pulse space $>0.1 \mathrm{~ms}$ is limited to lower voltages; otherwise Joule heating becomes a problem for treated cells, a factor also dependent on conductivity of the medium. Because voltages must be lower in this regime, the dependence on size of the membrane-bound body biases poration towards larger objects at their poles, therefore favoring plasma membrane disruption of larger cells (>tens of micron diameter) ${ }^{579}$. At these longer durations the membranes of larger cells such as skeletal muscle and nerve cells are much more responsive to electroporation. Taken together, data compiled from multiple reports suggest that manipulation of the pulse strength-duration parameter space is able to mediate a significant measure of control over the subcellular localization and distribution of membrane disruptions generated in cells (Figure 23).

\subsubsection{Cargo-Dependent Influx Mechanisms}

Electroporation has been used to deliver a diverse range of cargo molecules and materials to the intracellular space. This includes dyes ${ }^{100,742,1056,1078-1080}$, radiotracers ${ }^{1081,1082}$, sugars $^{79,470,534,1083}$, metabolites ${ }^{1081,1084}$, poorly permeable $\operatorname{drugs}^{55,56,1085,1086}$, ions ${ }^{1087,1088}$, molecular beacons ${ }^{1089,1090}$, proteins ${ }^{100,546,1091-1097}$, antibodies ${ }^{101,125,537,1098-1102}$, Cas9 protein or RNP complexes ${ }^{143,144,146,147,1103}$, antisense oligonucleotides ${ }^{1104}, \quad$ siRNA $^{235,1105-1109}, \quad$ mRNA $^{257,260,261,1110,1111}$, plasmid DNA $^{184,1112,1113}$, quantum dots $294,312,313,1114$, and gold nanoparticles ${ }^{1115}$. The mechanisms of uptake of these cargos vary as a function of their size, charge, and conformational flexibility (Figure 24).

\subsubsection{Cargo-Dependent Influx Mechanisms: Small Molecules}

Small neutral molecules enter cells via diffusion throughout the duration of a pore's lifetime ${ }^{163}$ (Figure 24B). If the molecules are charged, such as propidium iodide (PI, 660 Da), which carries two positive charges, there is an added electrophoretic component that can augment delivery during the pulse (Figure 24C). In this case, delivery will be augmented at the side of the cell facing the positive electrode, as PI will be attracted towards the negative electrode and into the cell ${ }^{1067,1116}$. Due to its small size and high diffusion coefficient, PI will also diffuse into the opposite side of the cell, but to a lesser extent. Because the lifetime of the electropores is much longer than the pulse duration, diffusion has been observed as the dominant mechanism of entry with only a minor contribution from electrophoresis ${ }^{1117,1118}$. Electropores have been reported to remain open to small molecule diffusion for up to several minutes after pulsing ${ }^{1084,1117}$. 

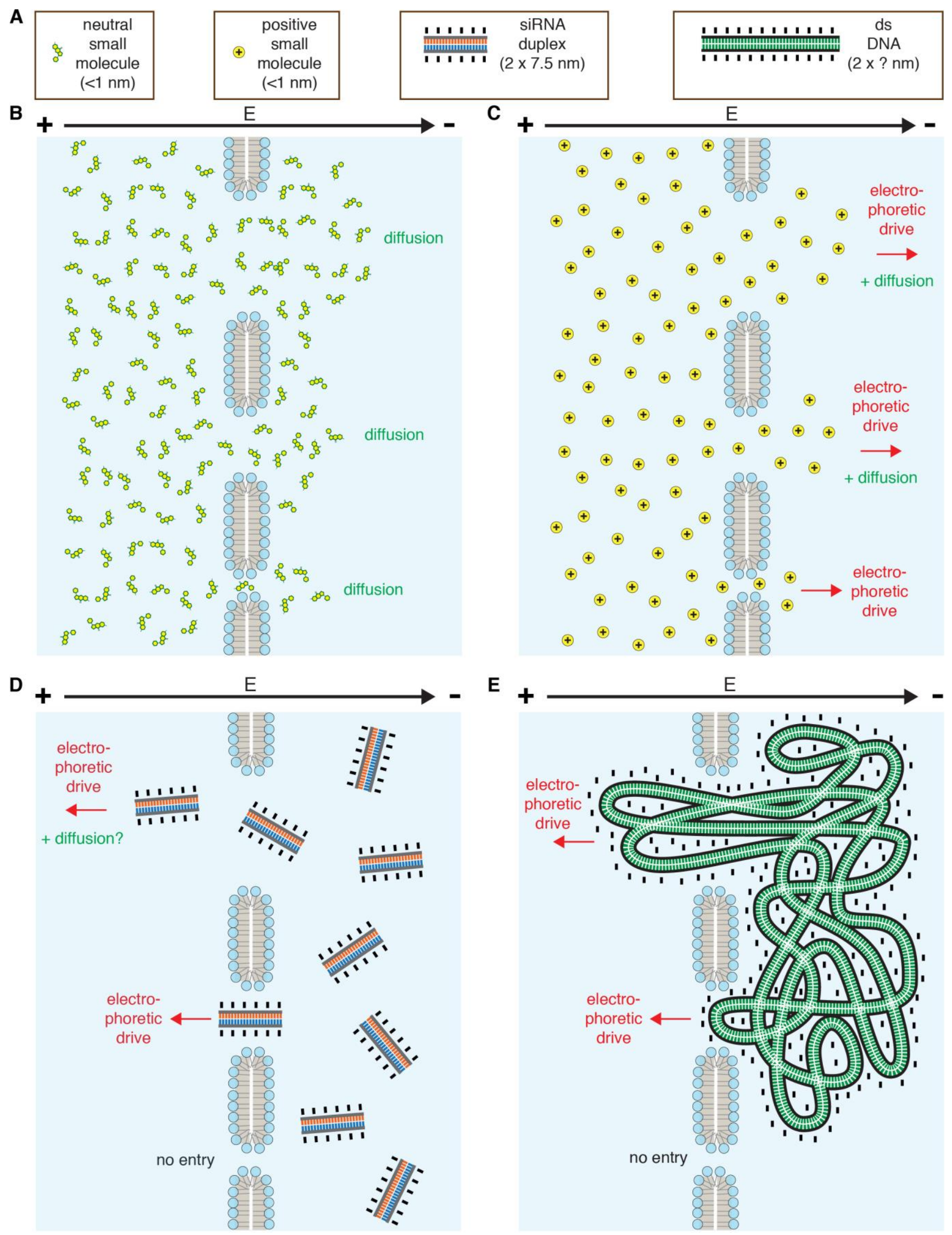
Figure 24. Relationship between size and charge of cargo molecule and mechanisms of entry through a given pore size for electroporation. (A) Depiction of approximate size and charge properties of molecules illustrated in scenarios from panels B to E. The depictions are based on knowledge from the literature and explained in the text.

For very small pore sizes $(\sim 1 \mathrm{~nm})$ diffusion alone may be insufficient for influx of charged molecules. This is because of Born's energy barrier, which describes the energetic cost of moving an ion or small charged molecule through a hole in a dielectric membrane ${ }^{163,1119}$. The charged entity interacts with the pore wall, increasing the energy required for translocation. For pore sizes close to the molecule size, the energy barrier for crossing the membrane strongly correlates with the charge number on the molecule. For example, Venslauskas et al. compared delivery of bleomycin (radius: $\sim 1.2$ $\mathrm{nm}$, charge: +1 ) to tetra-sulfonato-porphyrin (TSPP, radius: $\sim 1.0 \mathrm{~nm}$, charge: -4 ) under pulsing conditions designed to generate only small pores ${ }^{1120}$. Their experiments revealed that the electric field strength required to deliver the more highly charged molecule, TSPP, was several times greater than for bleomycin. Other groups claim to have identified ultrashort pulse electroporation conditions ( $\sim 60 \mathrm{~ns})$ where plasma membrane pores are so small that they do not allow transmission of PI, although they are conductive for smaller ions ${ }^{1121}$. In such a scenario, an electric field pulse can help overcome Born's energy barrier and promote influx.

\subsubsection{Cargo-Dependent Influx Mechanisms: Proteins \& Other Macromolecules}

Diffusion is the most likely mechanism underlying electroporation-mediated intracellular delivery of larger macromolecules $(\sim 10-1000 \mathrm{kDa})$, such as proteins, antibodies and dextrans ${ }^{1048}$. Most proteins and dextrans tend to be weakly charged or neutral, thus the electrophoretic contribution is thought to be minimal. Early experiments with proteins claimed efficient loading ( $>80 \%$ of cells), sometimes up to micromolar cytoplasmic concentrations, in a variety of mammalian cell lines at high survival rates $(>80 \%)^{101,1092,1122}$. Dye-conjugated dextrans of known molecular weights (from $3-2000 \mathrm{kDa}$ ) have also been electroporated into cells to analyze delivery efficiency and decipher the rules governing uptake $^{532,1080,1091,1123-1125}$. In comparison to small molecules, which can diffuse into cells for minutes, proteins and larger molecules (>10 kDa) exhibit a narrow window of opportunity to enter cells, constituting just a few seconds ${ }^{1095}$. It is known that electroporation produces mostly small pores with a subset of larger pores that grow as a function of the pulse duration ${ }^{419}$. When the electric field is turned off the large pores shrink almost instantly, while the small pores may linger in the plasma membrane for minutes ${ }^{163}$. Thus, the entry of larger cargo coincides with the pulse timing and is more 
efficient for longer pulse durations ${ }^{532}$. The smaller pores that prevail for minutes are unable to facilitate diffusive influx of proteins $^{1126}$.

Although less well-accepted, some researchers have proposed alternative delivery mechanisms. For example, the imposed electric field might augment macromolecule delivery through electrophoretic or electro-osmotic effects ${ }^{1123,1127,1128}$. The models based on electrophoresis, however, have not addressed how they would be relevant to uncharged molecules. The electro-osmotic explanation, on the other hand, proposes that the application of an electric field causes a convective flow of electrolytes and osmotically obliged solution that sweeps the cargo molecules along with it. Although discussed in some papers, the few studies that have sought to investigate electro-osmotic contributions to molecular delivery in live

cells are inconclusive ${ }^{1123,1127}$. Most of the electroporation literature favors explanations that emphasize cargo influx by diffusion or electrophoresis ${ }^{163,1048,1049,1056,1129}$.

Another idea is that electroporation-stimulated endocytosis via macropinocytosis may contribute to protein uptake in the minutes following electric field exposure ${ }^{1130}$. Strong electroporation treatments have sometimes been reported to cause proteins and dextrans to become aggregated or trapped at the plasma membrane ${ }^{1092,1122}$. Such membrane-bound proteins can be removed with the protease trypsin while dextrans could not, demonstrating that proteins were stuck to the cell surface and not inside the cell ${ }^{1122}$. If electroporation causes cargo to aggregate at the cell surface, this would make it amenable for uptake by endocytosis ${ }^{1130}$. The degree to which this occurs for different cargo molecules, however, has not been thoroughly investigated.

\subsubsection{Cargo-Dependent Influx Mechanisms: Plasmid DNA}

In contrast to small molecules, proteins and dextrans, the mechanisms of nucleic acid delivery via electroporation are regarded to be almost entirely dependent upon electrophoretic forces provided during the pulse ${ }^{163,1048,1131}$. In particular, the case of DNA plasmids has been extensively studied due to a broad interest in exogenous gene expression over the past decades ${ }^{1132,1133}$. After pioneering efforts demonstrating DNA transfection in mouse cells in the early $1980 \mathrm{~s}^{184,1134}$, it wasn't until a decade later that researchers realized that plasmids weren't immediately crossing the cell membrane, but rather aggregating at the cell surface as a result of electrophoretic forces (Figure 24E) ${ }^{531,1113,1135}$. A correlation between longer 
pulse durations, more prominent aggregates, and higher transfection efficiency also lent support to this view ${ }^{469,1135}$. Moreover, it was observed that pre-adsorption of DNA to the cell surface dramatically increased transfection efficiency and contributed to pore formation and stabilization, most likely by spearing of plasmid molecules into the membrane ${ }^{1135,1136}$.

In 2002 Golzio et al. advanced our understanding of electroporation-mediated plasmid transfection with single-cell imaging experiments that visualized the interaction of DNA at the cell surface during electroporation ${ }^{1137}$. It was found that DNA aggregated exclusively on the side of the cell facing the negative electrode (cathode) and formed localized clumps of $0.1-0.5 \mu \mathrm{m}$ in size. At the cell surface, it is believed that the highly negatively charged DNA plasmids are threaded through small pores where they become stuck in the negative electrode-facing region of the plasma membrane ${ }^{1067,1135,1136}$. These aggregates are then internalized via endocytosis over tens of minutes. Some of the plasmids eventually arrive at the nucleus over a timecourse of $\sim 2$ hours or longer ${ }^{1132}$. Collectively, these results led to the emergence of an endocytic model of plasmid electrotransfer that has gained significant attention (Figure 25). 


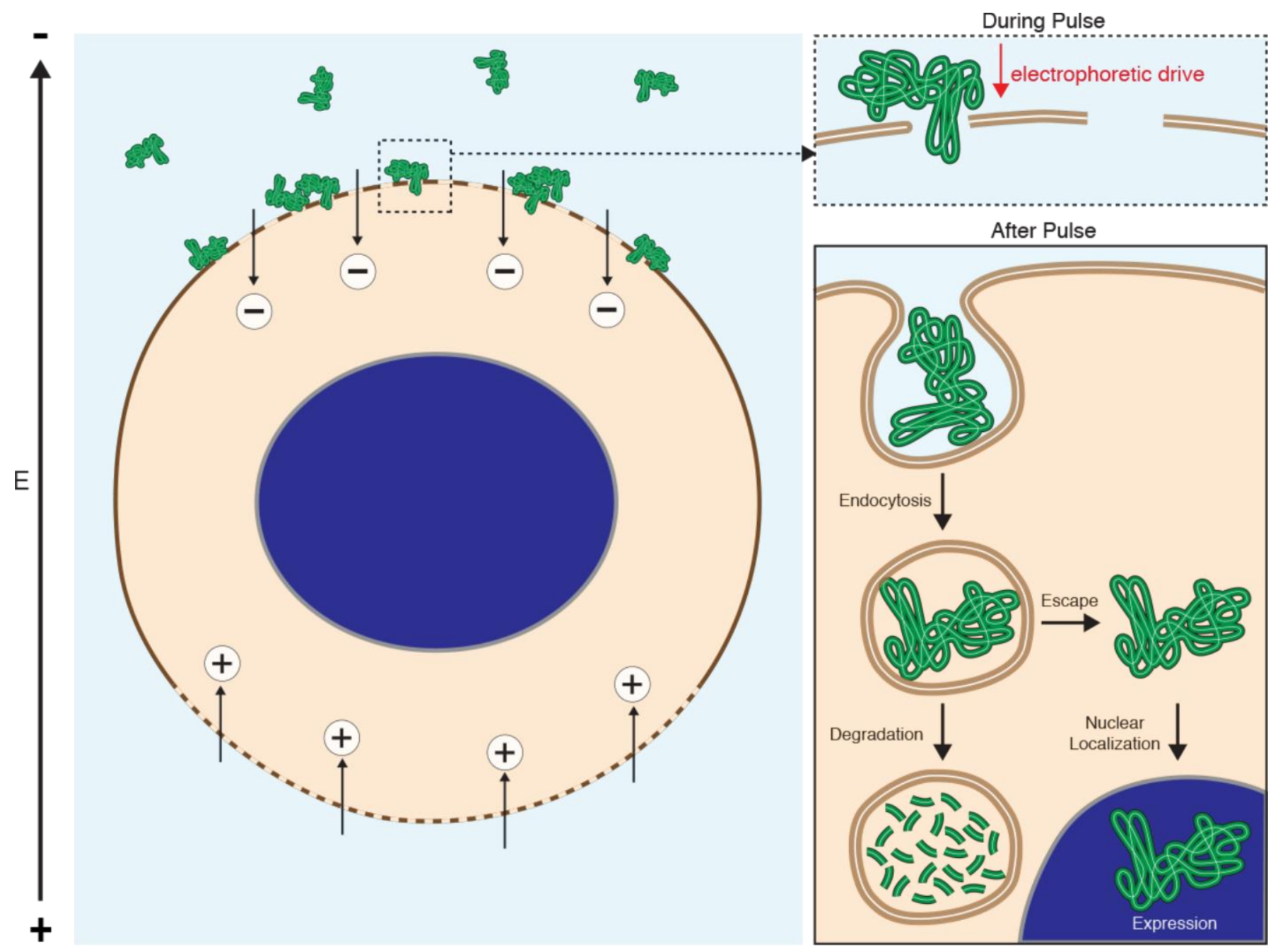

Figure 25. Model for endocytosis of electroporation-induced DNA aggregates at the cell surface. During the electric field pulse, negatively charged plasmid DNA is propelled into the side of the cell facing the negative electrode. Due to conformational flexibility some parts of the DNA may be threaded through pores in the cell membrane. Aggregates are then endocytosed, from which they either escape and find their way to the nucleus for the purpose of expression or are degraded by lysosomes.

As membrane remodeling via endocytosis is a core pathway used by cells to repair their membranes ${ }^{447,455}$, endocytic uptake could be an active cellular response to the perturbation caused by DNA entanglement in the membrane, as earlier predicted by Tsong and colleagues ${ }^{1070}$. Subsequent studies have shown that, in CHO cells for example, 50\% of DNA is internalized by caveolin/raft-mediated endocytosis, $\sim 25 \%$ by clathrin-mediated endocytosis, and $\sim 25 \%$ by macropinocytosis ${ }^{1138}$. Within 2 hours, more than half of the DNA ends up in lysosomes, as revealed by co-localization with the lysosomal marker LAMP1 ${ }^{1138}$. Furthermore, single-particle tracking experiments of fluorescently labeled plasmids indicate that cytoskeletal processes, involving both actin and microtubule networks, are involved in trafficking of DNA-associated endosomes toward the cell nucleus ${ }^{1138-1140}$. It is important to recognize, however, that many of the basic mechanisms of the plasmid trafficking from the membrane to the nucleus remain underexplored and poorly defined $^{1131,1132}$. 
How plasmids enter the nucleus is poorly understood, as DNA plasmids are invariably many times larger than the $\sim 40$ $\mathrm{kDa}$ cutoff for passive influx through nuclear pores. DNA transfection is known to be greater in proliferating cells that undergo transient nuclear envelope breakdown through mitosis, which allows plasmids to be entrapped inside the freshly reformed post-mitotic nucleus ${ }^{1141}$. The revelation that nuclear membrane disruptions are not an uncommon event in the life of a cell, and thus generate a stochastic pathway of exchange between cytosol and nucleus, could also provide clues $^{789,790}$. Alternatively, internalization motifs, such as nuclear targeting sequences, have been reported to promote import of plasmids into cell nuclei with varying success rates ${ }^{1142}$.

Overall, there are a protracted series of steps required for electroporation-mediated transfection and many of them require membrane trafficking and other active cellular processes. Only a small fraction of electroporated DNA vectors will arrive in the nucleus for successful expression ${ }^{1132}$. Despite this, electroporation is one of the few membrane disruption-based methods that can achieve high rates of DNA expression in millions of cells at acceptable throughputs. Several other methods are able to introduce DNA to the cytosol, but it is often unable to migrate through the tight cytoplasmic meshwork and is therefore degraded before reaching the nucleus, as has been shown for plasmids after microinjection ${ }^{202}$. In rare cases electroporation appears to mediate rapid expression of plasmids within an hour ${ }^{1143}$. Most often, however, it takes anywhere from 4-24 hours for peak expression ${ }^{1132,1140}$. Electroporation's paradigm of plasmid aggregation and endocytosis may thus serve to concentrate and protect DNA for the prolonged journey to the cell nucleus.

\subsubsection{Cargo-Dependent Influx Mechanisms: siRNA \& Other Oligonucleotides}

Electroporation-mediated delivery of oligonucleotides and siRNA is similar to the case of DNA in that it also relies on electrophoretic forces ${ }^{163}$. A key distinction, however, is that siRNA undergoes direct delivery into the cytoplasm without relying on endocytosis (Figure 24D). This is by virtue of its smaller dimensions $(2 \times 7.5 \mathrm{~nm})^{219}$ compared to DNA plasmids $(\sim 100-200 \mathrm{~nm})^{206}$ (see Table 1). Imaging of fluorescently labeled siRNA has shown that it enters during application of the electric field at the side of the cell facing the cathode and disperses throughout the cytosol within tens of seconds ${ }^{1144}$. siRNA influx was reported not to occur after cessation of applied voltage, indicating that electrophoretic forces are probably required for delivery ${ }^{144}$. For short $(10 \mathrm{~ns})$ pulses applied to GUVs, influx of siRNA also relied on 
electrophoretic drive and some siRNA remains trapped in the bilayer at the end of the electric pulse ${ }^{1145}$. However, electrophoretic driving forces might only be necessary for delivery through small pores of $<10 \mathrm{~nm}$ where there is a Born energy barrier ${ }^{163}$. Such a scenario is analogous to that discussed for small charged molecules (Figure 24C). It is likely that transient large pores $(>10 \mathrm{~nm})$ can facilitate entry of siRNA via free diffusion, since siRNA knockdown has been observed with membrane disruption-based methods that lack electrophoretic forces, including with pore-forming toxins ${ }^{238}$, microfluidic cell squeezing ${ }^{108,780}$, and laser-nucleated cavitation bubbles ${ }^{923}$. Therefore, one can speculate that siRNA delivery is mediated by a combination of electrophoretic and/or diffusive mechanisms depending on the size and lifetime of the pores.

\subsubsection{Summary of Cargo-Dependent Influx Mechanisms}

Taken together, the literature indicates electroporation-mediated intracellular delivery is influenced by the pore diameter $\left(\mathrm{d}_{\text {disruption }}\right)$ and the cargo dimensions $\left(\mathrm{d}_{\text {molecule }}\right)$, as well as the charge and conformational flexibility of the cargo molecule (Figure 26). For $\mathrm{d}_{\text {molecule }}<<\mathrm{d}_{\text {disruption }}$ both neutral and charged molecules should diffuse across their concentration gradient whilever the pore is large enough. Although the majority of delivery is via diffusion, electrophoretic or electro-osmotic phenomena may assist translocation during the pulse. For $\mathrm{d}_{\text {molecule }} \approx \mathrm{d}_{\text {disruption }}$ charge will play a critical role. Neutral molecules may diffuse through pores while their charged counterparts will face the Born's energy barrier, only being able to translocate while driven by sufficient electrophoretic forces. For the case of $d_{\text {molecule }}>>d_{\text {disruption }}$ only molecules that are both conformationally flexible and significantly charged will have a chance of penetrating. As exemplified by the case of DNA plasmids, parts of the molecule may be threaded into pores and therefore become embedded in the membrane. This makes the molecule available to be taken up via endocytosis, a result that may or may not be desirable for a given application. 


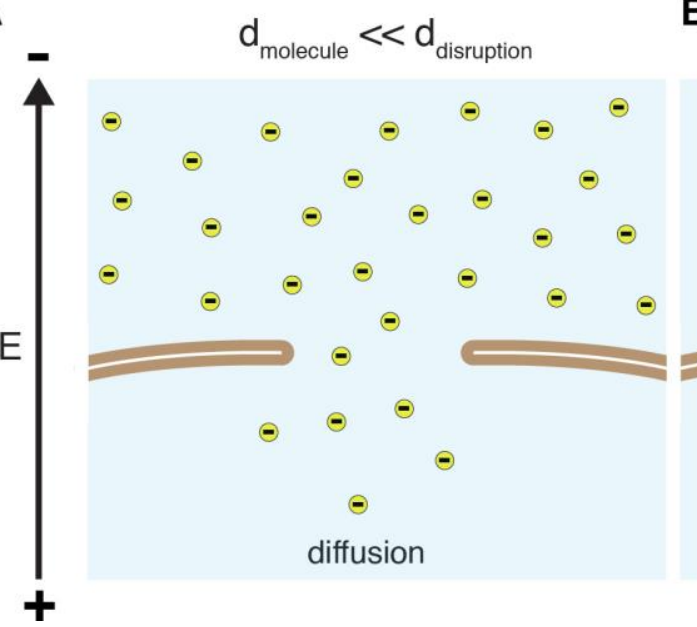

B

$\mathrm{d}_{\text {molecule }} \approx \mathrm{d}_{\text {disruption }}$

C

$d_{\text {molecule }}>d_{\text {disruption }}$
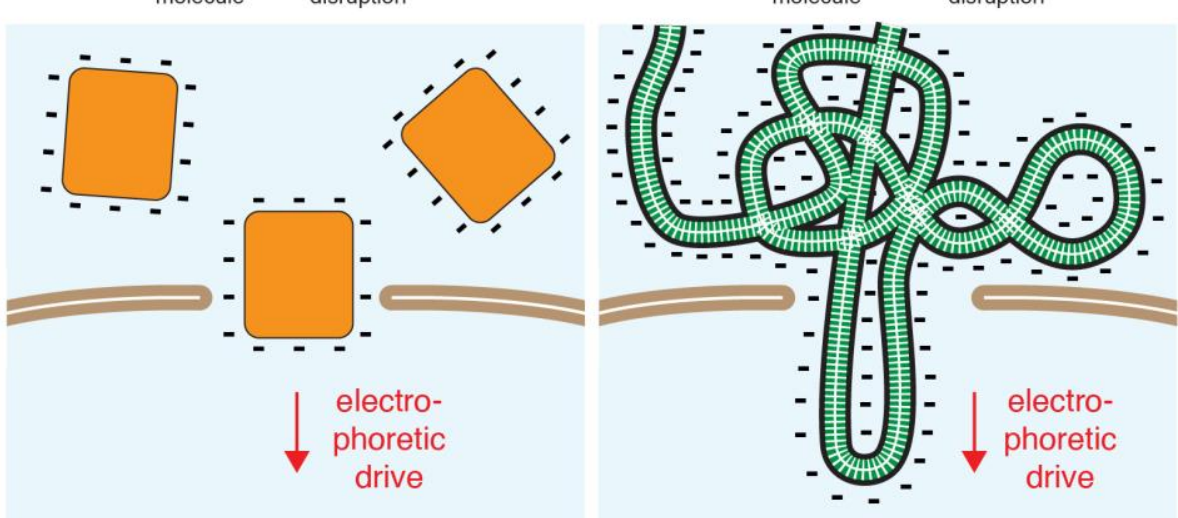

Figure 26. Schematic of the mechanisms of influx in relation to disruption size, molecule size, molecule charge, and conformational flexibility. For charged objects approaching the disruption size or larger, electrophoretic forces are crucial for delivery. (A) Shown is the case for a molecule much smaller the size of the membrane disruption. Regardless of charge, delivery is mostly via diffusion. (B) Shown is the case for a negatively charged molecule of similar size to the transient membrane disruption. Delivery requires an electrophoretic driving force. (C) Shown is the case for a flexible molecule (here a DNA plasmid) that is much larger than the membrane disruption. Electrophoretic force can thread part of the molecule into the cell.

\subsubsection{Tailoring Pulse Parameters for Optimal Delivery}

In the most elementary electroporation scenario, one wants to open up pores of sufficient size and duration to allow the desired influx of cargo molecules via diffusion. In more complicated cases, however, involving charged molecules close to or larger than the pore size (Figure 26), the efficiency of delivery depends critically on magnitude and duration of electrophoretic forces ${ }^{469,531,532,1113,1135}$. Regarding plasmid delivery, for example, longer pulse durations are often found to heavily improve transfection efficiency. Yet longer pulses can bring the problem of Joule heating and excessive cell damage $^{1146}$. One strategy to mitigate Joule heating is the use of low conductivity buffers that have lower electrolyte concentrations than standard physiological buffers or media. The osmolarity of the buffer will have an effect as well, because it can alter the size of the cell, tension on the plasma membrane, conformation of membrane reservoirs, and the interaction between cargo molecules and the cell surface ${ }^{1049}$. Temperature will also affect the properties of the membrane and energy barriers for electroporation, as well as the active cell response and membrane repair dynamics ${ }^{1049}$.

Electroporation can therefore be viewed as a balancing act between a large number of parameters and conditions. There is significant debate surrounding the optimal electroporation protocols for intracellular delivery and this is further complicated by variation between cell types ${ }^{1147,1148}$. Another issue is the lack of understanding associated with postelectroporation cell death, where loss of viability sometimes manifests after hours or even days ${ }^{490,529}$. This is an especially 
striking problem regarding electroporation of primary or sensitive cell types ${ }^{1149}$. When wanting to optimize the delivery of a particular cargo molecule into a specific cell type, the starting point is usually to screen three core parameters: (1) field strength (voltage), (2) pulse duration, and (3) number of pulses.

Based on a large number of electroporation studies, several types of pulsation strategies have been devised. In a review by $\mathrm{Gehl}^{1048}$, three categories of approaches for DNA transfection were described, all of which have achieved some measure of success: (1) Exclusively short, high-amplitude pulses ${ }^{184,1113,1150}$, for example, a series of six pulses of $100 \mu$ s at field strengths of $1.4 \mathrm{kV} \mathrm{cm}^{-1} .^{1151}$ (2) Exclusively long, low-amplitude pulses ${ }^{469,532,1112,1135}$, for example, eight pulses of $20 \mathrm{~ms}$ at field strengths of $0.2 \mathrm{kV} \mathrm{cm}^{-1}{ }^{1152}$ (3) A short, high-amplitude pulse followed by a long, low-amplitude pulse ${ }^{1153}$; for example, a first pulse of $10 \mu \mathrm{s}$ at $6 \mathrm{kV} \mathrm{cm}^{-1}$ followed up with a second pulse of $10 \mathrm{~ms}$ at $0.2 \mathrm{kV} \mathrm{cm}^{-1}$ as pioneered by

Sukharev et al. ${ }^{531}$. The rationale behind this dual pulse strategy is that the first pulse is thought to nucleate many pores over a large segment of the cell surface, while the second pulse should simultaneously grow the pores and electrophoretically propel charged molecules into the cell. Indeed, several studies have confirmed that the duration of the second low-voltage pulse correlates with DNA transfection efficiency $531,532,1135$.

\subsubsection{Dual Pulse Strategies}

The dual pulse strategy has captivated considerable attention from the field and inspired a number of further investigations ${ }^{1124,1125,1150,1154,1155}$. Figure 27 shows examples of pulse parameter sequences that constitute typical dual pulse strategies. The first example consists of two consecutive DC square wave pulses (Figure 27A) ${ }^{1124}$ while the second uses an AC signal for the first pulse followed by a delay then a second low-voltage DC pulse (Figure 27B) ${ }^{1125}$. The AC pulse is designed to increase the consistency of permeabilization at each pole of the cell and reduce side effects at the electrodes. These reports are a few among many to suggest that dual pulse strategies optimize delivery while preserving cell viability, not only for DNA transfection but also for delivery of other molecules like proteins and high molecular weight dextrans ${ }^{1124,1125}$. 

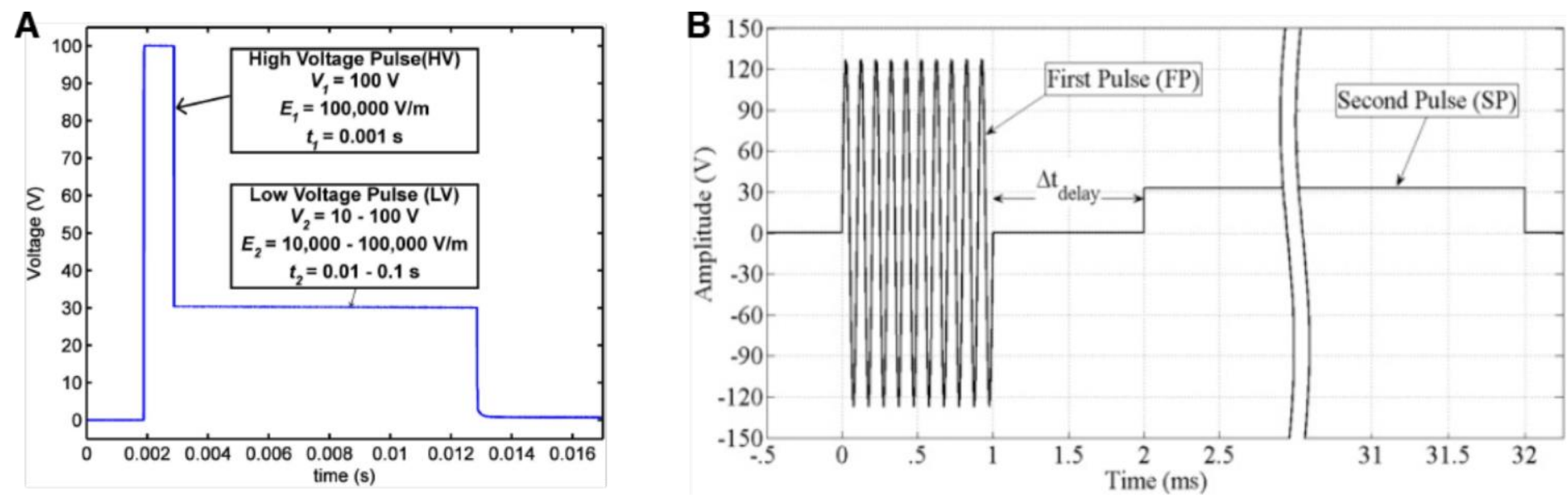

Figure 27. Examples of dual-pulse electroporation protocols from the literature. (A) The first pulse has a field strength of $1 \mathrm{kV} \mathrm{cm}{ }^{-1}$ and duration of $1 \mathrm{~ms}$. The second pulse $0.3 \mathrm{kV} \mathrm{cm}^{-1}$ in strength and $10 \mathrm{~ms}$ in duration. Figure reprinted from ref ${ }^{1124}$, Copyright 2014, with permission from Elsevier. (B) Schematic of a pulse sequence consisting of AC first followed by a pre-programmed delay then a second DC pulse of lower voltage. In this case, the first pulse is $1 \mathrm{~ms}$ and the second one is $30 \mathrm{~ms}$. Figure reprinted from ref ${ }^{1125}$, Copyright 2015, with permission from Elsevier.

\subsubsection{Nucleofection Mechanisms}

Nucleofection, one of the most popular electroporation systems of all time, was introduced in the early 2000s and rapidly gained traction as an effective intracellular delivery method. It is based upon a classical cuvette configuration with parallel plate electrodes, but the novelty comes from the systematic selection of optimal pulsing parameters and cell-type specific buffers $^{550,1156,1157}$. Although the exact pulsing parameters are proprietary, patents indicate that it is based around a dual pulse approach ${ }^{1158}$. The first pulse is administered at field strengths of $2-10 \mathrm{kV} \mathrm{cm}^{-1}$ for durations ranging from $10-100 \mu \mathrm{s}$. The second pulse lasts 1-100 ms at a lower, unspecified, field strength. Dozens of different pulsing protocols are programmed into the nucleofector control unit, presumably based on variations on this theme. The user then finds optimal electroporation conditions by screening the programs against delivery and viability outcomes for different cell types. To facilitate best results, cell type-specific buffers are also recommended. Patents on nucleofection buffers report nearphysiological concentrations of extracellular (high) $\mathrm{Na}^{+}$and (low) $\mathrm{K}^{+}$augmented by $>10 \mathrm{mM} \mathrm{Mg}^{2+}$ and robust $\mathrm{pH}$ buffering $^{550}$. This is in contrast with the trend of literature promoting the benefits of low conductivity buffers featuring organic osmolytes ${ }^{1148}$ or high $\mathrm{K}^{+}$cytoplasm-mimicking buffers ${ }^{548}$. The high conductivity of nucleofection buffers (ionic strength $>200 \mathrm{mM}$ ) is thought not to cause Joule heating problems due to the emphasis on small volumes and shorter pulse durations ${ }^{550,1157}$. Users have reported adapting nucleofection for use with phosphate buffered saline without a decline in performance ${ }^{52,554}$. A number of publications share protocols for homemade nucleofection buffer formulations to increase transparency of the protocols and lower costs ${ }^{551,552}$. 
A notable appeal of the nucleofector system has been the assertion that it delivers plasmid DNA rapidly and directly to the nucleus $^{1156,1159}$. This speculation, however, is controversial and difficult to find experimental support for it in the literature. An alternative explanation is that endocytic trafficking directs DNA to the nucleus, as has been observed for other types of electroporation ${ }^{1132}$. A number of factors lend credence to the endocytic explanation. First, the cytoplasm is a highly crowded and viscous environment laced with cytoskeletal filaments and organelles. The mobility of microinjected plasmid DNA is extremely small or even negligible in the cytoplasm or cell nucleus ${ }^{1160-1162}$. To be electrophoretically propelled through the cytoplasm into the nucleus, a combination of significant plasmid compaction and large electrophoretic forces would potentially be required, although this has not been directly proven ${ }^{1132}$. Second, the reported timing of gene expression is in the range of 6 hours after treatment ${ }^{1156,1159}$, which is actually longer than achieved with standard electroporation that relies on endocytosis ${ }^{1132}$. In contrast, microinjection of DNA directly into the nucleus can mediate gene expression within 30 minutes. Some authors have speculated whether nucleofection permeabilizes the nucleus with its first high-voltage pulse, thus assisting in nuclear delivery ${ }^{105,579,1157}$. This hypothesis has not been rigorously tested in experiments to date.

Regardless of the actual mechanisms, nucleofection has shown significant success rates for DNA transfection and expression in traditionally difficult-to-transfect cell types ${ }^{1108}$. This has been demonstrated in various types of stems cells, primary cells, and post-mitotic cells, for example, primary human melanocytes, smooth muscle cells, chondrocytes, and mesenchymal stem cells ${ }^{156,1163,1164}$, human monocyte-derived dendritic cells ${ }^{1165,1166}$, monocytic cell lines ${ }^{1159}$, primary leukemia cells and cell lines ${ }^{1167,1168}$, primary natural killer cells and cell lines ${ }^{1169,1170}$, primary lymphocytes ${ }^{1171,1172}$, embryonic and adult stem cells ${ }^{1173,1174}$, and mammalian neurons ${ }^{1157,1175}$. These papers and others contributed to the emergence of nucleofection as a leading method for transfection of recalcitrant cell types.

Overall, there are many examples of pulsing strategies that have been successfully employed to electroporate molecules into cells ${ }^{163,1048,1049,1132}$. Nucleofection is but one example of a dual pulse strategy that has been systematically honed for application with a wide range of cell types, including difficult-to-transfect cells. A deeper understanding of the mechanisms of electroporation phenomena on both cells and cargo molecules could yield even further advancements in delivery performance and cell health. 


\subsubsection{Electroporation Challenges \& Technical Advancements}

As with most membrane disruption-based intracellular delivery strategies, a major challenge with electroporation is cell mortality post-treatment. Cell death may occur immediately due to irreversible electroporation, lysis, or excessive thermal damage $^{490}$ (see Figure 11). Or it may take the form of a delayed necrosis, possibly due to failure of membrane repair, or prolonged apoptotic responses, taking place hours or days after treatment ${ }^{529}$. As an example of this problem, early reports on nucleofection of human monocyte-derived dendritic cells yielded unprecedented plasmid transfection results, with up to $60 \%$ gene expression. However, long-term functional assays indicated that cells were hampered by gradual loss of proliferative potential and poor viability ${ }^{1165}$. In this section, we discuss the problems with electroporation and the efforts that have gone into reducing its toxic burden on cells.

\subsubsection{The Problem of Joule Heating}

When electric current passes through an aqueous solution, it triggers temperature increase (Joule heating) concurrent with various chemical reactions at the solution-electrode interface (electrolysis). Electrolysis itself produces changes in the temperature, $\mathrm{pH}$, and the chemical composition of the adjacent solution. The degree of Joule heating is influenced by the conductivity of the buffer, electrode architecture, electric field parameters, and capacity of the system for dissipation. For cuvette style setups, temperature spikes of more than $30 \mathrm{~K}$ above ambient conditions have been measured in physiological saline at millisecond pulse durations ${ }^{1146}$. Such observations have led some researchers in the field to assert that Joule heating is a significant problem ${ }^{579}$. For example, an $8 \mathrm{kV} \mathrm{cm}^{-1}$ pulse of $100 \mu$ s has been calculated to lead to a temperature increase from $23{ }^{\circ} \mathrm{C}$ to $42{ }^{\circ} \mathrm{C}$ in PBS solution ${ }^{579}$. Lipid membranes and proteins are destabilized by temperatures above 42 ${ }^{\circ} \mathrm{C}^{1176}$. Therefore, Joule heating is not just an issue for the plasma membrane, but also for intracellular membranes and proteins throughout the cell. To mitigate the negative effects of Joule heating, electroporation procedures can be performed at room temperature $\left(20-25^{\circ} \mathrm{C}\right)$ or on ice $\left(0-4{ }^{\circ} \mathrm{C}\right)$. Lower temperatures, however, makes cells more resistant to pore formation ${ }^{574,575}$, thereby reducing delivery efficiency. Another approach to combat Joule heating is to use lowconductivity buffers, which feature lower concentrations of electrolytes and instead maintain osmolarity by inclusion of organic osmolytes or sugars like sucrose and mannitol ${ }^{532}$. Low-conductivity buffers reduce Joule heating while enabling the long pulses that are preferred for some protocols, such as for DNA transfection. 


\subsubsection{The Problem of Metal Contamination}

A number of publications have assessed the detrimental effects of metal ions released into solution by electrolysis ${ }^{574,1177-}$ ${ }^{1180}$. For large surface area electrodes, such as cuvette style electroporation chambers, the most commonly used materials are aluminium, copper, and stainless steel. Analysis of stainless steel and aluminium electrodes found that, after a train of pulses similar to a standard electroporation protocol, metal ions were found in solution at up to milliMolar concentrations $^{574,1178,1180,1181}$. Aluminium ions and aluminium hydroxides can wreak havoc on cellular processes, such as inositol phosphate activity ${ }^{574,1177}$. Moreover, Stapulionis et al. found that released copper, iron, and aluminium ions can interact with nucleic acids and cause their precipitation out of solution ${ }^{1178}$. Other studies have found $\mathrm{Fe}^{2+} / \mathrm{Fe}^{3+}$ to be toxic to in vitro cell cultures at milliMolar concentrations ${ }^{1180} . \mathrm{Fe}^{2+} / \mathrm{Fe}^{3+}$ released from the anode behave as Lewis acids and hydrolyze the water molecules in the solution. This effect can reduce $\mathrm{pH}$ and potentially alter the medium conductivity ${ }^{1181}$. Metal ions released from the electrodes can also contribute to local distortion of the electric field, further compounding the problems associated with metal ion contamination ${ }^{1182}$.

\subsubsection{The Problem of $\mathrm{pH}$ Changes}

As touched upon previously, $\mathrm{pH}$ changes that take place at the electrodes can have a substantial impact on cell health. The changes in $\mathrm{pH}$ values in solution have been measured to exceed 1-2 $\mathrm{pH}$ units under conditions similar to those used in standard electroporation ${ }^{1183}$. As with Joule heating, any shift in $\mathrm{pH}(\Delta \mathrm{pH})$ depends on the medium conductivity. $\Delta \mathrm{pH}$ of a solution in which sucrose was substituted for $\mathrm{NaCl}$, was reported to be about 5 times less than phosphate buffered saline. The electrode material also contributes, with aluminium cathodes yielding a two-fold greater $\Delta \mathrm{pH}$ in comparison with platinum, copper or stainless-steel cathodes. This led to the recommendation of stainless steel electrodes instead of aluminium $^{1183}$. Several studies have successfully visualized the changes in $\mathrm{pH}$ at electrodes by using $\mathrm{pH}$ sensitive dyes $^{574,1184,1185}$. Acidic fronts form at the anode while the cathode becomes basic. A study by Li et al. used microchipbased electroporation to determine that hydroxyl ions at the cathode are more toxic than protons at the anode ${ }^{1185}$. They observed that strong $\mathrm{pH}$ buffering can, to some extent, neutralize the problem, thereby bringing cell viability up above $90 \%$ in comparison with $60 \%$ for inadequately buffered and $40 \%$ for unbuffered solutions ${ }^{1185}$. The idea of switching the polarity of electrodes between pulses has also been suggested to prevent cumulative $\mathrm{pH}$ biases at the electrodes ${ }^{1183}$. 


\subsubsection{The Problem of Non-Uniformity in the Electric Field}

Non-uniformity of the electric field can cause some cells to be treated too harshly while others are insufficiently permeabilzied. Indeed, significant heterogeneity in electroporation arises due to a lack of consistency of the electric field $^{1049,1146}$. One effect of excessive electrolysis is degradation of the electrode performance. For example, a study with stainless steel electrodes in parallel plate geometry showed significant pitting of the anode ${ }^{1186}$. The increase in the roughness of the electrode was proposed to contribute heterogeneity and loss of consistency of the field applied across the cell suspension. Subsequent studies also showed that the pulsing frequency and the presence of chloride ions amplified the corrosion of iron electrodes ${ }^{1187}$. Furthermore, in a dense suspension of electroporated cells, neighboring cells will affect the geometry of the electric field due to mutual electrical shading ${ }^{1147,1188}$. When cells represent $1 \%$ of the volume fraction they behave as single cells, while for volume fractions greater than $10 \%$ (or for clusters of cells), the suspension density will distort the conferred transmembrane potential ${ }^{1087,1189}$.

\subsubsection{Counteracting Electrolysis}

The abovementioned studies show that electrolytic effects and electrode corrosion are a critical consideration for electroporation. This is especially important for cells and biological material bound for medical applications, such as cellbased therapies. Tactics that may be used to mitigate the negative effects of electrolysis include lowering solution conductivity, changing the pulsing schemes, buffering more strongly against $\mathrm{pH}$ changes, and reducing the surface area of electrodes adjacent to cells. Another strategy is to switch the polarity of electrodes between successive pulses, which has been shown to minimize cumulative electrolysis and decrease the contamination of metal ions in solution by an order of magnitude ${ }^{1180}$. The idea of using more inert gold or platinum, or replacing metal electrodes with plastic, graphite, or liquid ones has also been explored.

\subsubsection{Cell Damage from the Electric Field}

Aside from cell damage due to electrolytic effects (e.g. Joule heating, contamination via corrosion of electrodes, and pH changes), the electric field itself may harm cell components more directly. For example, the application of strong electric fields to cells has been suggested to trigger lipid peroxidation ${ }^{1190-1192}$, generation of reactive oxygen species ${ }^{1193,1194}$, protein 
denaturation, and DNA damage ${ }^{1195,1196}$ amongst other responses. Under electroporation conditions compatible with cell survival, it was shown that electroporation can trigger an "oxidative jump" where the level of reactive oxygen species (ROS) rises sharply ${ }^{1193}$. The measured generation of ROS was to some extent dependent on extracellular calcium and magnesium but could be prevented by addition of anti-oxidants. In subsequent studies, lipid peroxidation, as evidenced by the presence of lipid hydroperoxides, was observed in the membranes of both plant and animal cells following electroporation ${ }^{1191,1192}$. Further investigations using the chemiluminescent probe lucigenin found that CHO cells subject to millisecond pulses undergo a threshold level of oxidation of their plasma membrane lipids, but that this effect only partially correlates with cell survival ${ }^{194}$. Interestingly, lipid peroxidation of unsaturated phosphatidyl choline species has also been observed during electroformation of giant unilamellar vesicles ${ }^{1197}$. Membranes characterized by a high degree of peroxidized lipids tend to be weaker and more susceptible to disruption, including by electroporation ${ }^{1198}$. Indeed, lipid peroxidation is well known to influence membrane behaviour, including domain formation and mechanical properties, which could have implications for cell recovery post-electroporation.

The reactive oxygen species produced by electroporation will not only target lipids but can also degrade proteins and nucleic acids. DNA damage in proportion to the applied voltage and duration has been reported in HL60 cells, although no specific mechanisms were pinpointed ${ }^{1196}$. It could be that DNA damage is due to influx of oxidative agents from the extracellular environment. Regarding proteins, Chen and colleagues have suggested non-thermal electroconformational damage to ion channels following exposure to strong electric fields ${ }^{1199-1202}$. More general models describing electroconformational damage of membrane proteins and other cellular components have subsequently been described ${ }^{1203,1204}$. In particular, it is proposed that charged amino acids in membrane proteins or voltage-sensing segments in voltage-dependent transporters are vulnerable to sharp changes in electrical potential. These effects are thought to be more pronounced for shorter pulses of higher amplitude ${ }^{1205}$. Indeed, other studies showed that high voltage nanosecond pulses are likely to perturb the function of voltage-gated channel proteins ${ }^{1206}$, and possibly other proteins in general $^{1207}$.

Although not typically used for intracellular delivery, nanosecond pulsed electric fields are of interest for understanding how electric fields can affect cells on different timescales and in various compartments. One study examined generation of ROS in response to nanosecond pulsed electric fields $\left(30 \mathrm{kV} \mathrm{cm}^{-1} \text { at } 100 \mathrm{~ns}\right)^{1208}$. They found that ROS was inhibited by both calcium chelators, and the antioxidant trolox, in agreement with earlier observations that the presence of divalent 
ions appears to participate in ROS generation ${ }^{1193}$. Other reports have shown that $\mathrm{H}_{2} \mathrm{O}_{2}$ is among the damaging species generated by nanosecond pulsed electric fields ${ }^{1209}$. Although undesirable for intracellular delivery, non-thermal electrical destruction of proteins, cells and tissue have been proposed for a host of other medical and industrial applications ${ }^{1210}$.

Molecular dynamics simulations have shown that the presence of hydrophilic pores can augment the process of lipid flipflop, whereby lipids translocate from one leaflet of a bilayer to the other ${ }^{410}$. Partial abolition of the naturally uneven bilayer distribution of lipids has been observed in RBCs as a consequence of electric fields ${ }^{1211}$. Vernier and others found that nanosecond electric pulses can facilitate phosphatidylserine (PS) exposure to the outer leaflet within seconds ${ }^{1212-1214}$, indicating a biophysical mode of action rather than cell signaling. Rols and colleagues performed a follow-up study with millisecond permeabilizing pulses to examine membrane disorganization and phospholipid scrambling ${ }^{1215}$. Under the chosen conditions, PS exposure could not be detected. The threshold conditions that trigger PS exposure thus remain to be precisely determined, however, it appears that PS scrambling may only be relevant under regimes of very high field strength. Scrambling of the membrane asymmetry has implications for the long-term survival of cells, particularly in vivo where immune recognition mechanisms tend to destroy cells exhibiting wayward externalization of lipids.

\subsubsection{Cargo Damage from the Electric Field}

Apart from damage to the cell, administration of the field strengths commonly used for electroporation may also cause problems with the cargo molecules. Degradation and damage of electrically sensitive cargo has been suggested by some reports. For example, the Bhatia group reported aggregation of quantum dots upon electroporation, indicating it is not a suitable technique for intracellular delivery of quantum dots ${ }^{294}$. Electric pulse-induced precipitation of nucleic acids and other biological macromolecules has also been observed under certain conditions ${ }^{1178,1216}$, although it is unclear why other groups haven't seen such problems. These studies noted that nucleic acids aggregated into a non-functional state under the conditions of their experiment. If they can be identified, it seems likely that the conditions leading to precipitation must simply be avoided. Nevertheless, it is worth noting that not all molecular cargo can be assumed to be compatible with strong electric fields. 


\subsubsection{Technical Innovations: Bulk, Micro- \& Nano- Electroporation}

The early generation of electroporation systems were configured with a cuvette style geometry ${ }^{1134}$. Subsequently, the first commercial electroporator, the BioRad Gene Pulser, was launched with this configuration in the mid 1980s. Since then, the cuvette geometry has become the standard platform for electroporation, being simple, robust, and reasonably well understood (Figure 28A(i)). The nucleofector is no exception, and as discussed previously, its novelty arises not through a deviation from this geometry, but rather from the systematic use of optimized pulsing protocols and cell type-specific buffers. Despite its widespread adoption, the cuvette style geometry is not without problems. As discussed above, the large surface area of the metal electrodes presents issues concerning electrolysis, such as Joule heating, corrosion, $\mathrm{pH}$ deviations, and inconsistent field profile. Second, cuvette style electroporation is difficult to perform with low volumes $(<20 \mu \mathrm{l})$. As the intracellular delivery of a molecule via permeabilization is directly related to extracellular concentration, it is often advantageous to concentrate the cells into a minimal volume in the range of $10 \mu$ or less. This maximizes the concentration, which is especially useful for expensive or precious reagents. Below we discuss the innovations that have been produced in the electroporation field, including difference setups for bulk, micro- and nano-electroporation.

\subsubsection{Capillary Electroporation}

One of the first commercial setups to challenge the dominance of the cuvette style geometry came in the form of capillary electroporation (Figure 28A(ii)). This design was introduced by a company called NanoEntek in Korea and subsequently commercialized by Invitrogen/Thermo Fisher as the "Neon" electroporation system ${ }^{558}$. In the Neon system, cells and buffer solution are pipetted into a narrow capillary $(0.56 \mathrm{~mm}$ wide and $30 \mathrm{~mm}$ long) featuring a wire gold electrode with minimal surface area at the top. The other electrode, also made of gold, is located within a conductive electrolyte bath outside the capillary. Because of the small surface area and distance from the cells, bubbles, Joule heating, and pH waves are more effectively separated from the cells. The small size of the electrodes also means that gold plating becomes economical. Chemical stability of the electrodes is superior to those made from less inert metals like iron, aluminium, or copper. The inventors compared $\mathrm{pH}$ deviations in the capillary system to those of conventional cuvette style chambers and it appeared to confer substantial advantages in protecting cells from the toxic electrolytic processes that can occur at 
electrodes. Together these features are purported to increase the viability of cells treated in capillary electroporation system $^{558}$.

On the other hand, one disadvantage of the Neon system is the reduced flexibility in determining pulse parameters. The pulse duration is limited in the range 1-100 ms and voltage from 500-2000 V. Given the distance of the conductive path between the electrodes, this means the field strength does not exceed $1 \mathrm{kV} \mathrm{cm}^{-1}$. The user may increase the number of pulses but there is no option to program pulses of different voltage, or frequency. Thus, the dual pulse strategies that have become so popular with the Nucleofector system are not possible with the Neon platform. High cost of capillary tips, electrodes, and buffers is another factor that users dislike ${ }^{559}$. In response to this, some researchers have published protocols advising users on how to recycle the components and employ homemade buffers, such as one consisting of PBS supplemented with $250 \mathrm{mM}$ sucrose and $1 \mathrm{mM} \mathrm{MgCl}{ }^{559}$.

\subsubsection{Microfluidic Electroporation}

Motivated by the shortcomings of conventional electroporation equipment, a number of researchers and engineers have explored alternative solutions. Electroporation combined with microfabricated, microfluidic, and nanotechnology concepts has received a great deal of attention in the last two decades as evidenced by a spate of reviews on the topic ${ }^{1217-}$ ${ }^{1222}$. Compared to bulk electroporation systems, it has been argued that micro- and nano- electroporation can provide the

following advantages ${ }^{1220,1221}:$ 1) lower voltages due to smaller scale, thus obviating the need for high powered pulse generators, 2) ability to concentrate, trap, and position cells and cargoes for higher efficiency delivery, 3) real time monitoring of device performance at single cell level, and 4) scalable solutions from single cells up to large populations. 
A

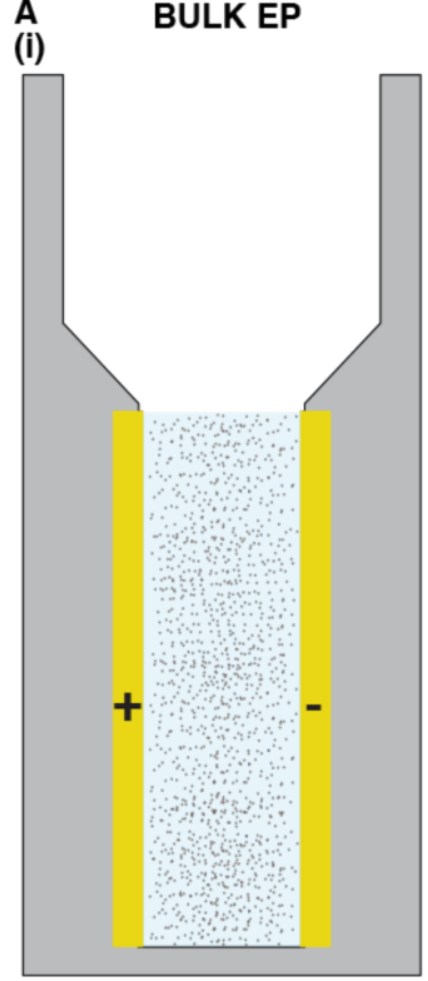

cuvette configuration

(ii)

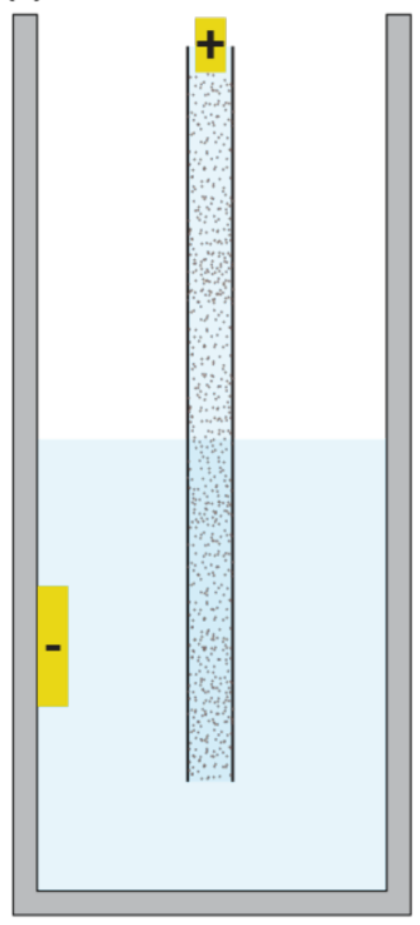

capillary configuration
B

(i)

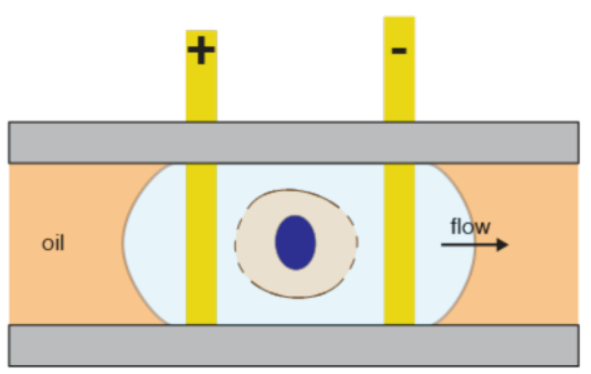

cells in microfluidic droplet

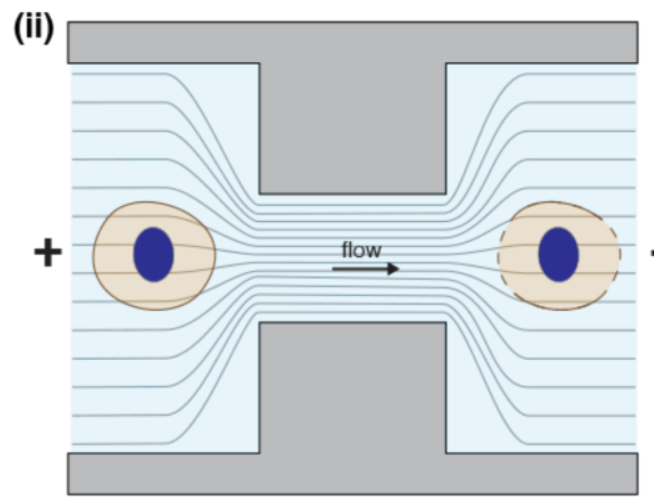

constant voltage + microfluidic constriction

(iii)

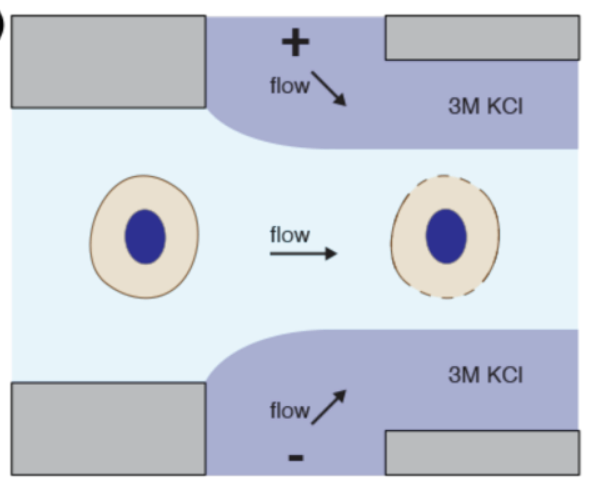

liquid electrodes + hydrodynamic focusing

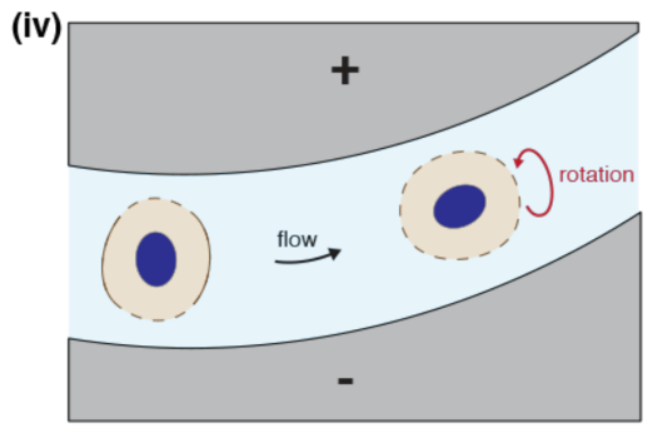

hydrodynamic vortex
C NANO EP

(i)

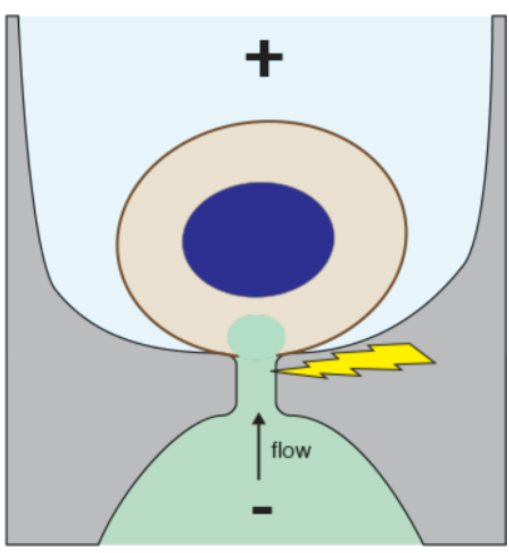

nanochannel electroporation

(ii)

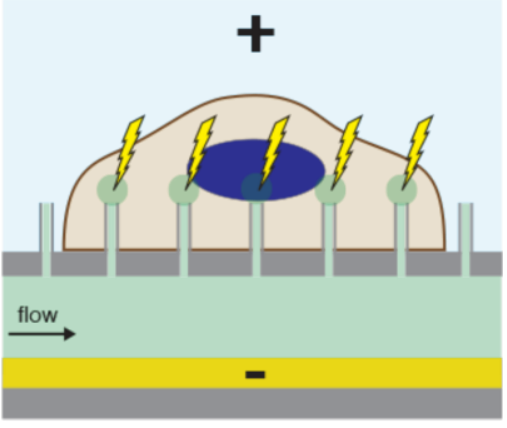

nanostraw electroporation

(iii)

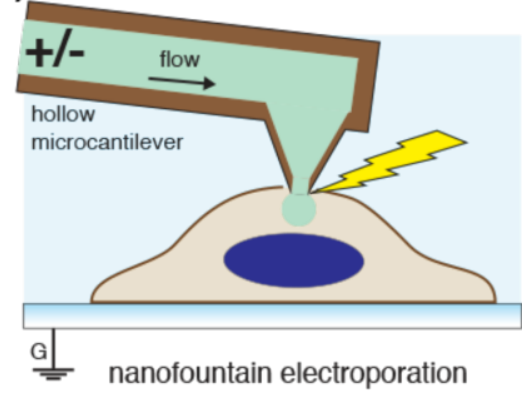

Figure 28. Electroporation (EP) configurations. (A) Bulk (conventional) electroporation in parallel plate cuvette (i) and capillary (ii) geometries. (B) Microscale electroporation examples showing (i) electroporation in droplets, (ii) the use of channel architecture to manipulate voltage pulses, (iii) hydrodynamic focusing to generate liquid electrodes, and (iv) hydrodynamic vortices to rotate cells through electric fields for more homogenous permeabilization. (C) Nanoscale electroporation with examples of (i) nanochannel electroporation, where cells are pressed against nanoscale apertures; (ii) nanostraw electroporation, in which the electric field is concentrated onto the end of a nanostraw; and (iii) nanofountain electroporation, which exploits a hollow AFM tip for addressing individual cells. 
One of the first microfluidic electroporation systems was constructed by Huang and Rubinsky in the late 1990 s $^{1223}$. It was essentially a small hole of $2-10 \mu \mathrm{m}$ diameter that a single cell could be sucked onto. The application of an electric pulse from below was used to permeabilize the basal side of the trapped cell and study the mechanisms of electroporation at single cell level. Although only demonstrated as a proof of concept, such developments spurred the field on toward further efforts. Several years later the first microfluidic flow electroporation devices appeared on the scene. Huang and Rubinsky were again pioneers in this department, demonstrating loading of small molecule dyes and transfection with GFPencoding plasmids, albeit at low throughput ${ }^{1224}$. In the following, we will highlight several examples of flow-based microfluidic electroporation.

Droplet-based microfluidics enables the use of microscale compartments to expose cells to a particular chemical environment within picoliter reaction volumes ${ }^{1225}$. Zhang et al. encapsulated cells in aqueous droplets before flowing them over a pair of electrodes subjected to a constant DC voltage ${ }^{1226}$. Due to the non-conductivity of the oil phase, cells only experience a transient electric pulse when the conductive droplets pass the electrodes (Figure 28B(i)). The cell is then permeabilized to the molecular cargo loaded within the droplet. In this case a DNA plasmid encoding for GFP was successfully delivered into $\mathrm{CHO}$ cells ${ }^{1226}$. The pulse parameters were governed by the flow speed, size of the droplet, distance between the electrode pair, and the positioning of the cell inside the droplet. Owing to the rise in droplet-based microfluidics for high-throughput single-cell manipulation, techniques that can perform intracellular delivery on cells within droplets are expected to be important.

In a second example of flow-based microfluidic electroporation, electric pulse parameters are again determined by the device geometry and flow speed under constant DC voltage. But in this case electroporation occurs at narrow constrictions within the main flow channel ${ }^{489}$. The geometry of the device channel controls the field amplification so that cells experience an electric pulse as they passage through a constriction (Figure 28B(ii)). Pulse duration imposed on the cell is therefore determined by flow speed, while amplitude is given by width ratio of the constriction to main channel. The number of constrictions in series will effectively determine the number of pulses. In subsequent efforts, Geng et al., scaled up this concept to process $20 \mathrm{~mL} \mathrm{~min}^{-1}$ of cells in continuous flow mode with a minimalist setup featuring low-cost components, a syringe pump, and a bench top DC power supply without the need for a pulse generator ${ }^{1227}$. For plasmid transfection in $\mathrm{CHO}$ cells a transfection efficiency of up to $75 \%$ was achieved. 
In a different microfluidic electroporation strategy, hydrodynamic flow focusing was exploited to create parallel laminar flow streams of different conductance (Figure 28B(iii)). Using a three-inlet approach, the top and bottom sheath flows were composed of highly conductive $3 \mathrm{M} \mathrm{KCl}$ solutions, which acted as liquid electrodes, while cells in standard aqueous solution were flowed through the center of the configuration ${ }^{1228}$. By applying a DC voltage of only $1.5 \mathrm{~V}$, electric field intensities of more than $1 \mathrm{kV} \mathrm{cm}^{-1}$ could be generated across the central zone to electroporate the passing cells. The device showed up to $70 \%$ delivery efficiency of fluorescein dyes into yeast cells ${ }^{1228}$. Moreover, distancing the metal electrodes from cells using hydrodynamic focusing had the advantage of separating cells from electrolysis issues such as heating, bubble generation, $\mathrm{pH}$ changes, and production of toxic ions ${ }^{1228}$. Thus, the use of non-metal liquid electrodes in hydrodynamic flow mode may mitigate some of the problems associated with cuvette-style electroporation.

In a fourth example of microfluidic ingenuity, a spiral-shaped microfluidic channel was implemented to generate flow vortices $^{1229}$. As cells traverse through the curved channels, vortices caused by Dean flows facilitate their rotation in reference to the electric field (Figure 28B(iv)). This results in permeabilizing the entire cell surface, rather than just the cell poles. By increasing the cell surface area that can be electropermeabilized high delivery efficiency was achieved with both dyes and DNA plasmids ${ }^{1229}$. Other vortex-based microfluidic systems have been implemented to achieve a similar effect $^{1230,1231}$ and have demonstrated intracellular deliver of dyes, miRNA, siRNA, proteins, and plasmids ${ }^{1232}$.

\subsubsection{Nanochannel Electroporation}

Inspired by early work on electroporation through micron-sized apertures ${ }^{1223}$, James Lee and colleagues introduced the concept of nanochannel electroporation ${ }^{1233}$. By scaling the aperture size down to $\sim 90 \mathrm{~nm}$, the membrane disruption effect of electroporation could be concentrated onto a very small spot on the cell surface (Figure 28C(i)). A significant claim of this strategy is dose control, i.e. the finding that the amount of delivered material directly correlates with the voltage pulse duration. Nanochannel electroporation also appears to introduce agents faster and deeper into the cytoplasm, a result attributed to enhanced and concentrated electrophoretic forces. In support of this, finite element simulations found that fringe fields extend into the cell and could possibly be used to propel molecular cargo through the permeabilized section of the cell periphery and deep into the cytoplasm. Compared to conventional electroporation and other forms of 
microfluidic electroporation, it was proposed that nanochannel delivery mechanism is based on electrophoretic forces rather than diffusion and/or endocytosis. Nanochannel electroporation was able to deliver dyes, oligonucleotides, siRNA, plasmids and quantum dots into recipient cells. Moreover, only nanochannel electroporation could deliver quantum dots into Jurkat cells, while conventional or microfluidic electroporation could not. One drawback of the method, however, was the low throughput nature of the technique. Prior to electroporation each single cell requires placement against the nanochannel with optical tweezers.

In 2016 the James Lee group published a scaled-up version of nanochannel electroporation able to process up to 40,000 cells on a single chip over a $1 \mathrm{~cm}^{2}$ area ${ }^{1234}$. In this version, termed "3D nanochannel electroporation", the aperture dimensions were expanded to $300-650 \mathrm{~nm}$. Positive dielectrophoresis was employed to simultaneously position thousands of cells across the array and press them against the array of nanochannels. This was necessary because a tight seal between the cell membrane and nanochannel is critical to ensure consistent electroporation performance across the device. Molecules to be loaded are filled into a reservoir below the substrate and delivered into cells concurrently with application of the electric field. The system was used for transfecting plasmid DNA into batches of natural killer cells, which are otherwise difficult to transfect. A predecessor to this idea was published in 2006 by Kurosawa et al. using an insulating substrate with an array of $2 \mu \mathrm{m}$ holes in it $^{1235}$. Just like in 3D nanochannel electroporation, the field was concentrated at the holes and molecules to be delivered were supplied from underneath. This design is essentially a scaled-up version of the original microfluidic electroporation system published by Huang in $1999^{1223}$.

The Luke Lee lab also published a series of papers where cells were sucked into microchannels made of PDMS. In effect, this design was not too dissimilar from a parallel array of micropipettes ${ }^{1236-1238}$. An electric field was introduced to focus the electroporation effects to the region of the cell sucked into the microchannels, thereby locally permeabilizing them ${ }^{1236-}$ 1238. The concept was later combined with electrophoresis for increasing the efficiency of delivery, where the delivery of molecules could be optically monitored in real time ${ }^{1239}$. Again, a similar concept to take advantage of using channels as trapping arrays was used to transfect plasmid DNA into stem cells ${ }^{1240}$. Collectively, these innovations show the power of localizing electric fields to the subcellular scale. If the problem of scale-up to high throughput can be solved at an acceptable cost, this approach can be expected to benefit the intracellular delivery toolkit. 


\subsubsection{Nanostraw Electroporation}

Another form of nanoscale electroporation takes the form of so called nanostraws (Figure 28C(ii)). The key difference is that the nanoscale aperture protrudes into the target cell as a hollow nanoneedle. Although cell membranes appear to be resistant to penetration by such nanoneedle under passive conditions, the addition of an electric field permeabilizes the cell membrane at the tip of the nanostraw ${ }^{702}$. One benefit of this approach is that active forces, such as optical tweezers or positive dielectrophoresis, are probably not required to establish optimal contact between cells and the nanostraw. Rather, a consistent period of settling might be required to facilitate uniform contact between cells and the substrate ${ }^{428}$. Furthermore, with sufficient adhesion to the nanostraw array, substantial pumping forces can presumably be used to flow molecules into the cell cytoplasm without cell detachment. In light of poor results with aluminium electrodes in bulk conditions, however, the choice of aluminium nanostraws as the fabrication material may need to be revised in future versions of this device.

\subsubsection{Nanofountain Probe Electroporation}

A scanning probe-based approach for localized electroporation, termed nanofountain probe electroporation, has been introduced by Espinosa and colleagues ${ }^{1241,1242}$. It is essentially an atomic force microscope cantilever engineering with a hollow channel for fluid flow. Target cells are cultured on a grounded coverslip and positive or negative voltages are applied to the conductive cantilever, thereby focusing the electric field at the site of contact between the cantilever and cell (Figure 28C(iii)). By coordinating the movement of the tip and the flow of fluid, introduction of dextrans and proteins into cells can be achieved ${ }^{1241}$. In follow-up applications of this system, it has successfully been employed to deliver molecular beacons to the cytoplasm for detection of mRNA transcription ${ }^{1242}$.

\subsubsection{Summary of Micro- and Nano-electroporation}

Innovations in micro- and nano-electroporation have showcased a number of interesting proof-of-concept prototypes. Diverse architectures have been developed, including the use of micro- or nano-channels smaller than the cell, channels larger than the cell, chambers, compartments, and droplets, and hydrodynamic effects such as sheath focusing and vortices $^{1218,1220}$. Some of these reports claim improved delivery efficiency and viability over conventional bulk 
electroporation. They have also provided elegant solutions to problems that have long troubled traditional electroporation setups, such as electrolytic reactions at the electrodes, gas bubble formation, $\mathrm{pH}$ deviations, Joule heating, inconsistent cell treatment, inability to scale down reagent volumes, excessive power consumption, and requirement for cumbersome equipment. Yet the technical advancements of miniaturized approaches have not translated to widespread adoption, most likely due to high cost, impractical throughput, lack of focus on clinical or industrially relevant problems, or lack of userfriendly designs ${ }^{1221}$. Thus, it remains to be seen what the next generation electroporation systems will look like, and whether they will challenge the dominance of existing methods. Apart from technical upgrades, recent literature emphasizes that further theoretical studies on mechanisms of cell membrane permeabilization and cargo uptake are needed to obtain further progress in the field ${ }^{163,1118,1131,1132,1138,1144,1219}$.

\subsubsection{In Vitro \& Ex Vivo Applications of Electroporation}

Of the membrane disruption-based approaches, electroporation is currently the most mature in regard to industrial applications and clinical translation. Electroporation-based technologies have been deployed in vivo as well as in vitro. The in vivo applications include electrochemotherapy, non-thermal tissue ablation, DNA vaccines, and transdermal drug delivery. These have already been discussed in other reviews ${ }^{1048,1050,1243-1247}$. In biotechnology, electroporation has also been used for extraction of biomolecules, sterilization/pasteurization of solutions, and transformation of microorganism ${ }^{1248}$. In keeping with the focus of this review, we will focus our discussion on the in vitro and ex vivo applications relevant to intracellular delivery in human and animal cells. Within this context, electroporation has been employed mainly for nucleic acid transfection, of which there are three main market areas: 1) biomedical research, 2) biomanufacture of biologics (proteins, antibodies, and viral vectors/particles), and 3) therapeutics (cell-based therapies, gene therapy, and cell manipulation for regenerative medicine)(see Figure 3). Furthermore, intracellular delivery of nonnucleic acid cargo is beginning to enjoy increased attention, especially with the rise of genome editing and new forms of cell-based therapies. Below we highlight a selection of key applications where electroporation had made an impact.

\subsubsection{Intracellular Delivery of Impermeable Drugs}

Permeabilization via electroporation has been proposed for pharmacological applications to identify the cytoplasmic activity of otherwise impermeable drugs and small molecules ${ }^{1249}$ (Figure 29A). In the 1980s a study by Melvik et al. 
showed that electroporation of cell lines significantly enhanced the efficacy of cisdichlorodiammineplatinum(II)(cisplatin) up to 3 -fold greater than controls ${ }^{1085}$. Using radiolabeled tracers, they found electroporation rendered cells permeable to small molecules for up to 10 minutes. Subsequently, electroporation has been used to screen for cytotoxicity of drugs that are otherwise susceptible to be pumped out of cells by the activity of cellular efflux pumps ${ }^{55,1086}$. Bleomycin $(\sim 1.4 \mathrm{kDa})$ represents a particularly striking example of a drug where activity is drastically increased with electroporation-mediated intracellular delivery ${ }^{55,1086}$. Thus, electroporation can be leveraged to test for the cytoplasmic activity of otherwise impermeable small molecules, peptides, and biochemical agents. 
A

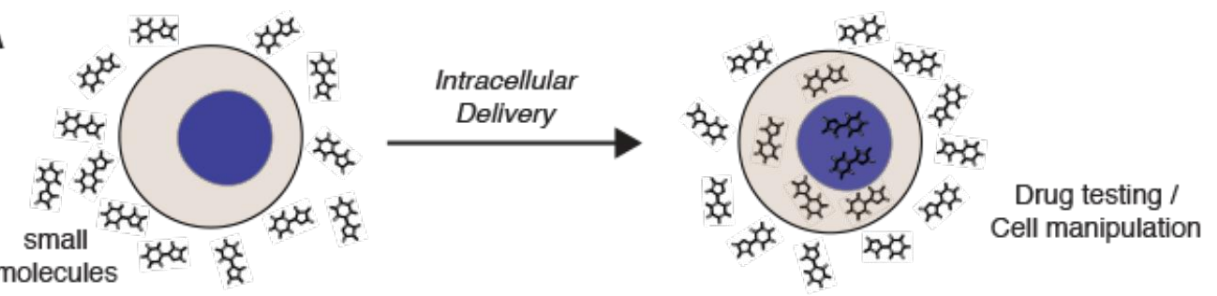

\section{B}
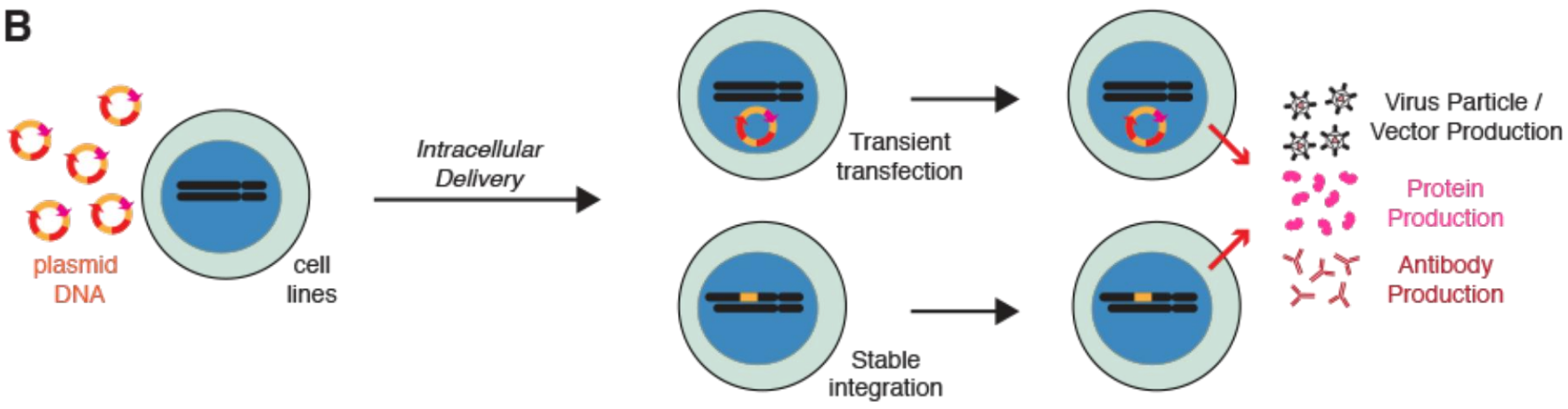

C
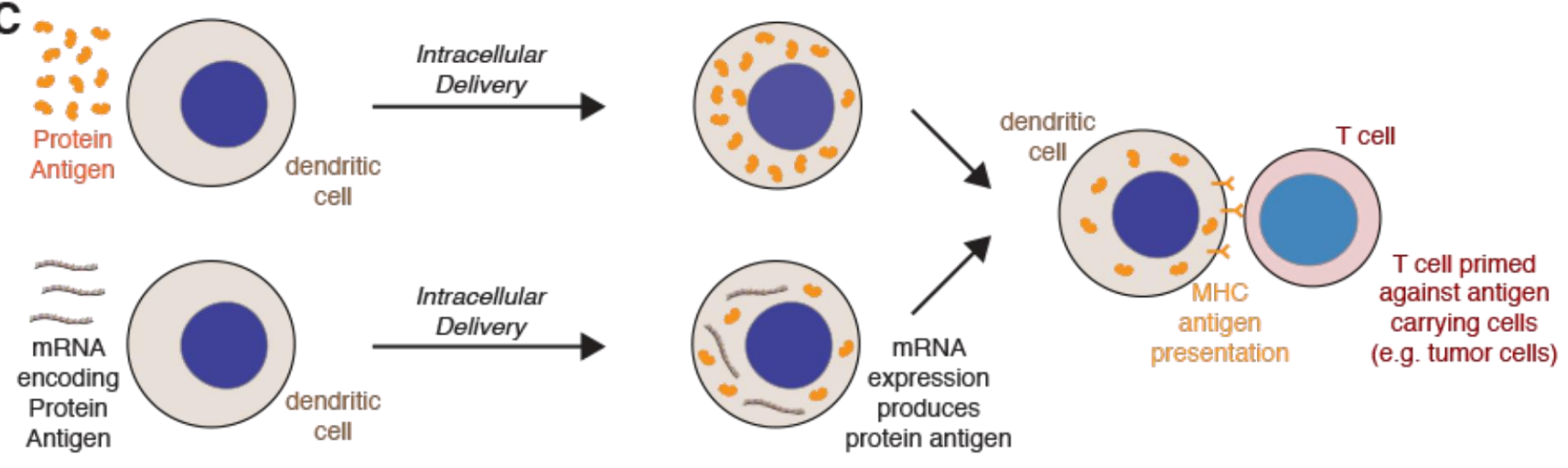

D
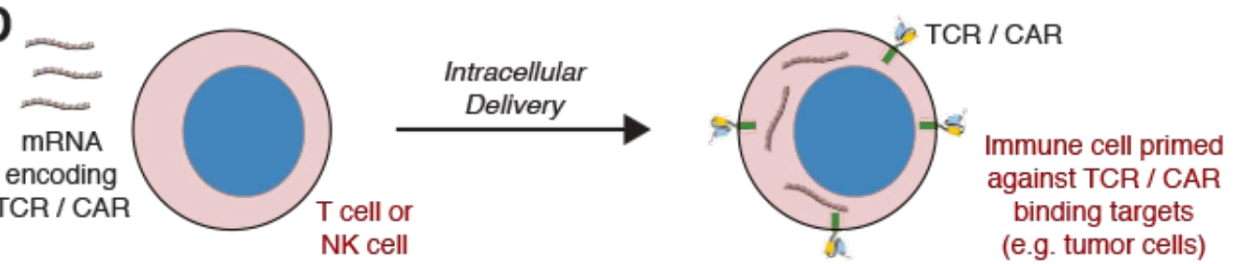

E

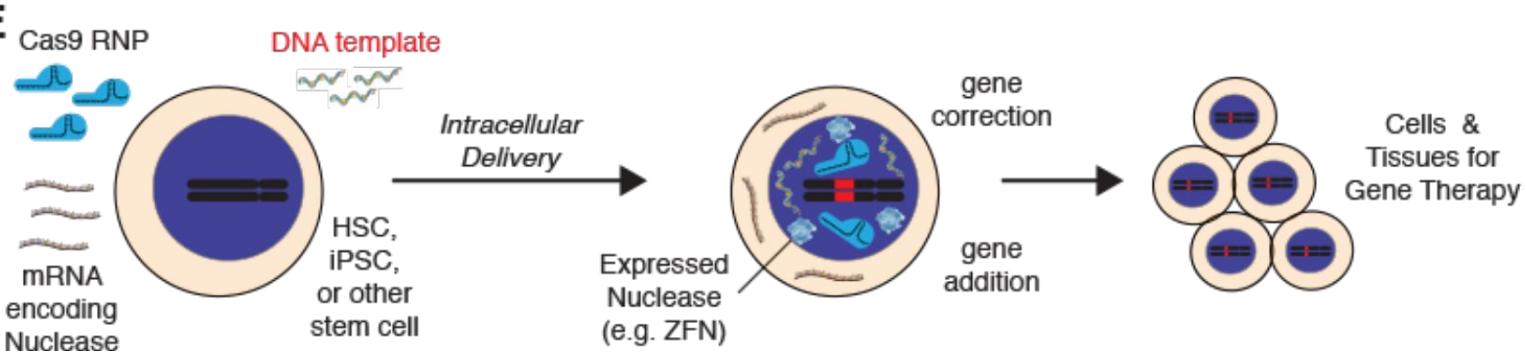

Figure 29. In vitro and ex vivo applications of intracellular delivery achieved with electroporation. (A) Delivery of impermeable drugs to the intracellular space for drug testing and/or cell manipulation. (B) Transfection with plasmid DNA encoding proteins, antibodies, and viral components for biomanufacturing purposes. (C) Loading of protein antigens or mRNA encoding such into dendritic cells. Presentation of antigen fragments through MHC pathways is able to prime $\mathrm{T}$ cells against cells carrying the antigens and may be useful for cancer immunotherapy. (D) Transfection of cytotoxic immune cells with mRNA encoding TCRs and/or CARs can be used to direct immune cells against specific cell targets, such as cancer cells. TCR $=\mathrm{T}$ cell receptor. $\mathrm{CAR}=$ chimeric antigen receptor. (E) Genome-editing molecules can be delivered into stem cells for the purposes of adding, deleting, or correcting genes. Modified stem cells can then be expanded for potential deployment in cell- and tissue-based gene therapy. Red signifies areas of the genome that have been edited. ZFN = zinc finger nuclease. 


\subsubsection{Biomanufacture Through Transfection}

Biomanufacture refers to the production of biomaterials or biomolecules by the harnessing of biological systems. Transfection of common cells lines can be used for production of proteins, antibodies, viral vectors, or viral particles ${ }^{1250-}$ 1255 (Figure 29B). These are often produced in mammalian cell lines such as CHO, HEK-293T, HeLa, A549 cells or insect cell lines, depending, for example, on the need for species-specific post-translational modifications. Significant efforts have gone into engineering these systems for maximum yield and economies of scale. Both stable genetically modified cell lines and transient transfection are key strategies for biomanufacture. Although lipid and polymer reagents are most commonly used for transfection in biomanufacture, electroporation is currently the leading option among membrane disruption-mediated methods.

\subsubsection{Large Volume Flow Electroporation}

The concept of large volume flow-based electroporation for cell processing emerged in the late 1980s. A flow-based electrofusion system for processing several milliliters of suspended cells per minute was introduced by Teissié and colleagues $^{1256,1257}$. Following that, the same group reported on a flow-based intracellular delivery system with similar throughput capable of transfecting plasmid DNA into different cell types at efficiencies of $25-35 \%{ }^{1258}$. In 2002 , a commercial large volume flow electroporation system for clinical and industrial bioprocessing was reported in the scientific literature by the company Maxcyte ${ }^{1259}$. Initial reports claimed that common suspension and adherent cells lines could be loaded with $500 \mathrm{kDa}$ dextran at $>90 \%$ efficiency and $>90 \%$ viability while gene transfection rates could reach up to $75 \%$. The latest versions of this technology are capable of tunable scale, from tens of thousands of cells up to 200 billion cells packed into liters of solution. The run time for a batch of 200 billion cells is approximately 30 minutes in a single run. Moreover, the system is sterile and compliant with current good manufacturing processes (cGMPs) for biological clean room facilities. In further demonstrations of its utility in manufacturing scenarios, the flow electroporation platform was used to batch transfect HEK293T cells for large-scale bio-production of lentiviral vectors ${ }^{1260}$.

Recently, Zhao et al. published a different strategy for large volume flow electroporation with a device that integrates a flow tube and a miniaturized needle electrode array with uniform spacing ${ }^{1261}$. The microfluidic design of the needle electrode array brings the benefit of lowering the required voltage. The system enables processing rates of 20 million cells 
per minute and was suggested to be suitable for in vitro and ex vivo batch mode applications. Another group published a similar concept constructed from custom-made microfluidics components as a solution for batch flow electroporation of mRNA into tens of millions of dendritic cells ${ }^{1262}$.

\subsubsection{Delivery of Genome-Editing Proteins and RNPs}

Recent advances in genome editing via programmable nuclease have spurred an interest in intracellular delivery of these proteins, particularly Cas9 RNPs. In the last few years RNP delivery has been successfully accomplished with electroporation $^{143,144,146,147}$, microinjection ${ }^{148,149}$, lipid nanoparticle formulations ${ }^{150}$, osmotically-induced endocytosis followed by endosome disruption ${ }^{151}$, microfluidic cell deformation ${ }^{152}$ and $\mathrm{CPPs}^{153}$. Electroporation, however, is reported to be more efficient with a number of primary, blood, and immune cell types in vitro. RNP delivery via electroporation has been demonstrated in a range of cell types, from common cell lines to blood and immune cells of clinical relevance, with both conventional cuvette style (Nucleofection) $)^{30,143,146,147,287}$ and capillary electroporation $(\text { Neon })^{45,144,145,1263}$ platforms.

The mechanisms of RNP entry via electroporation have not been heavily studied yet. Given what we already know about the influx behaviour of nucleic acids and proteins (Figures 24, 25, and 26), it is worth considering the possibilities. As discussed in section 2.2.2, an RNP complex should have about -80 negative charges, be $\sim 188 \mathrm{kDa}$, and up to $15 \mathrm{~nm}$ in size (Table 1). The mechanisms of electroporation-mediated delivery could thus be similar to siRNA, namely direct translocation of a highly negatively charged molecule into the cytoplasm at the side of the cell facing the negative electrode during the pulse (Figure 24D). Once in the cytoplasm a nuclear localization sequence (NLS) on the Cas9 would then promote its shuttling to the nucleus. Another possibility is that RNPs are endocytosed after being entangled in the destabilized plasma membrane, such as is the case for plasmid DNA (Figure 24E, Figure 25). Indeed, post-electroporation aggregation or trapping of proteins at the plasma membrane has been observed in several cases ${ }^{1092,1122}$. The groundbreaking potential of genome editing will no doubt stimulate the field toward studying mechanisms of protein and RNP delivery to the nucleus. For example, the optimal nuclear concentrations of Cas9 RNP needed for efficient genome editing are still unknown. In future, it will also be interesting to see how other membrane disruption-based delivery approaches (which do not supply electrophoretic forces) compare in their efficiencies of RNP delivery. 


\subsubsection{Hard-to-Transfect Cells}

A number of sensitive primary cell types do not easily tolerate foreign nucleic acids or the toxic side-effects of common transfection reagents. For example, dendritic cells, T lymphocytes (T cells), B lymphocytes (B cells), natural killer (NK) cells, leukemia cells, hematopoietic stem cells (HSCs), macrophages, and neurons have all been reported to be recalcitrant to polymer- or lipid-based transfection $233,260,261,282,286,290,1172,1264,1265$. Lentiviral transduction and electroporation have emerged as the two leading alternatives. However, procedures with viral vectors are sometimes unfavorable because they can: (1) be labor-intensive, inconsistent, and expensive, (2) present safety hazards, (3) cause untoward immune or inflammatory responses in vivo, and (4) carry a risk of insertional genotoxicity via genomic integration. Electroporation, on the other hand, is rapid and simple, but its core weakness is poor viability or loss of cell functionality, as has been reported for nucleofection of dendritic cells or T cells $\mathrm{s}^{279,1165,1264}$.

Nucleofection, in particular, has sought to build a reputation on effectiveness with hard-to-transfect cells ${ }^{289,1108}$. Nucleofection has demonstrated significant success with DNA and RNA transfection in various types of stem cells, primary cells, and post-mitotic cells. Published examples include primary human melanocytes, smooth muscles cells, chondrocytes, and mesenchymal stem cells ${ }^{1156,1163,1164}$, dendritic cells ${ }^{1165,1166}$, monocytic cell lines ${ }^{1159}$, primary leukemia cells and cell lines ${ }^{233,1167,1168}$, primary natural killer cells and their derivative cell lines ${ }^{1169,1170}$, primary lymphocytes ${ }^{1171,1172,1266}$, embryonic and adult stem cells ${ }^{1173,1174}$, and mammalian neurons ${ }^{1157,1175}$.

Other electroporation platforms have also achieved a measure of success in hard-to-transfect cells. Minimalist setups featuring standard 2 or $4 \mathrm{~mm}$ cuvettes, commercial pulse generators (such as the BioRad Gene Pulser or BTX units), and an electroporation buffer consisting of OPTIMEM media (or equivalent) have attained favorable results with macrophages $^{234,275}$, T lymphocytes ${ }^{187,257,551,1267-1271}$, dendritic cells ${ }^{186,260-262,1110,1272,1273}$, and B cells ${ }^{1274,1275}$. Some of these groups have even used such setups to perform small scale clinical trials ${ }^{560}$. In other cases, the Maxcyte system for largescale clinical-grade flow electroporation has demonstrated effectiveness with leukemia cells ${ }^{1276}$, natural killer cells ${ }^{1277,1278}$, dendritic cells ${ }^{136-138}, \mathrm{~T}$ cells ${ }^{187}$, and $\mathrm{CD} 34(+)$ hematopoietic cells ${ }^{185}$. The Neon capillary electroporation system has successfully delivered molecules into iPSCs ${ }^{45,145}, \mathrm{~T}$ cells ${ }^{144}$ and $\mathrm{HSCs}^{1263}$. Together these studies suggest that no one electroporation system has a monopoly on effectiveness with sensitive or difficult to treat cell types. 


\subsubsection{T Cells \& Other Immune Cells}

Immune cells are a key category of cells for biomedical investigations and therapeutic applications. In $\mathrm{T}$ cells it has been asserted that RNA delivery to cytoplasm is not difficult, but DNA plasmid transfection, which requires nuclear penetration, remains a significant hurdle ${ }^{279,1279}$. This is an example where primary cells may exhibit an innate toxic reaction against delivered material. $\mathrm{T}$ cells, in particular, appear to display little tolerance to plasmid transfection regardless of delivery technique ${ }^{146,279}$. Electroporation is counted among the techniques that perform well in delivering siRNA and mRNA into T cells, however, the margin of error leading to loss of viability can be narrow ${ }^{279}$, and changes in the activation state, signaling pathways, and transcriptional responses of cells must be taken into account ${ }^{1280,1281}$.

Many of the published electroporation protocols underscore the narrow window of appropriate parameters, emphasizing that there exists a fine line between effectiveness and cell death ${ }^{275,1168}$. The challenge for electroporation appears to be the long-term survival, potency and functionality of treated cells, not so much the initial delivery. Indeed, post-treatment loss of viability, proliferative potential or potency has been reported for immune cells and other primary cell types ${ }^{279,1165,1264}$. Moreover, electroporated immune cells have sometimes been observed to exhibit an unfavorable response or poor engraftment when infused back to the in vivo setting ${ }^{279}$. On the other hand, several studies have shown electroporated cells to recover well and exhibit decent potency in clinical contexts ${ }^{187,560,1282,1283}$.

\subsubsection{Ex Vivo Intracellular Delivery for Cell-Based Therapies}

Scientists have long envisaged the power of ex vivo cell manipulation for cell-based therapies, especially in regard to gene therapy, immunothereapy and regenerative medicine ${ }^{28,29,31,41}$. The concept is to remove cells or tissues from the patient, engineer their function, and re-implant them to confer a therapeutic effect. Many of the relevant cell types, however, fall into the category of "hard to transfect" cells as outlined above. In the following we will highlight several areas where electroporation has been attempted for ex vivo cell-based therapies. 


\subsubsection{Protein Loading for Antigen Display in Cancer Immunotherapy}

Loading of exogenous proteins into the cytoplasm of antigen-presenting cells leads to their processing and display through the MHC-I pathway95,1284 (Figure 29C). This primes cytotoxic T cells against any cells carrying the antigens, such as cancerous cells that produce mutant proteins (Figure 29C). Thus intracellular delivery of tumor proteins into antigen presenting cells, especially dendritic cells, has been proposed as a strategy for cancer immunotherapy ${ }^{1285}$. Kim et al. were among the first to use electroporation to load dendritic cells with exogenous antigens ex vivo before implanting them back into the body to elicit a robust anti-tumor response in mouse models ${ }^{135}$. The Maxcyte clinical electroporation system was also used to achieve similar results by loading tumor cell lysate into dendritic cells ${ }^{136}$. In recent years this concept has been put to the test in human clinical trials. In 2013, a Japanese group confirmed the safety and feasibility of administering dendritic cell vaccines generated by cytosolic loading of autologous tumor lysates via the Maxcyte system $^{137}$. This strategy was reported to produce a significant anti-tumor effect compared to passive incubation (pulsing) of dendritic cells with tumor lysate ${ }^{138}$.

\subsubsection{9 mRNA Transfection for Antigen Display in Cancer Immunotherapy}

For induction of the MHC-1 antigen presentation pathway, mRNA transfection may be preferred to protein loading ${ }^{1286}$ (Figure 29C). Van Tendeloo et al. published a paper in 2001 showcasing the efficacy of such an mRNA-based strategy in dendritic cells ${ }^{260}$. Using a basic cuvette style electroporation setup with OPTIMEM buffer, they were able to able to achieve $>80 \%$ expression with $>80 \%$ viability compared with much poorer results from plasmid DNA in earlier studies $^{1287}$. Their comparison of methods for mRNA transfection to dendritic cells suggested that electroporation was far superior to lipofection and other methods ${ }^{1288}$. Based on these studies, the idea of electroporation-mediated mRNA transfection for ex vivo immunotherapy and gene therapy gained significant momentum ${ }^{1289}$. Using similar electroporation methods as those described by Van Tendeloo et al. ${ }^{1273}$, several groups have pressed ahead with small-scale clinical trials to treat human patients suffering from melanoma and other cancers ${ }^{560,1290}$. Results gathered to date indicate positive longterm survival rates and safety of the treatments. 


\subsubsection{Electroporated B Cells for Antigen Display in Cancer Immunotherapy}

Apart from dendritic cells, several other types of professional antigen-presenting cells have been tested for their ability to prime $\mathrm{T}$ cells against a tumor antigen. Coughlin et al. employed nucleofection to demonstrate that $\mathrm{B}$ cells from pediatric patients can be efficient antigen presenting cells upon loading with tumor mRNA ${ }^{1266}$. As a proof of concept, mRNAtransfected B cells were used to successfully prime a $\mathrm{T}$ cell response against cultured neuroblastoma cells in vitro ${ }^{1266}$. According to another study, electroporation of multiple RNAs into activated B cells with a standard cuvette style system elicited in vitro antigen-specific cytotoxic $\mathrm{T}$ cell responses with similar efficiencies as those of mature dendritic cells ${ }^{1275}$. Thus, the use of intracellular delivery for ex vivo activation of B cells may represent an alternative source of antigen presenting cells in cancer immunotherapy, especially in pediatric cases where dendritic cells are not as readily available.

\subsubsection{Electroporation to Produce CAR-T Cells for Cancer Immunotherapy}

A more direct way of inducing an immune response against cancer is to express a $\mathrm{T}$ cell receptor (TCR) or chimeric antigen receptor (CAR) directly into cytotoxic immune cells, such as $\mathrm{T}$ cells or natural killer (NK) cells ${ }^{32,1283,1291,1292}$ (Figure 29D). A CAR is a genetically engineered immunoreceptor that endows modified cells with a novel specificity to kill any cell that carries molecules to which the CAR binds. The goal is to target the killing action of TCR- or CARmodified immune cells against cancer cells carrying complementary surface markers. Electroporation has been used to deliver mRNA for expression of TCRs or CARs, chemokine receptors, or cytokines in T cells ${ }^{187,257,1293,1294}$. Similar to the case of dendritic cells, switching from plasmid DNA to mRNA was reported to allow $>90 \%$ gene expression with $>80 \%$ viability in $\mathrm{T}$ cells post-electroporation, even while using a basic cuvette-style electroporation protocol in OPTIMEM buffer $^{257}$. Using such methods, it was shown that multiple infusions of mRNA-electroporated CAR-T cells mediated shrinkage of large vascularized flank mesothelioma tumors of human origin in a genetic mouse model ${ }^{187}$. CAR expression and anti-tumor activity of mRNA-electroporated T cells was detected up to a week after electroporation. This is important because mRNA electroporation for transient expression of CARs in T cells is seen as a far safer alternative to permanent integration of CAR genes into the genome ${ }^{1291,1295}$. T cells electroporated with mRNA encoding for a CAR against CD19 showed cancer killing capacity in immunodeficient mice bearing xenografted leukemia ${ }^{259}$. Even a single injection of CD19 mRNA CAR-T cells yielded a significant prolongation in survival in this model. Because mRNA electroporation is 
a cost-effective and efficient path to engineer $\mathrm{T}$ cells for pilot studies, this approach has been pursued for high-throughput and iterative testing of novel constructs and targets in small scale clinical trials in humans ${ }^{32,1283,1291}$.

\subsubsection{Electroporation to Produce Cytotoxic NK Cells for Cancer Immunotherapy}

Although most work with CARs has been carried out with T cells, NK cells represent an alternative option ${ }^{1292}$. Among the first attempts to investigate this possibility were a series of experiments in 2005 by Imai et al. that used retroviral transduction to guide the activity of NK cells expressing CD19 CARs against patient leukemia cells in in vitro assays ${ }^{1296}$. Next, electroporation of CAR mRNA into NK cells was attempted in 2010. Members of the Maxcyte team used their clinical-scale large-volume electroporation platform to transfect mRNA encoding a CD19 CAR into NK cells ${ }^{1277}$. The engineered cells demonstrated cytotoxic killing of acute lymphoblastic leukemia and B-lineage chronic lymphocytic leukemia cells for up to 3 days after electroporation ${ }^{1277}$. Shimasaki et al. then employed the Maxcyte system to scale up mRNA transfection to large batches of expanded NK cells with numbers reaching up to 250 million cells per run ${ }^{1278}$. Under these conditions CD19 CAR expression reached >80\% after 24 hours and mediated significant anti-tumor cytotoxicity in a mouse xenograft model of B cell leukemia.

\subsubsection{Electroporation for Ex Vivo Gene Therapy of Blood \& Immune Cells}

Ex vivo cell-based therapies have long been pursued as an avenue for treatment of blood cells to address hematological disease $^{29}$. But only recently have gene therapy clinical trials in T cells and HSCs shown significant progress. These trials mostly used lentiviral transduction, which can carry a risk of genotoxicity due to random genomic integration ${ }^{26,1297-1299}$. To address this problem, new approaches that deliver genome editing molecules directly into cells have attracted interest for ongoing studies ${ }^{1300}$. As discussed in previous sections of this review, electroporation is among the techniques that can deliver genome-editing molecules in the form of mRNA, sgRNA, proteins, and RNPs into clinically relevant cell types at reasonable efficiencies and viabilities.

Here are two examples where electroporation of one component is combined with non-integrating viral transduction of another. First, integrase-defective lentiviral expression of donor DNA combined with nucleofection of zinc finger mRNA was used for HDR-mediated correction of monogenic mutations in the $I L 2 R G$ gene of patient $\mathrm{HSCs}^{30}$. This strategy has 
the potential to provide a one-time cure for the immune disorder X-linked severe combined immunodeficiency (SCID-X1) as gene-edited HSCs give rise to functional lymphoid progenitors that exhibit a selective growth advantage over disease mutants. Second, a recent study by DeRavin et al. used targeted integration of a corrected gene into CD34(+) HSCs as a treatment strategy for X-linked chronic granulomatous disease ${ }^{185}$. Similar to the previous example, they used electroporation (in this case, the MaxCyte platform) to transfect zinc finger mRNA into cells while donor DNA for gene correction was supplied by adeno-associated viral (AAV) 6 vectors. By targeted integration of a corrected gene into the AAVS1 safe harbor locus of the genome, it was argued that genotoxicity associated with random integration can be avoided. In mice transplanted with corrected HSC progenitors, 4-11\% of human cells in the bone marrow expressed the therapeutically corrected gp91phox protein.

\subsubsection{Electroporation for Gene-Editing of Blood \& Immune Cells}

Other proof of concept studies for therapeutic genome editing in HSCs and T cells have been carried out with

Nucleofection $^{146}$, Neon electroporation ${ }^{144,1263}$, or standard BTX cuvette-based electroporation ${ }^{1271}$. In these cases, delivery of Cas9 RNPs ${ }^{144,146}$, or mRNA encoding Cas9, ZFNs, TALENS, or megaTAL nucleases was demonstrated ${ }^{146,1263,1271}$. In comparison, plasmid DNA encoding for these components usually led to comparatively lower efficiencies or poorer tolerance in these cell types ${ }^{146}$. Also of note, electroporation-mediated co-delivery of RNPs and a single-stranded oligonucleotide DNA template (HDR template) with 90 nucleotide homology arms mediated up to 20\% knock-in in primary human T cells ${ }^{144}$, obviating the need to express DNA template from plasmids or viral vectors.

\subsubsection{Electroporation for Genome Editing of Stem Cells}

iPSCs, HSCs and embryonic stem cells hold potential for regenerative medicine as a source of autologous cells and tissues for patients. By introducing genome-editing molecules through intracellular delivery, stem cells can be prepared for gene therapy (Figure 29E). Using nucleofection, Kim et al. were among the first to determine the advantages of RNP delivery versus plasmid transfection by observing higher site-specific editing rates with reduced off-target mutations in stem cells $^{143}$. They reported that RNP delivery is less stressful to human embryonic stem cells, producing at least two-fold more colonies than plasmid transfection strategies ${ }^{143}$. In keeping with this notion, recent CRISPR protocols for implementation in human stem cells and primary cells indicate a preference for Nucleofection of Cas9-sgRNA RNPs over plasmids ${ }^{287}$. 
Furthermore, Neon capillary-based electroporation was used to introduce CRISPR-Cas9 nucleases via plasmids and/or RNPs to correct disease-causing mutations in patient-derived iPSCs ${ }^{45}$. This strategy mediated functional correction of large factor VIII gene chromosomal inversions in patient cells, a mutation that underlies hemophilia A. Endothelial cells derived from these iPSCs were competent in rescuing factor VIII deficiency in an otherwise lethal mouse phenotype of hemophilia. Thus, direct intracellular delivery of genome editing molecules takes us closer to the long-standing goal of exploiting patient-derived autologously sourced iPSCs for therapeutic gene editing before re-implantation ${ }^{41}$.

\subsubsection{Electroporation Summary}

Electroporation can deliver a vast range of molecular cargo into a wide variety of cell types with precise temporal control. With conventional electroporation the pulse parameters (field strength, pulse duration, pulse number, frequency) are flexible, therefore the same piece of hardware can be programmed to address a large number of scenarios. Parameters can be manipulated to focus the membrane-perturbing effects on different regions of the cell, such as certain parts of the plasma membrane or membranes of intracellular organelles (Figure 23). Additionally, the dual mechanisms of pore formation and electrophoretic propulsion of cargo may be beneficial for delivery of charged cargos, such as plasmid DNA or mRNA (Figure 24). Fundamentally, it is not well understood how cell structure, cytoskeleton, membrane proteins, domain phases, and membrane reservoirs influence electroporation in live cells, making it difficult to decipher critical molecular events. Additionally, the intrinsic pore-formation mechanisms bias electroporation toward the formation of numerous small pores, somewhat limiting the delivery of large cargoes.

Electroporation has a number of challenges, especially post-treatment cell death. Indeed, the window for effective treatment can be quite narrow, especially in primary cells. Detrimental effects of electroporation can be attributed to electrochemical phenomena at the electrodes including Joule heating, $\mathrm{pH}$ waves, bubble formation, corrosion, and contamination of the solution. Other potential issues include electric field-based perturbation of native proteins, scrambling of lipid membranes, generation of ROS, and damage to cargo molecules. Technical innovations featuring different electrode designs or microfluidic and nanochannel designs have been developed to overcome some of these 
issues (Figure 28), but they have not yet superseded the basic cuvette-style electroporation, which remains the most widely used platform for common use.

The challenges of current electroporation techniques notwithstanding, for many applications the benefits outweigh the weaknesses. Consequently, it has become the most widely used membrane disruption-mediated intracellular delivery approach. Electroporation has shown promise for treatment of a wide variety of patient derived cells and stem cells, with even the most basic electroporation platforms finding use among in vitro and ex vivo medical and industrial applications, from biomanufacture and clinical trials of cancer immunotherapy to ex vivo cell-based gene therapy and regenerative medicine.

\subsection{Thermal Membrane Disruption}

Membrane formation, dynamics, and properties are temperature-dependent. At sufficiently high temperatures, lipid bilayers will dissociate due to kinetic energy of the constituent molecules being greater than the forces that maintain the membrane formation, namely the hydrophobic forces that repel water from the lipid tails. The thermodynamic considerations of lipid bilayer behaviour dictate that temperature is key in determining the energy required for a given membrane disruption event. They key role of temperature has been emphasized in the electroporation literature, for example, where theory posits that electric potential differences across membranes can tilt the energy landscape of stochastic thermally-driven defect formation ${ }^{419}$. The implications of temperature must be fully considered in any membrane disruption event. This applies both to the physical properties of lipid membranes and the active response of the cell (see section 4.3).

Membrane permeability is known to increase during thermal phase transitions ${ }^{73,1047,1301}$. Both magnitude and rate of temperature changes influence the molecular rearrangements in membrane domains that are linked to the stochastic formation of defects ${ }^{1302}$. Close to phase transitions, ion channel-like events are known to occur, even in the complete absence of proteins ${ }^{1301,1303,1304}$. The occurrence of purely lipid ion channels depends on temperature, hydrostatic pressure, lateral pressure, voltage, $\mathrm{pH}$, and ion concentrations. Such pore formation is expected to be especially probable adjacent to domain interfaces and protein clusters. 
Strategies for permeabilizing cells by thermal means include: 1) cycling cells through a cooling-heating cycle, which may or may not involve freezing; 2) heating cells to supraphysiological temperatures, and 3) transient intense heating of a small part of the cell. The literature includes examples of each of these approaches, which will be discussed here in this section. Overall though, thermal methods of membrane perturbation have not been widely employed with animal cells, despite being universal and obvious. This can probably be attributed to challenges in spatiotemporal control of temperature exposure and concerns related to off-target damage. In future there exists an opportunity to address these challenges with emerging lab-on-chip, microfluidic, optical, and nanotechnological systems ${ }^{19,104-107}$.

\subsubsection{Thermal Shock of Competent Bacteria}

In bacteria, thermal shock has been used for decades to transfect "competent" bacteria with DNA plasmids. The method was described in early papers from the 1980s where agents such as divalent cations (typically in the form of $\mathrm{CaCl}_{2}$ ) and dimethyl sulfoxide (DMSO) were added to make E. Coli amenable or "competent" to DNA transfection. Subsequently, the bacteria undergo transient incubation at $0{ }^{\circ} \mathrm{C}$, a brief pulse to $37-42{ }^{\circ} \mathrm{C}$, and subsequent return to normal growth conditions where the genes of interest are expressed ${ }^{1305,1306}$. Multiple cycles are sometimes conducted to boost efficiency. Mechanistic studies suggest that phase transitions of membrane lipids cause damage to the outer membrane, and are necessary for DNA entry ${ }^{1307,1308}$. Some data indicates that cold shock may not need to go down as low as $0{ }^{\circ} \mathrm{C}$, as the rate and magnitude of temperature changes are more critical than specific temperature extremes ${ }^{1307}$. However, more recent reports claim that a brief freeze in liquid nitrogen for 20 seconds increases the efficiency of freeze-thaw transfection, even obviating the need for standard pre-treatment steps normally employed to make bacteria competent ${ }^{1309}$. Interestingly, microwave irradiation of frozen bacteria/DNA samples was also found to improve DNA transfection ${ }^{1309}$. Finally, microfluidic reactors have been employed for temperature shock transfection of bacteria ${ }^{1310}$. The advantages include fewer materials, smaller sample volume, and increased precision compared to conventional bulk procedures ${ }^{1310}$.

\subsubsection{Freeze-Thaw \& Other Temperature Cycling Strategies}

Apart from bacteria, rapid freeze-thaw procedures have also been demonstrated to facilitate exchange between intracellular and extracellular solutions when conducted with animal cell membranes (Figure 30A). In 1989 this was shown with synaptosomes, which are vesicular sacs reconstituted from synaptic terminal membranes by 
mild homogenization of nervous tissue ${ }^{1311}$. In the reported procedure rat brain synaptosomes were frozen and thawed in the presence of $5 \% \mathrm{DMSO}^{1311}$. Impermeant proteins, inhibitors and metabolites were successfully introduced to study neural signaling processes ${ }^{1311}$. An updated 'cryoloading' procedure was reported by Nath et al. where molecules of at least $150 \mathrm{kDa}$ were successfully delivered into chick synaptosomes ${ }^{1312}$. After recovery $\sim 80 \%$ of the synaptosomes were properly functional and capable of recycling synaptic vesicles ${ }^{1312}$.

Intracellular delivery by cooling-heating cycles has rarely been attempted in animal cells, probably due to the delicate and complex nature of cell recovery and growth from the frozen state. In one of the few cases where it was tested, trehalose $(\sim 0.34 \mathrm{kDa})$ was loaded into suspensions of adult islet cells by cooling them through their membrane phase transition ${ }^{73}$. Under conditions where cells were cooled at a rate of $1{ }^{\circ} \mathrm{C}$ per minute, permeability to trehalose was greatest around the region $0-5{ }^{\circ} \mathrm{C}^{73}$. Loaded trehalose exhibited cryoprotectant properties and was able to significantly increase cell survival and insulin production of islet cells. Building on this approach, Puhlev et al. compared intracellular delivery via cooling in suspension versus adherent fibroblasts. In their procedure cells were exposed to $50 \mathrm{mM}$ trehalose for 5 minutes on ice, followed by 10 minutes at $37{ }^{\circ} \mathrm{C}^{1035}$. As with the previous paper, maximal delivery was estimated to occur below $5{ }^{\circ} \mathrm{C}$ and was more efficient in suspended cells versus their adherent counterparts. A similar strategy was also tested by the Mehmet Toner lab ${ }^{75}$. Temperature cycling from 0 to $39^{\circ} \mathrm{C}$ was able to load trehalose into a target cell population of suspended rat hepatocytes without compromising cell viability ${ }^{75}$. Using an extracellular concentration of $0.4 \mathrm{M}$ in diluted culture medium, 1 hour of temperature oscillations conducted every 10 minutes produced an average cytoplasmic concentration of $0.13 \mathrm{M}\left(\sim 3 \%\right.$ of extracellular concentration) as detected by high-performance liquid chromatography ${ }^{75}$. Extended periods of incubation at $39{ }^{\circ} \mathrm{C}$ increased loading efficiency but came with the caveat of harming cell survival. 
A

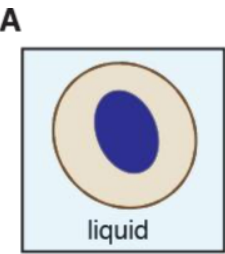

$\uparrow \Delta \mathrm{T}, \Delta \mathrm{V}$

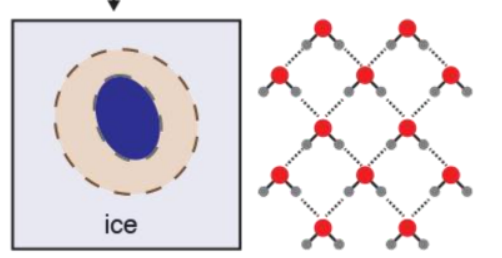

Freeze-thaw

D

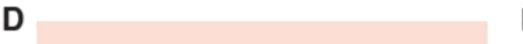

E

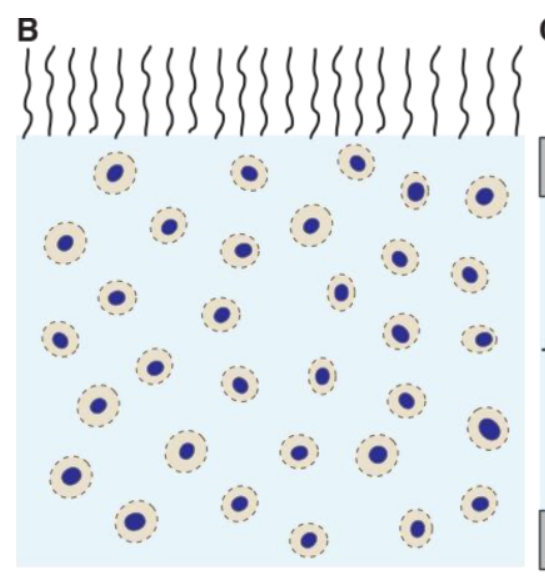

Bulk heating

E

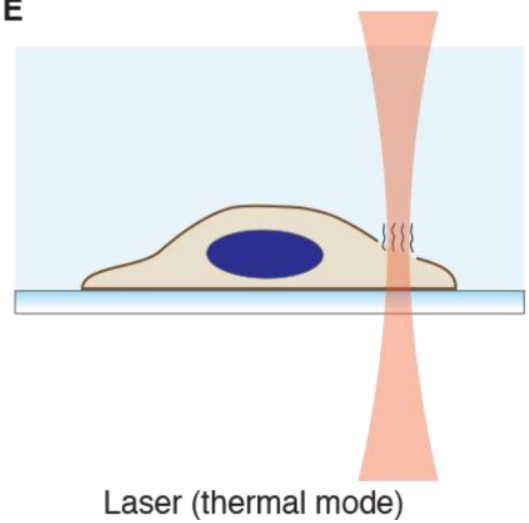

Laser (thermal mode)
C

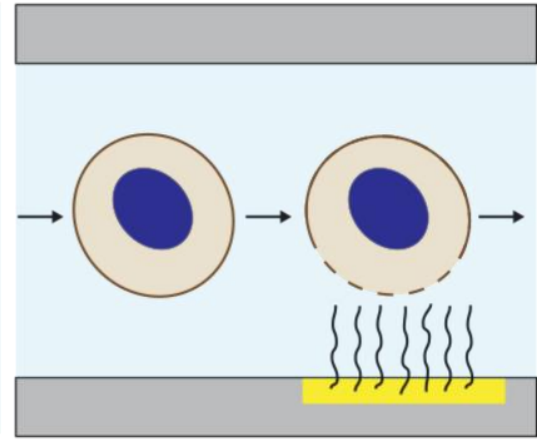

Localized microfluidic heating

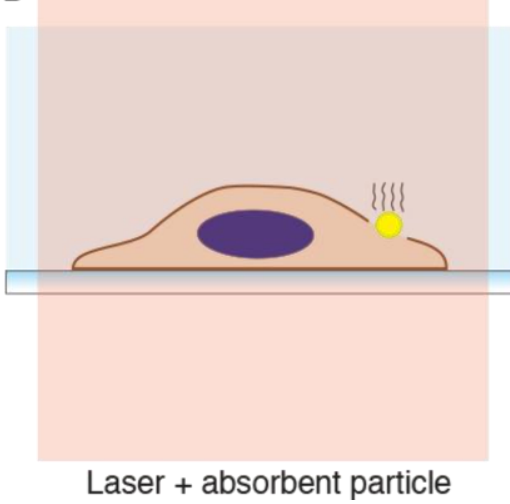

Figure 30. Thermal membrane disruption. (A) Membrane disruption by freeze-thaw cycles. Formation of ice crystals leads to volume expansion due to the changes in hydrogen bonding arrangement. Volume expansions are thought to be related to cracking of membranes during ice crystal formation. (B) Heating of cells above $42{ }^{\circ} \mathrm{C}$ increases the chances of spontaneous defect formation in membranes. (C) Microfluidic geometries may be used to confine the heating locally to a part of the cell, such as is possibly the case for thermal inkjet printing. (D) Absorbent nanoparticles may be used to locally convert laser power into local heating for membrane perturbation. (E) A focused laser can generate local heating at the membrane with selection of appropriate parameters.

\subsubsection{Supraphysiological Heating}

As temperature moves above $37{ }^{\circ} \mathrm{C}$, the probability of membrane defects arising increases. In experiments on mammalian cells, Bischof et al. exposed fibroblasts and muscle cells to temperatures ranging from 37 to $70{ }^{\circ} \mathrm{C}$ and monitored membrane integrity in real time. Permeability was assessed by tracking the leakage of calcein $(0.62 \mathrm{kDa})$ with timelapse fluorescence microscopy. Slow leakage, which starts above $40{ }^{\circ} \mathrm{C}$, was found to be a function of both temperature and time. Cells held at $45{ }^{\circ} \mathrm{C}$ were completely depleted of calcein within 25 minutes. This corroborates well with other data indicating cells must work harder to maintain their relatively high potassium concentrations during treatments at $43{ }^{\circ} \mathrm{C}^{1313}$. In Bishof et al.'s experiment, leakage takes slightly less than 10 minutes at $50{ }^{\circ} \mathrm{C}$. Above $55{ }^{\circ} \mathrm{C}$, almost $50 \%$ of calcein leaks out of the cell within one minute and efflux is fully complete by 2 minutes. To explain the increase in permeability, contributions from both protein denaturation and increased kinetic diffusion of lipid molecules were suggested. Other 
studies in red blood cells indicate that thermally-induced membrane disruption occurs at about $60{ }^{\circ} \mathrm{C}$ and protein denaturation temperature depends on the specific protein ${ }^{1314,1315}$. Interestingly, addition of poloxamers, which are both membrane-healing and anti-oxidant ${ }^{459}$, are able to rescue viability of thermally challenged cells ${ }^{1316}$. This indicates that loss of membrane integrity is a key aspect of immediate cell toxicity upon heating ${ }^{1316}$. For intracellular delivery purposes, supraphysiological temperatures have rarely been employed (Figure 30B), probably due to concerns of non-specific cell damage and toxicity as exemplified by the trehalose experiments discussed above ${ }^{75}$. Baseline temperature is a critical parameter for any delivery protocol, however, and there have been a few rare reports of supraphysiological regimes. For example, $43{ }^{\circ} \mathrm{C}$ was found employed in one study to make cell membranes more susceptible to fluid shear from laserinduced stress waves $^{1317}$.

\subsubsection{Thermal Inkjet Printers}

Thermal inkjet printers that disperse small volumes of fluid have been successfully deployed for mammalian cell gene transfection and intracellular delivery ${ }^{1318,1319}$. By replacing standard ink with media and cells, these printers not only perform intracellular delivery but can additionally pattern cells over a substrate. In thermal inkjet printers, a metal plate is heated at one side of the nozzle, which creates small air bubbles that collapse to provide pressure pulses to eject tiny drops of fluid. Over several microseconds the plate temperature may transiently rise to $300{ }^{\circ} \mathrm{C}$. It is not known whether membrane permeabilization is obtained by fluid shear forces or transient thermal disruption at the nozzle. In the studies performed so far Xu et al. achieved transfection efficiencies of 10\% with GFP plasmids in porcine aortic endothelial cells at $90 \%$ cell viability ${ }^{1318}$ while Cue and Boland obtained above $30 \%$ transfection efficiency in CHO cells with similar viabilities ${ }^{1319}$. Further mechanistic insights may improve the efficiency of this approach. A potential bonus of thermal inkjet printing is the ability to array cells into specific geometries and perform intracellular delivery in a single step, thereby facilitating the possibility of in vitro tissue engineering ${ }^{1320}$. The results with thermal inkjet printers point to an opportunity for future studies with microfabricated devices, where it should be possible to gain spatiotemporal control over temperature exposure through microfluidics (Figure 30C). 


\subsubsection{Laser-Particle Interactions}

As discussed in the sections on fluid shear, laser irradiance of an absorbent object in an aqueous environment can produce a variety of effects including cavitation, plasma production, chemical reactions, and heat ${ }^{1321-1323}$. Although it is sometimes difficult to be sure of the mechanisms, we report here on studies that claim to disrupt membranes by laser-mediating temperature changes. In most of cases nanoparticles are used as nucleation sites for intense local heating (Figure 30D). Umebayashi et al. showed that laser irradiation of unbound latex particles dispersed in solution leads to the uptake of impermeant dye molecules ${ }^{1324}$. The mechanism was proposed to be through thermal perturbation at the particle-membrane interface, pore formation, and subsequent diffusive influx of extracellular molecules ${ }^{1324}$. A similar thermal delivery concept was shown by Yao et al. with selectively bound antibody-conjugated gold nanoparticles, featuring a strong correlation between nanoparticle size and heating intensity ${ }^{1325}$. Follow up studies investigated the effects of laser pulsing parameters (pulse duration, exposure intensity, and irradiation mode) and found conditions where more than $50 \%$ of the treated suspension cells could take up a labeled $150-\mathrm{kDa}$ IgG antibody ${ }^{1326}$. In other studies, cancer cells were targeted by folate-conjugated gold nanorods. Under femtosecond laser irradiation the nanorods were shown to thermally disrupt the membranes as evidenced by flux of dye molecules across the plasma membrane ${ }^{1327}$. Gu et al. reported using low power continuous wave near-infrared (NIR) lasers to thermally excite inert crystalline magnetic carbon nanoparticles for delivery of impermeable dyes and plasmids ${ }^{1328}$. Gold nanoparticles have also been packed into a dense surface layer where $>10$ seconds of infrared laser irradiation heats the underside of cells to trigger permeabilization and delivery of dyes, dextrans and plasmids ${ }^{1329}$.

\subsubsection{Lasers-Membrane Interactions}

In the absence of absorbing structures, lasers alone can be harnessed for local heating of cell membranes within the focal region (Figure 30E). The mechanisms of laser interaction with lipid membranes are complex, usually being underpinned by mixture of thermal, chemical and mechanical components ${ }^{1321-1323}$. Hence, only under a narrow range of conditions are lasers thought to produce purely thermal membrane disruption. One example was published by Palumbo et al. where 0.25 seconds exposure to a $488 \mathrm{~nm}$ continuous wave argon laser of spot size 5-8 $\mu \mathrm{m}$ was focused onto the cell surface ${ }^{1330}$. Their 
report indicated that the poration mechanism was via heating, however other effects cannot be ruled out. More information on laser optoporation is presented the next section of this review.

\subsubsection{Summary of Thermal Membrane Disruption}

Baseline temperature is a basic consideration in any intracellular delivery technique. Moreover, fast temperature fluctuations within, and deviations outside, the physiological temperature range can result in thermally-driven membrane defects. Transfection assisted by thermal membrane disruption has been harnessed in bacteria for decades. In metazoan cells, strategies to control the spatiotemporal control of temperature exposures may yield fruit, as evidenced by encouraging reports with microfluidics, thermal inkjet printing, and laser-particle interactions.

\subsection{Optical Membrane Disruption (Optoporation)}

A wide variety of laser procedures have been implemented to selectively perform nanosurgery on cells and their components $^{1331}$. Targets include individual chromosomes, organelles, mitochondria, cytoskeletal structures, and lipid membranes. Optoporation is the permeabilization of lipid membranes by high intensity light. In some studies it has also been referred to by terms such as photoporation, optoinjection, laserfection, and optical transfection ${ }^{486,1332,1333}$. The aim of optoporation is to permeabilize the plasma membrane to cargo while leaving other cellular structures intact, thus preserving the health of the cell to the maximum extent possible. In this review, we define optoporation as membrane disruption arising from direct interaction of a laser focal region with the plasma membrane, and not absorption of laser energy by an intermediate structure such as nanoparticle or metal surface. Those strategies permeabilize membranes by secondary effects such as fluid shear (section 6.1.2) and chemical effects (section 6.5.5), and are covered in the respective sections dealing with those phenomena.

\subsubsection{Optoporation - Pioneering Studies}

DNA transfection by laser optoration was first reported in $1984^{1334}$. Nanosecond pulses of an Nd:YAG UV laser (wavelength $355 \mathrm{~nm}$ ) at an energy of $1 \mathrm{~mJ}$ with spot size of $\sim 0.5 \mu \mathrm{m}$ were focused on the surface of adherent NRK cells. A single pulse of 5-10 ns was sufficient to open up a hole several microns wide and promote the influx of DNA plasmids 
from an extracellular concentration of $10 \mu \mathrm{g} \mathrm{ml}^{-1}$ before closure of the wound. When manually targeting the laser pulse above the nucleus, $10 \%$ transfection efficiency was achieved while random scanning of the laser over the substrate resulted in only $0.6 \%$ chance of success ${ }^{1335}$. Laser transfection with a similar laser but different cell types was repeated several years later, this time establishing that a small percentage of target cells stably integrated the plasmid into their genome ${ }^{1336}$. Addition of dyes to change absorption properties of the media is another variable that was examined, with the presence of standard cell culture media additive phenol red shown to decrease the laser power needed for optoporation ${ }^{1330}$. A $488 \mathrm{~nm}$ continuous wave argon laser with nominal power of $2 \mathrm{~W}$ and spot size of 5-8 $\mu \mathrm{m}$ was focused onto the surface of NIH 3 T3 fibroblasts with an exposure time of 0.25 seconds to puncture the plasma membrane ${ }^{1330}$. After conducting the procedure in the presence of $10 \mu \mathrm{g} \mathrm{ml}^{-1}$ plasmid DNA, repair of a single large hole in the membrane took 1-2 minutes, followed by detectable gene expression after 2 hours ${ }^{1330}$. Plasma membrane disruption mechanisms were reported to be thermal and laser exposures of greater than 0.5 seconds were found to permanently damage cells ${ }^{1330}$.

The next major breakthrough in optoporation occurred in 2002, with the implementation of femtosecond-pulsed lasers ${ }^{1337}$. Tirlapur and König used a high-intensity, near-infrared (wavelength $800 \mathrm{~nm}$ ), femtosecond-pulsed laser beam from an 80 MHz titanium-sapphire laser, with a mean power of 50-100 mW. The laser was tightly focused to a sub-femtolitre focal volume just above the cell membrane. Under $16 \mathrm{~ms}$ exposure time, $\mathrm{CHO}$ and Ptk2 cells were transfected with GFP using only $0.4 \mu \mathrm{g} \mathrm{ml}{ }^{-1}$ of DNA plasmid in solution. Unprecedented high transfection efficiency and viability were reported, with both coming in at close to $100 \%$. A prime limitation of the procedure, however, was the need to manually refocus on each cell, yielding a throughput of only a few cells per minute. Since this landmark report 1) femtosecond lasers gained prominence as the most effective pulsing strategy for optoporation, and 2) the number of optoporation publications has increased dramatically. In terms of cargo delivery, the field has placed particular focus on delivery of small molecule dyes for mechanistic studies and DNA transfection to demonstrate applications. Indeed, laser optoporation have achieved successful delivery of plasmid DNA ${ }^{481,483,484,1330,1332-1358}$, mRNA $^{252,1353}$, siRNA ${ }^{1333,1343,1352}$, antisense morpholinos ${ }^{1353}$, $\operatorname{peptides}^{486,1359}$, proteins ${ }^{1333,1343}$, dextrans ${ }^{1333,1343,1349,1353,1360,1361}$, dyes ${ }^{252,480-484,486,1333,1340,1341,1343,1345,1346,1348,1354,1357,1361-1366}$, sucrose $^{485}$, molecular beacons ${ }^{1367}$, ions ${ }^{1333,1343,1368}$, semiconductor nanocrystals ${ }^{1333,1343}$, gold nanoparticles ${ }^{1369}$, quantum $\operatorname{dots}^{1370}$, and $\sim 1 \mu \mathrm{m}$ polystyrene beads ${ }^{1371}$. Moreover, many of these studies have sought to compare the mechanisms of various laser treatment regimes in order to optimize delivery efficiency and minimize off-target damage. 


\subsubsection{Mechanisms of Optoporation}

The mechanisms of laser-mediated membrane disruption are complex, involving combinations of mechanical, thermal, and chemical effects. Possibilities include burning/evaporation, thermoelastic mechanical stress, generation of low-density free-electron plasma and reactive oxygen specifies (ROS), and effects beyond the focal region, such as shock wave emission and growth/collapse of cavitation bubbles, which themselves produce fluid shear stress, extreme heat, and sonochemical phenomena ${ }^{1321-1323,1331}$ (Figure 31). The relative dominance of these phenomena depends on factors such as wavelength, frequency, whether the source is continuous wave or pulsed, laser power, exposure time, spot size, and absorbance properties of focal region. For example, membrane wounding from continuous wave irradiation is thought to arise primarily from local heating, which intensifies as a function of exposure time. Nanosecond pulsed lasers have been suggested to produce a combination of heating, bubble formation, and thermoelastic or di-electric mechanical stresses to damage the membrane. Femtosecond laser mechanisms appear tunable based on irradiance strength, pulse duration, and frequency. Mechanisms range from almost purely chemical degradation to combinations of thermal and mechanical. In cases where laser energy is transduced into fluid shear that travels far beyond the focal region, such as cavitation or shock waves, the mechanisms of membrane damage are not strictly optoporation and these scenarios are covered elsewhere in the section on fluid shear (section 6.1.2). Alternatively, if transmission of thermal energy from an absorbing object in immediate contact is the mechanism of membrane disruption, these accounts are covered in the thermal section (section 6.3).

\subsubsection{Femtosecond Optoporation}

Most recent work favors the use of a laser regime characterized by wavelengths $>700 \mathrm{~nm}$ administered at high frequencies ( $\sim \mathrm{MHz}$ range) and femtosecond pulse timings with a cumulative exposure of milliseconds or less ${ }^{1321}$. For example, a typical protocol might involve $5 \mathrm{~ms}$ of exposure to a cycle of $100 \mathrm{fs}$ pulses with gaps of $10 \mathrm{~ns}(\sim 100 \mathrm{MHz}$ frequency) for cooldown. When operating at wavelengths $>700 \mathrm{~nm}$ the mechanisms are related to multi-photon effects inherently concentrated within the focal region, thus offering increased precision and high spatial resolution ${ }^{1321}$. NIR and IR wavelengths also have the advantage of being less toxic to cells, as UV and blue light in particular are notorious for causing damage to DNA and other cellular structures. By using extremely short femtosecond pulses, absorbing material in focal region does not have sufficient time to transmit heat to adjacent regions. This enables extremely high-powered lasers 
to be deployed while avoiding excessive heating of cells. In such a scenario the resultant membrane disruption mechanisms have been reported to be due to chemical effects, such as the breakdown of bonds in lipid tails by low-energy plasma $^{1321-1323,1331}$ (Figure 31C). In other cases, femtosecond pulsing generates a well-controlled cavitation bubble originating within the focal region, the presence of which can destroy the membrane (Figure 31B). In many of these studies, distinctions between exact mechanisms are difficult to determine, and could be multifactorial.

A

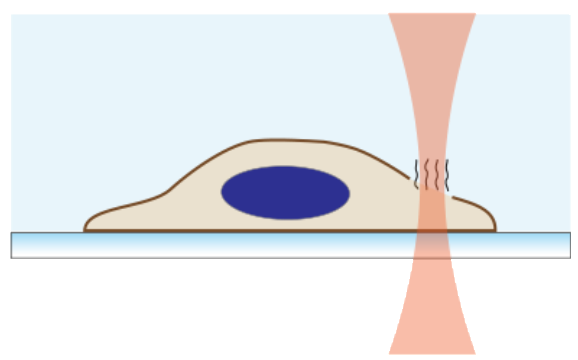

Laser (thermal)

C

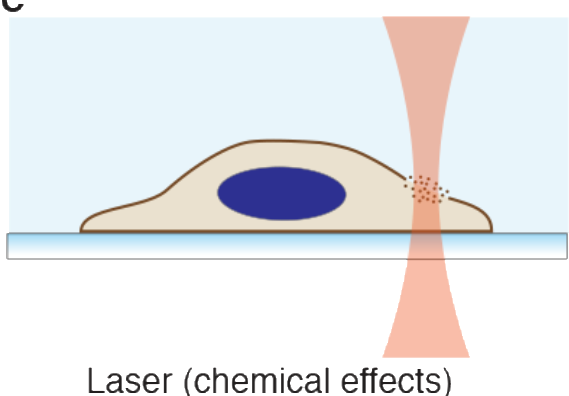

B

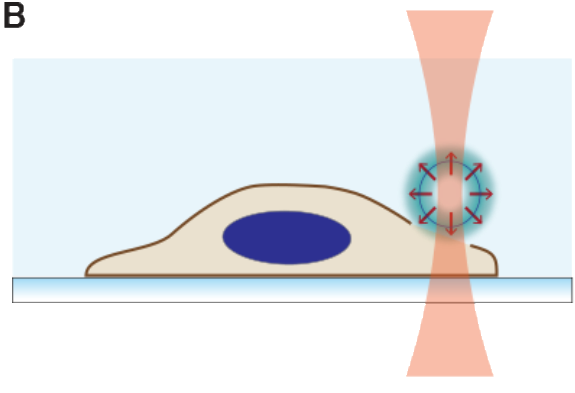

Laser (cavitation bubble)

D

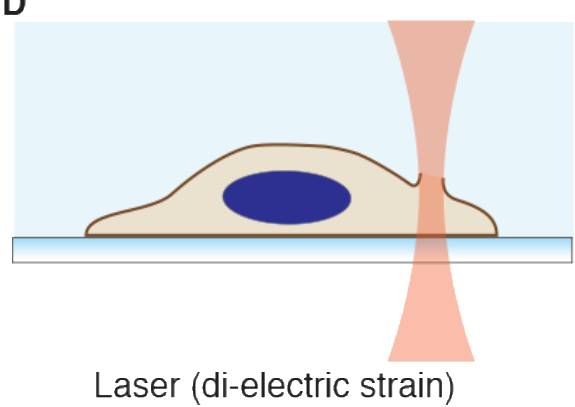

Figure 31 | Optoporation strategies for membrane disruption. Focused laser can inflict (A) thermal, (B) cavitation, (C) chemical, or (D) mechanical effects (such as di-electric strain) against lipid bilayers.

A number of elegant studies have been performed with femtosecond pulsed lasers. For example, in optical setups that combine laser tweezing and optoporation, optical tweezers may be used to guide a microbead $(\sim 1 \mu \mathrm{m})$ or nanoparticle through a hole formed by the laser, thus delivering large cargo ${ }^{1369,1371}$. In studies with frog embryos quantum dots were delivered by NIR femtosecond lasers. Neither the quantum dots nor optoporation retarded the ability of these embryos to grow into tadpoles. In another case, cargo was introduced into distinct regions of adherent primary rat neurons to assess localization-dependent biological functions ${ }^{252}$. mRNA-mediated expression of the transcription factor Elk-1 was found to produce different responses whether delivered to the soma or axon of the neurons ${ }^{252}$. This optoporation protocol involved an $840 \mathrm{~nm}$ titanium-sapphire laser delivering $100 \mathrm{fs}$ pulses at a repetition rate of $80 \mathrm{MHz}$ for $1-5 \mathrm{~ms}$ at a power of 30 $\mathrm{mW}^{252}$. Other studies have quantitatively measured the loading efficiency of femtosecond optoporation, and found that targeted cells can incorporate up to $40 \%$ of the concentration of extracellular molecules before resealing ${ }^{483}$. Furthermore, 
sub-20 femtosecond pulses at $\mathrm{MHz}$ frequencies with sub-millisecond exposure times have been demonstrated for the effective transfection of human primary pancreatic and salivary gland stem cells ${ }^{1348}$.

\subsubsection{Towards High Throughput \& More User-Friendly Optoporation}

A major rate-limiting step for optoporation is the reliance on precise positioning of the laser focal spot and alignment with target membranes ${ }^{1322,1337}$. A misfocus of as little as $3 \mu \mathrm{m}$ results in greater than 50 percent reduction in membrane disruption efficiency ${ }^{1344}$. One strategy to mitigate this limitation is the implementation of a "bessel beam", where the focal region is stretched into a rod of light over $100 \mu \mathrm{m}$ in length and a few microns wide ${ }^{1344}$. Bessel beam setups have been combined with microfluidics for hydrodynamic flow focusing to reach throughputs of tens of cells per second ${ }^{1365}$. However, cell viability and delivery efficiency were substantially less than standard femtosecond optoporation ${ }^{1365}$. Whether or not bessel beams cause off-target damage to non-membranous cellular structures is unknown ${ }^{1321}$.

Other attempts to increase throughput of optoporation include a user-friendly "point and click" touchscreen softwareintegrated approach ${ }^{1355}$. With this system throughputs of up to 100 cells per minute were obtained on adherent neurons ${ }^{1355}$. An extension of this strategy relies on automated image analysis of cell morphology, centering of the microscope stage to the laser focus, and execution of a femtosecond laser illumination protocol ${ }^{1357}$. With this system, software-controlled meandering of the sample stage allows adherent cells in a typical cell culture dish to be automatically targeted at a rate around 10,000 cells per hour ${ }^{1357}$. If optoporation is to be adopted by users outside of specialized labs, further efforts will need to address the challenge of how to precisely focus the laser spot onto thousands of cells for rapid treatment. Other issues that need to be addressed are portability, instrument complexity, and high cost.

\subsubsection{Summary of Optoporation}

Optoporation has captured a significant amount of attention over the last several decades, particularly for transfection.

One of the main problems is the high cost of lasers and the optical systems required to harness them, as well as poor adoption outside expert communities. A second main problem is how to increase the throughput of treatments, which is an area where microfluidics and computer automation have made positive contributions. Future progress in optoporation will need to develop creative ideas to move beyond traditional limitations. 


\subsection{Biochemical Membrane Disruption}

A range of chemical effects and biochemical agents can be used to disrupt cell membranes. These include synthetic detergents, surface-active agents (surfactants), organic solvents, and oxidizing agents to naturally secreted proteins and metabolites from a diversity of organisms. For example, organic solvents have been used for decades as penetration enhancers for transdermal delivery by fluidizing, destabilizing, or extracting components from lipid bilayers ${ }^{1372}$. Since the dawn of life, living organisms have evolved a range of potent molecules to attack and disrupt the membrane integrity of competing lifeforms. Pore-forming proteins (PFPs), which are produced by humans, animals, plants, fungi, protists, and bacteria for self-defense, are one such example ${ }^{399}$. Many plants synthesize and secrete metabolites like saponins to serve as an innate immune barrier to disrupt the membranes of invading microbes or other threatening organisms ${ }^{1373}$. These natural compounds tend to be relatively specific, relying on unique characteristics of the target membrane for their action, such as composition of membrane lipids and presence of external receptors. Several artificially produced detergents and solvents also exhibit a useful ability to disrupt plasma membranes in a relatively controlled manner. Furthermore, emerging concepts from nanotechnology, such as near-field ionizing plasmas, present opportunities to confine chemical destabilization phenomena to small membrane patches for short durations. This section will cover artificial and natural biochemical permeabilization strategies that hold demonstrated or theoretical potential for intracellular delivery applications.

\subsubsection{Organic Solvents \& Penetration Enhancers}

\subsubsection{DMSO}

Organic solvents are low-molecular weight compounds that can perturb bilayer structures by burying their hydrophobic residues into the membrane. A classic example of a membrane-active organic solvent is dimethyl sulfoxide (DMSO), often used as a penetration enhancer to increase the permeability of drugs and other small molecules ${ }^{54,1374}$. DMSO is amphiphilic, containing one hydrophilic sulfoxide group and two hydrophobic methyl groups. It is known to promote permeation of both hydrophilic ${ }^{54}$ and hydrophobic ${ }^{1374}$ species across lipid bilayers. DMSO's penetration enhancing effect can be attributed to two mechanisms. First, its ability to increase the solubility of small molecules, and second, because of 
increased incidence of membrane defects that allow passage of normally-impermeant molecules. Experiments with phospholipid vesicles have found leakage of carboxyfluorescein $(\sim 376 \mathrm{Da})$ at concentrations of DMSO $>10 \%{ }^{1375}$. For a given DMSO concentration, leakage also increases as a function of temperature ${ }^{1375}$.

Experiments have been used to understand the mechanisms by which DMSO increases lipid bilayer permeability. They have found that DMSO incorporation into the bilayer increases the distance between polar lipid headgroups and reduces membrane thickness, thereby weakening the membrane ${ }^{1374}$. This has been experimentally verified by the use of X-ray diffraction techniques ${ }^{1376}$. Second, the association of DMSO with solute molecules lowers the activation energy needed for them to enter into, and ultimately cross, the bilayer ${ }^{1374}$. This interpretation is supported by vibrational spectroscopic studies $^{1377}$.

In addition to physical measurements, simulations have been used to visualize the molecular events associated with membane disruption by $\mathrm{DMSO}^{410}$. Gurtovenko et al. observed that at low concentrations, DMSO causes membrane thinning and increases fluidity of the membrane's hydrophobic core ${ }^{1378}$. In agreement with experimental data, DMSO molecules are seen to penetrate into the bilayer, both expanding the distance between the lipids and reducing the thickness of the bilayer (Figure 32A). Consequently, the lipid-water interface becomes more prone to structural defects, especially due to thermal fluctuations. At higher DMSO concentrations, water molecules enter the membrane interior via DMSOmediated structural defects. As the number of penetrating water molecules increases, a significant re-orientation of lipid headgroups toward the membrane interior is required to minimize the free-energy of the system, resulting in the formation of hydrophilic channels spanning the membrane bilayer ${ }^{410}$. The emergence of hydrophilic channels occurs spontaneously between 10-20\% molar concentration of $\mathrm{DMSO}^{1378}$. The addition of sterols (i.e. cholesterol) can provide stabilization to the membrane and thus increase the DMSO concentration required for pore formation ${ }^{1379}$.

\subsubsection{Ethanol \& Other Alcohols}

In contrast to DMSO, ethanol's hydrophobicity is rather limited as a short-chain alcohol. Rather than embed deep, ethanol molecules tend to remain at the water-lipid interface forming hydrogen bonds with hydrophilic lipid headgroups ${ }^{1380,1381}$. Ethanol has a disordering effect on lipid hydrocarbon tails, increasing fluidity of the membrane and reducing rigidity. 
Simulations confirm that compromising the water-lipid interface induces ingression of water pockets into the membrane as inverse micelles, rather than pores that span the whole membrane ${ }^{1382}$ (Figure 32B). The bilayer structure is partly destroyed due to lipid desorption ${ }^{1382}$. Both experimental and simulation studies have shown that the bilayer structure cannot be maintained beyond an ethanol concentration around $12 \%$ molar or $30 \% \mathrm{v} / \mathrm{v}$ concentration. Correspondingly stronger results can be expected with longer chain alcohols, such as propanol, butanol, pentanol, as the concentration required for defect formation is inversely proportional to hydrocarbon chain length ${ }^{1383}$. As an example, significant membrane defects have been reported in membranes exposed to only $1 \%$ butanol $^{1384}$.

One case where ethanol was used for intracellular delivery purposes was reported recently by O'Dea et al ${ }^{1385}$. Reversible cell permeabilization was achieved by temporally and volumetrically controlling the contact of the target cells with a hypotonic solution of $75 \% \mathrm{H}_{2} \mathrm{O}, 25 \%$ ethanol, $32 \mathrm{mM}$ sucrose, $12 \mathrm{mM}$ potassium chloride, $12 \mathrm{mM}$ ammonium acetate and $5 \mathrm{mM}$ HEPES. An atomizer was employed to spray a small volume of this solution to form a thin film over a monolayer of cells. After 2 minutes, permeabilization was terminated with a neutralizing solution. Using this protocol intracellular delivery of proteins, mRNA, and plasmids was reported ${ }^{1385}$. The observation that ethanol permeabilization is optimal at $25 \% \mathrm{v} / \mathrm{v}$ fits well with the results from simulations that predict the effects of ethanol should be reversible at concentrations $<30 \% \mathrm{v} / \mathrm{v}^{1382}$.
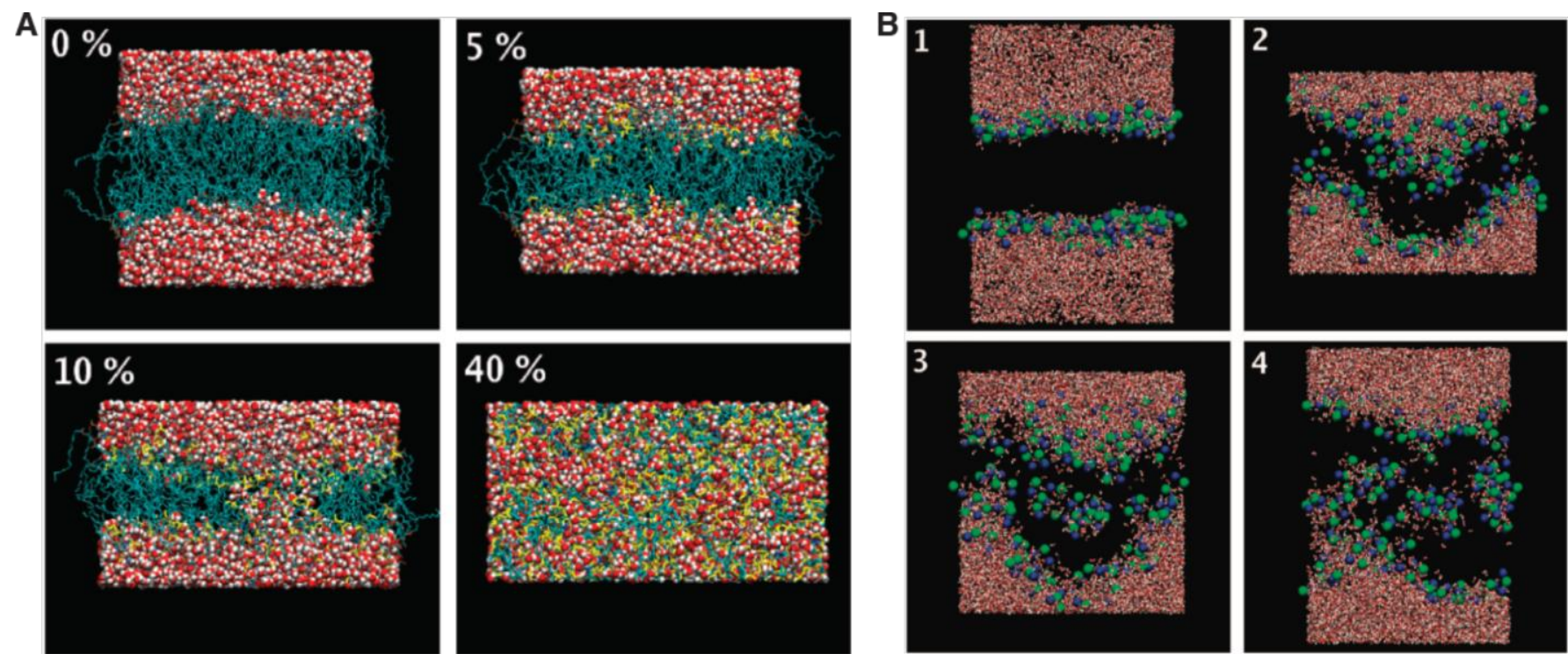

Figure 32. Simulations of membrane bilayer perturbation with DMSO and Ethanol. (A) Presented are side views of the final structures for the bilayer systems containing $0,5,10$, and 40 mol\% of DMSO. Lipids are shown in cyan, water in red, and DMSO in yellow. Reproduced from ref ${ }^{1378}$. Copyright 2007 American Chemical Society. (B) Formation of non-bilayer structures within the membrane interior with 15 mol\% of ethanol: (1) 3100 ps; (2) 13,180 ps; (3) 19,920 ps; (4) 30,000 ps. Shown are water molecules (red 
and white) and phosphorus (green) and nitrogen (blue) atoms of lipid head groups. The rest of the lipid atoms as well as ethanol molecules are not shown. Reproduced from ref ${ }^{1382}$. Copyright 2009 American Chemical Society.

\subsubsection{Organic Solvents \& Penetration Enhancers Summary}

Although widely used for permeabilizing fixed cells ${ }^{1386}$ and increasing the permeability of small molecules ${ }^{54}$, organic solvents and other low molecular weight penetration enhancers have generally not been used as the sole membrane disruption agents to deliver cargo molecules. This is probably due to their non-specific nature and lack of spatiotemporal control over the membrane disruption process. They may be useful as non-specific and relatively inert adjuvants to modify other membrane permeabilization strategies such as electroporation ${ }^{1387-1389}$.

\subsubsection{Detergents}

Detergents are water-soluble surfactants capable of solubilizing phospholipids found in biological membranes. Solubilization refers to the dissolution of the bilayer structure by sequestration into detergent-lipid micelles ${ }^{441,442}$. For the purposes of intracellular delivery, complete solubilization of membranes is lethal and undesirable, thus detergents must be used at intermediate concentrations for limited durations to yield optimal levels of cell permeabilization. Although the mechanisms of detergent solubilization of biological membranes have been discussed for decades ${ }^{441,1390-1392}$, the milder intermediate regime of non-lethal permeabilization is less well understood. As well as intracellular delivery applications, motivations to investigate this regime include understanding the action of membrane-perturbing secondary metabolites and characterizing new candidates for antimicrobials.

\subsubsection{Membrane Disruption by Detergents That Flip Flop}

Owing to their amphiphilic properties, detergent molecules integrate into lipid membranes. Most detergents are coneshaped, in that the head group of the detergent is disproportionately larger than the hydrophobic chains. They generally work by inserting into lipid bilayers and distorting their structure. Several mechanisms have been suggested for detergentmediated permeabilization of lipid bilayers depending on the type of detergent ${ }^{441}$. Those capable of flip-flopping to the inner leaflet will distribute throughout both leaflets of the bilayer (Figure 33). Because of the cone-shaped nature of detergents, the structure of the monolayer wants to assume a degree of convex intrinsic curvature. However, this is 
impossible if the monolayer is part of a bilayer, because it competes with the opposite spontaneous curvature of the other leaflet since are coupled with each other. Instead, the monolayers are 'bent straight' by an elastic deformation giving rise to a monolayer curvature strain. The major structural consequence of this curvature strain is a disordering of the hydrophobic chains. In turn, the membrane becomes thinner and more flexible. Monolayer curvature strain can be partially relaxed by the sequestering of surfactants into highly curved rims covering the hydrophobic edges of toroidal pores $^{441}$. Over time, thermal fluctuations will give rise to such events. Moreover, reduction of the pore's line tension by detergents may massively increase the lifetimes of induced pores or even stabilize them indefinitely. Above a critical surfactant concentration, pores appear spontaneously so that permeabilization becomes effectively persistent ${ }^{441}$.

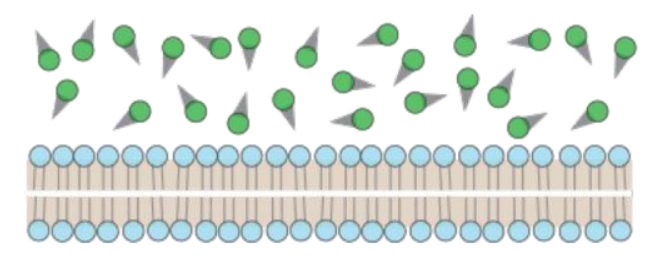

\section{detergents that flip flop}

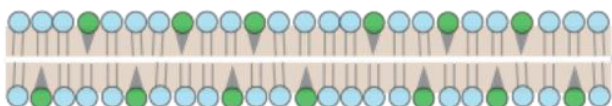

thinning, decreased packing

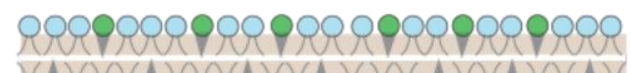

846006000600600060060060

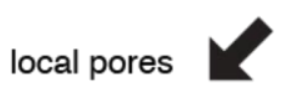

local budding
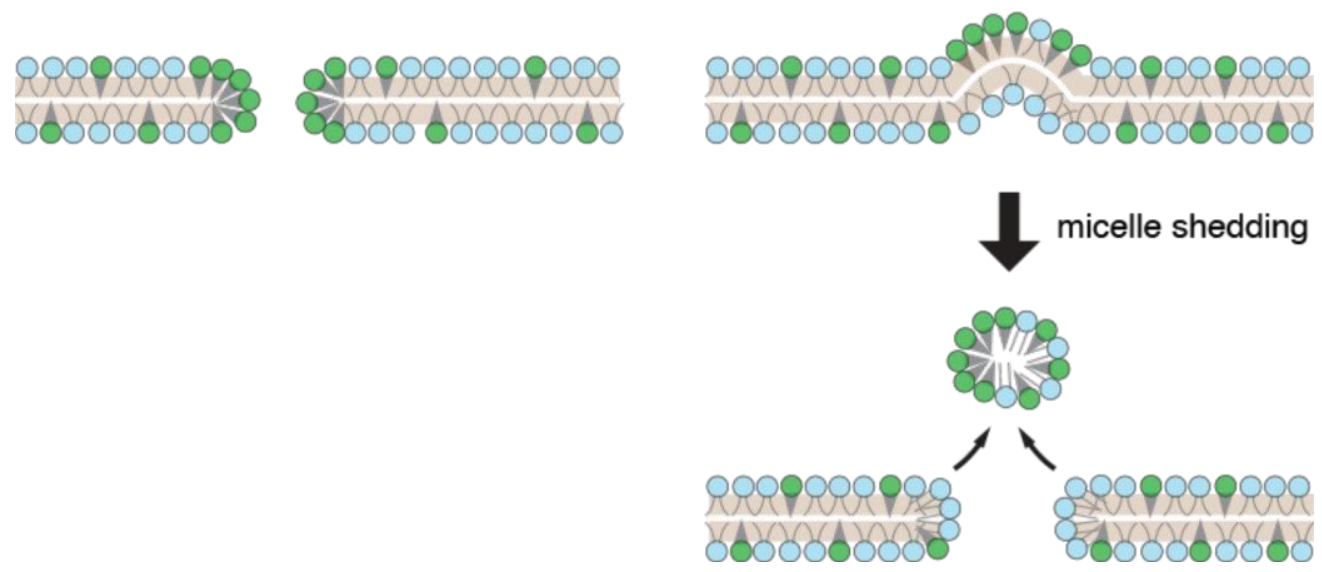

Figure 33. Proposed mechanisms of membrane permeabilization by detergents that flip flop. Integration of detergent monomers perturbs membrane integrity while stochastic local enrichment of detergents leads to formation of pores. 


\subsubsection{Membrane Disruption by Detergents That Do Not Flip Flop}

Detergents that embed into the outer leaflet but cannot flip flop expand the bilayer asymmetrically (Figure 34 ) ${ }^{441,442}$. If the bilayer is unable to bend to assume its spontaneous bilayer curvature, it develops a bilayer curvature strain by compressing the molecules in the overpopulated (outer) leaflet and/or expanding those in the underpopulated (inner) leaflet. Bilayer curvature eventually leads to mechanical failure of the membrane because the outer monolayer forms mixed micellar structures that bud off from the membrane. Shedding of these micelles into the aqueous solution results in emergence of defects and subsequent permeabilization ${ }^{442}$. These disruptions can have several effects. First, relaxation of the curvature strain allows the membrane leaflets to anneal, and second, they permit the passage of detergent molecules inside the cell to access the inner leaflet, thereby promoting further infiltration of the membrane by mechanisms akin to detergents that flip flop (Figure 33). 
detergents that don't flip flop

pop9o00900900009009000090

б000000000000000000000000

thinning, decreased packing

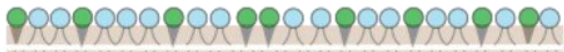

б000000000000000000000000

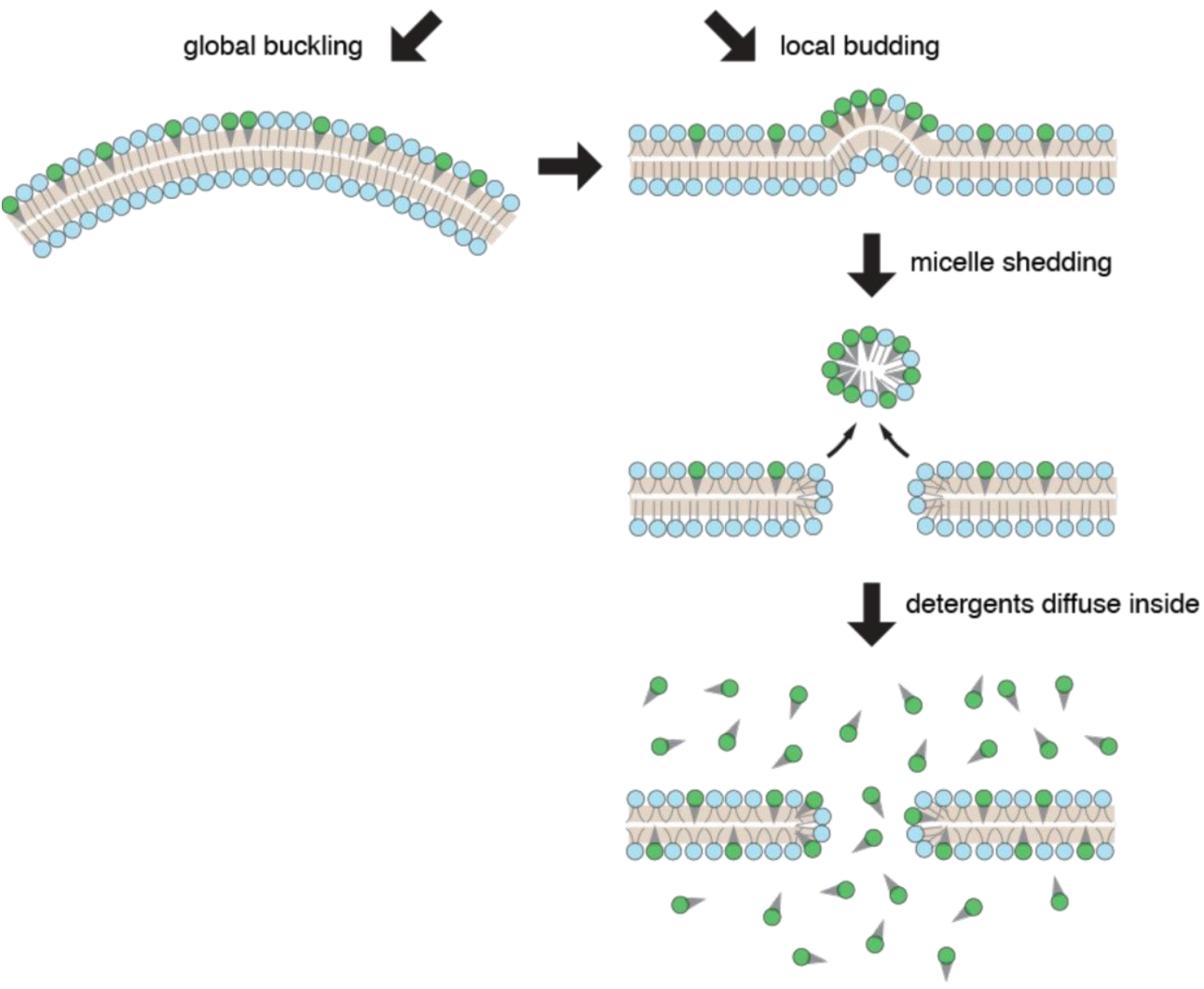

Figure 34. Proposed mechanisms of membrane permeabilization by detergents that do not flip flop. Once detergent monomers gain access to the interior side of the membrane, they can distribute to both leaflets and perturb the membrane by mechanisms similar to detergents that flip flop (see figure 33).

\subsubsection{Membrane Disruption by Detergents That Do Not Embed}

A third possibility is that collision of detergent micelles with the cell membranes recruits lipid units into the micelles, thereby generating defects in the membrane (Figure 35) 461,1393 . There is little theory to support this third possibility, however it should be mentioned as a possibility. In order to first achieve micelles, the detergent will need to be at 
concentrations above the critical micelle concentration (CMC). This will only be a realistic scenario in the case of detergents that don't readily embed into cell membranes. Thus, integration of individual detergent molecules into the target membrane may not be necessary to cause defect generation and subsequent permeabilization.
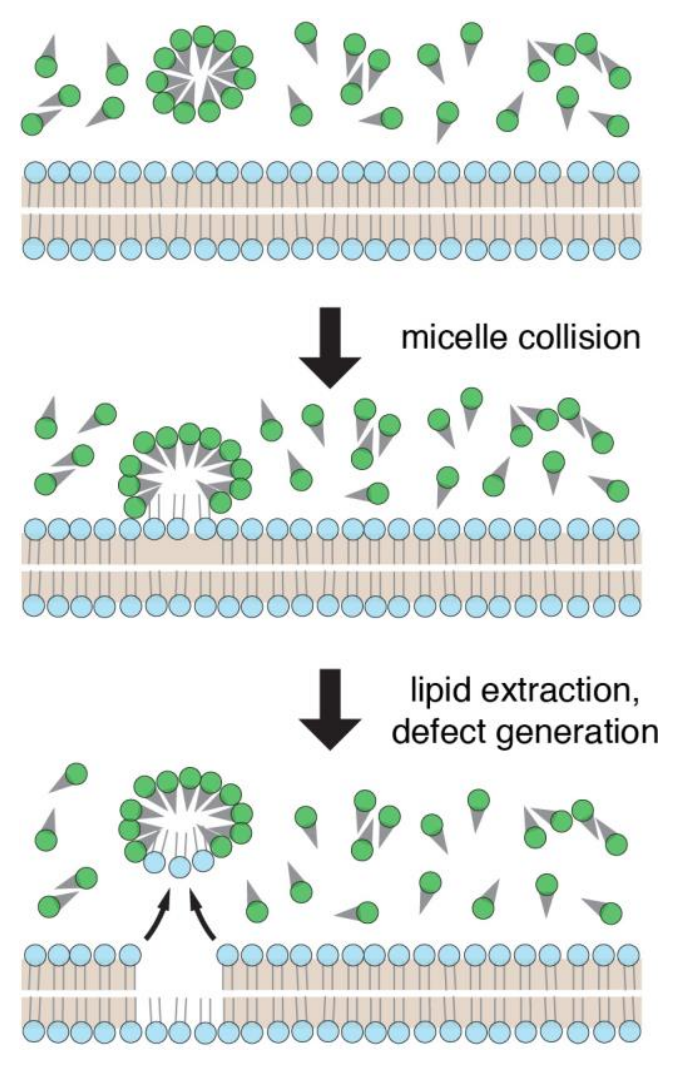

Figure 35. Proposed mechanisms of membrane permeabilization by detergent micelle collisions. Micelles colliding with the membrane may create defects by sequestering lipid molecules from the bilayer.

\subsubsection{Relationship Between Strain and Emergence of Defects}

Most of the detergents used to permeabilize biological membranes integrate into the bilayer ${ }^{441}$. Curvature-driven distortion and disordering of membranes leads to perturbation of the bilayer structure and subsequent permeabilization. As discussed, the key property of a micelle-forming amphiphile inserting into a lipid bilayer is its preference for a locally curved interface that is in conflict with the (on average) planar topology of a bilayer. Indeed, strongly curvature-inducing detergents are known to be far more effective in membrane permeabilization ${ }^{441}$. When local concentrations of detergents are high enough (perhaps due to random fluctuations), local mechanical distortions can cause defects in the form of spontaneous pores or shedding of micelles. 
A comprehensive study from Nazari et al. compared the membrane perturbing effects of a number of different detergents and surfactants on lipid vesicles, categorizing them into homogeneously and heterogeneously perturbing surfactants ${ }^{1394}$. In the homogeneous category were typical synthetic detergents, such as $\mathrm{C}_{12} \mathrm{EO}_{8}$, octyl glucoside, sodium dodecyl sulfate (SDS), and lauryl maltoside, which destroy the membrane through homogeneous disordering when a critical curvature stress is reached. In contrast, the hetergenous category included the fungicidal lipopeptides surfactin, fengycin, and iturin, as well as digitonin, CHAPS, and lysophosphatidylcholine, which perturb membranes without substantial overall disordering. Rather, they disrupt membranes locally in surfactant-rich defect structures. Nazari et al. proposed that such heterogeneous perturbation mechanisms may account for the superior activity, selectivity, and mutual synergism of antimicrobial biosurfactants, such as lipopeptides and saponins, to efficiently permeabilize target cell membranes in discrete loci at minimal concentrations ${ }^{1394}$.

\subsubsection{Detergent Permeabilization of Live Cells}

A further consideration influencing detergent-mediated membrane permeabilization is the composition of the target membrane of living cells. The permeabilizing activity of certain antimicrobial peptides and surfactants is strongly modulated by cholesterol, proteins and other raft domain components ${ }^{441}$. Owing to the heterogeneous and dynamic nature of living cell membranes, it has been a challenge to predict how detergents will permeabilize cells. One study by Vaidyanathan and colleagues used patch clamp to analyze permeabilization behaviour of detergents as a function of concentration $^{1395}$. They observed that anionic SDS, cationic cetyltrimethylammonium bromide (CTAB), and cationic fluorescent octadecyl rhodamine B (ORB) increased the membrane permeability of cells substantially within a second of exposure. It was reported that SDS $\leq 0.2 \mathrm{mM}$ (below SDS's CMC of $\sim 1 \mathrm{mM}$ ) and CTAB and ORB $\leq 1 \mathrm{mM}$ (above CTAB's CMC of $\sim 50 \mu \mathrm{M}$ ) induced transient cell membrane permeability without causing acute or permanent toxicity ${ }^{1395}$. Thus, careful titration of detergent concentration enabled the identification of conditions from which cells can recover after permeabilization.

In another study of detergent permeabilization in live cells, Koley and Bard used electrochemical microscopy to monitor the permeability of HeLa cells to the hydrophilic anion ferrocyanide $(\sim 0.2 \mathrm{kDa})$ in the presence of increasing concentrations of the nonionic detergent triton $\mathrm{X}-100^{1396}$. No effect on permeability was seen at triton $\mathrm{X}-100$ 
concentrations of $0.15 \mathrm{mM}$ for up to 1 hour. At $0.17 \mathrm{mM}$, initial permeabilization was observed followed by a recovery of cell viability. From $0.19 \mathrm{mM}$, which approaches the CMC, rapid irreversible permeabilization and cell death resulted. Thus, the effective concentration window of triton- X-100 on live cells is narrow under the tested set of experimental conditions. The above results underscore the importance of conducting systematic permeabilization studies in live cells.

\subsubsection{Saponins}

Saponins are steroid and triterpinoid glycosides produced by plants and certain marine organisms as secondary metabolites in response to environmental stimuli ${ }^{1373,1397}$. By perturbing the membranes of competing life forms, saponins constitute a form of innate immune system to poison threatening microbes, parasites, insects, and herbivores ${ }^{1373,1398}$. The detergent phenomena of saponins originates from their amphiphilic properties, featuring a lipophilic sapogenin part (usually a triterpene or steroid group) and a hydrophilic glycoside moiety. A wide range of applications for saponins relating to their membrane perturbing activity have been proposed. They include augmenting the penetration of drugs and cytotoxic agents to cancer cells, vaccine adjuvants, or deployment as microbials and pest control agents ${ }^{1399-1401}$.

For applications with mammalian cells, studies usually employ generic saponins or pure digitonin. Generic saponins are commercially available cocktails typified by a sapogenin content $>10 \%$ while digitonin is a prototype member of the saponin family isolated from the foxglove plant Digitalis purpurea. Other less-studied saponins that have been reported to disrupt membranes include $\alpha$-tomatine, glycyrrhizin, $\alpha$-chaconine, and $\alpha$-hederin ${ }^{1393}$. Saponins in general, and digitonin specifically, have been used with live cells for two main applications: 1) persistent permeabilization to produce "semiintact cells" for real-time manipulation of cytoplasmic constituents, and 2) to transiently disrupt the plasma membrane for intracellular delivery. Early work emphasized the first of these two applications.

\subsubsection{Characteristics \& Mechanisms of Saponin-Induced Membrane Disruption}

Saponins were initially characterized as membrane-perturbing agents in the scientific literature of the 1960s and $1970 \mathrm{~s}^{940,1402}$. Electron micrographs captured their membrane disrupting capabilities in reconstituted membranes, indicating arrays of holes around $8 \mathrm{~nm}^{1402}$. Serial section electron microscopy of fixed hemolysing erythrocytes revealed lesions of 4 $-5 \mathrm{~nm}$ after saponin treatment ${ }^{940}$. Most cell permeabilization studies have employed saponins in the concentration range 
$10-1000 \mu \mathrm{g} \mathrm{ml} l^{-1}$, which represents $\sim 8-800 \mu \mathrm{M}$. In this range, disruption sizes from a few nanometers up to one micron have been reported. Differences are probably related to variations in cell type, concentration, duration of exposure and other experimental conditions $397,1403,1404$. The inconsistency of these reports may also stem from the variety of analysis techniques. For example, misleading artifacts can occur during fixation of membranes for AFM and SEM imaging. Thus, our knowledge on saponin-based permeabilization and characteristics of holes formed may require revision with more current methods and stricter control of environmental conditions.

Most saponins preferentially interact with cholesterol- and hydroxysterol-rich membranes, a property that makes them relatively specific for the plasma membranes of animal cells. In this case the efficiency of their membrane perturbing effects are directly correlated with sterol content. Indeed, cholesterol-rich bilayers are thought to be about 20- to 100-fold more sensitive to saponins ${ }^{408}$. Hence, saponins can be been exploited to target the plasma membrane while leaving those of cholesterol-poor organelles, such as the ER and mitochondria, largely unaffected ${ }^{397,585,1405}$. Calcium stores within intracellular organelles are generally not eroded by the saponin concentrations that permeabilize plasma membranes ${ }^{583}$.

How do saponins interact with cholesterol to disrupt membranes? Frenkel et al. conducted investigations into the mechanism using quantitative physical techniques in model membranes. Their measurements indicate that digitonin extracts cholesterol out of the bilayer core to form a surface complex, which then induces curvature and disordering of the membrane ${ }^{1406}$. The magnitude of these effects was directly proportional to the amount of cholesterol in the bilayer (Figure 36). At $0 \%$ cholesterol, digitonin could not bind to the membrane and thus had no effect. At $5 \%$ cholesterol, exposure to digitonin triggered the formation of sterol-aglycone complexes without significant membrane distortion. At $20 \%$ cholesterol, digitonin extracts cholesterol into aggregates, thus removing it from the hydrophobic core region. The steric hindrance between saccharide residues in these aggregates may induce changes in the curvature of the membrane outer leaflet leading to compromisation of the membrane integrity and concomitant increase in membrane permeability ${ }^{1406}$. In essence, digitonin binds to cholesterol and transforms it into a detergent.

Beyond digitonin, studies have explored a wider range of individual saponins for membrane permeabilization. Recently a set of oleanane saponins (glycyrrhizic acid, Gypsophila, Saponaria and Quillaja saponins) and digitonin were tested in live cells. These saponins showed variable permeabilizing effects on cellular membranes from $6 \mu \mathrm{M}$, as measured by an 
impedance-based plate reader with ECV-304 human urinary bladder carcinoma cells ${ }^{1407}$. The results indicated that the molecular charge may be a relevant consideration in explaining the action of oleanane saponins. Further studies on $\alpha$ hederin indicate that the critical micelle concentration (CMC) plays a key role in its mechanism. At concentrations lower than the CMC, $\alpha$-hederin monomers bind to cholesterol and induce vesiculation and lateral phase separation ${ }^{1408,1409}$. These effects are analogous to the action of detergents that do not flip flop, as depicted in figure 34 . At concentrations higher than the $\mathrm{CMC}, \alpha$-hederin aggregates promote pore formation and the loss of membrane material by analogy to the scenario illustrated in figure 35. Thus, the self-aggregating properties and co-operative action of saponins may also be important for their effects. Most studies agree that the permeabilizing activity of saponins rely on the presence of sterols, from which they forms complexes to distort the membrane into non-bilayer structures. As an exception to this rule, some bidesmosidic saponins, such as avicin $\mathrm{D}^{1410}$, appear capable of porating cell membranes through detergent properties independent of cholesterol binding ${ }^{1393}$.
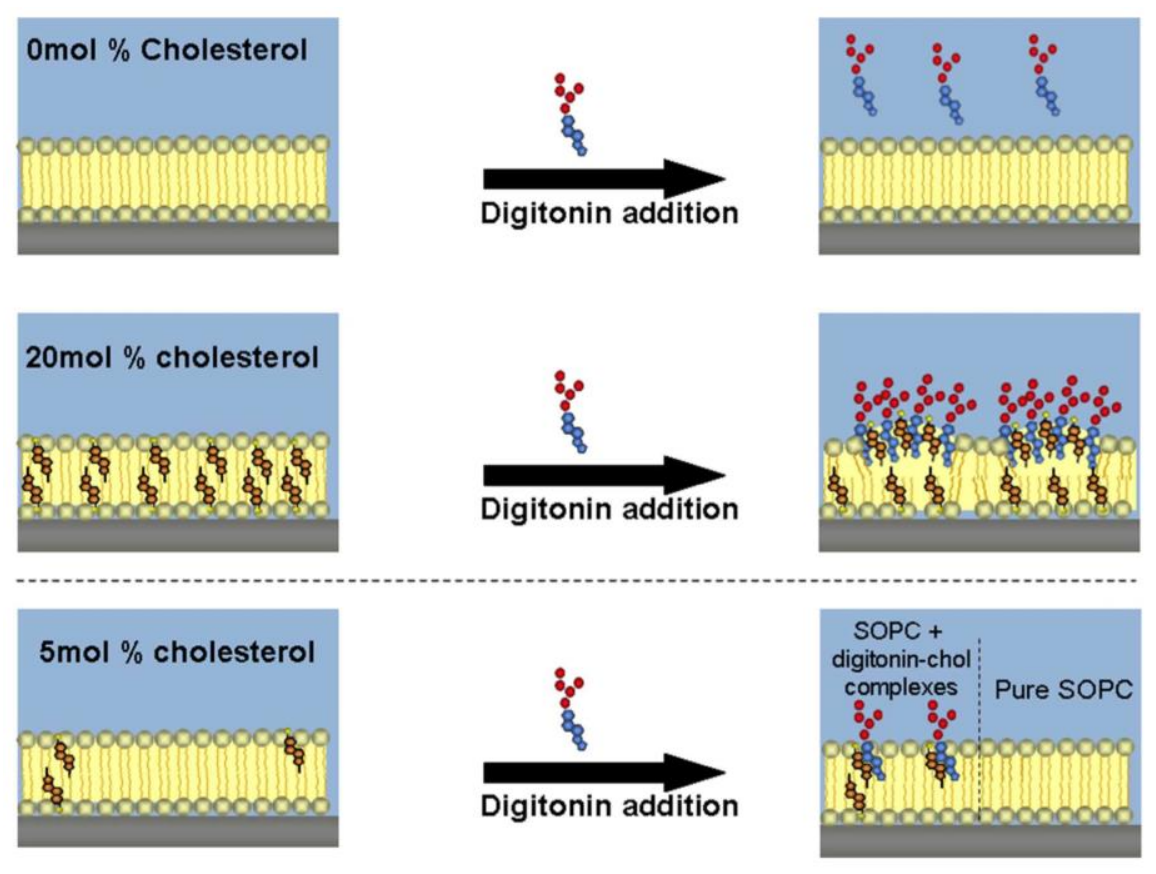

Figure 36. Interactions of $50 \mu \mathrm{M}$ digitonin ( $<$ CMC) with SOPC (1-Stearoyl-2-oleoyl-sn-glycero-3-phosphocholine) phospholipid bilayer membranes containing varying amounts of cholesterol. Reproduced from reference ${ }^{1406}$. Copyright 2014 American Chemical Society.

\subsubsection{Saponin-Mediated Permeabilization for Studies in Semi-Intact Cells}

Detergent-permeabilized semi-intact cells have led to advances in several areas of biology, including decoding the rules governing nuclear import of proteins and $\mathrm{DNA}^{592,593}$, studying mammalian protein synthesis and secretion 
machinery ${ }^{50,591}$, and the analysis of functional mitochondria in muscle fibers, tissues, and cells in situ ${ }^{594}$. The emergence of saponins for the production of semi-intact cells began around the early 1980s. In 1982 Wakasugi et al. used saponin or digitonin in the range $20-100 \mu \mathrm{g} \mathrm{ml}^{-1}(\sim 16-80 \mu \mathrm{M})$ to permeabilize acini from rat pancreases and probe the effect of ATP on intracellular calcium dynamics ${ }^{1411}$. A year later, the plasma membranes of isolated guinea pig hepatocytes were made permeable with $75 \mu \mathrm{g} \mathrm{ml}^{-1}(\sim 60 \mu \mathrm{M})$ saponin to study the ATP-dependent uptake of calcium into the endoplasmic reticulum ${ }^{583}$. Upon saponin treatment, cells were suspended in a medium resembling cytosol with an ATP-regenerating system consisting of ATP, creatine phosphate, and creatine phosphokinase. Dunn and Holz used $20 \mu \mathrm{M}$ digitonin to permeabilize chromaffin cells, and this protocol became a popular system to study intracellular processes in this cell type ${ }^{584,1412}$. Human platelets were also treated with saponins for the loading of the secondary messenger inositol $1,4,5-$ trisphosphate into the cytoplasm and studying of the metabolic signaling response ${ }^{1413}$. Several groups reported that with optimal conditions, $50 \%$ or more of the cytoplasmic enzyme lactate dehydrogenase $(\sim 140 \mathrm{kDa})$ is able to remain inside cells for extended periods, indicating the possibility of maintaining a feasible balance between plasma membrane permeabilization and cell function in these experiments ${ }^{584,585,1414}$. In most of these papers the plasma membrane resealing dynamics were not discussed. Thus, it is difficult to ascertain whether or not the cells were persistently permeabilized or whether they recovered due to plasma membrane repair.

\subsubsection{Saponin-Mediated Permeabilization for Intracellular Delivery}

An optimized protocol for peptide delivery into cardiac myocytes employed a 10 minute incubation at $4{ }^{\circ} \mathrm{C}$ with $50 \mu \mathrm{g}$ ml ${ }^{1}(\sim 40 \mu \mathrm{M}) \operatorname{saponin}^{539}$. Along with saponin, the permeabilization buffer was designed to mimic aspects of the intracellular environment by including high potassium, extracellular ATP to maintain energy stocks, and ascorbic acid as an antioxidant ${ }^{539}$. The authors reported loading of peptides at up to $\sim 10 \%$ of the extracellular concentration without loss of long-term viability. In another method, Miyamoto et al. used $7.5 \mu \mathrm{g} \mathrm{ml}^{-1}(\sim 6 \mu \mathrm{M})$ digitonin to induce reversible permeabilization of the plasma membrane in bovine, mouse, and porcine somatic cells ${ }^{1415}$. By optimizing the procedure, high efficiency ( 80\%) loading of $70 \mathrm{kDa}$ dextrans was achieved in bovine cumulus cells. It was also used to introduce cytoplasmic extracts from Xenopus laevas eggs into several mammalian cell types for successful induction of nuclear reprogramming and activation of pluripotent genes ${ }^{1415}$. 
More recently, saponins have been exploited for the delivery of quantum dots and nanoparticles. Lukyanenko published a protocol for the delivery of nanoparticles up to $20 \mathrm{~nm}$ with a transient $30-60$ second exposure to $0.01 \%$ saponin $(10 \mu \mathrm{g} \mathrm{ml}-$ ${ }^{1}$ or $\sim 8 \mu \mathrm{M}$ ) in high potassium low calcium permeabilization buffer ${ }^{1416}$. Depolymerization of cytoplasmic actin with cytochalasin D was reported to boost the efficiency of nanoparticle penetration into the cytoplasm, as the actin meshwork that underlies the plasma membrane may be considered another barrier to delivery ${ }^{1416}$. Medepalli and co-workers demonstrated quantum dot loading into adherent $\mathrm{H} 9 \mathrm{C} 2$ with a combination of $50 \mu \mathrm{g} \mathrm{ml}^{-1}(\sim 40 \mu \mathrm{M})$ saponin and 180 mOsm hypotonic media for 5 minutes at $4{ }^{\circ} \mathrm{C}^{311}$. Whether hypoosmotic shock produces a membrane tension to synergize with the membrane perturbing effect of saponin, or to generate inward fluid flux to promote delivery, remains to be determined $^{311}$.

For intracellular analysis with antibodies, Jacob et al. developed a saponin-based permeabilization protocol to load immune cells with monoclonal antibodies for the detection of cytoplasmic antigens by flow cytometry ${ }^{1417}$. They incubated primary lymphocytes and lymphoma cell lines at $4{ }^{\circ} \mathrm{C}$ in HBSS buffer with antibodies in a buffer containing $2 \%$ FBS and $0.1-0.3 \%$ saponin $\left(10-30 \mu \mathrm{g} \mathrm{ml}^{-1}\right.$ or $\left.\sim 8-24 \mu \mathrm{M}\right)$ for 30 minutes. As judged by flow cytometry analysis, monoclonal antibody delivery was achieved while cell integrity and morphology remained intact ${ }^{1417}$. Interestingly, this protocol did not rely on fixation with paraformaldehyde, a step that was only incorporated in later adaptations, presumably to prevent leakage of cytokines from the cell or avoid dealing with apoptotic cells ${ }^{1418-1420}$. An earlier method featuring lysophosphatidylcholine as permeabilization agent was similarly independent of fixation ${ }^{99}$.

\subsubsection{Detergent-Like Lipids \& Other Surfactants for Intracellular Delivery}

Surfactants include synthetic detergents, physiological compounds such as bile salts, lysolipids and certain amphiphilic peptides and amphiphiles. A widely used example is the naturally occurring lipid lysophosphatidylcholine (also known as lysolecithin). Miller et al. employed lysophosphatidylcholine exposures to permeabilize CHO cells and maintain them as semi-intact cells capable of DNA synthesis for several hours ${ }^{541}$. The protocol was used to explore for soluble factors that inhibit or stimulate DNA synthesis. A follow-up paper outlined generalized protocols for delivery of cargo molecules to a wide range of monolayer and suspension cells ${ }^{1421}$. In it, lysophosphatidylcholine concentrations from $30-250 \mu \mathrm{g} \mathrm{ml}{ }^{-1}(60$ - $500 \mu \mathrm{M})$ were chosen depending on the balance between delivery, viability, and leakage of the representative 
endogenous protein lactase dehydrogenase. Balinska employed lysophosphatidylcholine to introduce the exogenous nucleoside dTTP into the DNA of hepatoma cells via permeabilization-mediated intracellular delivery ${ }^{1422}$. Because there was only a slight loss (20-25\%) of lactate dehydrogenase, they concluded permeabilization of cells does not persistently disrupt membrane integrity and resealing could be achieved by exchanging back to standard media ${ }^{1422}$. Nomura and colleagues used lysophosphatidylcholine permeabilization for the delivery of larger proteins: diphtheria toxin (A fragment), horseradish peroxidase and antibodies against SV40 T-antigens ${ }^{1423}$. These macromolecules were successfully introduced into living mouse erythroleukemia cells, baby hamster kidney, and mouse fibroblast cells ${ }^{1423}$. Furthermore, lysophosphatidylcholine has been used to permeabilize primary human lymphocytes and monocytes for detection of intracellular antigens by flow cytometry ${ }^{99} .50 \mu \mathrm{g} \mathrm{ml}^{-1}(100 \mu \mathrm{M})$ of lysophosphatidylcholine was incubated at $4{ }^{\circ} \mathrm{C}$ for 5 min before recovery with antibodies inside, thus avoiding the need for fixation.

Along with lysophosphatidylcholine, similar compounds have been investigated for their detergent-like mechanisms. For example, simulations have been performed on plant-derived resorcinols ${ }^{1424}$ and dioctanoyl-phosphatidylcholine, a coneshaped counterpart of the native lipid DPPC ${ }^{1425}$. Studies with dioctanoyl-phosphatidylcholine reveal a curvature stress that can be relieved upon pore formation ${ }^{1425}$. Such mechanisms may also be applicable to lysophosphatidylcholine, which is also a cone-shaped lipid. In the case of resorcinols, micelles are observed to bind to the membrane. If micelles remain compact, they displace phospholipids head groups into the bilayer center, thereby disrupting the structure of the leaflet and causing the lipids to surround the micelle ${ }^{1424}$. However, if resorcinols are already embedded within the bi-layer their presence leads to stabilization instead, just like cholesterol. Thus, simulations are a useful tool to gain insight into the mechanisms and molecular events that underlie membrane disruption mechanisms that could be leveraged for intracellular delivery.

\subsubsection{Microfluidic \& Nanotechnological Control of Detergent Exposure}

For detergents and surfactants applied in bulk solution, a key weakness is that the nature of the membrane injury lacks precise spatiotemporal control. Molecules are added indiscriminately to solution, and it is difficult to get rid of them once then job is done. Thus, it is difficult to balance the required level of membrane permeabilization against excessive toxicity

(Figure 37A). Recently, Kilinc et al. used microfluidics to demonstrate controlled flux of localized saponins to perform 
precise axotomy (cut off an axon) on neurons cultured on microchips ${ }^{1426}$. In a variation on this theme, the detergent sodium dodecyl sulfate (SDS) was employed in laminar flow mode in a microfluidic device to damage specific sections of neurites and investigate the recovery process ${ }^{1427}$. Saponin has also been combined with nanostraws to localize membrane disruption to the nanostraw openings ${ }^{701}$. These examples showcase the potential of nano and microfluidic systems to localize and control damage conferred by detergents to subcellular regions (Figure 37B). It remains to be seen whether such strategies can be feasible for intracellular delivery at high throughput, although we anticipate that inventors will test this possibility in the near future.

Membrane-perturbing nanoparticles are another concept worth considering (Figure 37C). Multifunctional nanocarriers that switch to a membrane disrupting state are already being developed for endosomal escape purposes ${ }^{6}$. Similarly, conjugation with membrane-active peptides ${ }^{116}$ or pore-forming toxins ${ }^{119}$ can be harnessed to produce nanoscale cargo with more potent cell penetration properties. If membrane-perturbing nanoparticles can be made switchable by light or other environmental stimuli, they may confer the level of control required for reversible permeabilization at discrete locations on the cell surface.

A

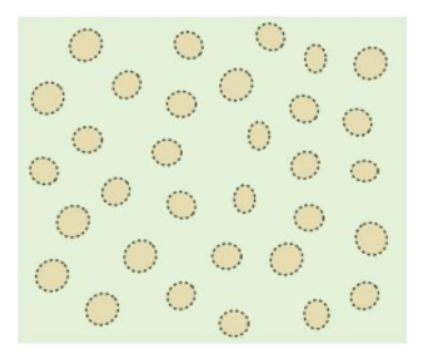

bulk detergent
B

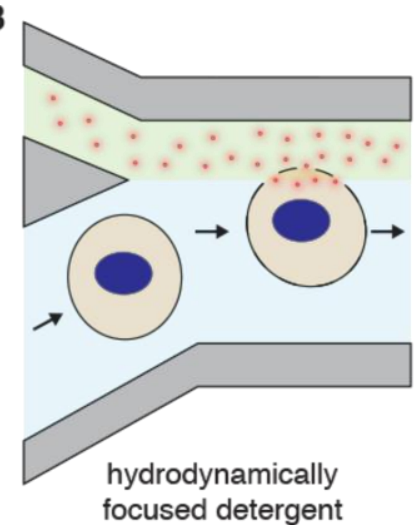

C

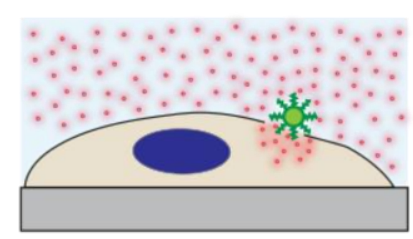

membrane-perturbing particle

Figure 37. Schematic of exposure to membrane-perturbing detergent and/or surfactants by (A) bulk mixing, (B) microfluidic hydrodynamic focusing, and (C) localization to a nanoscale particle.

\subsubsection{Summary of Permeabilization by Detergents}

The abovementioned studies suggest saponins, detergents, and other membrane permeabilizing surfactants can be used to introduce a wide range of cargo molecules into various cell types. The emergence of membrane defects depends on variables such as exposure time, temperature, diffusion, random fluctuations, mixing effects, and spontaneous 
interactions. This is in contrast to physical methods where a well-defined stimulus triggers a clean disruption event. Electroporation, in particular, has often been reported to achieve superior results in the hands of researchers when compared with detergents ${ }^{1098}$. The use of physically controllable or light-switchable surfactant systems may aid in developing more precise membrane perturbation strategies. Furthermore, it is worth considering that a wide range of organisms produce secondary metabolites with membrane-disrupting properties. As an increasing abundance of these natural detergents and lipopeptides are characterized, new possibilities for ideal membrane permeabilization agents may become available. For example, anabaenolysin lipopeptide toxins have recently been proposed as a potent alternative to digitonin for the selective disruption of cholesterol-containing biological membranes ${ }^{1428}$. Finally, using microfluidics and nanotechnology for local and transient exposure of cells to surfactants is another frontier where spatiotemporal control of membrane disruption may increase the effectiveness of intracellular delivery.

\subsubsection{Membrane-Active Peptides}

Various membrane-active peptides are known to disrupt lipid bilayer membranes ${ }^{433,440}$. Anti-microbial peptides (AMPs), which are usually both amphiphilic and cationic, can induce pore-formation at critical concentrations ${ }^{1429,1430}$. Under certain circumstances, cell-penetrating peptides (CPPs) and pathogenic amyloid peptides can also permeabilize lipid bilayers, although the mechanisms are less well-defined ${ }^{433}$. Most membrane-active peptides are thought to be intrinsically disordered in solution but adopt more defined structures upon contact with biological membranes, giving rise to their membrane-disrupting properties ${ }^{433}$. Membrane-active peptides are often conjugated to cargo to facilitate intracellular delivery ${ }^{1431,1432}$. However, there are also reports of membrane-active peptides permeabilizing cells to enable cytosolic delivery of dyes ${ }^{1433,1434}$, small proteins ${ }^{1435}$, low-molecular weight dextrans ${ }^{1436}$, and short oligonucleotides ${ }^{1437}$.

\subsubsection{Anti-Microbial Peptides}

The best-characterized membrane-active peptides are the AMPs. To date, more than 5,000 of them have been catalogued $^{1438,1439}$, with frog skin alone representing a source of more than 300 variants ${ }^{1440}$. Only a small selection of AMPs have been studied for their molecular mechanisms of action. A common feature is their ability to adopt a conformation with hydrophobic segments distinct from hydrophilic/cationic segments ${ }^{1429}$. For a given AMP, the ability to disrupt membranes also depends on the lipid composition of the target membrane. In contrast to the plasma membrane of 
animal cells, most microbial membranes, including those of gram-positive (monoderm) and gram-negative (diderm) bacteria, fungi, and protozoa, feature many negatively charged lipid headgroups on their outer leaflets ${ }^{1441}$. This allows a combination of electrostatic and hydrophobic interactions to drive adsorption of cationic AMPs to the surface of microbes with high affinity ${ }^{1429}$. Once at the interface, hydrophobic segments integrate into the membrane to disrupt it, with several different models proposed for how they generate pores ${ }^{440,1430,1442}$. Due to the higher affinity for microbial membranes, AMPs can lyse microbes at $\mu \mathrm{M}$ concentrations while having less effect on animal cell membranes. This enables them to kill microorganisms without being significantly toxic to mammalian cells. Moreover, in an opposite manner to saponins, cholesterol in the plasma membrane of animal cells serves to suppress the activity of AMPs due to its stabilizing effect. At high enough concentrations, however, AMPs will disrupt plasma membranes of mammalian cells, and this is the regime of interest for potential intracellular delivery applications.

\subsubsection{Mechanisms of Membrane Disruption by AMPs}

The main models used to describe AMP-mediated pore formation mechanisms share a common aspect, namely two distinct peptide-lipid states: an inactive surface-bound state and a pore-like insertion state ${ }^{1429,1443}$. One of the best studied AMPs is melittin, a peptide extracted from bee venom ${ }^{1444}$. It is a 26 amino acid chain containing +6 positive charges in total. Amino-terminal residues 1-20 are mostly hydrophobic while carboxyl-terminal residues 21-26 are hydrophilic due to a string of positive charges. Pores produced by melittin exposure have been estimated at $2.5-3 \mathrm{~nm}$ in palmitoyloleoylphosphatidylcholine (POPC) vesicles ${ }^{1445}$. Experiments with GUVs held by micropipettes revealed that melittin first increases the membrane surface area due to adsorption/integration before rearranging to induce stable pores without vesicle rupture ${ }^{1446}$. Later studies showed that melittin partitions to both sides of the bilayer, probably via transient defects, before finally reaching a concentration where stable pore formation occurs. The critical concentration lies in the $\mu \mathrm{M}$ range and corresponds to a peptide-to-lipid ratio of 1:100 or greater ${ }^{1447}$.

Another heavily studied AMP is magainin 2. Tamba et al. showed that pore-formation is triggered when magainin 2 reaches a critical concentration at the membrane interface ${ }^{1448}$. Their studies predicted that the initial disruption size could be as large as tens of nanometers before shrinking to a more stable pore of several nanometers ${ }^{1449}$. The pores are thought to be "chaotic", lined by a mixture of peptides and lipids acting in cooperation, rather than a well-defined peptide lined 
channel $^{1450}$. In keeping with the notion of a two-state model, the human LL37 peptide has been observed to first absorb parallel to the surface as an alpha-helix before inserting and rotating normal to the membrane to form pores with an estimated diameter of $2.3-3.3 \mathrm{~nm}^{1451}$. AMPs can to some extent exhibit detergent-like effects including membrane thinning, bilayer stresses, toroidal pore formation, micellization ${ }^{1452}$. Unlike detergents, however, they tend not to dissolve the membrane structure but rather induce smaller pores for the passage of low-molecular weight molecules ${ }^{1447}$. On the other hand, one report suggested that AMPs can form larger holes in certain types of membranes ${ }^{1453}$. Atomic force microscopy imaging of supported lipid bilayers was used to visualize a population of pores that could grow as a function of AMP concentration ${ }^{1453}$.

In many cases the exact structure of AMP-mediated pores is unknown. Multiple models have been proposed such as toroidal, disordered toroidal, and barrel stave. The depictions of these pore models are shown in Figure $38^{1454}$. Molecular dynamics simulations have played a part in elucidating possible molecular events ${ }^{410}$. They indicate that synergistic aggregation of several peptides together cooperatively results in defect formation ${ }^{410}$. AMP aggregation leads to a high local density of positive charges. This dense concentration of positive charges at the membrane interface can result in a highly localized electric field, which could destabilize the bilayer by an electroporation-like effect ${ }^{1455-1457}$. Interestingly, simulations indicate that the emerging defects appear to exhibit a significantly disordered shape, rather than a classic toroidal pore ${ }^{1454}$. Studies of magainin MG-H2 peptide reveal that its binding creates a local tension in the exposed leaflet, which creates a compressive stress that is relieved upon pore formation ${ }^{1458}$. Simulations of melittin ${ }^{1454}$ and $_{\text {cateslytin }}{ }^{1459}$ support a similar interpretation. Overall, the prerequisites for AMP-mediated pore formation appear to be: 1) a high concentration of peptides in solution and, 2) aggregation. The simulations used to visualize pore formation favor a model whereby membrane defects occur as disordered non-uniform pores ${ }^{1454}$. 


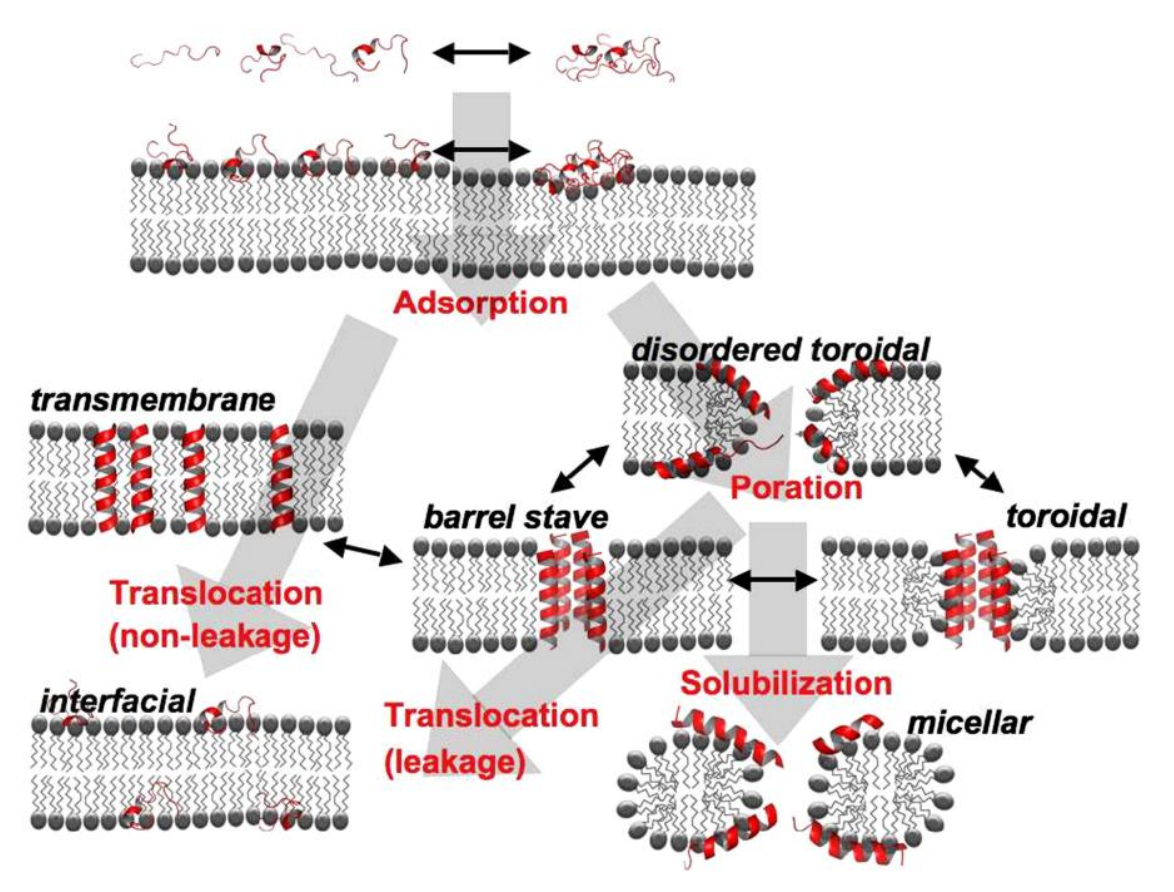

Figure 38. Schematic overview of the possible interaction pathways of an antimicrobial peptide with a lipid bilayer. Possible thermodynamic states (either stable or metastable) are indicated by black labels and the major kinetic pathways connecting them by gray arrows and red labels. Short black arrows represent additional inter-conversion pathways. Outside the target membrane, peptide monomers and small aggregates exist in equilibrium. At the target membrane, the peptides bind to the interface (Adsorption). At the interface an equilibrium may exist between monomeric and polymeric aggregation states. For a symmetric bilayer, the asymmetric membrane bound state is not thermodynamically stable. Eventually the peptides will distribute equally between the two monolayer leaflets. This can occur via two alternative translocation pathways. In the non-leaky variant the peptides are able to cross the bilayer without the formation of a pore. In some cases, the intermediate transmembrane state is thermodynamically stable (e.g. hydrophobic peptides which adopt a transmembrane orientation). The key feature of many antimicrobial peptides is that they permeabilize the membrane following a leaky translocation pathway. Above a certain peptidelipid ratio, the peptides insert into the bilayer to form a porated lamellar phase (poration). A variety of different pore structures may be formed, including the barrel-stave, the toroidal and the disordered toroidal state. These separate states should be interpreted as extreme cases with mixed varieties of these models, and conversion between alternative states is likely to occur. The porated states can be stable themselves, but they can also be transient structures in the translocation pathway. In that case, once enough peptides are adsorbed at the opposing monolayer leaflet, the pores seal. On the other hand, increased accumulation of certain peptides may lead to a detergent-like disintegration of the membrane resulting in formation of non-lamellar, e.g. micellar, systems (solubilization pathway). Note that the secondary structure of the peptides could vary along the various pathways. The helical or random configurations drawn here are merely illustrative of these processes and should not be taken literally. Figure and legend reproduced from ref ${ }^{1454}$, Copyright 2008, with permission from Elsevier.

\subsubsection{Cell-Penetrating Peptides \& Amyloid Peptides}

In contrast to the case of AMPs, cell-penetrating peptides (CPPs) and amyloid peptides do not adhere to the principle of well-defined hydrophilic/cationic and hydrophobic segments. Though most CPPs tend to be cationic, they may also be uncharged and hydrophilic. Well-studied CPPs include penetratin, HIV-1 TAT peptide, and poly-arginines of 8 or 9 units.

For these peptides molecular dynamics simulations have observed only very transient pores ${ }^{1460}$. Other simulations reveal deformations and bending phenomena without actual pore formation, although this is controversial and it has been argued that some simulations of CPP behaviour could be artifactual ${ }^{410}$. When attached to bulky cargo molecules, CPPs are 
believed to enter cells via endocytosis rather than direct translocation through the membrane, arguing that pore-formation

in the plasma membrane might have very little role in actual delivery ${ }^{116}$. Thus, the mechanisms could be different when CPPs are present as lone molecules versus when they are conjugated to a cargo molecule.

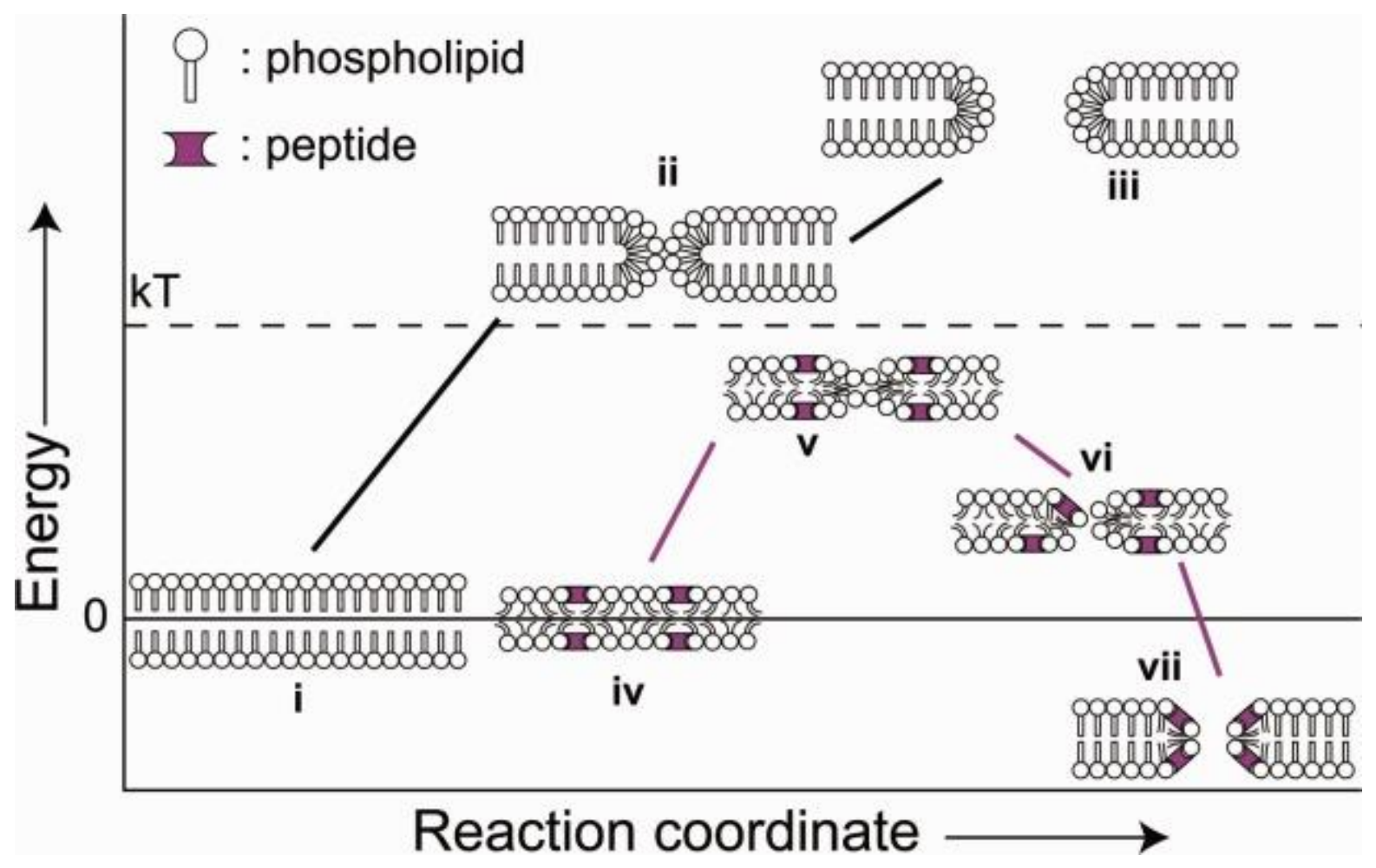

Figure 39. Schematic of the effect of peptide binding on lipid bilayer integrity. (i) The reference state for energy change is an intact phospholipid bilayer. (ii) Spontaneous fluctuations result in the sampling of membrane defects. These are energetically unfavorable and therefore sampled infrequently. (iii) Widening of the defect to permit leakage results in a further energetic penalty. (iv) In the presence of surface-bound peptides (magenta), membrane tension is induced. (v) Peptide binding increases the frequency of defect formation. (vi) Surface tension is released by pore formation ${ }^{1461}$ and stabilized by peptide binding resulting in equilibrium poration (vii). Note, many forms of defect, such as chaotic pores ${ }^{1462}$, can be accommodated by this model, and defect characteristics may differ between alternate peptides or the same peptide under alternate conditions. Figure and legend reproduced from ref ${ }^{1463}$, Copyright 2013, with permission from John Wiley and Sons.

To explain the observations gathered from various studies, Miranker and colleagues propose a common mechanistic landscape for membrane-active peptides ${ }^{433}$. The initial formation of a pore is catalyzed by peptide-induced membrane tension that lowers the activation energy of spontaneous poration to a regime more accessible by thermal fluctuations

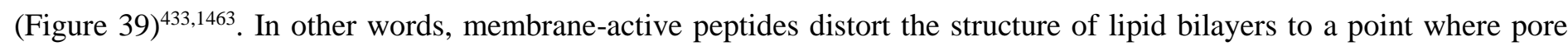
formation becomes the most energetically favorable option at a given temperature. The structure and lifetime of such pores in live cells remain to be determined. 


\subsubsection{Summary of Permeabilization by Membrane-Active Peptides}

Reviews of the literature increasingly look to examine common principles underlying the action of AMPs, CPPs, and amyloid peptides ${ }^{433,440,1452,1464}$. Further studies will be required to uncover their mechanisms of action in live cell membranes and to what degree they can be harnessed for intracellular delivery ${ }^{1432}$. So far, there are examples of membrane-active peptides permeabilizing cells to enable cytosolic delivery of dyes ${ }^{1433,1434}$, small proteins ${ }^{1435}$, lowmolecular weight dextrans ${ }^{1436}$, and short oligonucleotides ${ }^{1437}$. It remains to be seen whether membrane-active peptides can create pores large enough for siRNA, mRNA, RNPs or larger protein influx without excessive cell toxicity. Provided treatment with membrane-active peptides can be made sufficiently reversible and tolerable, their specificity for different types of membranes suggests they could be an intriguing strategy for intracellular delivery ${ }^{1465}$.

\subsubsection{Pore-Forming Proteins \& Toxins}

Organisms from all kingdoms have evolved pore-forming proteins (PFPs) that can permeabilize the membranes of competing lifeforms ${ }^{399}$. PFPs are produced by prokaryotes, eukaryotic parasites, fungi, marine organisms, and plants either as a defense mechanism or to access nutrients, especially under conditions of high competition or stress. Vertebrates also produce PFPs, such as the complement membrane attack complex (MAC) to kill bacteria, and the perforins expressed by immune killer cells to destroy malignant or infected cells. The best-characterized and largest class of PFPs, however, is that of the bacterial pore-forming toxins (PFTs).

PFTs are generally secreted as soluble monomers that can assemble into oligomers, undergo conformational changes, and insert into the membrane as an assembled pore complex (Figure 40$)^{399,439}$. Depending on the PFT, pore assembly may take place before reaching the target cell surface or via lateral diffusion and binding of monomers once embedded within the target cell plasma membrane. For many PFTs, the stoichiometry of the assembled pore is around 7 subunits, such as is the case for $S$. Aureus $\alpha$-hemolysin or the aerolysin family. These PFTs form $1-3 \mathrm{~nm}$ pores to permit the passage of ions, ATP, and nucleotides $399,439,1466$. 


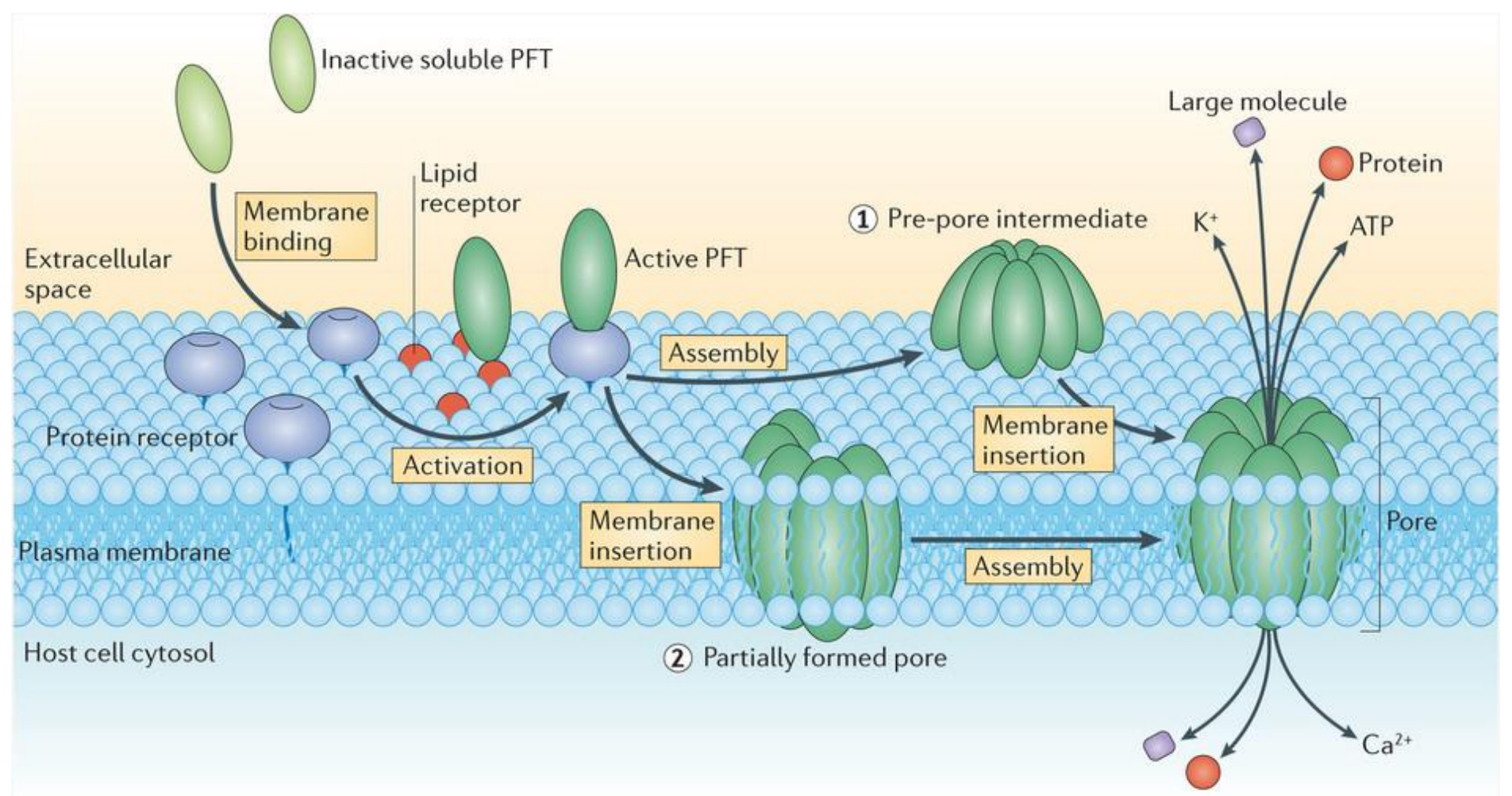

Figure 40. Schematic representation of the pore formation pathway of pore-forming toxins (PFTs). Soluble PFTs are recruited to the host membrane by protein receptors and/or specific interactions with lipids (for example, sphingomyelin for actinoporins or sterols for cholesterol-dependent cytolysins (CDCs)). Upon membrane binding, the toxins concentrate and start the oligomerization process, which usually follows one of two pathways. In the pathway followed by most $\beta$-PFTs, oligomerization occurs at the membrane surface, producing an intermediate structure known as a pre-pore (mechanism 1 ), which eventually undergoes conformational rearrangements that lead to concerted membrane insertion. In the pathway followed by most $\alpha$-PFTs, PFT insertion into the membrane occurs concomitantly with a sequential oligomerization mechanism, which can lead to the formation of either a partially formed, but active, pore (mechanism 2), or the formation of complete pores. Although classified as $\beta$-PFTs, CDCs also share some of the features of this second pathway, as they can also form intermediate structures (known as 'arcs', named after their shape) during pore formation. In both $\alpha$-PFT and $\beta$-PFT pathways, the final result is the formation of a transmembrane pore with different architecture, stoichiometry, size and conduction features, which promote the influx or efflux of ions, small molecules and proteins through the host membrane, and trigger various secondary responses involved in the repair of the host membrane. Note that, although the host membrane shown here is the eukaryotic plasma membrane, some PFTs are antibacterial and form pores in the inner membranes of gram-negative bacteria or the cell membranes of gram-positive bacteria. Figure and legend reproduced by permission from Springer Nature from ref ${ }^{439}$, Copyright 2015.

Alternatively, cholesterol-dependent cytolysins (CDCs) form multimeric assemblies of $>30$ units and generate large pores in the range of $20-50 \mathrm{~nm}$ (Figure 41A $)^{439}$. Atomic force microscopy images of prototype CDC perfringolysin $\mathrm{O}$ (PFO) embedded into cholesterol-containing supported lipid bilayers reveals the formation of ring-like pores with $\sim 25 \mathrm{~nm}$ diameter (Figure 41B) ${ }^{1467,1468}$. Many PFTs rely on the presence of specific surface receptors to bind and insert. CDCs, for example, exploit the presence of cholesterol or other lipid raft components, making them quite specific for the plasma membrane of animal cells ${ }^{407}$. This cholesterol-specific action makes CDCs reminiscent of saponins in their selectivity. Owing to this specificity and their large pore size, CDCs are the PFTs that have primarily been used for intracellular delivery of larger cargo (>1 nm) and will be the focus of subsequent discussion in this section. 
A
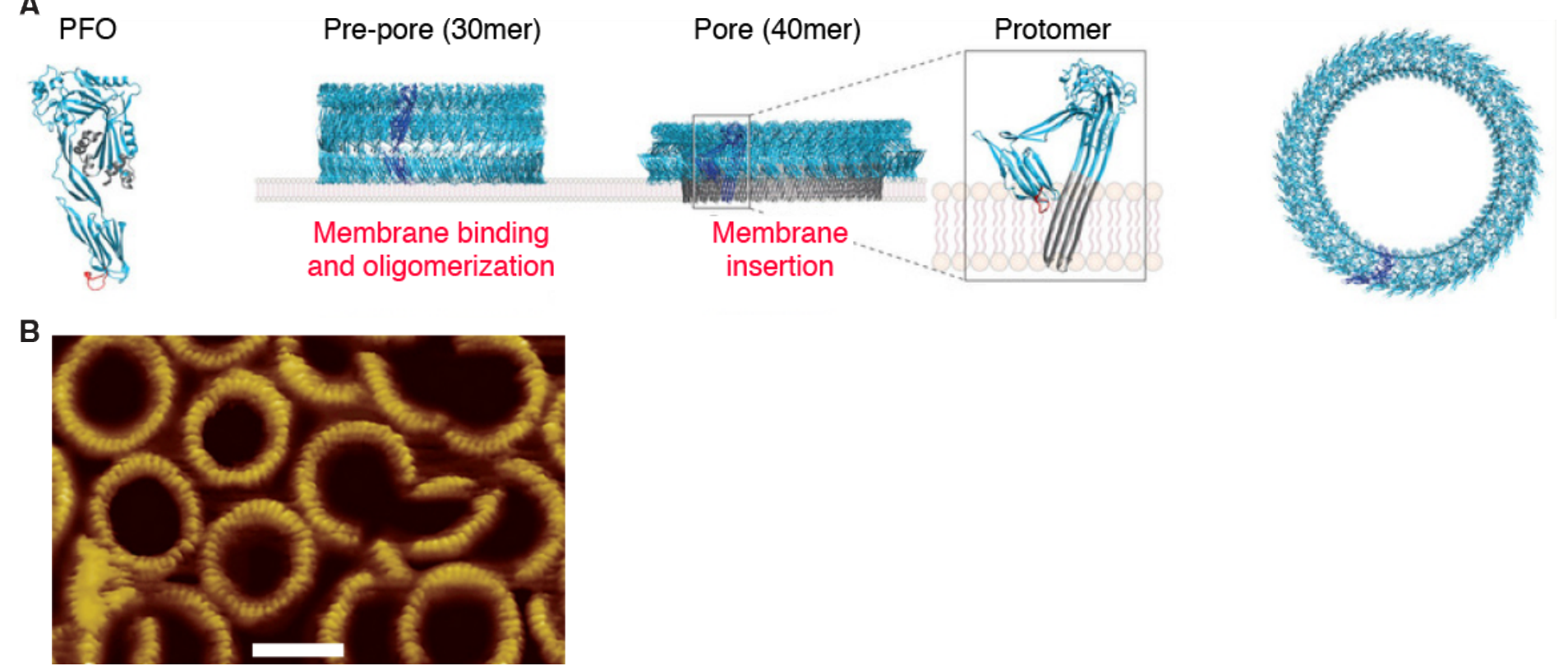

Figure 41. The structure of pores created by Perfringolysin O (PFO), a CDC pore-forming toxin. (A) PFO oligomerizes into large prepores, which, after an extended conformational change, form a membrane-inserted $\beta$-barrel pore. Top view of the pore is on the right. Figure adapted by permission from Springer Nature from ref ${ }^{439}$, Copyright 2015 . (B) AFM images of the PFO pore complexes in supported lipid bilayers that contain cholesterol. Scale bar $25 \mathrm{~nm}$. Figure reprinted from ref ${ }^{1467}$, Copyright 2004 , with permission from John Wiley and Sons.

\subsubsection{Cholesterol-Dependent Cytolysins for Intracellular Delivery}

The most widely used PFTs for permeabilization-mediated intracellular delivery are the cholesterol-dependent cytolysins (CDCs), of which Streptolysin O (SLO), Listeriolysin O (LLO) and Perfringolysin O (PFO) are the best-known examples. SLO is secreted by the bacteria Streptococcus pyogenes and has been used since the 1970 s to selectively permeabilize the plasma membrane for the study of intracellular processes in semi-intact cell models ${ }^{1469,1470}$. In the 1990 s SLO began to be used widely for intracellular delivery purposes ${ }^{1471}$. Barry et al. demonstrated that antisense phosphodiester oligodeoxynucleotides (ODN) could be introduced into cells during a brief permeabilization step with SLO ${ }^{1471}$. Cells were able to recover full function and showed maximum ODN-induced down regulation of gene expression at 18 hours before recovery to normal expression at 48 hours $^{1471}$. A subsequent study compared SLO-mediated permeabilization versus electroporation for delivery of a restriction enzyme, concluding that electroporation was more cytotoxic and SLO a better option for both $\mathrm{CHO}$ and human fibroblast cells ${ }^{1472}$. In their hands, SLO provided a more uniform permeabilization across the cell population, possibly because electroporation is to some extent cell size-dependent. In another comparative study, SLO treatment, electroporation, and lipid-carriers were tested for delivery of antisense ODNs that neutralize BCR-ABL mRNA to reduce protein expression ${ }^{1104}$. Contrasting the earlier report, greater variation in ODN uptake was seen for SLO 
permeabilized cells when compared with electroporated cells in the chronic myeloid leukemia model cell line KYO-1. The authors suggested that SLO exposure led to relatively under-permeabilized and over-permeabilized populations. Compared to SLO and electroporation, lipid delivery vehicles were found to be ineffective for KYO-1 cells. A separate study in primary rat ventricular myocytes used SLO to successfully deliver FITC-dextrans up to $148 \mathrm{kDa}$ and bovine albumin serum $(67 \mathrm{kDa})$, followed by full neutralization of toxin permeabilization and cell recovery ${ }^{1473}$.

In 2001, Bhakdi and co-workers published a report that significantly advanced our understanding of SLO-mediated intracellular delivery ${ }^{497}$. First, pre-titrated concentrations of high-quality SLO were administered to cells to determine precise concentrations for permeabilization in a variety of mammalian cell lines. Second, they deliberately employed calcium to trigger plasma membrane repair. With this approach, effective delivery of proteins and dextrans was achieved in $60-80 \%$ of cells with $>50 \%$ long-term viability. Third, they explored the size limits of cargo influx to estimate pore size. SLO permeabilization was able to deliver $150 \mathrm{kDa}$ dextrans but failed to mediate the passage of $250 \mathrm{kDa}$ dextrans (approximate diameter $\sim 23 \mathrm{~nm})^{866}$. These results suggest that SLO pores exhibit a cutoff size in the range $20 \mathrm{~nm}$. This is in reasonably good agreement with AFM images of another CDC family member perfringolysin O, which showed pore diameters of $\sim 25 \mathrm{~nm}^{1467}$. A fourth observation was that calmodulin activity, intact microtubules, and cytoplasmic ATP only returned to normal levels after $\sim 4$ hrs. Under various conditions screened, the method permitted proteins to be delivered to approximately $50 \%$ of the total cell population under near-full retention of viability, a performance level that has since been confirmed by others ${ }^{1474}$.

In subsequent studies it has been shown that delivery performance can be better for siRNA-mediated gene knockdown, where the molecule to be introduced is significantly smaller ( 13 kDa). Transfection with an optimized SLO permeabilization method showed $>80 \%$ RNAi-mediated knockdown in difficult to transfect myeloma cell lines (JIM-3, H929, RPMI8226 and U266 cell lines) with minimal effect on cell viability ( $<10 \%$ death) and cell cycle ${ }^{238}$. However, as noted by Bhakdi and colleagues ${ }^{497}$, several caveats exist for the use of SLO. Primary among them is that the quality of SLO preparations is important, because contaminations with proteases or DNAses may create deleterious artifacts. Due to variations in batch quality, the appropriate SLO concentration window usually needs to be pre-calibrated by titration experiments prior to cell treatment. Moreover, an oxygen-stable C530A substitution mutant obviates the need for a reducing agent to maintain SLO activity in the permeabilization buffer ${ }^{497}$. Thus, protein engineering efforts have 
contributed towards improved versions of pore-forming proteins for cell permeabilization. Despite these caveats, SLO permeabilization represents a relatively cheap, simple and effective method to introduce molecular cargo up to $\sim 20 \mathrm{~nm}$ into living cells. SLO has been used to perform cytoplasmic delivery of siRNA ${ }^{238,239,1475}$, antisense oligonucleotides $^{1104,1471,1476-1485}$, proteins ${ }^{102,121,497,1472,1473,1486-1488}$, peptides ${ }^{1474,1489,1490}$, cytoplasmic extracts ${ }^{508,540,1491-1506}$, dextrans $^{1473}$, PNA probes ${ }^{1507-1509}$, molecular beacons ${ }^{1089,1510-1516}$, photosensitizers ${ }^{1517}$, phosphatidic acid ${ }^{1518}, \mathrm{Rb}^{+}$ions $^{102}$, $\mathrm{ATP}^{102}$, various RNA probes ${ }^{1519-1521}$, lanthanum probes $^{72,1522}$ and gold nanoparticles ${ }^{1523}$.

Beyond SLO, permeabilization-based delivery with other CDC family members, such as LLO and PFO, have also been reported in the literature ${ }^{1524-1526}$. Recently LLO, which is produced by the bacteria Listeria monocytogenes, was found to be useful for delivery of small to mid-sized molecular cargo, $<10 \mathrm{kDa}^{1526}$. By screening for passage of dextrans of size 3 , 10, 40, 70, and $150 \mathrm{kDa}$, Murakami and co-authors found a size cutoff between 10 and $40 \mathrm{kDa}$. Thus, LLO-mediated permeabilization could be used to efficiently deliver the nucleotide analogue 8-OH-cAMP $(\sim 0.4 \mathrm{kDa}$, a PKA activator) and the small peptide Akt-in ( $2 \mathrm{kDa}$, an Akt inhibitor) inside cells without loss of proteins, such as GFP ( 28 kDa), from the cytosol ${ }^{1526}$.

\subsubsection{Pore-Forming Proteins as Endosome Disruptors}

There are a number of naturally occurring scenarios where organisms use pore-forming proteins to deliver cargo into target cells. So-called AB toxins can mediate this effect ${ }^{399}$. The B component permeabilizes membranes, often triggered by the acidic environment of endosomes, while the A subunit exerts separate enzymatic activity when unleashed into the cytoplasm $^{399}$. In other words, A is the cargo and B is the membrane disruptor. Under this principle, the vertebrate immune system has evolved perforins for the purpose of permeabilization to deliver toxic granzymes ${ }^{1527}$.

One model for how AB toxins operate was presented in an elegant study from Lieberman and colleagues. They observed that sublytic perforin permeabilization at the plasma membrane (featuring small $1-2 \mathrm{~nm}$ pores) induces endocytosis in response to calcium influx, thereby promoting endocytic uptake of the perforin plus cytotoxic granzymes ${ }^{1528}$. Perforins then lodge in the membrane of endosomes, inhibit maturation, and subsequently trigger rupture to release endosomal contents and cytotoxic granzymes, which then induce the death of target cells ${ }^{1528}$. In an analogous scenario, adenovirus 
employs the viral membrane lytic protein-VI to first generate small pores that trigger plasma membrane repair processes $^{1529}$. This is followed by its endocytosis into leaky compartments from which it, and potentially other viral components, can subsequently escape ${ }^{1529}$.

Recently, the natural AB-toxin mechanism has been repurposed for intracellular delivery through protein engineering efforts. Yang et al. showed that a neutralized version of perfringolysin (PFO) can be targeted to the EGF receptor of cancer cells and preferentially activated in endosomes to deliver toxic gelonin into the cytoplasm ${ }^{119}$. To do this, they designed a bi-specific antibody, where one terminal binds PFO while the other targets the EGF receptor for endocytosis. Once in endosomes, the acidic environment triggers PFO to disrupt the endosomal membrane. In another example of this strategy, Pentelute and colleagues showed that the protective antigen component of anthrax toxin generates a pore that can mediate egress of polypeptides, impermeable small molecule drugs, and antibody mimics from endosomes to the cytosol $^{1530}$. The power of these bio-inspired approaches is in their specificity against different types of membranes and endosomal compartments ${ }^{118}$. Such studies indicate the utility of pore-forming toxins and their components not just for plasma membrane permeabilization, but also controlled disruption of cargo-laden endosomes.

\subsubsection{Summary of Permeabilization by Pore-Forming Proteins \& Toxins}

Pore-forming proteins are produced by a wide range of organisms, many of which still remain to be characterized. While most of them produce small pores that are limited in their usefulness for intracellular delivery, some families, such as the cholesterol-dependent cytolysins, are commonly used for permeabilizing cell membranes to introduce molecular cargo. Limitations are similar to detergents, surfactants and membrane-active peptides, namely the delayed kinetics of pore formation /membrane disruption and the lack of spatiotemporal control. Significant improvements in cell treatment and intracellular delivery may be attainable if these problems are solved. Moreover, we anticipate that pore-forming proteins will continue to find utility in molecular conjugates and nanoparticles designed to overcome the various types of membranes barriers.

\subsubsection{Chemical Destabilization}


Chemical destabilization of lipid molecules can occur due to oxidative damage from a variety of sources. In fact, membrane disruptive lipid peroxidation events are thought to be a normal part of cell physiology. In a recent study, for example, endogenous production of reactive oxygen species (ROS) by the NOX2 enzyme mediates disruption of endosomal membranes to trigger leakage of antigens into the cytosol of dendritic cells for subsequent immune activation $^{1531}$. ROS and other free radicals cause peroxidation of lipid tails, which can lead to similar effects as those seen for surfactants, including distortion, buckling, curvature strain, and peeling off of micelles from lipid bilayers ${ }^{461,1532,1533}$. Common species of peroxidized lipids have been proposed to exist in two main classes: 1) phospatidylcholines with a hydroperoxide side chain, and 2) phospatidylcholines with oxidized and truncated chains terminated by an aldehyde or carboxylic group (Figure 42) ${ }^{1532}$. Lipid tails become more polar due to the presence of hydroperoxides, aldehyde or carboxyl groups. Consequently, these groups bend toward the water phase to allow hydrogen bonding with water and the lipid headgroups. The result of these distortions is an increase in area per lipid headgroup, which leads to membrane thinning, a decrease in lateral ordering, and membrane area expansion ${ }^{436}$.
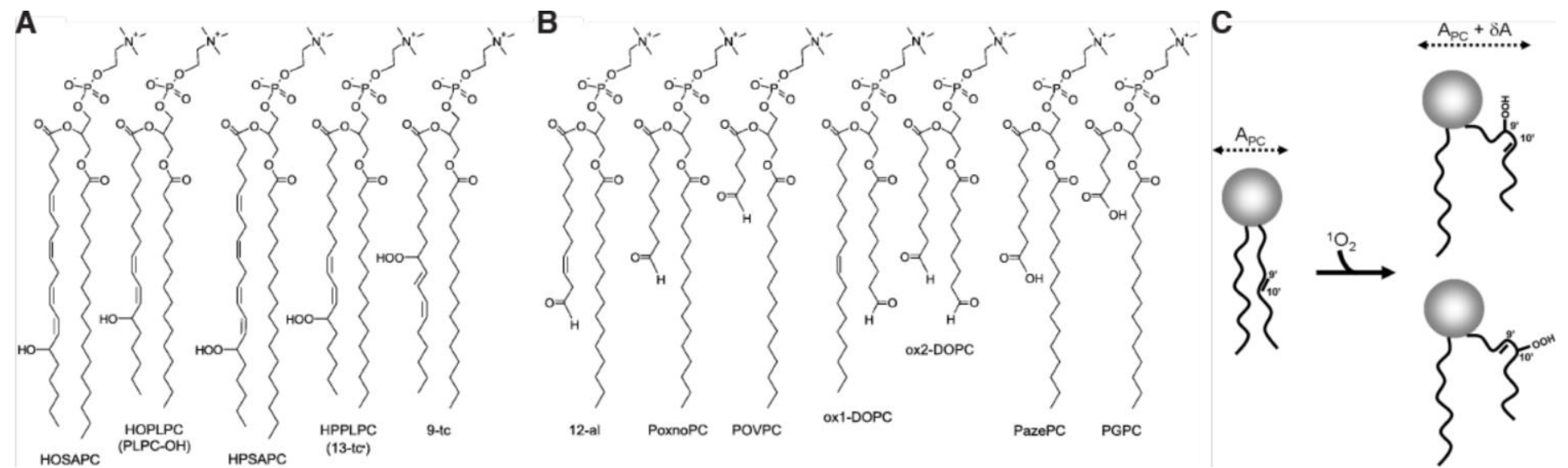

Figure 42. Chemical structures of oxidized phosphatidylcholines and their effects on bilayer packing. (A) Hydroxy- (HOSAPC and HOPLPC) and hydroperoxy-(HPSAPC, HPPLPC, and 9-tc) phospatidylcholines. Different cis/trans isomers are possible. 13-tc refers to trans-11, cis-9 isomer of HPPLPC. (B) Truncated (cleaved chain) phosphatidylcholines with aldehyde (12-al, PONPC, POVPC, ox1DOPC, and ox2-DOPC) and carboxylic (PAzPC and PGPC), functional groups. Figure reprinted from ref ${ }^{1532}$, Copyright 2012, with permission from Elsevier. (C) Example of conformation changes that lipid molecules undergo due to peroxidation. In this case singlet oxygen adds the more hydrophilic group - $\mathrm{OOH}$ at either 9 or 10 position, which migrates to the bilayer surface. This imposes a kink to the acyl chain, with an accompanying increase in area $\delta \mathrm{A}$ per lipid. Figure reprinted from ref ${ }^{436}$, Copyright 2009 , with permission from Elsevier.

Using GUVS as a model system, Riske et al. artificially converted the native lipid phospatidylcholine to an oxidized version with hydroperoxides groups at the 9 or 10 chain position (Figure 42C). This was accomplished by using a membrane-localized amphiphile photosensitizer that generates singlet oxidation under irradiation with visible light. They 
found a substantial increase in GUVs membrane surface area without membrane disruption or evidence of poration ${ }^{436}$. It was hypothesized that more intense treatment would eventually lead to compromisation of membrane integrity, just like with detergents. Compared to the oxidized lipids investigated by Riske et al., oxidized lipids with truncated chains featuring aldehydes or carboxyl termini are much more potent perturbants of membrane organization ${ }^{1532,1533}$. In the latter scenarios, simulations and experiments both observe pore formation and micellization as a function of concentration, concomitant with an increased susceptibility to bilayer rupture ${ }^{437,438}$.

\subsubsection{Confinement of Oxidative Damage through lonizing Plasmas}

How is it possible to confine lipid oxidation to subcellular regions? Under certain regimes, lasers exert a chemical oxidation effect on membranes through generation of near-field ionizing plasmas, as opposed to thermal or mechanical affects. For example, femtosecond lasers can produce near-field ionizing plasmas under specific intensities, pulse durations, and frequencies ${ }^{1321,1331}$ (see optoporation section 6.4 and figure 31D). Furthermore, near-field ionizing plasmas emanating from laser-irradiated gold nanoparticles have been proposed as a primary mechanism of membrane permeabilization in a recent study ${ }^{1534}$. Theoretical simulations and experiments both indicate that generation of a lowdensity plasma with multiphoton ionization of the surrounding environment perforates the cell membrane by oxidative effects. This strategy was reported to transfect siRNA into cells with $>90 \%$ efficiency and viability ${ }^{1534}$. Other delivery strategies that rely on fast pulse laser irradiation of metal nanoparticles or microscale features may work through a similar mechanism of plasma-induced damage ${ }^{310,923,925,926,929,930}$, and have been used to load cells with cargoes such as dyes, dextrans, siRNA, and quantum dots. The diffusive range of singlet oxygen species in aqueous environments has been estimated at about $100 \mathrm{~nm}^{436}$. Thus, local confinement of lipid oxidation may be feasible strategy for transient and precise membrane perforation without damage to the bulk of the cell.

\section{Gated Channels \& Valves}

So far, we have discussed membrane disruption approaches whereby cells recover through active plasma membrane repair (see section 4.3). In some cases, however, it may be possible to deliver cargo into cells by actuating opening and closing of 'windows' in the cell membrane. Such a strategy can be executed by external manipulation of native transmembrane 
proteins (e.g. channels and transporters), insertion of engineered molecular valves, or deployment of synthetic nanodevices.

\subsubsection{Endogenous Channels (ATP-activated)}

Since the 1980s several reports have demonstrated the influx of small molecules through the manipulation of particular endogenous membrane transporters and channels. Impermeable dyes have been observed to enter a number of cell types in the presence of high concentrations (up to $5 \mathrm{mM}$ ) of extracellular ATP ${ }^{1535}$. This is because ATP-gated channels permitting delivery are present in certain immortalized cell lines and primary immune cells ${ }^{1536}$. Steinberg et al. showed that only cargo of molecular weight less than 900 Dalton were able to enter cells in the presence of ATP ${ }^{1537}$. Specifically, it was found that ATP permeabilizes the plasma membrane of mouse macrophages to 6-carboxyfluorescein (376 Da), lucifer yellow (457 Da), and fura-2 (831 Da) but not to trypan blue (961 Da), evans blue (961 Da), or larger dye conjugates. These studies led to the idea that purinergic (i.e. ATP-mediated) activation of membrane channels can enable passage of cations and other small molecules. Toner and colleagues later used ATP-activated channels to load cells with trehalose ${ }^{1538}$, a 342 Da disaccharide with widespread applications in cryopreservation.

\subsubsection{Endogenous Channels (Swelling-activated)}

Osmotic swelling is another stimulus that can trigger the opening of mechanosensitive channels for influx of certain molecules. For example, osmotic swelling of Jurkat cells at 100 mOsm but not $200 \mathrm{mOsm}$ was found to trigger opening of channels for the delivery of monomeric sugars and sugar alcohols, but not larger molecules ${ }^{1539}$. It was found that extensive hypotonic swelling rendered the cell membrane permeable to PEG300-400, but not to PEG600-1500. By reference to the hydrodynamic radii of these PEG molecules, the size-selectivity of membrane permeation yielded an estimate of $\sim 0.74 \mathrm{~nm}$ for the cut-off radius of the swelling-activated channel ${ }^{1540}$. Further work identified SLC5A3 as an osmotically sensitive myo-inositol transporter that opens at imposed extracellular osmolarities of less than $200 \mathrm{mOsm}^{1541}$. Thus, this set of endogenous channels may be manipulated by osmotic stimuli to transport small molecules into cells. 


\subsubsection{Engineered Channels/Valves}

One of the first efforts towards engineering a switchable channel for intracellular delivery was reported by Toner, Bayley, and colleagues. Using a strategy that takes advantage of site-directed mutagenesis of $S$. Aureus $\alpha$-toxin, they developed a self-assembling, proteinaceous, $2 \mathrm{~nm}$ pore equipped with a $\mathrm{Zn}^{2+}$-actuated switch ${ }^{1542}$. Toxin monomers added to solution integrate into the plasma membranes of target cells and assemble to form an oligomeric pore complex. By adjusting the concentration of extracellular $\mathrm{Zn}^{2+}$, reversible permeabilization of the plasma membrane to small molecules $(1 \mathrm{kDa}$ or less) was achieved ${ }^{1542}$. In a follow-up study, the switchable pore was used to load trehalose at up to $0.5 \mathrm{M}$ concentration into fibroblasts ${ }^{77}$. These report were an intriguing demonstration of the idea that protein engineering could be leveraged to generate membranes with inbuilt permeability switches triggered by chemical, enzymatic, and physical stimuli ${ }^{1543,1544}$.

\subsubsection{Optogenetic Control of Cell Permeability}

The emergence of optogenetics heralded the concept of engineered light-activated transporters for manipulating cell permeability ${ }^{1545,1546}$. Kocer and colleagues modified the mechanosensitive channel of large conductance (MscL) from $E$. Coli into a light-addressable nanovalve sensitive to $366 \mathrm{~nm}$ UV irradiation ${ }^{1545}$. They verified the system by controlling the flux of calcein across proteoliposome membranes for both one-way and reversible nanovalves. In a parallel approach, Boyden et al. exploited the naturally occurring algal protein channelrhodopsin-2 as a rapidly gated light-sensitive cation channel in neurons ${ }^{1546}$. Lentiviral transduction was used to express these channels in neurons, whereby photostimulatation with blue light enabled cation influx and subsequent spatiotemporal actuation of neuron action potential firing, which was a long-sought goal in the field. Although limited to cations, this optogenetic proof of concept can conceivably be extended to a wider range of synthetic and bio-inspired nanovalves.

\subsubsection{Stimuli-Sensitive Channels for Larger Cargo Delivery}

Doerner et al. showed that the mechanosensitive bacterial MscL channel can be functionally expressed in mammalian cells to afford controlled uptake of membrane-impermeable molecules ${ }^{1547}$. The pore diameter of $>2.5 \mathrm{~nm}$ allows passage of large organic ions and small proteins up to $6.5 \mathrm{kDa}$. Furthermore, gating of the channel was found to be responsive to changes in membrane tension, both in native bacteria and mammalian cell membranes. To engineer more convenient gating, charges were engineered within the pore of MscL to induce spontaneous channel closure. The addition of charged 
methanethiosulphonate agents (such as MTSET) at $1 \mathrm{mM}$ were found to switch the channel between open and closed conformations. As a demonstration of utility, this system was exploited to load the bi-cyclic peptide phalloidin (789 Da) into $\mathrm{CHO}$ cells to label actin filaments.

\subsubsection{Nanodevice Gating}

More radical concepts for engineering switchable permeability have been demonstrated with synthetic nanodevices. Langecker et al. created an artificial membrane channel based on DNA origami nanostructures that anchor to the lipid membrane by cholesterol side chains (Figure 43A) ${ }^{1548}$. The shape of the DNA-based channel was inspired by the bacterial channel protein $\alpha$-hemolysin with some differences in physical properties such as charge, hydrophobicity, and size. Although not implemented in cells, future applications in cell membranes could include their deployment as antimicrobial agents, controlled interference of cellular homeostasis, or as intracellular delivery conduits ${ }^{1548}$.
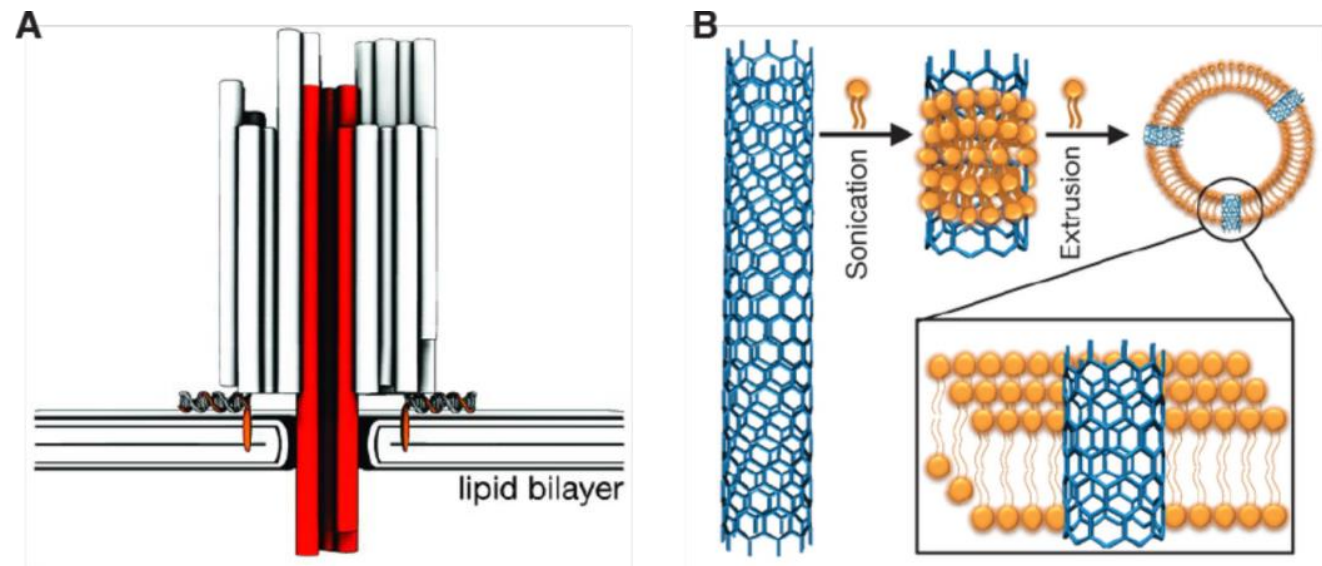

Figure 43. Synthetic nanodevices for use as membrane-embedded valves or channels. (A) DNA origami nanostructures assembled to form a membrane channel. Reprinted with permission from AAAS from ref ${ }^{1548}$. (B) Carbon nanotubes embedded within lipid bilayers for molecular transport. Figure reprinted by permission from Springer Nature from ref ${ }^{1549}$, Copyright 2014.

Carbon nanotubes (CNTs) represent another form of nanotechnology with engineering potential at the scale of the cell membrane. Geng et al. exploited the nature of their narrow hydrophobic inner pores that mimic structural motifs typical of biological channels ${ }^{1549}$. They developed a method to insert CNTs into lipid bilayers and live cell membranes to form conducting channels capable of transporting water, protons, small ions and DNA under physiological conditions (Figure 43B). It was found that the local channel and membrane charges control the conductance and ion selectivity of the CNT pores, thus suggesting potential starting points for engineering gating function. 
Recently one group devised molecular motors that can burrow through lipid membranes upon excitation with light ${ }^{1550}$. Upon physical adsorption of the molecular motors onto lipid bilayers and subsequent activation by ultraviolet light, holes were drilled in the cell membranes. They demonstrated intracellular delivery of the motors themselves, small molecule dyes such as PI, and accelerated cell death as a result of apoptosis or necrosis ${ }^{1550}$. Experimental results indicate an explanation based on the transduction of light energy into nanomechanical action rather than chemical or thermal effects $^{1550}$.

\section{Summary \& Outlook}

\subsubsection{Summary}

Motivations for better intracellular delivery range from basic research to the potential of therapeutic applications including cell-based therapies, gene therapy and regenerative medicine. Cargo of interest vary from small molecules that can naturally permeate the lipid bilayer to highly charged molecules and large complexes, genetic constructs, synthetic materials or organelles approaching the size of the cell itself. For the majority of these cargo, the plasma membrane is the primary barrier to intracellular delivery. Cells exhibit a distinct set of properties that can be exploited to overcome this barrier. For example, delivery methods can take advantage of the negative membrane potential, cholesterol-rich nature of the plasma membrane, or presence of specific extracellular receptors.

A broad assortment of approaches has been designed to deliver cargo into cells. They can be categorized as either carriermediated or membrane disruption-mediated strategies. Cells generally respond to the presence of carriers by processing them through endocytosis and other membrane trafficking pathways. On the other hand, they react to membrane disruption by deploying membrane repair processes to heal the plasma membrane and restore cell homeostasis. Due to their perturbing nature, most delivery strategies involve a trade-off between effective delivery and tolerable cell damage. Membrane disruption-mediated delivery strategies have the advantage of rapid and near-universal delivery of almost any cargo that can be dispersed in solution. The latest understanding of membrane repair pathways indicates that membrane disruption is a common event in the life of cells, and they are well equipped to deal with it. More challenging is the 
selection of appropriate membrane disruption modalities and their precise implementation to large batches of cells at high throughput. This is an engineering challenge that involves elements of both technological innovation and mechanistic understanding of the cell itself. Theories have been developed to explain defect formation in lipid bilayers and the phenomena that can be leveraged to achieve controlled disruption of cell membranes. In parallel, empirical studies have identified key modalities, such as electroporation and mechanical deformation, which can be deployed to achieve a relatively scalable and reproducible control of plasma membrane disruption.

Tables 5 and 6 summarize the membrane disruption approaches that have been discussed in this review. Table 5 lists each method with what is known about disruption mechanisms, size and distribution of resultant holes, treatment throughput, and whether it is applicable to adherent or suspension cells. If there is one theme that emerges from this analysis, it is that we still lack clear mechanistic understanding on how many membrane disruption-mediated intracellular delivery methods actually work. Indeed, many of the methods suffer from a lack of mechanistic insight to hone and optimize the salient parameters. Sonoporation is an example of a delivery strategy that has been challenging to optimize because of such complexity. In other cases, a membrane disruption method may work well but a lack of knowledge on appropriate environmental conditions leads to underperformance. For example, we have a limited understanding of how cell membranes behave and recover at different temperatures and osmolarities. Other methods feature clearly defined mechanisms but face intrinsic limitations because of the nature of the membrane disruption effect. For example, conventional electroporation and pore-forming toxins tend to generate membrane disruptions of less than $50 \mathrm{~nm}$ and are therefore limited in their ability to deliver large cargo.

Throughput and applicability to suspension or adherent cells are further considerations. In microinjection, for example, almost any cargo can be delivered to any cell type but only one at a time. The challenges involved in scale-up to high throughput are yet to be surmounted. Other methods, such as scrape loading, are low cost and high throughput but may lack consistency and precision across cell populations. In a further example, large cargo delivery can be accomplished with laser-controlled cavitation bubbles, but the systems require complex equipment and may only be applicable to adherent cells. Such a scenario rules out delivery to most immune and blood cells that naturally exist in suspension. Electroporation is currently the dominant high throughput method in the field. As covered in section 6.2.3, it has been demonstrated in applications ranging from testing of impermeable drugs and biomanufacture to engineering cells for 
cancer immunotherapy and stem cell-based gene therapy (Figure 29). However, electroporation is not without its limitations. Post-treatment cell death and inability to deliver large cargo are two such examples. Overall, no single method has a monopoly on all applications and further work is required to identify the optimal delivery strategies for a given application.

Table 6 compares membrane disruption approaches versus the cargoes they have been reported to deliver. It is important to note that many combinations have simply not been attempted. Moreover, many reports use a certain technique to deliver a particular cargo only because they adapt the protocol from an earlier publication. Thus, certain techniques seem to have an arbitrary emphasis on a particular cargo. For example, optoporation publications have tended to focus heavily on plasmid transfection while neglecting other cargoes. Filling out the table by screening all possible combinations would be extremely informative for the field. Comparisons of cost and cell type applicability would also add value to such an analysis and help to guide experimentalists toward optimal solutions. In future, we expect to see more publications move beyond trivial delivery of small molecules dyes $(<1 \mathrm{kDa})$ and showcase delivery of a smorgasbord of diverse cargo, especially proteins, nanomaterials, and larger cargo.

\subsubsection{Outlook}

Several membrane disruption-based methods are in widespread use in academic, industrial, and medical laboratories across the world, such as electroporation and microinjection. Yet the majority of modalities are either in nascent development or are yet to be pursued to their full potential. By identifying where the field can reduce costs and complexity, the potential exists to lower the barrier of entry to interdisciplinary scientists and researchers in resource-poor settings. This would no doubt strengthen global discovery. Overall, we believe that better and more streamlined intracellular delivery is more likely to arise out of a deeper understanding of current approaches and their capabilities.

The field has a number of frontiers where opportunities are ripe. One is the huge repository of unexplored membrane perturbing compounds in the form of natural and synthetic detergents, surfactants, pore-forming toxins, membrane-active peptides, and other secondary metabolites. Another is the rise of new microfluidic and nanotechnological tools that provide an unprecedented level of control to the membrane disruption process. This may be via high-throughput systems 
for mechanical deformation, such as microfluidic cell squeezing, or advanced fabrication of nanostructures, including nanowires and nanostraws. Combining the strengths of multiple modalities may be a prudent approach toward better technologies. For example, electroporation is biased toward producing small pores but provides a convenient electrophoretic force for the delivery of charged molecules. Methods that combine large disruption sizes with electrophoretic drive could potentially harness the benefits of both techniques. Future strategies could also be based on synthetic valves and nanodevices that embed within the membrane and enable remote control of permeability via external triggers. Light-gated methods that confer switchable control of membrane disruption are only beginning to be explored. In the coming years cost and convenience will be another important factor, as many of the current methods are either expensive or overly reliant on cumbersome equipment.

As our insight into membrane repair processes and cell recovery deepen, it may be possible to provide stimuli that switch membrane repair on and off, or to modulate stress responses that otherwise lead to untoward cell fate changes or death. How can we understand the energy landscape of defect formation to generate ideal membrane disruptions? What kinds of disruptions are optimal for delivery in specific cell-cargo combinations? How does the composition of external buffer determine which pathways are activated in response to permeabilization? The answers to these, and similar, questions will be more attainable with the establishment of better approaches to investigate plasma membrane homeostasis and the cellular response (Figure 11). Thus, along with technical advances in membrane disruption, our toolbox for studying cells must also improve.

For ex vivo cell-based therapies in particular, quality control procedures may be required to ensure the safety and efficacy of engineered cells. Methods for assessing DNA damage, fate changes, and cell functionality will possibly be required to avoid re-introduction of malignant or undesirable cells in cGMP settings. More accurate assays to evaluate cell function are expected to inform the appropriate and safe use of membrane disruption-based delivery methods going forward. Combined with further technological innovations in the way we disrupt membranes, we expect future progress in the field to catalyze breakthroughs in delivery applications ranging from fundamental research to ex vivo cell-based therapies. 
Table 5. Summary of membrane disruption approaches covered in this review. Several are widely used for intracellular delivery while others have barely been attempted. Techniques marked with red text represent methods that are either commercially available or accessible with common lab equipment.

\begin{tabular}{|c|c|c|c|c|c|c|}
\hline Modality & Methods & $\begin{array}{l}\text { Membrane Disruption } \\
\text { Mechanisms }\end{array}$ & $\begin{array}{l}\text { Spatial Distribution } \\
\text { Across Cell }\end{array}$ & Disruption Size & $\begin{array}{l}\text { Throughput / } \\
\text { Scalability }\end{array}$ & $\begin{array}{l}\text { Suspension / } \\
\text { Adherent }\end{array}$ \\
\hline \multicolumn{7}{|c|}{ DIRECT PENETRATION } \\
\hline \multirow[t]{3}{*}{ Mechanical } & Microinjection & \multirow[t]{3}{*}{$\begin{array}{l}\text { Mechanical forces at } \\
\text { contact zone. Membranes } \\
\text { only tolerate } 2-3 \% \text { lateral } \\
\text { strain }^{425} \text {. Can be strain } \\
\text { rate dependent (see } \\
\text { Figure } 9 \text { ) and refs } 421,1551\end{array}$} & \multirow[t]{3}{*}{ At contact zone } & $\begin{array}{l}\text { Depends on size } \\
\text { of injection tip, } \\
\text { usually } 0.3-1 \mu \mathrm{m}\end{array}$ & $\begin{array}{l}\text { Low, could be } \\
\text { improved via } \\
\text { automation }\end{array}$ & $\begin{array}{l}\text { Mostly } \\
\text { adherent. } \\
\text { Suspension } \\
\text { cells require } \\
\text { secondary } \\
\text { holding } \\
\text { pipette }\end{array}$ \\
\hline & $\begin{array}{l}\text { Penetrating } \\
\text { Projectiles } \\
\text { (Biolistics) }\end{array}$ & & & $\begin{array}{l}\text { Depends on size } \\
\text { of projectile - } \\
\text { usually micron- } \\
\text { size }\end{array}$ & $\begin{array}{l}\text { Potentially } \\
\text { high }\end{array}$ & $\begin{array}{l}\text { Primarily } \\
\text { adherent. } \\
\text { Some reports } \\
\text { on } \\
\text { suspension } \\
\text { cells }\end{array}$ \\
\hline & $\begin{array}{l}\text { Nanowires, } \\
\text { Nanoneedles \& } \\
\text { Nanostraws }\end{array}$ & & & $\begin{array}{l}\text { Depends on size } \\
\text { of tip: reported } \\
\text { range } 50-1000 \\
\mathrm{~nm}\end{array}$ & $\begin{array}{l}\text { Potentially } \\
\text { high }\end{array}$ & $\begin{array}{l}\text { Mostly } \\
\text { adherent. } \\
\text { Suspension } \\
\text { cells must be } \\
\text { forced onto } \\
\text { the array }\end{array}$ \\
\hline \multicolumn{7}{|c|}{ PERMEABILIZATION } \\
\hline \multirow{6}{*}{$\begin{array}{l}\text { Mechanical } \\
\text { (Solid Contact) }\end{array}$} & Cell Scraping & \multirow{5}{*}{$\begin{array}{l}\text { Mechanical forces } \\
\text { transmitted by direct } \\
\text { contact or cell } \\
\text { deformation. Membranes } \\
\text { only tolerate } 2-3 \% \text { lateral } \\
\text { strain }^{425} \text {. Can be strain } \\
\text { rate dependent (see } \\
\text { Figure 9) and refs } 421,1551\end{array}$} & \multirow{5}{*}{$\begin{array}{l}\text { Presumably at } \\
\text { contact zone } \\
\text { otherwise at weak } \\
\text { points/defects due } \\
\text { to global membrane } \\
\text { strain }\end{array}$} & \multirow{5}{*}{$\begin{array}{l}\text { Probably depends } \\
\text { on force, strain } \\
\text { rate, size of } \\
\text { contact zone, } \\
\text { direction of strain }\end{array}$} & High & Adherent \\
\hline & Bead Loading & & & & High & Adherent \\
\hline & Scratch Loading & & & & Low/Medium & Adherent \\
\hline & $\begin{array}{l}\text { Microfluidic Cell } \\
\text { Squeezing / } \\
\text { Constriction- } \\
\text { Mediated Cell } \\
\text { Deformation }\end{array}$ & & & & High & Suspension \\
\hline & $\begin{array}{l}\text { Nanowire } \\
\text { Permeabilization }\end{array}$ & & & & $\begin{array}{l}\text { Potentially } \\
\text { high }\end{array}$ & Adherent \\
\hline & $\begin{array}{l}\text { Sudden Cell Shape } \\
\text { Changes / Protease } \\
\text { Treatments }\end{array}$ & $\begin{array}{l}\text { Possibly tearing forces at } \\
\text { adhesion sites }\end{array}$ & $\begin{array}{l}\text { Possibly at adhesion } \\
\text { sites }\end{array}$ & Unknown & $\begin{array}{l}\text { Potentially } \\
\text { high }\end{array}$ & Adherent \\
\hline \multirow[t]{3}{*}{$\begin{array}{l}\text { Mechanical } \\
\text { (Fluid Shear) }\end{array}$} & $\begin{array}{l}\text { Syringe Loading / } \\
\text { Microfluidic / Bulk } \\
\text { Fluid Shear }\end{array}$ & $\begin{array}{l}\text { Fluid shear forces causing } \\
\text { membrane strain }\end{array}$ & Unknown & Unknown & $\begin{array}{l}\text { Potentially } \\
\text { high }\end{array}$ & Suspension \\
\hline & $\begin{array}{l}\text { Sonoporation / } \\
\text { Shockwaves }\end{array}$ & \multirow{2}{*}{$\begin{array}{l}\text { Stable Cavitation } \\
\text { (Microstreaming), Inertial } \\
\text { Cavitation (Jetting), or } \\
\text { other Acoustic Effects }\end{array}$} & \multirow{2}{*}{$\begin{array}{l}\text { Presumably a single } \\
\text { hole per cavitation } \\
\text { bubble }\end{array}$} & \multirow{2}{*}{$\begin{array}{l}\text { From nanometers } \\
\text { to several microns } \\
\text { depending on } \\
\text { cavitation } \\
\text { intensity and } \\
\text { stand-off distance }\end{array}$} & High & Both \\
\hline & $\begin{array}{l}\text { Laser-Induced } \\
\text { Cavitation }\end{array}$ & & & & $\begin{array}{l}\text { Medium to } \\
\text { High }\end{array}$ & Both \\
\hline \multirow{3}{*}{$\begin{array}{l}\text { Mechanical } \\
\text { (Pressure) }\end{array}$} & Hypo-Osmotic Shock & \multirow{3}{*}{$\begin{array}{l}\text { Mechanical forces } \\
\text { transmitted by } \\
\text { osmotic/hydrostatic } \\
\text { pressure. Membranes } \\
\text { only tolerate } 2-3 \% \text { lateral } \\
\text { strain }^{425} \text {. Can be strain } \\
\text { rate dependent (see } \\
\text { Figure } 9 \text { ) and refs }{ }^{421,1551}\end{array}$} & \multirow{3}{*}{$\begin{array}{l}\text { Presumably at weak } \\
\text { points or nucleating } \\
\text { at membrane } \\
\text { defects }\end{array}$} & \multirow[b]{2}{*}{$\begin{array}{l}\text { Probably } \\
\text { depending on } \\
\text { membrane } \\
\text { reservoirs, } \\
\text { attachment / } \\
\text { reinforcement of } \\
\text { membrane, and } \\
\text { magnitude / rate } \\
\text { of pressure }\end{array}$} & High & Both \\
\hline & Hydrostatic Pressure & & & & High & Both \\
\hline & $\begin{array}{l}\text { Osmotic Rupture of } \\
\text { Endosomes }\end{array}$ & & & $\begin{array}{l}\text { Limited by } \\
\text { endosome }\end{array}$ & High & Both \\
\hline Electroporation & Conventional & Probability of defect & At cell poles. More & Nucleate as small & High & Primarily \\
\hline
\end{tabular}




\begin{tabular}{|c|c|c|c|c|c|c|}
\hline & Electroporation & \multirow{3}{*}{$\begin{array}{l}\text { formation for given pulse- } \\
\text { strength duration at a } \\
\text { given temperature. See } \\
\text { section 6.2.1 for details. }\end{array}$} & $\begin{array}{l}\text { permeabilization } \\
\text { expected on } \\
\text { hyperpolarized side }\end{array}$ & \multirow{3}{*}{$\begin{array}{l}\text { defects then grow } \\
\text { as a function of } \\
\text { voltage and } \\
\text { duration }\end{array}$} & & $\begin{array}{l}\text { Suspension, } \\
\text { but Adherent } \\
\text { also possible }\end{array}$ \\
\hline & $\begin{array}{l}\text { Micro- } \\
\text { Electroporation }\end{array}$ & & $\begin{array}{l}\text { Depends on } \\
\text { geometry }\end{array}$ & & $\begin{array}{l}\text { Potentially } \\
\text { high }\end{array}$ & $\begin{array}{l}\text { Primarily } \\
\text { Suspension }\end{array}$ \\
\hline & $\begin{array}{l}\text { Nano- } \\
\text { Electroporation }\end{array}$ & & $\begin{array}{l}\text { Usually single hole } \\
\text { at nano-aperture }\end{array}$ & & $\begin{array}{l}\text { Currently Low } \\
\text { / Medium }\end{array}$ & $\begin{array}{l}\text { Both, } \\
\text { depending on } \\
\text { system }\end{array}$ \\
\hline \multirow[t]{4}{*}{ Thermal } & Freeze-Thaw & $\begin{array}{l}\text { Expansive mechanical } \\
\text { strain due to ice crystal } \\
\text { formation }\end{array}$ & $\begin{array}{l}\text { Location of ice } \\
\text { crystals }\end{array}$ & $\begin{array}{l}\text { Presumably } \\
\text { variable }\end{array}$ & High & Both \\
\hline & $\begin{array}{l}\text { Rapid Temperature } \\
\text { Transitions / Cycles }\end{array}$ & $\begin{array}{l}\text { Defect formation due to } \\
\text { phase transitions }\end{array}$ & $\begin{array}{l}\text { Probably near lipid } \\
\text { domain boundaries } \\
\text { and protein clusters }\end{array}$ & $\begin{array}{l}\text { Presumably small } \\
\text { defects }\end{array}$ & High & Both \\
\hline & $\begin{array}{l}\text { Supraphysiological } \\
\text { Heating }\end{array}$ & $\begin{array}{l}\text { Dissociation of bilayer } \\
\text { structure leading to } \\
\text { defect formation }\end{array}$ & $\begin{array}{l}\text { Site of maximal } \\
\text { heat }\end{array}$ & $\begin{array}{l}\text { Presumably small } \\
\text { defects }\end{array}$ & High & Both \\
\hline & $\begin{array}{l}\text { Laser Absorption at } \\
\text { Membrane or } \\
\text { Absorbent Particle / } \\
\text { Structure }\end{array}$ & $\begin{array}{l}\text { Absorption causes high } \\
\text { local temperature to } \\
\text { trigger membrane } \\
\text { disruption }\end{array}$ & $\begin{array}{l}\text { Laser focal point or } \\
\text { location of } \\
\text { absorbent structure }\end{array}$ & $\begin{array}{l}\text { Presumably } \\
\text { variable } \\
\text { depending on } \\
\text { local temperature } \\
\text { effects }\end{array}$ & High & Both \\
\hline Optoporation & Laser Optoporation & $\begin{array}{l}\text { Can be a mix of: } \\
\text { - Chemical (low energy } \\
\text { ionizing plasma) } \\
\text { - Mechanical (cavitation, } \\
\text { shock waves, } \\
\text { thermoelastic stress) } \\
\text { - Thermal (Heat in focal } \\
\text { region) }\end{array}$ & $\begin{array}{l}\text { Maximal in focal } \\
\text { region. Usually one } \\
\text { hole }\end{array}$ & $\begin{array}{l}\text { Depending on } \\
\text { parameters and } \\
\text { mechanisms. } \\
\text { Nanometers to } \\
\text { several microns }\end{array}$ & $\begin{array}{l}\text { Low to high - } \\
\text { limited by } \\
\text { laser focusing } \\
\text { approach }\end{array}$ & $\begin{array}{l}\text { Primarily } \\
\text { Adherent, but } \\
\text { suspension } \\
\text { also possible }\end{array}$ \\
\hline \multirow[t]{6}{*}{ Biochemical } & $\begin{array}{l}\text { Organic Solvents \& } \\
\text { Penetration } \\
\text { Enhancers }\end{array}$ & $\begin{array}{l}\text { Perturb bilayer structure } \\
\text { by burying their } \\
\text { hydrophobic residues into } \\
\text { the membrane }\end{array}$ & $\begin{array}{l}\text { Indiscriminate in } \\
\text { bulk, otherwise } \\
\text { depends on local } \\
\text { concentration }\end{array}$ & $\begin{array}{l}\text { Presumably small } \\
\text { defects then } \\
\text { disintegration of } \\
\text { the whole bilayer } \\
\text { at high } \\
\text { concentration }\end{array}$ & High & Both \\
\hline & $\begin{array}{l}\text { Detergents / } \\
\text { Surfactants: Generic }\end{array}$ & $\begin{array}{l}\text { Insert into bilayer and } \\
\text { distort the structure, } \\
\text { leading to defects, pore } \\
\text { formation, and } \\
\text { micellization }\end{array}$ & $\begin{array}{l}\text { Indiscriminate in } \\
\text { bulk, otherwise } \\
\text { depends on local } \\
\text { concentration }\end{array}$ & $\begin{array}{l}\text { Presumably small } \\
\text { defects then } \\
\text { disintegration of } \\
\text { the whole bilayer } \\
\text { at high } \\
\text { concentration }\end{array}$ & High & Both \\
\hline & $\begin{array}{l}\text { Detergents: Saponin } \\
\text { Family }\end{array}$ & $\begin{array}{l}\text { Extracts cholesterol out of } \\
\text { the bilayer core to form a } \\
\text { surface complex, induces } \\
\text { curvature and defect/pore } \\
\text { formation }\end{array}$ & $\begin{array}{l}\text { Cholesterol rich } \\
\text { sites. Indiscriminate } \\
\text { in bulk, otherwise } \\
\text { depends on local } \\
\text { concentration }\end{array}$ & $\begin{array}{l}\text { From nanometers } \\
\text { to micron }\end{array}$ & High & Both \\
\hline & $\begin{array}{l}\text { Pore-Forming } \\
\text { Toxins: CDC Family }\end{array}$ & $\begin{array}{l}\text { Insertion and } \\
\text { oligomerization into pore } \\
\text { structure in cholesterol- } \\
\text { rich membranes }\end{array}$ & $\begin{array}{l}\text { Cholesterol rich } \\
\text { sites. Indiscriminate } \\
\text { in bulk, otherwise } \\
\text { depends on local } \\
\text { concentration }\end{array}$ & $<30 \mathrm{~nm}$ & High & Both \\
\hline & $\begin{array}{l}\text { Membrane-Active } \\
\text { Peptides }\end{array}$ & $\begin{array}{l}\text { Adopt active } \\
\text { conformation upon } \\
\text { membrane binding. } \\
\text { Concentration dependent } \\
\text { aggregation / insertion }\end{array}$ & $\begin{array}{l}\text { Depend on } \\
\text { membrane } \\
\text { composition. } \\
\text { Indiscriminate in } \\
\text { bulk, otherwise } \\
\text { depends on local } \\
\text { concentration }\end{array}$ & $\begin{array}{l}\text { Presumably small } \\
\text { defects }\end{array}$ & High & Both \\
\hline & Lipid Peroxidation & $\begin{array}{l}\text { Lipid peroxidation leads to } \\
\text { structural interference / }\end{array}$ & $\begin{array}{l}\text { Depends on source } \\
\text { of oxidation. If local, }\end{array}$ & $\begin{array}{l}\text { Presumably small } \\
\text { defects, but large }\end{array}$ & High & Both \\
\hline
\end{tabular}




\begin{tabular}{|c|c|c|c|c|c|c|}
\hline & & $\begin{array}{l}\text { distortion of membranes } \\
\text { to form pores and defects }\end{array}$ & can be confined & $\begin{array}{l}\text { holes are } \\
\text { conceivable }\end{array}$ & & \\
\hline \multirow[t]{2}{*}{$\begin{array}{l}\text { Gated Channels } \\
\text { \& Valves }\end{array}$} & $\begin{array}{l}\text { Endogenous or } \\
\text { Engineered } \\
\text { Membrane } \\
\text { Transporters / } \\
\text { Channels }\end{array}$ & $\begin{array}{l}\text { Appropriate stimuli (e.g. } \\
\text { mechanical, chemical, } \\
\text { optical) to "gate" opening } \\
\text { and closing activity }\end{array}$ & $\begin{array}{l}\text { Depends on } \\
\text { location of the } \\
\text { membrane } \\
\text { transporters / } \\
\text { channels }\end{array}$ & $\begin{array}{l}\text { Limited by size of } \\
\text { the channel. } \\
\text { Usually only } \\
\text { amenable for } \\
\text { transport of small } \\
\text { molecules }<1 \mathrm{kDa}\end{array}$ & High & Both \\
\hline & $\begin{array}{l}\text { Synthetic } \\
\text { Nanodevices }\end{array}$ & $\begin{array}{l}\text { Insertion of constructs } \\
\text { into host membrane. } \\
\text { Gating may be engineered }\end{array}$ & $\begin{array}{l}\text { Depends on } \\
\text { location of the } \\
\text { nanodevices within } \\
\text { host membrane }\end{array}$ & $\begin{array}{l}\text { Limited by size of } \\
\text { the engineered } \\
\text { central channel }\end{array}$ & $\begin{array}{l}\text { Potentially } \\
\text { High }\end{array}$ & Both \\
\hline
\end{tabular}


Table 6. Cargo loaded versus membrane disruption approach. Techniques marked with red text represent methods that are either commercially available or accessible with common lab equipment. Cargoes are ordered across the table in approximate size order.

\begin{tabular}{|c|c|c|c|c|c|c|c|c|c|}
\hline \multirow[t]{3}{*}{ MODALITY } & \multirow[t]{3}{*}{ METHOD } & \multicolumn{8}{|c|}{ CARGO } \\
\hline & & \multirow{2}{*}{$\begin{array}{l}\text { Small } \\
\text { Molecule } \\
\text { Drugs / } \\
\text { Probes / } \\
\text { Dyes / } \\
\text { Sugars / } \\
\text { lons etc. }\end{array}$} & \multirow{2}{*}{$\begin{array}{l}\text { Peptides / } \\
\text { Proteins / } \\
\text { Antibodies / } \\
\text { RNPs etc. }\end{array}$} & \multirow{2}{*}{$\begin{array}{l}\text { Generic } \\
\text { Macro- } \\
\text { molecules } \\
\text { (e.g. } \\
\text { Dextrans) }\end{array}$} & \multicolumn{3}{|c|}{ Nucleic Acids and their Analogues } & \multirow{2}{*}{$\begin{array}{l}\text { Synthetic } \\
\text { Nano- } \\
\text { materials / } \\
\text { qDots / } \\
\text { CNTs etc. }\end{array}$} & \multirow{2}{*}{$\begin{array}{l}\text { Large Cargo: } \\
\text { Bacteria, } \\
\text { Organelles, } \\
\text { Beads etc. }\end{array}$} \\
\hline & & & & & Oligos & mRNA & vector DNA & & \\
\hline \multicolumn{10}{|c|}{ DIRECT PENETRATION } \\
\hline \multirow[t]{4}{*}{ Mechanical } & $\begin{array}{l}\text { Micro- } \\
\text { injection }\end{array}$ & $\begin{array}{l}\text { Dyes }^{595}, \\
\text { Mercury }^{324}, \\
\text { Trehalose }^{155} \\
2\end{array}$ & $\begin{array}{l}\text { Proteins } \\
82-86,604,1553, \\
\text { Antibodies }{ }^{15} \\
54, \\
\text { Peptides } \\
5503,1 \\
\text {, Cas9 } \\
\text { protein / } \\
\text { RNP149 }\end{array}$ & $\begin{array}{l}\text { Dextrans }{ }^{15} \\
56\end{array}$ & $\begin{array}{l}\text { Antisense } \\
\text { Oligonucle } \\
\text { otides }^{211,60} \\
{ }^{8} \text {, siRNA } \\
\end{array}$ & $\begin{array}{l}\mathrm{mRNA}^{240,15} \\
57\end{array}$ & $\begin{array}{l}\text { pDNA }^{200-} \\
202,607, \text { viral } \\
\text { DNA }^{200,606,1} \\
558\end{array}$ & $\begin{array}{l}\text { qDots }{ }^{294,1559,} \\
\text { 1560, MW- } \\
\text { CNTs }^{315}, \\
\text { SW-CNTs }\end{array}$ & $\begin{array}{l}\text { Bacteria }{ }^{324} \text {, } \\
\text { Nuclear } \\
\text { transplant } \\
\text { 6-32 } \\
\text { Chromosom } \\
\text { e } \\
\text { transplant } 33 \\
\text { 3, Sperm / } \\
\text { IVF }^{331,332,} \\
\text { Mito- } \\
\text { chondria }^{336} \text {, } \\
\text { Artificial } \\
\text { vesicles }{ }^{1561} \\
\text { Beads }{ }^{1562,156} \\
\text { 3, } \\
\text { Superparam } \\
\text { agnetic } \\
\text { beads }^{357}, \\
\text { Silicon } \\
\text { barcodes }^{358}\end{array}$ \\
\hline & $\begin{array}{l}\text { Penetrating } \\
\text { Projectiles } \\
\text { (Biolistics) }\end{array}$ & $\begin{array}{l}\text { Dyes }^{660,1564} \\
\text { Indicators }^{659}\end{array}$ & $\begin{array}{l}\text { Proteins }^{667-} \\
669, \text { Cas9 } \\
\text { protein / } \\
\text { RNP670 }\end{array}$ & & $\begin{array}{l}\operatorname{siRNA} 665,66 \\
6\end{array}$ & $\begin{array}{l}\text { Cas9 } \\
\text { mRNA }^{1565}, \\
\text { mRNA }^{662-} \\
664\end{array}$ & $\begin{array}{l}\text { pDNA }^{394,642,} \\
674,675\end{array}$ & $\begin{array}{l}\text { PEBBLE } \\
\text { nano- } \\
\text { sensors }^{1566}\end{array}$ & $\begin{array}{l}\text { Beads }^{351,353,} \\
\text { Latex } \\
\text { particles }^{352}\end{array}$ \\
\hline & $\begin{array}{l}\text { Nanowires } \\
\text { \& Nano- } \\
\text { needles }\end{array}$ & $\begin{array}{l}\text { Drugs }^{110}, \\
\text { Molecular } \\
\text { Beacons }^{683}\end{array}$ & $\begin{array}{l}\text { Proteins }{ }^{110,68} \\
0, \\
\text { Peptides }{ }^{110}, \\
\text { Cre } \\
\text { Recombinas } \\
\mathrm{e}^{682}, \\
\text { Antibodies } \\
7\end{array}$ & & $\begin{array}{l}\operatorname{siRNA}^{110,68} \\
0,681\end{array}$ & & $\begin{array}{l}\text { pDNA }^{109,110,} \\
676,684,713,156 \\
7\end{array}$ & $\begin{array}{l}\text { qDots }{ }^{684}, \\
\text { DNA Nano- } \\
\text { cages }^{685}\end{array}$ & \\
\hline & Nanostraws & $\begin{array}{l}\text { Dyes }^{700,702,} \\
\text { Co }^{2+} \text { ions } \\
\text { Ca }^{2+} \text { ions } \\
\end{array}$ & Proteins $^{707}$ & $\begin{array}{l}\text { Dextrans }{ }^{70} \\
1,707\end{array}$ & & & $\begin{array}{l}\text { pDNA }^{700,702,} \\
707\end{array}$ & qDots ${ }^{706}$ & \\
\hline \multicolumn{10}{|c|}{ PERMEABILIZATION } \\
\hline \multirow[t]{2}{*}{$\begin{array}{l}\text { Mechanical } \\
\text { (Solid } \\
\text { Contact) }\end{array}$} & Cell Scraping & Dyes $^{760-762}$ & $\begin{array}{l}\text { Proteins }^{96,543} \\
, 745-751, \\
\text { Antibodies }^{75} \\
2-754 \\
\text { Peptides } \\
56\end{array}$ & $\begin{array}{l}\text { Dextrans }{ }^{96,} \\
758, \\
\text { Lipopolysa } \\
\text { ccharide }^{759}\end{array}$ & $\begin{array}{l}\text { Antisense } \\
\text { Morpholin } \\
\text { os }^{757,1568-} \\
1570\end{array}$ & & pDNA $^{545}$ & & \\
\hline & $\begin{array}{l}\text { Bead } \\
\text { Loading }\end{array}$ & $\begin{array}{l}\text { Nucleotides }^{7} \\
31,956, \text { RNA } \\
\text { probes }^{1571} \text {, } \\
\text { PNA } \\
\text { probes }^{741} \text {, } \\
\text { SNAP- }\end{array}$ & $\begin{array}{l}\text { Proteins }{ }^{735-} \\
737, \text { Fab } \\
\text { Fragments }{ }^{73} \\
9,740, \\
\text { Antibodies } \\
\text { 2-734, }\end{array}$ & $\begin{array}{l}\text { Dextrans }^{97}, \\
735,1572\end{array}$ & & & $\mathrm{pDNA}^{729}$ & $\begin{array}{l}\text { qDots }^{744} \\
\text { SW-CNTs }^{743}\end{array}$ & \\
\hline
\end{tabular}




\begin{tabular}{|c|c|c|c|c|c|c|c|c|c|}
\hline & & $\begin{array}{l}\text { reactive } \\
\text { dyes }^{742}\end{array}$ & Peptides $^{738}$ & & & & & & \\
\hline & $\begin{array}{l}\text { Scratch } \\
\text { Loading }\end{array}$ & Dyes $^{764}$ & & $\begin{array}{l}\text { Dextrans }{ }^{76} \\
3\end{array}$ & & & & qDots $^{744}$ & \\
\hline & $\begin{array}{l}\text { Microfluidic } \\
\text { Cell } \\
\text { Squeezing / } \\
\text { Constriction- } \\
\text { Mediated } \\
\text { Cell Deform- } \\
\text { ation }\end{array}$ & $\begin{array}{l}\text { Dyes }^{108,777,79} \\
3, \text { Tags }^{785} \\
\text { Drugs }^{784}\end{array}$ & $\begin{array}{l}\text { Proteins }{ }^{108,78} \\
0,781,785,787, \\
\text { Cas9 } \\
\text { RNPs }^{152,793,79} \\
4\end{array}$ & $\begin{array}{l}\text { Dextrans }{ }^{10} \\
8,152,777,780,7 \\
88,794\end{array}$ & $\begin{array}{l}\text { siRNA }^{108,15} \\
2,780,788,792,7 \\
{ }^{94}, \text { tRNA }^{783}\end{array}$ & $\begin{array}{l}\text { mRNA }^{108,78} \\
7\end{array}$ & $\begin{array}{l}\text { pDNA }^{152,777,} \\
787,792-794\end{array}$ & $\begin{array}{l}\text { qDots }^{308} \\
\text { CNTs }^{108} \\
\text { DNA } \\
\text { nanostructu } \\
\text { res }^{794}\end{array}$ & \\
\hline & $\begin{array}{l}\text { Nanowire } \\
\text { Permeab- } \\
\text { ilization }\end{array}$ & Dyes $^{714,719}$ & $\begin{array}{l}\text { Antibodies } 69 \\
4\end{array}$ & $\begin{array}{l}\text { Dextrans }{ }^{80} \\
0\end{array}$ & & & $\begin{array}{l}\text { Lipid-pDNA } \\
\text { complexes }^{6} \\
94, \text { pDNA }^{800}\end{array}$ & qDots $^{694}$ & $\begin{array}{l}\sim 200 \mathrm{~nm} \\
\text { polystyrene } \\
\text { beads }^{694}\end{array}$ \\
\hline & $\begin{array}{l}\text { Sudden Cell } \\
\text { Shape } \\
\text { Changes / } \\
\text { Protease } \\
\text { Treatment }\end{array}$ & Dyes $^{769}$ & $\begin{array}{l}\text { Proteins }^{768,77} \\
1,773,774, \\
\text { Peptides }^{768}\end{array}$ & $\begin{array}{l}\text { Dextrans }{ }^{76} \\
6,768,1573\end{array}$ & $\begin{array}{l}\text { Oligonucle } \\
\text { otides }^{768} \text {. }\end{array}$ & & & & \\
\hline \multirow[t]{4}{*}{$\begin{array}{l}\text { Mechanical } \\
\text { (Fluid Shear) }\end{array}$} & $\begin{array}{l}\text { Syringe } \\
\text { Loading / } \\
\text { Microfluidic } \\
\text { / Bulk Fluid } \\
\text { Shear }\end{array}$ & $\begin{array}{l}\text { Dyes }^{824} \text {, } \\
\text { Nucleotides } \\
05,806\end{array}$ & $\begin{array}{l}\text { Proteins } \\
\text { 2,814-818,81, } \\
\text { Antibodies }^{80} \\
9-811,813,819\end{array}$ & $\begin{array}{l}\text { Dextrans } 98, \\
807,821,823,82 \\
5\end{array}$ & $\begin{array}{l}\text { Antinsens } \\
\text { e } \\
\text { morpholin } \\
\text { os }^{804}\end{array}$ & & pDNA $^{803,825}$ & & \\
\hline & $\begin{array}{l}\text { Sono- } \\
\text { poration }\end{array}$ & $\begin{array}{l}\text { Dyes }^{802,845,85} \\
0,851,857,865,871, \\
873-881,906, \\
\text { Drugs } \\
8871,882- \\
886,1574\end{array}$ & $\begin{array}{l}\text { Proteins }{ }^{828,86} \\
5, \\
\text { Antibodies } \\
88 \\
\text {, } \\
\text { Peptides }^{889}\end{array}$ & $\begin{array}{l}\text { Dextrans }{ }^{54} \\
5,827,828,849- \\
851,864,865,86 \\
7-872,890\end{array}$ & $\begin{array}{l}\text { siRNA } 861,86 \\
2, \\
\text { Antisense } \\
\text { oligonucle } \\
\text { otides }^{860}, \\
\text { ssDNA }^{1574}\end{array}$ & $\mathrm{mRNA}^{863}$ & $\begin{array}{l}\text { pDNA }^{545,830} \\
831,833,838,853- \\
859,890,1574\end{array}$ & $\begin{array}{l}25-75 \mathrm{~nm} \\
\text { nanoparticl } \\
\mathrm{es}^{867}\end{array}$ & $\begin{array}{l}\text { Viral } \\
\text { particles } \\
\text { 887 }\end{array}$ \\
\hline & $\begin{array}{l}\text { Shock Wave- } \\
\text { Mediated } \\
\text { Permeab- } \\
\text { ilization }\end{array}$ & $\begin{array}{l}\text { Dyes }^{899,903,90} \\
5,906,909, \\
\text { Drugs }^{1575}\end{array}$ & $\begin{array}{l}\text { Proteins }{ }^{901} \\
\text { Peptides }^{904}\end{array}$ & $\begin{array}{l}\text { Dextrans } 89 \\
7,899,900\end{array}$ & $\begin{array}{l}\text { Antisense } \\
\text { oligonucle } \\
\text { otides }^{1576}\end{array}$ & & $\begin{array}{l}\text { pDNA }^{1317,15} \\
\text { 77-1579 }\end{array}$ & & \\
\hline & $\begin{array}{l}\text { Laser- } \\
\text { Induced } \\
\text { Cavitation }\end{array}$ & $\begin{array}{l}\text { Dyes } 487,879,91 \\
4,918,919,922,926, \\
928,929,1580,1581\end{array}$ & $\begin{array}{l}\text { Proteins }{ }^{340,91} \\
9, \\
\text { Antibodies } \\
25\end{array}$ & $\begin{array}{l}\text { Dextrans }{ }^{91} \\
3,918,922,924,9 \\
27,929,1581- \\
1584\end{array}$ & $\begin{array}{l}\operatorname{siRNA}^{921,92} \\
3,924\end{array}$ & $\mathrm{mRNA}^{339}$ & $\begin{array}{l}\text { pDNA }^{339,913,} \\
917,919,1585\end{array}$ & $\begin{array}{l}\text { qDots } \\
589,310,1 \\
\text {, Gold } \\
\text { nanoparticl } \\
\text { es }{ }^{1586}\end{array}$ & $\begin{array}{l}\text { Bacteria }{ }^{339,3} \\
40 \\
\text { Mitochondri } \\
\mathrm{a}^{341}, \sim 200 \\
\mathrm{~nm} \\
\text { polystyrene } \\
\text { beads }\end{array}$ \\
\hline \multirow[t]{3}{*}{$\begin{array}{l}\text { Mechanical } \\
\text { (Pressure) }\end{array}$} & $\begin{array}{l}\text { Hypo- } \\
\text { Osmotic } \\
\text { Shock }\end{array}$ & $\begin{array}{l}\text { Dyes }^{951,1587} \text {, } \\
\text { Lanthanide } \\
\text { ions/comple } \\
\text { xes }^{967-972,} \\
\text { Nucleotides }^{9} \\
\text { 51-960, } \\
\text { Nucleosides } \\
\text { 51, BAPTA }\end{array}$ & $\begin{array}{l}\text { Proteins }{ }^{92,93,} \\
950,964,966,974, \\
\text { Peptides } \\
61\end{array}$ & $\begin{array}{l}\text { Dextrans }{ }^{96} \\
2,974,1588\end{array}$ & & & pDNA $^{975}$ & $\begin{array}{l}\text { qDots }^{311}, \sim 5 \\
\text { nm gold } \\
\text { nanoparticl } \\
\text { es }\end{array}$ & \\
\hline & $\begin{array}{l}\text { Hydrostatic } \\
\text { Pressure }\end{array}$ & Dyes $^{980,984}$ & $\begin{array}{l}\text { Proteins }{ }^{980,15} \\
89 \\
\text { Antibodies }^{15} \\
89\end{array}$ & & $\begin{array}{l}\text { ssODN }{ }^{988,9} \\
89 \\
\text { gRNA }^{991}, \\
\text { siRNA }^{981,98} \\
2,997,1590, \\
\text { Antisense } \\
\text { morpholin } \\
\text { os }^{1591} \text {, } \\
\text { Antisense } \\
\text { oligonucle } \\
\text { otides } \\
\text { 8-1066,99 }\end{array}$ & mRNA $^{984}$ & $\begin{array}{l}\text { pDNA }^{978,979,} \\
981,984,989,990, \\
998,1003,1004,1 \\
590,1592,1593, \\
\text { Bacterial } \\
\text { artificial } \\
\text { chromo- } \\
\text { somes } \\
\text { (BACs) }^{983}\end{array}$ & & $\begin{array}{l}\sim 100 \mathrm{~nm} \\
\text { Polystyrene } \\
\text { microsphere } \\
\mathrm{s}^{1005}\end{array}$ \\
\hline & $\begin{array}{l}\text { Osmotic } \\
\text { Rupture of }\end{array}$ & $\begin{array}{l}\text { Trehalose }{ }^{103} \\
5\end{array}$ & $\begin{array}{l}\text { Proteins }{ }^{94,95,} \\
\text { 1007-1021, }\end{array}$ & $\begin{array}{l}\text { Dextran }{ }^{94,1} \\
\text { 026-1028, }\end{array}$ & $\begin{array}{l}\text { siRNA }^{1042,1} \\
043\end{array}$ & & & $\begin{array}{l}\text { qDots }{ }^{1594} \text {, } \\
\text { Protein- }\end{array}$ & $\begin{array}{l}\text { Virus } \\
\text { particles }{ }^{1039}\end{array}$ \\
\hline
\end{tabular}




\begin{tabular}{|c|c|c|c|c|c|c|c|c|c|}
\hline & Endosomes & $\begin{array}{l}\text { Lanthanide } \\
\text { imaging } \\
\text { probes }^{71,72,} \\
\text { Dyes }{ }^{1026,1036} \text {, } \\
\text { UDP- } \\
\text { glucuronic } \\
\text { acid }^{1037}\end{array}$ & $\begin{array}{l}\text { Antibodies }^{10} \\
\text { 13,1022-1026, } \\
\text { Peptides }^{1029-} \\
1031, \text { Cell } \\
\text { lysate }^{1032}\end{array}$ & $\begin{array}{l}\text { Hyalurona } \\
n^{1033,1034}\end{array}$ & $\begin{array}{l}\text { Antisense } \\
\text { oligonucle } \\
\text { otides }^{1038}, \\
\text { Antisense } \\
\text { morpholin } \\
\text { os }^{804}\end{array}$ & & & $\begin{array}{l}\text { conjugated } \\
\text { qDots } \\
1041\end{array}$ & \\
\hline \multirow[t]{3}{*}{$\begin{array}{l}\text { Electro- } \\
\text { poration }\end{array}$} & $\begin{array}{l}\text { Conventiona } \\
\text { I Electro- } \\
\text { poration }\end{array}$ & 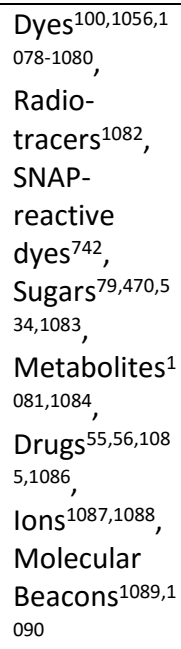 & $\begin{array}{l}\text { Proteins }^{100,13} \\
5,546,1091-1097 \\
\text { Antibodies }^{10} \\
1,125,537,1098- \\
1102, \text { Cas9 } \\
\text { protein / } \\
\text { RNP143,144,146 } \\
, 147,287,1103\end{array}$ & $\begin{array}{l}\text { Dextrans }{ }^{54} \\
6,1078,1126,12 \\
59\end{array}$ & $\begin{array}{l}\text { Antisense } \\
\text { oligonucle } \\
\text { otides }^{1104}, \\
\text { siRNA }^{235,11} \\
05-1109\end{array}$ & $\begin{array}{l}\mathrm{mRNA}^{30,146} \\
, 185,187,257,25 \\
9- \\
261,560,1110,1 \\
111,1263,1266, \\
1271,1275,1277 \\
, 1278,1290,129 \\
3,1294\end{array}$ & $\begin{array}{l}\operatorname{pDNA}^{184,111} \\
2,1113\end{array}$ & $\begin{array}{l}\text { qDots } 294,312,3 \\
13,1114,20 \\
\text { nm gold } \\
\text { nano- } \\
\text { particles }^{1115}\end{array}$ & \\
\hline & $\begin{array}{l}\text { Micro- } \\
\text { electroporat } \\
\text { ion }\end{array}$ & $\begin{array}{l}\text { Dyes } 489,1224,1 \\
228-1232\end{array}$ & Proteins ${ }^{1232}$ & $\begin{array}{l}\text { Dextrans }^{12} \\
31\end{array}$ & $\begin{array}{l}\operatorname{siRNA}^{1232,1} \\
595 \\
\text { miRNA }^{1232}\end{array}$ & & $\begin{array}{l}\text { pDNA }^{1224,12} \\
26,1227,1229,12 \\
30,1232,1595\end{array}$ & & \\
\hline & $\begin{array}{l}\text { Nano- } \\
\text { electroporat } \\
\text { ion }\end{array}$ & $\begin{array}{l}\text { Dyes }^{702,1233}, \\
\text { Molecular } \\
\text { Beacons } \\
242\end{array}$ & Proteins $^{1241}$ & $\begin{array}{l}\text { Dextrans }{ }^{12} \\
41,1242\end{array}$ & $\begin{array}{l}\text { siRNA }^{1233}, \\
\text { Oligonucle } \\
\text { otides }^{1233}\end{array}$ & & $\begin{array}{l}\text { pDNA }^{702,123} \\
3,1234\end{array}$ & qDots $^{1233}$ & \\
\hline \multirow[t]{3}{*}{ Thermal } & $\begin{array}{l}\text { Rapid } \\
\text { temperature } \\
\text { transitions / } \\
\text { cycles }\end{array}$ & $\begin{array}{l}\text { Trehalose }{ }^{73,7} \\
5,1035\end{array}$ & & & & & & & \\
\hline & $\begin{array}{l}\text { Supra- } \\
\text { physiological } \\
\text { Heating }\end{array}$ & Dyes $^{1176}$ & Proteins $^{1596}$ & & & & $\begin{array}{l}\text { pDNA }^{1318,13} \\
19\end{array}$ & & \\
\hline & $\begin{array}{l}\text { Laser } \\
\text { Absorption } \\
\text { Converted } \\
\text { to Heat }\end{array}$ & $\begin{array}{l}\text { Dyes }^{1324,1325,} \\
1327-1329,1597\end{array}$ & $\begin{array}{l}\text { Antibodies }{ }^{13} \\
26\end{array}$ & $\begin{array}{l}\text { Dextrans }{ }^{13} \\
25,1329\end{array}$ & siRNA $^{1598}$ & & $\begin{array}{l}\text { pDNA }^{1328,13} \\
29\end{array}$ & & \\
\hline $\begin{array}{l}\text { Opto- } \\
\text { poration }\end{array}$ & $\begin{array}{l}\text { Laser Opto- } \\
\text { poration }\end{array}$ & $\begin{array}{l}\text { Dyes }^{252,480-} \\
484,486,1333,1340 \\
, 1341,1343,1345,1 \\
346,1348,1354,135 \\
7,1361-1366, \\
\text { Sucrose } \\
\text { Molecular, } \\
\text { beacons } \\
\text { lons }{ }^{1333,1343,}, \\
368\end{array}$ & $\begin{array}{l}\text { Peptides }{ }^{486,1} \\
359 \\
\text { Proteins } \\
34333,1\end{array}$ & $\begin{array}{l}\text { Dextrans }{ }^{13} \\
33,1343,1349,1 \\
353,1360,1361\end{array}$ & $\begin{array}{l}\text { siRNA }^{1333,1} \\
343,1352, \\
\text { Antisense } \\
\text { morpholin } \\
\text { os }^{1353}\end{array}$ & $\begin{array}{l}\mathrm{mRNA}^{252,13} \\
53\end{array}$ & $\begin{array}{l}\text { pDNA }^{481,483,} \\
484,1330,1332- \\
1358\end{array}$ & $\begin{array}{l}\text { Semi- } \\
\text { conductor } \\
\text { nano- } \\
\text { crystals }^{1333,1} \\
343, \text { Gold } \\
\text { nano- } \\
\text { particles }^{1369}, \\
\text { qDots }^{1370}\end{array}$ & $\begin{array}{l}\sim 1 \mu \mathrm{m} \\
\text { Polystyrene } \\
\text { beads }^{1371}\end{array}$ \\
\hline \multirow[t]{2}{*}{$\begin{array}{l}\text { Bio- } \\
\text { chemical }\end{array}$} & $\begin{array}{l}\text { Organic } \\
\text { Solvents \& } \\
\text { Penetration } \\
\text { Enhancers } \\
\end{array}$ & & Proteins $^{1385}$ & & & $\mathrm{mRNA}^{1385}$ & $\mathrm{pDNA}^{1385}$ & & \\
\hline & $\begin{array}{l}\text { Detergents / } \\
\text { Surfactants: } \\
\text { Generic }\end{array}$ & $\begin{array}{l}\text { Nucleosides }^{1} \\
422, \text { Dyes }^{1422} \text {, } \\
\text { Nutrients \& } \\
\text { Metabolites }{ }^{1} \\
422, \text { Ferro- }\end{array}$ & $\begin{array}{l}\text { Proteins }^{1423}, \\
\text { Antibodies } \\
1423\end{array}$ & & & & & & \\
\hline
\end{tabular}




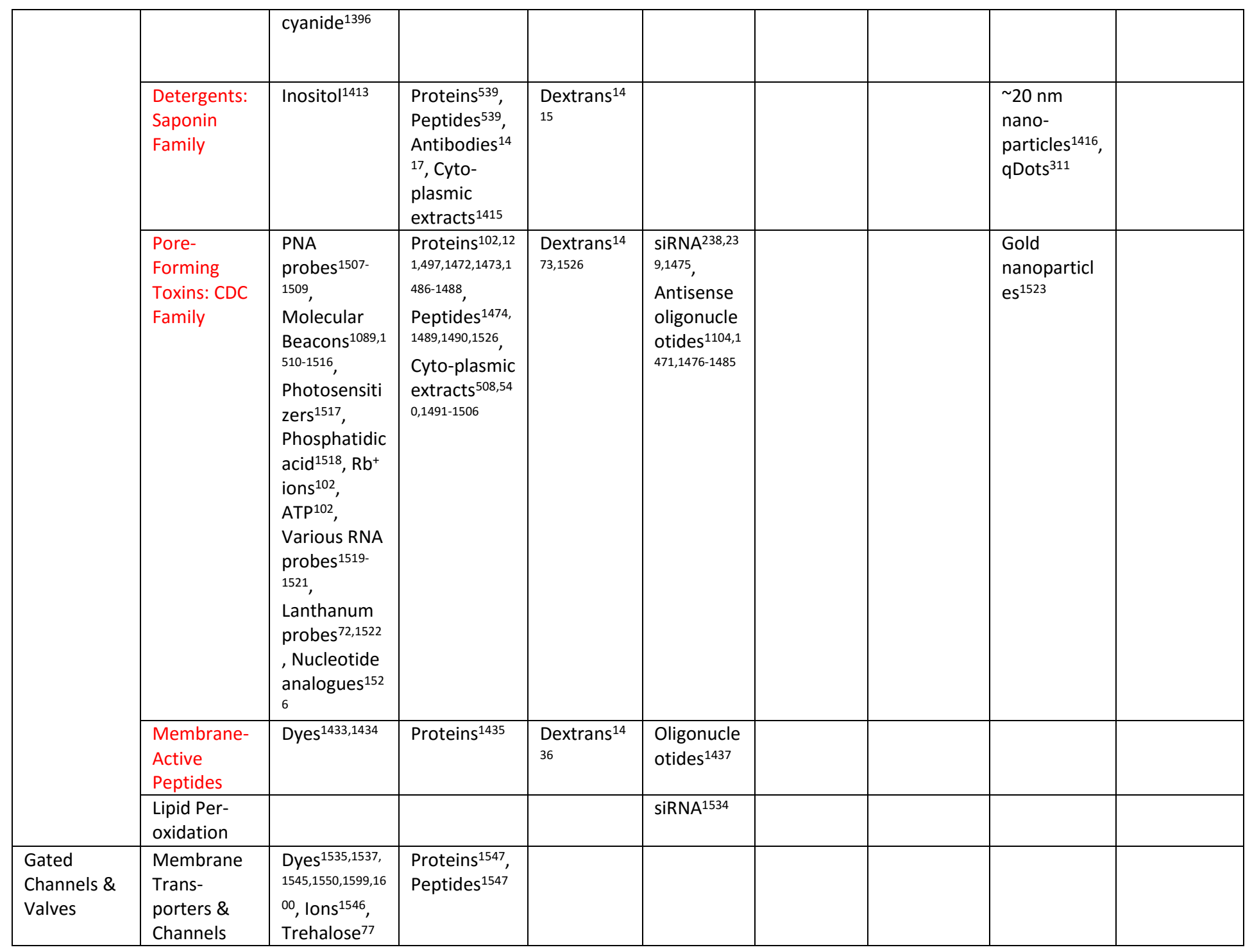




\section{AUTHOR INFORMATION}

\section{Corresponding Authors}

*M.P.S. Email:martstew@mit.edu.

*R.L. Email: rlanger@mit.edu.

*K.F.J. Email: kfjensen@ mit.edu

\section{ORCID ID}

Martin P. Stewart: 0000-0003-4112-6622

Robert Langer: 0000-0003-4255-0492

Klavs F. Jensen: 0000-0001-7192-580X

\section{Biographies}

Robert Langer is one of 13 Institute Professors at MIT (an Institute Professor is the highest honor awarded to a faculty member). His h-index of 242 is the highest of any engineer in history. He has over 1,300 issued and pending patents which have been licensed or sublicensed to over 350 companies. He served as Chairman of the FDA's SCIENCE BOARD (highest advisory board) from 1999-2002. Langer is one of very few individuals elected to the National Academy of Medicine, the National Academy of Engineering, the National Academy of Sciences and the National Academy of Inventors. He is one of four living individuals to receive both the US National Medal of Science and the US National Medal of Technology and Innovation. In 2015, Dr. Langer received the Queen Elizabeth Prize for Engineering. He has also received the Draper Prize (considered the engineering Nobel Prize), Albany Medical Center Prize, Wolf Prize for Chemistry, Millennium Technology Prize, Priestley Medal (highest award of the American Chemical Society), Gairdner Prize, Kyoto Prize, Breakthrough Prize and the Lemelson-MIT prize, for being “one of history's most prolific inventors in medicine.” He holds 31 honorary doctorates including honorary degrees from Harvard and Yale.

Klavs F. Jensen studied chemical engineering at the Technical University of Denmark and completed his $\mathrm{PhD}$ in chemical engineering at the University of Wisconsin-Madison. He started his independent career at the University of Minnesota in 1980 and moved to MIT in 1989 as professor in chemical engineering and materials science and engineering. In 2007 he became Warren K. Lewis Professor and head of MIT's department of chemical engineering until 2015. His research interests revolve around miniaturized systems for chemistry and biological discovery and 
manipulation. Professor Jensen is a member of the US National Academies of Engineering and Sciences, as well as the American Academy of Arts and Science.

Martin P. Stewart was born in Sydney, Australia in 1983. He received a B.Sc (Hons) from the University of Technology, Sydney in 2007. Martin then obtained his PhD from TU Dresden, Germany in 2012 working under the supervision of Professors Daniel Müller and Tony Hyman. His PhD research focused on the mechanisms of cell shape in mitosis. After a postdoctoral stint at ETH Zürich, Switzerland with Professor Daniel Müller, he joined the labs of Professors Klavs Jensen and Robert Langer at MIT, U.S.A. in 2014. Martin's current research interests are in cell manipulation and analysis, specifically in the areas of intracellular delivery and cell biophysics. He has been a recipient of postdoctoral fellowships from the Swiss National Science Foundation and the Life Sciences Research Foundation. He has also been awarded grants from the American Australian Association and the Broad Institute of MIT and Harvard.

\section{ACKNOWLEDGEMENTS}

This work was supported by the US National Institute of Health (R01GM101420-01A1). M.P.S. was supported by the Swiss NSF through the advanced postdoc mobility fellowship P300P3_151179. M.P.S. acknowledges support from a Keith Murdoch Fellowship via the American Australian Association, a Life Sciences Research Foundation Fellowship sponsored by Good Ventures, and a Broadnext 10 Catalytic Steps funding gift from the Broad Institute of MIT and Harvard. We are grateful for discussion and feedback from Xiaoyun Y. Ding, James C. Weaver, Eric Van Leen, and Ronan W. O’Connell. 


\section{REFERENCES}

(1) Peer, D.; Karp, J. M.; Hong, S.; Farokhzad, O. C.; Margalit, R.; Langer, R. Nanocarriers as an Emerging Platform for Cancer Therapy. Nature Nanotechnology 2007, 2, 751-760.

(2) Ganta, S.; Devalapally, H.; Shahiwala, A.; Amiji, M. A Review of Stimuli-Responsive Nanocarriers for Drug and Gene Delivery. J. Controlled Release 2008, 126, 187-204.

(3) Mura, S.; Nicolas, J.; Couvreur, P. Stimuli-Responsive Nanocarriers for Drug Delivery. Nat Mater 2013, 12, 991-1003.

(4) Prokop, A.; Davidson, J. M. Nanovehicular Intracellular Delivery Systems. J. Pharm. Sci. 2008, 97, 3518-3590.

(5) Blanco, E.; Shen, H.; Ferrari, M. Principles of Nanoparticle Design for Overcoming Biological Barriers to Drug Delivery. Nat. Biotechnol. 2015, 33, 941-951.

(6) Torchilin, V. P. Multifunctional, Stimuli-Sensitive Nanoparticulate Systems for Drug Delivery. Nat. Rev. Drug Discov. 2014, 13, 813-827.

(7) Yoo, J. W.; Irvine, D. J.; Discher, D. E.; Mitragotri, S. Bio-Inspired, Bioengineered and Biomimetic Drug Delivery Carriers. Nat. Rev. Drug Discov. 2011, 10, 521-535.

(8) Riley, M. K.; Vermerris, W. Recent Advances in Nanomaterials for Gene Delivery - a Review. Nanomaterials (Basel) 2017, 7, E94.

(9) Yin, H.; Kanasty, R. L.; Eltoukhy, A. A.; Vegas, A. J.; Dorkin, J. R.; Anderson, D. G. Non-Viral Vectors for Gene-Based Therapy. Nat. Rev. Genet. 2014, 15, 541-555.

(10) Mintzer, M. A.; Simanek, E. E. Nonviral Vectors for Gene Delivery. Chem. Rev. 2009, 109, $259-302$.

(11) Yang, J. P.; Zhang, Q.; Chang, H.; Cheng, Y. Y. Surface-Engineered Dendrimers in Gene Delivery. Chem. Rev. 2015, 115, 5274-5300.

(12) Li, Y. L.; Maciel, D.; Rodrigues, J.; Shi, X. Y.; Tomas, H. Biodegradable Polymer Nanogels for Drug/Nucleic Acid Delivery. Chem. Rev. 2015, 115, 8564-8608.

(13) Pattni, B. S.; Chupin, V. V.; Torchilin, V. P. New Developments in Liposomal Drug Delivery. Chem. Rev. 2015, 115, 1093810966.

(14) Lachelt, U.; Wagner, E. Nucleic Acid Therapeutics Using Polyplexes: A Journey of 50 Years (and Beyond). Chem. Rev. 2015, 115, 11043-11078. Wang, H.-X.; Li, M.; Lee, C. M.; Chakraborty, S.; Kim, H.-W.; Bao, G.; Leong, K. W. Crispr/Cas9-Based Genome Editing for Disease Modeling and Therapy: Challenges and Opportunities for Nonviral Delivery. Chem. Rev. 2017, 117, $9874-9906$.

(16) Cox, D. B.; Platt, R. J.; Zhang, F. Therapeutic Genome Editing: Prospects and Challenges. Nat. Med. 2015, 21, $121-131$.

(17) Doudna, J. A.; Charpentier, E. The New Frontier of Genome Engineering with Crispr-Cas9. Science 2014, 346, 1258096.

(18) Glass, Z.; Lee, M.; Li, Y. M.; Xu, Q. B. Engineering the Delivery System for Crispr-Based Genome Editing. Trends Biotechnol. 2018, 36, 173-185. 
Liu, J.; Wen, J.; Zhang, Z.; Liu, H.; Sun, Y. Voyage inside the Cell: Microsystems and Nanoengineering for Intracellular Measurement and Manipulation. Microsystems \& Nanoengineering 2015, 1, 15020.

(20) Chang, L. Q.; Hu, J. M.; Chen, F.; Chen, Z.; Shi, J. F.; Yang, Z. G.; Li, Y. W.; Lee, L. J. Nanoscale Bio-Platforms for Living Cell Interrogation: Current Status and Future Perspectives. Nanoscale 2016, 8, 3181-3206.

(21) Bruce, V. J.; Mcnaughton, B. R. Inside Job: Methods for Delivering Proteins to the Interior of Mammalian Cells. Cell Chem Biol 2017, 24, 924-934.

(22) Zhang, Y.; Røise, J. J.; Lee, K.; Li, J.; Murthy, N. Recent Developments in Intracellular Protein Delivery. Curr. Opin. Biotechnol. 2018, 52, 25-31.

(23) Restifo, N. P.; Dudley, M. E.; Rosenberg, S. A. Adoptive Immunotherapy for Cancer: Harnessing the T Cell Response. Nature Reviews Immunology 2012, 12, 269-281.

(24) Gross, G.; Waks, T.; Eshhar, Z. Expression of Immunoglobulin-T-Cell Receptor Chimeric Molecules as Functional Receptors with Antibody-Type Specificity. Proc. Natl. Acad. Sci. U. S. A. 1989, 86, 10024-10028.

(25) Fesnak, A. D.; June, C. H.; Levine, B. L. Engineered T Cells: The Promise and Challenges of Cancer Immunotherapy. Nat. Rev. Cancer 2016, 16, 566-581. Maude, S. L.; Frey, N.; Shaw, P. A.; Aplenc, R.; Barrett, D. M.; Bunin, N. J.; Chew, A.; Gonzalez, V. E.; Zheng, Z.; Lacey, S. F.et al. Chimeric Antigen Receptor T Cells for Sustained Remissions in Leukemia. N. Engl. J. Med. 2014, 371, 1507-1517.

(27) Sheridan, C. First Approval in Sight for Novartis' Car-T Therapy after Panel Vote. Nat. Biotechnol. 2017, 35, 691-693.

(28) Naldini, L. Ex Vivo Gene Transfer and Correction for Cell-Based Therapies. Nature Reviews Genetics 2011, 12, 301-315.

(29) Naldini, L. Gene Therapy Returns to Centre Stage. Nature 2015, 526, 351-360.

(30) Genovese, P.; Schiroli, G.; Escobar, G.; Di Tomaso, T.; Firrito, C.; Calabria, A.; Moi, D.; Mazzieri, R.; Bonini, C.; Holmes, M. C.et al. Targeted Genome Editing in Human Repopulating Haematopoietic Stem Cells. Nature 2014, 510, 235-240.

(31) Rosenberg, S. A.; Restifo, N. P. Adoptive Cell Transfer as Personalized Immunotherapy for Human Cancer. Science 2015, $348,62-68$.

(32) June, C. H.; Riddell, S. R.; Schumacher, T. N. Adoptive Cellular Therapy: A Race to the Finish Line. Sci. Transl. Med. 2015, 7, $280 p s 287$.

(33) Dever, D. P.; Bak, R. O.; Reinisch, A.; Camarena, J.; Washington, G.; Nicolas, C. E.; Pavel-Dinu, M.; Saxena, N.; Wilkens, A. B.; Mantri, S.et al. Crispr/Cas9 Beta-Globin Gene Targeting in Human Haematopoietic Stem Cells. Nature 2016, 539, 384-389.

(34) Maeder, M. L.; Gersbach, C. A. Genome-Editing Technologies for Gene and Cell Therapy. Mol. Ther. 2016, 24, 430-446.

(35) Takahashi, K.; Yamanaka, S. Induction of Pluripotent Stem Cells from Mouse Embryonic and Adult Fibroblast Cultures by Defined Factors. Cell 2006, 126, 663-676.

(36) Kim, D.; Kim, C. H.; Moon, J. I.; Chung, Y. G.; Chang, M. Y.; Han, B. S.; Ko, S.; Yang, E.; Cha, K. Y.; Lanza, R.et al. Generation of Human Induced Pluripotent Stem Cells by Direct Delivery of Reprogramming Proteins. Cell Stem Cell 2009, 4, 472-476. 
Warren, L.; Manos, P. D.; Ahfeldt, T.; Loh, Y. H.; Li, H.; Lau, F.; Ebina, W.; Mandal, P. K.; Smith, Z. D.; Meissner, A.et al. Highly Efficient Reprogramming to Pluripotency and Directed Differentiation of Human Cells with Synthetic Modified Mrna. Cell Stem Cell 2010, 7, 618-630.

(38) Rohani, L.; Fabian, C.; Holland, H.; Naaldijk, Y.; Dressel, R.; Loffler-Wirth, H.; Binder, H.; Arnold, A.; Stolzing, A. Generation of Human Induced Pluripotent Stem Cells Using Non-Synthetic Mrna. Stem Cell Research 2016, 16, 662-672.

Anokye-Danso, F.; Trivedi, C. M.; Juhr, D.; Gupta, M.; Cui, Z.; Tian, Y.; Zhang, Y. Z.; Yang, W. L.; Gruber, P. J.; Epstein, J. A.et al. Highly Efficient Mirna-Mediated Reprogramming of Mouse and Human Somatic Cells to Pluripotency. Cell Stem Cell 2011, 8, 376-388.

(40) Shi, Y.; Desponts, C.; Do, J. T.; Hahm, H. S.; Scholer, H. R.; Ding, S. Induction of Pluripotent Stem Cells from Mouse Embryonic Fibroblasts by Oct4 and Klf4 with Small-Molecule Compounds. Cell Stem Cell 2008, 3, 568-574.

(41) Robinton, D. A.; Daley, G. Q. The Promise of Induced Pluripotent Stem Cells in Research and Therapy. Nature 2012, 481, 295-305.

Tabar, V.; Studer, L. Pluripotent Stem Cells in Regenerative Medicine: Challenges and Recent Progress. Nature Reviews Genetics 2014, 15, 82-92.

Takebe, T.; Sekine, K.; Enomura, M.; Koike, H.; Kimura, M.; Ogaeri, T.; Zhang, R. R.; Ueno, Y.; Zheng, Y. W.; Koike, N.et al. Vascularized and Functional Human Liver from an Ipsc-Derived Organ Bud Transplant. Nature 2013, 499, $481-484$.

Themeli, M.; Riviere, I.; Sadelain, M. New Cell Sources for T Cell Engineering and Adoptive Immunotherapy. Cell Stem Cell 2015, 16, 357-366.

Park, C. Y.; Kim, D. H.; Son, J. S.; Sung, J. J.; Lee, J.; Bae, S.; Kim, J. H.; Kim, D. W.; Kim, J. S. Functional Correction of Large Factor Viii Gene Chromosomal Inversions in Hemophilia a Patient-Derived Ipscs Using Crispr-Cas9. Cell Stem Cell 2015, 17, 213-220.

(46) Cottet, M.; Faklaris, O.; Maurel, D.; Scholler, P.; Doumazane, E.; Trinquet, E.; Pin, J. P.; Durroux, T. Bret and Time-Resolved Fret Strategy to Study Gpcr Oligomerization: From Cell Lines toward Native Tissues. Front. Endocrinol. (Lausanne) 2012, 3, 92.

(47) Galeone, A.; Vecchio, G.; Malvindi, M. A.; Brunetti, V.; Cingolani, R.; Pompa, P. P. In Vivo Assessment of Cdse-Zns Quantum Dots: Coating Dependent Bioaccumulation and Genotoxicity. Nanoscale 2012, 4, 6401-6407.

(48) Tian, Y.; Wang, T.; Liu, W. Y.; Xin, H. L.; Li, H. L.; Ke, Y. G.; Shih, W. M.; Gang, O. Prescribed Nanoparticle Cluster Architectures and Low-Dimensional Arrays Built Using Octahedral DNA Origami Frames. Nature Nanotechnology 2015, 10, 637-644.

(49) Gomez-Martinez, R.; Hernandez-Pinto, A. M.; Duch, M.; Vazquez, P.; Zinoviev, K.; De La Rosa, E. J.; Esteve, J.; Suarez, T.; Plaza, J. A. Silicon Chips Detect Intracellular Pressure Changes in Living Cells. Nat Nanotechnol 2013, 8, 517-521.

(50) Drews, J. Drug Discovery: A Historical Perspective. Science 2000, 287, 1960-1964.

(51) Lipinski, C. A.; Lombardo, F.; Dominy, B. W.; Feeney, P. J. Experimental and Computational Approaches to Estimate Solubility and Permeability in Drug Discovery and Development Settings. Adv. Drug Del. Rev. 1997, 23, 3-25. 
Keller, T. H.; Pichota, A.; Yin, Z. A Practical View of 'Druggability'. Curr. Opin. Chem. Biol. 2006, 10, 357-361.

(53) Yun, J.; Mullarky, E.; Lu, C. Y.; Bosch, K. N.; Kavalier, A.; Rivera, K.; Roper, J.; Chio, I. I. C.; Giannopoulou, E. G.; Rago, C.et al. Vitamin C Selectively Kills Kras and Braf Mutant Colorectal Cancer Cells by Targeting Gapdh. Science 2015, 350, 13911396.

Williams, A. C.; Barry, B. W. Penetration Enhancers. Adv. Drug Del. Rev. 2004, 56, 603-618.

Orlowski, S.; Belehradek, J., Jr.; Paoletti, C.; Mir, L. M. Transient Electropermeabilization of Cells in Culture. Increase of the Cytotoxicity of Anticancer Drugs. Biochem. Pharmacol. 1988, 37, 4727-4733.

Gothelf, A.; Mir, L. M.; Gehl, J. Electrochemotherapy: Results of Cancer Treatment Using Enhanced Delivery of Bleomycin by Electroporation. Cancer Treat. Rev. 2003, 29, 371-387.

Li, X. H.; Gao, X. H.; Shi, W.; Ma, H. M. Design Strategies for Water-Soluble Small Molecular Chromogenic and Fluorogenic Probes. Chem. Rev. 2014, 114, 590-659.

Dallasta, V.; Gatti, R.; Orlandini, G.; Rossi, P. A.; Rotoli, B. M.; Sala, R.; Bussolati, O.; Gazzola, G. C. Membrane Potential Changes Visualized in Complete Growth Media through Confocal Laser Scanning Microscopy Bis-Oxonol-Loaded Cells. Exp. Cell Res. 1997, 231, 260-268.

Wolff, C.; Fuks, B.; Chatelain, P. Comparative Study of Membrane Potential-Sensitive Fluorescent Probes and Their Use in Ion Channel Screening Assays. J. Biomol. Screen. 2003, 8, 533-543.

Nakata, E.; Yukimachi, Y.; Uto, Y.; Hori, H.; Morii, T. Latent Ph-Responsive Ratiometric Fluorescent Cluster Based on SelfAssembled Photoactivated Snarf Derivatives. Science and Technology of Advanced Materials 2016, 17, 431-436.

Buckler, K. J.; Vaughan-Jones, R. D. Application of a New Ph-Sensitive Fluoroprobe (Carboxy-Snarf-1) for Intracellular Ph Measurement in Small, Isolated Cells. Pflugers Archiv-European Journal of Physiology 1990, 417, 234-239.

Meuwis, K.; Boens, N.; De Schryver, F. C.; Gallay, J.; Vincent, M. Photophysics of the Fluorescent K+ Indicator Pbfi. Biophys. J. 1995, 68, 2469-2473.

Kasner, S. E.; Ganz, M. B. Regulation of Intracellular Potassium in Mesangial Cells - a Fluorescence Analysis Using the Dye, Pbfi. Am. J. Physiol. 1992, 262, F462-F467.

Minta, A.; Tsien, R. Y. Fluorescent Indicators for Cytosolic Sodium. J. Biol. Chem. 1989, 264, 19449-19457.

Jezek, P.; Mahdi, F.; Garlid, K. D. Reconstitution of the Beef-Heart and Rat-Liver Mitochondrial $\mathrm{K}+/ \mathrm{H}+(\mathrm{Na}+/ \mathrm{H}+)$ Antiporter Quantitation of K+ Transport with the Novel Fluorescent-Probe, Pbfi. J. Biol. Chem. 1990, 265, 10522-10526.

Grynkiewicz, G.; Poenie, M.; Tsien, R. Y. A New Generation of Ca-2+ Indicators with Greatly Improved Fluorescence Properties. J. Biol. Chem. 1985, 260, 3440-3450.

Minta, A.; Kao, J. P. Y.; Tsien, R. Y. Fluorescent Indicators for Cytosolic Calcium Based on Rhodamine and Fluorescein Chromophores. J. Biol. Chem. 1989, 264, 8171-8178.

(69) Kantevari, S.; Gordon, G. R. J.; Macvicar, B. A.; Ellis-Davies, G. C. R. A Practical Guide to the Synthesis and Use of Membrane-Permeant Acetoxymethyl Esters of Caged Inositol Polyphosphates. Nat. Protoc. 2011, 6, 327-337. 
(70) Heffern, M. C.; Matosziuk, L. M.; Meade, T. J. Lanthanide Probes for Bioresponsive Imaging. Chem. Rev. 2014, 114, 44964539.

(71) Gahlaut, N.; Miller, L. W. Time-Resolved Microscopy for Imaging Lanthanide Luminescence in Living Cells. Cytometry Part A 2010, $77 A, 1113-1125$.

(72) Rajapakse, H. E.; Gahlaut, N.; Mohandessi, S.; Yu, D.; Turner, J. R.; Miller, L. W. Time-Resolved Luminescence Resonance Energy Transfer Imaging of Protein-Protein Interactions in Living Cells. Proc. Natl. Acad. Sci. U. S. A. 2010, 107, 1358213587.

(73) Beattie, G. M.; Crowe, J. H.; Lopez, A. D.; Cirulli, V.; Ricordi, C.; Hayek, A. Trehalose: A Cryoprotectant That Enhances Recovery and Preserves Function of Human Pancreatic Islets after Long-Term Storage. Diabetes 1997, 46, 519-523.

(74) Eroglu, A.; Russo, M. J.; Bieganski, R.; Fowler, A.; Cheley, S.; Bayley, H.; Toner, M. Intracellular Trehalose Improves the Survival of Cryopreserved Mammalian Cells. Nat. Biotechnol. 2000, 18, 163-167.

He, X. M.; Amin, A. A.; Fowler, A.; Toner, M. Thermally Induced Introduction of Trehalose into Primary Rat Hepatocytes. Cell Preserv. Technol. 2006, 4, 178-187.

(76) Zhang, W. J.; Rong, J. H.; Wang, Q.; He, X. M. The Encapsulation and Intracellular Delivery of Trehalose Using a Thermally Responsive Nanocapsule. Nanotechnology 2009, 20, 275101.

(77) Acker, J. P.; Lu, X. M.; Young, V.; Cheley, S.; Bayley, H.; Fowler, A.; Toner, M. Measurement of Trehalose Loading of Mammalian Cells Porated with a Metal-Actuated Switchable Pore. Biotechnol. Bioeng. 2003, 82, 525-532.

(78) Dovgan, B.; Barlic, A.; Knezevic, M.; Miklavcic, D. Cryopreservation of Human Adipose-Derived Stem Cells in Combination with Trehalose and Reversible Electroporation. J. Membr. Biol. 2017, 250, 1-9.

(79) Shirakashi, R.; Kostner, C. M.; Muller, K. J.; Kurschner, M.; Zimmermann, U.; Sukhorukov, V. L. Intracellular Delivery of Trehalose into Mammalian Cells by Electropermeabilization. J. Membr. Biol. 2002, 189, 45-54.

(80) Feldherr, C. M. The Intracellular Distribution of Ferritin Following Microinjection. J. Cell Biol. 1962, 12, $159-167$.

(81) Lin, T. P. Microinjection of Mouse Eggs. Science 1966, 151, 333-337.

(82) Paine, P. L.; Feldherr, C. M. Nucleocytoplasmic Exchange of Macromolecules. Exp. Cell Res. $1972,74,81-98$.

(83) Paine, P. L. Nucleocytoplasmic Movement of Fluorescent Tracers Microinjected into Living Salivary-Gland Cells. J. Cell Biol. $1975,66,652-657$.

(84) Stacey, D. W.; Allfrey, V. G. Evidence for the Autophagy of Microinjected Proteins in Hela Cells. J. Cell Biol. 1977, 75, 807817.

(85) Kreis, T. E.; Winterhalter, K. H.; Birchmeier, W. In Vivo Distribution and Turnover of Fluorescently Labeled Actin Microinjected into Human Fibroblasts. Proc. Natl. Acad. Sci. U. S. A. 1979, 76, 3814-3818.

(86) Feramisco, J. R. Microinjection of Fluorescently Labeled Alpha-Actinin into Living Fibroblasts. Proc. Natl. Acad. Sci. U. S. A. 1979, 76, 3967-3971.

(87) Schlegel, R. A.; Rechsteiner, M. C. Microinjection of Thymidine Kinase and Bovine Serum-Albumin into Mammalian-Cells by Fusion with Red Blood-Cells. Cell 1975, 5, 371-379. 
Loyter, A.; Zakai, N.; Kulka, R. G. Ultramicroinjection of Macromolecules or Small Particles into Animal-Cells - New Technique Based on Virus-Induced Cell-Fusion. J. Cell Biol. 1975, 66, 292-304.

(89) Furusawa, M.; Nishimur.T; Yamaizum.M; Okada, Y. Injection of Foreign Substances into Single Cells by Cell-Fusion. Nature $1974,249,449-450$.

Poste, G.; Papahadjopoulos, D. Lipid Vesicles as Carriers for Introducing Materials into Cultured-Cells - Influence of Vesicle Lipid-Composition on Mechanism(S) of Vesicle Incorporation into Cells. Proc. Natl. Acad. Sci. U. S. A. 1976, 73, 1603-1607.

(91) Gregoriadis, G.; Buckland, R. A. Enzyme-Containing Liposomes Alleviate a Model for Storage Disease. Nature 1973, 244, 170-172.

$1982,217,252-254$.

Borle, A. B.; Snowdowne, K. W. Measurement of Intracellular Free Calcium in Monkey Kidney-Cells with Aequorin. Science

Snowdowne, K. W.; Borle, A. B. Measurement of Cytosolic Free Calcium in Mammalian-Cells with Aequorin. Am. J. Physiol. 1984, 247, C396-C408.

Okada, C. Y.; Rechsteiner, M. Introduction of Macromolecules into Cultured Mammalian-Cells by Osmotic Lysis of Pinocytic Vesicles. Cell 1982, 29, 33-41.

Moore, M. W.; Carbone, F. R.; Bevan, M. J. Introduction of Soluble-Protein into the Class-I Pathway of Antigen Processing and Presentation. Cell 1988, 54, 777-785.

Mcneil, P. L.; Murphy, R. F.; Lanni, F.; Taylor, D. L. A Method for Incorporating Macromolecules into Adherent Cells. J. Cell Biol. 1984, 98, 1556-1564.

Mcneil, P. L.; Warder, E. Glass-Beads Load Macromolecules into Living Cells. J. Cell Sci. 1987, 88, 669-678.

Clarke, M. S. F.; Mcneil, P. L. Syringe Loading Introduces Macromolecules into Living Mammalian-Cell Cytosol. J. Cell Sci. 1992, 102, 533-541.

Schroff, R. W.; Bucana, C. D.; Klein, R. A.; Farrell, M. M.; Morgan, A. C. Detection of Intracytoplasmic Antigens by FlowCytometry. J. Immunol. Methods 1984, 70, 167-177.

Mir, L. M.; Banoun, H.; Paoletti, C. Introduction of Definite Amounts of Nonpermeant Molecules into Living Cells after Electropermeabilization - Direct Access to the Cytosol. Exp. Cell Res. 1988, 175, 15-25.

Chakrabarti, R.; Wylie, D. E.; Schuster, S. M. Transfer of Monoclonal-Antibodies into Mammalian-Cells by Electroporation. J. Biol. Chem. 1989, 264, 15494-15500.

Ahnert-Hilger, G.; Bader, M. F.; Bhakdi, S.; Gratzl, M. Introduction of Macromolecules into Bovine Adrenal Medullary Chromaffin Cells and Rat Pheochromocytoma Cells (Pc12) by Permeabilization with Streptolysin O: Inhibitory Effect of Tetanus Toxin on Catecholamine Secretion. J. Neurochem. 1989, 52, 1751-1758.

(103) Bryant, P. E. Induction of Chromosomal Damage by Restriction Endonuclease in Cho Cells Porated with Streptolysin-O. Mutat. Res. 1992, 268, 27-34.

(104) Yan, L.; Zhang, J.; Lee, C. S.; Chen, X. Micro- and Nanotechnologies for Intracellular Delivery. Small 2014, 10, $4487-4504$. 
Meacham, J. M.; Durvasula, K.; Degertekin, F. L.; Fedorov, A. G. Physical Methods for Intracellular Delivery: Practical Aspects from Laboratory Use to Industrial-Scale Processing. Jala 2014, 19, 1-18.

(106) Kang, W.; Mcnaughton, R. L.; Espinosa, H. D. Micro- and Nanoscale Technologies for Delivery into Adherent Cells. Trends Biotechnol. 2016, 34, 665-678.

(107) Stewart, M. P.; Sharei, A.; Ding, X. Y.; Sahay, G.; Langer, R.; Jensen, K. F. In Vitro and Ex Vivo Strategies for Intracellular Delivery. Nature 2016, 538, 183-192.

(108) Sharei, A.; Zoldan, J.; Adamo, A.; Sim, W. Y.; Cho, N.; Jackson, E.; Mao, S.; Schneider, S.; Han, M. J.; Lytton-Jean, A.et al. A Vector-Free Microfluidic Platform for Intracellular Delivery. Proc. Natl. Acad. Sci. U. S. A. 2013, $110,2082-2087$.

(109) Mcknight, T. E.; Melechko, A. V.; Griffin, G. D.; Guillorn, M. A.; Merkulov, V. I.; Serna, F.; Hensley, D. K.; Doktycz, M. J.; Lowndes, D. H.; Simpson, M. L. Intracellular Integration of Synthetic Nanostructures with Viable Cells for Controlled Biochemical Manipulation. Nanotechnology 2003, 14, 551-556.

(110) Shalek, A. K.; Robinson, J. T.; Karp, E. S.; Lee, J. S.; Ahn, D. R.; Yoon, M. H.; Sutton, A.; Jorgolli, M.; Gertner, R. S.; Gujral, T. S.et al. Vertical Silicon Nanowires as a Universal Platform for Delivering Biomolecules into Living Cells. Proc. Natl. Acad. Sci. U. S. A. 2010, 107, 1870-1875.

(111) Marschall, A. L. J.; Frenzel, A.; Schirrmann, T.; Schungel, M.; Dubel, S. Targeting Antibodies to the Cytoplasm. Mabs 2011, 3, 3-16.

(112) Tinsley, J. H.; Hawker, J.; Yuan, Y. Efficient Protein Transfection of Cultured Coronary Venular Endothelial Cells. American Journal of Physiology-Heart and Circulatory Physiology 1998, 275, H1873-H1878.

(113) Zelphati, O.; Wang, Y.; Kitada, S.; Reed, J. C.; Felgner, P. L.; Corbeil, J. Intracellular Delivery of Proteins with a New LipidMediated Delivery System. J. Biol. Chem. 2001, 276, 35103-35110.

(114) Weill, C. O.; Biri, S.; Adib, A.; Erbacher, P. A Practical Approach for Intracellular Protein Delivery. Cytotechnology 2008, 56, 41-48.

(115) Erazo-Oliveras, A.; Najjar, K.; Dayani, L.; Wang, T. Y.; Johnson, G. A.; Pellois, J. P. Protein Delivery into Live Cells by Incubation with an Endosomolytic Agent. Nat. Methods 2014, 11, 861-867.

(116) Lonn, P.; Dowdy, S. F. Cationic Ptd/Cpp-Mediated Macromolecular Delivery: Charging into the Cell. Expert Opin Drug Deliv 2015, 1-10.

(117) Liao, X. L.; Rabideau, A. E.; Pentelute, B. L. Delivery of Antibody Mimics into Mammalian Cells Via Anthrax Toxin Protective Antigen. ChemBioChem 2014, 15, 2458-2466.

(118) Guillard, S.; Minter, R. R.; Jackson, R. H. Engineering Therapeutic Proteins for Cell Entry: The Natural Approach. Trends Biotechnol. 2015, 33, 163-171.

(119) Yang, N. J.; Liu, D. V.; Sklaviadis, D.; Gui, D. Y.; Vander Heiden, M. G.; Wittrup, K. D. Antibody-Mediated Neutralization of Perfringolysin O for Intracellular Protein Delivery. Mol. Pharm. 2015, 12, 1992-2000.

(120) Ryou, J. H.; Sohn, Y. K.; Hwang, D. E.; Park, W. Y.; Kim, N.; Heo, W. D.; Kim, M. Y.; Kim, H. S. Engineering of Bacterial Exotoxins for Highly Efficient and Receptor-Specific Intracellular Delivery of Diverse Cargos. Biotechnol. Bioeng. 2016. 
(121) Beilhartz, G. L.; Sugiman-Marangos, S. N.; Melnyk, R. A. Repurposing Bacterial Toxins for Intracellular Delivery of Therapeutic Proteins. Biochem. Pharmacol. 2017, 15, 13-20.

(122) Gu, Z.; Biswas, A.; Zhao, M. X.; Tang, Y. Tailoring Nanocarriers for Intracellular Protein Delivery. Chem. Soc. Rev. 2011, 40, 3638-3655.

(123) Lu, Y.; Sun, W. J.; Gu, Z. Stimuli-Responsive Nanomaterials for Therapeutic Protein Delivery. J. Controlled Release 2014, $194,1-19$

Ray, M.; Lee, Y. W.; Scaletti, F.; Yu, R.; Rotello, V. M. Intracellular Delivery of Proteins by Nanocarriers. Nanomedicine (Lond) 2017, 12, 941-952.

Marschall, A. L. J.; Zhang, C. C.; Frenzel, A.; Schirrmann, T.; Hust, M.; Perez, F.; Dubel, S. Delivery of Antibodies to the Cytosol Debunking the Myths. Mabs 2014, 6, 943-956.

Choi, Y. S.; David, A. E. Cell Penetrating Peptides and the Mechanisms for Intracellular Entry. Curr. Pharm. Biotechnol. 2014, $15,192-199$.

Bechara, C.; Sagan, S. Cell-Penetrating Peptides: 20 Years Later, Where Do We Stand? FEBS Lett. 2013, 587, 1693-1702.

Dewitt, M. A.; Corn, J. E.; Carroll, D. Genome Editing Via Delivery of Cas9 Ribonucleoprotein. Methods 2017, 121-122, 9-15.

Helma, J.; Cardoso, M. C.; Muyldermans, S.; Leonhardt, H. Nanobodies and Recombinant Binders in Cell Biology. J. Cell Biol. 2015, 209, 633-644.

Rothbauer, U.; Zolghadr, K.; Tillib, S.; Nowak, D.; Schermelleh, L.; Gahl, A.; Backmann, N.; Conrath, K.; Muyldermans, S.; Cardoso, M. C.et al. Targeting and Tracing Antigens in Live Cells with Fluorescent Nanobodies. Nat. Methods 2006, 3, 887889.

(131) Fu, A. L.; Tang, R.; Hardie, J.; Farkas, M. E.; Rotello, V. M. Promises and Pitfalls of Intracellular Delivery of Proteins. Bioconjugate Chem. 2014, 25, 1602-1608.

Leader, B.; Baca, Q. J.; Golan, D. E. Protein Therapeutics: A Summary and Pharmacological Classification. Nature Reviews Drug Discovery 2008, 7, 21-39.

Dimitrov, D. S. Therapeutic Proteins. Methods Mol. Biol. 2012, 899, 1-26.

Uhlen, M.; Fagerberg, L.; Hallstrom, B. M.; Lindskog, C.; Oksvold, P.; Mardinoglu, A.; Sivertsson, A.; Kampf, C.; Sjostedt, E.; Asplund, A.et al. Tissue-Based Map of the Human Proteome. Science 2015, 347, 1260419.

Kim, K. W.; Kim, S. H.; Jang, J. H.; Lee, E. Y.; Park, S. W.; Um, J. H.; Lee, Y. J.; Lee, C. H.; Yoon, S.; Seo, S. Y.et al. Dendritic Cells Loaded with Exogenous Antigen by Electroporation Can Enhance Mhc Class I-Mediated Antitumor Immunity. Cancer Immunol. Immunother. 2004, 53, 315-322.

Weiss, J. M.; Allen, C.; Shivakumar, R.; Feller, S.; Li, L. H.; Liu, L. N. Efficient Responses in a Murine Renal Tumor Model by Electroloading Dendritic Cells with Whole-Tumor Lysate. J. Immunother. 2005, 28, 542-550.

Kamigaki, T.; Kaneko, T.; Naitoh, K.; Takahara, M.; Kondo, T.; Ibe, H.; Matsuda, E.; Maekawa, R.; Goto, S. Immunotherapy of Autologous Tumor Lysate-Loaded Dendritic Cell Vaccines by a Closed-Flow Electroporation System for Solid Tumors.

Anticancer Res. 2013, 33, 2971-2976. 
Wolfraim, L. A.; Takahara, M.; Viley, A. M.; Shivakumar, R.; Nieda, M.; Maekawa, R.; Liu, L. N.; Peshwa, M. V. Clinical Scale Electroloading of Mature Dendritic Cells with Melanoma Whole Tumor Cell Lysate Is Superior to Conventional Lysate CoIncubation in Triggering Robust in Vitro Expansion of Functional Antigen-Specific CtI. Int. Immunopharmacol. 2013, 15, 488497.

(139) Jinek, M.; Chylinski, K.; Fonfara, I.; Hauer, M.; Doudna, J. A.; Charpentier, E. A Programmable Dual-Rna-Guided DNA Endonuclease in Adaptive Bacterial Immunity. Science 2012, 337, 816-821.

(140) Ran, F. A.; Hsu, P. D.; Wright, J.; Agarwala, V.; Scott, D. A.; Zhang, F. Genome Engineering Using the Crispr-Cas9 System. Nat. Protoc. 2013, 8, 2281-2308.

(141) Shao, Y. J.; Guan, Y. T.; Wang, L. R.; Qiu, Z. W.; Liu, M. Z.; Chen, Y. T.; Wu, L. J.; Li, Y. M.; Ma, X. Y.; Liu, M. Y.et al. Crispr/Cas-Mediated Genome Editing in the Rat Via Direct Injection of One-Cell Embryos. Nat. Protoc. 2014, 9, $2493-2512$.

(142) Sung, Y. H.; Kim, J. M.; Kim, H. T.; Lee, J.; Jeon, J.; Jin, Y.; Choi, J. H.; Ban, Y. H.; Ha, S. J.; Kim, C. H.et al. Highly Efficient Gene Knockout in Mice and Zebrafish with Rna-Guided Endonucleases. Genome Res. 2014, 24, 125-131.

(143) Kim, S.; Kim, D.; Cho, S. W.; Kim, J.; Kim, J. S. Highly Efficient Rna-Guided Genome Editing in Human Cells Via Delivery of Purified Cas9 Ribonucleoproteins. Genome Res. 2014, 24, 1012-1019.

(144) Schumann, K.; Lin, S.; Boyer, E.; Simeonov, D. R.; Subramaniam, M.; Gate, R. E.; Haliburton, G. E.; Yee, C. J.; Bluestone, J. A.; Doudna, J. A.et al. Generation of Knock-in Primary Human T Cells Using Cas9 Ribonucleoproteins. Proc. Natl. Acad. Sci. U. S. A. 2015, 112, 10437-10442.

(145) Liang, X. Q.; Potter, J.; Kumar, S.; Zou, Y. F.; Quintanilla, R.; Sridharan, M.; Carte, J.; Chen, W.; Roark, N.; Ranganathan, S.et al. Rapid and Highly Efficient Mammalian Cell Engineering Via Cas9 Protein Transfection. J. Biotechnol. 2015, 208, 44-53.

(146) Hendel, A.; Bak, R. O.; Clark, J. T.; Kennedy, A. B.; Ryan, D. E.; Roy, S.; Steinfeld, I.; Lunstad, B. D.; Kaiser, R. J.; Wilkens, A. B.et al. Chemically Modified Guide Rnas Enhance Crispr-Cas Genome Editing in Human Primary Cells. Nat. Biotechnol. 2015, 33, 985-989.

(147) Lin, S.; Staahl, B.; Alla, R. K.; Doudna, J. A. Enhanced Homology-Directed Human Genome Engineering by Controlled Timing of Crispr/Cas9 Delivery. Elife 2014, 3, e04766.

(148) Moreno-Mateos, M. A.; Vejnar, C. E.; Beaudoin, J. D.; Fernandez, J. P.; Mis, E. K.; Khokha, M. K.; Giraldez, A. J. Crisprscan: Designing Highly Efficient Sgrnas for Crispr-Cas9 Targeting in Vivo. Nat. Methods 2015, 12, 982-988.

(149) Jacobi, A. M.; Rettig, G. R.; Turk, R.; Collingwood, M. A.; Zeiner, S. A.; Quadros, R. M.; Harms, D. W.; Bonthuis, P. J.; Gregg, C.; Ohtsuka, M.et al. Simplified Crispr Tools for Efficient Genome Editing and Streamlined Protocols for Their Delivery into Mammalian Cells and Mouse Zygotes. Methods 2017, 121, 16-28.

(150) Zuris, J. A.; Thompson, D. B.; Shu, Y.; Guilinger, J. P.; Bessen, J. L.; Hu, J. H.; Maeder, M. L.; Joung, J. K.; Chen, Z. Y.; Liu, D. R. Cationic Lipid-Mediated Delivery of Proteins Enables Efficient Protein-Based Genome Editing in Vitro and in Vivo. Nat. Biotechnol. 2015, 33, 73-80.

(151) D'astolfo, D. S.; Pagliero, R. J.; Pras, A.; Karthaus, W. R.; Clevers, H.; Prasad, V.; Lebbink, R. J.; Rehmann, H.; Geijsen, N. Efficient Intracellular Delivery of Native Proteins. Cell 2015, 161, 674-690. 
Han, X.; Liu, Z.; Ma, Y.; Zhang, K.; Qin, L. Cas9 Ribonucleoprotein Delivery Via Microfluidic Cell-Deformation Chip for Human T-Cell Genome Editing and Immunotherapy. Advanced Biosystems 2017, 1, 1600007.

(153) Ramakrishna, S.; Dad, A. K.; Beloor, J.; Gopalappa, R.; Lee, S. K.; Kim, H. Gene Disruption by Cell-Penetrating PeptideMediated Delivery of Cas9 Protein and Guide Rna. Genome Res. 2014, 24, 1020-1027.

(154) Nishimasu, H.; Ran, F. A.; Hsu, P. D.; Konermann, S.; Shehata, S. I.; Dohmae, N.; Ishitani, R.; Zhang, F.; Nureki, O. Crystal Structure of Cas9 in Complex with Guide Rna and Target DNA. Cell 2014, 156, 935-949. Jinek, M.; Jiang, F. G.; Taylor, D. W.; Sternberg, S. H.; Kaya, E.; Ma, E. B.; Anders, C.; Hauer, M.; Zhou, K. H.; Lin, S.et al. Structures of Cas9 Endonucleases Reveal Rna-Mediated Conformational Activation. Science 2014, 343, 1247997.

(156) Yin, H.; Kauffman, K. J.; Anderson, D. G. Delivery Technologies for Genome Editing. Nat. Rev. Drug Discov. 2017, 16, 387399.

(157) Bruns, A. M.; Leser, G. P.; Lamb, R. A.; Horvath, C. M. The Innate Immune Sensor Lgp2 Activates Antiviral Signaling by Regulating Mda5-Rna Interaction and Filament Assembly. Mol. Cell 2014, 55, 771-781.

Ma, H.; Marti-Gutierrez, N.; Park, S. W.; Wu, J.; Lee, Y.; Suzuki, K.; Koski, A.; Ji, D.; Hayama, T.; Ahmed, R.et al. Correction of a Pathogenic Gene Mutation in Human Embryos. Nature 2017, 548, 413-419.

Sarma, V. R.; E.W., S.; Davies, D. R.; Terry, W. D. The Three Dimensional Structure at 6 a Resolution of a Human Gamma G1 Immunoglobulin Molecule. J. Biol. Chem. 1971, 246, 3753-3759.

Yin, T.; Bader, A. R.; Hou, T. K.; Maron, B. A.; Kao, D. D.; Qian, R.; Kohane, D. S.; Handy, D. E.; Loscalzo, J.; Zhang, Y. Y. Sdf-1 Alpha in Glycan Nanoparticles Exhibits Full Activity and Reduces Pulmonary Hypertension in Rats. Biomacromolecules 2013, 14, 4009-4020.

(161) Mcnaughton, B. R.; Cronican, J. J.; Thompson, D. B.; Liu, D. R. Mammalian Cell Penetration, Sirna Transfection, and DNA Transfection by Supercharged Proteins. Proc. Natl. Acad. Sci. U. S. A. 2009, 106, 6111-6116.

(162) Vives, E.; Brodin, P.; Lebleu, B. A Truncated Hiv-1 Tat Protein Basic Domain Rapidly Translocates through the Plasma Membrane and Accumulates in the Cell Nucleus. J. Biol. Chem. 1997, 272, 16010-16017.

(163) Venslauskas, M. S.; Satkauskas, S. Mechanisms of Transfer of Bioactive Molecules through the Cell Membrane by Electroporation. European Biophysics Journal with Biophysics Letters 2015, 44, 277-289.

(164) Bockus, A. T.; Mcewen, C. M.; Lokey, R. S. Form and Function in Cyclic Peptide Natural Products: A Pharmacokinetic Perspective. Curr. Top. Med. Chem. 2013, 13, 821-836.

(165) Passioura, T.; Katoh, T.; Goto, Y.; Suga, H. Selection-Based Discovery of Druglike Macrocyclic Peptides. Annu. Rev. Biochem 2014, 83, 727-752.

(166) Hewitt, W. M.; Leung, S. S.; Pye, C. R.; Ponkey, A. R.; Bednarek, M.; Jacobson, M. P.; Lokey, R. S. Cell-Permeable Cyclic Peptides from Synthetic Libraries Inspired by Natural Products. J. Am. Chem. Soc. 2015, 137, 715-721.

(167) Chu, Q.; Moellering, R. E.; Hilinski, G. J.; Kim, Y. W.; Grossmann, T. N.; Yeh, J. T. H.; Verdine, G. L. Towards Understanding Cell Penetration by Stapled Peptides. Medchemcomm 2015, 6, 111-119. 
(168) Yang, N. J.; Hinner, M. J. Getting across the Cell Membrane: An Overview for Small Molecules, Peptides, and Proteins. Methods Mol. Biol. 2015, 1266, 29-53.

(169) Schafmeister, C. E.; Po, J.; Verdine, G. L. An All-Hydrocarbon Cross-Linking System for Enhancing the Helicity and Metabolic Stability of Peptides. JACS 2000, 122, 5891-5892.

(170) Verdine, G. L.; Hilinski, G. J. Stapled Peptides for Intracellular Drug Targets. Method Enzymol 2012 , $503,3-33$.

(171) Moellering, R. E.; Cornejo, M.; Davis, T. N.; Del Bianco, C.; Aster, J. C.; Blacklow, S. C.; Kung, A. L.; Gilliland, D. G.; Verdine, G. L.; Bradner, J. E. Direct Inhibition of the Notch Transcription Factor Complex. Nature 2009, 462, $182-188$.

(172) Warden, D.; Thorne, H. V. The Infectivity of Polyoma Virus DNA for Mouse Embryo Cells in the Presence of Diethylaminoethyl-Dextran. J. Gen. Virol. 1968, 3, 371-377.

Mccutchan, J. H.; Pagano, J. S. Enhancement of Infectivity of Simian Virus 40 Deoxyribonucleic Acid with DiethylaminoethylDextran. J. Natl. Cancer Inst. 1968, 41, 351-357.

Pagano, J. S.; Vaheri, A. Enhancement of Infectivity of Poliovirus Rna with Diethylaminoethyl-Dextran (Deae-D). Arch. Gesamte Virusforsch. 1965, 17, 456-464.

Graham, F. L.; Van Der Eb, A. J. A New Technique for the Assay of Infectivity of Human Adenovirus 5 DNA. Virology 1973, $52,456-467$.

Fraley, R.; Subramani, S.; Berg, P.; Papahadjopoulos, D. Introduction of Liposome-Encapsulated Sv40 DNA into Cells. J. Biol. Chem. 1980, 255, 431-435.

Wong, T. K.; Nicolau, C.; Hofschneider, P. H. Appearance of Beta-Lactamase Activity in Animal-Cells Upon LiposomeMediated Gene-Transfer. Gene 1980, 10, 87-94.

Felgner, P. L.; Gadek, T. R.; Holm, M.; Roman, R.; Chan, H. W.; Wenz, M.; Northrop, J. P.; Ringold, G. M.; Danielsen, M. Lipofection - a Highly Efficient, Lipid-Mediated DNA-Transfection Procedure. Proc. Natl. Acad. Sci. U. S. A. 1987, 84, 74137417.

(179) Boussif, O.; Lezoualch, F.; Zanta, M. A.; Mergny, M. D.; Scherman, D.; Demeneix, B.; Behr, J. P. A Versatile Vector for Gene and Oligonucleotide Transfer into Cells in Culture and in-Vivo - Polyethylenimine. Proc. Natl. Acad. Sci. U. S. A. 1995, 92, 7297-7301.

(180) Haensler, J.; Szoka, F. C. Polyamidoamine Cascade Polymers Mediate Efficient Transfection of Cells in Culture. Bioconjugate Chem. 1993, 4, 372-379.

(181) Kawai, S.; Nishizawa, M. New Procedure for DNA Transfection with Polycation and Dimethylsulfoxide. Mol. Cell. Biol. 1984, 4, 1172-1174.

(182) Wu, G. Y.; Wu, C. H. Receptor-Mediated Gene Delivery and Expression in Vivo. J. Biol. Chem. 1988, 263, $14621-14624$.

(183) Wu, G. Y.; Wu, C. H. Receptor-Mediated in Vitro Gene Transformation by a Soluble DNA Carrier System. J. Biol. Chem. 1987, 262, 4429-4432.

(184) Neumann, E.; Schaeferridder, M.; Wang, Y.; Hofschneider, P. H. Gene Transfer into Mouse Lyoma Cells by Electroporation in High Electric-Fields. EMBO J. 1982, 1, 841-845. 
De Ravin, S. S.; Reik, A.; Liu, P. Q.; Li, L.; Wu, X.; Su, L.; Raley, C.; Theobald, N.; Choi, U.; Song, A. H.et al. Targeted Gene Addition in Human Cd34(+) Hematopoietic Cells for Correction of X-Linked Chronic Granulomatous Disease. Nat. Biotechnol. 2016, 34, 424-429.

(186) Schuurhuis, D. H.; Verdijk, P.; Schreibelt, G.; Aarntzen, E. H. J. G.; Scharenborg, N.; De Boer, A.; Van De Rakt, M. W. M. M.; Kerkhoff, M.; Gerritsen, M. J. P.; Eijckeler, F.et al. In Situ Expression of Tumor Antigens by Messenger Rna-Electroporated Dendritic Cells in Lymph Nodes of Melanoma Patients. Cancer Res. 2009, 69, 2927-2934.

Zhao, Y. B.; Moon, E.; Carpenito, C.; Paulos, C. M.; Liu, X. J.; Brennan, A. L.; Chew, A.; Carroll, R. G.; Scholler, J.; Levine, B. L.et al. Multiple Injections of Electroporated Autologous T Cells Expressing a Chimeric Antigen Receptor Mediate Regression of Human Disseminated Tumor. Cancer Res. 2010, 70, 9053-9061.

Benteyn, D.; Heirman, C.; Bonehill, A.; Thielemans, K.; Breckpot, K. Mrna-Based Dendritic Cell Vaccines. Expert Review of Vaccines 2015, 14, 161-176.

(189) Lederberg, J. Cell Genetics and Hereditary Symbiosis. Physiol. Rev. 1952, 32, 403-430.

(190) Collins, J.; Hohn, B. Cosmids - Type of Plasmid Gene-Cloning Vector That Is Packageable Invitro in Bacteriophage LambdaHeads. Proc. Natl. Acad. Sci. U. S. A. 1978, 75, 4242-4246.

(191) Thomas, C. E.; Ehrhardt, A.; Kay, M. A. Progress and Problems with the Use of Viral Vectors for Gene Therapy. Nature Reviews Genetics 2003, 4, 346-358.

Kouprina, N.; Tomilin, A. N.; Masumoto, H.; Earnshaw, W. C.; Larionov, V. Human Artificial Chromosome-Based Gene Delivery Vectors for Biomedicine and Biotechnology. Expert Opin Drug Deliv 2014, 11, 517-535.

Jackson, D. A.; Berg, P.; Symons, R. H. Biochemical Method for Inserting New Genetic Information into DNA of Simian Virus 40 - Circular Sv40 DNA Molecules Containing Lambda Phage Genes and Galactose Operon of Escherichia-Coli. Proc. Natl. Acad. Sci. U. S. A. 1972, 69, 2904-2909.

Cohen, S. N.; Chang, A. C.; Boyer, H. W.; Helling, R. B. Construction of Biologically Functional Bacterial Plasmids in Vitro. Proc. Natl. Acad. Sci. U. S. A. 1973, 70, 3240-3244.

Cohen, S. N. DNA Cloning: A Personal View after 40 Years. Proc. Natl. Acad. Sci. U. S. A. 2013, 110, $15521-15529$.

Wensink, P. C.; Finnegan, D. J.; Donelson, J. E.; Hogness, D. S. A System for Mapping DNA Sequences in the Chromosomes of Drosophila Melanogaster. Cell 1974, 3, 315-325.

(197) Chang, A. C.; Nunberg, J. H.; Kaufman, R. J.; Erlich, H. A.; Schimke, R. T.; Cohen, S. N. Phenotypic Expression in E. Coli of a DNA Sequence Coding for Mouse Dihydrofolate Reductase. Nature 1978, 275, 617-624.

(198) Wigler, M.; Sweet, R.; Sim, G. K.; Wold, B.; Pellicer, A.; Lacy, E.; Maniatis, T.; Silverstein, S.; Axel, R. Transformation of Mammalian Cells with Genes from Procaryotes and Eucaryotes. Cell 1979, 16, 777-785.

(199) Wigler, M.; Pellicer, A.; Silverstein, S.; Axel, R.; Urlaub, G.; Chasin, L. DNA-Mediated Transfer of the Adenine Phosphoribosyltransferase Locus into Mammalian-Cells. Proc. Natl. Acad. Sci. U. S. A. 1979, 76, $1373-1376$.

(200) Mertz, J. E.; Gurdon, J. B. Purified Dnas Are Transcribed after Microinjection into Xenopus Oocytes. Proc. Natl. Acad. Sci. U. S. A. $1977,74,1502-1506$. 
(201) Anderson, W. F.; Killos, L.; Sandershaigh, L.; Kretschmer, P. J.; Diacumakos, E. G. Replication and Expression of Thymidine Kinase and Human Globin Genes Micro-Injected into Mouse Fibroblasts. Proceedings of the National Academy of Sciences of the United States of America-Biological Sciences 1980, 77, 5399-5403.

(202) Capecchi, M. R. High Efficiency Transformation by Direct Microinjection of DNA into Cultured Mammalian Cells. Cell 1980, 22, 479-488.

(203) Cohen, S. N.; Miller, C. A. Multiple Molecular Species of Circular R-Factor DNA Isolated from Escherichia-Coli. Nature 1969, $224,1273-1277$.

(204) Tsoi, M.; Do, T. T.; Tang, V.; Aguilera, J. A.; Perry, C. C.; Milligan, J. R. Characterization of Condensed Plasmid DNA Models for Studying the Direct Effect of lonizing Radiation. Biophys. Chem. 2010, 147, 104-110.

(205) Tang, M. X.; Szoka, F. C. The Influence of Polymer Structure on the Interactions of Cationic Polymers with DNA and Morphology of the Resulting Complexes. Gene Ther. 1997, 4, 823-832.

(206) Vijayanathan, V.; Thomas, T.; Shirahata, A.; Thomas, T. J. DNA Condensation by Polyamines: A Laser Light Scattering Study of Structural Effects. Biochemistry 2001, 40, 13644-13651.

(207) Catanese, D. J.; Fogg, J. M.; Schrock, D. E.; Gilbert, B. E.; Zechiedrich, L. Supercoiled Minivector DNA Resists Shear Forces Associated with Gene Therapy Delivery. Gene Ther. 2012, 19, 94-100.

(208) Hornstein, B. D.; Roman, D.; Arevalo-Soliz, L. M.; Engevik, M. A.; Zechiedrich, L. Effects of Circular DNA Length on Transfection Efficiency by Electroporation into Hela Cells. PLoS One 2016, 11, e0167537.

(209) Stephenson, M. L.; Zamecnik, P. C. Inhibition of Rous-Sarcoma Viral-Rna Translation by a Specific Oligodeoxyribonucleotide. Proc. Natl. Acad. Sci. U. S. A. 1978, 75, 285-288.

(210) Izant, J. G.; Weintraub, H. Inhibition of Thymidine Kinase Gene-Expression by Anti-Sense Rna - a Molecular Approach to Genetic-Analysis. Cell 1984, 36, 1007-1015.

(211) Rosenberg, U. B.; Preiss, A.; Seifert, E.; Jackle, H.; Knipple, D. C. Production of Phenocopies by Kruppel Antisense Rna Injection into Drosophila Embryos. Nature 1985, 313, 703-706.

(212) Knecht, D. A.; Loomis, W. F. Antisense Rna Inactivation of Myosin Heavy-Chain Gene-Expression in DictyosteliumDiscoideum. Science 1987, 236, 1081-1086.

(213) Marwick, C. First "Antisense" Drug Will Treat Cmv Retinitis. Jama-Journal of the American Medical Association 1998, 280, $871-871$.

(214) De Smet, M. D.; Meenken, C. J.; Van Den Horn, G. J. Fomivirsen - a Phosphorothioate Oligonucleotide for the Treatment of Cmv Retinitis. Ocul. Immunol. Inflamm. 1999, 7, 189-198.

(215) Fire, A.; Xu, S. Q.; Montgomery, M. K.; Kostas, S. A.; Driver, S. E.; Mello, C. C. Potent and Specific Genetic Interference by Double-Stranded Rna in Caenorhabditis Elegans. Nature 1998, 391, 806-811.

(216) Elbashir, S. M.; Harborth, J.; Lendeckel, W.; Yalcin, A.; Weber, K.; Tuschl, T. Duplexes of 21-Nucleotide Rnas Mediate Rna Interference in Cultured Mammalian Cells. Nature 2001, 411, 494-498. 
(217) Lee, R. C.; Feinbaum, R. L.; Ambros, V. The C-Elegans Heterochronic Gene Lin-4 Encodes Small Rnas with Antisense Complementarity to Lin-14. Cell 1993, 75, 843-854.

(218) Lundin, K. E.; Gissberg, O.; Smith, C. I. E. Oligonucleotide Therapies: The Past and the Present. Hum. Gene Ther. 2015, 26, 475-485.

Schroeder, A.; Levins, C. G.; Cortez, C.; Langer, R.; Anderson, D. G. Lipid-Based Nanotherapeutics for Sirna Delivery. J. Intern. Med. 2010, 267, 9-21.

Juliano, R. L. The Delivery of Therapeutic Oligonucleotides. Nucleic Acids Res. 2016, 44, 6518-6548.

Crooke, S. T.; Wang, S.; Vickers, T. A.; Shen, W.; Liang, X. H. Cellular Uptake and Trafficking of Antisense Oligonucleotides. Nat. Biotechnol. 2017, 35, 230-237.

Summerton, J.; Weller, D. Morpholino Antisense Oligomers: Design, Preparation, and Properties. Antisense Nucleic Acid Drug Dev. 1997, 7, 187-195.

Nielsen, P. E.; Egholm, M.; Berg, R. H.; Buchardt, O. Sequence-Selective Recognition of DNA by Strand Displacement with a Thymine-Substituted Polyamide. Science 1991, 254, 1497-1500.

Meade, B. R.; Gogoi, K.; Hamil, A. S.; Palm-Apergi, C.; Berg, A.; Hagopian, J. C.; Springer, A. D.; Eguchi, A.; Kacsinta, A. D.; Dowdy, C. F.et al. Efficient Delivery of Rnai Prodrugs Containing Reversible Charge-Neutralizing Phosphotriester Backbone Modifications. Nat. Biotechnol. 2014, 32, 1256-1261.

(225) Dowdy, S. F. Overcoming Cellular Barriers for Rna Therapeutics. Nat. Biotechnol. 2017, 35, $222-229$.

(226) Stewart, M. P.; Lorenz, A.; Dahlman, J.; Sahay, G. Challenges in Carrier-Mediated Intracellular Delivery: Moving Beyond Endosomal Barriers. Wires Nanomed Nanobi 2016, 8, 465-478.

(227) Yang, B.; Ming, X.; Cao, C.; Laing, B.; Yuan, A.; Porter, M. A.; Hull-Ryde, E. A.; Maddry, J.; Suto, M.; Janzen, W. P.et al. HighThroughput Screening Identifies Small Molecules That Enhance the Pharmacological Effects of Oligonucleotides. Nucleic Acids Res. 2015, 43, 1987-1996.

(228) Wittrup, A.; Lieberman, J. Knocking Down Disease: A Progress Report on Sirna Therapeutics. Nature Reviews Genetics 2015, $16,543-552$.

(229) Kanasty, R.; Dorkin, J. R.; Vegas, A.; Anderson, D. Delivery Materials for Sirna Therapeutics. Nat Mater 2013, $12,967-977$.

(230) Liu, Y. M.; Kuan, C. T.; Mi, J.; Zhang, X. W.; Clary, B. M.; Bigner, D. D.; Sullenger, B. A. Aptamers Selected against the Unglycosylated Egfrviii Ectodomain and Delivered Intracellularly Reduce Membrane-Bound Egfrviii and Induce Apoptosis. Biol. Chem. 2009, 390, 137-144.

(231) Leung, A. K. K.; Tam, Y. Y. C.; Cullis, P. R. Lipid Nanoparticles for Short Interfering Rna Delivery. Nonviral Vectors for Gene Therapy Lipid- and Polymer-Based Gene Transfer 2014, 88, 71-110.

(232) Mcmanus, M. T.; Haines, B. B.; Dillon, C. P.; Whitehurst, C. E.; Van Parijs, L.; Chen, J. Z.; Sharp, P. A. Small Interfering RnaMediated Gene Silencing in T Lymphocytes. J. Immunol. 2002, 169, 5754-5760.

(233) Merkerova, M.; Klamova, H.; Brdicka, R.; Bruchova, H. Targeting of Gene Expression by Sirna in Cml Primary Cells. Mol. Biol. Rep. 2007, 34, 27-33. 
Wiese, M.; Castiglione, K.; Hensel, M.; Schleicher, U.; Bogdan, C.; Jantsch, J. Small Interfering Rna (Sirna) Delivery into Murine Bone Marrow-Derived Macrophages by Electroporation. J. Immunol. Methods 2010, 353, $102-110$.

Chabot, S.; Teissie, J.; Golzio, M. Targeted Electro-Delivery of Oligonucleotides for Rna Interference: Sirna and Antimir. Adv. Drug Del. Rev. 2015, 81, 161-168.

(236) Garcia-Sanchez, A.; Marques-Garcia, F. Gene Silencing Delivery Methods: Lipid-Mediated and Electroporation Transfection Protocols. Methods Mol. Biol. 2016, 1434, 139-151.

Tasfaout, H.; Buono, S.; Guo, S.; Kretz, C.; Messaddeq, N.; Booten, S.; Greenlee, S.; Monia, B. P.; Cowling, B. S.; Laporte, J. Antisense Oligonucleotide-Mediated Dnm2 Knockdown Prevents and Reverts Myotubular Myopathy in Mice. Nat Commun 2017, 8, 15661.

(238) Brito, J. L. R.; Davies, F. E.; Gonzalez, D.; Morgan, G. J. Streptolysin-O Reversible Permeabilisation Is an Effective Method to Transfect Sirnas into Myeloma Cells. J. Immunol. Methods 2008, 333, 147-155.

(239) Zhou, P.; Ma, X.; lyer, L.; Chaulagain, C.; Comenzo, R. L. One Sirna Pool Targeting the Lambda Constant Region Stops Lambda Light-Chain Production and Causes Terminal Endoplasmic Reticulum Stress. Blood 2014, 123, 3440-3451.

(240) Brachet, J.; Huez, G.; Hubert, E. Microinjection of Rabbit Hemoglobin Messenger-Rna into Amphibian Oocytes and Embryos. Proc. Natl. Acad. Sci. U. S. A. 1973, 70, 543-547.

(241) Woodland, H. R.; Gurdon, J. B.; Lingrel, J. B. The Translation of Mammalian Globin Mrna Injected into Fertilized Eggs of Xenopus Laevis. li. The Distribution of Globin Synthesis in Different Tissues. Dev. Biol. 1974, 39, 134-140.

(242) Gurdon, J. B.; Woodland, H. R.; Lingrel, J. B. The Translation of Mammalian Globin Mrna Injected into Fertilized Eggs of Xenopus Laevis I. Message Stability in Development. Dev. Biol. 1974, 39, 125-133.

(243) Mizutani, S.; Colonno, R. J. In Vitro Synthesis of an Infectious Rna from Cdna Clones of Human Rhinovirus Type 14. J. Virol. $1985,56,628-632$.

(244) Vanderwerf, S.; Bradley, J.; Wimmer, E.; Studier, F. W.; Dunn, J. J. Synthesis of Infectious Poliovirus Rna by Purified T7 RnaPolymerase. Proc. Natl. Acad. Sci. U. S. A. 1986, 83, 2330-2334.

(245) Malone, R. W.; Felgner, P. L.; Verma, I. M. Cationic Liposome-Mediated Rna Transfection. Proc. Natl. Acad. Sci. U. S. A. $1989,86,6077-6081$.

(246) Lai, C. J.; Zhao, B.; Hori, H.; Bray, M. Infectious Rna Transcribed from Stably Cloned Full-Length Cdna of Dengue Type-4 Virus. Proc. Natl. Acad. Sci. U. S. A. 1991, 88, 5139-5143.

(247) Islam, M. A.; Reesor, E. K.; Xu, Y.; Zope, H. R.; Zetter, B. R.; Shi, J. Biomaterials for Mrna Delivery. Biomater Sci 2015, 3, 1519-1533.

(248) Gallie, D. R. The Cap and Poly(a) Tail Function Synergistically to Regulate Mrna Translational Efficiency. Genes Dev. 1991, 5, 2108-2116.

(249) Yokoe, H.; Meyer, T. Spatial Dynamics of Gfp-Tagged Proteins Investigated by Local Fluorescence Enhancement. Nat. Biotechnol. 1996, 14, 1252-1256. 
Tavernier, G.; Andries, O.; Demeester, J.; Sanders, N. N.; De Smedt, S. C.; Rejman, J. Mrna as Gene Therapeutic: How to Control Protein Expression. J. Controlled Release 2011, 150, 238-247.

Yamamoto, A.; Kormann, M.; Rosenecker, J.; Rudolph, C. Current Prospects for Mrna Gene Delivery. Eur. J. Pharm. Biopharm. 2009, 71, 484-489.

(252) Barrett, L. E.; Sul, J. Y.; Takano, H.; Van Bockstaele, E. J.; Haydon, P. G.; Eberwine, J. H. Region-Directed Phototransfection Reveals the Functional Significance of a Dendritically Synthesized Transcription Factor. Nat. Methods 2006, 3, 455-460.

Rabinovich, P. M.; Komarovskaya, M. E.; Ye, Z. J.; Imai, C.; Campana, D.; Bahceci, E.; Weissman, S. M. Synthetic Messenger Rna as a Tool for Gene Therapy. Hum. Gene Ther. 2006, 17, 1027-1035.

(254) Sahin, U.; Kariko, K.; Tureci, O. Mrna-Based Therapeutics - Developing a New Class of Drugs. Nature Reviews Drug Discovery 2014, 13, 759-780.

(255) Kauffman, K. J.; Webber, M. J.; Anderson, D. G. Materials for Non-Viral Intracellular Delivery of Messenger Rna Therapeutics. J. Control. Release 2016, 240, 227-234.

(256) Gilboa, E.; Vieweg, J. Cancer Immunotherapy with Mrna-Transfected Dendritic Cells. Immunol. Rev. 2004, 199, $251-263$.

(257) Zhao, Y. B.; Zheng, Z. L.; Cohen, C. J.; Gattinoni, L.; Palmer, D. C.; Restifo, N. P.; Rosenberg, S. A.; Morgan, R. A. HighEfficiency Transfection of Primary Human and Mouse T Lymphocytes Using Rna Electroporation. Mol. Ther. 2006, 13, 151159.

(258) Kranz, L. M.; Diken, M.; Haas, H.; Kreiter, S.; Loquai, C.; Reuter, K. C.; Meng, M.; Fritz, D.; Vascotto, F.; Hefesha, H.et al. Systemic Rna Delivery to Dendritic Cells Exploits Antiviral Defence for Cancer Immunotherapy. Nature 2016, 534, 396-401. Barrett, D. M.; Zhao, Y. B.; Liu, X. J.; Jiang, S. G.; Carpenito, C.; Kalos, M.; Carroll, R. G.; June, C. H.; Grupp, S. A. Treatment of Advanced Leukemia in Mice with Mrna Engineered T Cells. Hum. Gene Ther. 2011, 22, 1575-1586.

(260) Van Tendeloo, V. F. I.; Ponsaerts, P.; Lardon, F.; Nijs, G.; Lenjou, M.; Van Broeckhoven, C.; Van Bockstaele, D. R.; Berneman, Z. N. Highly Efficient Gene Delivery by Mrna Electroporation in Human Hematopoietic Cells: Superiority to Lipofection and Passive Pulsing of Mrna and to Electroporation of Plasmid Cdna for Tumor Antigen Loading of Dendritic Cells. Blood 2001, 98, 49-56.

(261) Van Meirvenne, S.; Straetman, L.; Heirman, C.; Dullaers, M.; De Greef, C.; Van Tendeloo, V.; Thielemans, K. Efficient Genetic Modification of Murine Dendritic Cells by Electroporation with Mrna. Cancer Gene Ther. 2002, 9, 787-797.

(262) Benteyn, D.; Van Nuffel, A. M. T.; Wilgenhof, S.; Bonehill, A. Single-Step Antigen Loading and Maturation of Dendritic Cells through Mrna Electroporation of a Tumor-Associated Antigen and a Trimix of Costimulatory Molecules. Cancer Vaccines: Methods and Protocols 2014, 1139, 3-15.

(263) Gopal, A.; Zhou, Z. H.; Knobler, C. M.; Gelbart, W. M. Visualizing Large Rna Molecules in Solution. Rna-a Publication of the Rna Society 2012, 18, 284-299.

(264) Kormann, M. S. D.; Hasenpusch, G.; Aneja, M. K.; Nica, G.; Flemmer, A. W.; Herber-Jonat, S.; Huppmann, M.; Mays, L. E.; Illenyi, M.; Schams, A.et al. Expression of Therapeutic Proteins after Delivery of Chemically Modified Mrna in Mice. Nat. Biotechnol. 2011, 29, 154-157. 
(265)

Rothemund, P. W. K. Folding DNA to Create Nanoscale Shapes and Patterns. Nature 2006, 440, $297-302$.

Bhatia, D.; Arumugam, S.; Nasilowski, M.; Joshi, H.; Wunder, C.; Chambon, V.; Prakash, V.; Grazon, C.; Nadal, B.; Maiti, P. K.et al. Quantum Dot-Loaded Monofunctionalized DNA Icosahedra for Single-Particle Tracking of Endocytic Pathways. Nat Nanotechnol 2016, 11, 1112-1119.

Zanacchi, F. C.; Manzo, C.; Alvarez, A. S.; Derr, N. D.; Garcia-Parajo, M. F.; Lakadamyali, M. A DNA Origami Platform for Quantifying Protein Copy Number in Super-Resolution. Nat. Methods 2017, 14, 789-792.

Tyagi, S.; Kramer, F. R. Molecular Beacons: Probes That Fluoresce Upon Hybridization. Nat. Biotechnol. 1996, 14, 303-308.

Tan, W.; Wang, K.; Drake, T. J. Molecular Beacons. Curr. Opin. Chem. Biol. 2004, 8, 547-553.

Hamaguchi, N.; Ellington, A.; Stanton, M. Aptamer Beacons for the Direct Detection of Proteins. Anal. Biochem. 2001, 294, 126-131.

Lee, J. F.; Stovall, G. M.; Ellington, A. D. Aptamer Therapeutics Advance. Curr. Opin. Chem. Biol. 2006, 10, $282-289$.

Song, S. P.; Wang, L. H.; Li, J.; Zhao, J. L.; Fan, C. H. Aptamer-Based Biosensors. Trac-Trends in Analytical Chemistry 2008, $27,108-117$.

Iliuk, A. B.; Hu, L. H.; Tao, W. A. Aptamer in Bioanalytical Applications. Anal. Chem. 2011, 83, 4440-4452.

Siegert, I.; Schatz, V.; Prechtel, A. T.; Steinkasserer, A.; Bogdan, C.; Jantsch, J. Electroporation of Sirna into Mouse Bone Marrow-Derived Macrophages and Dendritic Cells. Electroporation Protocols: Preclinical and Clinical Gene Medicine, 2nd Edition 2014, 1121, 111-119.

Jensen, K.; Anderson, J. A.; Glass, E. J. Comparison of Small Interfering Rna (Sirna) Delivery into Bovine Monocyte-Derived Macrophages by Transfection and Electroporation. Vet. Immunol. Immunopathol. 2014, 158, $224-232$.

He, W.; Bennett, M. J.; Luistro, L.; Carvajal, D.; Nevins, T.; Smith, M.; Tyagi, G.; Cai, J.; Wei, X.; Lin, T. A.et al. Discovery of Sirna Lipid Nanoparticles to Transfect Suspension Leukemia Cells and Provide in Vivo Delivery Capability. Mol. Ther. 2014, 22, 359-370.

Gul-Uludag, H.; Valencia-Serna, J.; Kucharski, C.; Marquez-Curtis, L. A.; Jiang, X. Y.; Larratt, L.; Janowska-Wieczorek, A.; Uludag, H. Polymeric Nanoparticle-Mediated Silencing of Cd44 Receptor in Cd34(+) Acute Myeloid Leukemia Cells. Leukemia Res. 2014, 38, 1299-1308.

Peer, D. A Daunting Task: Manipulating Leukocyte Function with Rnai. Immunol. Rev. 2013, 253, 185-197.

Freeley, M.; Long, A. Advances in Sirna Delivery to T-Cells: Potential Clinical Applications for Inflammatory Disease, Cancer and Infection. Biochem. J 2013, 455, 133-147.

Novobrantseva, T. I.; Borodovsky, A.; Wong, J.; Klebanov, B.; Zafari, M.; Yucius, K.; Querbes, W.; Ge, P.; Ruda, V. M.; Milstein, S.et al. Systemic Rnai-Mediated Gene Silencing in Nonhuman Primate and Rodent Myeloid Cells. Mol Ther Nucleic Acids 2012, 1, e4.

Humbert, J. M.; Halary, F. Viral and Non-Viral Methods to Genetically Modify Dendritic Cells. Curr. Gene Ther. 2012, 12, 127136. 

Methods Mol. Biol. 2009, 531, 123-143.

Li, G. B.; Lu, G. X. Gene Delivery Efficiency in Bone Marrow-Derived Dendritic Cells: Comparison of Four Methods and Optimization for Lentivirus Transduction. Mol. Biotechnol. 2009, 43, 250-256.

(284) Lee, J. S.; Reiner, N. E. Stable Lentiviral Vector-Mediated Gene Silencing in Human Monocytic Cell Lines. Methods Mol. Biol. 2009, 531, 287-300.

(285) Ceppi, M.; Schmidt, E.; Pierre, P. Genetic Modification of Murine Dendritic Cells by Rna Transfection. Methods Mol. Biol. 2009, 531, 145-156.

(286) Met, O.; Eriksen, J.; Svane, I. M. Studies on Mrna Electroporation of Immature and Mature Dendritic Cells: Effects on Their Immunogenic Potential. Mol. Biotechnol. 2008, 40, 151-160.

(287) Liu, J.; Gaj, T.; Yang, Y.; Wang, N.; Shui, S.; Kim, S.; Kanchiswamy, C. N.; Kim, J. S.; Barbas, C. F., 3rd. Efficient Delivery of Nuclease Proteins for Genome Editing in Human Stem Cells and Primary Cells. Nat. Protoc. 2015, 10, $1842-1859$.

Mandal, Pankaj k.; Ferreira, L. M. R.; Collins, R.; Meissner, Torsten b.; Boutwell, Christian I.; Friesen, M.; Vrbanac, V.; Garrison, Brian s.; Stortchevoi, A.; Bryder, D.et al. Efficient Ablation of Genes in Human Hematopoietic Stem and Effector Cells Using Crispr/Cas9. Cell Stem Cell 2014, 15, 643-652.

Gresch, O.; Altrogge, L. Transfection of Difficult-to-Transfect Primary Mammalian Cells. Protein Expression in Mammalian Cells: Methods and Protocols 2012, 801, 65-74.

(290) Karra, D.; Dahm, R. Transfection Techniques for Neuronal Cells. J. Neurosci. 2010, 30, 6171-6177.

(291) So, H. M.; Won, K.; Kim, Y. H.; Kim, B. K.; Ryu, B. H.; Na, P. S.; Kim, H.; Lee, J. O. Single-Walled Carbon Nanotube Biosensors Using Aptamers as Molecular Recognition Elements. JACS 2005, 127, 11906-11907.

(292) Heller, D. A.; Baik, S.; Eurell, T. E.; Strano, M. S. Single-Walled Carbon Nanotube Spectroscopy in Live Cells: Towards LongTerm Labels and Optical Sensors. Adv. Mater. 2005, 17, 2793-2799.

(293) Fakhri, N.; Wessel, A. D.; Willms, C.; Pasquali, M.; Klopfenstein, D. R.; Mackintosh, F. C.; Schmidt, C. F. High-Resolution Mapping of Intracellular Fluctuations Using Carbon Nanotubes. Science 2014, 344, 1031-1035.

Derfus, A. M.; Chan, W. C. W.; Bhatia, S. N. Intracellular Delivery of Quantum Dots for Live Cell Labeling and Organelle Tracking. Adv. Mater. 2004, 16, 961-966.

Michalet, X.; Pinaud, F. F.; Bentolila, L. A.; Tsay, J. M.; Doose, S.; Li, J. J.; Sundaresan, G.; Wu, A. M.; Gambhir, S. S.; Weiss, S. Quantum Dots for Live Cells, in Vivo Imaging, and Diagnostics. Science 2005, 307, 538-544.

(296) Chinen, A. B.; Guan, C. M.; Ferrer, J. R.; Barnaby, S. N.; Merkel, T. J.; Mirkin, C. A. Nanoparticle Probes for the Detection of Cancer Biomarkers, Cells, and Tissues by Fluorescence. Chem. Rev. 2015, 115, 10530-10574.

Schulz, A.; Mcdonagh, C. Intracellular Sensing and Cell Diagnostics Using Fluorescent Silica Nanoparticles. Soft Matter 2012, 8, 2579-2585.

Liu, J. W.; Cao, Z. H.; Lu, Y. Functional Nucleic Acid Sensors. Chem. Rev. 2009, 109, 1948-1998. 
(299) Lee, S. E.; Liu, G. L.; Kim, F.; Lee, L. P. Remote Optical Switch for Localized and Selective Control of Gene Interference. Nano Lett. 2009, 9, 562-570.

(300) Breger, J.; Delehanty, J. B.; Medintz, I. L. Continuing Progress toward Controlled Intracellular Delivery of Semiconductor Quantum Dots. Wiley Interdiscip Rev Nanomed Nanobiotechnol 2014, 7, 131-151.

(301) Hong, G. S.; Diao, S. O.; Antaris, A. L.; Dai, H. J. Carbon Nanomaterials for Biological Imaging and Nanomedicinal Therapy. Chem. Rev. 2015, 115, 10816-10906.

(302) Ajayan, P. M. Nanotubes from Carbon. Chem. Rev. 1999, 99, 1787-1799.

(303) Zhang, Y. B.; Petibone, D.; Xu, Y.; Mahmood, M.; Karmakar, A.; Casciano, D.; Ali, S.; Biris, A. S. Toxicity and Efficacy of Carbon Nanotubes and Graphene: The Utility of Carbon-Based Nanoparticles in Nanomedicine. Drug Metab. Rev. 2014, 46, 232-246.

(304) Holt, B. D.; Shawky, J. H.; Dahl, K. N.; Davidson, L. A.; Islam, M. F. Distribution of Single Wall Carbon Nanotubes in the Xenopus Laevis Embryo after Microinjection. J. Appl. Toxicol. 2016, 36, 568-578.

(305) Medintz, I. L.; Uyeda, H. T.; Goldman, E. R.; Mattoussi, H. Quantum Dot Bioconjugates for Imaging, Labelling and Sensing. Nature Materials 2005, 4, 435-446.

(306) Courty, S.; Luccardini, C.; Bellaiche, Y.; Cappello, G.; Dahan, M. Tracking Individual Kinesin Motors in Living Cells Using Single Quantum-Dot Imaging. Nano Lett. 2006, 6, 1491-1495.

(307) Liu, B. R.; Huang, Y. W.; Chiang, H. J.; Lee, H. J. Cell-Penetrating Peptide-Functionalized Quantum Dots for Intracellular Delivery. Journal of Nanoscience and Nanotechnology 2010, 10, 7897-7905.

(308) Lee, J.; Sharei, A.; Sim, W. Y.; Adamo, A.; Langer, R.; Jensen, K. F.; Bawendi, M. G. Nonendocytic Delivery of Functional Engineered Nanoparticles into the Cytoplasm of Live Cells Using a Novel, High-Throughput Microfluidic Device. Nano Lett. 2012, 12, 6322-6327.

(309) Xu, J. M.; Teslaa, T.; Wu, T. H.; Chiou, P. Y.; Teitell, M. A.; Weiss, S. Nanoblade Delivery and Incorporation of Quantum Dot Conjugates into Tubulin Networks in Live Cells. Nano Lett. 2012, 12, 5669-5672.

(310) Xiong, R. H.; Joris, F.; De Cock, I.; Demeester, J.; De Smedt, S. C.; Skirtach, A. G.; Braeckmans, K. Efficient Delivery of Quantum Dots in Live Cells by Gold Nanoparticle Mediated Photoporation. Colloidal Nanoparticles for Biomedical Applications $X 2015,9338,93380 X$

(311) Medepalli, K.; Alphenaar, B. W.; Keynton, R. S.; Sethu, P. A New Technique for Reversible Permeabilization of Live Cells for Intracellular Delivery of Quantum Dots. Nanotechnology 2013, 24, 205101.

(312) Sun, C.; Cao, Z. N.; Wu, M.; Lu, C. Intracellular Tracking of Single Native Molecules with Electroporation-Delivered Quantum Dots. Anal. Chem. 2014, 86, 11403-11409.

(313) Ma, Y.; Wang, M.; Li, W.; Zhang, Z.; Zhang, X.; Tan, T.; Zhang, X.-E.; Cui, Z. Live Cell Imaging of Single Genomic Loci with Quantum Dot-Labeled Tales. Nat Commun 2017, 8, 15318.

(314) Biju, V.; Itoh, T.; Ishikawa, M. Delivering Quantum Dots to Cells: Bioconjugated Quantum Dots for Targeted and Nonspecific Extracellular and Intracellular Imaging. Chem. Soc. Rev. 2010, 39, 3031-3056. 
Delehanty, J. B.; Mattoussi, H.; Medintz, I. L. Delivering Quantum Dots into Cells: Strategies, Progress and Remaining Issues. Anal. Bioanal. Chem. 2009, 393, 1091-1105.

(316) Yoo, D.; Lee, J. H.; Shin, T. H.; Cheon, J. Theranostic Magnetic Nanoparticles. Acc. Chem. Res. 2011, 44, 863-874.

(317) Lewin, M.; Carlesso, N.; Tung, C. H.; Tang, X. W.; Cory, D.; Scadden, D. T.; Weissleder, R. Tat Peptide-Derivatized Magnetic Nanoparticles Allow in Vivo Tracking and Recovery of Progenitor Cells. Nat. Biotechnol. 2000, 18, 410-414.

(318) Nitin, N.; Laconte, L. E. W.; Zurkiya, O.; Hu, X.; Bao, G. Functionalization and Peptide-Based Delivery of Magnetic Nanoparticles as an Intracellular Mri Contrast Agent. J. Biol. Inorg. Chem. 2004, 9, 706-712.

(319) Kievit, F. M.; Veiseh, O.; Bhattarai, N.; Fang, C.; Gunn, J. W.; Lee, D.; Ellenbogen, R. G.; Olson, J. M.; Zhang, M. Q. Pei-PegChitosan-Copolymer-Coated Iron Oxide Nanoparticles for Safe Gene Delivery: Synthesis, Complexation, and Transfection. Adv. Funct. Mater. 2009, 19, 2244-2251.

(320) Jeon, S.; Subbiah, R.; Bonaedy, T.; Van, S.; Park, K.; Yun, K. Surface Functionalized Magnetic Nanoparticles Shift Cell Behavior with on/Off Magnetic Fields. J. Cell. Physiol. 2018, 233, 1168-1178.

Baffou, G.; Rigneault, H.; Marguet, D.; Jullien, L. A Critique of Methods for Temperature Imaging in Single Cells. Nat. Methods 2014, 11, 899-901.

Kucsko, G.; Maurer, P. C.; Yao, N. Y.; Kubo, M.; Noh, H. J.; Lo, P. K.; Park, H.; Lukin, M. D. Nanometre-Scale Thermometry in a Living Cell. Nature 2013, 500, 54-58.

Okabe, K.; Inada, N.; Gota, C.; Harada, Y.; Funatsu, T.; Uchiyama, S. Intracellular Temperature Mapping with a Fluorescent Polymeric Thermometer and Fluorescence Lifetime Imaging Microscopy. Nat Commun 2012, 3.

Barber, M. A. A Technic for the Inoculation of Bacteria and Other Substances into Living Cells. J. Infect. Dis. 1911, 8, 348-360. Korzh, V.; Strahle, U. Marshall Barber and the Century of Microinjection: From Cloning of Bacteria to Cloning of Everything. Differentiation 2002, 70, 221-226.

Briggs, R.; King, T. J. Transplantation of Living Nuclei from Blastula Cells into Enucleated Frogs Eggs. Proc. Natl. Acad. Sci. U. S. A. $1952,38,455-463$.

Fischberg, M.; Gurdon, J. B.; Elsdale, T. R. Nuclear Transplantation in Xenopus Laevis. Nature 1958, 181, 424-424.

Gurdon, J. B. Adult Frogs Derived from Nuclei of Single Somatic Cells. Dev. Biol. 1962, 4, 256-273.

Wilmut, I.; Schnieke, A. E.; Mcwhir, J.; Kind, A. J.; Campbell, K. H. S. Viable Offspring Derived from Fetal and Adult Mammalian Cells. Nature 1997, 385, 810-813.

Craven, L.; Tuppen, H. A.; Greggains, G. D.; Harbottle, S. J.; Murphy, J. L.; Cree, L. M.; Murdoch, A. P.; Chinnery, P. F.; Taylor, R. W.; Lightowlers, R. N.et al. Pronuclear Transfer in Human Embryos to Prevent Transmission of Mitochondrial DNA Disease. Nature 2010, 465, 82-85.

Hiramoto, Y. Microinjection of Live Spermatozoa into Sea Urchin Eggs. Exp. Cell Res. 1962, 27, 416-426.

Palermo, G.; Joris, H.; Devroey, P.; Van Steirteghem, A. C. Pregnancies after Intracytoplasmic Injection of Single Spermatozoon into an Oocyte. Lancet 1992, 340, 17-18.

Diacumakos, E. G. Methods for Micromanipulation of Human Somatic Cells in Culture. Methods Cell Biol. 1973, 7, $287-311$. 
Co, D. O.; Borowski, A. H.; Leung, J. D.; Van Der Kaa, J.; Hengst, S.; Platenburg, G. J.; Pieper, F. R.; Perez, C. F.; Jirik, F. R.; Drayer, J. I. Generation of Transgenic Mice and Germline Transmission of a Mammalian Artificial Chromosome Introduced into Embryos by Pronuclear Microinjection. Chromosome Res. 2000, 8, 183-191.

Monteith, D. P.; Leung, J. D.; Borowski, A. H.; Co, D. O.; Praznovszky, T.; Jirik, F. R.; Hadlaczky, G.; Perez, C. F. Pronuclear Microinjection of Purified Artificial Chromosomes for Generation of Transgenic Mice: Pick-and-Inject Technique. Methods Mol. Biol. 2004, 240, 227-242.

(336) Knowles, J. K. An Improved Microinjection Technique in Paramecium Aurelia. Transfer of Mitochondria Conferring Erythromycin-Resistance. Exp. Cell Res. 1974, 88, 79-87.

King, M. P.; Attardi, G. Injection of Mitochondria into Human-Cells Leads to a Rapid Replacement of the Endogenous Mitochondrial-DNA. Cell 1988, 52, 811-819.

Pinkert, C. A.; Irwin, M. H.; Johnson, L. W.; Moffatt, R. J. Mitochondria Transfer into Mouse Ova by Microinjection. Transgenic Res. 1997, 6, 379-383.

Wu, T. H.; Teslaa, T.; Kalim, S.; French, C. T.; Moghadam, S.; Wall, R.; Miller, J. F.; Witte, O. N.; Teitell, M. A.; Chiou, P. Y. Photothermal Nanoblade for Large Cargo Delivery into Mammalian Cells. Anal. Chem. 2011, 83, 1321-1327.

Wu, Y. C.; Wu, T. H.; Clemens, D. L.; Lee, B. Y.; Wen, X. M.; Horwitz, M. A.; Teitell, M. A.; Chiou, P. Y. Massively Parallel Delivery of Large Cargo into Mammalian Cells with Light Pulses. Nat. Methods 2015, 12, 439-444.

Wu, T. H.; Sagullo, E.; Case, D.; Zheng, X.; Li, Y. J.; Hong, J. S.; Teslaa, T.; Patananan, A. N.; Mccaffery, J. M.; Niazi, K.et al. Mitochondrial Transfer by Photothermal Nanoblade Restores Metabolite Profile in Mammalian Cells. Cell Metab. 2016, 23, 921-929.

King, M. P.; Attardi, G. Human-Cells Lacking Mtdna - Repopulation with Exogenous Mitochondria by Complementation. Science 1989, 246, 500-503.

Moraes, C. T.; Dey, R.; Barrientos, A. Transmitochondrial Technology in Animal Cells. Methods in Cell Biology, Vol 65 2001, $65,397-412$.

Fournier, R. E. K.; Ruddle, F. H. Microcell-Mediated Transfer of Murine Chromosomes into Mouse, Chinese-Hamster, and Human Somatic-Cells. Proc. Natl. Acad. Sci. U. S. A. 1977, 74, 319-323.

Tomizuka, K.; Yoshida, H.; Uejima, H.; Kugoh, H.; Sato, K.; Ohguma, A.; Hayasaka, M.; Hanaoka, K.; Oshimura, M.; Ishida, I. Functional Expression and Germline Transmission of a Human Chromosome Fragment in Chimaeric Mice. Nat. Genet. 1997, $16,133-143$.

(346) Kazuki, Y.; Hoshiya, H.; Takiguchi, M.; Abe, S.; lida, Y.; Osaki, M.; Katoh, M.; Hiratsuka, M.; Shirayoshi, Y.; Hiramatsu, K.et al. Refined Human Artificial Chromosome Vectors for Gene Therapy and Animal Transgenesis. Gene Ther. 2011, 18, 384-393.

(347) Tedesco, F. S.; Hoshiya, H.; D'antona, G.; Gerli, M. F. M.; Messina, G.; Antonini, S.; Tonlorenzi, R.; Benedetti, S.; Berghella, L.; Torrente, Y.et al. Stem Cell-Mediated Transfer of a Human Artificial Chromosome Ameliorates Muscular Dystrophy. Sci. Transl. Med. 2011, 3, 96ra78. 
Tseng, Y.; Kole, T. P.; Wirtz, D. Micromechanical Mapping of Live Cells by Multiple-Particle-Tracking Microrheology. Biophys. J. 2002, 83, 3162-3176.

(349) Ehrenberg, M.; Mcgrath, J. L. Binding between Particles and Proteins in Extracts: Implications for Microrheology and Toxicity. Acta Biomater. 2005, 1, 305-315.

(350) Wirtz, D. Particle-Tracking Microrheology of Living Cells: Principles and Applications. Annual Review of Biophysics 2009, 38, 301-326.

(351) Thompson, M. S.; Wirtz, D. Sensing Cytoskeletal Mechanics by Ballistic Intracellular Nanorheology (Bin) Coupled with Cell Transfection. Biophysical Tools for Biologists, Vol 2: In Vivo Techniques 2008, 89, 467-486.

Li, Y. X.; Vanapalli, S. A.; Duits, M. H. G. Dynamics of Ballistically Injected Latex Particles in Living Human Endothelial Cells. Biorheology 2009, 46, 309-321.

Wu, P. H.; Hale, C. M.; Chen, W. C.; Lee, J. S. H.; Tseng, Y.; Wirtz, D. High-Throughput Ballistic Injection Nanorheology to Measure Cell Mechanics. Nat. Protoc. 2012, 7, 155-170.

Guo, M.; Ehrlicher, A. J.; Jensen, M. H.; Renz, M.; Moore, J. R.; Goldman, R. D.; Lippincott-Schwartz, J.; Mackintosh, F. C.; Weitz, D. A. Probing the Stochastic, Motor-Driven Properties of the Cytoplasm Using Force Spectrum Microscopy. Cell 2014, $158,822-832$.

(355) Nishizawa, K.; Bremerich, M.; Ayade, H.; Schmidt, C. F.; Ariga, T.; Mizuno, D. Feedback-Tracking Microrheology in Living Cells. Science Advances 2017, 3, e1700318.

(356) Moch, M.; Windoffer, R.; Schwarz, N.; Pohl, R.; Omenzetter, A.; Schnakenberg, U.; Herb, F.; Chaisaowong, K.; Merhof, D.; Ramms, L.et al. Effects of Plectin Depletion on Keratin Network Dynamics and Organization. PLoS One 2016, 11, e0149106.

Garzon-Coral, C.; Fantana, H. A.; Howard, J. A Force-Generating Machinery Maintains the Spindle at the Cell Center During Mitosis. Science 2016, 352, 1124-1127.

Novo, S.; Barrios, L.; Santalo, J.; Gomez-Martinez, R.; Duch, M.; Esteve, J.; Plaza, J. A.; Nogues, C.; Ibanez, E. A Novel Embryo Identification System by Direct Tagging of Mouse Embryos Using Silicon-Based Barcodes. Hum. Reprod. 2011, 26, 96-105.

Mellott, A. J.; Forrest, M. L.; Detamore, M. S. Physical Non-Viral Gene Delivery Methods for Tissue Engineering. Ann. Biomed. Eng. 2013, 41, 446-468.

(360) Lakshmanan, S.; Gupta, G. K.; Avci, P.; Chandran, R.; Sadasivam, M.; Jorge, A. E.; Hamblin, M. R. Physical Energy for Drug Delivery; Poration, Concentration and Activation. Adv Drug Deliv Rev 2013, 71, 98-114.

(361) Villemejane, J.; Mir, L. M. Physical Methods of Nucleic Acid Transfer: General Concepts and Applications. Br. J. Pharmacol. 2009, 157, 207-219.

(362) Mehier-Humbert, S.; Guy, R. H. Physical Methods for Gene Transfer: Improving the Kinetics of Gene Delivery into Cells. Adv. Drug Del. Rev. 2005, 57, 733-753.

(363) Sahay, G.; Alakhova, D. Y.; Kabanov, A. V. Endocytosis of Nanomedicines. J. Controlled Release 2010, 145, 182-195.

(364) Luo, D.; Saltzman, W. M. Synthetic DNA Delivery Systems. Nat. Biotechnol. 2000, 18, 33-37. 

Polyplex Gene Delivery. Biotechnol. Bioeng. 2000, 67, 598-606.

(366) Lv, H. T.; Zhang, S. B.; Wang, B.; Cui, S. H.; Yan, J. Toxicity of Cationic Lipids and Cationic Polymers in Gene Delivery. J. Controlled Release 2006, 114, 100-109.

(367) Goff, S. P.; Berg, P. Construction of Hybrid Viruses Containing Sv40 and Lambda Phage DNA Segments and Their Propagation in Cultured Monkey Cells. Cell 1976, 9, 695-705.

(368) Williams, D. A.; Lemischka, I. R.; Nathan, D. G.; Mulligan, R. C. Introduction of New Genetic Material into Pluripotent Haematopoietic Stem Cells of the Mouse. Nature 1984, 310, 476-480.

Cepko, C. L.; Roberts, B. E.; Mulligan, R. C. Construction and Applications of a Highly Transmissible Murine Retrovirus Shuttle Vector. Cell 1984, 37, 1053-1062.

Mingozzi, F.; High, K. A. Therapeutic in Vivo Gene Transfer for Genetic Disease Using Aav: Progress and Challenges. Nature Reviews Genetics 2011, 12, 341-355.

(371) Kay, M. A. State-of-the-Art Gene-Based Therapies: The Road Ahead. Nature Reviews Genetics 2011, $12,316-328$.

(372) Waehler, R.; Russell, S. J.; Curiel, D. T. Engineering Targeted Viral Vectors for Gene Therapy. Nature Reviews Genetics 2007, 8, 573-587.

Van Der Loo, J. C.; Wright, J. F. Progress and Challenges in Viral Vector Manufacturing. Hum. Mol. Genet. 2015, 25, R42-52. Jafari, M.; Soltani, M.; Naahidi, S.; Karunaratne, D. N.; Chen, P. Nonviral Approach for Targeted Nucleic Acid Delivery. Curr. Med. Chem. 2012, 19, 197-208.

Mitragotri, S.; Burke, P. A.; Langer, R. Overcoming the Challenges in Administering Biopharmaceuticals: Formulation and Delivery Strategies. Nature Reviews Drug Discovery 2014, 13, 655-672.

Iversen, T. G.; Skotland, T.; Sandvig, K. Endocytosis and Intracellular Transport of Nanoparticles: Present Knowledge and Need for Future Studies. Nano Today 2011, 6, 176-185.

Duncan, R.; Richardson, S. C. W. Endocytosis and Intracellular Trafficking as Gateways for Nanomedicine Delivery: Opportunities and Challenges. Mol. Pharm. 2012, 9, 2380-2402.

Akinc, A.; Battaglia, G. Exploiting Endocytosis for Nanomedicines. Cold Spring Harb. Perspect. Biol. 2013, 5, a016980.

Sahay, G.; Querbes, W.; Alabi, C.; Eltoukhy, A.; Sarkar, S.; Zurenko, C.; Karagiannis, E.; Love, K.; Chen, D. L.; Zoncu, R.et al. Efficiency of Sirna Delivery by Lipid Nanoparticles Is Limited by Endocytic Recycling. Nat. Biotechnol. 2013, 31, 653-658.

Gilleron, J.; Querbes, W.; Zeigerer, A.; Borodovsky, A.; Marsico, G.; Schubert, U.; Manygoats, K.; Seifert, S.; Andree, C.; Stoter, M.et al. Image-Based Analysis of Lipid Nanoparticle-Mediated Sirna Delivery, Intracellular Trafficking and Endosomal Escape. Nat. Biotechnol. 2013, 31, 638-646.

(381) Wittrup, A.; Ai, A.; Liu, X.; Hamar, P.; Trifonova, R.; Charisse, K.; Manoharan, M.; Kirchhausen, T.; Lieberman, J. Visualizing Lipid-Formulated Sirna Release from Endosomes and Target Gene Knockdown. Nat. Biotechnol. 2015, 33, 870-876.

(382) Felgner, P. L. Particulate Systems and Polymers for in Vitro and in Vivo Delivery of Polynucleotides. Adv. Drug Del. Rev. 1990, 5, 163-187. 
Rehman, Z. U.; Hoekstra, D.; Zuhorn, I. S. Mechanism of Polyplex- and Lipoplex-Mediated Delivery of Nucleic Acids: RealTime Visualization of Transient Membrane Destabilization without Endosomal Lysis. Acs Nano 2013, 7, 3767-3777. Helenius, A.; Doxsey, S.; Mellman, I. Viruses as Tools in Drug Delivery. Ann. N.Y. Acad. Sci. 1987, 507, 1-6.

Hoffmann, B.et al. Fusogenic Liposomes as Nanocarriers for the Delivery of Intracellular Proteins. Langmuir 2017, 33, 1051 1059.

(388) Kolasinac, R.; Kleusch, C.; Braun, T.; Merkel, R.; Csiszar, A. Deciphering the Functional Composition of Fusogenic Liposomes. Int. J. Mol. Sci. 2018, 19, E346.

Montecalvo, A.; Larregina, A. T.; Shufesky, W. J.; Stolz, D. B.; Sullivan, M. L. G.; Karlsson, J. M.; Baty, C. J.; Gibson, G. A.; Erdos, G.; Wang, Z. L.et al. Mechanism of Transfer of Functional Micrornas between Mouse Dendritic Cells Via Exosomes. Blood 2012, 119, 756-766.

(390) El Andaloussi, S.; Maeger, I.; Breakefield, X. O.; Wood, M. J. A. Extracellular Vesicles: Biology and Emerging Therapeutic Opportunities. Nature Reviews Drug Discovery 2013, 12, 348-358.

(391) Yim, N.; Ryu, S. W.; Choi, K.; Lee, K. R.; Lee, S.; Choi, H.; Kim, J.; Shaker, M. R.; Sun, W.; Park, J. H.et al. Exosome Engineering for Efficient Intracellular Delivery of Soluble Proteins Using Optically Reversible Protein-Protein Interaction Module. Nat Commun 2016, 7, 12277.

(392) Feramisco, J.; Perona, R.; Lacal, J. C. In Microinjection; Lacal, J.;Feramisco, J.;Perona, R., Eds.; Birkhäuser Basel, 1999. Mcallister, D. V.; Wang, P. M.; Davis, S. P.; Park, J. H.; Canatella, P. J.; Allen, M. G.; Prausnitz, M. R. Microfabricated Needles for Transdermal Delivery of Macromolecules and Nanoparticles: Fabrication Methods and Transport Studies. Proc. Natl. Acad. Sci. U. S. A. 2003, 100, 13755-13760.

(394) Klein, T. M.; Wolf, E. D.; Wu, R.; Sanford, J. C. High-Velocity Microprojectiles for Delivering Nucleic-Acids into Living Cells. Nature 1987, 327, 70-73.

(395) Stephens, D. J.; Pepperkok, R. The Many Ways to Cross the Plasma Membrane. Proc. Natl. Acad. Sci. U. S. A. 2001, 98, 4295-4298.

(396) Hapala, I. Breaking the Barrier: Methods for Reversible Permeabilization of Cellular Membranes. Crit. Rev. Biotechnol. 1997, $17,105-122$.

Schulz, I. Permeabilizing Cells - Some Methods and Applications for the Study of Intracellular Processes. Methods Enzymol. $1990,192,280-300$.

Cooper, S. T.; Mcneil, P. L. Membrane Repair: Mechanisms and Pathophysiology. Physiol. Rev. 2015, 95, 1205-1240. 
(399) Bischofberger, M.; lacovache, I.; Van Der Goot, F. G. Pathogenic Pore-Forming Proteins: Function and Host Response. Cell Host \& Microbe 2012, 12, 266-275.

(400) Shinoda, W. Permeability across Lipid Membranes. Biochim. Biophys. Acta 2016, 1858, 2254-2265.

(401) Lingrel, J. B.; Kuntzweiler, T. Na+,K+-Atpase. J. Biol. Chem. 1994, 269, 19659-19662.

(402) Holthuis, J. C.; Menon, A. K. Lipid Landscapes and Pipelines in Membrane Homeostasis. Nature 2014, 510, 48-57.

(403) Van Meer, G.; Voelker, D. R.; Feigenson, G. W. Membrane Lipids: Where They Are and How They Behave. Nature Reviews Molecular Cell Biology 2008, 9, 112-124.

(404) Simons, K.; Sampaio, J. L. Membrane Organization and Lipid Rafts. Cold Spring Harb. Perspect. Biol. 2011,3 , a004697.

(405) Lingwood, D.; Simons, K. Lipid Rafts as a Membrane-Organizing Principle. Science 2010, 327, 46-50.

(406) Parton, R. G.; Simons, K. The Multiple Faces of Caveolae. Nature Reviews Molecular Cell Biology 2007, 8, $185-194$.

(407) Tweten, R. K.; Hotze, E. M.; Wade, K. R. The Unique Molecular Choreography of Giant Pore Formation by the CholesterolDependent Cytolysins of Gram-Positive Bacteria. Annu. Rev. Microbiol. 2015, 69, 323-340.

(408) Gogelein, H.; Huby, A. Interaction of Saponin and Digitonin with Black Lipid-Membranes and Lipid Monolayers. Biochim. Biophys. Acta 1984, 773, 32-38.

(409) Pfaff, R. T.; Liu, J.; Gao, D.; Peter, A. T.; Li, T. K.; Critser, J. K. Water and Dmso Membrane Permeability Characteristics of inVivo- and in-Vitro-Derived and Cultured Murine Oocytes and Embryos. Mol. Hum. Reprod. 1998, 4, 51-59.

(410) Gurtovenko, A. A.; Anwar, J.; Vattulainen, I. Defect-Mediated Trafficking across Cell Membranes: Insights from in Silico Modeling. Chem. Rev. 2010, 110, 6077-6103.

(411) King, L. S.; Kozono, D.; Agre, P. From Structure to Disease: The Evolving Tale of Aquaporin Biology. Nature Reviews Molecular Cell Biology 2004, 5, 687-698.

(412) Dobson, P. D.; Patel, Y.; Kell, D. B. 'Metabolite-Likeness' as a Criterion in the Design and Selection of Pharmaceutical Drug Libraries. Drug Discovery Today 2009, 14, 31-40.

(413) Reitsma, S.; Slaaf, D. W.; Vink, H.; Van Zandvoort, M. a. M. J.; Egbrink, M. G. a. O. The Endothelial Glycocalyx: Composition, Functions, and Visualization. Pflugers Archiv-European Journal of Physiology 2007, 454, 345-359.

(414) Clark, A. G.; Wartlick, O.; Salbreux, G.; Paluch, E. K. Stresses at the Cell Surface During Animal Cell Morphogenesis. Curr. Biol. 2014, 24, R484-R494.

(415) Groulx, N.; Boudreault, F.; Orlov, S. N.; Grygorczyk, R. Membrane Reserves and Hypotonic Cell Swelling. J. Membr. Biol. 2006, 214, 43-56.

(416) Sinha, B.; Koster, D.; Ruez, R.; Gonnord, P.; Bastiani, M.; Abankwa, D.; Stan, R. V.; Butler-Browne, G.; Vedie, B.; Johannes, L.et al. Cells Respond to Mechanical Stress by Rapid Disassembly of Caveolae. Cell 2011, 144, 402-413.

(417) Aalipour, A.; Xu, A. M.; Leal-Ortiz, S.; Garner, C. C.; Melosh, N. A. Plasma Membrane and Actin Cytoskeleton as Synergistic Barriers to Nanowire Cell Penetration. Langmuir 2014, 30, 12362-12367.

(418) Xu, A. M.; Aalipour, A.; Leal-Ortiz, S.; Mekhdjian, A. H.; Xie, X.; Dunn, A. R.; Garner, C. C.; Melosh, N. A. Quantification of Nanowire Penetration into Living Cells. Nat Commun 2014, 5, 3613. 
(419) Weaver, J. C.; Chizmadzhev, Y. A. Theory of Electroporation: A Review. Bioelectrochem. Bioenerg. 1996, 41, 135-160.

(420) Neu, J. C.; Krassowska, W. Asymptotic Model of Electroporation. Physical Review E 1999, 59, 3471-3482.

(421) Evans, E.; Heinrich, V.; Ludwig, F.; Rawicz, W. Dynamic Tension Spectroscopy and Strength of Biomembranes. Biophys. J. 2003, 85, 2342-2350.

(422) Dickey, A.; Faller, R. Examining the Contributions of Lipid Shape and Headgroup Charge on Bilayer Behavior. Biophys. J. 2008, 95, 2636-2646.

(423) Karatekin, E.; Sandre, O.; Guitouni, H.; Borghi, N.; Puech, P. H.; Brochard-Wyart, F. Cascades of Transient Pores in Giant Vesicles: Line Tension and Transport. Biophys. J. 2003, 84, 1734-1749.

(424) Mcneil, P. L.; Steinhardt, R. A. Plasma Membrane Disruption: Repair, Prevention, Adaptation. Annu. Rev. Cell Dev. Biol. 2003, 19, 697-731.

(425) Bloom, M.; Evans, E.; Mouritsen, O. G. Physical-Properties of the Fluid Lipid-Bilayer Component of Cell-Membranes - a Perspective. Q. Rev. Biophys. 1991, 24, 293-397.

(426) Marmottant, P.; Biben, T.; Hilgenfeldt, S. Deformation and Rupture of Lipid Vesicles in the Strong Shear Flow Generated by Ultrasound-Driven Microbubbles. Proceedings of the Royal Society a-Mathematical Physical and Engineering Sciences 2008, $464,1781-1800$

(427) Kawamura, R.; Shimizu, K.; Matsumoto, Y.; Yamagishi, A.; Silberberg, Y. R.; lijima, M.; Kuroda, S.; Fukazawa, K.; Ishihara, K.; Nakamura, C. High Efficiency Penetration of Antibody-Immobilized Nanoneedle Thorough Plasma Membrane for in Situ Detection of Cytoskeletal Proteins in Living Cells. Journal of Nanobiotechnology 2016, 14, 74.

(428) Xie, X.; Aalipour, A.; Gupta, S. V.; Melosh, N. A. Determining the Time Window for Dynamic Nanowire Cell Penetration Processes. ACS Nano 2015, 9, 11667-11677.

(429) Vakarelski, I. U.; Brown, S. C.; Higashitani, K.; Moudgil, B. M. Penetration of Living Cell Membranes with Fortified Carbon Nanotube Tips. Langmuir 2007, 23, 10893-10896.

(430) Obataya, I.; Nakamura, C.; Han, S.; Nakamura, N.; Miyake, J. Nanoscale Operation of a Living Cell Using an Atomic Force Microscope with a Nanoneedle. Nano Lett. 2005, 5, 27-30.

(431) Obataya, F.; Nakamura, C.; Han, S. W.; Nakamura, N.; Miyake, J. Mechanical Sensing of the Penetration of Various Nanoneedles into a Living Cell Using Atomic Force Microscopy. Biosens. Bioelectron. 2005, 20, 1652-1655.

(432) Angle, M. R.; Wang, A.; Thomas, A.; Schaefer, A. T.; Melosh, N. A. Penetration of Cell Membranes and Synthetic Lipid Bilayers by Nanoprobes. Biophys. J. 2014, 107, 2091-2100.

(433) Last, N. B.; Schlamadinger, D. E.; Miranker, A. D. A Common Landscape for Membrane-Active Peptides. Protein Sci. 2013, $22,870-882$.

(434) Bennett, W. F. D.; Tieleman, D. P. The Importance of Membrane Defects-Lessons from Simulations. Acc. Chem. Res. 2014, 47, 2244-2251.

(435) Wang, T. Y.; Libardo, M. D.; Angeles-Boza, A. M.; Pellois, J. P. Membrane Oxidation in Cell Delivery and Cell Killing Applications. ACS Chem. Biol. 2017, 12, 1170-1182. 
(436) Riske, K. A.; Sudbrack, T. P.; Archilha, N. L.; Uchoa, A. F.; Schroder, A. P.; Marques, C. M.; Baptista, M. S.; Itri, R. Giant Vesicles under Oxidative Stress Induced by a Membrane-Anchored Photosensitizer. Biophys. J. 2009, 97, 1362-1370. Makky, A.; Tanaka, M. Impact of Lipid Oxidization on Biophysical Properties of Model Cell Membranes. J. Phys. Chem. B 2015, 119, 5857-5863.

Boonnoy, P.; Jarerattanachat, V.; Karttunen, M.; Wong-Ekkabut, J. Bilayer Deformation, Pores, and Micellation Induced by Oxidized Lipids. J. Phys. Chem. Lett. 2015, 6, 4884-4888.

Peraro, M. D.; Van Der Goot, F. G. Pore-Forming Toxins: Ancient, but Never Really out of Fashion. Nat Rev Micro 2015, 14, 77-92.

(440) Sun, D. L.; Forsman, J.; Woodward, C. E. Current Understanding of the Mechanisms by Which Membrane-Active Peptides Permeate and Disrupt Model Lipid Membranes. Curr. Top. Med. Chem. 2016, 16, 170-186.

(441) Heerklotz, H. Interactions of Surfactants with Lipid Membranes. Q. Rev. Biophys. 2008, 41, $205-264$.

(442) Lichtenberg, D.; Ahyayauch, H.; Goni, F. M. The Mechanism of Detergent Solubilization of Lipid Bilayers. Biophys. J. 2013, $105,289-299$

(443) Steinhardt, R. A.; Bi, G. Q.; Alderton, J. M. Cell-Membrane Resealing by a Vesicular Mechanism Similar to Neurotransmitter Release. Science 1994, 263, 390-393.

(444) Mcneil, P. L.; Kirchhausen, T. An Emergency Response Team for Membrane Repair. Nature Reviews Molecular Cell Biology 2005, 6, 499-505.

(445) Jimenez, A. J.; Perez, F. Plasma Membrane Repair: The Adaptable Cell Life-Insurance. Curr. Opin. Cell Biol. 2017, 47, 99107.

(446) Demonbreun, A. R.; Mcnally, E. M. Plasma Membrane Repair In health and Disease. Curr. Top. Membr. 2016, 77, 67-96.

(447) Moe, A.; Golding, A. E.; Bement, W. M. Cell Healing: Calcium, Repair and Regeneration. Semin. Cell Dev. Biol. 2015, 45, 1823.

(448) Lauritzen, S. P.; Boye, T. L.; Nylandsted, J. Annexins Are Instrumental for Efficient Plasma Membrane Repair in Cancer Cells. Semin. Cell Dev. Biol. 2015, 45, 32-38.

(449) Jimenez, A. J.; Perez, F. Physico-Chemical and Biological Considerations for Membrane Wound Evolution and Repair in Animal Cells. Semin. Cell Dev. Biol. 2015, 45, 2-9.

(450) Cheng, X.; Zhang, X.; Yu, L.; Xu, H. Calcium Signaling in Membrane Repair. Semin. Cell Dev. Biol. 2015, 45, 24-31.

(451) Boucher, E.; Mandato, C. A. Plasma Membrane and Cytoskeleton Dynamics During Single-Cell Wound Healing. Biochim. Biophys. Acta 2015, 1853, 2649-2661.

(452) Babiychuk, E. B.; Draeger, A. Defying Death: Cellular Survival Strategies Following Plasmalemmal Injury by Bacterial Toxins. Semin. Cell Dev. Biol. 2015, 45, 39-47.

(453) Andrews, N. W.; Corrotte, M.; Castro-Gomes, T. Above the Fray: Surface Remodeling by Secreted Lysosomal Enzymes Leads to Endocytosis-Mediated Plasma Membrane Repair. Semin. Cell Dev. Biol. 2015, 45, 10-17. 
Jimenez, A. J.; Maiuri, P.; Lafaurie-Janvore, J.; Divoux, S.; Piel, M.; Perez, F. Escrt Machinery Is Required for Plasma Membrane Repair. Science 2014, 343, 1247136.

Andrews, N. W.; Almeida, P. E.; Corrotte, M. Damage Control: Cellular Mechanisms of Plasma Membrane Repair. Trends Cell Biol. 2014, 24, 734-742.

Draeger, A.; Schoenauer, R.; Atanassoff, A. P.; Wolfmeier, H.; Babiychuk, E. B. Dealing with Damage: Plasma Membrane Repair Mechanisms. Biochimie 2014, 107, 66-72.

Bhakdi, S.; Weller, U.; Walev, I.; Martin, E.; Jonas, D.; Palmer, M. A Guide to the Use of Pore-Forming Toxins for Controlled Permeabilization of Cell-Membranes. Med. Microbiol. Immunol. 1993, 182, 167-175.

Weaver, J. C. Electroporation of Biological Membranes from Multicellular to Nano Scales. Dielectrics and Electrical Insulation, IEEE Transactions on 2003, 10, 754-768.

Marks, J. D.; Pan, C. Y.; Bushell, T.; Cromie, W.; Lee, R. C. Amphiphilic, Tri-Block Copolymers Provide Potent MembraneTargeted Neuroprotection. FASEB J. 2001, 15, 1107-1109.

Yasuda, S.; Townsend, D.; Michele, D. E.; Favre, E. G.; Day, S. M.; Metzger, J. M. Dystrophic Heart Failure Blocked by Membrane Sealant Poloxamer. Nature 2005, 436, 1025-1029.

Agarwal, J.; Walsh, A.; Lee, R. C. Multimodal Strategies for Resuscitating Injured Cells. Cell Injury: Mechanisms, Responses, and Repair 2005, 1066, 295-309.

Sengupta, A.; Dwivedi, N.; Kelly, S. C.; Tucci, L.; Thadhani, N. N.; Prausnitz, M. R. Poloxamer Surfactant Preserves Cell Viability During Photoacoustic Delivery of Molecules into Cells. Biotechnol. Bioeng. 2014, 112, 405-415.

Serbest, G.; Horwitz, J.; Barbee, K. The Effect of Poloxamer-188 on Neuronal Cell Recovery from Mechanical Injury. J. Neurotrauma 2005, 22, 119-132.

Hartikka, J.; Sukhu, I.; Buchner, C.; Hazard, D.; Bozoukova, V.; Margalith, M.; Nishioka, W. K.; Wheeler, C. J.; Manthorpe, M.; Sawdey, M. Electroporation-Facilitated Delivery of Plasmid DNA in Skeletal Muscle: Plasmid Dependence of Muscle Damage and Effect of Poloxamer 188. Mol. Ther. 2001, 4, 407-415.

Bittner, G.; Spaeth, C.; Poon, A.; Burgess, Z.; Mcgill, C. Repair of Traumatic Plasmalemmal Damage to Neurons and Other Eukaryotic Cells. Neural Regeneration Research 2016, 11, 1033-1042.

Howard, A. C.; Mcneil, A. K.; Mcneil, P. L. Promotion of Plasma Membrane Repair by Vitamin E. Nat Commun $2011,2,597$.

Labazi, M.; Mcneil, A. K.; Kurtz, T.; Lee, T. C.; Pegg, R. B.; Friedmann, J.; Conrad, M.; Mcneil, P. L. The Antioxidant Requirement for Plasma Membrane Repair in Skeletal Muscle. Free Radic. Biol. Med. 2015, 84, $246-253$.

Duan, X.; Chan, K. T.; Lee, K. K.; Mak, A. F. Oxidative Stress and Plasma Membrane Repair in Single Myoblasts after Femtosecond Laser Photoporation. Ann. Biomed. Eng. 2015, 43, 2735-2744.

Golzio, M.; Mora, M. P.; Raynaud, C.; Delteil, C.; Teissie, J.; Rols, M. P. Control by Osmotic Pressure of Voltage-Induced Permeabilization and Gene Transfer in Mammalian Cells. Biophys. J. 1998, 74, 3015-3022.

Ferret, E.; Evrard, C.; Foucal, A.; Gervais, P. Volume Changes of Isolated Human K562 Leukemia Cells Induced by Electric Field Pulses. Biotechnol. Bioeng. 2000, 67, 520-528. 
Shirakashi, R.; Sukhorukov, V. L.; Tanasawa, I.; Zimmermann, U. Measurement of the Permeability and Resealing Time Constant of the Electroporated Mammalian Cell Membranes. Int. J. Heat Mass Transfer 2004, 47, 4517-4524.

Pavlin, M.; Kanduser, M.; Rebersek, M.; Pucihar, G.; Hart, F. X.; Magjarevic, R.; Miklavcic, D. Effect of Cell Electroporation on the Conductivity of a Cell Suspension. Biophys. J. 2005, 88, 4378-4390.

(473) Wang, H. Y.; Lu, C. High-Throughput and Real-Time Study of Single Cell Electroporation Using Microfluidics: Effects of Medium Osmolarity. Biotechnol. Bioeng. 2006, 95, 1116-1125.

(474) Usaj, M.; Trontelj, K.; Hudej, R.; Kanduser, M.; Miklavcic, D. Cell Size Dynamics and Viability of Cells Exposed to Hypotonic Treatment and Electroporation for Electrofusion Optimization. Radiology and Oncology 2009, 43, 108-119.

Nesin, O. M.; Pakhomova, O. N.; Xiao, S.; Pakhomov, A. G. Manipulation of Cell Volume and Membrane Pore Comparison Following Single Cell Permeabilization with 60-and 600-Ns Electric Pulses. Biochimica Et Biophysica Acta-Biomembranes 2011, 1808, 792-801.

Pakhomov, A. G.; Xiao, S.; Pakhomova, O. N.; Semenov, I.; Kuipers, M. A.; Ibey, B. L. Disassembly of Actin Structures by Nanosecond Pulsed Electric Field Is a Downstream Effect of Cell Swelling. Bioelectrochemistry 2014, 100, 88-95.

Romeo, S.; Wu, Y. H.; Levine, Z. A.; Gundersen, M. A.; Vernier, P. T. Water Influx and Cell Swelling after Nanosecond Electropermeabilization. Biochim. Biophys. Acta 2013, 1828, 1715-1722.

Sozer, E. B.; Wu, Y. H.; Romeo, S.; Vernier, P. T. Nanometer-Scale Permeabilization and Osmotic Swelling Induced by 5-Ns Pulsed Electric Fields. J. Membr. Biol. 2017, 250, 21-30.

Anderson, S. E.; Bau, H. H. Electrical Detection of Cellular Penetration During Microinjection with Carbon Nanopipettes. Nanotechnology 2014, 25, 245102.

Beier, H. T.; Tolstykh, G. P.; Musick, J. D.; Thomas, R. J.; Ibey, B. L. Plasma Membrane Nanoporation as a Possible Mechanism Behind Infrared Excitation of Cells. Journal of Neural Engineering 2014, 11, 066006.

Davis, A. A.; Farrar, M. J.; Nishimura, N.; Jin, M. M.; Schaffer, C. B. Optoporation and Genetic Manipulation of Cells Using Femtosecond Laser†Pulses. Biophys. J. 2013, 105, 862-871.

Antkowiak, M.; Torres-Mapa, M. L.; Dholakia, K.; Gunn-Moore, F. J. Quantitative Phase Study of the Dynamic Cellular Response in Femtosecond Laser Photoporation. Biomedical Optics Express 2010, 1, 414-424.

Baumgart, J.; Bintig, W.; Ngezahayo, A.; Willenbrock, S.; Murua Escobar, H.; Ertmer, W.; Lubatschowski, H.; Heisterkamp, A. Quantified Femtosecond Laser Based Opto-Perforation of Living Gfshr-17 and Mth53 a Cells. Opt. Express 2008, 16, 30213031.

(484) Stevenson, D.; Agate, B.; Tsampoula, X.; Fischer, P.; Brown, C. T. A.; Sibbett, W.; Riches, A.; Gunn-Moore, F.; Dholakia, K. Femtosecond Optical Transfection of Cells: Viability and Efficiency. Opt. Express 2006, 14, 7125-7133.

(485) Kohli, V.; Acker, J. P.; Elezzabi, A. Y. Reversible Permeabilization Using High-Intensity Femtosecond Laser Pulses: Applications to Biopreservation. Biotechnol. Bioeng. 2005, 92, 889-899. 
Krasieva, T. B.; Chapman, C. F.; Lamorte, V. J.; Venugopalan, V.; Berns, M. W.; Tromberg, B. J. Cell Permeabilization and Molecular Transport by Laser Microirradiation. Optical Investigations of Cells in Vitro and in Vivo, Proceedings Of 1998, 3260, 38-44.

(487) Li, Z. G.; Liu, A. Q.; Klaseboer, E.; Zhang, J. B.; Ohl, C. D. Single Cell Membrane Poration by Bubble-Induced Microjets in a Microfluidic Chip. Lab on a Chip 2013, 13, 1144-1150.

Miyake, K.; Mcneil, P. L. Vesicle Accumulation and Exocytosis at Sites of Plasma Membrane Disruption. J. Cell Biol. 1995, $131,1737-1745$

(489) Wang, H. Y.; Lu, C. Electroporation of Mammalian Cells in a Microfluidic Channel with Geometric Variation. Anal. Chem. 2006, $78,5158-5164$

(490) Hui, S. W.; Li, L. H. In Vitro and Ex Vivo Gene Delivery to Cells by Electroporation. Methods Mol. Med. 2000, 37, $157-171$.

(491) Hoffmann, E. K.; Lambert, I. H.; Pedersen, S. F. Physiology of Cell Volume Regulation in Vertebrates. Physiol. Rev. 2009, 89, 193-277.

(492) Fink, S. L.; Cookson, B. T. Apoptosis, Pyroptosis, and Necrosis: Mechanistic Description of Dead and Dying Eukaryotic Cells. Infect. Immun. 2005, 73, 1907-1916.

(493) Fulda, S.; Gorman, A. M.; Hori, O.; Samali, A. Cellular Stress Responses: Cell Survival and Cell Death. Int. J. Cell Biol. 2010, $2010,214074$.

(494) Abidor, I. G.; Li, L. H.; Hui, S. W. Studies of Cell Pellets .2. Osmotic Properties, Electroporation, and Related Phenomena Membrane Interactions. Biophys. J. 1994, 67, 427-435.

Gonzalez, M. R.; Bischofberger, M.; Freche, B.; Ho, S.; Parton, R. G.; Van Der Goot, F. G. Pore-Forming Toxins Induce Multiple Cellular Responses Promoting Survival. Cell. Microbiol. 2011, 13, 1026-1043.

(496) Kosowski, H.; Matthias, R.; Schild, L.; Halangk, W. Electropulsing of Acinar-Cells Isolated from Rat Pancreas - Dependence of Reversible Membrane Perforation on Cellular-Energy State. Bioelectrochem. Bioenerg. 1995, 38, $377-381$.

Walev, I.; Bhakdi, S. C.; Hofmann, F.; Djonder, N.; Valeva, A.; Aktories, K.; Bhakdi, S. Delivery of Proteins into Living Cells by Reversible Membrane Permeabilization with Streptolysin-O. Proc. Natl. Acad. Sci. U. S. A. 2001, 98, 3185-3190.

Wald, T.; Petry-Podgorska, I.; Fiser, R.; Matousek, T.; Dedina, J.; Osicka, R.; Sebo, P.; Masin, J. Quantification of Potassium Levels in Cells Treated with Bordetella Adenylate Cyclase Toxin. Anal. Biochem. 2014, 450, 57-62.

(499) Orrenius, S.; Mcconkey, D. J.; Bellomo, G.; Nicotera, P. Role of Ca2+ in Toxic Cell Killing. Trends Pharmacol. Sci. 1989, 10, 281-285.

(500) Babiychuk, E. B.; Monastyrskaya, K.; Potez, S.; Draeger, A. Intracellular Ca2+ Operates a Switch between Repair and Lysis of Streptolysin O-Perforated Cells. Cell Death Differ. 2009, 16, 1126-1134.

(501) Wolfmeier, H.; Schoenauer, R.; Atanassoff, A. P.; Neill, D. R.; Kadioglu, A.; Draeger, A.; Babiychuk, E. B. Ca-Dependent Repair of Pneumolysin Pores: A New Paradigm for Host Cellular Defense against Bacterial Pore-Forming Toxins. Biochim. Biophys. Acta 2014, 1853, 2045-2054. 
Blangero, C.; Rols, M. P.; Teissie, J. Cytoskeletal Reorganization During Electric-Field-Induced Fusion of Chinese Hamster Ovary Cells Grown in Monolayers. Biochim. Biophys. Acta 1989, 981, 295-302.

Harkin, D. G.; Hay, E. D. Effects of Electroporation on the Tubulin Cytoskeleton and Directed Migration of Corneal Fibroblasts Cultured within Collagen Matrices. Cell Motil. Cytoskeleton 1996, 35, 345-357.

(504) Kanthou, C.; Kranjc, S.; Sersa, G.; Tozer, G.; Zupanic, A.; Cemazar, M. The Endothelial Cytoskeleton as a Target of Electroporation-Based Therapies. Mol. Cancer Ther. 2006, 5, 3145-3152.

(505) Thompson, G. L.; Roth, C. C.; Dalzell, D. R.; Kuipers, M.; Ibey, B. L. Calcium Influx Affects Intracellular Transport and Membrane Repair Following Nanosecond Pulsed Electric Field Exposure. Journal of Biomedical Optics 2014, $19,055005$.

(506) Keith, C.; Dipaola, M.; Maxfield, F. R.; Shelanski, M. L. Microinjection of Ca++-Calmodulin Causes a Localized Depolymerization of Microtubules. J. Cell Biol. 1983, 97, 1918-1924.

(507) Togo, T. Disruption of the Plasma Membrane Stimulates Rearrangement of Microtubules and Lipid Traffic toward the Wound Site. J. Cell Sci. 2006, 119, 2780-2786.

(508) Kano, F.; Nakatsu, D.; Noguchi, Y.; Yamamoto, A.; Murata, M. A Resealed-Cell System for Analyzing Pathogenic Intracellular Events: Perturbation of Endocytic Pathways under Diabetic Conditions. PLoS One 2012, 7, e44127.

(509) Saklayen, N.; Kalies, S.; Madrid, M.; Nuzzo, V.; Huber, M.; Shen, W.; Sinanan-Singh, J.; Heinemann, D.; Heisterkamp, A.; Mazur, E. Analysis of Poration-Induced Changes in Cells from Laser-Activated Plasmonic Substrates. Biomedical Optics Express 2017, 8, 4756-4771.

(510) Bischof, L. J.; Kao, C. Y.; Los, F. C. O.; Gonzalez, M. R.; Shen, Z. X.; Briggs, S. P.; Van Der Goot, F. G.; Aroian, R. V. Activation of the Unfolded Protein Response Is Required for Defenses against Bacterial Pore-Forming Toxin in Vivo. PLoS Path. 2008, 4, e1000176.

(511) Pillich, H.; Loose, M.; Zimmer, K. P.; Chakraborty, T. Activation of the Unfolded Protein Response by Listeria Monocytogenes. Cell. Microbiol. 2012, 14, 949-964.

(512) Stassen, M.; Muller, C.; Richter, C.; Neudorfl, C.; Hultner, L.; Bhakdi, S.; Walev, I.; Schmitt, E. The Streptococcal Exotoxin Streptolysin O Activates Mast Cells to Produce Tumor Necrosis Factor Alpha by P38 Mitogen-Activated Protein Kinase- and Protein Kinase C-Dependent Pathways. Infect. Immun. 2003, 71, 6171-6177.

(513) Cassidy, S. K.; Hagar, J. A.; Kanneganti, T. D.; Franchi, L.; Nunez, G.; O'riordan, M. X. Membrane Damage During Listeria Monocytogenes Infection Triggers a Caspase-7 Dependent Cytoprotective Response. PLoS Pathog. 2012 , 8, e1002628.

(514) Tang, P.; Rosenshine, I.; Cossart, P.; Finlay, B. B. Listeriolysin O Activates Mitogen-Activated Protein Kinase in Eucaryotic Cells. Infect. Immun. 1996, 64, 2359-2361.

(515) Kao, C. Y.; Los, F. C. O.; Huffman, D. L.; Wachi, S.; Kloft, N.; Husmann, M.; Karabrahimi, V.; Schwartz, J. L.; Bellier, A.; Ha, C.et al. Global Functional Analyses of Cellular Responses to Pore-Forming Toxins. PLoS Path. 2011, 7, e1001314.

(516) Porta, H.; Cancino-Rodezno, A.; Soberon, M.; Bravo, A. Role of Mapk P38 in the Cellular Responses to Pore-Forming Toxins. Peptides 2011, 32, 601-606. 
Cabezas, S.; Ho, S.; Ros, U.; Lanio, M. E.; Alvarez, C.; Van Der Goot, F. G. Damage of Eukaryotic Cells by the Pore-Forming Toxin Sticholysin li: Consequences of the Potassium Efflux. Biochim. Biophys. Acta 2017, 1859, 982-992.

(518) Kloft, N.; Busch, T.; Neukirch, C.; Weis, S.; Boukhallouk, F.; Bobkiewicz, W.; Cibis, I.; Bhakdi, S.; Husmann, M. Pore-Forming Toxins Activate Mapk P38 by Causing Loss of Cellular Potassium. Biochem. Biophys. Res. Commun. 2009, 385, 503-506.

(519) Nagahama, M.; Shibutani, M.; Seike, S.; Yonezaki, M.; Takagishi, T.; Oda, M.; Kobayashi, K.; Sakurai, J. The P38 Mapk and Jnk Pathways Protect Host Cells against Clostridium Perfringens Beta-Toxin. Infect. Immun. 2013, 81, 3703-3708.

(520) Grembowicz, K. P.; Sprague, D.; Mcneil, P. L. Temporary Disruption of the Plasma Membrane Is Required for C-Fos Expression in Response to Mechanical Stress. Mol. Biol. Cell 1999, 10, 1247-1257.

(521) Kayal, S.; Lilienbaum, A.; Poyart, C.; Memet, S.; Israel, A.; Berche, P. Listeriolysin O-Dependent Activation of Endothelial Cells During Infection with Listeria Monocytogenes: Activation of Nf-Kappa B and Upregulation of Adhesion Molecules and Chemokines. Mol. Microbiol. 1999, 31, 1709-1722.

(522) Togo, T.; Alderton, J. M.; Bi, G. Q.; Steinhardt, R. A. The Mechanism of Facilitated Cell Membrane Resealing. J. Cell Sci. 1999, 112, 719-731.

(523) Togo, T.; Alderton, J. M.; Steinhardt, R. A. Long-Term Potentiation of Exocytosis and Cell Membrane Repair in Fibroblasts. Mol. Biol. Cell 2003, 14, 93-106.

(524) Togo, T. Long-Term Potentiation of Wound-Induced Exocytosis and Plasma Membrane Repair Is Dependant on CampResponse Element-Mediated Transcription Via a Protein Kinase C- and P38 Mapk-Dependent Pathway. J. Biol. Chem. 2004, 279, 44996-45003.

(525) Tolstykh, G. P.; Beier, H. T.; Roth, C. C.; Thompson, G. L.; Payne, J. A.; Kuipers, M. A.; Ibey, B. L. Activation of Intracellular Phosphoinositide Signaling after a Single 600 Nanosecond Electric Pulse. Bioelectrochemistry 2013, 94, 23-29.

(526) Morotomi-Yano, K.; Akiyama, H.; Yano, K. Nanosecond Pulsed Electric Fields Activate Mapk Pathways in Human Cells. Arch. Biochem. Biophys. 2011, 515, 99-106.

(527) Roth, C. C.; Glickman, R. D.; Tolstykh, G. P.; Estlack, L. E.; Moen, E. K.; Echchgadda, I.; Beier, H. T.; Barnes, R. A., Jr.; Ibey, B. L. Evaluation of the Genetic Response of U937 and Jurkat Cells to 10-Nanosecond Electrical Pulses (Nsep). PLoS One 2016, 11, e0154555.

(528) Ullery, J. C.; Tarango, M.; Roth, C. C.; Ibey, B. L. Activation of Autophagy in Response to Nanosecond Pulsed Electric Field Exposure. Biochem. Biophys. Res. Commun. 2015, 458, 411-417.

(529) Pinero, J.; Lopezbaena, M.; Ortiz, T.; Cortes, F. Apoptotic and Necrotic Cell Death Are Both Induced by Electroporation in HI60 Human Promyeloid Leukaemia Cells. Apoptosis 1997, 2, 330-336.

(530) Li, L. H.; Sen, A.; Murphy, S. P.; Jahreis, G. P.; Fuji, H.; Hui, S. W. Apoptosis Induced by DNA Uptake Limits Transfection Efficiency. Exp. Cell Res. 1999, 253, 541-550.

(531) Sukharev, S. I.; Klenchin, V. A.; Serov, S. M.; Chernomordik, L. V.; Chizmadzhev, Y. A. Electroporation and Electrophoretic DNA Transfer into Cells - the Effect of DNA Interaction with Electropores. Biophys. J. 1992, 63, 1320-1327. 
Rols, M. P.; Teissie, J. Electropermeabilization of Mammalian Cells to Macromolecules: Control by Pulse Duration. Biophys. J. 1998, 75, 1415-1423.

Kinosita, K.; Tsong, T. Y. Formation and Resealing of Pores of Controlled Sizes in Human Erythrocyte-Membrane. Nature $1977,268,438-441$.

(534) Kinosita, K., Jr.; Tsong, T. Y. Survival of Sucrose-Loaded Erythrocytes in the Circulation. Nature 1978, 272, $258-260$.

(535) Rols, M. P.; Teissie, J. Electropermeabilization of Mammalian-Cells - Quantitative-Analysis of the Phenomenon. Biophys. J. 1990, 58, 1089-1098.

(536) Rols, M. P.; Teissie, J. Modulation of Electrically Induced Permeabilization and Fusion of Chinese Hamster Ovary Cells by Osmotic-Pressure. Biochemistry 1990, 29, 4561-4567.

(537) Kwee, S.; Nielsen, H. V.; Celis, J. E. Electropermeabilization of Human Cultured-Cells Grown in Monolayers - Incorporation of Monoclonal-Antibodies. Bioelectrochem. Bioenerg. 1990, 23, 65-80.

(538) Blangero, C.; Teissie, J. Ionic Modulation of Electrically Induced Fusion of Mammalian Cells. J. Membr. Biol. 1985, 86, 247253.

(539) Johnson, J. A.; Gray, M. O.; Karliner, J. S.; Chen, C. H.; Mochlyrosen, D. An Improved Permeabilization Protocol for the Introduction of Peptides into Cardiac Myocytes - Application to Protein Kinase C Research. Circul. Res. 1996, 79, 1086-1099.

(540) Bru, T.; Clarke, C.; Mcgrew, M. J.; Sang, H. M.; Wilmut, I.; Blow, J. J. Rapid Induction of Pluripotency Genes after Exposure of Human Somatic Cells to Mouse Es Cell Extracts. Exp. Cell Res. 2008, 314, 2634-2642.

(541) Miller, M. R.; Castellot, J. J., Jr.; Pardee, A. B. A Permeable Animal Cell Preparation for Studying Macromolecular Synthesis. DNA Synthesis and the Role of Deoxyribonucleotides in S Phase Initiation. Biochemistry 1978, 17, 1073-1080.

(542) Baker, P. F.; Knight, D. E. High-Voltage Techniques for Gaining Access to the Interior of Cells - Application to the Study of Exocytosis and Membrane Turnover. Methods Enzymol. 1983, 98, 28-37.

(543) Mcneil, P. L.; Taylor, D. L. Aequorin Entrapment in Mammalian-Cells. Cell Calcium 1985, 6, 83-93.

(544) Knight, D. E.; Scrutton, M. C. Gaining Access to the Cytosol - the Technique and Some Applications of Electropermeabilization. Biochem. J 1986, 234, 497-506.

(545) Fechheimer, M.; Boylan, J. F.; Parker, S.; Sisken, J. E.; Patel, G. L.; Zimmer, S. G. Transfection of Mammalian-Cells with Plasmid DNA by Scrape Loading and Sonication Loading. Proc. Natl. Acad. Sci. U. S. A. 1987, 84, 8463-8467.

(546) Michel, M. R.; Elgizoli, M.; Koblet, H.; Kempf, C. Diffusion Loading Conditions Determine Recovery of Protein-Synthesis in Electroporated P3x63ag8 Cells. Experientia 1988, 44, 199-203.

(547) Van Den Hoff, M. J. B.; Moorman, A. F. M.; Lamers, W. H. Electroporation in Intracellular Buffer Increases Cell-Survival. Nucleic Acids Res. 1992, 20, 2902-2902.

(548) Van Den Hoff, M. J.; Christoffels, V. M.; Labruyere, W. T.; Moorman, A. F.; Lamers, W. H. Electrotransfection with "Intracellular" Buffer. Methods Mol. Biol. 1995, 48, 185-197.

(549) Baron, S.; Poast, J.; Rizzo, D.; Mcfarland, E.; Kieff, E. Electroporation of Antibodies, DNA, and Other Macromolecules into Cells: A Highly Efficient Method. J. Immunol. Methods 2000, 242, 115-126. 
(550) Riemen, G.; Lorbach, E.; Helfrich, J.; Siebenkotten, G.; Muller-Hartmann, H.; Rothmann-Cosic, K.; Thiel, C.; Weigel, M.; Wessendorf, H.; Brosterbus, H.; LONZA COLOGNE AG: United States, 2005.

(551) Chicaybam, L.; Sodre, A. L.; Curzio, B. A.; Bonamino, M. H. An Efficient Low Cost Method for Gene Transfer to T Lymphocytes. PLoS One 2013, 8, e60298.

(552) Parreno, J.; Delve, E.; Andrejevic, K.; Paez-Parent, S.; Wu, P. H.; Kandel, R. Efficient, Low-Cost Nucleofection of Passaged Chondrocytes. Cartilage 2016, 7, 82-91.

(553) Chicaybam, L.; Barcelos, C.; Peixoto, B.; Carneiro, M.; Limia, C. G.; Redondo, P.; Lira, C.; Paraguassu-Braga, F.; Vasconcelos, Z. F.; Barros, L.et al. An Efficient Electroporation Protocol for the Genetic Modification of Mammalian Cells. Front Bioeng Biotechnol 2016, 4, 99.

(554) Kang, J.; Ramu, S.; Lee, S.; Aguilar, B.; Ganesan, S. K.; Yoo, J.; Kalra, V. K.; Koh, C. J.; Hong, Y. K. Phosphate-Buffered Saline-Based Nucleofection of Primary Endothelial Cells. Anal. Biochem. 2009, 386, 251-255.

Patel, N.; Kalra, V. K. Placenta Growth Factor-Induced Early Growth Response 1 (Egr-1) Regulates Hypoxia-Inducible Factor1 Alpha (Hif-1 Alpha) in Endothelial Cells. J. Biol. Chem. 2010, 285, 20570-20579.

Potter, H. Transfection by Electroporation. Curr. Protoc. Mol. Biol. 2003, 62, 9.3.1-9.3.6.

Potter, H.; Heller, R. Transfection by Electroporation. Curr. Protoc. Cell Biol. 2011, Chapter 2057, A.1E.1-A.1E.11.

Kim, J. A.; Cho, K. C.; Shin, M. S.; Lee, W. G.; Jung, N. C.; Chung, C. I.; Chang, J. K. A Novel Electroporation Method Using a Capillary and Wire-Type Electrode. Biosens. Bioelectron. 2008, 23, 1353-1360.

Brees, C.; Fransen, M. A Cost-Effective Approach to Microporate Mammalian Cells with the Neon Transfection System. Anal. Biochem. 2014, 466, 49-50.

Wilgenhof, S.; Corthals, J.; Van Nuffel, A. M. T.; Benteyn, D.; Heirman, C.; Bonehill, A.; Thielemans, K.; Neyns, B. Long-Term Clinical Outcome of Melanoma Patients Treated with Messenger Rna-Electroporated Dendritic Cell Therapy Following Complete Resection of Metastases. Cancer Immunology Immunotherapy 2015, 64, 381-388.

(561) Soneru, A. P.; Beckett, M. A.; Weichselbaum, R. R.; Lee, R. C. Mg Atp and Antioxidants Augment the Radioprotective Effect of Surfactant Copolymers. Health Phys. 2011, 101, 731-738.

(562) Volpe, S. L. Magnesium in Disease Prevention and Overall Health. Adv. Nutr. 2013, 4, 378S-383S.

(563) Schoenauer, R.; Atanassoff, A. P.; Wolfmeier, H.; Pelegrin, P.; Babiychuk, E. B.; Draeger, A. P2x7 Receptors Mediate Resistance to Toxin-Induced Cell Lysis. Biochimica Et Biophysica Acta-Molecular Cell Research 2014, 1843, $915-922$.

(564) Rols, M. P.; Delteil, C.; Golzio, M.; Teissie, J. Control by Atp and Adp of Voltage-Induced Mammalian-Cell-Membrane Permeabilization, Gene Transfer and Resulting Expression. Eur. J. Biochem. 1998, 254, 382-388.

(565) Draeger, A.; Babiychuk, E. B. In Sphingolipids in Disease; 2013/04/09 ed.; Gulbins, E.;Petrache, I., Eds.; Springer, Vienna: Vienna, 2013; Vol. 216.

(566) Potez, S.; Luginbul, M.; Monastyrskaya, K.; Hostettler, A.; Draeger, A.; Babiychuk, E. B. Tailored Protection against Plasmalemmal Injury by Annexins with Different Ca2+ Sensitivities. J. Biol. Chem. 2011, 286, 17982-17991. 
Draeger, A.; Monastyrskaya, K.; Babiychuk, E. B. Plasma Membrane Repair and Cellular Damage Control: The Annexin Survival Kit. Biochem. Pharmacol. 2011, 81, 703-712.

(568) Cai, C.; Lin, P.; Zhu, H.; Ko, J. K.; Hwang, M.; Tan, T.; Pan, Z.; Korichneva, I.; Ma, J. Zinc Binding to Mg53 Facilitates Repair of Injury to Cell Membrane. J. Biol. Chem. 2015, 209, 13830-13839.

(569) Li, H.; Duann, P.; Lin, P. H.; Zhao, L.; Fan, Z.; Tan, T.; Zhou, X.; Sun, M.; Fu, M.; Orange, M.et al. Modulation of Wound Healing and Scar Formation by Mg53-Mediated Cell Membrane Repair. J. Biol. Chem. 2015, 290, 24592-24603.

(570) Kim, S. C.; Kellett, T.; Wang, S.; Nishi, M.; Nagre, N.; Zhou, B.; Flodby, P.; Shilo, K.; Ghadiali, S. N.; Takeshima, H.et al. Modulation of Trim72 Alters the Repair Capacity of Lung Epithelial Cells. Ann Am Thorac Soc 2015, 12, S72.

Jia, Y.; Chen, K.; Lin, P.; Lieber, G.; Nishi, M.; Yan, R.; Wang, Z.; Yao, Y.; Li, Y.; Whitson, B. A.et al. Treatment of Acute Lung Injury by Targeting Mg53-Mediated Cell Membrane Repair. Nat Commun 2014, 5, 4387.

Bouter, A.; Gounou, C.; Berat, R.; Tan, S.; Gallois, B.; Granier, T.; D'estaintot, B. L.; Poschl, E.; Brachvogel, B.; Brisson, A. R. Annexin-A5 Assembled into Two-Dimensional Arrays Promotes Cell Membrane Repair. Nat Commun 2011, 2, 270.

(573) Miller, H.; Castro-Gomes, T.; Corrotte, M.; Tam, C.; Maugel, T. K.; Andrews, N. W.; Song, W. Lipid Raft-Dependent Plasma Membrane Repair Interferes with the Activation of B Lymphocytes. J. Cell Biol. 2015, 211, 1193-1205.

(574) Friedrich, U.; Stachowicz, N.; Simm, A.; Fuhr, G.; Lucas, K.; Zimmermann, U. High Efficiency Electrotransfection with Aluminum Electrodes Using Microsecond Controlled Pulses. Bioelectrochem. Bioenerg. 1998, 47, 103-111.

(575) Kanduser, M.; Sentjurc, M.; Miklavcic, D. The Temperature Effect During Pulse Application on Cell Membrane Fluidity and Permeabilization. Bioelectrochemistry 2008, 74, 52-57.

(576) Corrotte, M.; Castro-Gomes, T.; Koushik, A. B.; Andrews, N. W. Approaches for Plasma Membrane Wounding and Assessment of Lysosome-Mediated Repair Responses. Methods Cell Biol. 2015, 126, 139-158.

(577) Babiychuk, E. B.; Monastyrskaya, K.; Potez, S.; Draeger, A. Blebbing Confers Resistance against Cell Lysis. Cell Death Differ. 2011, 18, 80-89.

(578) Carmeille, R.; Degrelle, S. A.; Plawinski, L.; Bouvet, F.; Gounou, C.; Evain-Brion, D.; Brisson, A. R.; Bouter, A. Annexin-A5 Promotes Membrane Resealing in Human Trophoblasts. Biochim. Biophys. Acta 2015, 1853, 2033-2044.

(579) Weaver, J. C.; Smith, K. C.; Esser, A. T.; Son, R. S.; Gowrishankar, T. R. A Brief Overview of Electroporation Pulse StrengthDuration Space: A Region Where Additional Intracellular Effects Are Expected. Bioelectrochemistry 2012, 87, 236-243.

(580) Beckers, C. J. M.; Keller, D. S.; Balch, W. E. Semi-Intact Cells Permeable to Macromolecules - Use in Reconstitution of Protein-Transport from the Endoplasmic-Reticulum to the Golgi-Complex. Cell 1987, 50, 523-534.

(581) Donaldson, J. G.; Lippincottschwartz, J.; Klausner, R. D. Guanine-Nucleotides Modulate the Effects of Brefeldin-a in Semipermeable Cells - Regulation of the Association of a 110-Kd Peripheral Membrane-Protein with the Golgi-Apparatus. J. Cell Biol. 1991, 112, 579-588.

(582) Simons, K.; Virta, H. Perforated Mdck Cells Support Intracellular-Transport. EMBO J. 1987, 6, 2241-2247.

(583) Burgess, G. M.; Mckinney, J. S.; Fabiato, A.; Leslie, B. A.; Putney, J. W. Calcium Pools in Saponin-Permeabilized Guinea-Pig Hepatocytes. J. Biol. Chem. 1983, 258, 5336-5345. 
Holz, R. W.; Senter, R. A. Plasma-Membrane and Chromaffin Granule Characteristics in Digitonin-Treated Chromaffin Cells. J. Neurochem. 1985, 45, 1548-1557.

(585) Wassler, M.; Jonasson, I.; Persson, R.; Fries, E. Differential Permeabilization of Membranes by Saponin Treatment of Isolated Rat Hepatocytes - Release of Secretory Proteins. Biochem. J 1987, 247, 407-415.

(586) Mick, G. J.; Bonn, T.; Steinberg, J.; Mccormick, K. Preservation of Intermediary Metabolism in Saponin-Permeabilized Rat Adipocytes. J. Biol. Chem. 1988, 263, 10667-10673.

(587) Mooney, R. A. Use of Digitonin-Permeabilized Adipocytes for Camp Studies. Methods Enzymol. 1988, 159, $193-202$.

(588) Miller, S. G.; Moore, H. P. H. Reconstitution of Constitutive Secretion Using Semi-Intact Cells - Regulation by Gtp but Not Calcium. J. Cell Biol. 1991, 112, 39-54.

(589) Plutner, H.; Davidson, H. W.; Saraste, J.; Balch, W. E. Morphological Analysis of Protein-Transport from the Er to Golgi Membranes in Digitonin-Permeabilized Cells - Role of the P58 Containing Compartment. J. Cell Biol. 1992, 119, 1097-1116. Wilson, R.; Allen, A. J.; Oliver, J.; Brookman, J. L.; High, S.; Bulleid, N. J. The Translocation, Folding, Assembly and RedoxDependent Degradation of Secretory and Membrane-Proteins in Semi-Permeabilized Mammalian-Cells. Biochem. J 1995, 307, 679-687.

(591) Negrutskii, B. S.; Stapulionis, R.; Deutscher, M. P. Supramolecular Organization of the Mammalian Translation System. Proc. Natl. Acad. Sci. U. S. A. 1994, 91, 964-968.

(592) Adam, S. A.; Marr, R. S.; Gerace, L. Nuclear-Protein Import in Permeabilized Mammalian-Cells Requires Soluble Cytoplasmic Factors. J. Cell Biol. 1990, 111, 807-816.

(593) Hagstrom, J. E.; Ludtke, J. J.; Bassik, M. C.; Sebestyen, M. G.; Adam, S. A.; Wolff, J. A. Nuclear Import of DNA in DigitoninPermeabilized Cells. J. Cell Sci. 1997, 110, 2323-2331.

(594) Kuznetsov, A. V.; Veksler, V.; Gellerich, F. N.; Saks, V.; Margreiter, R.; Kunz, W. S. Analysis of Mitochondrial Function in Situ in Permeabilized Muscle Fibers, Tissues and Cells. Nat. Protoc. 2008, 3, 965-976.

(595) Kite, G. L. Studies on the Permeability of the Internal Cytoplasm of Animal and Plant Cells. Am. J. Physiol. 1915, 37, $282-299$.

(596) Chambers, R. New Apparatus and Methods for the Dissection and Injection of Living Cells. Anat. Rec. 1922, 24, 1-19.

(597) Hildebrand, E. M. Micrurgy and the Plant Cell. Bot. Rev. 1960, 26, 277-330.

(598) Chambers, R.; Chambers, E. L. Explorations into the Nature of the Living Cell. Acad. Med. 1961, 36, 966.

(599) Wilson, J. F. Micrurgical Techniques for Neurospora. Am. J. Bot. 1961, 48, 46-51.

(600) Jeon, K. W.; Danielli, J. F. Micrurgical Studies with Large Free-Living Amebas. Int. Rev. Cytol. 1971, 30, $49-89$.

(601) Terreros, D. A.; Grantham, J. J. Marshall Barber and the Origins of Micropipet Methods. Am. J. Physiol. 1982, 242, F293F296.

(602) Llinas, R.; Nicholson, C.; Blinks, J. R. Calcium Transient in Presynaptic Terminal of Squid Giant Synapse - Detection with Aequorin. Science 1972, 176, 1127-1129.

(603) Maller, J. L.; Kemp, B. E.; Krebs, E. G. In Vivo Phosphorylation of a Synthetic Peptide Substrate of Cyclic Amp-Dependent Protein Kinase. Proc. Natt. Acad. Sci. U. S. A. 1978, 75, 248-251. 

and Fibronectin. Cell 1980, 19, 587-595.

(605) Stacey, D. W.; Allfrey, V. G. Microinjection Studies of Duck Globin Messenger-Rna Translation in Human and Avian Cells. Cell 1976, 9, 725-732.

(606) Graessmann, M.; Graessmann, A.; Hoffmann, E.; Niebel, J.; Pilaski, K. The Biological Activity of Different Forms of Polyoma Virus DNA and Viral DNA Fragments. Mol. Biol. Rep. 1973, 1, 233-241.

(607) Gordon, J. W.; Scangos, G. A.; Plotkin, D. J.; Barbosa, J. A.; Ruddle, F. H. Genetic Transformation of Mouse Embryos by Microinjection of Purified DNA. Proc. Natl. Acad. Sci. U. S. A. 1980, 77, 7380-7384.

(608) Wormington, W. M. Stable Repression of Ribosomal-Protein L1 Synthesis in Xenopus Oocytes by Microinjection of Antisense Rna. Proc. Natl. Acad. Sci. U. S. A. 1986, 83, 8639-8643.

(609) Zhang, Y. Y.; Ballas, C. B.; Rao, M. P. Towards Ultrahigh Throughput Microinjection: Mems-Based Massively-Parallelized Mechanoporation. leee Eng Med Bio 2012, 2012, 594-597.

(610) Knoblauch, M.; Hibberd, J. M.; Gray, J. C.; Van Bel, A. J. E. A Galinstan Expansion Femtosyringe for Microinjection of Eukaryotic Organelles and Prokaryotes. Nat. Biotechnol. 1999, 17, 906-909.

(611) Laforge, F. O.; Carpino, J.; Rotenberg, S. A.; Mirkin, M. V. Electrochemical Attosyringe. Proc. Natl. Acad. Sci. U. S. A. 2007, $104,11895-11900$.

(612) Singhal, R.; Orynbayeva, Z.; Sundaram, R. V. K.; Niu, J. J.; Bhattacharyya, S.; Vitol, E. A.; Schrlau, M. G.; Papazoglou, E. S.; Friedman, G.; Gogotsi, Y. Multifunctional Carbon-Nanotube Cellular Endoscopes. Nature Nanotechnology 2011, 6, 57-64.

(613) Simonis, M.; Hubner, W.; Wilking, A.; Huser, T.; Hennig, S. Survival Rate of Eukaryotic Cells Following Electrophoretic Nanoinjection. Sci. Rep. 2017, 7, 41277.

(614) Guillaume-Gentil, O.; Potthoff, E.; Ossola, D.; Franz, C. M.; Zambelli, T.; Vorholt, J. A. Force-Controlled Manipulation of Single Cells: From Afm to Fluidfm. Trends Biotechnol. 2014, 32, 381-388.

(615) Meister, A.; Gabi, M.; Behr, P.; Studer, P.; Voros, J.; Niedermann, P.; Bitterli, J.; Polesel-Maris, J.; Liley, M.; Heinzelmann, H.et al. Fluidfm: Combining Atomic Force Microscopy and Nanofluidics in a Universal Liquid Delivery System for Single Cell Applications and Beyond. Nano Lett. 2009, 9, 2501-2507.

(616) Guillaume-Gentil, O.; Grindberg, R. V.; Kooger, R.; Dorwling-Carter, L.; Martinez, V.; Ossola, D.; Pilhofer, M.; Zambelli, T.; Vorholt, J. A. Tunable Single-Cell Extraction for Molecular Analyses. Cell 2016, 166, 506-516.

(617) Guillaume-Gentil, O.; Rey, T.; Kiefer, P.; Ibanez, A. J.; Steinhoff, R.; Bronnimann, R.; Dorwling-Carter, L.; Zambelli, T.; Zenobi, R.; Vorholt, J. A. Single-Cell Mass Spectrometry of Metabolites Extracted from Live Cells by Fluidic Force Microscopy. Anal. Chem. 2017, 89, 5017-5023.

(618) Guillaume-Gentil, O.; Potthoff, E.; Ossola, D.; Dorig, P.; Zambelli, T.; Vorholt, J. A. Force-Controlled Fluidic Injection into Single Cell Nuclei. Small 2013, 9, 1904-1907.

(619) Seger, R. A.; Actis, P.; Penfold, C.; Maalouf, M.; Vilozny, B.; Pourmand, N. Voltage Controlled Nano-Injection System for Single-Cell Surgery. Nanoscale 2012, 4, 5843-5846. 

System. Exp. Cell Res. 1988, 178, 369-376.

Ansorge, W.; Pepperkok, R. Performance of an Automated-System for Capillary Microinjection into Living Cells. J. Biochem. Bioph. Methods 1988, 16, 283-292.

(622) Wang, W.; Liu, X.; Gelinas, D.; Ciruna, B.; Sun, Y. A Fully Automated Robotic System for Microinjection of Zebrafish Embryos. PLoS One 2007, 2, e862.

(623) Wang, W. H.; Sun, Y.; Zhang, M.; Anderson, R.; Langille, L.; Chan, W. A System for High-Speed Microinjection of Adherent Cells. Rev. Sci. Instrum. 2008, 79, 104302.

(624) Adamo, A.; Jensen, K. F. Microfluidic Based Single Cell Microinjection. Lab on a Chip 2008, 8, 1258-1261.

Adamo, A.; Roushdy, O.; Dokov, R.; Sharei, A.; Jensen, K. F. Microfluidic Jet Injection for Delivering Macromolecules into Cells. Journal of Micromechanics and Microengineering 2013, 23, 035026.

Sanford, J. C.; Smith, F. D.; Russell, J. A. Optimizing the Biolistic Process for Different Biological Applications. Methods Enzymol. 1993, 217, 483-509.

Klein, T. M.; Fitzpatrick-Mcelligott, S. Particle Bombardment: A Universal Approach for Gene Transfer to Cells and Tissues. Curr. Opin. Biotechnol. 1993, 4, 583-590.

O'brien, J. A.; Lummis, S. C. R. Biolistic Transfection of Neuronal Cultures Using a Hand-Held Gene Gun. Nat. Protoc. 2006, $1,977-981$.

(629) Williams, R. S.; Johnston, S. A.; Riedy, M.; Devit, M. J.; Mcelligott, S. G.; Sanford, J. C. Introduction of Foreign Genes into Tissues of Living Mice by DNA-Coated Microprojectiles. Proc. Natl. Acad. Sci. U. S. A. 1991, 88, 2726-2730.

(630) Yang, N. S.; Burkholder, J.; Roberts, B.; Martinell, B.; Mccabe, D. In Vivo and in Vitro Gene Transfer to Mammalian Somatic Cells by Particle Bombardment. Proc. Natl. Acad. Sci. U. S. A. 1990, 87, 9568-9572.

Zelenin, A. V.; Titomirov, A. V.; Kolesnikov, V. A. Genetic Transformation of Mouse Cultured Cells with the Help of HighVelocity Mechanical DNA Injection. FEBS Lett. 1989, 244, 65-67.

(632) Russell, J. A.; Roy, M. K.; Sanford, J. C. Physical Trauma and Tungsten Toxicity Reduce the Efficiency of Biolistic Transformation. Plant Physiol. 1992, 98, 1050-1056.

(633) Fitzpatrick-Mcelligott, S. Gene-Transfer to Tumor-Infiltrating Lymphocytes and Other Mammalian Somatic Cells by Microprojectile Bombardment. Bio-Technology 1992, 10, 1036-1040.

(634) Burkholder, J. K.; Decker, J.; Yang, N. S. Rapid Transgene Expression in Lymphocyte and Macrophage Primary Cultures after Particle Bombardment-Mediated Gene Transfer. J. Immunol. Methods 1993, 165, 149-156.

(635) Woffendin, C.; Yang, Z. Y.; Udaykumar; Xu, L.; Yang, N. S.; Sheehy, M. J.; Nabel, G. J. Nonviral and Viral Delivery of a Human-Immunodeficiency-Virus Protective Gene into Primary Human T-Cells. Proc. Natl. Acad. Sci. U. S. A. 1994, 91, 1158111585.

(636) Verma, S.; Woffendin, C.; Bahner, I.; Ranga, U.; Xu, L.; Yang, Z. Y.; King, S. R.; Kohn, D. B.; Nabel, G. J. Gene Transfer into Human Umbilical Cord Blood-Derived Cd34(+) Cells by Particle-Mediated Gene Transfer. Gene Ther. 1998, 5, 692-699. 
Ye, Z. Q.; Qiu, P.; Burkholder, J. K.; Turner, J.; Culp, J.; Roberts, T.; Shahidi, N. T.; Yang, N. S. Cytokine Transgene Expression and Promoter Usage in Primary Cd34(+) Cells Using Particle-Mediated Gene Delivery. Hum. Gene Ther. 1998, 9, 2197-2205.

(638) Mahvi, D. M.; Burkholder, J. K.; Turner, J.; Culp, J.; Malter, J. S.; Sondel, P. M.; Yang, N. S. Particle-Mediated Gene Transfer of Granulocyte-Macrophage Colony-Stimulating Factor Cdna to Tumor Cells: Implications for a Clinically Relevant Tumor Vaccine. Hum. Gene Ther. 1996, 7, 1535-1543.

(639) Uchida, M.; Li, X. W.; Mertens, P.; Alpar, H. O. Transfection by Particle Bombardment: Delivery of Plasmid DNA into Mammalian Cells Using Gene Gun. Biochimica Et Biophysica Acta-General Subjects 2009, 1790, 754-764.

(640) Zhang, S. B.; Gu, J.; Yang, N. S.; Kao, C. H.; Gardner, T. A.; Eble, J. N.; Cheng, L. Relative Promoter Strengths in Four Human Prostate Cancer Cell Lines Evaluated by Particle Bombardment-Mediated Gene Transfer. Prostate 2002, 51, $286-292$.

(641) Antolik, C.; De Deyne, P. G.; Bloch, R. J. Biolistic Transfection of Cultured Myotubes. Sci. STKE 2003, 2003, pl11.

(642) Heiser, W. C. Gene-Transfer into Mammalian-Cells by Particle Bombardment. Anal. Biochem. 1994, 217, $185-196$.

(643) Johnston, S. A.; Tang, D. C. Gene Gun Transfection of Animal-Cells and Genetic Immunization. Methods Cell Biol. 1994, 43, 353-365.

(644) Thompson, T. A.; Gould, M. N.; Burkholder, J. K.; Yang, N. S. Transient Promoter Activity in Primary Rat Mammary EpithelialCells Evaluated Using Particle Bombardment Gene-Transfer. In Vitro Cellular \& Developmental Biology-Animal 1993, 29, 165170.

(645) Bridgman, P. C.; Brown, M. E.; Balan, I. Biolistic Transfection. Neurons: Methods and Applications for the Cell Biologist 2003, 71, 353-368.

(646) O'brien, J. A.; Lummis, S. C. Diolistic Labeling of Neuronal Cultures and Intact Tissue Using a Hand-Held Gene Gun. Nat. Protoc. 2006, 1, 1517-1521.

(647) Klimaschewski, L.; Nindl, W.; Pimpl, M.; Waltinger, P.; Pfaller, K. Biolistic Transfection and Morphological Analysis of Cultured Sympathetic Neurons. J. Neurosci. Methods 2002, 113, 63-71.

(648) Usachev, Y. M.; Khammanivong, A.; Campbell, C.; Thayer, S. A. Particle-Mediated Gene Transfer to Rat Neurons in Primary Culture. Pflugers Archiv-European Journal of Physiology 2000, 439, 730-738.

(649) Mcallister, A. K. Biolistic Transfection of Neurons. Sci. STKE 2000, 2000, pl1.

(650) Wellmann, H.; Kaltschmidt, B.; Kaltschmidt, C. Optimized Protocol for Biolistic Transfection of Brain Slices and Dissociated Cultured Neurons with a Hand-Held Gene Gun. J. Neurosci. Methods 1999, 92, 55-64.

(651) Biewenga, J. E.; Destree, O. H. J.; Schrama, L. H. Plasmid-Mediated Gene Transfer in Neurons Using the Biolistics Technique. J. Neurosci. Methods 1997, 71, 67-75.

(652) Lin, M. T. S.; Pulkkinen, L.; Uitto, J.; Yoon, K. The Gene Gun: Current Applications in Cutaneous Gene Therapy. Int. J. Dermatol. 2000, 39, 161-170.

(653) Fuller, D. H.; Loudon, P.; Schmaljohn, C. Preclinical and Clinical Progress of Particle-Mediated DNA Vaccines for Infectious Diseases. Methods 2006, 40, 86-97. 
Lin, C. C.; Yen, M. C.; Lin, C. M.; Huang, S. S.; Yang, H. J.; Chow, N. H.; Lai, M. D. Delivery of Noncarrier Naked DNA Vaccine into the Skin by Supersonic Flow Induces a Polarized T Helper Type 1 Immune Response to Cancer. J. Gene Med. 2008, 10, 679-689.

(655) Fynan, E. F.; Webster, R. G.; Fuller, D. H.; Haynes, J. R.; Santoro, J. C.; Robinson, H. L. DNA Vaccines - Protective Immunizations by Parenteral, Mucosal, and Gene-Gun Inoculations. Proc. Natl. Acad. Sci. U. S. A. 1993, 90, $11478-11482$.

Raju, P. A.; Mcsloy, N.; Truong, N. K.; Kendall, M. a. F. Assessment of Epidermal Cell Viability by near Infrared Multi-Photon Microscopy Following Ballistic Delivery of Gold Micro-Particles. Vaccine 2006, 24, 4644-4647.

(657) Yang, N. S.; Sun, W. H.; Mccabe, D. Developing Particle-Mediated Gene-Transfer Technology for Research into Gene Therapy of Cancer. Mol. Med. Today 1996, 2, 476-481.

(658) Benediktsson, A. M.; Schachtele, S. J.; Green, S. H.; Dailey, M. E. Ballistic Labeling and Dynamic Imaging of Astrocytes in Organotypic Hippocampal Slice Cultures. J. Neurosci. Methods 2005, 141, 41-53.

(659) Kettunen, P.; Demas, J.; Lohmann, C.; Kasthuri, N.; Gong, Y. D.; Wong, R. O. L.; Gan, W. B. Imaging Calcium Dynamics in the Nervous System by Means of Ballistic Delivery of Indicators. J. Neurosci. Methods 2002, 119, 37-43.

(660) Gan, W. B.; Grutzendler, J.; Wong, W. T.; Wong, R. O. L.; Lichtman, J. W. Multicolor "Diolistic" Labeling of the Nervous System Using Lipophilic Dye Combinations. Neuron 2000, 27, 219-225.

(661) Grutzendler, J.; Tsai, J.; Gan, W. B. Rapid Labeling of Neuronal Populations by Ballistic Delivery of Fluorescent Dyes. Methods 2003, 30, 79-85.

(662) Davis, R. E.; Parra, A.; Loverde, P. T.; Ribeiro, E.; Glorioso, G.; Hodgson, S. Transient Expression of DNA and Rna in Parasitic Helminths by Using Particle Bombardment. Proc. Natl. Acad. Sci. U. S. A. 1999, 96, 8687-8692.

(663) Sohn, R. L.; Murray, M. T.; Schwarz, K.; Nyitray, J.; Purray, P.; Franko, A. P.; Palmer, K. C.; Diebel, L. N.; Dulchavsky, S. A. In-Vivo Particle Mediated Delivery of Mrna to Mammalian Tissues: Ballistic and Biologic Effects. Wound Repair Regen. 2001, 9, 287-296.

(664) Schwarz, K. W.; Murray, M. T.; Sylora, R.; Sohn, R. L.; Dulchavsky, S. A. Augmentation of Wound Healing with Translation Initiation Factor Eif4e Mrna. J. Surg. Res. 2002, 103, 175-182.

(665) Svarovsky, S.; Borovkov, A.; Sykes, K. Cationic Gold Microparticles for Biolistic Delivery of Nucleic Acids. BioTechniques 2008, 45, 535-540.

(666) Belyantseva, I. A. Helios Gene Gun-Mediated Transfection of the Inner Ear Sensory Epithelium. Methods Mol. Biol. 2009, 493, 103-123.

(667) Wu, J.; Du, H. W.; Liao, X. W.; Zhao, Y.; Li, L. G.; Yang, L. Y. An Improved Particle Bombardment for the Generation of Transgenic Plants by Direct Immobilization of Relleasable Tn5 Transposases onto Gold Particles. Plant Mol. Biol. 2011, 77, 117-127.

(668) Martin-Ortigosa, S.; Valenstein, J. S.; Lin, V. S. Y.; Trewyn, B. G.; Wang, K. Gold Functionalized Mesoporous Silica Nanoparticle Mediated Protein and DNA Codelivery to Plant Cells Via the Biolistic Method. Adv. Funct. Mater. 2012, 22, 35763582. 
Martin-Ortigosa, S.; Wang, K. Proteolistics: A Biolistic Method for Intracellular Delivery of Proteins. Transgenic Res. 2014, 23, 743-756.

(670) Liang, Z.; Chen, K. L.; Li, T. D.; Zhang, Y.; Wang, Y. P.; Zhao, Q.; Liu, J. X.; Zhang, H. W.; Liu, C. M.; Ran, Y. D.et al. Efficient DNA-Free Genome Editing of Bread Wheat Using Crispr/Cas9 Ribonucleoprotein Complexes. Nat Commun 2017, 8, 14261.

(671) Zhang, D. W.; Das, D. B.; Rielly, C. D. Potential of Microneedle-Assisted Micro-Particle Delivery by Gene Guns: A Review. Drug Deliv. 2014, 21, 571-587.

(672) O'brien, J. A.; Lummis, S. C. R. Nano-Biolistics: A Method of Biolistic Transfection of Cells and Tissues Using a Gene Gun with Novel Nanometer-Sized Projectiles. BMC Biotechnol. 2011, 11, 66.

(673) Roizenblatt, R.; Weiland, J. D.; Carcieri, S.; Qiu, G.; Behrend, M.; Humayun, M. S.; Chow, R. H. Nanobiolistic Delivery of Indicators to the Living Mouse Retina. J. Neurosci. Methods 2006, 153, 154-161.

(674) Cai, D.; Mataraza, J. M.; Qin, Z. H.; Huang, Z. P.; Huang, J. Y.; Chiles, T. C.; Carnahan, D.; Kempa, K.; Ren, Z. F. Highly Efficient Molecular Delivery into Mammalian Cells Using Carbon Nanotube Spearing. Nat. Methods 2005, 2, $449-454$. Xu, X.; Hou, S.; Wattanatorn, N.; Wang, F.; Yang, Q.; Zhao, C.; Yu, X.; Tseng, H. R.; Jonas, S. J.; Weiss, P. S. PrecisionGuided Nanospears for Targeted and High-Throughput Intracellular Gene Delivery. ACS Nano 2018, $12,4503-4511$.

Mcknight, T. E.; Melechko, A. V.; Hensley, D. K.; Mann, D. G. J.; Griffin, G. D.; Simpson, M. L. Tracking Gene Expression after DNA Delivery Using Spatially Indexed Nanofiber Arrays. Nano Lett. 2004, 4, 1213-1219.

Mann, D. G. J.; Mcknight, T. E.; Mcpherson, J. T.; Hoyt, P. R.; Melechko, A. V.; Simpson, M. L.; Sayler, G. S. Inducible Rna Interference-Mediated Gene Silencing Using Nanostructured Gene Delivery Arrays. Acs Nano 2008, 2, 69-76.

Kim, W.; Ng, J. K.; Kunitake, M. E.; Conklin, B. R.; Yang, P. D. Interfacing Silicon Nanowires with Mammalian Cells. JACS 2007, 129, 7228-7229.

Shalek, A. K.; Gaublomme, J. T.; Wang, L. L.; Yosef, N.; Chevrier, N.; Andersen, M. S.; Robinson, J. T.; Pochet, N.; Neuberg, D.; Gertner, R. S.et al. Nanowire-Mediated Delivery Enables Functional Interrogation of Primary Immune Cells: Application to the Analysis of Chronic Lymphocytic Leukemia. Nano Lett. 2012, 12, 6498-6504.

(680) Choi, M.; Lee, S. H.; Kim, W. B.; Gujrati, V.; Kim, D.; Lee, J.; Kim, J.-I.; Kim, H.; Saw, P. E.; Jon, S. Intracellular Delivery of Bioactive Cargos to Hard-to-Transfect Cells Using Carbon Nanosyringe Arrays under an Applied Centrifugal G-Force. Advanced Healthcare Materials 2016, 5, 101-107.

(681) Nair, B. G.; Hagiwara, K.; Ueda, M.; Yu, H. H.; Tseng, H. R.; Ito, Y. High Density of Aligned Nanowire Treated with Polydopamine for Efficient Gene Silencing by Sirna According to Cell Membrane Perturbation. ACS Appl Mater Interfaces 2016, 8, 18693-18700.

(682) Matsumoto, D.; Rao Sathuluri, R.; Kato, Y.; Silberberg, Y. R.; Kawamura, R.; Iwata, F.; Kobayashi, T.; Nakamura, C. Oscillating High-Aspect-Ratio Monolithic Silicon Nanoneedle Array Enables Efficient Delivery of Functional BioMacromolecules into Living Cells. Sci. Rep. 2015, 5, 15325. 
Kim, K. H.; Kim, J.; Choi, J. S.; Bae, S.; Kwon, D.; Park, I.; Kim, D. H.; Seo, T. S. Rapid, High-Throughput, and Direct Molecular Beacon Delivery to Human Cancer Cells Using a Nanowire-Incorporated and Pneumatic Pressure-Driven Microdevice. Small 2015, 11, 6215-6224.

(684) Park, S.; Kim, Y. S.; Kim, W. B.; Jon, S. Carbon Nanosyringe Array as a Platform for Intracellular Delivery. Nano Lett. 2009, 9, 1325-1329.

(685) Chan, M. S.; Lo, P. K. Nanoneedle-Assisted Delivery of Site-Selective Peptide-Functionalized DNA Nanocages for Targeting Mitochondria and Nuclei. Small 2014, 10, 1255-1260.

(686) Yosef, N.; Shalek, A. K.; Gaublomme, J. T.; Jin, H. L.; Lee, Y. J.; Awasthi, A.; Wu, C.; Karwacz, K.; Xiao, S.; Jorgolli, M.et al. Dynamic Regulatory Network Controlling T(H)17 Cell Differentiation. Nature 2013, 496, 461-468.

Elnathan, R.; Delalat, B.; Brodoceanu, D.; Alhmoud, H.; Harding, F. J.; Buehler, K.; Nelson, A.; Isa, L.; Kraus, T.; Voelcker, N. H. Maximizing Transfection Efficiency of Vertically Aligned Silicon Nanowire Arrays. Adv. Funct. Mater. 2015, $25,7215-7225$. Mumm, F.; Beckwith, K. M.; Bonde, S.; Martinez, K. L.; Sikorski, P. A Transparent Nanowire-Based Cell Impalement Device Suitable for Detailed Cell-Nanowire Interaction Studies. Small 2013, 9, 263-272.

Hanson, L.; Lin, Z. C.; Xie, C.; Cui, Y.; Cui, B. X. Characterization of the Cell-Nanopillar Interface by Transmission Electron Microscopy. Nano Lett. 2012, 12, 5815-5820.

(690) Berthing, T.; Bonde, S.; Rostgaard, K. R.; Madsen, M. H.; Sorensen, C. B.; Nygard, J.; Martinez, K. L. Cell Membrane Conformation at Vertical Nanowire Array Interface Revealed by Fluorescence Imaging. Nanotechnology 2012, $23,415102$.

(691) Xie, X.; Xu, A. M.; Angle, M. R.; Tayebi, N.; Verma, P.; Melosh, N. A. Mechanical Model of Vertical Nanowire Cell Penetration. Nano Lett. 2013, 13, 6002-6008.

(692) Bae, S.; Park, S.; Kim, J.; Choi, J. S.; Kim, K. H.; Kwon, D.; Jin, E.; Park, I.; Kim, D. H.; Seo, T. S. Exogenous Gene Integration for Microalgal Cell Transformation Using a Nanowire-Incorporated Microdevice. ACS Appl Mater Interfaces 2015, 7, 27554-27561.

(693) Lee, D.; Lee, D.; Won, Y.; Hong, H.; Kim, Y.; Song, H.; Pyun, J. C.; Cho, Y. S.; Ryu, W.; Moon, J. Insertion of Vertically Aligned Nanowires into Living Cells by Inkjet Printing of Cells. Small 2016, 12, 1446-1457.

(694) Wang, Y.; Yang, Y.; Yan, L.; Kwok, S. Y.; Li, W.; Wang, Z. G.; Zhu, X. Y.; Zhu, G. Y.; Zhang, W. J.; Chen, X. F.et al. Poking Cells for Efficient Vector-Free Intracellular Delivery. Nat Commun 2014, 5, 4466.

(695) Han, S. W.; Nakamura, C.; Obataya, I.; Nakamura, N.; Miyake, J. Gene Expression Using an Ultrathin Needle Enabling Accurate Displacement and Low Invasiveness. Biochem. Biophys. Res. Commun. 2005, 332, 633-639.

(696) Prinz, C. N. Interactions between Semiconductor Nanowires and Living Cells. Journal of Physics-Condensed Matter 2015, 27, 233103.

Persson, H.; Kobler, C.; Molhave, K.; Samuelson, L.; Tegenfeldt, J. O.; Oredsson, S.; Prinz, C. N. Fibroblasts Cultured on Nanowires Exhibit Low Motility, Impaired Cell Division, and DNA Damage. Small 2013, 9, 4006-4016.

Persson, H.; Li, Z.; Tegenfeldt, J. O.; Oredsson, S.; Prinz, C. N. From Immobilized Cells to Motile Cells on a Bed-of-Nails: Effects of Vertical Nanowire Array Density on Cell Behaviour. Sci. Rep. 2015, 5, 18535 
Bonde, S.; Berthing, T.; Madsen, M. H.; Andersen, T. K.; Buch-Manson, N.; Guo, L.; Li, X. M.; Badique, F.; Anselme, K.; Nygard, J.et al. Tuning Inas Nanowire Density for Hek293 Cell Viability, Adhesion, and Morphology: Perspectives for Nanowire-Based Biosensors. Acs Applied Materials \& Interfaces 2013, 5, 10510-10519.

(700) Vandersarl, J. J.; Xu, A. M.; Melosh, N. A. Nanostraws for Direct Fluidic Intracellular Access. Nano Lett. 2012, 12, $3881-3886$.

(701) Peer, E.; Artzy-Schnirman, A.; Gepstein, L.; Sivan, U. Hollow Nanoneedle Array and Its Utilization for Repeated Administration of Biomolecules to the Same Cells. Acs Nano 2012, 6, 4940-4946.

(702) Xie, X.; Xu, A. M.; Leal-Ortiz, S.; Cao, Y. H.; Garner, C. C.; Melosh, N. A. Nanostraw-Electroporation System for Highly Efficient Intracellular Delivery and Transfection. ACS Nano 2013, 7, 4351-4358.

(703) Xu, A. M.; Kim, S. A.; Wang, D. S.; Aalipour, A.; Melosh, N. A. Temporally Resolved Direct Delivery of Second Messengers into Cells Using Nanostraws. Lab on a Chip 2016, 16, 2434-2439.

(704) Xu, A. M.; Wang, D. S.; Shieh, P.; Cao, Y. H.; Melosh, N. A. Direct Intracellular Delivery of Cell-Impermeable Probes of Protein Glycosylation by Using Nanostraws. ChemBioChem 2017, 18, 623-628.

(705) Cao, Y.; Hjort, M.; Chen, H.; Birey, F.; Leal-Ortiz, S. A.; Han, C. M.; Santiago, J. G.; Pasca, S. P.; Wu, J. C.; Melosh, N. A. Nondestructive Nanostraw Intracellular Sampling for Longitudinal Cell Monitoring. Proc. Natl. Acad. Sci. U. S. A. 2017, 114, E1866-E1874.

(706) Durney, A. R.; Frenette, L. C.; Hodvedt, E. C.; Krauss, T. D.; Mukaibo, H. Fabrication of Tapered Microtube Arrays and Their Application as a Microalgal Injection Platform. Acs Applied Materials \& Interfaces 2016, 8, 34198-34208.

(707) Golshadi, M.; Wright, L. K.; Dickerson, I. M.; Schrlau, M. G. High-Efficiency Gene Transfection of Cells through Carbon Nanotube Arrays. Small 2016, 12, 3014-3020.

(708) Chen, X.; Kis, A.; Zettl, A.; Bertozzi, C. R. A Cell Nanoinjector Based on Carbon Nanotubes. Proc. Natl. Acad. Sci. U. S. A. 2007, 104, 8218-8222.

(709) Han, S. W.; Nakamura, C.; Obataya, I.; Nakamura, N.; Miyake, J. A Molecular Delivery System by Using Afm and Nanoneedle. Biosens. Bioelectron. 2005, 20, 2120-2125.

(710) Cuerrier, C. M.; Lebel, R.; Grandbois, M. Single Cell Transfection Using Plasmid Decorated Afm Probes. Biochem. Biophys. Res. Commun. 2007, 355, 632-636.

(711) Aten, Q. T.; Jensen, B. D.; Tamowski, S.; Wilson, A. M.; Howell, L. L.; Burnett, S. H. Nanoinjection: Pronuclear DNA Delivery Using a Charged Lance. Transgenic Res. 2012, 21, 1279-1290.

(712) Yoo, S. M.; Kang, M.; Kang, T.; Kim, D. M.; Lee, S. Y.; Kim, B. Electrotriggered, Spatioselective, Quantitative Gene Delivery into a Single Cell Nucleus by Au Nanowire Nanoinjector. Nano Lett. 2013, 13, 2431-2435.

(713) Park, K.; Kim, K. C.; Lee, H.; Sung, Y.; Kang, M.; Lee, Y. M.; Ahn, J. Y.; Lim, J. M.; Kang, T.; Kim, B.et al. Suppressing Mosaicism by Au Nanowire Injector-Driven Direct Delivery of Plasmids into Mouse Embryos. Biomaterials 2017, 138, $169-178$.

(714) Hara, C.; Tateyama, K.; Akamatsu, N.; Imabayashi, H.; Karaki, K.; Nomura, N.; Okano, H.; Miyawaki, A. A Practical Device for Pinpoint Delivery of Molecules into Multiple Neurons in Culture. Brain Cell Biol. 2006, 35, 229-237. 
(715) Yamamoto, F.; Furusawa, M. A Simple Microinjection Technique Not Employing a Micromanipulator. Exp. Cell Res. 1978, 117, 441-445.

(716) Yamamoto, F.; Furusawa, M.; Takamatsu, K.; Miura, N.; Uchida, T. Intracellular Introduction of a Fixed Quantity of Substances by Pricking Cells Using a Modified Microscope. Exp. Cell Res. 1981, 135, 341-345.

(717) Yamamoto, F.; Furusawa, M.; Furusawa, I.; Obinata, M. The 'Pricking' Method. A New Efficient Technique for Mechanically Introducing Foreign DNA into the Nuclei of Culture Cells. Exp. Cell Res. 1982, 142, 79-84.

(718) Kudo, A.; Yamamoto, F.; Furusawa, M.; Kuroiwa, A.; Natori, S.; Obinata, M. Structure of Thymidine Kinase Gene Introduced into Mouse Ltk-Cells by a New Injection Method. Gene 1982, 19, 11-19.

(719) Teichert, G. H.; Burnett, S.; Jensen, B. D. A Microneedle Array Able to Inject Tens of Thousands of Cells Simultaneously. Journal of Micromechanics and Microengineering 2013, 23, 095003.

(720) Lee, K.; Lingampalli, N.; Pisano, A. P.; Murthy, N.; So, H. Physical Delivery of Macromolecules Using High-Aspect Ratio Nanostructured Materials. ACS Applied Materials \& Interfaces 2015, 7, 23387-23397.

(721) Kwak, M.; Han, L.; Chen, J. J.; Fan, R. Interfacing Inorganic Nanowire Arrays and Living Cells for Cellular Function Analysis. Small 2015, 11, 5600-5610.

(722) Sharma, P.; Cho, H. A.; Lee, J. W.; Ham, W. S.; Park, B. C.; Cho, N. H.; Kim, Y. K. Efficient Intracellular Delivery of Biomacromolecules Employing Clusters of Zinc Oxide Nanowires. Nanoscale 2017, 9, 15371-15378

(723) Pan, J.; Yuan, Y.; Wang, H.; Liu, F.; Xiong, X.; Chen, H.; Yuan, L. Efficient Transfection by Using Pdmaema Modified Sinwas as a Platform for Ca2+-Dependent Gene Delivery. ACS Applied Materials \& Interfaces 2016, 8, $15138-15144$.

(724) Nateri, A. S.; Tzavelas, C.; Bildirici, L.; Rickwood, D. Transfection of Human Peripheral Blood Mononuclear Cells Using Immunoporation. J. Immunoassay Immunochem. 2005, 26, 169-177.

(725) Tzavelas, C.; Bildirici, L.; Rickwood, D. Factors That Affect the Efficiency of Cell Transfection by Immunoporation. Anal. Biochem. 2004, 328, 219-224.

(726) Tzavelas, C.; Bildirici, L.; Rickwood, D. Production of Stably Transfected Cell Lines Using Immunoporation. BioTechniques 2004, 37, 276-278.

(727) Bildirici, L.; Tzaveles, C.; Rickwood, D. Immunoporation of Adherent Cells in Situ. Mol. Biol. Cell 2004, 15, 446A-446A.

(728) Bildirici, L.; Rickwood, D. Comparisons of Different Types of Antibody-Coated Beads for Cell Transfection Using Immunoporation. Mol. Biol. Cell 2004, 15, 446A-446A.

(729) Bildirici, L.; Smith, P.; Tzavelas, C.; Horefti, E.; Rickwood, D. Biotechniques - Transfection of Cells by Immunoporation. Nature 2000, 405, 298.

(730) Rickwood, D.; Bildirici, L.; Smith, P.; Tromberg, H. Immunoporation: A Novel Method for Transfecting Cells Selectively and at High Efficiency. Mol. Biol. Cell 1999, 10, 271A-271A.

(731) Manders, E. M. M.; Kimura, H.; Cook, P. R. Direct Imaging of DNA in Living Cells Reveals the Dynamics of Chromosome Formation. J. Cell Biol. 1999, 144, 813-821. 
(732) Cox, D.; Berg, J. S.; Cammer, M.; Chinegwundoh, J. O.; Dale, B. M.; Cheney, R. E.; Greenberg, S. Myosin X Is a Downstream Effector of $\mathrm{Pi}(3) \mathrm{K}$ During Phagocytosis. Nat. Cell Biol. 2002, 4, 469-477.

(733) Santic, M.; Molmeret, M.; Barker, J. R.; Klose, K. E.; Dekanic, A.; Doric, M.; Abu Kwaik, Y. A Francisella Tularensis Pathogenicity Island Protein Essential for Bacterial Proliferation within the Host Cell Cytosol. Cell. Microbiol. 2007, 9, 23912403.

(734) Besteiro, S.; Michelin, A.; Poncet, J.; Dubremetz, J. F.; Lebrun, M. Export of a Toxoplasma Gondii Rhoptry Neck Protein Complex at the Host Cell Membrane to Form the Moving Junction During Invasion. PLoS Path. 2009, 5, e1000309.

(735) Rosqvist, R.; Forsberg, A.; Wolfwatz, H. Intracellular Targeting of the Yersinia Yope Cytotoxin in Mammalian-Cells Induces Actin Microfilament Disruption. Infect. Immun. 1991, 59, 4562-4569.

(736) Gilmore, A. P.; Romer, L. H. Inhibition of Focal Adhesion Kinase (Fak) Signaling in Focal Adhesions Decreases Cell Motility and Proliferation. Mol. Biol. Cell 1996, 7, 1209-1224.

(737) Memedula, S.; Belmont, A. S. Sequential Recruitment of Hat and Swi/Snf Components to Condensed Chromatin by Vp16. Curr. Biol. 2003, 13, 241-246.

(738) Rai, A. K.; Rai, A.; Ramaiya, A. J.; Jha, R.; Mallik, R. Molecular Adaptations Allow Dynein to Generate Large Collective Forces inside Cells. Cell 2013, 152, 172-182.

(739) Becker, T.; Volchuk, A.; Rothman, J. E. Differential Use of Endoplasmic Reticulum Membrane for Phagocytosis in J774 Macrophages. Proc. Natl. Acad. Sci. U. S. A. 2005, 102, 4022-4026.

(740) Morisaki, T.; Lyon, K.; Deluca, K. F.; Deluca, J. G.; English, B. P.; Zhang, Z. J.; Lavis, L. D.; Grimm, J. B.; Viswanathan, S.; Looger, L. L.et al. Real-Time Quantification of Single Rna Translation Dynamics in Living Cells. Science 2016, 352, 14251429.

(741) Molenaar, C.; Wiesmeijer, K.; Verwoerd, N. P.; Khazen, S.; Eils, R.; Tanke, H. J.; Dirks, R. W. Visualizing Telomere Dynamics in Living Mammalian Cells Using Pna Probes. EMBO J. 2003, 22, 6631-6641.

(742) Jones, S. A.; Shim, S. H.; He, J.; Zhuang, X. W. Fast, Three-Dimensional Super-Resolution Imaging of Live Cells. Nat. Methods 2011, 8, 499-505.

(743) Cheng, J. P.; Fernando, K. a. S.; Veca, L. M.; Sun, Y. P.; Lamond, A. I.; Lam, Y. W.; Cheng, S. H. Reversible Accumulation of Pegylated Single-Walled Carbon Nanotubes in the Mammalian Nucleus. Acs Nano 2008, 2, 2085-2094.

(744) Emerson, N. T.; Hsia, C. H.; Rafalska-Metcalf, I. U.; Yang, H. Mechanodelivery of Nanoparticles to the Cytoplasm of Living Cells. Nanoscale 2014, 6, 4538-4543.

(745) Frankel, A. D.; Pabo, C. O. Cellular Uptake of the Tat Protein from Human Immunodeficiency Virus. Cell 1988, 55, $1189-1193$.

(746) Gentz, R.; Chen, C. H.; Rosen, C. A. Bioassay for Trans-Activation Using Purified Human Immunodeficiency Virus TatEncoded Protein - Trans-Activation Requires Messenger-Rna Synthesis. Proc. Natl. Acad. Sci. U. S. A. 1989, 86, 821-824.

(747) Malcolm, K. C.; Elliott, C. M.; Exton, J. H. Evidence for Rho-Mediated Agonist Stimulation of Phospholipase D in Rat1 Fibroblasts - Effects of Clostridium Botulinum C3 Exoenzyme. J. Biol. Chem. 1996, 271, 13135-13139. 
(748) Flinn, H. M.; Ridley, A. J. Rho Stimulates Tyrosine Phosphorylation of Focal Adhesion Kinase, P130 and Paxillin. J. Cell Sci. 1996, 109, 1133-1141.

(749) Ubezio, P.; Civoli, F. Flow Cytometric Detection of Hydrogen-Peroxide Production Induced by Doxorubicin in Cancer-Cells. Free Radical Biol. Med. 1994, 16, 509-516.

(750) Cusato, K.; Bosco, A.; Rozental, R.; Guimaraes, C. A.; Reese, B. E.; Linden, R.; Spray, D. C. Gap Junctions Mediate Bystander Cell Death in Developing Retina. J. Neurosci. 2003, 23, 6413-6422.

(751) Kamijo, K.; Ohara, N.; Abe, M.; Uchimura, T.; Hosoya, H.; Lee, J. S.; Miki, T. Dissecting the Role of Rho-Mediated Signaling in Contractile Ring Formation. Mol. Biol. Cell 2006, 17, 43-55.

(752) Bernat, R. L.; Borisy, G. G.; Rothfield, N. F.; Earnshaw, W. C. Injection of Anticentromere Antibodies in Interphase Disrupts Events Required for Chromosome Movement at Mitosis. J. Cell Biol. 1990, 111, 1519-1533.

(753) Hollenbeck, P. J.; Swanson, J. A. Radial Extension of Macrophage Tubular Lysosomes Supported by Kinesin. Nature 1990, 346, 864-866.

(754) Araki, N.; Hatae, T.; Yamada, T.; Hirohashi, S. Actinin-4 Is Preferentially Involved in Circular Ruffling and Macropinocytosis in Mouse Macrophages: Analysis by Fluorescence Ratio Imaging. J. Cell Sci. 2000, 113, 3329-3340.

Adler, V.; Pincus, M. R.; Polotskaya, A.; Montano, X.; Friedman, F. K.; Ronai, Z. Activation of C-Jun-Nh2-Kinase by Uv Irradiation Is Dependent on P21(Ras). J. Biol. Chem. 1996, 271, 23304-23309.

Riedl, J.; Crevenna, A. H.; Kessenbrock, K.; Yu, J. H.; Neukirchen, D.; Bista, M.; Bradke, F.; Jenne, D.; Holak, T. A.; Werb, Z.et al. Lifeact: A Versatile Marker to Visualize F-Actin. Nat. Methods 2008, 5, 605-607.

Partridge, M.; Vincent, A.; Matthews, P.; Puma, J.; Stein, D.; Summerton, J. A Simple Method for Delivering Morpholino Antisense Oligos into the Cytoplasm of Cells. Antisense Nucleic Acid Drug Dev. 1996, 6, 169-175.

Altan, N.; Chen, Y.; Schindler, M.; Simon, S. M. Tamoxifen Inhibits Acidification in Cells Independent of the Estrogen Receptor. Proc. Natl. Acad. Sci. U. S. A. 1999, 96, $4432-4437$.

O'riordan, M.; Yi, C. H.; Gonzales, R.; Lee, K. D.; Portnoy, D. A. Innate Recognition of Bacteria by a Macrophage Cytosolic Surveillance Pathway. Proc. Natl. Acad. Sci. U. S. A. 2002, 99, 13861-13866.

Steinberg, T. H.; Newman, A. S.; Swanson, J. A.; Silverstein, S. C. Macrophages Possess Probenecid-Inhibitable Organic Anion Transporters That Remove Fluorescent Dyes from the Cytoplasmic Matrix. J. Cell Biol. 1987, 105, $2695-2702$.

(761) Cheng, B. X.; Zhao, S. J.; Luo, J.; Sprague, E.; Bonewald, L. F.; Jiang, J. X. Expression of Functional Gap Junctions and Regulation by Fluid Flow in Osteocyte-Like Mlo-Y4 Cells. J. Bone Miner. Res. 2001, 16, 249-259.

(762) Wu, M. M.; Grabe, M.; Adams, S.; Tsien, R. Y.; Moore, H. P. H.; Machen, T. E. Mechanisms of Ph Regulation in the Regulated Secretory Pathway. J. Biol. Chem. 2001, 276, 33027-33035.

(763) Swanson, J. A.; Mcneil, P. L. Nuclear Reassembly Excludes Large Macromolecules. Science 1987, $238,548-550$.

(764) Legenzov, E. A.; Dirda, N. D. A.; Hagen, B. M.; Kao, J. P. Y. Synthesis and Characterization of 8-O-Carboxymethylpyranine (Cm-Pyranine) as a Bright, Violet-Emitting, Fluid-Phase Fluorescent Marker in Cell Biology. PLoS One 2015, 10 , e0133518. 

Chromatin in Living Cells. Chromosome Res. 2001, 9, 77-80.

(766) Lin, Y. C.; Ho, C. H.; Grinnell, F. Fibroblasts Contracting Collagen Matrices Form Transient Plasma Membrane Passages through Which the Cells Take up Fluorescein Isothiocyanate-Dextran and Ca2+. Mol. Biol. Cell 1997, 8, 59-71.

(767) Grinnell, F. Fibroblast-Collagen-Matrix Contraction: Growth-Factor Signalling and Mechanical Loading. Trends Cell Biol. 2000, $10,362-365$.

(768) Pellegrin, P.; Fernandez, A.; Lamb, N. J. C.; Bennes, R. Macromolecular Uptake Is a Spontaneous Event During Mitosis in Cultured Fibroblasts: Implications for Vector-Dependent Plasmid Transfection. Mol. Biol. Cell 2002, 13, $570-578$.

(769) Sit, K. H.; Bay, B. H.; Wong, K. P. Distinctive Uptake of Neutral Red by Mitotic Cancer-Cells. Biotech. Histochem. 1992, 67, $196-201$

(770) Sit, K. H. Cell Rounding with "Rip Off" Detachment. Histol. Histopathol. 1996, 11, 215-227.

(771) Lemons, R.; Forster, S.; Thoene, J. Protein Microinjection by Protease Permeabilization of Fibroblasts. Anal. Biochem. 1988, $172,219-227$.

(772) Brugmans, M.; Cassiman, J. J.; Vanleuven, F.; Vandenberghe, H. Quantitative Assessment of the Amount and the Activity of Trypsin Associated with Trypsinized Cells. Cell Biol. Int. Rep. 1979, 3, 257-263.

Borowski, P.; Oehlmann, K.; Heiland, M.; Laufs, R. Nonstructural Protein 3 of Hepatitis C Virus Blocks the Distribution of the Free Catalytic Subunit of Cyclic Amp-Dependent Protein Kinase. J. Virol. 1997, 71, 2838-2843.

(774) Borowski, P.; Zur Wiesch, J. S.; Resch, K.; Feucht, H.; Laufs, R.; Schmitz, H. Protein Kinase C Recognizes the Protein Kinase a-Binding Motif of Nonstructural Protein 3 of Hepatitis C Virus. J. Biol. Chem. 1999, 274, 30722-30728.

(775) Stewart, M. P., TU Dresden, 2012.

(776) Sautter, C.; Waldner, H.; Neuhausurl, G.; Galli, A.; Neuhaus, G.; Potrykus, I. Micro-Targeting - High-Efficiency Gene-Transfer Using a Novel-Approach for the Acceleration of Micro-Projectiles. Bio-Technology 1991, 9, 1080-1085.

(777) Williams, A. R.; Bao, S.; Miller, D. L. Filtroporation: A Simple, Reliable Technique for Transfection and Macromolecular Loading of Cells in Suspension. Biotechnol. Bioeng. 1999, 65, 341-346.

(778) Sharei, A.; Cho, N.; Mao, S.; Jackson, E.; Poceviciute, R.; Adamo, A.; Zoldan, J.; Langer, R.; Jensen, K. F. Cell Squeezing as a Robust, Microfluidic Intracellular Delivery Platform. J Vis Exp 2013, 81, e50980.

(779) Sharei, A.; Poceviciute, R.; Jackson, E. L.; Cho, N.; Mao, S.; Hartoularos, G. C.; Jang, D. Y.; Jhunjhunwala, S.; Eyerman, A.; Schoettle, T.et al. Plasma Membrane Recovery Kinetics of a Microfluidic Intracellular Delivery Platform. Integr. Biol. (Camb.) 2014, 6, 470-475.

(780) Sharei, A.; Trifonova, R.; Jhunjhunwala, S.; Hartoularos, G. C.; Eyerman, A. T.; Lytton-Jean, A.; Angin, M.; Sharma, S.; Poceviciute, R.; Mao, S.et al. Ex Vivo Cytosolic Delivery of Functional Macromolecules to Immune Cells. PLoS One 2015, 10, e0118803.

(781) Szeto, G. L.; Van Egeren, D.; Worku, H.; Sharei, A.; Alejandro, B.; Park, C.; Frew, K.; Brefo, M.; Mao, S.; Heimann, M.et al. Microfluidic Squeezing for Intracellular Antigen Loading in Polyclonal B-Cells as Cellular Vaccines. Sci. Rep. 2015, 5, 10276 
Griesbeck, M.; Ziegler, S.; Laffont, S.; Smith, N.; Chauveau, L.; Tomezsko, P.; Sharei, A.; Kourjian, G.; Porichis, F.; Hart, M.et al. Sex Differences in Plasmacytoid Dendritic Cell Levels of Irf5 Drive Higher Ifn-Alpha Production in Women. J. Immunol. 2015, 195, 5327-5336.

Tu, C.; Santo, L.; Mishima, Y.; Raje, N.; Smilansky, Z.; Zoldan, J. Monitoring Protein Synthesis in Single Live Cancer Cells. Integr. Biol. (Camb.) 2016, 8, 645-653.

Li, J.; Wang, B.; Juba, B. M.; Vazquez, M.; Kortum, S. W.; Pierce, B. S.; Pacheco, M.; Roberts, L.; Strohbach, J. W.; Jones, L. H.et al. Microfluidic-Enabled Intracellular Delivery of Membrane Impermeable Inhibitors to Study Target Engagement in Human Primary Cells. ACS Chem. Biol. 2017, 12, 2970-2974.

Kollmannsperger, A.; Sharei, A.; Raulf, A.; Heilemann, M.; Langer, R.; Jensen, K. F.; Wieneke, R.; Tampe, R. Live-Cell Protein Labelling with Nanometre Precision by Cell Squeezing. Nat Commun 2016, 7, 10372.

(786) Saung, M. T.; Sharei, A.; Adalsteinsson, V. A.; Cho, N.; Kamath, T.; Ruiz, C.; Kirkpatrick, J.; Patel, N.; Mino-Kenudson, M.; Thayer, S. P.et al. A Size-Selective Intracellular Delivery Platform. Small 2016, 12, 5873-5881.

Ding, X.; Stewart, M. P.; Sharei, A.; Weaver, J. C.; Langer, R. S.; Jensen, K. F. High-Throughput Nuclear Delivery and Rapid Expression of DNA Via Mechanical and Electrical Cell-Membrane Disruption. Nature Biomedical Engineering 2017, 1, 0039. Liu, Z.; Han, X.; Zhou, Q.; Chen, R.; Fruge, S.; Jo, M. C.; Ma, Y.; Li, Z.; Yokoi, K.; Qin, L. Integrated Microfluidic System for Gene Silencing and Cell Migration. Advanced Biosystems 2017, 1, 1700054.

Raab, M.; Gentili, M.; De Belly, H.; Thiam, H. R.; Vargas, P.; Jimenez, A. J.; Lautenschlaeger, F.; Voituriez, R.; LennonDumenil, A. M.; Manel, N.et al. Escrt lii Repairs Nuclear Envelope Ruptures During Cell Migration to Limit DNA Damage and Cell Death. Science 2016, 352, 359-362.

(790) Denais, C. M.; Gilbert, R. M.; Isermann, P.; Mcgregor, A. L.; Te Lindert, M.; Weigelin, B.; Davidson, P. M.; Friedl, P.; Wolf, K.; Lammerding, J. Nuclear Envelope Rupture and Repair During Cancer Cell Migration. Science 2016, 352, 353-358.

Olmos, Y.; Hodgson, L.; Mantell, J.; Verkade, P.; Carlton, J. G. Escrt-lii Controls Nuclear Envelope Reformation. Nature 2015, 522, 236-239.

(792) Han, X.; Liu, Z. B.; Jo, M. C.; Zhang, K.; Li, Y.; Zeng, Z. H.; Li, N.; Zu, Y. L.; Qin, L. D. Crispr-Cas9 Delivery to Hard-toTransfect Cells Via Membrane Deformation. Science Advances 2015, 1, e1500454.

(793) Ma, Y.; Han, X.; Bustamante, O. Q.; De Castro, R. B.; Zhang, K.; Zhang, P. C.; Li, Y.; Liu, Z. B.; Liu, X. W.; Ferrari, M.et al. Highly Efficient Genome Editing of Human Hematopoietic Stem Cells Via a Nano-Silicon-Blade Delivery Approach. Integrative Biology 2017, 9, 548-554.

(794) Deng, Y.; Kizer, M.; Rada, M.; Sage, J.; Wang, X.; Cheon, D.-J.; Chung, A. J. Intracellular Delivery of Nanomaterials Via an Inertial Microfluidic Cell Hydroporator. Nano Lett. 2018, 18, 2705-2710. Versaevel, M.; Riaz, M.; Grevesse, T.; Gabriele, S. Cell Confinement: Putting the Squeeze on the Nucleus. Soft Matter 2013, 9, 6665-6676. 
Rowat, A. C.; Jaalouk, D. E.; Zwerger, M.; Ung, W. L.; Eydelnant, I. A.; Olins, D. E.; Olins, A. L.; Herrmann, H.; Weitz, D. A.; Lammerding, J. Nuclear Envelope Composition Determines the Ability of Neutrophil-Type Cells to Passage through MicronScale Constrictions. J. Biol. Chem. 2013, 288, 8610-8618.

(797) Harada, T.; Swift, J.; Irianto, J.; Shin, J. W.; Spinler, K. R.; Athirasala, A.; Diegmiller, R.; Dingal, P. C. D. P.; Ivanovska, I. L.; Discher, D. E. Nuclear Lamin Stiffness Is a Barrier to 3d Migration, but Softness Can Limit Survival. J. Cell Biol. 2014, 204, 669-682.

(798) Mayr, M.; Hu, Y. H.; Hainaut, P.; Xu, Q. B. Mechanical Stress-Induced DNA Damage and Rac-P38mapk Signal Pathways Mediate P53-Dependent Apoptosis in Vascular Smooth Muscle Cells. FASEB J. 2002, 16, 1423-1425.

(799) Wood, D. K.; Weingeist, D. M.; Bhatia, S. N.; Engelward, B. P. Single Cell Trapping and DNA Damage Analysis Using Microwell Arrays. Proc. Natl. Acad. Sci. U. S. A. 2010, 107, 10008-10013.

(800) Matsumoto, D.; Yamagishi, A.; Saito, M.; Sathuluri, R. R.; Silberberg, Y. R.; Iwata, F.; Kobayashi, T.; Nakamura, C. Mechanoporation of Living Cells for Delivery of Macromolecules Using Nanoneedle Array. J. Biosci. Bioeng. 2016, 122, 748752.

(801) Hanasaki, I.; Walther, J. H.; Kawano, S.; Koumoutsakos, P. Coarse-Grained Molecular Dynamics Simulations of ShearInduced Instabilities of Lipid Bilayer Membranes in Water. Physical Review E 2010, 82, 051602.

Yuan, F.; Yang, C.; Zhong, P. Cell Membrane Deformation and Bioeffects Produced by Tandem Bubble-Induced Jetting Flow. Proc. Natl. Acad. Sci. U. S. A. 2015, 112, E7039-7047.

(803) Waldman, A. S.; Waldman, B. C. Stable Transfection of Mammalian Cells by Syringe-Mediated Mechanical Loading of DNA. Anal. Biochem. 1998, 258, 216-222.

Ghosh, C.; Iversen, P. L. Intracellular Delivery Strategies for Antisense Phosphorodiamidate Morpholino Oligomers. Antisense Nucleic Acid Drug Dev. 2000, 10, 263-274.

Laudanna, C.; Campbell, J. J.; Butcher, E. C. Role of Rho in Chemoattractant-Activated Leukocyte Adhesion through Integrins. Science 1996, 271, 981-983.

(806) Meyer, C. J.; Alenghat, F. J.; Rim, P.; Fong, J. H. J.; Fabry, B.; Ingber, D. E. Mechanical Control of Cyclic Amp Signalling and Gene Transcription through Integrins. Nat. Cell Biol. 2000, 2, 666-668.

(807) Hollenbeck, P. J. Products of Endocytosis and Autophagy Are Retrieved from Axons by Regulated Retrograde Organelle Transport. J. Cell Biol. 1993, 121, 305-315.

(808) Tachibana, K.; Sato, T.; Davirro, N.; Morimoto, C. Direct Association of Pp125(Fak) with Paxillin, the Focal Adhesion-Targeting Mechanism of Pp125(Fak). J. Exp. Med. 1995, 182, 1089-1099.

(809) Vannhieu, G. T.; Krukonis, E. S.; Reszka, A. A.; Horwitz, A. F.; Isberg, R. R. Mutations in the Cytoplasmic Domain of the Integrin Beta(1) Chain Indicate a Role for Endocytosis Factors in Bacterial Internalization. J. Biol. Chem. 1996, 271, 76657672.

(810) Sydor, A. M.; Su, A. L.; Wang, F. S.; Xu, A.; Jay, D. G. Talin and Vinculin Play Distinct Roles in Filopodial Motility in the Neuronal Growth Cone. J. Cell Biol. 1996, 134, 1197-1207. 
De Vos, K.; Goossens, V.; Boone, E.; Vercammen, D.; Vancompernolle, K.; Vandenabeele, P.; Haegeman, G.; Fiers, W.; Grooten, J. The 55-Kda Tumor Necrosis Factor Receptor Induces Clustering of Mitochondria through Its Membrane-Proximal Region. J. Biol. Chem. 1998, 273, 9673-9680.

Kaiser, D. A.; Vinson, V. K.; Murphy, D. B.; Pollard, T. D. Profilin Is Predominantly Associated with Monomeric Actin in Acanthamoeba. J. Cell Sci. 1999, 112, 3779-3790.

(813) Adams, J. C.; Schwartz, M. A. Stimulation of Fascin Spikes by Thrombospondin-1 Is Mediated by the Gtpases Rac and Cdc42. J. Cell Biol. 2000, 150, 807-822.

(814) Tzima, E.; Del Pozo, M. A.; Kiosses, W. B.; Mohamed, S. A.; Li, S.; Chien, S.; Schwartz, M. A. Activation of Rac1 by Shear Stress in Endothelial Cells Mediates Both Cytoskeletal Reorganization and Effects on Gene Expression. EMBO J. 2002, 21, 6791-6800.

(815) Katsumi, A.; Milanini, J.; Kiosses, W. B.; Del Pozo, M. A.; Kaunas, R.; Chien, S.; Hahn, K. M.; Schwartz, M. A. Effects of Cell Tension on the Small Gtpase Rac. J. Cell Biol. 2002, 158, 153-164.

(816) Shoeman, R. L.; Huttermann, C.; Hartig, R.; Traub, P. Amino-Terminal Polypeptides of Vimentin Are Responsible for the Changes in Nuclear Architecture Associated with Human Immunodeficiency Virus Type 1 Protease Activity in Tissue Culture Cells. Mol. Biol. Cell 2001, 12, 143-154.

(817) Phillips, R. M.; Six, D. A.; Dennis, E. A.; Ghosh, P. In Vivo Phospholipase Activity of the Pseudomonas Aeruginosa Cytotoxin Exou and Protection of Mammalian Cells with Phospholipase a(2) Inhibitors. J. Biol. Chem. 2003, 278, 41326-41332.

(818) Xu, L.; Shen, X. H.; Bryan, A.; Banga, S.; Swanson, M. S.; Luo, Z. Q. Inhibition of Host Vacuolar H+-Atpase Activity by a Legionella Pneumophila Effector. PLoS Path. 2010, 6, e1000822.

(819) Copeland, A. M.; Newcomb, W. W.; Brown, J. C. Herpes Simplex Virus Replication: Roles of Viral Proteins and Nucleoporins in Capsid-Nucleus Attachment. J. Virol. 2009, 83, 1660-1668.

(820) Mcneil, P. L. Direct Introduction of Molecules into Cells. Curr. Protoc. Cell Biol. 2001, 18, 20.21.21-20.21.27.

(821) Hallow, D. M.; Seeger, R. A.; Kamaev, P. P.; Prado, G. R.; Laplaca, M. C.; Prausnitz, M. R. Shear-Induced Intracellular Loading of Cells with Molecules by Controlled Microfluidics. Biotechnol. Bioeng. 2008, 99, 846-854.

Laplaca, M. C.; Lee, V. M. Y.; Thibault, L. E. An in Vitro Model of Traumatic Neuronal Injury: Loading Rate-Dependent Changes in Acute Cytosolic Calcium and Lactate Dehydrogenase Release. J. Neurotrauma 1997, 14, 355-368.

(823) Blackman, B. R.; Barbee, K. A.; Thibault, L. E. In Vitro Cell Shearing Device to Investigate the Dynamic Response of Cells in a Controlled Hydrodynamic Environment. Ann. Biomed. Eng. 2000, 28, 363-372.

(824) Kilinc, D.; Gallo, G.; Barbee, K. A. Mechanically-Induced Membrane Poration Causes Axonal Beading and Localized Cytoskeletal Damage. Exp. Neurol. 2008, 212, 422-430.

(825) Chouinard-Pelletier, G.; Leduc, M.; Guay, D.; Coulombe, S.; Leask, R. L.; Jones, E. a. V. Use of Inert Gas Jets to Measure the Forces Required for Mechanical Gene Transfection. Biomedical Engineering Online 2012, 11, 67.

(826) Cooper, S.; Jonak, P.; Chouinard-Pelletier, G.; Coulombe, S.; Jones, E.; Leask, R. L. Permeabilization of Adhered Cells Using an Inert Gas Jet. J. Vis. Exp. 2013, 79, e50612. 
Fechheimer, M.; Denny, C.; Murphy, R. F.; Taylor, D. L. Measurement of Cytoplasmic Ph in Dictyostelium Discoideum by Using a New Method for Introducing Macromolecules into Living Cells. Eur. J. Cell Biol. 1986, 40, 242-247.

Fechheimer, M.; Taylor, D. L. Introduction of Exogenous Molecules into the Cytoplasm of Dictyostelium-Discoideum Amebas by Controlled Sonication. Methods Cell Biol. 1987, 28, 179-190.

(829) Furukawa, R.; Wampler, J. E.; Fechheimer, M. Measurement of the Cytoplasmic Ph of Dictyostelium-Discoideum Using a Low Light Level Microspectrofluorometer. J. Cell Biol. 1988, 107, 2541-2549.

(830) Wyber, J. A.; Andrews, J.; Demanuele, A. The Use of Sonication for the Efficient Delivery of Plasmid DNA into Cells. Pharm. Res. 1997, 14, 750-756.

(831) Bao, S. P.; Thrall, B. D.; Miller, D. L. Transfection of a Reporter Plasmid into Cultured Cells by Sonoporation in Vitro. Ultrasound Med. Biol. 1997, 23, 953-959.

(832) Kim, H. J.; Greenleaf, J. F.; Kinnick, R. R.; Bronk, J. T.; Bolander, M. E. Ultrasound-Mediated Transfection of Mammalian Cells. Hum. Gene Ther. 1996, 7, 1339-1346.

(833) Miller, D. L.; Pislaru, S. V.; Greenleaf, J. E. Sonoporation: Mechanical DNA Delivery by Ultrasonic Cavitation. Somat. Cell Mol. Genet. 2002, 27, 115-134.

(834) Kennedy, J. E.; Ter Haar, G. R.; Cranston, D. High Intensity Focused Ultrasound: Surgery of the Future? Br. J. Radiol. 2003, 76, 590-599.

(835) Hill, C. R.; Terhaar, G. R. Review Article: High Intensity Focused Ultrasound-Potential for Cancer Treatment. Br. J. Radiol. $1995,68,1296-1303$.

(836) Mitragotri, S. Innovation - Healing Sound: The Use of Ultrasound in Drug Delivery and Other Therapeutic Applications. Nature Reviews Drug Discovery 2005, 4, 255-260.

(837) Miller, M. W.; Miller, D. L.; Brayman, A. A. A Review of in Vitro Bioeffects of Inertial Ultrasonic Cavitation from a Mechanistic Perspective. Ultrasound Med. Biol. 1996, 22, 1131-1154.

(838) Greenleaf, W. J.; Bolander, M. E.; Sarkar, G.; Goldring, M. B.; Greenleaf, J. F. Artificial Cavitation Nuclei Significantly Enhance Acoustically Induced Cell Transfection. Ultrasound Med. Biol. 1998, 24, 587-595.

(839) Oberli, M. A.; Schoellhammer, C. M.; Langer, R.; Blankschtein, D. Ultrasound-Enhanced Transdermal Delivery: Recent Advances and Future Challenges. Ther. Deliv. 2014, 5, 843-857.

(840) Lentacker, I.; De Cock, I.; Deckers, R.; De Smedt, S. C.; Moonen, C. T. Understanding Ultrasound Induced Sonoporation: Definitions and Underlying Mechanisms. Adv. Drug. Deliv. Rev. 2014, 72, 49-64.

(841) Kooiman, K.; Vos, H. J.; Versluis, M.; De Jong, N. Acoustic Behavior of Microbubbles and Implications for Drug Delivery. Adv Drug Deliv Rev 2014, 72C, 28-48.

(842) Fan, Z.; Kumon, R. E.; Deng, C. X. Mechanisms of Microbubble-Facilitated Sonoporation for Drug and Gene Delivery. Ther. Deliv. 2014, 5, 467-486.

(843) Sutton, J. T.; Haworth, K. J.; Pyne-Geithman, G.; Holland, C. K. Ultrasound-Mediated Drug Delivery for Cardiovascular Disease. Expert Opinion on Drug Delivery 2013, 10, 573-592. 
Liu, Y.; Yan, J.; Prausnitz, M. R. Can Ultrasound Enable Efficient Intracellular Uptake of Molecules? A Retrospective Literature Review and Analysis. Ultrasound Med. Biol. 2012, 38, 876-888.

(845) Van Wamel, A.; Kooiman, K.; Harteveld, M.; Emmer, M.; Ten Cate, F. J.; Versluis, M.; De Jong, N. Vibrating Microbubbles Poking Individual Cells: Drug Transfer into Cells Via Sonoporation. J. Controlled Release 2006, 112, 149-155.

(846) Delalande, A.; Kotopoulis, S.; Postema, M.; Midoux, P.; Pichon, C. Sonoporation: Mechanistic Insights and Ongoing Challenges for Gene Transfer. Gene 2013, 525, 191-199.

(847) Guo, X. S.; Cai, C. L.; Xu, G. Y.; Yang, Y. Y.; Tu, J.; Huang, P. T.; Zhang, D. Interaction between Cavitation Microbubble and Cell: A Simulation of Sonoporation Using Boundary Element Method (Bem). Ultrason. Sonochem. 2017, 39, 863-871.

(848) Marmottant, P.; Hilgenfeldt, S. Controlled Vesicle Deformation and Lysis by Single Oscillating Bubbles. Nature 2003, 423, 153156.

(849) Forbes, M. M.; Steinberg, R. L.; O'brien, W. D. Examination of Inertial Cavitation of Optison in Producing Sonoporation of Chinese Hamster Ovary Cells. Ultrasound Med. Biol. 2008, 34, 2009-2018.

(850) Schlicher, R. K.; Radhakrishna, H.; Tolentino, T. P.; Apkarian, R. P.; Zarnitsyn, V.; Prausnitz, M. R. Mechanism of Intracellular Delivery by Acoustic Cavitation. Ultrasound Med. Biol. 2006, 32, 915-924.

(851) Zarnitsyn, V.; Rostad, C. A.; Prausnitz, M. R. Modeling Transmembrane Transport through Cell Membrane Wounds Created by Acoustic Cavitation. Biophys. J. 2008, 95, 4124-4138.

(852) Kudo, N.; Okada, K.; Yamamoto, K. Sonoporation by Single-Shot Pulsed Ultrasound with Microbubbles Adjacent to Cells. Biophys. J. 2009, 96, 4866-4876.

(853) Huber, P. E.; Pfisterer, P. In Vitro and in Vivo Transfection of Plasmid DNA in the Dunning Prostate Tumor R3327-At1 Is Enhanced by Focused Ultrasound. Gene Ther. 2000, 7, 1516-1525.

(854) Frenkel, P. A.; Chen, S. Y.; Thai, T.; Shohet, R. V.; Grayburn, P. A. DNA-Loaded Albumin Microbubbles Enhance UltrasoundMediated Transfection in Vitro. Ultrasound Med. Biol. 2002, 28, 817-822.

(855) Zarnitsyn, V. G.; Prausnitz, M. R. Physical Parameters Influencing Optimization of Ultrasound-Mediated DNA Transfection. Ultrasound Med. Biol. 2004, 30, 527-538.

(856) Meijering, B. D. M.; Henning, R. H.; Van Gilst, W. H.; Gavrilovic, I.; Van Wamel, A.; Deelman, L. E. Optimization of Ultrasound and Microbubbles Targeted Gene Delivery to Cultured Primary Endothelial Cells. J. Drug Targeting 2007, 15, 664-671.

(857) Zarnitsyn, V. G.; Meacham, J. M.; Varady, M. J.; Hao, C. H.; Degertekin, F. L.; Fedorov, A. G. Electrosonic Ejector Microarray for Drug and Gene Delivery. Biomed. Microdevices 2008, 10, 299-308.

(858) Fan, Z.; Chen, D.; Deng, C. X. Improving Ultrasound Gene Transfection Efficiency by Controlling Ultrasound Excitation of Microbubbles. J. Control. Release 2013, 170, 401-413.

(859) Liu, Y.; Yan, J.; Santangelo, P. J.; Prausnitz, M. R. DNA Uptake, Intracellular Trafficking and Gene Transfection after Ultrasound Exposure. J. Control. Release 2016, 234, 1-9.

(860) Miura, S.; Tachibana, K.; Okamoto, T.; Saku, K. In Vitro Transfer of Antisense Oligodeoxynucleotides into Coronary Endothelial Cells by Ultrasound. Biochem. Biophys. Res. Commun. 2002, 298, 587-590. 

Ultrasound. Biochem. Biophys. Res. Commun. 2005, 335, 393-399.

Vandenbroucke, R. E.; Lentacker, I.; Demeester, J.; De Smedt, S. C.; Sanders, N. N. Ultrasound Assisted Sirna Delivery Using Peg-Siplex Loaded Microbubbles. J. Controlled Release 2008, 126, 265-273.

(863) De Temmerman, M. L.; Dewitte, H.; Vandenbroucke, R. E.; Lucas, B.; Libert, C.; Demeester, J.; De Smedt, S. C.; Lentacker, I.; Rejman, J. Mrna-Lipoplex Loaded Microbubble Contrast Agents for Ultrasound-Assisted Transfection of Dendritic Cells. Biomaterials 2011, 32, 9128-9135.

(864) Saito, K.; Miyake, K.; Mcneil, P. L.; Kato, K.; Yago, K.; Sugai, N. Plasma Membrane Disruption Underlies Injury of the Corneal Endothelium by Ultrasound. Exp. Eye Res. 1999, 68, 431-437.

(865) Guzman, H. R.; Nguyen, D. X.; Mcnamara, A. J.; Prausnitz, M. R. Equilibrium Loading of Cells with Macromolecules by Ultrasound: Effects of Molecular Size and Acoustic Energy. J. Pharm. Sci. 2002, 91, 1693-1701.

(866) Armstrong, J. K.; Wenby, R. B.; Meiselman, H. J.; Fisher, T. C. The Hydrodynamic Radii of Macromolecules and Their Effect on Red Blood Cell Aggregation. Biophys. J. 2004, 87, 4259-4270.

(867) Mehier-Humbert, S.; Bettinger, T.; Yan, F.; Guy, R. H. Plasma Membrane Poration Induced by Ultrasound Exposure: Implication for Drug Delivery. J. Controlled Release 2005, 104, 213-222.

(868) Meijering, B. D. M.; Juffermans, L. J. M.; Van Wamel, A.; Henning, R. H.; Zuhorn, I. S.; Emmer, M.; Versteilen, A. M. G.; Paulus, W. J.; Van Gilst, W. H.; Kooiman, K.et al. Ultrasound and Microbubble-Targeted Delivery of Macromolecules Is Regulated by Induction of Endocytosis and Pore Formation. Circul. Res. 2009, 104, 679-687.

(869) Karshafian, R.; Bevan, P. D.; Williams, R.; Samac, S.; Burns, P. N. Sonoporation by Ultrasound-Activated Microbubble Contrast Agents: Effect of Acoustic Exposure Parameters on Cell Membrane Permeability and Cell Viability. Ultrasound Med. Biol. 2009, 35, 847-860.

(870) Karshafian, R.; Samac, S.; Bevan, P. D.; Burns, P. N. Microbubble Mediated Sonoporation of Cells in Suspension: Clonogenic Viability and Influence of Molecular Size on Uptake. Ultrasonics 2010, 50, 691-697.

(871) Carugo, D.; Ankrett, D. N.; Glynne-Jones, P.; Capretto, L.; Boltryk, R. J.; Zhang, X. L.; Townsend, P. A.; Hill, M. Contrast Agent-Free Sonoporation: The Use of an Ultrasonic Standing Wave Microfluidic System for the Delivery of Pharmaceutical Agents. Biomicrofluidics 2011, 5, 44108-4410815.

(872) Yoon, S.; Kim, M. G.; Chiu, C. T.; Hwang, J. Y.; Kim, H. H.; Wang, Y.; Shung, K. K. Direct and Sustained Intracellular Delivery of Exogenous Molecules Using Acoustic-Transfection with High Frequency Ultrasound. Sci. Rep. 2016, 6, 20477.

(873) Furukawa, R.; Wampler, J. E.; Fechheimer, M. Cytoplasmic Ph of Dictyostelium-Discoideum Amebae During Early Development - Identification of 2 Cell Subpopulations before the Aggregation Stage. J. Cell Biol. 1990, 110, 1947-1954. Keyhani, K.; Guzman, H. R.; Parsons, A.; Lewis, T. N.; Prausnitz, M. R. Intracellular Drug Delivery Using Low-Frequency Ultrasound: Quantification of Molecular Uptake and Cell Viability. Pharm. Res. 2001, 18, 1514-1520.

(875) Guzman, H. R.; Nguyen, D. X.; Khan, S.; Prausnitz, M. R. Ultrasound-Mediated Disruption of Cell Membranes. li. Heterogeneous Effects on Cells. J. Acoust. Soc. Am. 2001, 110, 597-606. 
Hallow, D. M.; Mahajan, A. D.; Mccutchen, T. E.; Prausnitz, M. R. Measurement and Correlation of Acoustic Cavitation with Cellular Bioeffects. Ultrasound Med. Biol. 2006, 32, 1111-1122.

Hutcheson, J. D.; Schlicher, R. K.; Hicks, H. K.; Prausnitz, M. R. Saving Cells from Ultrasound-Induced Apoptosis:

Quantification of Cell Death and Uptake Following Sonication and Effects of Targeted Calcium Chelation. Ultrasound Med.

Biol. 2010, 36, 1008-1021.

(878) Schlicher, R. K.; Hutcheson, J. D.; Radhakrishna, H.; Apkarian, R. P.; Prausnitz, M. R. Changes in Cell Morphology Due to Plasma Membrane Wounding by Acoustic Cavitation. Ultrasound Med. Biol. 2010, 36, 677-692.

(879) Fan, Z. Z.; Liu, H. Y.; Mayer, M.; Deng, C. X. Spatiotemporally Controlled Single Cell Sonoporation. Proc. Natl. Acad. Sci. U. S. A. 2012, 109, 16486-16491.

(880) Dixon, A. J.; Dhanaliwala, A. H.; Chen, J. L.; Hossack, J. A. Enhanced Intracellular Delivery of a Model Drug Using Microbubbles Produced by a Microfluidic Device. Ultrasound Med. Biol. 2013, 39, 1267-1276.

Helfield, B.; Chen, X. C.; Watkins, S. C.; Villanueva, F. S. Biophysical Insight into Mechanisms of Sonoporation. Proc. Natl. Acad. Sci. U. S. A. 2016, 113, 9983-9988.

Tachibana, K.; Uchida, T.; Tamura, K.; Eguchi, H.; Yamashita, N.; Ogawa, K. Enhanced Cytotoxic Effect of Ara-C by Low Intensity Ultrasound to HI-60 Cells. Cancer Lett. 2000, 149, 189-194.

(883) Tachibana, K.; Uchida, T.; Ogawa, K.; Yamashita, N.; Tamura, K. Induction of Cell-Membrane Porosity by Ultrasound. Lancet 1999, 353, p1409.

(884) Tachibana, K.; Uchida, T.; Hisano, S.; Morioka, E. Eliminating Adult T-Cell Leukaemia Cells with Ultrasound. Lancet 1997, 349, p325.

(885) Paliwal, S.; Sundaram, J.; Mitragotri, S. Induction of Cancer-Specific Cytotoxicity Towards Human Prostate and Skin Cells Using Quercetin and Ultrasound. Br. J. Cancer 2005, 92, 499-502.

(886) Escoffre, J. M.; Piron, J.; Novell, A.; Bouakaz, A. Doxorubicin Delivery into Tumor Cells with Ultrasound and Microbubbles. Mol. Pharm. 2011, 8, 799-806.

(887) Joersbo, M.; Brunstedt, J. Inoculation of Sugar-Beet Protoplasts with Beet Necrotic Yellow Vein Virus-Particles by Mild Sonication. J. Virol. Methods 1990, 29, 63-69.

(888) Furukawa, R.; Butz, S.; Fleischmann, E.; Fechheimer, M. The Dictyostelium-Discoideum 30,000 Dalton Protein Contributes to Phagocytosis. Protoplasma 1992, 169, 18-27.

(889) Van Wamel, A.; Bouakaz, A.; Bernard, B.; Ten Cate, F.; De Jong, N. Radionuclide Tumour Therapy with Ultrasound Contrast Microbubbles. Ultrasonics 2004, 42, 903-906.

(890) Meacham, J. M.; Durvasula, K.; Degertekin, F. L.; Fedorov, A. G. Enhanced Intracellular Delivery Via Coordinated Acoustically Driven Shear Mechanoporation and Electrophoretic Insertion. Sci. Rep. 2018, 8, 3727.

(891) Li, Y. S.; Davidson, E.; Reid, C. N.; Mchale, A. P. Optimising Ultrasound-Mediated Gene Transfer (Sonoporation) in Vitro and Prolonged Expression of a Transgene in Vivo: Potential Applications for Gene Therapy of Cancer. Cancer Lett. 2009, 273, 6269. 
Tlaxca, J. L.; Anderson, C. R.; Klibanov, A. L.; Lowrey, B.; Hossack, J. A.; Alexander, J. S.; Lawrence, M. B.; Rychak, J. J. Analysis of in Vitro Transfection by Sonoporation Using Cationic and Neutral Microbubbles. Ultrasound Med. Biol. 2010, 36, 1907-1918.

(893) Juffermans, L. J. M.; Dijkmans, P. A.; Musters, R. J. P.; Visser, C. A.; Kamp, O. Transient Permeabilization of Cell Membranes by Ultrasound-Exposed Microbubbles Is Related to Formation of Hydrogen Peroxide. American Journal of Physiology-Heart and Circulatory Physiology 2006, 291, H1595-H1601.

(894) Hutchins, D. A. Ultrasonic Generation by Pulsed Lasers. Physical Acoustics 1988, 18, 21-123.

(895) Lokhandwalla, M.; Sturtevant, B. Mechanical Haemolysis in Shock Wave Lithotripsy (Swl): I. Analysis of Cell Deformation Due to Swl Flow-Fields. Phys. Med. Biol. 2001, 46, 413-437.

(896) Lokhandwalla, M.; Mcateer, J. A.; Williams, J. C.; Sturtevant, B. Mechanical Haemolysis in Shock Wave Lithotripsy (Swl): li. In Vitro Cell Lysis Due to Shear. Phys. Med. Biol. 2001, 46, 1245-1264.

(897) Gambihler, S.; Delius, M.; Ellwart, J. W. Permeabilization of the Plasma-Membrane of L1210 Mouse Leukemia-Cells Using Lithotripter Shock-Waves. J. Membr. Biol. 1994, 141, 267-275.

(898) Gambihler, S.; Delius, M.; Brendel, W. Biological Effects of Shock-Waves - Cell Disruption, Viability, and Proliferation of L1210-Cells Exposed to Shock-Waves Invitro. Ultrasound Med. Biol. 1990, 16, 587-594.

Kodama, T.; Doukas, A. G.; Hamblin, M. R. Shock Wave-Mediated Molecular Delivery into Cells. Biochimica Et Biophysica Acta-Molecular Cell Research 2002, 1542, 186-194.

(900) Kodama, T.; Hamblin, M. R.; Doukas, A. G. Cytoplasmic Molecular Delivery with Shock Waves: Importance of Impulse. Biophys. J. 2000, 79, 1821-1832.

(901) Kodama, T.; Doukas, A. G.; Hamblin, M. R. Delivery of Ribosome-Inactivating Protein Toxin into Cancer Cells with Shock Waves. Cancer Lett. 2003, 189, 69-75.

(902) Brummer, F.; Brenner, J.; Brauner, T.; Hulser, D. F. Effect of Shock Waves on Suspended and Immobilized L1210 Cells. Ultrasound Med. Biol. 1989, 15, 229-239.

(903) Sonden, A.; Svensson, B.; Roman, N.; Brismar, B.; Palmblad, J.; Kjellstrom, B. T. Mechanisms of Shock Wave Induced Endothelial Cell Injury. Lasers Surg. Med. 2002, 31, 233-241.

(904) Soughayer, J. S.; Krasieva, T.; Jacobson, S. C.; Ramsey, J. M.; Tromberg, B. J.; Allbritton, N. L. Characterization of Cellular Optoporation with Distance. Anal. Chem. 2000, 72, 1342-1347.

(905) Mulholland, S. E.; Lee, S.; Mcauliffe, D. J.; Doukas, A. G. Cell Loading with Laser-Generated Stress Waves: The Role of the Stress Gradient. Pharm. Res. 1999, 16, 514-518.

(906) Lee, S.; Anderson, T.; Zhang, H.; Flotte, T. J.; Doukas, A. G. Alteration of Cell Membrane by Stress Waves in Vitro. Ultrasound Med. Biol. 1996, 22, 1285-1293.

(907) Doukas, A. G.; Flotte, T. J. Physical Characteristics and Biological Effects of Laser-Induced Stress Waves. Ultrasound Med. Biol. 1996, 22, 151-164. 
Doukas, A. G.; Mcauliffe, D. J.; Lee, S.; Venugopalan, V.; Flotte, T. J. Physical Factors Involved in Stress-Wave-Induced Cell Injury - the Effect of Stress Gradient. Ultrasound Med. Biol. 1995, 21, 961-967.

(909) Doukas, A. G.; Mcauliffe, D. J.; Flotte, T. J. Biological Effects of Laser-Induced Shock-Waves - Structural and Functional CellDamage in Vitro. Ultrasound Med. Biol. 1993, 19, 137-146.

(910) Ohl, C. D.; Arora, M.; Ikink, R.; De Jong, N.; Versluis, M.; Delius, M.; Lohse, D. Sonoporation from Jetting Cavitation Bubbles. Biophys. J. 2006, 91, 4285-4295.

(911) Boulais, E.; Lachaine, R.; Hatef, A.; Meunier, M. Plasmonics for Pulsed-Laser Cell Nanosurgery: Fundamentals and Applications. Journal of Photochemistry and Photobiology C-Photochemistry Reviews 2013, 17, 26-49.

Xiong, R. H.; Samal, S. K.; Demeester, J.; Skirtach, A. G.; De Smedt, S. C.; Braeckmans, K. Laser-Assisted Photoporation: Fundamentals, Technological Advances and Applications. Advances in Physics-X 2016, 1, 596-620.

(913) Fan, Q.; Hu, W.; Ohta, A. T. Efficient Single-Cell Poration by Microsecond Laser Pulses. Lab on a Chip 2015, 15, 581-588.

(914) Le Gac, S.; Zwaan, E.; Van Den Berg, A.; Ohl, C. D. Sonoporation of Suspension Cells with a Single Cavitation Bubble in a Microfluidic Confinement. Lab on a Chip 2007, 7, 1666-1672.

(915) Quinto-Su, P. A.; Suzuki, M.; Ohl, C. D. Fast Temperature Measurement Following Single Laser-Induced Cavitation inside a Microfluidic Gap. Sci. Rep. 2014, 4, 5445.

(916) Pitsillides, C. M.; Joe, E. K.; Wei, X. B.; Anderson, R. R.; Lin, C. P. Selective Cell Targeting with Light-Absorbing Microparticles and Nanoparticles. Biophys. J. 2003, 84, 4023-4032.

(917) Lukianova-Hleb, E. Y.; Samaniego, A. P.; Wen, J. G.; Metelitsa, L. S.; Chang, C. C.; Lapotko, D. O. Selective Gene Transfection of Individual Cells in Vitro with Plasmonic Nanobubbles. J. Controlled Release 2011, 152, $286-293$.

(918) Lukianova-Hleb, E. Y.; Wagner, D. S.; Brenner, M. K.; Lapotko, D. O. Cell-Specific Transmembrane Injection of Molecular Cargo with Gold Nanoparticle-Generated Transient Plasmonic Nanobubbles. Biomaterials 2012, 33, 5441-5450.

Chakravarty, P.; Qian, W.; El-Sayed, M. A.; Prausnitz, M. R. Delivery of Molecules into Cells Using Carbon Nanoparticles Activated by Femtosecond Laser Pulses. Nat Nanotechnol 2010, 5, 607-611.

(920) Sengupta, A.; Gray, M. D.; Kelly, S. C.; Holguin, S. Y.; Thadhani, N. N.; Prausnitz, M. R. Energy Transfer Mechanisms During Molecular Delivery to Cells by Laser-Activated Carbon Nanoparticles. Biophys. J. 2017, 112, 1258-1269.

(921) Sengupta, A.; Mezencev, R.; Mcdonald, J. F.; Prausnitz, M. R. Delivery of Sirna to Ovarian Cancer Cells Using LaserActivated Carbon Nanoparticles. Nanomedicine 2015, 10, 1775-1784.

(922) Sengupta, A.; Kelly, S. C.; Dwivedi, N.; Thadhani, N.; Prausnitz, M. R. Efficient Intracellular Delivery of Molecules with High Cell Viability Using Nanosecond-Pulsed Laser-Activated Carbon Nanoparticles. ACS Nano 2014, 8, 2889-2899.

(923) Xiong, R. H.; Raemdonck, K.; Peynshaert, K.; Lentacker, I.; De Cock, I.; Demeester, J.; De Smedt, S. C.; Skirtach, A. G.; Braeckmans, K. Comparison of Gold Nanoparticle Mediated Photoporation: Vapor Nanobubbles Outperform Direct Heating for Delivering Macromolecules in Live Cells. ACS Nano 2014, 8, 6288-6296.

(924) Wayteck, L.; Xiong, R.; Braeckmans, K.; De Smedt, S. C.; Raemdonck, K. Comparing Photoporation and Nucleofection for Delivery of Small Interfering Rna to Cytotoxic T Cells. J. Control. Release 2017, 267, 154-162. 
Boulais, E.; Lachaine, R.; Meunier, M. Plasma Mediated Off-Resonance Plasmonic Enhanced Ultrafast Laser-Induced Nanocavitation. Nano Lett. 2012, 12, 4763-4769.

(926) St-Louis Lalonde, B.; Boulais, E.; Lebrun, J. J.; Meunier, M. Visible and near Infrared Resonance Plasmonic Enhanced Nanosecond Laser Optoporation of Cancer Cells. Biomed Opt Express 2013, 4, 490-499.

(927) Fan, Q. H.; Hu, W. Q.; Ohta, A. T. Laser-Induced Microbubble Poration of Localized Single Cells. Lab on a Chip 2014, 14, 1572-1578.

(928) Courvoisier, S.; Saklayen, N.; Huber, M.; Chen, J.; Diebold, E. D.; Bonacina, L.; Wolf, J. P.; Mazur, E. Plasmonic Tipless Pyramid Arrays for Cell Poration. Nano Lett. 2015, 15, 4461-4466.

(929) Saklayen, N.; Huber, M.; Madrid, M.; Nuzzo, V.; Vulis, D. I.; Shen, W.; Nelson, J.; Mcclelland, A. A.; Heisterkamp, A.; Mazur, E. Intracellular Delivery Using Nanosecond-Laser Excitation of Large-Area Plasmonic Substrates. ACS Nano 2017, 11, 36713680.

(930) Chen, J.; Saklayen, N.; Courvoisier, S.; Shen, Z. H.; Lu, J.; Ni, X. W.; Mazur, E. Dynamics of Transient Microbubbles Generated by Fs-Laser Irradiation of Plasmonic Micropyramids. Appl. Phys. Lett. 2017, 110, 153102.

(931) Yamane, D.; Wu, Y. C.; Wu, T. H.; Toshiyoshi, H.; Teitell, M. A.; Chiou, P. Y. Electrical Impedance Monitoring of Photothermal Porated Mammalian Cells. Jala 2014, 19, 50-59.

Chakrabarti, R.; Pfeiffer, N. E.; Wylie, D. E.; Schuster, S. M. Incorporation of Monoclonal-Antibodies into Cells by Osmotic Permeabilization - Effect on Cellular-Metabolism. J. Biol. Chem. 1989, 264, 8214-8221.

Hoffman, J. F. Active Transport of Sodium by Ghosts of Human Red Blood Cells. J. Gen. Physiol. 1962, 45, 837-859.

Dodge, J. T.; Hanahan, D. J.; Mitchell, C. Preparation and Chemical Characteristics of Hemoglobin-Free Ghosts of Human Erythrocytes. Arch. Biochem. Biophys. 1963, 100, 119-130.

Baker, R. F. Entry of Ferritin into Human Red Cells During Hypotonic Haemolysis. Nature 1967, 215, 424-425.

Bodemann, H.; Passow, H. Factors Controlling the Resealing of the Membrane of Human Erythrocyte Ghosts after Hypotonic Hemolysis. J. Membr. Biol. 1972, 8, 1-26.

Schwoch, G.; Passow, H. Preparation and Properties of Human Erythrocyte-Ghosts. Mol. Cell. Biochem. 1973, 2, $197-218$.

Rechsteiner, M. C. In Techniques in Somatic Cell Genetics; Shay, J. W., Ed.; Springer US: Boston, MA, 1982.

Seeman, P. Transient Holes in the Erythrocyte Membrane During Hypotonic Hemolysis and Stable Holes in the Membrane after Lysis by Saponin and Lysolecithin. J. Cell Biol. 1967, 32, 55-70.

Seeman, P.; Cheng, D.; Iles, G. H. Structure of Membrane Holes in Osmotic and Saponin Hemolysis. J. Cell Biol. 1973, 56, 519-527.

Lieber, M. R.; Steck, T. L. A Description of the Holes in Human-Erythrocyte Membrane Ghosts. J. Biol. Chem. 1982, 257, 1651-1659.

Ihler, G. M.; Glew, R. H.; Schnure, F. W. Enzyme Loading of Erythrocytes. Proc. Natl. Acad. Sci. U. S. A. 1973, 70, 26632666. 
(943) Dale, G. L.; Villacorte, D. G.; Beutler, E. High-Yield Entrapment of Proteins into Erythrocytes. Biochem. Med. 1977, 18, 220225.

(944) Ihler, G. M. Erythrocyte Carriers. Pharmacol. Ther. 1983, 20, 151-169.

(945) Hamidi, M.; Tajerzadeh, H. Carrier Erythrocytes: An Overview. Drug Deliv. 2003, 10, 9-20.

(946) Millan, C. G.; Marinero, M. L. S.; Castaneda, A. Z.; Lanao, J. M. Drug, Enzyme and Peptide Delivery Using Erythrocytes as Carriers. J. Controlled Release 2004, 95, 27-49.

(947) Shi, J. H.; Kundrat, L.; Pishesha, N.; Bilate, A.; Theile, C.; Maruyama, T.; Dougan, S. K.; Ploegh, H. L.; Lodish, H. F. Engineered Red Blood Cells as Carriers for Systemic Delivery of a Wide Array of Functional Probes. Proc. Natl. Acad. Sci. U. S. A. 2014, 111, 10131-10136.

(948) Furusawa, M.; Yamaizumi, M.; Nishimura, T.; Uchida, T.; Okada, Y. Chapter 5 Use of Erythrocyte Ghosts for Injection of Substances into Animal Cells by Cell Fusion. Methods Cell Biol. 1976, 14, 73-80.

(949) Kaltoft, K.; Celis, J. E. Ghost-Mediated Transfer of Human Hypoxanthine-Guanine Phosphoribosyl Transferase into Deficient Chinese Hamster Ovary Cells by Means of Polyethylene Glycol-Induced Fusion. Exp. Cell Res. 1978, 115, 423-428.

(950) Klabusay, M.; Skopalik, J.; Erceg, S.; Hrdlicka, A. Aequorin as Intracellular Ca2+ Indicator Incorporated in Follicular Lymphoma Cells by Hypoosmotic Shock Treatment. Folia Biol. (Praha) 2015, 61, 134-139.

(951) Koberna, K.; Stanek, D.; Malinsky, J.; Eltsov, M.; Pliss, A.; Ctrnacta, V.; Cermanova, S.; Raska, I. Nuclear Organization Studied with the Help of a Hypotonic Shift: Its Use Permits Hydrophilic Molecules to Enter into Living Cells. Chromosoma $1999,108,325-335$.

Malinsky, J.; Koberna, K.; Stanek, D.; Masata, M.; Votruba, I.; Raska, I. The Supply of Exogenous Deoxyribonucleotides Accelerates the Speed of the Replication Fork in Early S-Phase. J. Cell Sci. 2001, 114, 747-750.

(953) Ahmad, K.; Henikoff, S. Centromeres Are Specialized Replication Domains in Heterochromatin. J. Cell Biol. 2001, 153, 101109.

Koberna, K.; Ligasova, A.; Malinsky, J.; Pliss, A.; Siegel, A. J.; Cvackova, Z.; Fidlerova, H.; Masata, M.; Fialova, M.; Raska, I.et al. Electron Microscopy of DNA Replication in 3-D: Evidence for Similar-Sized Replication Foci Throughout S-Phase. J. Cell. Biochem. 2005, 94, 126-138.

(955) Panning, M. M.; Gilbert, D. M. Spatio-Temporal Organization of DNA Replication in Murine Embryonic Stem, Primary, and Immortalized Cells. J. Cell. Biochem. 2005, 95, 74-82.

(956) Takebayashi, S.; Tamura, T.; Matsuoka, C.; Okano, M. Major and Essential Role for the DNA Methylation Mark in Mouse Embryogenesis and Stable Association of Dnmt1 with Newly Replicated Regions. Mol. Cell. Biol. 2007, 27, 8243-8258.

(957) Sharif, J.; Muto, M.; Takebayashi, S. I.; Suetake, I.; Iwamatsu, A.; Endo, T. A.; Shinga, J.; Mizutani-Koseki, Y.; Toyoda, T.; Okamura, K.et al. The Sra Protein Np95 Mediates Epigenetic Inheritance by Recruiting Dnmt1 to Methylated DNA. Nature 2007, 450, 908-912. 
Hori, M.; Satou, K.; Harashima, H.; Kamiya, H. Suppression of Mutagenesis by 8-Hydroxy-2 '-Deoxyguanosine 5 'Triphosphate (7,8-Dihydro-8-Oxo-2 '-Deoxyguanosine 5 '-Triphosphate) by Human Mth1, Mth2, and Nudt5. Free Radical Biol. Med. 2010, 48, 1197-1201.

(959) Yamazaki, S.; Ishii, A.; Kanoh, Y.; Oda, M.; Nishito, Y.; Masai, H. Rif1 Regulates the Replication Timing Domains on the Human Genome. EMBO J. 2012, 31, 3667-3677.

Alabert, C.; Bukowski-Wills, J. C.; Lee, S. B.; Kustatscher, G.; Nakamura, K.; Alves, F. D.; Menard, P.; Mejlvang, J.; Rappsilber, J.; Groth, A. Nascent Chromatin Capture Proteomics Determines Chromatin Dynamics During DNA Replication and Identifies Unknown Fork Components. Nat. Cell Biol. 2014, 16, 281-293.

Pliss, A.; Koberna, K.; Vecerova, J.; Malinsky, J.; Masata, M.; Fialova, M.; Raska, I.; Berezney, R. Spatio-Temporal Dynamics at Rdna Foci: Global Switching between DNA Replication and Transcription. J. Cell. Biochem. 2005, 94, 554-565.

Bhattacharya, D.; Mazumder, A.; Miriam, S. A.; Shivashankar, G. V. Egfp-Tagged Core and Linker Histones Diffuse Via Distinct Mechanisms within Living Cells. Biophys. J. 2006, 91, 2326-2336.

Mazumder, A.; Shivashankar, G. V. Gold-Nanoparticle-Assisted Laser Perturbation of Chromatin Assembly Reveals Unusual Aspects of Nuclear Architecture within Living Cells. Biophys. J. 2007, 93, 2209-2216.

Mills, C. L.; Pereira, M. M. C.; Dormer, R. L.; Mcpherson, M. A. An Antibody against a Cftr-Derived Synthetic Peptide, Incorporated into Living Submandibular Cells, Inhibits Beta-Adrenergic Stimulation of Mucin Secretion. Biochem. Biophys.

Res. Commun. 1992, 188, 1146-1152.

(965) Mills, C. L.; Dormer, R. L.; Mcpherson, M. A. Introduction of Bapta into Intact Rat Submandibular Acini Inhibits Mucin Secretion in Response to Cholinergic and Beta-Adrenergic Agonists. FEBS Lett. 1991, 289, 141-144.

(966) Bradbury, N. A.; Dormer, R. L.; Mcpherson, M. A. Introduction of Cyclic-Amp Phosphodiesterase into Rat Submandibular Acini Prevents Isoproterenol-Stimulated Cyclic-Amp Rise without Affecting Mucin Secretion. Biochem. Biophys. Res. Commun. 1989, $161,661-671$.

(967) Di Gregorio, E.; Ferrauto, G.; Gianolio, E.; Aime, S. Gd Loading by Hypotonic Swelling: An Efficient and Safe Route for Cellular Labeling. Contrast Media Mol. Imaging 2013, 8, 475-486.

(968) Yang, Y.; Yang, F.; Gong, Y. J.; Chen, J. L.; Goldfarb, D.; Su, X. C. A Reactive, Rigid Gdiii Labeling Tag for in-Cell Epr Distance Measurements in Proteins. Angew. Chem. Int. Ed. Engl. 2017, 56, 2914-2918.

(969) Markov, D. E.; Boeve, H.; Gleich, B.; Borgert, J.; Antonelli, A.; Sfara, C.; Magnani, M. Human Erythrocytes as Nanoparticle Carriers for Magnetic Particle Imaging. Phys. Med. Biol. 2010, 55, 6461-6473.

(970) Martorana, A.; Bellapadrona, G.; Feintuch, A.; Di Gregorio, E.; Aime, S.; Goldfarb, D. Probing Protein Conformation in Cells by Epr Distance Measurements Using Gd3+ Spin Labeling. JACS 2014, 136, 13458-13465.

(971) Ferrauto, G.; Castelli, D. D.; Di Gregorio, E.; Langereis, S.; Burdinski, D.; Grull, H.; Terreno, E.; Aime, S. Lanthanide-Loaded Erythrocytes as Highly Sensitive Chemical Exchange Saturation Transfer Mri Contrast Agents. JACS 2014, 136, 638-641.

(972) Ferrauto, G.; Di Gregorio, E.; Dastru, W.; Lanzardo, S.; Aime, S. Gd-Loaded-Rbcs for the Assessment of Tumor Vascular Volume by Contrast-Enhanced-Mri. Biomaterials 2015, 58, 82-92. 
Di Gregorio, E.; Ferrauto, G.; Gianolio, E.; Lanzardo, S.; Carrera, C.; Fedeli, F.; Aime, S. An Mri Method to Map Tumor Hypoxia Using Red Blood Cells Loaded with a Po(2)-Responsive Gd-Agent. Acs Nano 2015, 9, 8239-8248.

Widdicombe, J. H.; Azizi, F.; Kang, T.; Pittet, J. F. Transient Permeabilization of Airway Epithelium by Mucosal Water. J. Appl. Physiol. 1996, 81, 491-499.

(975) Sawa, T.; Miyazaki, H.; Pittet, J. F.; Widdicombe, J. H.; Gropper, M. A.; Hashimoto, S.; Conrad, D. J.; Folkesson, H. G.; Debs, R.; Forsayeth, J. R.et al. Intraluminal Water Increases Expression of Plasmid DNA in Rat Lung. Hum. Gene Ther. 1996, 7, 933-941.

(976) Lemoine, J. L.; Farley, R.; Huang, L. Mechanism of Efficient Transfection of the Nasal Airway Epithelium by Hypotonic Shock. Gene Ther. 2005, 12, 1275-1282.

(977) Rudolph, C.; Schillinger, U.; Ortiz, A.; Plank, C.; Golas, M. M.; Sander, B.; Stark, H.; Rosenecker, J. Aerosolized Nanogram Quantities of Plasmid DNA Mediate Highly Efficient Gene Delivery to Mouse Airway Epithelium. Mol. Ther. 2005, 12, 493-501.

Zhang, G. F.; Budker, V.; Wolff, J. A. High Levels of Foreign Gene Expression in Hepatocytes after Tail Vein Injections of Naked Plasmid DNA. Hum. Gene Ther. 1999, 10, 1735-1737.

Liu, F.; Song, Y. K.; Liu, D. Hydrodynamics-Based Transfection in Animals by Systemic Administration of Plasmid DNA. Gene Ther. 1999, 6, 1258-1266.

Zhang, G.; Gao, X.; Song, Y. K.; Vollmer, R.; Stolz, D. B.; Gasiorowski, J. Z.; Dean, D. A.; Liu, D. Hydroporation as the Mechanism of Hydrodynamic Delivery. Gene Ther. 2004, 11, 675-682.

(981) Chang, J. H.; Sigal, L. J.; Lerro, A.; Taylor, J. Replication of the Human Hepatitis Delta Virus Genome Is Initiated in Mouse Hepatocytes Following Intravenous Injection of Naked DNA or Rna Sequences. J. Virol. 2001, 75, 3469-3473.

Mccaffrey, A. P.; Meuse, L.; Pham, T. T. T.; Conklin, D. S.; Hannon, G. J.; Kay, M. A. Gene Expression - Rna Interference in Adult Mice. Nature 2002, 418, 38-39.

Magin-Lachmann, C.; Kotzamanis, G.; D'aiuto, L.; Cooke, H.; Huxley, C.; Wagner, E. In Vitro and in Vivo Delivery of Intact Bac DNA - Comparison of Different Methods. J. Gene Med. 2004, 6, 195-209.

Kobayashi, N.; Nishikawa, M.; Hirata, K.; Takakura, Y. Hyrodynamics-Based Procedure Involves Transient Hyperpermeability in the Hepatic Cellular Membrane: Implication of a Nonspecific Process in Efficient Intracellular Gene Delivery. J. Gene Med. 2004, 6, 584-592.

(985) Al-Dosari, M. S.; Knapp, J. E.; Liu, D. X. Hydrodynamic Delivery. Non-Viral Vectors for Gene Therapy, Second Edition: Part 2 2005, 54, 65-82.

(986) Herweijer, H.; Wolff, J. A. Gene Therapy Progress and Prospects: Hydrodynamic Gene Delivery. Gene Ther. 2007, 14, 99107.

Bonamassa, B.; Hai, L.; Liu, D. X. Hydrodynamic Gene Delivery and Its Applications in Pharmaceutical Research. Pharm. Res. 2011, 28, 694-701.

Yin, H.; Xue, W.; Chen, S.; Bogorad, R. L.; Benedetti, E.; Grompe, M.; Koteliansky, V.; Sharp, P. A.; Jacks, T.; Anderson, D. G. Genome Editing with Cas9 in Adult Mice Corrects a Disease Mutation and Phenotype. Nat. Biotechnol. 2014, 32, 551-553. 
Xue, W.; Chen, S. D.; Yin, H.; Tammela, T.; Papagiannakopoulos, T.; Joshi, N. S.; Cai, W. X.; Yang, G. L.; Bronson, R.; Crowley, D. G.et al. Crispr-Mediated Direct Mutation of Cancer Genes in the Mouse Liver. Nature 2014, 514, $380-384$.

Zhen, S.; Hua, L.; Liu, Y. H.; Gao, L. C.; Fu, J.; Wan, D. Y.; Dong, L. H.; Song, H. F.; Gao, X. Harnessing the Clustered Regularly Interspaced Short Palindromic Repeat (Crispr)/Crispr-Associated Cas9 System to Disrupt the Hepatitis B Virus. Gene Ther. 2015, 22, 404-412.

Sakurai, T.; Kamiyoshi, A.; Kawate, H.; Mori, C.; Watanabe, S.; Tanaka, M.; Uetake, R.; Sato, M.; Shindo, T. A Non-Inheritable Maternal Cas9-Based Multiple-Gene Editing System in Mice. Sci. Rep. 2016, 6, 20011.

Wolff, J. A.; Budker, V. The Mechanism of Naked DNA Uptake and Expression. Adv. Genet. 2005, 54, 1-20.

Budker, V.; Budker, T.; Zhang, G. F.; Subbotin, V.; Loomis, A.; Wolff, J. A. Hypothesis: Naked Plasmid DNA Is Taken up by Cells in Vivo by a Receptor-Mediated Process. J. Gene Med. 2000, 2, 76-88.

(994) Gao, X.; Kim, K. S.; Liu, D. X. Nonviral Gene Delivery: What We Know and What Is Next. Aaps Journal 2007, 9, E92-E104.

(995) Suda, T.; Liu, D. Hydrodynamic Gene Delivery: Its Principles and Applications. Mol. Ther. 2007, 15, 2063-2069.

(996) Mann, M. J.; Gibbons, G. H.; Hutchinson, H.; Poston, R. S.; Hoyt, E. G.; Robbins, R. C.; Dzau, V. J. Pressure-Mediated Oligonucleotide Transfection of Rat and Human Cardiovascular Tissues. Proc. Natl. Acad. Sci. U. S. A. 1999, 96, 6411-6416. Andersen, N. D.; Chopra, A.; Monahan, T. S.; Malek, J. Y.; Jain, M.; Pradhan, L.; Ferran, C.; Logerfo, F. W. Endothelial Cells Are Susceptible to Rapid Sirna Transfection and Gene Silencing Ex Vivo. J. Vasc. Surg. 2010, 52, 1608-1615.

Von Der Leyen, H. E.; Braun-Dullaeus, R.; Mann, M. J.; Zhang, L. N.; Niebauer, J.; Dzau, V. J. A Pressure-Mediated Nonviral Method for Efficient Arterial Gene and Oligonucleotide Transfer. Hum. Gene Ther. 1999, 10, 2355-2364.

Mann, M. J.; Whittemore, A. D.; Donaldson, M. C.; Belkin, M.; Conte, M. S.; Polak, J. F.; Orav, E. J.; Ehsan, A.; Dell'acqua, G.; Dzau, V. J. Ex-Vivo Gene Therapy of Human Vascular Bypass Grafts with E2f Decoy: The Prevent Single-Centre, Randomised, Controlled Trial. Lancet 1999, 354, 1493-1498.

(1000) Miniati, D. N.; Hoyt, E. G.; Feeley, B. T.; Poston, R. S.; Robbins, R. C. Ex Vivo Antisense Oligonucleotides to Proliferating Cell Nuclear Antigen and Cdc2 Kinase Inhibit Graft Coronary Artery Disease. Circulation 2000, 102, $237-242$.

(1001) Feeley, B. T.; Miniati, D. N.; Park, A. K.; Hoyt, E. G.; Robbins, R. C. Nuclear Factor-Kappab Transcription Factor Decoy Treatment Inhibits Graft Coronary Artery Disease after Cardiac Transplantation in Rodents. Transplantation 2000, 70, 15601568.

(1002) Miyake, T.; Aoki, M.; Shiraya, S.; Tanemoto, K.; Ogihara, T.; Kaneda, Y.; Morishita, R. Inhibitory Effects of Nfkappab Decoy Oligodeoxynucleotides on Neointimal Hyperplasia in a Rabbit Vein Graft Model. J. Mol. Cell. Cardiol. 2006, 41, 431-440.

(1003) Suzuki, M.; Ishizaka, N.; Tsukamoto, K.; Minami, K.; Taguchi, J.; Nagai, R.; Ohno, M. Pressurization Facilitates AdenovirusMediated Gene Transfer into Vein Graft. FEBS Lett. 2000, 470, 370-374.

(1004) Vecchione, C.; Aretini, A.; Marino, G.; Bettarini, U.; Poulet, R.; Maffei, A.; Sbroggio, M.; Pastore, L.; Gentile, M. T.; Notte, A.et al. Selective Rac-1 Inhibition Protects from Diabetes-Induced Vascular Injury. Circul. Res. 2006, 98, $218-225$.

(1005) Ander, S.; Maclennan, M.; Bentil, S.; Leavitt, B.; Chesler, N. Pressure-Induced Vector Transport in Human Saphenous Vein. Ann. Biomed. Eng. 2005, 33, 202-208. 
(1006) Park, R. D.; Sullivan, P. C.; Storrie, B. Hypertonic Sucrose Inhibition of Endocytic Transport Suggests Multiple Early Endocytic Compartments. J. Cell. Physiol. 1988, 135, 443-450.

(1007) Durante, M.; Grossi, G. F.; Napolitano, M.; Gialanella, G. Repair of Potentially Lethal Damage by Introduction of T4 DNA Ligase in Eucaryotic Cells. Int. J. Radiat Biol. 1991, 59, 963-971.

(1008) Chin, D. T.; Kuehl, L.; Rechsteiner, M. Conjugation of Ubiquitin to Denatured Hemoglobin Is Proportional to the Rate of Hemoglobin Degradation in Hela Cells. Proceedings of the National Academy of Sciences of the United States of AmericaBiological Sciences 1982, 79, 5857-5861.

(1009) Hough, R.; Rechsteiner, M. Effects of Temperature on the Degradation of Proteins in Rabbit Reticulocyte Lysates and after Injection into Hela Cells. Proceedings of the National Academy of Sciences of the United States of America-Biological Sciences 1984, 81, 90-94.

(1010) Schmid, D. S.; Tite, J. P.; Ruddle, N. H. DNA Fragmentation - Manifestation of Target-Cell Destruction Mediated by Cytotoxic T-Cell Lines, Lymphotoxin-Secreting Helper T-Cell Clones, and Cell-Free Lymphotoxin-Containing Supernatant. Proc. Natl. Acad. Sci. U. S. A. 1986, 83, 1881-1885.

(1011) Rubin, E. J.; Gill, D. M.; Boquet, P.; Popoff, M. R. Functional Modification of a 21-Kilodalton G-Protein When Adp-Ribosylated by Exoenzyme-C3 of Clostridium-Botulinum. Mol. Cell. Biol. 1988, 8, 418-426.

(1012) Winegar, R. A.; Preston, R. J. The Induction of Chromosome-Aberrations by Restriction Endonucleases That Produce BluntEnd or Cohesive-End Double-Strand Breaks. Mutat. Res. 1988, 197, 141-149.

(1013) Cornwell, T. L.; Lincoln, T. M. Regulation of Intracellular Ca-2+ Levels in Cultured Vascular Smooth-Muscle Cells - Reduction of Ca-2+ by Atriopeptin and 8-Bromo-Cyclic Gmp Is Mediated by Cyclic Gmp-Dependent Protein-Kinase. J. Biol. Chem. 1989, 264, 1146-1155.

(1014) Rock, K. L.; Gamble, S.; Rothstein, L. Presentation of Exogenous Antigen with Class-I Major Histocompatibility ComplexMolecules. Science 1990, 249, 918-921.

(1015) Rock, K. L.; Rothstein, L. E.; Gamble, S. R.; Benacerraf, B. Reassociation with Beta-2-Microglobulin Is Necessary for Kb Class-I Major Histocompatibility Complex Binding of Exogenous Peptides. Proc. Natl. Acad. Sci. U. S. A. 1990, 87, 7517-7521.

(1016) Bowen, J. C.; Nair, S. K.; Reddy, R.; Rouse, B. T. Cholera-Toxin Acts as a Potent Adjuvant for the Induction of Cytotoxic TLymphocyte Responses with Nonreplicating Antigens. Immunology 1994, 81, 338-342.

(1017) Williams, M. S.; Henkart, P. A. Apoptotic Cell-Death Induced by Intracellular Proteolysis. J. Immunol. $1994,153,4247-4255$.

(1018) Zeng, Q.; Lagunoff, D.; Masaracchia, R.; Goeckeler, Z.; Cote, G.; Wysolmerski, R. Endothelial Cell Retraction Is Induced by Pak2 Monophosphorylation of Myosin li. J. Cell Sci. 2000, 113, 471-482.

(1019) Geluk, A.; Van Meijgaarden, K. E.; Franken, K. L. M. C.; Drijfhout, J. W.; D'souza, S.; Necker, A.; Huygen, K.; Ottenhoff, T. H. M. Identification of Major Epitopes of Mycobacterium Tuberculosis Ag85b That Are Recognized by Hla-a*0201-Restricted Cd8(+) T Cells in Hla-Transgenic Mice and Humans. J. Immunol. 2000, 165, 6463-6471.

(1020) Calautti, E.; Grossi, M.; Mammucari, C.; Aoyama, Y.; Pirro, M.; Ono, Y.; Li, J.; Dotto, G. P. Fyn Tyrosine Kinase Is a Downstream Mediator of Rho/Prk2 Function in Keratinocyte Cell-Cell Adhesion. J. Cell Biol. 2002, 156, $137-148$. 
(1021) Gonciarz-Swiatek, M.; Rechsteiner, M. Proteasomes and Antigen Presentation: Evidence That a Keke Motif Does Not Promote Presentation of the Class I Epitope Siinfekl. Mol. Immunol. 2006, 43, 1993-2001.

(1022) Shii, K.; Roth, R. A. Inhibition of Insulin Degradation by Hepatoma-Cells after Microinjection of Monoclonal-Antibodies to a Specific Cytosolic Protease. Proc. Natl. Acad. Sci. U. S. A. 1986, 83, 4147-4151.

(1023) Morgan, D. O.; Roth, R. A. Acute Insulin Action Requires Insulin-Receptor Kinase-Activity - Introduction of an Inhibitory Monoclonal-Antibody into Mammalian-Cells Blocks the Rapid Effects of Insulin. Proc. Natl. Acad. Sci. U. S. A. 1987, 84, 41-45.

(1024) Ahmad, F.; Li, P. M.; Meyerovitch, J.; Goldstein, B. J. Osmotic Loading of Neutralizing Antibodies Demonstrates a Role for Protein-Tyrosine-Phosphatase 1b in Negative Regulation of the Insulin Action Pathway. J. Biol. Chem. 1995, 270, 2050320508.

(1025) Agazie, Y. M.; Burkholder, G. D.; Lee, J. S. Triplex DNA in the Nucleus: Direct Binding of Triplex-Specific Antibodies and Their Effect on Transcription, Replication and Cell Growth. Biochem. J 1996, 316, 461-466.

(1026) Mather, S.; Dora, K. A.; Sandow, S. L.; Winter, P.; Garland, C. J. Rapid Endothelial Cell-Selective Loading of Connexin 40 Antibody Blocks Endothelium-Derived Hyperpolarizing Factor Dilation in Rat Small Mesenteric Arteries. Circul. Res. 2005, 97, $399-407$.

(1027) Lee, G.; Delohery, T. M.; Ronai, Z.; Brandtrauf, P. W.; Pincus, M. R.; Murphy, R. B.; Weinstein, I. B. A Comparison of Techniques for Introducing Macromolecules into Living Cells. Cytometry 1993, 14, 265-270.

(1028) Hughey, J. J.; Wikswo, J. P.; Seale, K. T. Intra-Microfluidic Pinocytic Loading of Human T Cells. 2007 leee/Nih Life Science Systems and Applications Workshop 2007, 132-135.

(1029) Kawashima, I.; Tsai, V.; Southwood, S.; Takesako, K.; Sette, A.; Celis, E. Identification of Hla-A3-Restricted Cytotoxic T Lymphocyte Epitopes from Carcinoembryonic Antigen and Her-2/Neu by Primary in Vitro Immunization with Peptide-Pulsed Dendritic Cells. Cancer Res. 1999, 59, 431-435.

(1030) Matsura, T.; Kai, M.; Fujii, Y.; Ito, H.; Yamada, K. Hydrogen Peroxide-Induced Apoptosis in HI-60 Cells Requires Caspase-3 Activation. Free Radical Res. 1999, 30, 73-83.

(1031) Li, H.; Sims, C. E.; Kaluzova, M.; Stanbridge, E. J.; Allbritton, N. L. A Quantitative Single-Cell Assay for Protein Kinase B Reveals Important Insights into the Biochemical Behavior of an Intracellular Substrate Peptide. Biochemistry 2004, 43, 15991608.

(1032) Tjoa, B.; Boynton, A.; Kenny, G.; Ragde, H.; Misrock, S. L.; Murphy, G. Presentation of Prostate Tumor Antigens by Dendritic Cells Stimulates T-Cell Proliferation and Cytotoxicity. Prostate 1996, 28, 65-69.

(1033) Prehm, P. Hyaluronate Is Synthesized at Plasma-Membranes. Biochem. J 1984, 220, 597-600.

(1034) Schulz, T.; Schumacher, U.; Prehm, P. Hyaluronan Export by the Abc Transporter Mrp5 and Its Modulation by Intracellular Cgmp. J. Biol. Chem. 2007, 282, 20999-21004.

(1035) Puhlev, I.; Guo, N.; Brown, D. R.; Levine, F. Desiccation Tolerance in Human Cells. Cryobiology 2001, 42, $207-217$.

(1036) Jones, R. A.; Smail, A.; Wilson, M. R. Detecting Mitochondrial Permeability Transition by Confocal Imaging of Intact Cells Pinocytically Loaded with Calcein. Eur. J. Biochem. 2002, 269, 3990-3997. 
(1037) Brecht, M.; Mayer, U.; Schlosser, E.; Prehm, P. Increased Hyaluronate Synthesis Is Required for Fibroblast Detachment and Mitosis. Biochem. J 1986, 239, 445-450.

(1038) Summerton, J. Morpholino Antisense Oligomers: The Case for an Rnase H-Independent Structural Type. Biochimica Et Biophysica Acta-Gene Structure and Expression 1999, 1489, 141-158.

(1039) Tewari, M. K.; Sinnathamby, G.; Rajagopal, D.; Eisenlohr, L. C. A Cytosolic Pathway for Mhc Class li-Restricted Antigen Processing That Is Proteasome and Tap Dependent. Nat. Immunol. 2005, 6, 287-294.

(1040) Nelson, S. R.; Ali, M. Y.; Trybus, K. M.; Warshaw, D. M. Random Walk of Processive, Quantum Dot-Labeled Myosin Va Molecules within the Actin Cortex of Cos-7 Cells. Biophys. J. 2009, 97, 509-518.

(1041) Pierobon, P.; Achouri, S.; Courty, S.; Dunn, A. R.; Spudich, J. A.; Dahan, M.; Cappello, G. Velocity, Processivity, and Individual Steps of Single Myosin V Molecules in Live Cells. Biophys. J. 2009, 96, 4268-4275.

(1042) Gruber, J.; Boese, G.; Tuschl, T.; Osborn, M.; Weber, K. Rna Interference by Osmotic Lysis of Pinosomes: LiposomeIndependent Transfection of Sirnas into Mammalian Cells. BioTechniques 2004, 37, 96-102.

(1043) Aoki, M.; Ishii, T.; Kanaoka, M.; Kimura, T. Rna Interference in Immune Cells by Use of Osmotic Delivery of Sirna. Biochem. Biophys. Res. Commun. 2006, 341, 326-333.

(1044) Rechsteiner, M. Osmotic Lysis of Pinosomes. Methods Enzymol. 1987, 149, 42-48.

(1045) Neumann, E.; Rosenhec.K. Permeability Changes Induced by Electric Impulses in Vesicular Membranes. J. Membr. Biol. 1972, 10, 279-290.

(1046) Abidor, I. G.; Arakelyan, V. B.; Chernomordik, L. V.; Chizmadzhev, Y. A.; Pastushenko, V. F.; Tarasevich, M. R. Electric Breakdown of Bilayer Lipid-Membranes .1. Main Experimental Facts and Their Qualitative Discussion. Bioelectrochem. Bioenerg. 1979, 6, 37-52.

(1047) Kanehisa, M. I.; Tsong, T. Y. Cluster Model of Lipid Phase-Transitions with Application to Passive Permeation of Molecules and Structure Relaxations in Lipid Bilayers. JACS 1978, 100, 424-432.

(1048) Gehl, J. Electroporation: Theory and Methods, Perspectives for Drug Delivery, Gene Therapy and Research. Acta Physiol. Scand. 2003, 177, 437-447.

(1049) Kanduser, M.; Miklavcic, D. In Electrotechnologies for Extraction from Food Plants and Biomaterials; Vorobiev, E.;Lebovka, N., Eds.; Springer: New York, 2008.

(1050) Yarmush, M. L.; Golberg, A.; Sersa, G.; Kotnik, T.; Miklavcic, D. Electroporation-Based Technologies for Medicine: Principles, Applications, and Challenges. Annu. Rev. Biomed. Eng. 2014, 16, 295-320.

(1051) Weaver, J. C. Electroporation Theory. Concepts and Mechanisms. Methods Mol. Biol. 1995, 55, 3-28.

(1052) Smith, K. C.; Son, R. S.; Gowrishankar, T. R.; Weaver, J. C. Emergence of a Large Pore Subpopulation During Electroporating Pulses. Bioelectrochemistry 2014, 100, 3-10.

(1053) Bockmann, R. A.; De Groot, B. L.; Kakorin, S.; Neumann, E.; Grubmuller, H. Kinetics, Statistics, and Energetics of Lipid Membrane Electroporation Studied by Molecular Dynamics Simulations. Biophys. J. 2008, 95, 1837-1850. 
(1054) Neumann, E.; Kakorin, S.; Toensing, K. Principles of Membrane Electroporation and Transport of Macromolecules. Methods Mol. Med. 2000, 37, 1-35.

(1055) Neumann, E.; Kakorin, S.; Toensing, K. Fundamentals of Electroporative Delivery of Drugs and Genes. Bioelectrochem. Bioenerg. 1999, 48, 3-16.

(1056) Neumann, E.; Toensing, K.; Kakorin, S.; Budde, P.; Frey, J. Mechanism of Electroporative Dye Uptake by Mouse B Cells. Biophys. J. 1998, 74, 98-108.

(1057) Neumann, E. Membrane Electroporation and Direct Gene Transfer. Bioelectrochem. Bioenerg. $1992,28,247-267$.

(1058) Sugar, I. P.; Neumann, E. Stochastic Model for Electric Field-Induced Membrane Pores Electroporation. Biophys. Chem. 1984, $19,211-225$.

(1059) Sukhorukov, V. L.; Mussauer, H.; Zimmermann, U. The Effect of Electrical Deformation Forces on the Electropermeabilization of Erythrocyte Membranes in Low- and High-Conductivity Media. J. Membr. Biol. 1998, 163, 235-245.

(1060) Akinlaja, J.; Sachs, F. The Breakdown of Cell Membranes by Electrical and Mechanical Stress. Biophys. J. 1998, 74, 247-254.

(1061) Barrau, C.; Teissie, J.; Gabriel, B. Osmotically Induced Membrane Tension Facilitates the Triggering of Living Cell Electropermeabilization. Bioelectrochemistry 2004, 63, 327-332.

(1062) Coster, H. G.; Zmmermann, U. The Mechanism of Electrical Breakdown in the Membranes of Valonai Utricularis. J. Membr. Biol. 1975, 22, 73-90.

(1063) Son, R. S.; Smith, K. C.; Gowrishankar, T. R.; Vernier, P. T.; Weaver, J. C. Basic Features of a Cell Electroporation Model: Illustrative Behavior for Two Very Different Pulses. J. Membr. Biol. 2014, 247, 1209-1228.

(1064) Tieleman, D. P.; Leontiadou, H.; Mark, A. E.; Marrink, S. J. Simulation of Pore Formation in Lipid Bilayers by Mechanical Stress and Electric Fields. JACS 2003, 125, 6382-6383.

(1065) Gurtovenko, A. A.; Lyulina, A. S. Electroporation of Asymmetric Phospholipid Membranes. J. Phys. Chem. B 2014, 118, 99099918.

(1066) Gabriel, B.; Teissie, J. Time Courses of Mammalian Cell Electropermeabilization Observed by Millisecond Imaging of Membrane Property Changes During the Pulse. Biophys. J. 1999, 76, 2158-2165.

(1067) Rols, M. P. Electropermeabilization, a Physical Method for the Delivery of Therapeutic Molecules into Cells. Biochimica Et Biophysica Acta-Biomembranes 2006, 1758, 423-428.

(1068) Sengel, J. T.; Wallace, M. I. Imaging the Dynamics of Individual Electropores. Proc. Natl. Acad. Sci. U. S. A. 2016, 113, 52815286.

(1069) Teissie, J.; Golzio, M.; Rols, M. P. Mechanisms of Cell Membrane Electropermeabilization: A Minireview of Our Present (Lack Of ?) Knowledge. BBA - Gen. Subjects 2005, 1724, 270-280.

(1070) Tsong, T. Y. Electroporation of Cell-Membranes. Biophys. J. 1991, 60, 297-306.

(1071) Teissie, J.; Eynard, N.; Gabriel, B.; Rols, M. P. Electropermeabilization of Cell Membranes. Adv. Drug Del. Rev. 1999, 35, 319. 
(1072) Gissel, H.; Lee, R. C.; Gehl, J. In Clinical Aspects of Electroporation; Kee, S.;Gehl, J.;Lee, E., Eds.; Springer: New York, NY, 2011.

(1073) Schoenbach, K. H.; Beebe, S. J.; Buescher, E. S. Intracellular Effect of Ultrashort Electrical Pulses. Bioelectromagnetics 2001, $22,440-448$.

(1074) Beebe, S. J.; Fox, P. M.; Rec, L. J.; Willis, L. K.; Schoenbach, K. H. Nanosecond, High-Intensity Pulsed Electric Fields Induce Apoptosis in Human Cells. FASEB J. 2003, 17, 1493-1495.

(1075) Nuccitelli, R.; Pliquett, U.; Chen, X. H.; Ford, W.; Swanson, R. J.; Beebe, S. J.; Kolb, J. F.; Schoenbach, K. H. Nanosecond Pulsed Electric Fields Cause Melanomas to Self-Destruct. Biochem. Biophys. Res. Commun. 2006, 343, 351-360.

(1076) Batista Napotnik, T.; Rebersek, M.; Vernier, P. T.; Mali, B.; Miklavcic, D. Effects of High Voltage Nanosecond Electric Pulses on Eukaryotic Cells (in Vitro): A Systematic Review. Bioelectrochemistry 2016, 110, 1-12.

(1077) Pakhomova, O. N.; Gregory, B. W.; Semenov, I.; Pakhomov, A. G. Two Modes of Cell Death Caused by Exposure to Nanosecond Pulsed Electric Field. PLoS One 2013, 8, e70278.

(1078) Bartoletti, D. C.; Harrison, G. I.; Weaver, J. C. The Number of Molecules Taken up by Electroporated Cells: Quantitative Determination. FEBS Lett. 1989, 256, 4-10.

(1079) Dinchuk, J. E.; Kelley, K. A.; Callahan, G. N. Flow Cytometric Analysis of Transport Activity in Lymphocytes Electroporated with a Fluorescent Organic Anion Dye. J. Immunol. Methods 1992, 155, 257-265.

(1080) He, H. Q.; Chang, D. C.; Lee, Y. K. Using a Micro Electroporation Chip to Determine the Optimal Physical Parameters in the Uptake of Biomolecules in Hela Cells. Bioelectrochemistry 2007, 70, 363-368.

(1081) Jones, P. M.; Salmon, D. M. W.; Howell, S. L. Protein-Phosphorylation in Electrically Permeabilized Islets of Langerhans Effects of Ca-2+, Cyclic-Amp, a Phorbol Ester and Noradrenaline. Biochem. J 1988, 254, 397-403.

(1082) Engstrom, P. E.; Persson, B. R. R.; Salford, L. G. Studies of in Vivo Electropermeabilization by Gamma Camera Measurements of Tc-99m-Dtpa. Biochimica Et Biophysica Acta-General Subjects 1999, 1473, 321-328.

(1083) Gordon, P. B.; Tolleshaug, H.; Seglen, P. O. Autophagic Sequestration of [C-14]Sucrose Introduced into Isolated Rat Hepatocytes by Electrical and Non-Electrical Methods. Exp. Cell Res. 1985, 160, 449-458.

(1084) Saulis, G.; Venslauskas, M. S.; Naktinis, J. Kinetics of Pore Resealing in Cell-Membranes after Electroporation. Bioelectrochem. Bioenerg. 1991, 26, 1-13.

(1085) Melvik, J. E.; Pettersen, E. O.; Gordon, P. B.; Seglen, P. O. Increase in Cis-Dichlorodiammineplatinum(li) Cytotoxicity Upon Reversible Electropermeabilization of the Plasma-Membrane in Cultured Human Nhik-3025 Cells. Eur. J. Cancer Clin. Oncol. $1986,22,1523-1530$.

(1086) Gehl, J.; Skovsgaard, T.; Mir, L. M. Enhancement of Cytotoxicity by Electropermeabilization: An Improved Method for Screening Drugs. Anti-Cancer Drugs 1998, 9, 319-325.

(1087) Pavlin, M.; Leben, V.; Miklavcic, D. Electroporation in Dense Cell Suspension--Theoretical and Experimental Analysis of lon Diffusion and Cell Permeabilization. Biochim. Biophys. Acta 2007, 1770, 12-23. 
(1088) Bowman, A. M.; Nesin, O. M.; Pakhomova, O. N.; Pakhomov, A. G. Analysis of Plasma Membrane Integrity by Fluorescent Detection of TI+ Uptake. J. Membr. Biol. 2010, 236, 15-26.

(1089) Chen, A. K.; Behlke, M. A.; Tsourkas, A. Sub-Cellular Trafficking and Functionality of 2'-O-Methyl and 2'-O-MethylPhosphorothioate Molecular Beacons. Nucleic Acids Res. 2009, 37, e149.

(1090) Chen, A. K.; Davydenko, O.; Behlke, M. A.; Tsourkas, A. Ratiometric Bimolecular Beacons for the Sensitive Detection of Rna in Single Living Cells. Nucleic Acids Res. 2010, 38, e148.

(1091) Graziadei, L.; Burfeind, P.; Barsagi, D. Introduction of Unlabeled Proteins into Living Cells by Electroporation and Isolation of Viable Protein-Loaded Cells Using Dextran Fluorescein Isothiocyanate as a Marker for Protein-Uptake. Anal. Biochem. 1991, 194, 198-203.

(1092) Wilson, A. K.; Horwitz, J.; Delanerolle, P. Evaluation of the Electroinjection Method for Introducing Proteins into Living Cells. Am. J. Physiol. 1991, 260, C355-C363.

(1093) Dagher, S. F.; Conrad, S. E.; Werner, E. A.; Patterson, R. J. Phenotypic Conversion of Tk-Deficient Cells Following Electroporation of Functional Tk Enzyme. Exp. Cell Res. 1992, 198, 36-42.

(1094) Li, Y.; Ke, Y.; Gottlieb, P. D.; Kapp, J. A. Delivery of Exogenous Antigen into the Major Histocompatibility Complex Class-I and Class-li Pathways by Electroporation. J. Leukocyte Biol. 1994, 56, 616-624.

(1095) Prausnitz, M. R.; Milano, C. D.; Gimm, J. A.; Langer, R.; Weaver, J. C. Quantitative Study of Molecular-Transport Due to Electroporation - Uptake of Bovine Serum-Albumin by Erythrocyte-Ghosts. Biophys. J. 1994, 66, 1522-1530.

(1096) Morgan, W. F.; Day, J. P. In Animal Cell Electroporation and Electrofusion Protocols; Nickoloff, J. A., Ed.; Humana Press: New York, 1995; Vol. 48

(1097) Sontag, R. L.; Mihai, C.; Orr, G.; Savchenko, A.; Skarina, T.; Cui, H.; Cort, J. R.; Adkins, J. N.; Brown, R. N. Electroporation of Functional Bacterial Effectors into Mammalian Cells. Jove-Journal of Visualized Experiments 2015, 95, 52296.

(1098) Berglund, D. L.; Starkey, J. R. Isolation of Viable Tumor Cells Following Introduction of Labeled Antibody to an Intracellular Oncogene Product Using Electroporation. J. Immunol. Methods 1989, 125, 79-87.

(1099) Lukas, J.; Bartek, J.; Strauss, M. Efficient Transfer of Antibodies into Mammalian Cells by Electroporation. J. Immunol. Methods 1994, 170, 255-259.

(1100) Campbell, P. L.; Mccluskey, J.; Yeo, J.; Toh, B.-H. In Animal Cell Electroporation and Electrofusion Protocols; 1 ed.; Nickoloff, J. A., Ed.; Humana Press: New York, 1995; Vol. 48.

(1101) Verspohl, E. J.; Kaiserlingbuddemeier, I.; Wienecke, A. Introducing Specific Antibodies into Electropermeabilized Cells Is a Valuable Tool for Eliminating Specific Cell Functions. Cell Biochem. Funct. 1997, 15, 127-134.

(1102) Rui, M.; Chen, Y. Y.; Zhang, Y. M.; Ma, D. L. Transfer of Anti-Tfar19 Monoclonal Antibody into Hela Cells by in Situ Electroporation Can Inhibit the Apoptosis. Life Sci. 2002, 71, 1771-1778.

(1103) Hou, P.; Chen, S.; Wang, S.; Yu, X.; Chen, Y.; Jiang, M.; Zhuang, K.; Ho, W.; Hou, W.; Huang, J.et al. Genome Editing of Cxcr4 by Crispr/Cas9 Confers Cells Resistant to Hiv-1 Infection. Sci. Rep. 2015, 5, 15577. 
(1104) Spiller, D. G.; Giles, R. V.; Grzybowski, J.; Tidd, D. M.; Clark, R. E. Improving the Intracellular Delivery and Molecular Efficacy of Antisense Oligonucleotides in Chronic Myeloid Leukemia Cells: A Comparison of Streptolysin-O Permeabilization, Electroporation, and Lipophilic Conjugation. Blood 1998, 91, 4738-4746.

(1105) Walters, D. K.; Jelinek, D. F. The Effectiveness of Double-Stranded Short Inhibitory Rnas (Sirnas) May Depend on the Method of Transfection. Antisense Nucleic Acid Drug Dev. 2002, 12, 411-418.

(1106) Calegari, F.; Haubensak, W.; Yang, D.; Huttner, W. B.; Buchholz, F. Tissue-Specific Rna Interference in Postimplantation Mouse Embryos with Endoribonuclease-Prepared Short Interfering Rna. Proc. Natl. Acad. Sci. U. S. A. 2002, 99, 1423614240.

(1107) Pekarik, V.; Bourikas, D.; Miglino, N.; Joset, P.; Preiswerk, S.; Stoeckli, E. T. Screening for Gene Function in Chicken Embryo Using Rnai and Electroporation. Nat. Biotechnol. 2003, 21, 93-96.

(1108) Gresch, O.; Engel, F. B.; Nesic, D.; Tran, T. T.; England, H. M.; Hickman, E. S.; Korner, I.; Gan, L.; Chen, S.; Castro-Obregon, S.et al. New Non-Viral Method for Gene Transfer into Primary Cells. Methods 2004, 33, 151-163.

(1109) Prechtel, A. T.; Turza, N. M.; Theodoridis, A. A.; Kummer, M.; Steinkasserer, A. Small Interfering Rna (Sirna) Delivery into Monocyte-Derived Dendritic Cells by Electroporation. J. Immunol. Methods 2006, 311, 139-152.

(1110) Saeboe-Larssen, S.; Fossberg, E.; Gaudernack, G. Mrna-Based Electrotransfection of Human Dendritic Cells and Induction of Cytotoxic T Lymphocyte Responses against the Telomerase Catalytic Subunit (Htert). J. Immunol. Methods 2002, 259, 191203.

(1111) Takahashi, M.; Narita, M.; Ayres, F.; Satoh, N.; Abe, T.; Yanao, T.; Furukawa, T.; Toba, K.; Hirohashi, T.; Aizawa, Y. Cytoplasmic Expression of Egfp in Dendritic Cells Transfected with in Vitro Transcribed Mrna or Cellular Total Rna Extracted from Egfp Expressing Leukemia Cells. Med. Oncol. 2003, 20, 335-348.

(1112) Chu, G.; Hayakawa, H.; Berg, P. Electroporation for the Efficient Transfection of Mammalian Cells with DNA. Nucleic Acids Res. 1987, 15, 1311-1326.

(1113) Klenchin, V. A.; Sukharev, S. I.; Serov, S. M.; Chernomordik, L. V.; Chizmadzhev Yu, A. Electrically Induced DNA Uptake by Cells Is a Fast Process Involving DNA Electrophoresis. Biophys. J. 1991, 60, 804-811.

(1114) Katrukha, E. A.; Mikhaylova, M.; Van Brakel, H. X.; Henegouwen, P. M. V. E.; Akhmanova, A.; Hoogenraad, C. C.; Kapitein, L. C. Probing Cytoskeletal Modulation of Passive and Active Intracellular Dynamics Using Nanobody-Functionalized Quantum Dots. Nat Commun 2017, 8, 14772.

(1115) Lambert, H.; Pankov, R.; Gauthier, J.; Hancock, R. Electroporation-Mediated Uptake of Proteins into Mammalian-Cells. Biochem. Cell Biol. 1990, 68, 729-734.

(1116) Gabriel, B.; Teissie, J. Direct Observation in the Millisecond Time Range of Fluorescent Molecule Asymmetrical Interaction with the Electropermeabilized Cell Membrane. Biophys. J. 1997, 73, 2630-2637.

(1117) Pucihar, G.; Kotnik, T.; Miklavcic, D.; Teissie, J. Kinetics of Transmembrane Transport of Small Molecules into Electropermeabilized Cells. Biophys. J. 2008, 95, 2837-2848. 
(1118) Escoffre, J. M.; Portet, T.; Favard, C.; Teissie, J.; Dean, D. S.; Rols, M. P. Electromediated Formation of DNA Complexes with Cell Membranes and Its Consequences for Gene Delivery. Biochim. Biophys. Acta 2011, 1808, 1538-1543.

(1119) Parsegian, A. Energy of an Ion Crossing a Low Dielectric Membrane - Solutions to 4 Relevant Electrostatic Problems. Nature $1969,221,844-846$.

(1120) Venslauskas, M. S.; Satkauskas, S.; Rodaite-Riseviciene, R. Efficiency of the Delivery of Small Charged Molecules into Cells in Vitro. Bioelectrochemistry 2010, 79, 130-135.

(1121) Pakhomov, A. G.; Shevin, R.; White, J. A.; Kolb, J. F.; Pakhomova, O. N.; Joshi, R. P.; Schoenbach, K. H. Membrane Permeabilization and Cell Damage by Ultrashort Electric Field Shocks. Arch. Biochem. Biophys. 2007, 465, $109-118$.

(1122) Glogauer, M.; Mcculloch, C. a. G. Introduction of Large Molecules into Viable Fibroblasts by Electroporation - Optimization of Loading and Identification of Labeled Cellular Compartments. Exp. Cell Res. 1992, 200, 227-234.

(1123) Zaharoff, D. A.; Henshaw, J. W.; Mossop, B.; Yuan, F. Mechanistic Analysis of Electroporation-Induced Cellular Uptake of Macromolecules. Exp. Biol. Med. 2008, 233, 94-105.

(1124) Sadik, M. M.; Yu, M.; Zheng, M. D.; Zahn, J. D.; Shan, J. W.; Shreiber, D. I.; Lin, H. Scaling Relationship and Optimization of Double-Pulse Electroporation. Biophys. J. 2014, 106, 801-812.

(1125) Demiryurek, Y.; Nickaeen, M.; Zheng, M. D.; Yu, M.; Zahn, J. D.; Shreiber, D. I.; Lin, H.; Shan, J. W. Transport, Resealing, and Re-Poration Dynamics of Two-Pulse Electroporation-Mediated Molecular Delivery. Biochimica Et Biophysica ActaBiomembranes 2015, 1848, 1706-1714.

(1126) Liang, H.; Purucker, W. J.; Stenger, D. A.; Kubiniec, R. T.; Hui, S. W. Uptake of Fluorescence-Labeled Dextrans by $10 t$ 1/2 Fibroblasts Following Permeation by Rectangular and Exponential-Decay Electric Field Pulses. BioTechniques 1988, 6, 550552.

(1127) Dimitrov, D. S.; Sowers, A. E. Membrane Electroporation - Fast Molecular-Exchange by Electroosmosis. Biochim. Biophys. Acta 1990, 1022, 381-392.

(1128) Prausnitz, M. R.; Corbett, J. D.; Gimm, J. A.; Golan, D. E.; Langer, R.; Weaver, J. C. Millisecond Measurement of Transport During and after an Electroporation Pulse. Biophys. J. 1995, 68, 1864-1870.

(1129) Prausnitz, M. R. A Practical Assessment of Transdermal Drug Delivery by Skin Electroporation. Adv. Drug Del. Rev. 1999, 35, $61-76$.

(1130) Rols, M. P.; Femenia, P.; Teissie, J. Long-Lived Macropinocytosis Takes Place in Electropermeabilized Mammalian-Cells. Biochem. Biophys. Res. Commun. 1995, 208, 26-35.

(1131) Escoffre, J. M.; Portet, T.; Wasungu, L.; Teissie, J.; Dean, D.; Rols, M. P. What Is (Still Not) Known of the Mechanism by Which Electroporation Mediates Gene Transfer and Expression in Cells and Tissues. Mol. Biotechnol. 2009, 41, $286-295$.

(1132) Rosazza, C.; Meglic, S. H.; Zumbusch, A.; Rols, M. P.; Miklavcic, D. Gene Electrotransfer: A Mechanistic Perspective. Curr. Gene Ther. 2016, 16, 98-129.

(1133) Lambricht, L.; Lopes, A.; Kos, S.; Sersa, G.; Preat, V.; Vandermeulen, G. Clinical Potential of Electroporation for Gene Therapy and DNA Vaccine Delivery. Expert Opinion on Drug Delivery 2016, 13, 295-310. 
(1134) Potter, H.; Weir, L.; Leder, P. Enhancer-Dependent Expression of Human Kappa-Immunoglobulin Genes Introduced into Mouse Pre-B Lymphocytes by Electroporation. Proceedings of the National Academy of Sciences of the United States of America-Biological Sciences 1984, 81, 7161-7165.

(1135) Wolf, H.; Rols, M. P.; Boldt, E.; Neumann, E.; Teissie, J. Control by Pulse Parameters of Electric Field-Mediated Gene Transfer in Mammalian-Cells. Biophys. J. 1994, 66, 524-531.

(1136) Spassova, M.; Tsoneva, I.; Petrov, A. G.; Petkova, J. I.; Neumann, E. Dip Patch-Clamp Currents Suggest Electrodiffusive Transport of the Polyelectrolyte DNA through Lipid Bilayers. Biophys. Chem. 1994, 52, 267-274.

(1137) Golzio, M.; Teissie, J.; Rols, M. P. Direct Visualization at the Single-Cell Level of Electrically Mediated Gene Delivery. Proc. Natl. Acad. Sci. U. S. A. 2002, 99, 1292-1297.

(1138) Rosazza, C.; Deschout, H.; Buntz, A.; Braeckmans, K.; Rols, M. P.; Zumbusch, A. Endocytosis and Endosomal Trafficking of DNA after Gene Electrotransfer in Vitro. Mol Ther Nucleic Acids 2016, 5, e286.

(1139) Rosazza, C.; Escoffre, J. M.; Zumbusch, A.; Rols, M. P. The Actin Cytoskeleton Has an Active Role in the Electrotransfer of Plasmid DNA in Mammalian Cells. Mol. Ther. 2011, 19, 913-921.

(1140) Rosazza, C.; Buntz, A.; Riess, T.; Woll, D.; Zumbusch, A.; Rols, M. P. Intracellular Tracking of Single-Plasmid DNA Particles after Delivery by Electroporation. Mol. Ther. 2013, 21, 2217-2226.

(1141) Brunner, S.; Furtbauer, E.; Sauer, T.; Kursa, M.; Wagner, E. Overcoming the Nuclear Barrier: Cell Cycle Independent Nonviral Gene Transfer with Linear Polyethylenimine or Electroporation. Mol. Ther. 2002, 5, 80-86.

(1142) Badding, M. A.; Lapek, J. D.; Friedman, A. E.; Dean, D. A. Proteomic and Functional Analyses of Protein-DNA Complexes During Gene Transfer. Mol. Ther. 2013, 21, 775-785.

(1143) Mendoza, J. M.; Amante, D. H.; Kichaev, G.; Knott, C. L.; Kiosses, W. B.; Smith, T. R.; Sardesai, N. Y.; Broderick, K. E. Elucidating the Kinetics of Expression and Immune Cell Infiltration Resulting from Plasmid Gene Delivery Enhanced by Surface Dermal Electroporation. Vaccines (Basel) 2013, 1, 384-397.

(1144) Paganin-Gioanni, A.; Bellard, E.; Escoffre, J. M.; Rols, M. P.; Teissie, J.; Golzio, M. Direct Visualization at the Single-Cell Level of Sirna Electrotransfer into Cancer Cells. Proc. Natl. Acad. Sci. U. S. A. 2011, 108, 10443-10447.

(1145) Breton, M.; Delemotte, L.; Silve, A.; Mir, L. M.; Tarek, M. Transport of Sirna through Lipid Membranes Driven by Nanosecond Electric Pulses: An Experimental and Computational Study. JACS 2012, 134, 13938-13941.

(1146) Pliquett, U.; Gift, E. A.; Weaver, J. C. Determination of the Electric Field and Anomalous Heating Caused by Exponential Pulses with Aluminum Electrodes in Electroporation Experiments. Bioelectrochem. Bioenerg. 1996, 39, 39-53.

(1147) Canatella, P. J.; Karr, J. F.; Petros, J. A.; Prausnitz, M. R. Quantitative Study of Electroporation-Mediated Molecular Uptake and Cell Viability. Biophys. J. 2001, 80, 755-764.

(1148) Sukhorukov, V. L.; Reuss, R.; Zimmermann, D.; Held, C.; Muller, K. J.; Kiesel, M.; Gessner, P.; Steinbach, A.; Schenk, W. A.; Bamberg, E.et al. Surviving High-Intensity Field Pulses: Strategies for Improving Robustness and Performance of Electrotransfection and Electrofusion. J. Membr. Biol. 2005, 206, 187-201. 
(1149) Jordan, E. T.; Collins, M.; Terefe, J.; Ugozzoli, L.; Rubio, T. Optimizing Electroporation Conditions in Primary and Other Difficult-to-Transfect Cells. J Biomol Tech 2008, 19, 328-334.

(1150) Kanduser, M.; Miklavcic, D.; Pavlin, M. Mechanisms Involved in Gene Electrotransfer Using High- and Low-Voltage Pulses an in Vitro Study. Bioelectrochemistry 2009, 74, 265-271.

(1151) Heller, R.; Jaroszeski, M.; Atkin, A.; Moradpour, D.; Gilbert, R.; Wands, J.; Nicolau, C. In Vivo Gene Electroinjection and Expression in Rat Liver. FEBS Lett. 1996, 389, 225-228.

(1152) Mir, L. M.; Bureau, M. F.; Gehl, J.; Rangara, R.; Rouy, D.; Caillaud, J. M.; Delaere, P.; Branellec, D.; Schwartz, B.; Scherman, D. High-Efficiency Gene Transfer into Skeletal Muscle Mediated by Electric Pulses. Proc. Natl. Acad. Sci. U. S. A. 1999, 96, $4262-4267$.

(1153) Andreason, G. L.; Evans, G. A. Optimization of Electroporation for Transfection of Mammalian-Cell Lines. Anal. Biochem. $1989,180,269-275$.

(1154) Yockell-Lelievre, J.; Riendeau, V.; Gagnon, S. N.; Garenc, C.; Audette, M. Efficient Transfection of Endothelial Cells by a Double-Pulse Electroporation Method. DNA Cell Biol. 2009, 28, 561-566.

(1155) Stroh, T.; Erben, U.; Kuhl, A. A.; Zeitz, M.; Siegmund, B. Combined Pulse Electroporation - a Novel Strategy for Highly Efficient Transfection of Human and Mouse Cells. PLoS One 2010, 5, e9488.

(1156) Hamm, A.; Krott, N.; Breibach, I.; Blindt, R.; Bosserhoff, A. K. Efficient Transfection Method for Primary Cells. Tissue Eng. 2002, 8, 235-245.

(1157) Zeitelhofer, M.; Vessey, J. P.; Xie, Y. L.; Tubing, F.; Thomas, S.; Kiebler, M.; Dahm, R. High-Efficiency Transfection of Mammalian Neurons Via Nucleofection. Nat. Protoc. 2007, 2, 1692-1704.

(1158) Muller-Hartmann, H.; Riemen, G.; Rothmann-Cosic, K.; Thiel, C.; Altrogge, L.; Weigel, M.; Christine, R.; Lorbach, E.; Helfrich, J.; Wessendorf, H.; LONZA COLOGNE AG: United States, 2004.

(1159) Martinet, W.; Schrijvers, D. M.; Kockx, M. M. Nucleofection as an Efficient Nonviral Transfection Method for Human Monocytic Cells. Biotechnol. Lett 2003, 25, 1025-1029.

(1160) Dauty, E.; Verkman, A. S. Actin Cytoskeleton as the Principal Determinant of Size-Dependent DNA Mobility in Cytoplasm. J. Biol. Chem. 2005, 280, 7823-7828.

(1161) Lukacs, G. L.; Haggie, P.; Seksek, O.; Lechardeur, D.; Freedman, N.; Verkman, A. S. Size-Dependent DNA Mobility in Cytoplasm and Nucleus. J. Biol. Chem. 2000, 275, 1625-1629.

(1162) Luby-Phelps, K. Cytoarchitecture and Physical Properties of Cytoplasm: Volume, Viscosity, Diffusion, Intracellular Surface Area. International Review of Cytology - a Survey of Cell Biology 2000, 192, 189-221.

(1163) Aluigi, M.; Fogli, M.; Curti, A.; Isidori, A.; Gruppioni, E.; Chiodoni, C.; Colombo, M. P.; Versura, P.; D'errico-Grigioni, A.; Ferri, E.et al. Nucleofection Is an Efficient Nonviral Transfection Technique for Human Bone Marrow-Derived Mesenchymal Stem Cells. Stem Cells 2006, 24, 454-461. 
(1164) Aslan, H.; Zilberman, Y.; Arbeli, V.; Sheyn, D.; Matan, Y.; Liebergall, M.; Li, J. Z.; Helm, G. A.; Gazit, D.; Gazit, Z. Nucleofection-Based Ex Vivo Nonviral Gene Delivery to Human Stem Cells as a Platform for Tissue Regeneration. Tissue Eng. 2006, 12, 877-889.

(1165) Lenz, P.; Bacot, S. M.; Frazier-Jessen, M. R.; Feldman, G. M. Nucleoporation of Dendritic Cells: Efficient, Gene Transfer by Electroporation into Human Monocyte-Derived Dendritic Cells. FEBS Lett. 2003, 538, 149-154.

(1166) Landi, A.; Babiuk, L. A.; Littel-Van Den Hurk, S. V. High Transfection Efficiency, Gene Expression, and Viability of MonocyteDerived Human Dendritic Cells after Nonviral Gene Transfer. J. Leukocyte Biol. 2007, 82, 849-860.

(1167) Schakowski, F.; Buttgereit, P.; Mazur, M.; Marten, A.; Schottker, B.; Gorschluter, M.; Schmidt-Wolf, I. G. Novel Non-Viral Method for Transfection of Primary Leukemia Cells and Cell Lines. Genet. Vaccines Ther. 2004, 2, 1.

(1168) Van Bockstaele, F.; Pede, V.; Naessens, E.; Van Coppernolle, S.; Van Tendeloo, V.; Verhasselt, B.; Philippe, J. Efficient Gene Transfer in Cll by Mrna Electroporation. Leukemia 2008, 22, 323-329.

(1169) Trompeter, H. I.; Weinhold, S.; Thiel, C.; Wernet, P.; Uhrberg, M. Rapid and Highly Efficient Gene Transfer into Natural Killer Cells by Nucleofection. J. Immunol. Methods 2003, 274, 245-256.

(1170) Maasho, K.; Marusina, A.; Reynolds, N. M.; Coligan, J. E.; Borrego, F. Efficient Gene Transfer into the Human Natural Killer Cell Line, Nkl, Using the Amaxa Nucleofection System (Tm). J. Immunol. Methods 2004, 284, 133-140.

(1171) Lai, W.; Chang, C. H.; Farber, D. L. Gene Transfection and Expression in Resting and Activated Murine Cd4 T Cell Subsets. J. Immunol. Methods 2003, 282, 93-102.

(1172) Goffinet, C.; Keppler, O. T. Efficient Nonviral Gene Delivery into Primary Lymphocytes from Rats and Mice. FASEB J. 2006, $20,500-502$.

(1173) Lakshmipathy, U.; Pelacho, B.; Sudo, K.; Linehan, J. L.; Coucouvanis, E.; Kaufman, D. S.; Verfaillie, C. M. Efficient Transfection of Embryonic and Adult Stem Cells. Stem Cells 2004, 22, 531-543.

(1174) Siemen, H.; Nix, M.; Endl, E.; Koch, P.; Itskovitz-Eldor, J.; Brustle, O. Nucleofection of Human Embryonic Stem Cells. Stem Cells and Development 2005, 14, 378-383.

(1175) Leclere, P. G.; Panjwani, A.; Docherty, R.; Berry, M.; Pizzey, J.; Tonge, D. A. Effective Gene Delivery to Adult Neurons by a Modified Form of Electroporation. J. Neurosci. Methods 2005, 142, 137-143.

(1176) Bischof, J. C.; Padanilam, J.; Holmes, W. H.; Ezzell, R. M.; Lee, R. C.; Tompkins, R. G.; Yarmush, M. L.; Toner, M. Dynamics of Cell-Membrane Permeability Changes at Supraphysiological Temperatures. Biophys. J. 1995, 68, $2608-2614$.

(1177) Loomishusselbee, J. W.; Cullen, P. J.; Irvine, R. F.; Dawson, A. P. Electroporation Can Cause Artifacts Due to Solubilization of Cations from the Electrode Plates - Aluminum Ions Enhance Conversion of Inositol 1,3,4,5-Tetrakisphosphate into Inositol 1,4,5-Trisphosphate in Electroporated L1210 Cells. Biochem. J 1991, 277, 883-885.

(1178) Stapulionis, R. Electric Pulse-Induced Precipitation of Biological Macromolecules in Electroporation. Bioelectrochem. Bioenerg. 1999, 48, 249-254.

(1179) Tomov, T.; Tsoneva, I. Are the Stainless Steel Electrodes Inert? Bioelectrochemistry 2000, 51, $207-209$. 
(1180) Kotnik, T.; Miklavcic, D.; Mir, L. M. Cell Membrane Electropermeabilization by Symmetrical Bipolar Rectangular Pulses - Part li. Reduced Electrolytic Contamination. Bioelectrochemistry 2001, 54, 91-95.

(1181) Rodaite-Riseviciene, R.; Saule, R.; Snitka, V.; Saulis, G. Release of Iron lons from the Stainless Steel Anode Occurring During High-Voltage Pulses and Its Consequences for Cell Electroporation Technology. leee Transactions on Plasma Science 2014, 42, $249-254$.

(1182) Chafai, D. E.; Mehle, A.; Tilmatine, A.; Maouche, B.; Miklacic, D. Assessment of the Electrochemical Effects of Pulsed Electric Fields in a Biological Cell Suspension. Bioelectrochemistry 2015, 106, 249-257.

(1183) Saulis, G.; Lape, R.; Praneviciute, R.; Mickevicius, D. Changes of the Solution Ph Due to Exposure by High-Voltage Electric Pulses. Bioelectrochemistry 2005, 67, 101-108.

(1184) Turjanski, P.; Olaiz, N.; Maglietti, F.; Michinski, S.; Suarez, C.; Molina, F. V.; Marshall, G. The Role of Ph Fronts in Reversible Electroporation. PLoS One 2011, 6, e17303.

(1185) Li, Y.; Wu, M.; Zhao, D.; Wei, Z.; Zhong, W.; Wang, X.; Liang, Z.; Li, Z. Electroporation on Microchips: The Harmful Effects of Ph Changes and Scaling Down. Sci. Rep. 2015, 5, 17817.

(1186) Saulis, G.; Rodaite-Riseviciene, R.; Snitka, V. Increase of the Roughness of the Stainless-Steel Anode Surface Due to the Exposure to High-Voltage Electric Pulses as Revealed by Atomic Force Microscopy. Bioelectrochemistry 2007, 70, 519-523.

(1187) Pataro, G.; Falcone, M.; Donsi, G.; Ferrari, G. Metal Release from Stainless Steel Electrodes of a Pef Treatment Chamber: Effects of Electrical Parameters and Food Composition. Innov. Food Sci. Emerg. Technol. 2014, 21, 58-65.

(1188) Pucihar, G.; Kotnik, T.; Valic, B.; Miklavcic, D. Numerical Determination of Transmembrane Voltage Induced on Irregularly Shaped Cells. Ann. Biomed. Eng. 2006, 34, 642-652.

(1189) Swezey, R. R.; Epel, D. Stable, Resealable Pores Formed in Sea-Urchin Eggs by Electric-Discharge (Electroporation) Permit Substrate Loading for Assay of Enzymes Invivo. Cell Regul. 1989, 1, 65-74.

(1190) Benov, L. C.; Antonov, P. A.; Ribarov, S. R. Oxidative Damage of the Membrane-Lipids after Electroporation. Gen. Physiol. Biophys. 1994, 13, 85-97.

(1191) Maccarrone, M.; Rosato, N.; Agro, A. F. Electroporation Enhances Cell-Membrane Peroxidation and Luminescence. Biochem. Biophys. Res. Commun. 1995, 206, 238-245.

(1192) Maccarrone, M.; Bladergroen, M. R.; Rosato, N.; Agro, A. F. Role of Lipid-Peroxidation in Electroporation-Induced CellPermeability. Biochem. Biophys. Res. Commun. 1995, 209, 417-425.

(1193) Gabriel, B.; Teissie, J. Generation of Reactive-Oxygen Species Induced by Electropermeabilization of Chinese-Hamster Ovary Cells and Their Consequence on Cell Viability. Eur. J. Biochem. 1994, 223, 25-33.

(1194) Bonnafous, P.; Vernhes, M. C.; Teissie, J.; Gabriel, B. The Generation of Reactive-Oxygen Species Associated with LongLasting Pulse-Induced Electropermeabilisation of Mammalian Cells Is Based on a Non-Destructive Alteration of the Plasma Membrane. Biochimica Et Biophysica Acta-Biomembranes 1999, 1461, 123-134.

(1195) Vatteroni, L.; Piras, A.; Simi, S.; Mariani, L.; Moretti, A.; Citti, L.; Mariani, T.; Rainaldi, G. Analysis of Electroporation-Induced Genetic Damages in V79/Ap4 Chinese-Hamster Cells. Mutat. Res. 1993, 291, 163-169. 
(1196) Meaking, W. S.; Edgerton, J.; Wharton, C. W.; Meldrum, R. A. Electroporation-Induced Damage in Mammalian Cell DNA. Biochimica Et Biophysica Acta-Gene Structure and Expression 1995, 1264, 357-362.

(1197) Zhou, Y.; Berry, C. K.; Storer, P. A.; Raphael, R. M. Peroxidation of Polyunsaturated Phosphatidyl-Choline Lipids During Electroformation. Biomaterials 2007, 28, 1298-1306.

(1198) Vernier, P. T.; Levine, Z. A.; Wu, Y. H.; Joubert, V.; Ziegler, M. J.; Mir, L. M.; Tieleman, D. P. Electroporating Fields Target Oxidatively Damaged Areas in the Cell Membrane. PLoS One 2009, 4, e7966.

(1199) Chen, W.; Lee, R. C. Altered Ion-Channel Conductance and Ionic Selectivity Induced by Large Imposed Membrane-Potential Pulse. Biophys. J. 1994, 67, 603-612.

(1200) Chen, W.; Han, Y.; Chen, Y.; Astumian, D. Electric Field-Induced Functional Reductions in the K+ Channels Mainly Resulted from Supramembrane Potential-Mediated Electroconformational Changes. Biophys. J. 1998, 75, $196-206$.

(1201) Chen, W.; Zhang, Z. S.; Lee, R. C. Supramembrane Potential-Induced Electroconformational Changes in Sodium Channel Proteins: A Potential Mechanism Involved in Electric Injury. Burns 2006, 32, 52-59.

(1202) Chen, W.; Han, Y.; Chen, Y.; Lee, R. C. In Electricity and Magnetism in Biology and Medicine; F., B., Ed.; Springer: Boston, 1999.

(1203) Lee, R. C. Cell Injury by Electric Forces. Cell Injury: Mechanisms, Responses, and Repair 2005, 1066, 85-91.

(1204) Chen, W. Electroconformational Denaturation of Membrane Proteins. Cell Injury: Mechanisms, Responses, and Repair 2005, $1066,92-105$.

(1205) Huang, F. R.; Fang, Z. H.; Mast, J.; Chen, W. Comparison of Membrane Electroporation and Protein Denature in Response to Pulsed Electric Field with Different Durations. Bioelectromagnetics 2013, 34, 253-263.

(1206) Nesin, V.; Bowman, A. M.; Xiao, S.; Pakhomov, A. G. Cell Permeabilization and Inhibition of Voltage-Gated Ca2+and Na+ Channel Currents by Nanosecond Pulsed Electric Field. Bioelectromagnetics 2012, 33, 394-404.

(1207) Beebe, S. J. Considering Effects of Nanosecond Pulsed Electric Fields on Proteins. Bioelectrochemistry 2015, 103, 52-59.

(1208) Nuccitelli, R.; Lui, K. Y.; Kreis, M.; Athos, B.; Nuccitelli, P. Nanosecond Pulsed Electric Field Stimulation of Reactive Oxygen Species in Human Pancreatic Cancer Cells Is Ca2+-Dependent. Biochem. Biophys. Res. Commun. 2013, 435, $580-585$.

(1209) Pakhomova, O. N.; Khorokhorina, V. A.; Bowman, A. M.; Rodaite-Riseviciene, R.; Saulis, G.; Xiao, S.; Pakhomov, A. G. Oxidative Effects of Nanosecond Pulsed Electric Field Exposure in Cells and Cell-Free Media. Arch. Biochem. Biophys. 2012, $527,55-64$

(1210) Golberg, A.; Yarmush, M. L. Nonthermal Irreversible Electroporation: Fundamentals, Applications, and Challenges. IEEE Trans. Biomed. Eng. 2013, 60, 707-714.

(1211) Schwarz, S.; Haest, C. W. M.; Deuticke, B. Extensive Electroporation Abolishes Experimentally Induced Shape Transformations of Erythrocytes: A Consequence of Phospholipid Symmetrization. Biochimica Et Biophysica ActaBiomembranes 1999, 1421, 361-379.

(1212) Vernier, P. T.; Sun, Y. H.; Marcu, L.; Craft, C. M.; Gundersen, M. A. Nanoelectropulse-Induced Phosphatidylserine Translocation. Biophys. J. 2004, 86, 4040-4048. 
(1213) Vernier, P. T.; Ziegler, M. J.; Sun, Y. H.; Chang, W. V.; Gundersen, M. A.; Tieleman, D. P. Nanopore Formation and Phosphatidylserine Externalization in a Phospholipid Bilayer at High Transmembrane Potential. JACS 2006, 128, 6288-6289.

(1214) Vincelette, R. L.; Roth, C. C.; Mcconnell, M. P.; Payne, J. A.; Beier, H. T.; Ibey, B. L. Thresholds for Phosphatidylserine Externalization in Chinese Hamster Ovarian Cells Following Exposure to Nanosecond Pulsed Electrical Fields (Nspef). PLoS One 2013, 8, e63122.

(1215) Escoffre, J. M.; Bellard, E.; Faurie, C.; Sebai, S. C.; Golzio, M.; Teissie, J.; Rols, M. P. Membrane Disorder and Phospholipid Scrambling in Electropermeabilized and Viable Cells. Biochimica Et Biophysica Acta-Biomembranes 2014, 1838, $1701-1709$.

(1216) Kooijmans, S. a. A.; Stremersch, S.; Braeckmans, K.; De Smedt, S. C.; Hendrix, A.; Wood, M. J. A.; Schiffelers, R. M.; Raemdonck, K.; Vader, P. Electroporation-Induced Sirna Precipitation Obscures the Efficiency of Sirna Loading into Extracellular Vesicles. J. Controlled Release 2013, 172, 229-238.

(1217) Fox, M. B.; Esveld, D. C.; Valero, A.; Luttge, R.; Mastwijk, H. C.; Bartels, P. V.; Van Den Berg, A.; Boom, R. M. Electroporation of Cells in Microfluidic Devices: A Review. Anal. Bioanal. Chem. 2006, 385, 474-485.

(1218) Wang, M. Y.; Orwar, O.; Olofsson, J.; Weber, S. G. Single-Cell Electroporation. Anal. Bioanal. Chem. 2010, 397, 3235-3248.

(1219) Movahed, S.; Li, D. Q. Microfluidics Cell Electroporation. Microfluid. Nanofluid. 2011, 10, 703-734.

(1220) Geng, T.; Lu, C. Microfluidic Electroporation for Cellular Analysis and Delivery. Lab on a Chip 2013, $13,3803-3821$.

(1221) Wang, S.; Lee, L. J. Micro-/Nanofluidics Based Cell Electroporation. Biomicrofluidics 2013, 7, 11301.

(1222) Yang, Z. G.; Chang, L. Q.; Chiang, C. L.; Lee, L. J. Micro-/Nano-Electroporation for Active Gene Delivery. Curr. Pharm. Des. $2015,21,6081-6088$.

(1223) Huang, Y.; Rubinsky, B. Micro-Electroporation: Improving the Efficiency and Understanding of Electrical Permeabilization of Cells. Biomed. Microdevices 1999, 2, 145-150.

(1224) Huang, Y.; Rubinsky, B. Flow-through Micro-Electroporation Chip for High Efficiency Single-Cell Genetic Manipulation. Sensors and Actuators a-Physical 2003, 104, 205-212.

(1225) Seemann, R.; Brinkmann, M.; Pfohl, T.; Herminghaus, S. Droplet Based Microfluidics. Rep. Prog. Phys. 2012, 75, 016601.

(1226) Zhan, Y. H.; Wang, J.; Bao, N.; Lu, C. Electroporation of Cells in Microfluidic Droplets. Anal. Chem. 2009, 81, 2027-2031.

(1227) Geng, T.; Zhan, Y. H.; Wang, H. Y.; Witting, S. R.; Cornetta, K. G.; Lu, C. Flow-through Electroporation Based on Constant Voltage for Large-Volume Transfection of Cells. J. Controlled Release 2010, 144, 91-100.

(1228) Zhu, T.; Luo, C. X.; Huang, J. Y.; Xiong, C. Y.; Ouyang, Q.; Fang, J. Electroporation Based on Hydrodynamic Focusing of Microfluidics with Low Dc Voltage. Biomed. Microdevices 2010, 12, 35-40.

(1229) Wang, J.; Zhan, Y. H.; Ugaz, V. M.; Lu, C. Vortex-Assisted DNA Delivery. Lab on a Chip 2010, 10, $2057-2061$.

(1230) Yun, H.; Hur, S. C. Sequential Multi-Molecule Delivery Using Vortex-Assisted Electroporation. Lab on a Chip 2013, 13, 27642772.

(1231) Zheng, M. D.; Shan, J. W.; Lin, H.; Shreiber, D. I.; Zahn, J. D. Hydrodynamically Controlled Cell Rotation in an Electroporation Microchip to Circumferentially Deliver Molecules into Single Cells. Microfluid. Nanofluid. 2016, 20, 16. 
(1232) Ouyang, M.; Hill, W.; Lee, J. H.; Hur, S. C. Microscale Symmetrical Electroporator Array as a Versatile Molecular Delivery System. Sci. Rep. 2017, 7, 44757.

(1233) Boukany, P. E.; Morss, A.; Liao, W. C.; Henslee, B.; Jung, H. C.; Zhang, X. L.; Yu, B.; Wang, X. M.; Wu, Y.; Li, L.et al. Nanochannel Electroporation Delivers Precise Amounts of Biomolecules into Living Cells. Nat Nanotechnol 2011, 6, 747-754.

(1234) Chang, L. Q.; Bertani, P.; Gallego-Perez, D.; Yang, Z. G.; Chen, F.; Chiang, C. L.; Malkoc, V.; Kuang, T. R.; Gao, K. L.; Lee, L. J.et al. 3d Nanochannel Electroporation for High-Throughput Cell Transfection with High Uniformity and Dosage Control. Nanoscale 2016, 8, 243-252.

(1235) Kurosawa, O.; Oana, H.; Matsuoka, S.; Noma, A.; Kotera, H.; Washizu, M. Electroporation through a Micro-Fabricated Orifice and Its Application to the Measurement of Cell Response to External Stimuli. Meas. Sci. Technol. 2006, 17, 3127-3133.

(1236) Seo, J.; lonescu-Zanetti, C.; Diamond, J.; Lal, R.; Lee, L. P. Integrated Multiple Patch-Clamp Array Chip Via Lateral Cell Trapping Junctions. Appl. Phys. Lett. 2004, 84, 1973-1975.

(1237) Khine, M.; Lau, A.; Ionescu-Zanetti, C.; Seo, J.; Lee, L. P. A Single Cell Electroporation Chip. Lab on a Chip 2005, 5, $38-43$.

(1238) Khine, M.; Ionescu-Zanetti, C.; Blatz, A.; Wang, L. P.; Lee, L. P. Single-Cell Electroporation Arrays with Real-Time Monitoring and Feedback Control. Lab on a Chip 2007, 7, 457-462.

(1239) Ionescu-Zanetti, C.; Blatz, A.; Khine, M. Electrophoresis-Assisted Single-Cell Electroporation for Efficient Intracellular Delivery. Biomed. Microdevices 2008, 10, 113-116.

(1240) Valero, A.; Post, J. N.; Van Nieuwkasteele, J. W.; Ter Braak, P. M.; Kruijer, W.; Van Den Berg, A. Gene Transfer and Protein Dynamics in Stem Cells Using Single Cell Electroporation in a Microfluidic Device. Lab on a Chip 2008, 8, 62-67.

(1241) Kang, W. M.; Yavari, F.; Minary-Jolandan, M.; Giraldo-Vela, J. P.; Safi, A.; Mcnaughton, R. L.; Parpoil, V.; Espinosa, H. D. Nanofountain Probe Electroporation (Nfp-E) of Single Cells. Nano Lett. 2013, 13, 2448-2457.

(1242) Giraldo-Vela, J. P.; Kang, W.; Mcnaughton, R. L.; Zhang, X. M.; Wile, B. M.; Tsourkas, A.; Bao, G.; Espinosa, H. D. Single-Cell Detection of Mrna Expression Using Nanofountain-Probe Electroporated Molecular Beacons. Small 2015, $11,2386-2391$.

(1243) Charoo, N. A.; Rahman, Z.; Repka, M. A.; Murthy, S. N. Electroporation: An Avenue for Transdermal Drug Delivery. Curr Drug Deliv 2010, 7, 125-136.

(1244) Sardesai, N. Y.; Weiner, D. B. Electroporation Delivery of DNA Vaccines: Prospects for Success. Curr. Opin. Immunol. 2011, 23, 421-429.

(1245) Mali, B.; Jarm, T.; Snoj, M.; Sersa, G.; Miklavcic, D. Antitumor Effectiveness of Electrochemotherapy: A Systematic Review and Meta-Analysis. Ejso 2013, 39, 4-16.

(1246) Jiang, C. L.; Davalos, R. V.; Bischof, J. C. A Review of Basic to Clinical Studies of Irreversible Electroporation Therapy. IEEE Trans. Biomed. Eng. 2015, 62, 4-20.

(1247) Calvet, C. Y.; Mir, L. M. The Promising Alliance of Anti-Cancer Electrochemotherapy with Immunotherapy. Cancer Metastasis Rev. 2016, 35, 165-177.

(1248) Kotnik, T.; Frey, W.; Sack, M.; Meglic, S. H.; Peterka, M.; Miklavcic, D. Electroporation-Based Applications in Biotechnology. Trends Biotechnol. 2015, 33, 480-488. 
(1249) Orlowski, S.; Mir, L. M. Cell Electropermeabilization - a New Tool for Biochemical and Pharmacological Studies. Biochim. Biophys. Acta 1993, 1154, 51-63.

(1250) Pham, P. L.; Kamen, A.; Durocher, Y. Large-Scale Transfection of Mammalian Cells for the Fast Production of Recombinant Protein. Mol. Biotechnol. 2006, 34, 225-237.

(1251) Wurm, F. M. Production of Recombinant Protein Therapeutics in Cultivated Mammalian Cells. Nat. Biotechnol. 2004, 22, 13931398.

(1252) Baldi, L.; Hacker, D. L.; Adam, M.; Wurm, F. M. Recombinant Protein Production by Large-Scale Transient Gene Expression in Mammalian Cells: State of the Art and Future Perspectives. Biotechnol. Lett 2007, 29, 677-684.

(1253) Merten, O. W.; Charrier, S.; Laroudie, N.; Fauchille, S.; Dugue, C.; Jenny, C.; Audit, M.; Zanta-Boussif, M. A.; Chautard, H.; Radrizzani, M.et al. Large-Scale Manufacture and Characterization of a Lentiviral Vector Produced for Clinical Ex Vivo Gene Therapy Application. Hum. Gene Ther. 2011, 22, 343-356.

(1254) Lock, M.; Alvira, M.; Vandenberghe, L. H.; Samanta, A.; Toelen, J.; Debyser, Z.; Wilson, J. M. Rapid, Simple, and Versatile Manufacturing of Recombinant Adeno-Associated Viral Vectors at Scale. Hum. Gene Ther. 2010, 21, $1259-1271$.

(1255) Zeltins, A. Construction and Characterization of Virus-Like Particles: A Review. Mol. Biotechnol. 2013, 53, 92-107.

(1256) Teissie, J.; Rols, M. P. Electrofusion of Large Volumes of Cells in Culture .2. Cells Growing in Suspension. Bioelectrochem. Bioenerg. 1988, 19, 59-66.

(1257) Teissie, J.; Conte, P. Electrofusion of Large Volumes of Cells in Culture .1. Anchorage-Dependent Strains. Bioelectrochem. Bioenerg. 1988, 19, 49-57.

(1258) Rols, M. P.; Coulet, D.; Teissie, J. Highly Efficient Transfection of Mammalian-Cells by Electric-Field Pulses - Application to Large Volumes of Cell-Culture by Using a Flow System. Eur. J. Biochem. 1992, 206, 115-121.

(1259) Li, L. H.; Shivakumar, R.; Feller, S.; Allen, C.; Weiss, J. M.; Dzekunov, S.; Singh, V.; Holaday, J.; Fratantoni, J.; Liu, L. N. Highly Efficient, Large Volume Flow Electroporation. Technol. Cancer Res. Treat. 2002, 1, 341-349.

(1260) Witting, S. R.; Li, L. H.; Jasti, A.; Allen, C.; Cornetta, K.; Brady, J.; Shivakumar, R.; Peshwa, M. V. Efficient Large Volume Lentiviral Vector Production Using Flow Electroporation. Hum. Gene Ther. 2012, 23, 243-249.

(1261) Zhao, D. Y.; Huang, D.; Li, Y.; Wu, M. X.; Zhong, W. F.; Cheng, Q.; Wang, X. X.; Wu, Y. D.; Zhou, X.; Wei, Z. W.et al. A Flowthrough Cell Electroporation Device for Rapidly and Efficiently Transfecting Massive Amounts of Cells in Vitro and Ex Vivo. Sci. Rep. 2016, 6, 18469.

(1262) Selmeczi, D.; Hansen, T. S.; Met, O.; Svane, I. M.; Larsen, N. B. Efficient Large Volume Electroporation of Dendritic Cells through Micrometer Scale Manipulation of Flow in a Disposable Polymer Chip. Biomed. Microdevices 2011, 13, $383-392$.

(1263) Sather, B. D.; Romano Ibarra, G. S.; Sommer, K.; Curinga, G.; Hale, M.; Khan, I. F.; Singh, S.; Song, Y.; Gwiazda, K.; Sahni, J.et al. Efficient Modification of Ccr5 in Primary Human Hematopoietic Cells Using a Megatal Nuclease and Aav Donor Template. Sci. Transl. Med. 2015, 7, 307ra156. 
(1264) Dullaers, M.; Breckpot, K.; Van Meirvenne, S.; Bonehill, A.; Tuyaerts, S.; Michiels, A.; Straetman, L.; Heirman, C.; De Greef, C.; Van Der Bruggen, P.et al. Side-by-Side Comparison of Lentivirally Transduced and Mrna-Electroporated Dendritic Cells: Implications for Cancer Immunotherapy Protocols. Mol. Ther. 2004, 10, 768-779.

(1265) Dalby, B.; Cates, S.; Harris, A.; Ohki, E. C.; Tilkins, M. L.; Price, P. J.; Ciccarone, V. C. Advanced Transfection with Lipofectamine 2000 Reagent: Primary Neurons, Sirna, and High-Throughput Applications. Methods 2004, 33, 95-103.

(1266) Coughlin, C. M.; Vance, B. A.; Grupp, S. A.; Vonderheide, R. H. Rna-Transfected Cd40-Activated B Cells Induce Functional TCell Responses against Viral and Tumor Antigen Targets: Implications for Pediatric Immunotherapy. Blood 2004, 103, 20462054.

(1267) Schaft, N.; Dorrie, J.; Muller, I.; Beck, V.; Baumann, S.; Schunder, T.; Kampgen, E.; Schuler, G. A New Way to Generate Cytolytic Tumor-Specific T Cells: Electroporation of Rna Coding for a T Cell Receptor into T Lymphocytes. Cancer Immunology Immunotherapy 2006, 55, 1132-1141.

(1268) Kunii, N.; Zhao, Y. B.; Jiang, S. G.; Liu, X. J.; Scholler, J.; Balagopalan, L.; Samelson, L. E.; Milone, M. C.; June, C. H. Enhanced Function of Redirected Human T Cells Expressing Linker for Activation of T Cells That Is Resistant to Ubiquitylation. Hum. Gene Ther. 2013, 24, 27-37.

(1269) Riet, T.; Holzinger, A.; Dorrie, J.; Schaft, N.; Schuler, G.; Abken, H. Nonviral Rna Transfection to Transiently Modify T Cells with Chimeric Antigen Receptors for Adoptive Therapy. Methods Mol. Biol. 2013, 969, 187-201.

(1270) Krug, C.; Wiesinger, M.; Abken, H.; Schuler-Thurner, B.; Schuler, G.; Dorrie, J.; Schaft, N. A Gmp-Compliant Protocol to Expand and Transfect Cancer Patient T Cells with Mrna Encoding a Tumor-Specific Chimeric Antigen Receptor. Cancer Immunology Immunotherapy 2014, 63, 999-1008.

(1271) Wang, J.; Declercq, J. J.; Hayward, S. B.; Li, P. W.; Shivak, D. A.; Gregory, P. D.; Lee, G.; Holmes, M. C. Highly Efficient Homology-Driven Genome Editing in Human T Cells by Combining Zinc-Finger Nuclease Mrna and Aav6 Donor Delivery. Nucleic Acids Res. 2016, 44, e30.

(1272) Grunebach, F.; Muller, M. R.; Nencioni, A.; Brossart, P. Delivery of Tumor-Derived Rna for the Induction of Cytotoxic TLymphocytes. Gene Ther. 2003, 10, 367-374.

(1273) Van Nuffel, A. M. T.; Corthals, J.; Neyns, B.; Heirman, C.; Thielemans, K.; Bonehill, A. Immunotherapy of Cancer with Dendritic Cells Loaded with Tumor Antigens and Activated through Mrna Electroporation. Rna Therapeutics: Function, Design, and Delivery 2010, 629, 403-450.

(1274) Van Den Bosch, G. A.; Ponsaerts, P.; Nijs, G.; Lenjou, M.; Vanham, G.; Van Bockstaele, D. R.; Berneman, Z. N.; Van Tendeloo, V. F. I. Ex Vivo Induction of Viral Antigen-Specific Cd8(+) T Cell Responses Using Mrna-Electroporated Cd40Activated B Cells. Clin. Exp. Immunol. 2005, 139, 458-467.

(1275) Lee, J.; Dollins, C. M.; Boczkowski, D.; Sullenger, B. A.; Nair, S. Activated B Cells Modified by Electroporation of Multiple Mrnas Encoding Immune Stimulatory Molecules Are Comparable to Mature Dendritic Cells in Inducing in Vitro Antigen-Specific T-Cell Responses. Immunology 2008, 125, 229-240. 
(1276) Li, L. H.; Biagi, E.; Allen, C.; Shivakumar, R.; Weiss, J. M.; Feller, S.; Yvon, E.; Fratantoni, J. C.; Liu, L. N. Rapid and Efficient Nonviral Gene Delivery of Cd154 to Primary Chronic Lymphocytic Leukemia Cells. Cancer Gene Ther. 2006, $13,215-224$.

(1277) Li, L.; Liu, L. N.; Feller, S.; Allen, C.; Shivakumar, R.; Fratantoni, J.; Wolfraim, L. A.; Fujisaki, H.; Campana, D.; Chopas, N.et al. Expression of Chimeric Antigen Receptors in Natural Killer Cells with a Regulatory-Compliant Non-Viral Method. Cancer Gene Ther. 2010, 17, 147-154.

(1278) Shimasaki, N.; Fujisaki, H.; Cho, D.; Masselli, M.; Lockey, T.; Eldridge, P.; Leung, W.; Campana, D. A Clinically Adaptable Method to Enhance the Cytotoxicity of Natural Killer Cells against B-Cell Malignancies. Cytotherapy 2012, 14, 830-840.

(1279) Bilal, M. Y.; Vacaflores, A.; Houtman, J. C. D. Optimization of Methods for the Genetic Modification of Human T Cells. Immunol. Cell Biol. 2015, 93, 896-908.

(1280) Zhang, M.; Ma, Z.; Selliah, N.; Weiss, G.; Genin, A.; Finkel, T. H.; Cron, R. Q. The Impact of Nucleofection (R) on the Activation State of Primary Human Cd4 T Cells. J. Immunol. Methods 2014, 408, 123-131.

(1281) Anderson, B. R.; Kariko, K.; Weissman, D. Nucleofection Induces Transient Eif2alpha Phosphorylation by Gcn2 and Perk. Gene Ther. 2013, 20, 136-142.

(1282) Wilgenhof, S.; Van Nuffel, A. M. T.; Benteyn, D.; Corthals, J.; Aerts, C.; Heirman, C.; Van Riet, I.; Bonehill, A.; Thielemans, K.; Neyns, B. A Phase lb Study on Intravenous Synthetic Mrna Electroporated Dendritic Cell Immunotherapy in Pretreated Advanced Melanoma Patients. Ann. Oncol. 2013, 24, 2686-2693.

(1283) Gill, S.; June, C. H. Going Viral: Chimeric Antigen Receptor T-Cell Therapy for Hematological Malignancies. Immunol. Rev. 2015, 263, 68-89.

(1284) Yewdell, J. W.; Bennink, J. R.; Hosaka, Y. Cells Process Exogenous Proteins for Recognition by Cytotoxic T Lymphocytes. Science 1988, 239, 637-640.

(1285) Tacken, P. J.; De Vries, I. J. M.; Torensma, R.; Figdor, C. G. Dendritic-Cell Immunotherapy: From Ex Vivo Loading to in Vivo Targeting. Nature Reviews Immunology 2007, 7, 790-802.

(1286) Mitchell, D. A.; Nair, S. K. Rna-Transfected Dendritic Cells in Cancer Immunotherapy. J. Clin. Invest. 2000, 106, 1065-1069.

(1287) Van Tendeloo, V. F. I.; Snoeck, H. W.; Lardon, F.; Vanham, G. L. E. E.; Nijs, G.; Lenjou, M.; Hendriks, L.; Van Broeckhoven, C.; Moulijn, A.; Rodrigus, I.et al. Nonviral Transfection of Distinct Types of Human Dendritic Cells: High-Efficiency Gene Transfer by Electroporation into Hematopoietic Progenitor- but Not Monocyte-Derived Dendritic Cells. Gene Ther. 1998, 5, 700-707.

(1288) Ponsaerts, P.; Van Tendeloo, V. F. I.; Berneman, Z. N. Cancer Immunotherapy Using Rna-Loaded Dendritic Cells. Clin. Exp. Immunol. 2003, 134, 378-384.

(1289) Li, S. Electroporation Gene Therapy: New Developments in Vivo and in Vitro. Curr. Gene Ther. 2004, 4, 309-316.

(1290) Wilgenhof, S.; Van Nuffel, A. M. T.; Corthals, J.; Heirman, C.; Tuyaerts, S.; Benteyn, D.; De Coninck, A.; Van Riet, I.; Verfaillie, G.; Vandeloo, J.et al. Therapeutic Vaccination with an Autologous Mrna Electroporated Dendritic Cell Vaccine in Patients with Advanced Melanoma. J. Immunother. 2011, 34, 448-456. 
(1291) Kalos, M.; June, C. H. Adoptive T Cell Transfer for Cancer Immunotherapy in the Era of Synthetic Biology. Immunity 2013, 39, 49-60.

(1292) Morvan, M. G.; Lanier, L. L. Nk Cells and Cancer: You Can Teach Innate Cells New Tricks. Nature Reviews Cancer 2016, 16, 7-19.

(1293) Mitchell, D. A.; Karikari, I.; Cui, X. Y.; Xie, W. H.; Schmittling, R.; Sampson, J. H. Selective Modification of Antigen-Specific T Cells by Rna Electroporation. Hum. Gene Ther. 2008, 19, 511-521.

(1294) Yoon, S. H.; Lee, J. M.; Cho, H. I.; Kim, E. K.; Kim, H. S.; Park, M. Y.; Kim, T. G. Adoptive Immunotherapy Using Human Peripheral Blood Lymphocytes Transferred with Rna Encoding Her-2/Neu-Specific Chimeric Immune Receptor in Ovarian Cancer Xenograft Model. Cancer Gene Ther. 2009, 16, 489-497.

(1295) Maus, M. V.; Haas, A. R.; Beatty, G. L.; Albelda, S. M.; Levine, B. L.; Liu, X. J.; Zhao, Y. B.; Kalos, M.; June, C. H. T Cells Expressing Chimeric Antigen Receptors Can Cause Anaphylaxis in Humans. Cancer Immunology Research $2013,1,26-31$.

(1296) Imai, C.; Iwamoto, S.; Campana, D. Genetic Modification of Primary Natural Killer Cells Overcomes Inhibitory Signals and Induces Specific Killing of Leukemic Cells. Blood 2005, 106, 376-383.

(1297) Tebas, P.; Stein, D.; Tang, W. W.; Frank, I.; Wang, S. Q.; Lee, G.; Spratt, S. K.; Surosky, R. T.; Giedlin, M. A.; Nichol, G.et al. Gene Editing of Ccr5 in Autologous Cd4 T Cells of Persons Infected with Hiv. New Engl. J. Med. 2014, 370, 901-910.

(1298) Biffi, A.; Montini, E.; Lorioli, L.; Cesani, M.; Fumagalli, F.; Plati, T.; Baldoli, C.; Martino, S.; Calabria, A.; Canale, S.et al. Lentiviral Hematopoietic Stem Cell Gene Therapy Benefits Metachromatic Leukodystrophy. Science 2013, 341, 1233158.

(1299) Aiuti, A.; Biasco, L.; Scaramuzza, S.; Ferrua, F.; Cicalese, M. P.; Baricordi, C.; Dionisio, F.; Calabria, A.; Giannelli, S.; Castiello, M. C.et al. Lentiviral Hematopoietic Stem Cell Gene Therapy in Patients with Wiskott-Aldrich Syndrome. Science 2013, 341, 1233151.

(1300) Lombardo, A.; Naldini, L. Genome Editing: A Tool for Research and Therapy: Targeted Genome Editing Hits the Clinic. Nat. Med. 2014, 20, 1101-1103.

(1301) Heimburg, T. In Thermal Biophysics of Membranes; Heimburg, T., Ed.; Wiley-VCH Verlag GmbH \& Co. KGaA, 2007.

(1302) Jacobson, K.; Papahadjopoulos, D. Phase-Transitions and Phase Separations in Phospholipid Membranes Induced by Changes in Temperature, $\mathrm{Ph}$, and Concentration of Bivalent-Cations. Biochemistry 1975, 14, 152-161.

(1303) Boheim, G.; Hanke, W.; Eibl, H. Lipid Phase-Transition in Planar Bilayer-Membrane and Its Effect on Carrier-Mediated and Pore-Mediated Ion-Transport. Proceedings of the National Academy of Sciences of the United States of America-Biological Sciences 1980, 77, 3403-3407.

(1304) Antonov, V. F.; Petrov, V. V.; Molnar, A. A.; Predvoditelev, D. A.; Ivanov, A. S. Appearance of Single-lon Channels in Unmodified Lipid Bilayer-Membranes at the Phase-Transition Temperature. Nature 1980, 283, 585-586.

(1305) Mandel, M.; Higa, A. Calcium-Dependent Bacteriophage DNA Infection. J. Mol. Biol. 1970, 53, $159-162$.

(1306) Hanahan, D. Studies on Transformation of Escherichia-Coli with Plasmids. J. Mol. Biol. 1983, 166, 557-580.

(1307) Vandie, I. M.; Bergmans, H. E. N.; Hoekstra, W. P. M. Transformation in Escherichia-Coli - Studies on the Role of the HeatShock in Induction of Competence. J. Gen. Microbiol. 1983, 129, 663-670. 
(1308) Panja, S.; Aich, P.; Jana, B.; Basu, T. How Does Plasmid DNA Penetrate Cell Membranes in Artificial Transformation Process of Escherichia Coli? Mol. Membr. Biol. 2008, 25, 411-422.

(1309) Tripp, V. T.; Maza, J. C.; Young, D. D. Development of Rapid Microwave-Mediated and Low-Temperature Bacterial Transformations. J. Chem. Biol. 2013, 6, 135-140.

(1310) Li, S.; Anderson, L. M.; Yang, J. M.; Lin, L.; Yang, H. DNA Transformation Via Local Heat Shock. Appl. Phys. Lett. 2007, 91, 013902.

(1311) Nichols, R. A.; Wu, W. C. S.; Haycock, J. W.; Greengard, P. Introduction of Impermeant Molecules into Synaptosomes Using Freeze Thaw Permeabilization. J. Neurochem. 1989, 52, 521-529.

(1312) Nath, A. R.; Chen, R. H. C.; Stanley, E. F. Cryoloading: Introducing Large Molecules into Live Synaptosomes. Front. Cell. Neurosci. 2014, 8, 4.

(1313) Yi, P. N.; Chang, C. S.; Tallen, M.; Bayer, W.; Ball, S. Hyperthermia-Induced Intracellular lonic Level Changes in Tumor-Cells. Radiat. Res. 1983, 93, 534-544.

(1314) Ivanov, I. T.; Todorova, R.; Zlatanov, I. Spectrofluorometric and Microcalorimetric Study of the Thermal Poration Relevant to the Mechanism of Thermohaemolysis. Int. J. Hyperthermia 1999, 15, 29-43.

(1315) Ivanov, I. T. Investigation of Surface and Shape Changes Accompanying the Membrane Alteration Responsible for the HeatInduced Lysis of Human Erythrocytes. Colloids and Surfaces B-Biointerfaces 1999, 13, 311-323.

(1316) Merchant, F. A.; Holmes, W. H.; Capelli-Schellpfeffer, M.; Lee, R. C.; Toner, M. Poloxamer 188 Enhances Functional Recovery of Lethally Heat-Shocked Fibroblasts. J. Surg. Res. 1998, 74, 131-140.

(1317) Terakawa, M.; Ogura, M.; Sato, S.; Wakisaka, H.; Ashida, H.; Uenoyama, M.; Masaki, Y.; Obara, M. Gene Transfer into Mammalian Cells by Use of a Nanosecond Pulsed Laser-Induced Stress Wave. Opt. Lett. 2004, 29, 1227-1229.

(1318) Xu, T.; Rohozinski, J.; Zhao, W. X.; Moorefield, E. C.; Atala, A.; Yoo, J. J. Inkjet-Mediated Gene Transfection into Living Cells Combined with Targeted Delivery. Tissue Engineering Part A 2009, 15, 95-101.

(1319) Cui, X.; Dean, D.; Ruggeri, Z. M.; Boland, T. Cell Damage Evaluation of Thermal Inkjet Printed Chinese Hamster Ovary Cells. Biotechnol. Bioeng. 2010, 106, 963-969.

(1320) Cui, X. F.; Boland, T. Human Microvasculature Fabrication Using Thermal Inkjet Printing Technology. Biomaterials 2009, 30, $6221-6227$.

(1321) Stevenson, D. J.; Gunn-Moore, F. J.; Campbell, P.; Dholakia, K. Single Cell Optical Transfection. Journal of the Royal Society Interface 2010, 7, 863-871.

(1322) Yao, C. P.; Zhang, Z. X.; Rahmanzadeh, R.; Huettmann, G. Laser-Based Gene Transfection and Gene Therapy. IEEE Trans. NanoBiosci. 2008, 7, 111-119.

(1323) Vogel, A.; Linz, N.; Freidank, S.; Paltauf, G. Femtosecond-Laser-Induced Nanocavitation in Water: Implications for Optical Breakdown Threshold and Cell Surgery. Phys. Rev. Lett. 2008, 100, 038102.

(1324) Umebayashi, Y.; Miyamoto, Y.; Wakita, M.; Kobayashi, A.; Nishisaka, T. Elevation of Plasma Membrane Permeability on Laser Irradiation of Extracellular Latex Particles. J. Biochem. 2003, 134, 219-224. 
(1325) Yao, C. P.; Rahmanzadeh, R.; Endl, E.; Zhang, Z. X.; Gerdes, J.; Huttmann, G. Elevation of Plasma Membrane Permeability by Laser Irradiation of Selectively Bound Nanoparticles. Journal of Biomedical Optics 2005, 10, 064012.

(1326) Yao, C. P.; Qu, X. C.; Zhang, Z. X.; Huttmann, G.; Rahmanzadeh, R. Influence of Laser Parameters on Nanoparticle-Induced Membrane Permeabilization. Journal of Biomedical Optics 2009, 14, 054034.

(1327) Tong, L.; Zhao, Y.; Huff, T. B.; Hansen, M. N.; Wei, A.; Cheng, J. X. Gold Nanorods Mediate Tumor Cell Death by Compromising Membrane Integrity. Adv. Mater. 2007, 19, 3136-+.

(1328) Gu, L.; Koymen, A. R.; Mohanty, S. K. Crystalline Magnetic Carbon Nanoparticle Assisted Photothermal Delivery into Cells Using Cw near-Infrared Laser Beam. Sci. Rep. 2014, 4, 5106

(1329) Lyu, Z. L.; Zhou, F.; Liu, Q.; Xue, H.; Yu, Q.; Chen, H. A Universal Platform for Macromolecular Delivery into Cells Using Gold Nanoparticle Layers Via the Photoporation Effect. Adv. Funct. Mater. 2016, 26, 5787-5795.

(1330) Palumbo, G.; Caruso, M.; Crescenzi, E.; Tecce, M. F.; Roberti, G.; Colasanti, A. Targeted Gene Transfer in Eucaryotic Cells by Dye-Assisted Laser Optoporation. Journal of Photochemistry and Photobiology B-Biology 1996, 36, 41-46.

(1331) Vogel, A.; Noack, J.; Huttman, G.; Paltauf, G. Mechanisms of Femtosecond Laser Nanosurgery of Cells and Tissues. Applied Physics B-Lasers and Optics 2005, 81, 1015-1047.

(1332) Paterson, L.; Agate, B.; Comrie, M.; Ferguson, R.; Lake, T. K.; Morris, J. E.; Carruthers, A. E.; Brown, C. T. A.; Sibbett, W.; Bryant, P. E.et al. Photoporation and Cell Transfection Using a Violet Diode Laser. Opt. Express 2005, 13, 595-600.

(1333) Rhodes, K.; Clark, I.; Zatcoff, M.; Eustaquio, T.; Hoyte, K. L.; Koller, M. R. Cellular Laserfection. Laser Manipulation of Cells and Tissues 2007, 82, 309-333.

(1334) Tsukakoshi, M.; Kurata, S.; Nomiya, Y.; Ikawa, Y.; Kasuya, T. A Novel Method of DNA Transfection by Laser Microbeam Cell Surgery. Applied Physics B-Photophysics and Laser Chemistry 1984, 35, 135-140.

(1335) Kurata, S.; Tsukakoshi, M.; Kasuya, T.; Ikawa, Y. The Laser Method for Efficient Introduction of Foreign DNA into CulturedCells. Exp. Cell Res. 1986, 162, 372-378.

(1336) Tao, W.; Wilkinson, J.; Stanbridge, E. J.; Berns, M. W. Direct Gene-Transfer into Human Cultured-Cells Facilitated by Laser Micropuncture of the Cell-Membrane. Proc. Natl. Acad. Sci. U. S. A. 1987, 84, 4180-4184.

(1337) Tirlapur, U. K.; Konig, K. Targeted Transfection by Femtosecond Laser. Nature 2002, 418, $290-291$.

(1338) Guo, Y. D.; Liang, H.; Berns, M. W. Laser-Mediated Gene-Transfer in Rice. Physiol. Plant. 1995, 93, $19-24$.

(1339) Shirahata, Y.; Ohkohchi, N.; Itagak, H.; Satomi, S. New Technique for Gene Transfection Using Laser Irradiation. J. Investig. Med. 2001, 49, 184-190.

(1340) Schneckenburger, H.; Hendinger, A.; Sailer, R.; Strauss, W. S.; Schmitt, M. Laser-Assisted Optoporation of Single Cells. J Biomed Opt 2002, 7, 410-416.

(1341) Mohanty, S. K.; Sharma, M.; Gupta, P. K. Laser-Assisted Microinjection into Targeted Animal Cells. Biotechnol. Lett 2003, 25, 895-899.

(1342) Sagi, S.; Knoll, T.; Trojan, L.; Schaaf, A.; Alken, P.; Michel, M. S. Gene Delivery into Prostate Cancer Cells by Holmium Laser Application. Prostate Cancer Prostatic Dis. 2003, 6, 127-130. 
(1343) Clark, I. B.; Hanania, E. G.; Stevens, J.; Gallina, M.; Fieck, A.; Brandes, R.; Palsson, B. O.; Koller, M. R. Optoinjection for Efficient Targeted Delivery of a Broad Range of Compounds and Macromolecules into Diverse Cell Types. Journal of Biomedical Optics 2006, 11, 014034.

(1344) Tsampoula, X.; Garces-Chavez, V.; Comrie, M.; Stevenson, D. J.; Agate, B.; Brown, C. T. A.; Gunn-Moore, F.; Dholakia, K. Femtosecond Cellular Transfection Using a Nondiffracting Light Beam. Appl. Phys. Lett. 2007, 91, 053902.

(1345) He, H.; Kong, S. K.; Lee, R. K.; Suen, Y. K.; Chan, K. T. Targeted Photoporation and Transfection in Human Hepg2 Cells by a Fiber Femtosecond Laser at 1554 Nm. Opt. Lett. 2008, 33, 2961-2963.

(1346) Schinkel, H.; Jacobs, P.; Schillberg, S.; Wehner, M. Infrared Picosecond Laser for Perforation of Single Plant Cells. Biotechnol. Bioeng. 2008, 99, 244-248.

(1347) Tsampoula, X.; Taguchi, K.; Cizmar, T.; Garces-Chavez, V.; Ma, N.; Mohanty, S.; Mohanty, K.; Gunn-Moore, F.; Dholakia, K. Fibre Based Cellular Transfection. Opt. Express 2008, 16, 17007-17013.

(1348) Uchugonova, A.; Konig, K.; Bueckle, R.; Isemann, A.; Tempea, G. Targeted Transfection of Stem Cells with Sub-20 Femtosecond Laser Pulses. Opt. Express 2008, 16, 9357-9364.

(1349) Hosokawa, Y.; Iguchi, S.; Yasukuni, R.; Hiraki, Y.; Shukunami, C.; Masuhara, H. Gene Delivery Process in a Single Animal Cell after Femtosecond Laser Microinjection. Appl. Surf. Sci. 2009, 255, 9880-9884.

(1350) Antkowiak, M.; Torres-Mapa, M. L.; Gunn-Moore, F.; Dholakia, K. Application of Dynamic Diffractive Optics for Enhanced Femtosecond Laser Based Cell Transfection. Journal of Biophotonics 2010, 3, 696-705.

(1351) Mthunzi, P.; Dholakia, K.; Gunn-Moore, F. Phototransfection of Mammalian Cells Using Femtosecond Laser Pulses: Optimization and Applicability to Stem Cell Differentiation. Journal of Biomedical Optics 2010, $15,041507$.

(1352) Torres-Mapa, M. L.; Angus, L.; Ploschner, M.; Dholakia, K.; Gunn-Moore, F. J. Transient Transfection of Mammalian Cells Using a Violet Diode Laser. Journal of Biomedical Optics 2010, 15, 041506.

(1353) Hosokawa, Y.; Ochi, H.; lino, T.; Hiraoka, A.; Tanaka, M. Photoporation of Biomolecules into Single Cells in Living Vertebrate Embryos Induced by a Femtosecond Laser Amplifier. PLoS One 2011, 6, e27677.

(1354) Soman, P.; Zhang, W. D.; Umeda, A.; Zhang, Z. J.; Chen, S. C. Femtosecond Laser-Assisted Optoporation for Drug and Gene Delivery into Single Mammalian Cells. Journal of Biomedical Nanotechnology 2011, 7, 334-341.

(1355) Antkowiak, M.; Torres-Mapa, M. L.; Witts, E. C.; Miles, G. B.; Dholakia, K.; Gunn-Moore, F. J. Fast Targeted Gene Transfection and Optogenetic Modification of Single Neurons Using Femtosecond Laser Irradiation. Sci. Rep. $2013,3,3281$.

(1356) Breunig, H. G.; Uchugonova, A.; Batista, A.; Konig, K. High-Throughput Continuous Flow Femtosecond Laser-Assisted Cell Optoporation and Transfection. Microsc. Res. Tech. 2014, 77, 974-979.

(1357) Breunig, H. G.; Uchugonova, A.; Batista, A.; Konig, K. Software-Aided Automatic Laser Optoporation and Transfection of Cells. Sci. Rep. 2015, 5, 11185

(1358) Uchugonova, A.; Breunig, H. G.; Batista, A.; Konig, K. Optical Reprogramming of Human Cells in an Ultrashort Femtosecond Laser Microfluidic Transfection Platform. J Biophotonics 2015, 9, 942-947. 
(1359) Dhakal, K.; Black, B.; Mohanty, S. Introduction of Impermeable Actin-Staining Molecules to Mammalian Cells by Optoporation. Sci. Rep. 2014, 4, 6553.

(1360) Stracke, F.; Rieman, I.; Konig, K. Optical Nanoinjection of Macromolecules into Vital Cells. Journal of Photochemistry and Photobiology B-Biology 2005, 81, 136-142.

(1361) Torres-Mapa, M. L.; Antkowiak, M.; Cizmarova, H.; Ferrier, D. E. K.; Dholakia, K.; Gunn-Moore, F. J. Integrated Holographic System for All-Optical Manipulation of Developing Embryos. Biomedical Optics Express 2011, 2, 1564-1575.

(1362) Peng, C.; Palazzo, R. E.; Wilke, I. Laser Intensity Dependence of Femtosecond near-Infrared Optoinjection. Physical Review $E$ 2007, 75, 041903.

(1363) Lei, M.; Xu, H. P.; Yang, H.; Yao, B. L. Femtosecond Laser-Assisted Microinjection into Living Neurons. J. Neurosci. Methods 2008, 174, 215-218.

(1364) Marchington, R. F.; Arita, Y.; Tsampoula, X.; Gunn-Moore, F. J.; Dholakia, K. Optical Injection of Mammalian Cells Using a Microfluidic Platform. Biomedical Optics Express 2010, 1, 527-536.

(1365) Rendall, H. A.; Marchington, R. F.; Praveen, B. B.; Bergmann, G.; Arita, Y.; Heisterkamp, A.; Gunn-Moore, F. J.; Dholakia, K. High-Throughput Optical Injection of Mammalian Cells Using a Bessel Light Beam. Lab on a Chip 2012, 12, 4816-4820.

(1366) Rudhall, A. P.; Antkowiak, M.; Tsampoula, X.; Mazilu, M.; Metzger, N. K.; Gunn-Moore, F.; Dholakia, K. Exploring the Ultrashort Pulse Laser Parameter Space for Membrane Permeabilisation in Mammalian Cells. Sci. Rep. $2012,2,858$.

(1367) Foldes-Papp, Z.; Konig, K.; Studier, H.; Buckle, R.; Breunig, H. G.; Uchugonova, A.; Kostner, G. M. Trafficking of Mature Mirna-122 into the Nucleus of Live Liver Cells. Curr. Pharm. Biotechnol. 2009, 10, 569-578.

(1368) Roth, C. C.; Barnes, R. A.; Ibey, B. L.; Glickman, R. D.; Beier, H. T. Short Infrared (Ir) Laser Pulses Can Induce Nanoporation. Proc SPIE 2016, 9690, 96901L.

(1369) Mcdougall, C.; Stevenson, D. J.; Brown, C. T. A.; Gunn-Moore, F.; Dholakia, K. Targeted Optical Injection of Gold Nanoparticles into Single Mammalian Cells. Journal of Biophotonics 2009, 2, 736-743.

(1370) Umanzor-Alvarez, J.; Wade, E. C.; Gifford, A.; Nontapot, K.; Cruz-Reese, A.; Gotoh, T.; Sible, J. C.; Khodaparast, G. A. NearInfrared Laser Delivery of Nanoparticles to Developing Embryos: A Study of Efficacy and Viability. Biotechnology Journal 2011, 6, 519-524.

(1371) Waleed, M.; Hwang, S. U.; Kim, J. D.; Shabbir, I.; Shin, S. M.; Lee, Y. G. Single-Cell Optoporation and Transfection Using Femtosecond Laser and Optical Tweezers. Biomedical Optics Express 2013, 4, 1533-1547.

(1372) Karande, P.; Jain, A.; Ergun, K.; Kispersky, V.; Mitragotri, S. Design Principles of Chemical Penetration Enhancers for Transdermal Drug Delivery. Proc. Natl. Acad. Sci. U. S. A. 2005, 102, 4688-4693.

(1373) Faizal, A.; Geelen, D. Saponins and Their Role in Biological Processes in Plants. Phytochem. Rev. 2013, 12, 877-893.

(1374) Yu, Z. W.; Quinn, P. J. The Modulation of Membrane Structure and Stability by Dimethyl Sulphoxide (Review). Mol. Membr. Biol. 1998, 15, 59-68.

(1375) Anchordoguy, T. J.; Carpenter, J. F.; Crowe, J. H.; Crowe, L. M. Temperature-Dependent Perturbation of PhospholipidBilayers by Dimethylsulfoxide. Biochim. Biophys. Acta 1992, 1104, 117-122. 
(1376) Yu, Z. W.; Quinn, P. J. Solvation Effects of Dimethyl Sulphoxide on the Structure of Phospholipid Bilayers. Biophys. Chem. 1998, 70, 35-39.

(1377) Lin, S. Y.; Duan, K. J.; Lin, T. C. Direct or Indirect Skin Lipid-Ordering Effect of Pyrrolidone Carboxylate Sodium after Topical Treatment with Penetration Enhancers. Biomed. Mater. Eng. 1995, 5, 9-20.

(1378) Gurtovenko, A. A.; Anwar, J. Modulating the Structure and Properties of Cell Membranes: The Molecular Mechanism of Action of Dimethyl Sulfoxide. J. Phys. Chem. B 2007, 111, 10453-10460.

(1379) Hughes, Z. E.; Mancera, R. L. Molecular Dynamics Simulations of Mixed Dopc-Beta-Sitosterol Bilayers and Their Interactions with Dmso. Soft Matter 2013, 9, 2920-2935.

(1380) Holte, L. L.; Gawrisch, K. Determining Ethanol Distribution in Phospholipid Multilayers with Mas-Noesy Spectra. Biochemistry $1997,36,4669-4674$

(1381) Feller, S. E.; Brown, C. A.; Nizza, D. T.; Gawrisch, K. Nuclear Overhauser Enhancement Spectroscopy Cross-Relaxation Rates and Ethanol Distribution across Membranes. Biophys. J. 2002, 82, 1396-1404.

(1382) Gurtovenko, A. A.; Anwar, J. Interaction of Ethanol with Biological Membranes: The Formation of Non-Bilayer Structures within the Membrane Interior and Their Significance. J. Phys. Chem. B 2009, 113, 1983-1992.

(1383) Ly, H. V.; Longo, M. L. The Influence of Short-Chain Alcohols on Interfacial Tension, Mechanical Properties, Area/Molecule, and Permeability of Fluid Lipid Bilayers. Biophys. J. 2004, 87, 1013-1033.

(1384) Dickey, A. N.; Faller, R. How Alcohol Chain-Length and Concentration Modulate Hydrogen Bond Formation in a Lipid Bilayer. Biophys. J. 2007, 92, 2366-2376.

(1385) O'dea, S.; Annibaldi, V.; Gallagher, L.; Mulholland, J.; Molloy, E. L.; Breen, C. J.; Gilbert, J. L.; Martin, D. S.; Maguire, M.; Curry, F. R. Vector-Free Intracellular Delivery by Reversible Permeabilization. PLoS One 2017, 12, e0174779.

(1386) Jamur, M. C.; Oliver, C. Permeabilization of Cell Membranes. Immunocytochemical Methods and Protocols, Third Edition 2010, 588, 63-66.

(1387) Melkonyan, H.; Sorg, C.; Klempt, M. Electroporation Efficiency in Mammalian Cells Is Increased by Dimethyl Sulfoxide (Dmso). Nucleic Acids Res. 1996, 24, 4356-4357.

(1388) Assad-Garcia, J. S.; Bonnin-Jusserand, M.; Garmyn, D.; Guzzo, J.; Alexandre, H.; Grandvalet, C. An Improved Protocol for Electroporation of Oenococcus Oeni Atcc Baa-1163 Using Ethanol as Immediate Membrane Fluidizing Agent. Lett. Appl. Microbiol. 2008, 47, 333-338.

(1389) Fernandez, M. L.; Reigada, R. Effects of Dimethyl Sulfoxide on Lipid Membrane Electroporation. J. Phys. Chem. B 2014, 118, 9306-9312.

(1390) Helenius, A.; Simons, K. Solubilization of Membranes by Detergents. Biochim. Biophys. Acta 1975, $415,29-79$.

(1391) Linke, D. Detergents: An Overview. Methods Enzymol. 2009, 463, 603-617.

(1392) Lichtenberg, D.; Ahyayauch, H.; Alonso, A.; Goni, F. M. Detergent Solubilization of Lipid Bilayers: A Balance of Driving Forces. Trends Biochem. Sci 2013, 38, 85-93. 
(1393) Lorent, J. H.; Quetin-Leclercq, J.; Mingeot-Leclercq, M. P. The Amphiphilic Nature of Saponins and Their Effects on Artificial and Biological Membranes and Potential Consequences for Red Blood and Cancer Cells. Org. Biomol. Chem. 2014, 12, 88038822.

(1394) Nazari, M.; Kurdi, M.; Heerklotz, H. Classifying Surfactants with Respect to Their Effect on Lipid Membrane Order. Biophys. J. 2012, 102, 498-506.

(1395) Vaidyanathan, S.; Orr, B. G.; Holl, M. M. B. Detergent Induction of Hek 293a Cell Membrane Permeability Measured under Quiescent and Superfusion Conditions Using Whole Cell Patch Clamp. J. Phys. Chem. B 2014, 118, $2112-2123$.

(1396) Koley, D.; Bard, A. J. Triton X-100 Concentration Effects on Membrane Permeability of a Single Hela Cell by Scanning Electrochemical Microscopy (Secm). Proc. Natl. Acad. Sci. U. S. A. 2010, 107, 16783-16787.

(1397) Francis, G.; Kerem, Z.; Makkar, H. P. S.; Becker, K. The Biological Action of Saponins in Animal Systems: A Review. Br. J. Nutr. 2002, 88, 587-605.

(1398) Papadopoulou, K.; Melton, R. E.; Leggett, M.; Daniels, M. J.; Osbourn, A. E. Compromised Disease Resistance in SaponinDeficient Plants. Proc. Natl. Acad. Sci. U. S. A. 1999, 96, 12923-12928.

(1399) Podolak, I.; Galanty, A.; Sobolewska, D. Saponins as Cytotoxic Agents: A Review. Phytochem. Rev. 2010, 9, $425-474$.

(1400) Sun, H. X.; Xie, Y.; Ye, Y. P. Advances in Saponin-Based Adjuvants. Vaccine 2009, 27, 1787-1796.

(1401) Fuchs, H.; Bachran, D.; Panjideh, H.; Schellmann, N.; Weng, A.; Melzig, M. F.; Sutherland, M.; Bachran, C. Saponins as Tool for Improved Targeted Tumor Therapies. Curr. Drug Targets 2009, 10, 140-151.

(1402) Bangham, A. D.; Glauert, A. M.; Horne, R. W.; Dingle, J. T.; Lucy, J. A. Action of Saponin on Biological Cell Membranes. Nature 1962, 196, 952-955.

(1403) Lepers, A.; Cacan, R.; Verbert, A. Permeabilized Cells as a Way of Gaining Access to Intracellular Organelles - an Approach to Glycosylation Reactions. Biochimie 1990, 72, 1-5.

(1404) Keeney, S.; Linn, S. A Critical-Review of Permeabilized Cell Systems for Studying Mammalian DNA-Repair. Mutat. Res. 1990, 236, 239-252.

(1405) Elias, P. M.; Friend, D. S.; Goerke, J. Membrane Sterol Heterogeneity - Freeze-Fracture Detection with Saponins and Filipin. J. Histochem. Cytochem. 1979, 27, 1247-1260.

(1406) Frenkel, N.; Makky, A.; Sudji, I. R.; Wink, M.; Tanaka, M. Mechanistic Investigation of Interactions between Steroidal Saponin Digitonin and Cell Membrane Models. J. Phys. Chem. B 2014, 118, 14632-14639.

(1407) Gilabert-Oriol, R.; Mergel, K.; Thakur, M.; Von Mallinckrodt, B.; Melzig, M. F.; Fuchs, H.; Weng, A. Real-Time Analysis of Membrane Permeabilizing Effects of Oleanane Saponins. Biorg. Med. Chem. 2013, 21, 2387-2395.

(1408) Lorent, J.; Lins, L.; Domenech, O.; Quetin-Leclercq, J.; Brasseur, R.; Mingeot-Leclercq, M. P. Domain Formation and Permeabilization Induced by the Saponin Alpha-Hederin and Its Aglycone Hederagenin in a Cholesterol-Containing Bilayer. Langmuir 2014, 30, 4556-4569. 
(1409) Lorent, J.; Le Duff, C. S.; Quetin-Leclercq, J.; Mingeot-Leclercq, M. P. Induction of Highly Curved Structures in Relation to Membrane Permeabilization and Budding by the Triterpenoid Saponins, Alpha- and Delta-Hederin. J. Biol. Chem. 2013, 288, $14000-14017$.

(1410) Li, X. X.; Davis, B.; Haridas, V.; Gutterman, J. U.; Colombini, M. Proapoptotic Triterpene Electrophiles (Avicins) Form Channels in Membranes: Cholesterol Dependence. Biophys. J. 2005, 88, 2577-2584.

(1411) Wakasugi, H.; Kimura, T.; Haase, W.; Kribben, A.; Kaufmann, R.; Schulz, I. Calcium-Uptake into Acini from Rat Pancreas Evidence for Intracellular Atp-Dependent Calcium Sequestration. J. Membr. Biol. 1982, 65, 205-220.

(1412) Dunn, L. A.; Holz, R. W. Catecholamine Secretion from Digitonin-Treated Adrenal-Medullary Chromaffin Cells. J. Biol. Chem. 1983, 258, 4989-4993.

(1413) Authi, K. S.; Evenden, B. J.; Crawford, N. Metabolic and Functional Consequences of Introducing Inositol 1,4,5-Trisphosphate into Saponin-Permeabilized Human-Platelets. Biochem. J 1986, 233, 707-718.

(1414) Weigel, P. H.; Ray, D. A.; Oka, J. A. Quantitation of Intracellular Membrane-Bound Enzymes and Receptors in DigitoninPermeabilized Cells. Anal. Biochem. 1983, 133, 437-449.

(1415) Miyamoto, K.; Yamashita, T.; Tsukiyama, T.; Kitamura, N.; Minami, N.; Yamada, M.; Imai, H. Reversible Membrane Permeabilization of Mammalian Cells Treated with Digitonin and Its Use for Inducing Nuclear Reprogramming by Xenopus Egg Extracts. Cloning Stem Cells 2008, 10, 535-542.

(1416) Lukyanenko, V. Permeabilization of Cell Membrane for Delivery of Nano-Objects to Cellular Sub-Domains. Methods Mol. Biol. 2013, 991, 57-63.

(1417) Jacob, M. C.; Favre, M.; Bensa, J. C. Membrane Cell Permeabilization with Saponin and Multiparametric Analysis by FlowCytometry. Cytometry 1991, 12, 550-558.

(1418) Sander, B.; Andersson, J.; Andersson, U. Assessment of Cytokines by Immunofluorescence and the ParaformaldehydeSaponin Procedure. Immunol. Rev. 1991, 119, 65-93.

(1419) Jung, T.; Schauer, U.; Heusser, C.; Neumann, C.; Rieger, C. Detection of Intracellular Cytokines by Flow-Cytometry. J. Immunol. Methods 1993, 159, 197-207.

(1420) Pala, P.; Hussell, T.; Openshaw, P. J. M. Flow Cytometric Measurement of Intracellular Cytokines. J. Immunol. Methods 2000, 243, 107-124.

(1421) Miller, M. R.; Castellot, J. J.; Pardee, A. B. General-Method for Permeabilizing Monolayer and Suspension Cultured AnimalCells. Exp. Cell Res. 1979, 120, 421-425.

(1422) Balinska, M.; Samsonoff, W. A.; Galivan, J. Reversibly Permeable Hepatoma Cells in Culture. Biochim. Biophys. Acta 1982, $721,253-261$.

(1423) Nomura, S.; Kamiya, T.; Oishi, M. A Procedure to Introduce Protein Molecules into Living Mammalian Cells. Exp. Cell Res. 1986, 163, 434-444.

(1424) Siwko, M. E.; De Vries, A. H.; Mark, A. E.; Kozubek, A.; Marrink, S. J. Disturb or Stabilize? A Molecular Dynamics Study of the Effects of Resorcinolic Lipids on Phospholipid Bilayers. Biophys. J. 2009, 96, 3140-3153. 
(1425) Esteban-Martin, S.; Risselada, H. J.; Salgado, J.; Marrink, S. J. Stability of Asymmetric Lipid Bilayers Assessed by Molecular Dynamics Simulations. JACS 2009, 131, 15194-15202.

(1426) Kilinc, D.; Peyrin, J. M.; Soubeyre, V.; Magnifico, S.; Saias, L.; Viovy, J. L.; Brugg, B. Wallerian-Like Degeneration of Central Neurons after Synchronized and Geometrically Registered Mass Axotomy in a Three-Compartmental Microfluidic Chip. Neurotox. Res. 2011, 19, 149-161.

(1427) Lee, C. Y.; Romanova, E. V.; Sweedler, J. V. Laminar Stream of Detergents for Subcellular Neurite Damage in a Microfluidic Device: A Simple Tool for the Study of Neuroregeneration. Journal of Neural Engineering 2013, $10,036020$.

(1428) Oftedal, L.; Myhren, L.; Jokela, J.; Gausdal, G.; Sivonen, K.; Doskeland, S. O.; Herfindal, L. The Lipopeptide Toxins Anabaenolysin a and B Target Biological Membranes in a Cholesterol-Dependent Manner. Biochimica Et Biophysica ActaBiomembranes 2012, 1818, 3000-3009.

(1429) Zasloff, M. Antimicrobial Peptides of Multicellular Organisms. Nature 2002, 415, 389-395.

(1430) Brogden, K. A. Antimicrobial Peptides: Pore Formers or Metabolic Inhibitors in Bacteria? Nature Reviews Microbiology 2005, 3, 238-250.

(1431) Dinca, A.; Chien, W. M.; Chin, M. T. Intracellular Delivery of Proteins with Cell-Penetrating Peptides for Therapeutic Uses in Human Disease. Int. J. Mol. Sci. 2016, 17, 263.

(1432) Plank, C.; Zauner, W.; Wagner, E. Application of Membrane-Active Peptides for Drug and Gene Delivery across Cellular Membranes. Adv. Drug Del. Rev. 1998, 34, 21-35.

(1433) Midoux, P.; Mayer, R.; Monsigny, M. Membrane Permeabilization by Alpha-Helical Peptides: A Flow Cytometry Study. Biochim. Biophys. Acta 1995, 1239, 249-256.

(1434) Lioi, A. B.; Rodriguez, A. L.; Funderburg, N. T.; Feng, Z.; Weinberg, A.; Sieg, S. F. Membrane Damage and Repair in Primary Monocytes Exposed to Human Beta-Defensin-3. J. Leukoc. Biol. 2012, 92, 1083-1091.

(1435) Kyung, H.; Kim, H.; Lee, H.; Lee, S. J. Enhanced Intracellular Delivery of Macromolecules by Melittin Derivatives Mediated Cellular Uptake. J Ind Eng Chem 2018, 58, 290-295.

(1436) Plank, C.; Oberhauser, B.; Mechtler, K.; Koch, C.; Wagner, E. The Influence of Endosome-Disruptive Peptides on GeneTransfer Using Synthetic Virus-Like Gene-Transfer Systems. J. Biol. Chem. 1994, 269, 12918-12924.

(1437) Wyman, T. B.; Nicol, F.; Zelphati, O.; Scaria, P. V.; Plank, C.; Szoka, F. C. Design, Synthesis, and Characterization of a Cationic Peptide That Binds to Nucleic Acids and Permeabilizes Bilayers. Biochemistry 1997, 36, 3008-3017.

(1438) Zhao, X. W.; Wu, H. Y.; Lu, H. R.; Li, G. D.; Huang, Q. S. Lamp: A Database Linking Antimicrobial Peptides. PLoS One 2013, 8, e66557.

(1439) Waghu, F. H.; Gopi, L.; Barai, R. S.; Ramteke, P.; Nizami, B.; Idicula-Thomas, S. Camp: Collection of Sequences and Structures of Antimicrobial Peptides. Nucleic Acids Res. 2014, 42, D1154-D1158.

(1440) Bahar, A. A.; Ren, D. Antimicrobial Peptides. Pharmaceuticals (Basel) 2013, 6, 1543-1575.

(1441) Matsuzaki, K. Why and How Are Peptide-Lipid Interactions Utilized for Self-Defense? Magainins and Tachyplesins as Archetypes. Bba-Biomembranes 1999, 1462, 1-10. 
(1442) Wimley, W. C. Describing the Mechanism of Antimicrobial Peptide Action with the Interfacial Activity Model. ACS Chem. Biol. 2010, 5, 905-917.

(1443) Huang, H. W. Action of Antimicrobial Peptides: Two-State Model. Biochemistry 2000, 39, 8347-8352.

(1444) Raghuraman, H.; Chattopadhyay, A. Melittin: A Membrane-Active Peptide with Diverse Functions. Biosci. Rep. 2007, 27, 189223.

(1445) Ladokhin, A. S.; Selsted, M. E.; White, S. H. Sizing Membrane Pores in Lipid Vesicles by Leakage of Co-Encapsulated Markers: Pore Formation by Melittin. Biophys. J. 1997, 72, 1762-1766.

(1446) Lee, M. T.; Hung, W. C.; Chen, F. Y.; Huang, H. W. Mechanism and Kinetics of Pore Formation in Membranes by WaterSoluble Amphipathic Peptides. Proc. Natl. Acad. Sci. U. S. A. 2008, 105, 5087-5092.

(1447) Lee, M. T.; Sun, T. L.; Hung, W. C.; Huang, H. W. Process of Inducing Pores in Membranes by Melittin. Proc. Natl. Acad. Sci. U. S. A. 2013, 110, 14243-14248.

(1448) Tamba, Y.; Yamazaki, M. Magainin 2-Induced Pore Formation in the Lipid Membranes Depends on Its Concentration in the Membrane Interface. J. Phys. Chem. B 2009, 113, 4846-4852.

(1449) Tamba, Y.; Ariyama, H.; Levadny, V.; Yamazaki, M. Kinetic Pathway of Antimicrobial Peptide Magainin 2-Induced Pore Formation in Lipid Membranes. J. Phys. Chem. B 2010, 114, 12018-12026.

(1450) Gregory, S. M.; Pokorny, A.; Almeida, P. F. F. Magainin 2 Revisited: A Test of the Quantitative Model for the All-or-None Permeabilization of Phospholipid Vesicles. Biophys. J. 2009, 96, 116-131.

(1451) Lee, C. C.; Sun, Y.; Qian, S.; Huang, H. W. Transmembrane Pores Formed by Human Antimicrobial Peptide LI-37. Biophys. J. 2011, 100, 1688-1696.

(1452) Patel, H.; Huynh, Q.; Barlehner, D.; Heerklotz, H. Additive and Synergistic Membrane Permeabilization by Antimicrobial (Lipo)Peptides and Detergents. Biophys. J. 2014, 106, 2115-2125.

(1453) Rakowska, P. D.; Jiang, H. B.; Ray, S.; Pyne, A.; Lamarre, B.; Carr, M.; Judge, P. J.; Ravi, J.; Gerling, U. I. M.; Koksch, B.et al. Nanoscale Imaging Reveals Laterally Expanding Antimicrobial Pores in Lipid Bilayers. Proc. Natl. Acad. Sci. U. S. A. 2013, $110,8918-8923$.

(1454) Sengupta, D.; Leontiadou, H.; Mark, A. E.; Marrink, S. J. Toroidal Pores Formed by Antimicrobial Peptides Show Significant Disorder. Biochimica Et Biophysica Acta-Biomembranes 2008, 1778, 2308-2317.

(1455) Binder, H.; Lindblom, G. Charge-Dependent Translocation of the Trojan Peptide Penetratin across Lipid Membranes. Biophys. J. 2003, 85, 982-995.

(1456) Miteva, M.; Andersson, M.; Karshikoff, A.; Otting, G. Molecular Electroporation: A Unifying Concept for the Description of Membrane Pore Formation by Antibacterial Peptides, Exemplified with Nk-Lysin. FEBS Lett. 1999, 462, $155-158$.

(1457) Gurtovenko, A. A.; Vattulainen, I. Pore Formation Coupled to Ion Transport through Lipid Membranes as Induced by Transmembrane Ionic Charge Imbalance: Atomistic Molecular Dynamics Study. JACS 2005, 127, $17570-17571$.

(1458) Leontiadou, H.; Mark, A. E.; Marrink, S. J. Antimicrobial Peptides in Action. JACS 2006, 128, $12156-12161$. 
(1459) Jean-Francois, F.; Elezgaray, J.; Berson, P.; Vacher, P.; Dufourc, E. J. Pore Formation Induced by an Antimicrobial Peptide: Electrostatic Effects. Biophys. J. 2008, 95, 5748-5756.

(1460) Herce, H. D.; Garcia, A. E. Molecular Dynamics Simulations Suggest a Mechanism for Translocation of the Hiv-1 Tat Peptide across Lipid Membranes. Proc. Natl. Acad. Sci. U. S. A. 2007, 104, 20805-20810.

(1461) Lee, M. T.; Chen, F. Y.; Huang, H. W. Energetics of Pore Formation Induced by Membrane Active Peptides. Biochemistry 2004, 43, 3590-3599.

(1462) Gregory, S. M.; Cavenaugh, A.; Journigan, V.; Pokorny, A.; Almeida, P. F. F. A Quantitative Model for the All-or-None Permeabilization of Phospholipid Vesicles by the Antimicrobial Peptide Cecropin A. Biophys. J. 2008, 94, 1667-1680.

(1463) Last, N. B.; Miranker, A. D. Common Mechanism Unites Membrane Poration by Amyloid and Antimicrobial Peptides. Proc. Natl. Acad. Sci. U. S. A. 2013, 110, 6382-6387.

(1464) Henriques, S. T.; Melo, M. N.; Castanho, M. a. R. B. Cell-Penetrating Peptides and Antimicrobial Peptides: How Different Are They? Biochem. J 2006, 399, 1-7.

(1465) Ferrer-Miralles, N.; Vazquez, E.; Villaverde, A. Membrane-Active Peptides for Non-Viral Gene Therapy: Making the Safest Easier. Trends Biotechnol. 2008, 26, 267-275.

(1466) Kasianowicz, J. J.; Brandin, E.; Branton, D.; Deamer, D. W. Characterization of Individual Polynucleotide Molecules Using a Membrane Channel. Proc. Natl. Acad. Sci. U. S. A. 1996, 93, 13770-13773.

(1467) Czajkowsky, D. M.; Hotze, E. M.; Shao, Z. F.; Tweten, R. K. Vertical Collapse of a Cytolysin Prepore Moves Its Transmembrane Beta-Hairpins to the Membrane. EMBO J. 2004, 23, 3206-3215.

(1468) Hodel, A. W.; Leung, C.; Dudkina, N. V.; Saibil, H. R.; Hoogenboom, B. W. Atomic Force Microscopy of Membrane Pore Formation by Cholesterol Dependent Cytolysins. Curr. Opin. Struct. Biol. 2016, 39, 8-15.

(1469) Ahnerthilger, G.; Mach, W.; Fohr, K. J.; Gratzl, M. Poration by Alpha-Toxin and Streptolysin-O - an Approach to Analyze Intracellular Processes. Methods Cell Biol. 1989, 31, 63-90.

(1470) Wendland, M.; Subramani, S. Cytosol-Dependent Peroxisomal Protein Import in a Permeabilized Cell System. J. Cell Biol. 1993, 120, 675-685.

(1471) Barry, E. L. R.; Gesek, F. A.; Friedman, P. A. Introduction of Antisense Oligonucleotides into Cells by Permeabilization with Streptolysin O. BioTechniques 1993, 15, 1016-1018.

(1472) Harvey, A. N.; Costa, N. D.; Savage, J. R. K. Electroporation and Streptolysin-O - a Comparison of Poration Techniques. Mutat. Res. 1994, 315, 17-23.

(1473) Fawcett, J. M.; Harrison, S. M.; Orchard, C. H. A Method for Reversible Permeabilization of Isolated Rat Ventricular Myocytes. Exp. Physiol. 1998, 83, 293-303.

(1474) Ogino, S.; Kubo, S.; Umemoto, R.; Huang, S. X.; Nishida, N.; Shimada, I. Observation of Nmr Signals from Proteins Introduced into Living Mammalian Cells by Reversible Membrane Permeabilization Using a Pore-Forming Toxin, Streptolysin O. JACS 2009, 131, 10834-10835. 
(1475) Ma, X.; Zhou, P.; Wong, S. W.; Warner, M.; Chaulagain, C.; Comenzo, R. L. Sirna Targeting the Kappa Light Chain Constant Region: Preclinical Testing of an Approach to Nonfibrillar and Fibrillar Light Chain Deposition Diseases. Gene Ther. 2016, 23, 727-733.

(1476) Spiller, D. G.; Tidd, D. M. Nuclear Delivery of Antisense Oligodeoxynucleotides through Reversible Permeabilization of Human Leukemia-Cells with Streptolysin-O. Antisense Res. Dev. 1995, 5, $13-21$.

(1477) Broughton, C. M.; Spiller, D. G.; Pender, N.; Komorovskaya, M.; Grzybowski, J.; Giles, R. V.; Tidd, D. M.; Clark, R. E. Preclinical Studies of Streptolysin-O in Enhancing Antisense Oligonucleotide Uptake in Harvests from Chronic Myeloid Leukaemia Patients. Leukemia 1997, 11, 1435-1441.

(1478) Giles, R. V.; Grzybowski, J.; Spiller, D. G.; Tidd, D. M. Enhanced Antisense Effects Resulting from an Improved Streptolysin-O Protocol for Oligodeoxynucleotide Delivery into Human Leukaemia Cells. Nucleosides Nucleotides 1997, 16, $1155-1163$.

(1479) Giles, R. V.; Spiller, D. G.; Clark, R. E.; Tidd, D. M. C-Myc Antisense Morpholino Oligonucleotide Analogue Induces MisSplicing of Mrna in Living Cells. Blood 1998, 92, 244b-245b.

(1480) Giles, R. V.; Spiller, D. G.; Grzybowski, J.; Clark, R. E.; Tidd, D. M. Selecting Optimal Oligonucleotide Composition for Maximal Antisense Effect Following Streptolysin O-Mediated Delivery into Human Leukaemia Cells. Nucleic Acids Res. 1998, $26,1567-1575$

(1481) Clark, R. E.; Grzybowski, J.; Broughton, C. M.; Pender, N. T.; Spiller, D. G.; Brammer, C. G.; Giles, R. V.; Tidd, D. M. Clinical Use of Streptolysin-O to Facilitate Antisense Oligodeoxyribonucleotide Delivery for Purging Autografts in Chronic Myeloid Leukaemia. Bone Marrow Transplant. 1999, 23, 1303-1308.

(1482) Giles, R. V.; Spiller, D. G.; Clark, R. E.; Tidd, D. M. Antisense Morpholino Oligonucleotide Analog Induces Missplicing of CMyc Mrna. Antisense Nucleic Acid Drug Dev. 1999, 9, 213-220.

(1483) Giles, R. V.; Spiller, D. G.; Tidd, D. M. Chimeric Oligodeoxynucleotide Analogs: Chemical Synthesis, Purification, and Molecular and Cellular Biology Protocols. Methods Enzymol. 2000, 313, 95-135.

(1484) Lin, Y. P.; Ma, W. L.; Benchimol, S. Pidd, a New Death-Domain-Containing Protein, Is Induced by P53 and Promotes Apoptosis. Nat. Genet. 2000, 26, 122-125.

(1485) Faria, M.; Spiller, D. G.; Dubertret, C.; Nelson, J. S.; White, M. R. H.; Scherman, D.; Helene, C.; Giovannangeli, C. Phosphoramidate Oligonucleotides as Potent Antisense Molecules in Cells and in Vivo. Nat. Biotechnol. 2001, $19,40-44$.

(1486) Wu, J. R.; Berland, K. M. Comparing the Intracellular Mobility of Fluorescent Proteins Following in Vitro Expression or Cell Loading with Streptolysin-O. Journal of Biomedical Optics 2008, 13, 031214.

(1487) Nagahama, M.; Ohkubo, A.; Oda, M.; Kobayashi, K.; Amimoto, K.; Miyamoto, K.; Sakurai, J. Clostridium Perfringens Tpel Glycosylates the Rac and Ras Subfamily Proteins. Infect. Immun. 2011, 79, 905-910.

(1488) Teng, K. W.; Ishitsuka, Y.; Ren, P.; Youn, Y. A.; Deng, X.; Ge, P. H.; Belmont, A. S.; Selvin, P. R. Labeling Proteins inside Living Cells Using External Fluorophores for Microscopy. Elife 2016, 5, e20378.

(1489) Fu, H. M.; Ding, J.; Flutter, B.; Gao, B. Investigation of Endogenous Antigen Processing by Delivery of an Intact Protein into Cells. J. Immunol. Methods 2008, 335, 90-97. 
(1490) Bekei, B.; Rose, H. M.; Herzig, M.; Selenko, P. In-Cell Nmr in Mammalian Cells: Part 2. Methods Mol. Biol. 2012, 895, 55-66.

(1491) Hakelien, A. M.; Landsverk, H. B.; Robl, J. M.; Skalhegg, B. S.; Collas, P. Reprogramming Fibroblasts to Express T-Cell Functions Using Cell Extracts. Nat. Biotechnol. 2002, 20, 460-466.

(1492) Hakelien, A. M.; Gaustad, K. G.; Collas, P. Transient Alteration of Cell Fate Using a Nuclear and Cytoplasmic Extract of an Insulinoma Cell Line. Biochem. Biophys. Res. Commun. 2004, 316, 834-841.

(1493) Taranger, C. K.; Noer, A.; Sorensen, A. L.; Hakelien, A. M.; Boquest, A. C.; Collas, P. Induction of Dedifferentiation, Genomewide Transcriptional Programming, and Epigenetic Reprogramming by Extracts of Carcinoma and Embryonic Stem Cells. Mol. Biol. Cell 2005, 16, 5719-5735.

(1494) Håkelien, A.-M.; Gaustad, K. G.; Collas, P. In Nuclear Reprogramming: Methods and Protocols; Pells, S., Ed.; Humana Press: Totowa, NJ, 2006; Vol. 325.

(1495) Neri, T.; Monti, M.; Rebuzzini, P.; Merico, V.; Garagna, S.; Redi, C. A.; Zuccott, M. Mouse Fibroblasts Are Reprogrammed to Oct-4 and Rex-1 Gene Expression and Alkaline Phosphatase Activity by Embryonic Stem Cell Extracts. Cloning Stem Cells 2007, 9, 394-406.

(1496) Miyamoto, K.; Furusawa, T.; Ohnuki, M.; Goel, S.; Tokunaga, T.; Minami, N.; Yamada, M.; Ohsumi, K.; Imai, H. Reprogramming Events of Mammalian Somatic Cells Induced by Xenopus Laevis Egg Extracts. Mol. Reprod. Dev 2007, 74, 1268-1277.

(1497) Bui, H. T.; Wakayama, S.; Kishigami, S.; Kim, J. H.; Van Thuan, N.; Wakayama, T. The Cytoplasm of Mouse Germinal Vesicle Stage Oocytes Can Enhance Somatic Cell Nuclear Reprogramming. Development 2008, 135, 3935-3945.

(1498) Singhal, N.; Graumann, J.; Wu, G. M.; Arauzo-Bravo, M. J.; Han, D. W.; Greber, B.; Gentile, L.; Mann, M.; Scholer, H. R. Chromatin-Remodeling Components of the Baf Complex Facilitate Reprogramming. Cell 2010, 141, $943-955$.

(1499) Zhan, W. J.; Liu, Z. P.; Liu, Y.; Ke, Q. C.; Ding, Y. Y.; Lu, X. Y.; Wang, Z. C. Modulation of Rabbit Corneal Epithelial Cells Fate Using Embryonic Stem Cell Extract. Mol. Vis. 2010, 16, 1154-1161.

(1500) Cho, H. J.; Lee, C. S.; Kwon, Y. W.; Paek, J. S.; Lee, S. H.; Hur, J.; Lee, E. J.; Roh, T. Y.; Chu, I. S.; Leem, S. H.et al. Induction of Pluripotent Stem Cells from Adult Somatic Cells by Protein-Based Reprogramming without Genetic Manipulation. Blood 2010, 116, 386-395.

(1501) Han, J. N.; Sachdev, P. S.; Sidhu, K. S. A Combined Epigenetic and Non-Genetic Approach for Reprogramming Human Somatic Cells. PLoS One 2010, 5, e12297.

(1502) Ganier, O.; Bocquet, S.; Peiffer, I.; Brochard, V.; Arnaud, P.; Puy, A.; Jouneau, A.; Feil, R.; Renard, J. P.; Mechali, M. Synergic Reprogramming of Mammalian Cells by Combined Exposure to Mitotic Xenopus Egg Extracts and Transcription Factors. Proc. Natl. Acad. Sci. U. S. A. 2011, 108, 17331-17336.

(1503) Ostrup, O.; Hyttel, P.; Klaerke, D. A.; Collas, P. Remodeling of Ribosomal Genes in Somatic Cells by Xenopus Egg Extract. Biochem. Biophys. Res. Commun. 2011, 412, 487-493. 
(1504) Bui, H. T.; Kwon, D. N.; Kang, M. H.; Oh, M. H.; Park, M. R.; Park, W. J.; Paik, S. S.; Thuan, N. V.; Kim, J. H. Epigenetic Reprogramming in Somatic Cells Induced by Extract from Germinal Vesicle Stage Pig Oocytes. Development 2012, 139, 4330-4340.

(1505) Rathbone, A. J.; Liddell, S.; Campbell, K. H. S. Proteomic Analysis of Early Reprogramming Events in Murine Somatic Cells Incubated with Xenopus Laevis Oocyte Extracts Demonstrates Network Associations with Induced Pluripotency Markers. Cellular Reprogramming 2013, 15, 269-280.

(1506) Xiong, X. R.; Lan, D. L.; Li, J.; Zi, X. D.; Ma, L.; Wang, Y. Cellular Extract Facilitates Nuclear Reprogramming by Altering DNA Methylation and Pluripotency Gene Expression. Cellular Reprogramming 2014, 16, 215-222.

(1507) Faruqi, A. F.; Egholm, M.; Glazer, P. M. Peptide Nucleic Acid-Targeted Mutagenesis of a Chromosomal Gene in Mouse Cells. Proc. Natl. Acad. Sci. U. S. A. 1998, 95, 1398-1403.

(1508) Boutimah-Hamoudi, F.; Leforestier, E.; Senamaud-Beaufort, C.; Nielsen, P. E.; Giovannangeli, C.; Saison-Behmoaras, T. E. Cellular Antisense Activity of Peptide Nucleic Acid (Pnas) Targeted to Hiv-1 Polypurine Tract (Ppt) Containing Rna. Nucleic Acids Res. 2007, 35, 3907-3917.

(1509) Kummer, S.; Knoll, A.; Socher, E.; Bethge, L.; Herrmann, A.; Seitz, O. Fluorescence Imaging of Influenza H1n1 Mrna in Living Infected Cells Using Single-Chromophore Fit-Pna. Angewandte Chemie-International Edition 2011, 50, 1931 -1934.

(1510) Paillasson, S.; Vandecorput, M.; Dirks, R. W.; Tanke, H. J.; Robertnicoud, M.; Ronot, X. In Situ Hybridization in Living Cells: Detection of Rna Molecules. Exp. Cell Res. 1997, 231, 226-233.

(1511) Santangelo, P. J.; Nix, B.; Tsourkas, A.; Bao, G. Dual Fret Molecular Beacons for Mrna Detection in Living Cells. Nucleic Acids Res. 2004, 32, e57.

(1512) Santangelo, P. J.; Nitin, N.; Bao, G. Direct Visualization of Mrna Colocalization with Mitochondria in Living Cells Using Molecular Beacons. Journal of Biomedical Optics 2005, 10, 44025.

(1513) Santangelo, P.; Nitin, N.; Laconte, L.; Woolums, A.; Bao, G. Live-Cell Characterization and Analysis of a Clinical Isolate of Bovine Respiratory Syncytial Virus, Using Molecular Beacons. J. Virol. 2006, 80, 682-688.

(1514) Abe, H.; Kool, E. T. Flow Cytometric Detection of Specific Rnas in Native Human Cells with Quenched Autoligating Fret Probes. Proc. Natl. Acad. Sci. U. S. A. 2006, 103, 263-268.

(1515) Santangelo, P. J.; Bao, G. Dynamics of Filamentous Viral Rnps Prior to Egress. Nucleic Acids Res. 2007, 35, 3602-3611.

(1516) Rhee, W. J.; Santangelo, P. J.; Jo, H. J.; Bao, G. Target Accessibility and Signal Specificity in Live-Cell Detection of Bmp-4 Mrna Using Molecular Beacons. Nucleic Acids Res. 2008, 36, e30.

(1517) Arian, D.; Clo, E.; Gothelf, K. V.; Mokhir, A. A Nucleic Acid Dependent Chemical Photocatalysis in Live Human Cells. Chemistry-a European Journal 2010, 16, 288-295.

(1518) Schatter, B.; Walev, I.; Klein, J. Mitogenic Effects of Phospholipase D and Phosphatidic Acid in Transiently Permeabilized Astrocytes: Effects of Ethanol. J. Neurochem. 2003, 87, 95-100.

(1519) Furukawa, K.; Abe, H.; Hibino, K.; Sako, Y.; Tsuneda, S.; Ito, Y. Reduction-Triggered Fluorescent Amplification Probe for the Detection of Endogenous Rnas in Living Human Cells. Bioconjugate Chem. 2009, 20, 1026-1036. 
(1520) Lifland, A. W.; Zurla, C.; Santangelo, P. J. Single Molecule Sensitive Multivalent Polyethylene Glycol Probes for Rna Imaging. Bioconjugate Chem. 2010, 21, 483-488.

(1521) Liang, Y.; Zhang, Z. P.; Wei, H. P.; Hu, Q. X.; Deng, J. Y.; Guo, D. Y.; Cui, Z. Q.; Zhang, X. E. Aptamer Beacons for Visualization of Endogenous Protein Hiv-1 Reverse Transcriptase in Living Cells. Biosens. Bioelectron. 2011, $28,270-276$.

(1522) Rajapakse, H. E.; Miller, L. W. Time-Resolved Luminescence Resonance Energy Transfer Imaging of Protein-Protein Interactions in Living Cells. Methods in Enzymology: Imaging and Spectroscopic Analysis of Living Cells 2012, 505, 329-345.

(1523) Levy, R.; Shaheen, U.; Cesbron, Y.; See, V. Gold Nanoparticles Delivery in Mammalian Live Cells: A Critical Review. Nano Rev 2010, 1, 4889.

(1524) Provoda, C. J.; Lee, K. D. Bacterial Pore-Forming Hemolysins and Their Use in the Cytosolic Delivery of Macromolecules. Adv. Drug Del. Rev. 2000, 41, 209-221.

(1525) Provoda, C. J.; Stier, E. M.; Lee, K. D. Tumor Cell Killing Enabled by Listeriolysin O-Liposome-Mediated Delivery of the Protein Toxin Gelonin. J. Biol. Chem. 2003, 278, 35102-35108.

(1526) Murakami, M.; Kano, F.; Murata, M. Llo-Mediated Cell Resealing System for Analyzing Intracellular Activity of MembraneImpermeable Biopharmaceuticals of Mid-Sized Molecular Weight. Sci. Rep. 2018, 8, 1946.

(1527) Thiery, J.; Lieberman, J. Perforin: A Key Pore-Forming Protein for Immune Control of Viruses and Cancer. Subcell. Biochem. 2014, 80, 197-220.

(1528) Thiery, J.; Keefe, D.; Boulant, S.; Boucrot, E.; Walch, M.; Martinvalet, D.; Goping, I. S.; Bleackley, R. C.; Kirchhausen, T.; Lieberman, J. Perforin Pores in the Endosomal Membrane Trigger the Release of Endocytosed Granzyme B into the Cytosol of Target Cells. Nat. Immunol. 2011, 12, 770-777.

(1529) Luisoni, S.; Suomalainen, M.; Boucke, K.; Tanner, L. B.; Wenk, M. R.; Guan, X. L.; Grzybek, M.; Coskun, U.; Greber, U. F. CoOption of Membrane Wounding Enables Virus Penetration into Cells. Cell Host Microbe 2015, 18, 75-85.

(1530) Rabideau, A. E.; Liao, X. L.; Akcay, G.; Pentelute, B. L. Translocation of Non-Canonical Polypeptides into Cells Using Protective Antigen. Sci. Rep. 2015, 5, 11944.

(1531) Dingjan, I.; Verboogen, D. R.; Paardekooper, L. M.; Revelo, N. H.; Sittig, S. P.; Visser, L. J.; Mollard, G. F.; Henriet, S. S.; Figdor, C. G.; Ter Beest, M.et al. Lipid Peroxidation Causes Endosomal Antigen Release for Cross-Presentation. Sci. Rep. 2016, 6, 22064.

(1532) Jurkiewicz, P.; Olzynska, A.; Cwiklik, L.; Conte, E.; Jungwirth, P.; Megli, F. M.; Hof, M. Biophysics of Lipid Bilayers Containing Oxidatively Modified Phospholipids: Insights from Fluorescence and Epr Experiments and from Md Simulations. Biochimica Et Biophysica Acta-Biomembranes 2012, 1818, 2388-2402.

(1533) Itri, R.; Junqueira, H. C.; Mertins, O.; Baptista, M. S. Membrane Changes under Oxidative Stress: The Impact of Oxidized Lipids. Biophysical Reviews 2014, 6, 47-61.

(1534) Schomaker, M.; Heinemann, D.; Kalies, S.; Willenbrock, S.; Wagner, S.; Nolte, I.; Ripken, T.; Escobar, H. M.; Meyer, H.; Heisterkamp, A. Characterization of Nanoparticle Mediated Laser Transfection by Femtosecond Laser Pulses for Applications in Molecular Medicine. Journal of Nanobiotechnology 2015, 13, 1. 
(1535) Gomperts, B. D. Involvement of Guanine Nucleotide-Binding Protein in the Gating of Ca-2+ by Receptors. Nature 1983, 306, 64-66.

(1536) Gomperts, B. D.; Fernandez, J. M. Techniques for Membrane Permeabilization. Trends Biochem. Sci $1985,10,414-417$.

(1537) Steinberg, T. H.; Newman, A. S.; Swanson, J. A.; Silverstein, S. C. Atp4- Permeabilizes the Plasma-Membrane of Mouse Macrophages to Fluorescent Dyes. J. Biol. Chem. 1987, 262, 8884-8888.

(1538) Elliott, G. D.; Liu, X. H.; Cusick, J. L.; Menze, M.; Vincent, J.; Witt, T.; Hand, S.; Toner, M. Trehalose Uptake through P2x(7) Purinergic Channels Provides Dehydration Protection. Cryobiology 2006, 52, 114-127.

(1539) Reuss, R.; Ludwig, J.; Shirakashi, R.; Ehrhart, F.; Zimmermann, H.; Schneider, S.; Weber, M. M.; Zimmermann, U.; Schneider, H.; Sukhorukov, V. L. Intracellular Delivery of Carbohydrates into Mammalian Cells through Swelling-Activated Pathways. J. Membr. Biol. 2004, 200, 67-81.

(1540) Sukhorukov, V. L.; Imes, D.; Woellhaf, M. W.; Andronic, J.; Kiesel, M.; Shirakashi, R.; Zimmermann, U.; Zimmermann, H. Pore Size of Swelling-Activated Channels for Organic Osmolytes in Jurkat Lymphocytes, Probed by Differential Polymer Exclusion. Biochimica Et Biophysica Acta-Biomembranes 2009, 1788, 1841-1850.

(1541) Andronic, J.; Shirakashi, R.; Pickel, S. U.; Westerling, K. M.; Klein, T.; Holm, T.; Sauer, M.; Sukhorukov, V. L. Hypotonic Activation of the Myo-Inositol Transporter Slc5a3 in Hek293 Cells Probed by Cell Volumetry, Confocal and Super-Resolution Microscopy. PLoS One 2015, 10, e0119990.

(1542) Russo, M. J.; Bayley, H.; Toner, M. Reversible Permeabilization of Plasma Membranes with an Engineered Switchable Pore. Nat. Biotechnol. 1997, 15, 278-282.

(1543) Bayley, H.; Jayasinghe, L. Functional Engineered Channels and Pores - (Review). Mol. Membr. Biol. 2004, 21, 209-220.

(1544) Bonardi, F.; Nouwen, N.; Feringa, B. L.; Driessen, A. J. M. Protein Conducting Channels-Mechanisms, Structures and Applications. Mol. Biosyst. 2012, 8, 709-719.

(1545) Kocer, A.; Walko, M.; Meijberg, W.; Feringa, B. L. A Light-Actuated Nanovalve Derived from a Channel Protein. Science 2005, 309, 755-758.

(1546) Boyden, E. S.; Zhang, F.; Bamberg, E.; Nagel, G.; Deisseroth, K. Millisecond-Timescale, Genetically Targeted Optical Control of Neural Activity. Nat. Neurosci. 2005, 8, 1263-1268.

(1547) Doerner, J. F.; Febvay, S.; Clapham, D. E. Controlled Delivery of Bioactive Molecules into Live Cells Using the Bacterial Mechanosensitive Channel Mscl. Nat Commun 2012, 3, 990.

(1548) Langecker, M.; Arnaut, V.; Martin, T. G.; List, J.; Renner, S.; Mayer, M.; Dietz, H.; Simmel, F. C. Synthetic Lipid Membrane Channels Formed by Designed DNA Nanostructures. Science 2012, 338, 932-936.

(1549) Geng, J.; Kim, K.; Zhang, J.; Escalada, A.; Tunuguntla, R.; Comolli, L. R.; Allen, F. I.; Shnyrova, A. V.; Cho, K. R.; Munoz, D.et al. Stochastic Transport through Carbon Nanotubes in Lipid Bilayers and Live Cell Membranes. Nature 2014, 514, $612-615$.

(1550) Garcia-Lopez, V.; Chen, F.; Nilewski, L. G.; Duret, G.; Aliyan, A.; Kolomeisky, A. B.; Robinson, J. T.; Wang, G.; Pal, R.; Tour, J. M. Molecular Machines Open Cell Membranes. Nature 2017, 548, 567-572. 
(1551) Evans, E.; Heinrich, V.; Rawicz, W. Using Dynamic Tension Spectroscopy to Explore Destabilization of Membranes by Antimicrobial Peptides. Biophys. J. 2004, 86, 330A.

(1552) Eroglu, A.; Toner, M.; Toth, T. L. Beneficial Effect of Microinjected Trehalose on the Cryosurvival of Human Oocytes. Fertil. Steril. 2002, 77, 152-158.

(1553) Kreis, T. E.; Birchmeier, W. Microinjection of Fluorescently Labeled Proteins into Living Cells with Emphasis on Cytoskeletal Proteins. Int. Rev. Cytol. 1982, 75, 209-214.

(1554) Klymkowsky, M. W. Intermediate Filaments in 3t3 Cells Collapse after Intracellular Injection of a Monoclonal Anti-Intermediate Filament Antibody. Nature 1981, 291, 249-251.

(1555) Wehland, J.; Osborn, M.; Weber, K. Phalloidin-Induced Actin Polymerization in the Cytoplasm of Cultured Cells Interferes with Cell Locomotion and Growth. Proc. Natl. Acad. Sci. U. S. A. 1977, 74, 5613-5617.

(1556) Paine, P. L.; Moore, L. C.; Horowitz, S. B. Nuclear-Envelope Permeability. Nature 1975, 254, $109-114$.

(1557) Kreis, T. E.; Geiger, B.; Schmid, E.; Jorcano, J. L.; Franke, W. W. De Novo Synthesis and Specific Assembly of Keratin Filaments in Nonepithelial Cells after Microinjection of Mrna for Epidermal Keratin. Cell 1983, 32, 1125-1137.

(1558) Jaenisch, R.; Mintz, B. Simian Virus 40 DNA Sequences in DNA of Healthy Adult Mice Derived from Preimplantation Blastocysts Injected with Viral DNA. Proc. Natl. Acad. Sci. U. S. A. 1974, 71, 1250-1254.

(1559) Dubertret, B.; Skourides, P.; Norris, D. J.; Noireaux, V.; Brivanlou, A. H.; Libchaber, A. In Vivo Imaging of Quantum Dots Encapsulated in Phospholipid Micelles. Science 2002, 298, 1759-1762.

(1560) Rieger, S.; Kulkarni, R. P.; Darcy, D.; Fraser, S. E.; Koster, R. W. Quantum Dots Are Powerful Multipurpose Vital Labeling Agents in Zebrafish Embryos. Dev. Dyn. 2005, 234, 670-681.

(1561) Koike, S.; Jahn, R. Probing and Manipulating Intracellular Membrane Traffic by Microinjection of Artificial Vesicles. Proceedings of the National Academy of Sciences 2017, 114, E9883-E9892.

(1562) Adams, R. J.; Bray, D. Rapid Transport of Foreign Particles Microinjected into Crab Axons. Nature 1983, 303, 718-720.

(1563) Beckerle, M. C. Microinjected Fluorescent Polystyrene Beads Exhibit Saltatory Motion in Tissue Culture Cells. J. Cell Biol. 1984, 98, 2126-2132.

(1564) O'brien, J. A.; Lummis, S. C. R. Diolistics: Incorporating Fluorescent Dyes into Biological Samples Using a Gene Gun. Trends Biotechnol. 2007, 25, 530-534.

(1565) Zhang, Y.; Liang, Z.; Zong, Y.; Wang, Y. P.; Liu, J. X.; Chen, K. L.; Qiu, J. L.; Gao, C. X. Efficient and Transgene-Free Genome Editing in Wheat through Transient Expression of Crispr/Cas9 DNA or Rna. Nat Commun 2016, 7, 12617.

(1566) Webster, A.; Coupland, P.; Houghton, F. D.; Leese, H. J.; Aylott, J. W. The Delivery of Pebble Nanosensors to Measure the Intracellular Environment. Biochem. Soc. Trans. 2007, 35, 538-543.

(1567) Sessions, J. W.; Skousen, C. S.; Price, K. D.; Hanks, B. W.; Hope, S.; Alder, J. K.; Jensen, B. D. Crispr-Cas9 Directed Knockout of a Constitutively Expressed Gene Using Lance Array Nanoinjection. Springerplus 2016, 5, 1521.

(1568) Stein, D. A.; Skilling, D. E.; Iversen, P. L.; Smith, A. W. Inhibition of Vesivirus Infections in Mammalian Tissue Culture with Antisense Morpholino Oligomers. Antisense Nucleic Acid Drug Dev. 2001, 11, 317-325. 
(1569) Amantana, A.; London, C. A.; Iversen, P. L.; Devi, G. R. X-Linked Inhibitor of Apoptosis Protein Inhibition Induces Apoptosis and Enhances Chemotherapy Sensitivity in Human Prostate Cancer Cells. Mol. Cancer Ther. 2004, 3, 699-707.

(1570) Neuman, B. W.; Stein, D. A.; Kroeker, A. D.; Paulino, A. D.; Moulton, H. M.; Iversen, P. L.; Buchmeier, M. J. Antisense Morpholino-Oligomers Directed against the 5 ' End of the Genome Inhibit Coronavirus Proliferation and Growth. J. Virol. 2004, $78,5891-5899$.

(1571) Dirks, R. W.; Molenaar, C.; Tanke, H. J. Methods for Visualizing Rna Processing and Transport Pathways in Living Cells. Histochem. Cell Biol. 2001, 115, 3-11.

(1572) Seksek, O.; Biwersi, J.; Verkman, A. S. Translational Diffusion of Macromolecule-Sized Solutes in Cytoplasm and Nucleus. J. Cell Biol. 1997, 138, 131-142.

(1573) Geddes, D. M.; Cargill, R. S.; Laplaca, M. C. Mechanical Stretch to Neurons Results in a Strain Rate and MagnitudeDependent Increase in Plasma Membrane Permeability. J. Neurotrauma 2003, 20, 1039-1049.

(1574) Zhang, Z.; Wang, Y.; Zhang, H.; Tang, Z.; Liu, W.; Lu, Y.; Wang, Z.; Yang, H.; Pang, W.; Zhang, H.et al. Hypersonic Poration: A New Versatile Cell Poration Method to Enhance Cellular Uptake Using a Piezoelectric Nano-Electromechanical Device. Small 2017, 13, 1602962.

(1575) Frairia, R.; Catalano, M. G.; Fortunati, N.; Fazzari, A.; Raineri, M.; Berta, L. High Energy Shock Waves (Hesw) Enhance Paclitaxel Cytotoxicity in Mcf-7 Cells. Breast Cancer Res. Treat. 2003, 81, 11-19.

(1576) Tschoep, K.; Hartmann, G.; Jox, R.; Thompson, S.; Eigler, A.; Krug, A.; Erhardt, S.; Adams, G.; Endres, S.; Delius, M. Shock Waves: A Novel Method for Cytoplasmic Delivery of Antisense Oligonucleotides. J. Mol. Med. 2001, 79, 306-313.

(1577) Terakawa, M.; Sato, S.; Ashida, H.; Aizawa, K.; Uenoyama, M.; Masaki, Y.; Obara, M. In Vitro Gene Transfer to Mammalian Cells by the Use of Laser-Induced Stress Waves: Effects of Stress Wave Parameters, Ambient Temperature, and Cell Type. Journal of Biomedical Optics 2006, 11, 014026.

(1578) Magana-Ortiz, D.; Coconi-Linares, N.; Ortiz-Vazquez, E.; Fernandez, F.; Loske, A. M.; Gomez-Lim, M. A. A Novel and Highly Efficient Method for Genetic Transformation of Fungi Employing Shock Waves. Fungal Genet. Biol. 2013, 56, 9-16.

(1579) Loske, A. M.; Fernandez, F.; Magana-Ortiz, D.; Coconi-Linares, N.; Ortiz-Vazquez, E.; Gomez-Lim, M. A. Tandem Shock Waves to Enhance Genetic Transformation of Aspergillus Niger. Ultrasonics 2014, 54, 1656-1662.

(1580) Dijkink, R.; Le Gac, S.; Nijhuis, E.; Van Den Berg, A.; Vermes, I.; Poot, A.; Ohl, C. D. Controlled Cavitation-Cell Interaction: Trans-Membrane Transport and Viability Studies. Phys. Med. Biol. 2008, 53, 375-390.

(1581) Chakravarty, P.; Lane, C. D.; Orlando, T. M.; Prausnitz, M. R. Parameters Affecting Intracellular Delivery of Molecules Using Laser-Activated Carbon Nanoparticles. Nanomed. Nanotechnol. Biol. Med. 2016, 12, 1003-1011.

(1582) Hellman, A. N.; Rau, K. R.; Yoon, H. H.; Venugopalan, V. Biophysical Response to Pulsed Laser Microbeam-Induced Cell Lysis and Molecular Delivery. Journal of Biophotonics 2008, 1, 24-35.

(1583) Compton, J. L.; Hellman, A. N.; Venugopalan, V. Hydrodynamic Determinants of Cell Necrosis and Molecular Delivery Produced by Pulsed Laser Microbeam Irradiation of Adherent Cells. Biophys. J. 2013, 105, 2221-2231. 
(1584) Xiong, R.; Drullion, C.; Verstraelen, P.; Demeester, J.; Skirtach, A. G.; Abbadie, C.; De Vos, W. H.; De Smedt, S. C.; Braeckmans, K. Fast Spatial-Selective Delivery into Live Cells. J. Controlled Release 2017, 266.

(1585) Arita, Y.; Ploschner, M.; Antkowiak, M.; Gunn-Moore, F.; Dholakia, K. Laser-Induced Breakdown of an Optically Trapped Gold Nanoparticle for Single Cell Transfection. Opt. Lett. 2013, 38, 3402-3405.

(1586) Li, M.; Lohmuller, T.; Feldmann, J. Optical Injection of Gold Nanoparticles into Living Cells. Nano Lett. 2015, 15, 770-775.

(1587) Gan, B. S.; Krump, E.; Shrode, L. D.; Grinstein, S. Loading Pyranine Via Purinergic Receptors or Hypotonic Stress for Measurement of Cytosolic Ph by Imaging. Am J Physiol-Cell Ph 1998, 275, C1158-C1166.

(1588) Pedrini, M. R. D.; Dupont, S.; Camara, A. D.; Beney, L.; Gervais, P. Osmoporation: A Simple Way to Internalize Hydrophilic Molecules into Yeast. Appl. Microbiol. Biotechnol. 2014, 98, 1271-1280.

(1589) Kobayashi, N.; Kuramoto, T.; Yamaoka, K.; Hashida, M.; Takakura, Y. Hepatic Uptake and Gene Expression Mechanisms Following Intravenous Administration of Plasmid DNA by Conventional and Hydrodynamics-Based Procedures. J. Pharmacol. Exp. Ther. 2001, 297, 853-860.

(1590) Bartlett, D. W.; Davis, M. E. Insights into the Kinetics of Sirna-Mediated Gene Silencing from Live-Cell and Live-Animal Bioluminescent Imaging. Nucleic Acids Res. 2006, 34, 322-333.

(1591) Mccaffrey, A. P.; Meuse, L.; Karimi, M.; Contag, C. H.; Kay, M. A. A Potent and Specific Morpholino Antisense Inhibitor of Hepatitis C Translation in Mice. Hepatology 2003, 38, 503-508.

(1592) Grunenfelder, J.; Miniati, D. N.; Murata, S.; Falk, V.; Hoyt, E. G.; Robbins, R. C. Up-Regulation of Bcl-2 through Hyperbaric Pressure Transfection of Tgf-Beta 1 Ameliorates Ischemia-Reperfusion Injury in Rat Cardiac Allografts. J. Heart Lung Transplant. 2002, 21, 244-250.

(1593) Lin, S. R.; Yang, H. C.; Kuo, Y. T.; Liu, C. J.; Yang, T. Y.; Sung, K. C.; Lin, Y. Y.; Wang, H. Y.; Wang, C. C.; Shen, Y. C.et al. The Crispr/Cas9 System Facilitates Clearance of the Intrahepatic Hbv Templates in Vivo. Mol Ther-Nucl Acids 2014,3 , e186.

(1594) Pons, T.; Lequeux, N.; Mahler, B.; Sasnouski, S.; Fragola, A.; Dubertret, B. Synthesis of near-Infrared-Emitting, Water-Soluble Cdtese/Cdzns Core/Shell Quantum Dots. Chem. Mater. 2009, 21, 1418-1424.

(1595) Wei, Z. W.; Zhao, D. Y.; Li, X. M.; Wu, M. X.; Wang, W.; Huang, H.; Wang, X. X.; Du, Q.; Liang, Z. C.; Li, Z. H. A Laminar Flow Electroporation System for Efficient DNA and Sirna Delivery. Anal. Chem. 2011, 83, 5881-5887.

(1596) Owczarczak, A. B.; Shuford, S. O.; Wood, S. T.; Deitch, S.; Dean, D. Creating Transient Cell Membrane Pores Using a Standard Inkjet Printer. J Vis Exp 2012, 61, 3681.

(1597) Bhattacharyya, K.; Mehta, S.; Viator, J. Optically Absorbing Nanoparticle Mediated Cell Membrane Permeabilization. Opt. Lett. 2012, 37, 4474-4476.

(1598) Heinemann, D.; Schomaker, M.; Kalies, S.; Schieck, M.; Carlson, R.; Escobar, H. M.; Ripken, T.; Meyer, H.; Heisterkamp, A. Gold Nanoparticle Mediated Laser Transfection for Efficient Sirna Mediated Gene Knock Down. PLoS One 2013,8, e58604.

(1599) Bukhari, M.; Deng, H.; Jones, N.; Towne, Z.; Woodworth, C. D.; Samways, D. S. K. Selective Permeabilization of Cervical Cancer Cells to an lonic DNA-Binding Cytotoxin by Activation of P2y Receptors. FEBS Lett. 2015, 589, $1498-1504$. 
(1600) Bukhari, M.; Burm, H.; Samways, D. S. K. Ion Channel-Mediated Uptake of Cationic Vital Dyes into Live Cells: A Potential Source of Error When Assessing Cell Viability. Cell Biol. Toxicol. 2016, 32, 363-371. 


\section{TOC FIGURE}

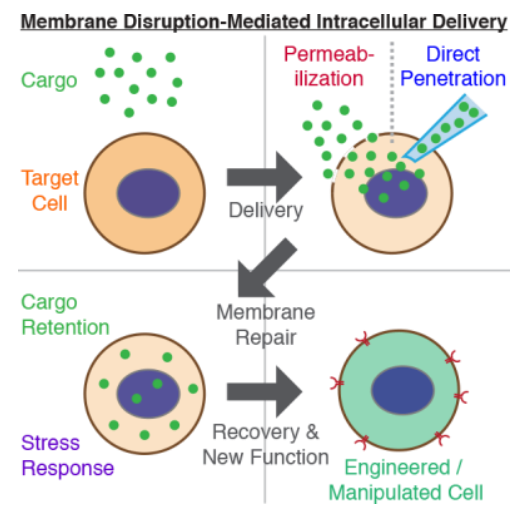

